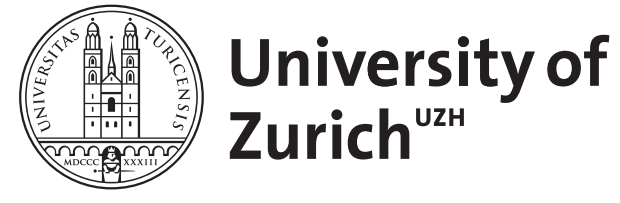

\title{
Les grands prêtres de Ptah de Memphis
}

\author{
Maystre, Charles
}

\begin{abstract}
Considéré dans son ensemble, le présent livre est une recherche sur les grands prêtres du dieu égyptien Ptah. Une première partie comprend l'étude du pontificat memphite, une seconde présente l'histoire des pontifes memphites, tandis qu'une dernière parties donne les sources. Pour chacune d'elles, le lecteur rencontre d'abord le texte hiéroglyphique dans la grande majorité, puis la traduction en français et un commentaire. Les hiéroglyphes ont été dessinés par l'auteur.
\end{abstract}

Posted at the Zurich Open Repository and Archive, University of Zurich ZORA URL: https://doi.org/10.5167/uzh-156843

Monograph

Published Version

Originally published at:

Maystre, Charles (1992). Les grands prêtres de Ptah de Memphis. Freiburg, Göttingen: Universitätsverlag, Vandenhoeck Ruprecht. 
Charles Maystre

Les grands prêtres de Ptah de Memphis 


\section{ORBIS BIBLICUS ET ORIENTALIS}

Im Auftrag des Biblischen Instituts

der Universität Freiburg Schweiz, des Seminars für Biblische Zeitgeschichte der Universität Münster i. W.

und der Schweizerischen Gesellschaft

für orientalische Altertumswissenschaft

herausgegeben von

Othmar Keel

unter Mitarbeit von Erich Zenger und Albert de Pury

Notice biographique

Charles Maystre est né à Genève le 30 janvier 1907. Baccalauréat de I'Université de Genève 1925. Licence ès lettres 1930. Diplôme de l'Ecole pratique des hautes études, $5^{e}$ section (des sciences religieuses) Paris 1934. Attaché étranger à l'Institut français d'archéologie orientale, Le Caire 1933 1940 et 1945-1947. Doctorat ès lettres d'Etat, Paris Sorbonne 1948.

Chargé de cours d'égyptologie, Faculté des lettres, Genève 1948-1950. Ibidem, professeur extraordinaire d'égyptologie 1950-1962. Attaché à la collection égyptienne du Musée d'art et d'histoire, Genève 1952-1978. Créateur et administrateur du Centre d'études orientales, Genève 1956-1977. Professeur ordinaire d'égyptologie à la Faculté des lettres, Genève 19621977. Directeur de la Mission archéologique Henry M. Blackmer, New York et du Centre d'études orientales de I'Université de Genève dès 1965. Professeur honoraire dès 1977.

Principales publications

La tombe de Nebenmât IFAO, Le Caire 1936 - Les déclarations d'innocence IFAO, Le Caire 1937 - Akasha I, Genève 1981, Tabo I, Genève 1986. 


\section{Orbis Biblicus et Orientalis 113}

Charles Maystre

\section{Les grands prêtres de Ptah \\ de Memphis}

Universitätsverlag Freiburg Schweiz Vandenhoeck \& Ruprecht Göttingen 
Die Deutsche Bibliothek - CIP-Einheitsaufnahme

\section{Maystre, Charles:}

Les grands prêtres de Ptah de Memphis/Charles Maystre. - Freiburg, Schweiz: Univ.-Verl.; Göttingen: Vandenhoeck und Ruprecht, 1992

(Orbis biblicus et orientalis; 113)

ISBN 3-525-53747-6 (Vandenhoeck und Ruprecht)

ISBN 3-7278-0794-6 (Univ.-Verl.)

NE: GT

Die Druckvorlagen wurden vom Herausgeber

als reprofertige Dokumente zur Verfügung gestellt

(C) 1992 by Universitätsverlag Freiburg Schweiz

Vandenhoeck \& Ruprecht Göttingen

Paulusdruckerei Freiburg Schweiz

ISBN 3-7278-0794-6 (Universitätsverlag)

ISBN 3-525-53747-6 (Vandenhoeck \& Ruprecht)

Digitalisat erstellt durch Florina Tischhauser,

Religionswissenschaftliches Seminar, Universität Zürich 
A la mémoire de

G. LEFEBVRE 



\title{
TABLE DES MATIERES
}

Pages

AVANT-PROPOS

xi

PREFACE

xiii

\author{
PREMIERE PARTIE
}

\section{LE PONTIFICAT MEMPHITE}

CHAPITRE I: Le grand prêtre de Ptah

$\S \S 1$. Introduction. 2. Le titre hiéroglyphique du grand prêtre de Ptah. 3. Our kherep hemout. 4. Traduction de our kherep hemout. 5. Sem. 6. Le recrutement des grands prêtres. 7. L'accession au pontificat. 8. La fin de la carrière du grand prêtre. 9. Costumes et insignes des grands prêtres. 10. Revenus et personnel des grands prêtres.

CHAPITRE II: Les fonctions du grand prêtre

$\S \S 11$. Fonctions civiles. 12. Service de Ptah. 13. Service de Sokar. 14. Service du roi. 15. Service de Rê. 16. Service d'Apis. 17. Service d'autres divinités.

CHAPITRE III: La dualité du pontificat de la IVème à la VIème dynastie

$\S \S 18$. Les mentions des deux grands prêtres. 19. Les rapports des deux grands prêtres. 20. La raison de l'existence simultanée 
viii

simultanée de deux grands prêtres. 21. Hypothèse sur l'origine des grands des chefs des artisans.

CHAPITRE IV: La réforme du pontificat sous la VIème dynastie

§ 22. Le pontificat antérieurement à Pépi Ier. 23. La stèle de Sabou-Tjéti. 24. La carrière de Sabou-Tjéti. 25. La réforme, oeuvre du roi. 26. L'unité probable du pontificat depuis Pépi Ier.

CHAPITRE V: Les modifications du pontificat au Nouvel Empire

$\$ \S 27$. Le pontificat au cours des deux périodes intermédiaires. 28. Le pontificat memphite et le pontificat thébain. 29. Le pontificat pendant le règne d'Akhenaton. 30. L'évolution du pontificat au début de la XIXème dynastie. 31. L'importance respective des deux titres du grand prêtre. 32 . Ramsès II et les grands prêtres de Memphis.

\section{SECONDE PARTIE}

\section{LES GRAND PRETRES MEMPHITES}

§§ 33. La généalogie d'Ankhef-ny-sekhmet. 34. La généalogie d'Achaout-akh. 35. La généalogie de Petobastis.

CHAPITRE VII: Les grands prêtres de l'Ancien Empire

$\S$ 36. Ptahchepses I. 37. Setjou. 38. Ptahchepses II, Rênefer et Neferefrê-ankh. 39. Ptahchepses III et Sabou-kem. 40. SabouIbebi. 41. Ptahchepses IV. 42. Sabou-Tjéti et Ptahchepses-Impy. 43. Appendice au Chapitre VII. 
$\S \S$ 44. Hapidjefa. 45. Senousert-ankh. 46. Sehetepibrê-ankhnedjem, Nebpou et Sehetepibrê-cheri. 47. Appendice au Chapitre VIII.

CHAPITRE IX: Les grands prêtres du Nouvel Empire

§§ 48. Les Ptahmès. 49. Ptahmès I. 50. Ptahmès II. 51. Le prince Thoutmôsis. 52. Ptahmès III. 53. Méryptah. 54. Ptahemhat-Ty. 55. Nebmehyt, Khamouas I, Hori I et II. 56. Sennefer, Dedia et Pahemneter. 57. Rêhetep et Hy. 58. Le prince Khamouas. 59. Neferrenpet. 60. Ptahmès IV et Iyry. 61. Appendice au Chapitre IX.

CHAPITRE X: Les grands prêtres de la Basse Epoque

$\S \S$ 62. Paoupaou, Horsaïset I et Ankhef-ny-sekhmet. 63. Chedsounefertoum. 64. Chechanq et Osorkon. 65. Le prince Chechanq. 66. Merenptah et Takelot. 67. Padiiset. 68. Peftjaouaouibastet et Horsaïset II. 69. Bakenrenef. 70. Hekairâa, Neferibrêmen et Nekaomen. 71. Ahmèsmen. 72. Appendice au Chapitre X.

CHAPITRE XI: Les grands prêtres de l'époque ptolémaïque

$\S \S 73 . \quad$ Nesqed-Petobastis. 74. Anemher. 75. Teos. 76. Harmachis. 77. Psenptaïs I. 78. Petobastis et Psenptaïs. 79. Petobastis II. 80. Psenptaïs II. 81. Imouthès-Petobastis. 82. Appendice au Chapitre XI.

CHAPITRE XII: Un grand prêtre de l'époque romaine § 83. Psenamounis. 


\section{TROISIEME PARTIE}

\section{LES INSCRIPTIONS}

$\begin{array}{ll}\text { INTRODUCTION } & 221\end{array}$

CHAPITRE XIII: Les inscriptions de l'Ancien Empire (1 - 29) 223

CHAPITRE XIV: Les inscriptions du Moyen Empire (30 - 35) 251

CHAPITRE XV: Les inscriptions du Nouvel Empire (36 - 162b) 257

CHAPITRE XVI: Les inscriptions de la Basse Epoque

$(163-189 \mathrm{e})$

CHAPITRE XVII: Les inscriptions de l'Epoque Ptolémaïque (190 - 206)

APPENDICE: APERCU DU PERSONNEL DE PTAH

ADDENDUM

INDEX DES NOMS PROPRES EGYPTIENS DE PERSONNES 449

LISTE ALPHABETIQUE DES NOMS DE "GRANDS DES

CHEFS DES ARTISANS"

EXPLICATIONS DES PLANCHES I ET II 


\section{AVANT-PROPOS}

Le présent travail sur les grands prêtres de Ptah de Memphis est la reproduction des deux thèses - principale et secondaire - que j'ai présentées et soutenues en Sorbonne au mois de février 1948, et qui m'ont valu la mention summa cum laude.

Sans modifier l'ouvrage, j'ai jugé bon de donner, autant que faire se peut, la référence bibliographique la plus récente pour chacun des monuments des grands prêtres de Ptah publiés ou republiés après 1947 ainsi que d'ajouter, à la fin de la troisième partie, un addendum à notre documentation.

Mademoiselle Frédérique von Känel a bien voulu compléter les références que j'avais moi-même rassemblées au fil des années et mettre l'ouvrage sur ordinateur. Je l'en remercie vivement.

Genève, juin 1991 



\section{P R E F A C E}

Les dieux de l'Egypte ancienne ont suscité beaucoup de travaux dans lesquels des savants ont abordé, et souvent résolu, de nombreux problèmes posés par l'examen des croyances religieuses de ce pays.

On ne peut en dire autant des sacerdoces égyptiens et de leurs dirigeants. A part les grands prêtres d'Amon, si bien étudiés par M. Gustave Lefebvre, les chefs des autres clergés n'ont pas encore été l'objet de monographies.

Cette lacune demande à être comblée d'autant que la seule différence entre les titres portés par des grands prêtres qui existaient déjà sous l'Ancien Empire, tels ceux de Rê, de Thot ou de Ptah, et l'appellation plus récente des grands prêtres d'Amon laisse déjà entrevoir que la dignité du chef d'un des anciens clergés n'était pas identique à celle des "premiers prophètes d'Amon".

Mais on ne saurait, à notre avis, entreprendre de comparer les grands prêtres de divers dieux avant de les avoir examinés séparément et à fond.

C'est pourquoi nous ne nous sommes occupés que des grands prêtres de Ptah de Memphis, dont M. Georges Posener nous avait signalé l'intérêt possible, il y a plusieurs années. Nous les avons choisis parce que nous pouvions les suivre assez régulièrement depuis le temps des grandes pyramides de Gizeh jusqu'aux premières années de la domination romaine; ainsi le pontificat memphite apparaît comme une des rares institutions religieuses dont on puisse prendre une vue d'ensemble embrassant toutes les grandes périodes historiques de l'antiquité égyptienne. 
xiv

Notre manuscrit était déjà rédigé lorsque nous avons eu connaissance de l'étude que l'égyptologue suédoise Maj. Sandman Holmberg a consacrée récemment au dieu Ptah. Mme Sandman Holmberg a été amenée à traiter quelques-unes des questions soulevées par les grands prêtres de Memphis. Elle s'est alors basée sur une documentation réduite, ce dont on ne peut lui tenir rigueur puisqu'il s'agissait de problèmes secondaires dans ses recherches. Mais cette circonstance ne lui a pas permis d'entrevoir les modifications apportées par le temps à la dignité de grand prêtre et lui a suggéré des conclusions auxquelles nous ne souscrivons pas sans réserve.

La documentation à laquelle nous avons puisé est réunie dans la Troisième Partie "Inscriptions des grands prêtres memphites". Pour simplifier les renvois à ces textes, nous nous servons d'un chiffre en caractère gras pour indiquer le numéro de l'inscription : par exemple, (26,5-11) signifie: cf. inscription 26, lignes 5 à 11.

Comme nous n'avons pas pu utiliser les fiches du dictionnaire de Berlin pour notre travail, plusieurs sources nous auraient échappé si nos camarades de l'Institut Français d'Archéologie Orientale ne nous les avaient pas signalées. Qu'ils veuillent, ainsi que tous ceux qui nous ont aidé d'autres manières, trouver ici l'expression de notre profonde gratitude!

Genève, février 1948 


\section{PREMIERE PARTIE}

L E P O N T I F I CA T M E M P H I T E 



\section{H A P I T R E I}

\section{LE GRAND PRETRE DE PTAH}

\section{§ 1. INTRODUCTION}

Les documents dont nous disposons sont assez abondants pour que nous puissions définir la dignité du grand prêtre de Ptah de Memphis et l'étudier aux principales époques de l'antiquité égyptienne, depuis l'Ancien Empire jusqu'au début de la domination romaine. C'est ce que nous faisons dans la première partie de notre ouvrage.

D'autre part, les grands prêtres dont nous avons connaissance sont assez nombreux pour nous permettre de dresser leur catalogue historique et d'esquisser la biographie de plusieurs d'entre eux. Nous traitons ce sujet dans la seconde partie de ce volume.

\section{§ 2. LE TITRE HIEROGLYPHIQUE DU GRAND PRETRE DE PTAH}

Avant d'employer les termes "grand prêtre de Ptah de Memphis" que nous abrégeons souvent en "grand prêtre de Ptah", "grand prêtre memphite" ou "de Memphis", ou même en "grand prêtre", nous devons déterminer l'expression hiéroglyphique correspondante.

On admet communément ${ }^{1}$ que le $\frac{7}{9} \Phi$ our kherep hemout est le grand prêtre de Ptah de Memphis. Il nous paraît cependant utile d'interroger les monuments à ce propos.

${ }^{1}$ M. STOLK, Ptah, 1911, p. 37; A.M. BLACKMAN, Priest, Priesthood (Egyptian), dans HASTINGS, Encyclopaedia of Religion and Ethics, Vol. 10 (1918), p. 295, 1ère col.; ERMAN-GRAPOW, Wörterbuch der Ägyptischen Sprache, Vol. I (1926), p. 329, réf. 12. 
Seules, des époques relativement récentes nous fournissent des documents où nous sommes certains de lire l'appellation de grand prêtre memphite. Au temple d'Edfou, la liste géographique lui donne les titres de our kherep hemout et sem, et le papyrus Hood, dont on sait qu'il est "postérieur à la XXIème dynastie et sans doute assez proche de la XXVIème"1, et son duplicata, le papyrus Golénischeff, mentionnent, après les grands prêtres d'Amon et de Rê, le "Our kherep hemout de Celui qui est au sud de son Mur (épithète de Ptah) et sem de Celui dont le visage est beau (autre épithète de Ptah)" (cf. nos Inscriptions, $N^{\circ} \mathbf{2 0 5}$, 184, et 185). Au cours du premier millénaire avant J.-C., l'appellation complète du grand prêtre de Ptah semble comprendre à la fois our kherep hemout et sem.

\section{§ 3. OUR KHEREP HEMOUT}

Si nous nous tournons vers les plus anciennes mentions des titres du grand prêtre, nous rencontrons le premier à plusieurs reprises sous l'Ancien Empire, plus exactement de la IVème à la VIème dynastie, soit dans des textes qui jettent quelque jour sur le rôle des our kherep hemout soit dans des titulatures individuelles. Nos constatations sur le rôle des our kherep hemout sont pareilles à celles de Maj. SANDMAN HOLMBERG, The God Ptah, Lund et Copenhague, 1946, p. 52-53 (abrégé plus loin en "SANDMAN, Ptah").

Trois sources qui sont les inscriptions du tombeau que le roi Mycérinus offrit à Debehen, la stèle de Ny-ankh-sekhmet, médecin du roi Sahourê (1 et 2) et la stèle de Sabou-Tjéti $(\mathbf{2 6 , 7})$, enseignent qu'il y a eu deux ${ }^{2}$ our kherep hemout à la fin de la IVème dynastie, au début de la Vème et dans la première moitié de la VIème dynastie. Leur rayon d'action débordait Saqqara et s'étendait jusqu'à Gizeh. Leur tâche consistait, au

1 G. LEFEBVRE, Histoire des grands prêtres d'Amon de Karnak jusqu'à la XXIème dynastie, 1929, p. 13. D'après A.H. GARDINER, Ancient Egyptian Onomastica, Oxford, 1947, p. 24, les papyri Hood et Golénischeff sont des copies, faites à la XXIème ou à la XXIIème dynastie, de l'onomasticon composé par Amenemope, et on peut supposer que celui-ci a vécu à la fin de la XXIème dynastie.

2 Dans la seule documentation sur les wr hrp ḥmwt, il y a la mention de "2 trésoriers du dieu" $(\mathbf{2 , 6 )}$ et celle de "2 ouabet" $(\mathbf{2 6 , 2 )}$. 
moins en partie, à diriger le travail effectué par les ouvriers-sculpteurs sur des blocs de calcaire destinés à des monuments funéraires.

Rien ne nous autorise, dans les textes de Debehen et de Ny-ankhsekhmet, à voir dans les deux our kherep hemout des personnages qui ont eu un rapport quelconque avec le culte de Ptah à cette époque, et nous serions réduits à des conjectures sur ce sujet si les fouilles de Mariette à Saqqara n'avaient pas révélé quelques our kherep hemout de l'Ancien Empire dont les titres et même, parfois, la biographie, montrent l'appartenance au clergé de Ptah.

Sur les onze our kherep hemout de cette époque, huit se déclarent prophètes de Ptah, indiquant par là qu'ils ont appartenu à la plus haute classe de ses prêtres. Les trois autres, Neferef-rê-ankh, Sabou-Tjéti et Ptahchepses-Impy paraissent privés de cette qualité. Mais le premier ne nous est connu que par une courte mention sur un monument qui ne lui appartient pas (8); le second affirme clairement ses relations avec le temple de Ptah dans sa biographie $(\mathbf{2 6 , 6})$ et le troisième n'est sauvé de l'oubli que par les courtes titulatures de sa statue conservée au Louvre (29). Il est donc possible qu'ils aient aussi été "prophètes de Ptah".

Les tombeaux découverts par Mariette révèlent aussi le prix que les our kherep hemout attachaient à la possession de leur titre. Chaque fois que Ptahchepses I énumère quelques-unes de ses qualités, que ce soit dans les lignes horizontales ou les colonnes verticales de sa stèle (4), ou encore sur son linteau (3), il ne manque pas de s'appeler our kherep hemout; sur les huit fois où il le fait, il place quatre fois ce titre en tête des autres, dans les lignes horizontales et la colonne médiane de sa stèle, ainsi que sur son linteau. D'autre part, il ne mentionne aucun de ses nombreux autres titres aussi souvent; les plus fréquents ne se rencontrent, en effet, que quatre fois au maximum. Ptahchepses I estimait donc que our kherep hemout était sa principale qualité. Un examen des monuments laissés par les autres our kherep hemout de l'Ancien Empire aboutit à la même constatation: our kherep hemout est le titre par excellence de ces personnages. 


\section{§ 4. TRADUCTION DE OUR KHEREP HEMOUT}

Plusieurs traductions de our kherep hemout ont été données à ce jour. En voici quelques-unes, classées chronologiquement:

- "chef de l'oeuvre"1

- "le grand chef des artisans (the great leader of the artisans) ${ }^{2}$

- "celui qui est grand à la direction des artisans" (he who is great at directing the craftsmen $)^{3}$

- "chef des maîtres-artisans" (chief of the master-artificers) ${ }^{4}$

- "chef des maîtres-ouvriers" (Oberster der Werkmeister) ${ }^{5}$

- "grand des chefs d'atelier" (atelier doit être, pensons-nous, pris dans le sens d'"ouvriers travaillant sous un maître") $)^{6}$

- "le plus grand chef des arts" (der grösste Leiter der Künste) ${ }^{7}$

- "directeur en chef des artistes"

- "le grand pour diriger les artistes"

- "le chef des artisans du Grand" (der Leiter der Handwerker des Grossen $)^{10}$

${ }^{1}$ G. MASPERO, Un manuel de hiérarchie égyptienne, dans Etudes égyptiennes, II, 1888 , p. 9.

${ }^{2}$ K. SETHE, dans M.A. MURRAY, Saqqara Mastabas, Part II, 1937, p. 12, § 14, 3; le manuscrit de SETHE a été terminé en 1905, cf. op. cit., p. 11.

3 A.M. BLACKMAN, dans HASTINGS, Encyclopaedia of Religion and Ethics, vol. X, 1918, p. 295, 1ère col.

4 A.M. BLACKMAN, Position of women in the Egyptian hierarchy, dans Journal of Egyptian Archaeology, VII, 1921, p. 16.

${ }^{5}$ A. ERMAN et H. GRAPOW, Wörterbuch der Ägyptischen Sprache, I, 1926, p. 329, 12 et III, 1929, p. 86, 1.

${ }^{6}$ G. LEFEBVRE, Histoire des grands prêtres d'Amon de Karnak jusqu'à la XXIème dynastie, 1929, p. 135.

${ }^{7}$ H. KEES, Memphis, dans PAULY-WISSOWA, Real-Encyclopädie der klassischen Altertumswissenschaft, vol. XXIX, 1931, p. 677, 42.

${ }^{8}$ A. ERMAN, La religion des Egyptiens, 1937 (traduction par H. Wild de l'édition de 1934), p. 83.

9 ibidem, p. 224.

${ }^{10}$ H. JUNKER, Die Götterlehre von Memphis, dans Abhandlungen der Preuss. Akad. der Wissenschaften, Jahrgang 1939, phil.-hist. Klasse, Nr. 23, 1940, p. 29. 
- "grand directeur des artistes" (Grosser Vorsteher der Künstler) ${ }^{1}$

- "chef suprême des métiers" (supreme leader of handicraft) ${ }^{2}$

- "le plus grand des artisans" (Greatest of artificers) ${ }^{3}$.

Les désaccords portent sur les trois éléments du titre hiéroglyphique, mais c'est sur le premier seulement qu'il y a opposition entre les traducteurs. La plupart voient, dans cet adjectif employé comme un substantif, la désignation d'un être humain et leur interprétation est confirmée par les titres "grand des dix de Haute-Egypte", "grand des cinq de la maison de Thot"4, et plus encore par "grand des médecins" ${ }^{15} . \mathrm{H}$. Junker regarde "Grand" comme le nom d'une divinité. Son interprétation, qui est nouvelle, demande un examen attentif. Après avoir "établi solidement que $W r$ (our) peut aussi être employé comme nom d'un dieu" (p. 27), H. Junker est amené à réexaminer quelques-uns des anciens titres sacerdotaux contenant our. Sa recherche porte sur khety-our, qu'il traduit "celui qui est à la suite du Grand", sur le grand prêtre d'Héliopolis dont il lit le titre ma-our et le traduit "celui qui voit le Grand", puis sur le grand prêtre de Memphis. Ici, nous traduisons le texte même de H. Junker : "Le grand prêtre de Memphis avait aussi un titre commençant par our, 步 $\$$ \& , auquel on donne ordinairement la signification de our kherep hemout $=$ grand chef des artisans. Cela signifierait qu'il y avait une pluralité de chefs des artisans à la tête desquels il faudrait voir notre our kherepou hemout. On attendrait, à dire vrai, un imy-ra, sechem (conducteur), hery ou un terme analogue, dont l'emploi est ordinaire pour des directeurs et des inspecteurs, tandis que our désigne davantage le premier parmi des gens de même rang. Et lorsqu'on parcourt les citations de MURRAY, Noms et titres, 19 et 44, on rencontre d'autres difficultés. Ainsi, Ptahchepses et Sabou sont à la

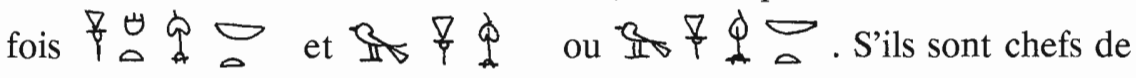

${ }^{1}$ A. RUSCH, Phthas, dans PAULY-WISSOWA, Real-Encyclopädie der klassischen Altertumswissenschaft, Vol. XXIX, 1941, p. 935,1,1, qui adopte la traduction d'Ed. MEYER, Geschichte...

${ }^{2}$ SANDMAN, Ptah, p. 25.

${ }^{3}$ A.H. GARDINER, Ancient Egyptian Onomastica, 1947, Text, Vol. I, p. $38^{*}$.

${ }^{4}$ M.A. MURRAY, Index of names and titles of the Old Kingdom, 1908, pl. XIX.

${ }^{5}$ Bibliographie dans G. POSENER, La première domination perse en Egypte, 1936, p. 4 , note (c). 
tous les artisans, cela exprime déjà suffisamment leur position de grands chefs. Si par contre nous comprenons our comme nom de dieu, toute difficulté est supprimée. Le chef des artisans du Grand désigne alors le directeur des artisans travaillant au service du Grand. Our irait, en outre, bien pour Ptah qui, en tant que $\begin{gathered}\square \\ 0\end{gathered}$ pourrait peut-être encore alléguer une autre raison pour le choix de la désignation. Une assez grande partie des artisans était occupée à Toura, en face de Memphis et E. Meyer supposait que Ptah avait été d'abord là, précisément, le dieu protecteur des carriers (STOLK, Ptah, p. 13 et remarque 1). Mais l'ancienne ville dans laquelle le Grand avait un lieu principal de culte était située autrefois à Ayn, dans le territoire de Toura (voir Mitteilungen des Instituts Kairo IX, p. 37, remarque 1). Si nous comprenons our dans le titre comme $d u$ Grand, le fait que le kherep hemout our remplisse une fonction sacerdotale et ne soit pas seulement un fonctionnaire supérieur se trouve également expliqué."1

Reprenons point par point au moins le début de cette argumentation. Notons d'abord que H. Junker ne combat qu'une interprétation de our kherep hemout et que celle-ci est impossible; en effet, our ne peut pas être un adjectif employé comme épithète du substantif kherep puisqu'il le précède au lieu de le suivre ${ }^{2}$.

En second lieu, la pluralité des chefs des artisans semble indiquée par l'existence de personnages qui, tout en n'étant jamais our kherep hemout ont porté le titre de imy-ra hemout "directeur des artisans"; plusieurs d'entre eux ont été aussi des prophètes de $\mathrm{Ptah}^{3}$.

Ensuite, pourquoi faudrait-il que la pluralité de chefs des artisans eût à sa tête un directeur, un conducteur ou un supérieur qui l'eût commandée ? Les appellations des grands prêtres de Thot et d'Amon, "grand des cinq de la maison de Thot" et "premier prophète d'Amon" suggèrent que le grand prêtre de ces divinités était, au moins à l'origine, un primus inter pares qui aurait présidé plutôt que dirigé un collège de

${ }^{1}$ H. JUNKER, op. cit., p. 28-29.

${ }^{2}$ G. LEFEBVRE, Grammaire, § 167.

${ }^{3}$ Voir l'Aperçu du personnel de Ptah" à la fin du volume. Pour des directeurs des artisans que les monuments connus ne désignent pas comme prophètes de Ptah, comparer les deux titres dans M.A. MURRAY, Index, pl. XXII et XXVIII. 
prêtres à la tête des affaires du dieu. Le our kherep hemout, interprété comme "grand des chefs des artisans" aurait alors été sur le même pied que le "grand des cinq de la maison de Thot". H. Junker ne parle pas de ce titre également composé avec our.

La difficulté soulevée ensuite n'est qu'apparente puisque les deux premiers mots de our kherep hemout ne peuvent pas dire "grand chef", comme nous venons de le noter, et qu'ainsi our kherep hemout indique une autre fonction que "chef de tous les artisans"1. Quant à our kherep hemout nebet, c'est un titre qui n'existe pas bien que Miss M.A. Murray en donne deux références ${ }^{2}$. La première concerne Sabou'; nulle part, dans Mariette, que nous avons relu attentivement à ce sujet, Sabou ne porte un titre pareil.

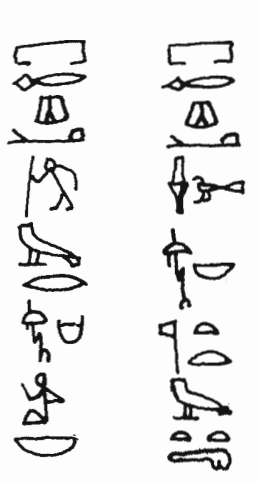

La seconde référence se rapporte à Iny-ankh, dont nous reproduisons les inscriptions ci-contre ${ }^{4}$. Dans la colonne de gauche, il est "khery-â our per-âa, imy-ra hemout nebet, grand adjoint du Palais, directeur de tous les artisans". Dans la colonne de droite où Miss M.A. Murray a relevé un soi-disant our kherep hemout nebet nous pourrions voir, à la rigueur, un kherep our hemout nebet que nous ne saurions toutefois assimiler à un grand prêtre de Ptah car aucun des nombreux our kherep hemout que nous avons rassemblés n'a jamais écrit son titre autrement qu'en commençant par our. Mais même la lecture kherep our hemout nebet ne nous paraît pas juste. En effet, comme le titre khery-âa our per-âa est attribué à Iny-ankh par la colonne de gauche et que kherep hemout nebet est attesté dans les inscriptions des grands prêtres, il nous semble qu'il faut lire dans la colonne de droite: khery-âa our per-âa, kherep hemout nebet netery-metout (?) "grand adjoint du Palais, chef de tous les artisans

1 Our kherep hemout, interprété comme "grand des chefs des artisans" et "chef de tous les artisans" nous paraissent désigner deux degrés d'une même fonction; voir cidessous $\S 6$.

\footnotetext{
2 op. cit., pl. XIX.

${ }^{3}$ A. MARIETTE, Mastabas, E 1 et 2, E 12.

${ }^{4}$ ibidem, D 26.
} 
de (?) netery-metout ${ }^{\prime 1}$. Cette lecture suppose un déplacement du signe our que le graveur aura placé derrière kherep pour éviter une ambiguïté; si our était devant ou au-dessus de kherep, on pourrait aussi bien lire khery-â per-âa, our kherep hemout etc., que ce que nous proposons ${ }^{2}$. Quel que soit, d'ailleurs, le sens des titres d'Iny-ankh, il est certain qu'ils ne comprennent pas celui de our kherep hemout nebet.

Les arguments de $\mathrm{H}$. Junker contre l'interprétation ordinaire du premier mot de our kherep hemout nous paraissent insuffisamment fondés. Ceux qu'il avance ensuite pour appuyer sa nouvelle traduction ne sont pas convaincants. En admettant comme lui qu'il y a eu un dieu Our et que c'est ce dieu et non le simple adjectif "grand" que nous rencontrons dans Ptah-Our, il n'en résulte qu'une possibilité; celle de supposer que cette divinité apparaît dans le titre our kherep hemout. En ce qui concerne les carriers de Toura, pouvons-nous savoir s'ils étaient sous la dépendance du our kherep hemout ? Les deux seuls textes d'après lesquels nous pouvons connaître l'activité du our kherep hemout sont ceux de Debehen (1) et de Ny-ankh-Sekhmet (2). Dans le premier, la destruction de toute une partie des lignes 9 et 11, probablement tracées jadis à l'encre noire mais pas encore sculptées et recouvertes aujourd'hui de noir de fumée, ne permet aucune conjecture sur les rapports des our kherep hemout et de Toura; SANDMAN, Ptah, p. 52, traduit ainsi les lignes 9 et 10 de l'inscription de Debehen (1,9-10): "(Sa Majesté ordonna) que de la pierre fût rapportée de Toura pour en parer les parois du temple (probablement le temple de la pyramide) et aussi deux faussesportes et une porte tombale pour cette (= de Debehen) tombe par (l'inspecteur) de la flotte et les deux chefs suprêmes des métiers et l'architecte (?) du roi qui étaient venus...".

${ }^{1} \mathrm{Si}$ la lecture netery-metout est juste, ces mots signifieraient "divin de semence", c'est à dire "celui qui est semence divine", et désigneraient soit le roi en général (cf. "semence divine" appliqué au roi-Horus dans Pyr. § 1417, cité dans Wörterbuch, II, Belegstellen, 169,3 ) soit un roi particulier (cf. les noms Netery-khet, Netery-mou). A l'appui du rôle grammatical joué ici par netery-metout, cf. d'après M.A. Murray, op. cit., pl. XXII un "directeur des artisans du Palais (per-âa)" (L. D., II, 115 b).

${ }^{2} \mathrm{Si}$ notre interprétation des inscriptions d'Iny-ankh est exacte, elles permettent d'établir une équivalence entre "chef de tous les artisans" et "directeur de tous les artisans", et fournissent ainsi un argument en faveur de la pluralité des chefs des artisans. 
On peut, en effet, lire: in.t(w) (passif). Par contre, le texte et les enseignements de la stèle de Ny-ankh-sekhmet (2) nous font maintenir notre interprétation de in au milieu de la ligne 10 comme une particule proclitique. D'ailleurs, même si Mycérinus a chargé les deux grands des chefs des artisans de "rapporter" la pierre de Toura, cela ne signifie pas que ces personnages aient eu un pouvoir quelconque sur les carrières et sur les carriers de Toura, et la traduction ci-dessus ne peut pas servir à appuyer l'assertion d'H. Junker sur Toura. Enfin, l'existence d'un culte de Ptah à Toura, attestée seulement à partir du Nouvel Empire (SANDMAN, Ptah, p. 216-217) ne prouve pas davantage que le clergé de Ptah de Toura ait été sous la dépendance du grand prêtre de Memphis. La ligne 2 montre les our kherep hemout en activité sur le chantier de la pyramide de Mycérinus. La stèle de Ny-ankh-sekhmet est plus explicite: ce n'est qu'après avoir été apportés de Toura que les deux blocs de pierre sont remis aux mains des our kherep hemout, qui dirigent les artisans de la ouabet mais pas les carriers. Sandman, Ptah, p. 52-53, traduit ainsi une partie de l'inscription de Ny-ankh-sekhmet (2,3-4): "( $S a$ Majesté ordonna) ... qu'elles (= les fausses-portes) fussent placées dans le vestibule dans la maison "Sahourê apparaît avec des couronnes" et que les deux chefs suprêmes des métiers et des hommes d'atelier y fussent mis afin que le travail sur elles pût être exécuté dans le voisinage immédiat du roi lui-même ...". On peut, en effet, faire dépendre tous les verbes des lignes 3 et 4 de "Sa Majesté fit" au milieu de la ligne 2. Par contre, il nous semble difficile d'admettre que le mot ouabet puisse dépendre du substantif qui le suit.

La dernière phrase de $\mathrm{H}$. Junker touche à un sujet que nous traiterons à propos de la dualité des our kherep hemout. C'est pourquoi nous dirons seulement que les our kherep hemout ne nous semblent pas avoir revêtu à l'origine une fonction sacerdotale quelconque en tant que our kherep hemout.

Après cet examen de l'argumentation de $\mathrm{H}$. Junker, nous avons encore une remarque à faire. Le titre our kherep hemout apparait parfois au duel et au pluriel. Le duel est toujours indiqué à la fin du titre et ne nous est donc ici d'aucune utilité. Le pluriel est aussi marqué quelquefois seulement à la fin du titre $(157,158,189,3)$, mais on le rencontre indiqué à la fois par trois traits à la fin du titre et par trois autres traits après our $(\mathbf{1 1 5 , 3}$ et $\mathbf{1 8 2 , 6 1 )}$. Comme le contexte donne clairement à 
entendre qu'il s'agit de plusieurs grands prêtres, nous voyons dans our au pluriel une désignation de ceux-ci et traduisons "les grands etc.". Pour les auteurs de ces inscriptions, il n'était donc pas question d'un dieu appelé "Grand". Faudrait-il penser, comme H. Junker le fait dans le cas analogue des grands prêtres d'Héliopolis ${ }^{1}$, que l'interprétation "les grands etc." était, à l'époque de ces inscriptions, "pas originelle et fausse" ? Nous avons de la peine à l'admettre à cause de la personnalité des auteurs de ces textes; le premier, Khamouas, était lui-même grand prêtre de Memphis; il s'est occupé des anciens monuments de Saqqara et eut plus tard la renommée d'un chercheur de vieux textes ${ }^{2}$; l'autre n'a pas pu établir une généalogie de soixante générations, sur laquelle il y a du reste des remarques à faire ${ }^{3}$, sans consulter d'anciens manuscrits ${ }^{4}$.

En résumé, nous constatons que rien ne permet de condamner l'ancienne interprétation de our "le grand de..." et que, d'autre part, la nouvelle traduction "... du Grand" est possible à condition d'admettre que la notion de ce dieu nommé Grand était perdue au Nouvel Empire. Pour nous, nous préférons nous en tenir au sens "le grand de...". Notre conclusion sur l'hypothèse d'H. Junker est analogue à celle de SANDMAN, Ptah, p. 51-52, qui, après avoir brièvement examiné l'interprétation d'H. Junker, écrit que celle-ci "peut difficilement être considérée comme suffisamment bien établie".

Le second élément de our kherep hemout a été pris parfois pour un verbe, mais, sans parler du caractère de la construction our + infinitif, l'expression qu'on obtient ainsi, "grand pour commander les artisans", a une signification semblable à "directeur des artisans" et paraît à écarter pour cette raison. Le mot kherep disparaît dans le titre démotique du grand prêtre de Memphis, qui ne conserve plus que our et hemout ${ }^{5}$ soit "grand des artisans". Nous pensons que cette forme, qui était celle du langage courant sous les Ptolémée, est à l'origine de la graphie

${ }^{1}$ H. JUNKER, op. cit., p. 28.

2 Cf. p. 156.

${ }^{3}$ Cf. p. 93 sq.

${ }^{4}$ On pourrait aussi tirer argument du titre "our kherep hemout de Ptah" qui apparaît à la XIXème dynastie. Sur son origine, voir Chapitre $\mathbf{V}$.

5 W.M. MULLER, Über einige Hieroglyphenzeichen, dans Rec. Trav., IX, (1887), p. 166-168; H. BRUGSCH, Thesaurus, p. 887. 
hiéroglyphique our hemout kherep; celle-ci apparaît sur les monuments ptolémaïques et nous proposons de la rendre par "grand des artisanschefs" (196 et suivantes). D'autre part, comme kherep est toujours écrit au moyen d'un seul signe, sans complément phonétique, alors qu'on en rencontre pour our et hemout, ce second élément du titre a peut-être commencé à s'affaiblir de bonne heure dans le langage parlé. Cette particularité graphique nous fait transcrire kherep au lieu de kherep(ou).

Pour le troisième mot de our kherep hemout, nous repoussons les traductions oeuvre et art à cause du déterminatif de l'homme assis écrit après hemout dans le titre kherep hemout nebet $(\mathbf{4 , 7})^{1}$ et nous adoptons celle de troupe d'artisans ou corps de métier que nous abrégeons en "artisans". Nous avons hésité à accepter le terme "atelier" à cause de son double sens. Nous traduisons donc our kherep hemout "grand des chefs des artisans".

\section{§ 5. SEM}

Le second titre donné au grand prêtre de Ptah par la liste géographique du temple d'Edfou et par le papyrus Hood appelle quelques remarques en ce qui concerne le grand prêtre de $\mathrm{Ptah}^{2}$.

Sem avec la graphie $\bigcap$ se montre en tout cas sous les IIème et IIIème dynasties; il est alors en relation avec Oupouaout et Anubis et les personnages ainsi intitulés n'ont aucune attache avec $\mathrm{Ptah}^{3}$.

Ecrit de la même façon, sem est totalement absent des titulatures des grands des chefs des artisans de la Vème et du début de la VIème dynasties (3 à 25); on peut évidemment supposer qu'il a été porté par un grand prêtre tel que Neferef-rê-ankh, connu seulement par une courte mention (8), mais la même supposition est très invraisemblable pour

${ }^{1}$ La comparaison de hemout dans les deux titres est justifiée par le fait que le mot s'abrège peu à peu et finit par s'écrire avec un seul signe dans kherep hemout nebet (comparer 4,7, 5,4 et 21,5) comme dans our kherep hemout.

${ }^{2}$ Une étude complète de sem dépasserait beaucoup les limites de ce travail.

${ }^{3}$ K. SETHE, dans J. GARSTANG, Mahâsna and Bêt Khallâf, 1903, p. 19; ibidem, pl. VIII,1 et XXVI,7. 
Sabou-Ibebi et d'autres dont nous avons des monuments importants. Les grands des chefs des artisans de l'époque en question ont bien dans leur titulature un titre "sem", mais ce dernier s'écrit 949, 9198 ou ก 2424 et n'est employé que comme complément de nom de kherep dans l'expression kherep semou (3, 4 et suivantes), kherep semou neb $(\mathbf{1 0 , 3})$, kherep semou seker $(\mathbf{6 , 2})$, "chef des sem", "de tous les sem", "des sem de Sokar"; de plus, ce mot sem est toujours écrit avec 149 accompagné ou non des compléments phonétiques $\bigcap$ ou $\bigcap$, tandis que le titre 15 sem n'est jamais écrit avec 994 chez les grands prêtres de Ptah. Une équivalence entre les deux mots ${ }^{1}$ nous semble donc exclue chez les grands prêtres de Ptah.

Sem, 7 appliqué à un grand des chefs des artisans apparaît chez Sabou-Tjéti $(\mathbf{2 6 , 4 )}$ dans le courant de la VIème dynastie; c'est alors un titre beaucoup moins important que grand des chefs des artisans. Au Moyen Empire, il vient en contact avec ce dernier dans l'ordre sem - our kherep hemout, régulièrement en usage jusqu'à la XIXème dynastie; our kherep hemout termine ainsi souvent les énumérations de titres et a une place en vue à côté du nom propre du grand prêtre. On retrouve le même ordre de succession à la XXVIème dynastie $(\mathbf{1 7 9 , 1 8 )}$ et à l'époque ptolémaïque $(\mathbf{1 9 3 , 9 )}$.

L'expression inverse, our kherep hemout - sem, existe exceptionnellement à la XVIIIème dynastie chez le grand prêtre et prince Thoutmôsis $(\mathbf{6 2 , 1 - 2 )}$. Elle réapparaît pendant la XIXème dynastie et supplante même l'ancienne manière jusqu'à la veille de l'époque saïte $(\mathbf{1 8 4})^{2}$. C'est aussi au cours de la XIXème dynastie que naît la graphie setem $(\mathbf{1 0 3 , 1 4})$.

${ }^{1}$ Elle a été proposée par A. VOLTEN, Demotische Traumdeutung, 1942, p. 26, note 3.

${ }^{2}$ Our kherep hemout est aussi employé seul à toutes les époques, avec ou sans d'autres titres. 
Enfin, quatre grands prêtres ont eu une prédilection marquée pour le titre sem: les princes Thoutmôsis (63 à 65) et Khamouas (109 et suivantes) aux XVIIIème et XIXème dynasties, Iyry (156) à la fin de la XIXème, Anemher (192,12 et suivantes) à l'époque ptolémaïque ${ }^{1}$.

Sem n'est donc pas un titre initial du grand prêtre de Memphis; son importance et son emploi parfois presqu'exclusif se font jour au Nouvel Empire.

Pour la signification du titre sem, que nous ne traduisons pas, nous n'avons qu'à rappeler le rôle du sem dans les rites funéraires et l'étroite parenté de ceux-ci et du rituel du culte divin journalier ${ }^{2}$.

Arrivés au terme de notre enquête sur les titres du grand prêtre de Ptah dans l'inscription géographique du temple d'Edfou et le papyrus Hood, nous concluons: les grands des chefs des artisans qui ont fait au moins une partie de leur carrière à Memphis peuvent être regardés comme d'authentiques grands prêtres de Ptah dans ce lieu ${ }^{3}$, mais il est prudent d'écarter de leur liste les personnages memphites qui sont seulement "sem", tant qu'un document de leur époque ne vient pas modifier nos connaissances.

${ }^{1}$ La qualité princière des deux premiers pourrait avoir déterminé leur préférence. Voir Chapitre V, § 31.

2 E. SCHIAPARELLI, Il libro dei funerali, 1881-90; A. MORET, Le rituel du culte divin journalier, 1902.

${ }^{3}$ En dehors de Memphis, nous connaissons deux grands des chefs des artisans thébains: le grand prêtre d'Amon Bakenkhonsou II qui a été sem et grand des chefs des artisans de Ptah "en sa qualité d'administrateur du culte thébain de Ptah" (G. LEFEBVRE, Histoire, p. 135) et le sem dans le temple de Ptah et grand des chefs des artisans dans Héliopolis de Haute-Egypte, Méryptah (G. DARESSY, Recueil de cônes funéraires, dans Mémoires de la Mission Française au Caire, tome VIII, 2ème fascicule, 1893, p. 291, $\mathrm{n}^{\circ}$ 208). Il y a eu aussi des sem de Ptah thébain: le grand prêtre d'Amon Nebneterou; le prince Khamouas, fils de Ramsès III, dont le tombeau est à la Vallée des Reines. 


\section{§ 6. LE RECRUTEMENT DES GRANDS PRETRES}

La moitié environ des grands des chefs des artisans que nous avons rassemblés ne donnent pas leur filiation ${ }^{1}$. Une partie de l'autre moitié est formée par des hommes dont le père n'était pas grand prêtre: trois sont fils de rois $^{2}$; deux, fils de vizirs ${ }^{3}$; un, peut-être fils de juge ${ }^{4}$; deux, fils de prophète et père divin ${ }^{5}$; deux, sans doute fils de gens de condition modeste dont le nom propre seul est cité 6 . Le grand prêtre de Ptah a donc pu être recruté dans des classes sociales très différentes.

Les grands prêtres, fils de grands prêtres, apparaissent ainsi: deux au Moyen Empire; ; deux à la XIXème dynastie ${ }^{8}$; trois à la fin de la XXIème et au début de la XXIIème dynastie ${ }^{9}$; quatre sous la XXIIème dynastie $^{10}$; un sous la XXVIème dynastie ${ }^{11}$; sept sous les Ptolémées ${ }^{12}$.

1 Nous utilisons les généalogies dans la mesure fixée au chapitre qui leur est consacré: nous admettons la filiation Paoupaou-Horsaïset I et l'appartenance de tous les grands des chefs des artisans ptolémaïques à une seule famille.

${ }^{2}$ Thoutmôsis, Khamouas, Chechanq.

${ }^{3}$ Ptahmès II, Khamouas I.

${ }^{4}$ Neferrenpet.

${ }^{5}$ Ptahmès III, Ptahemhat-Ty.

${ }^{6}$ Hori II, Pahemneter.

${ }^{7}$ Nebpou et son fils Sehetepibrê-cheri; avec le père de Nebpou, Sehetepibrê-ankhnedjem, cela fait trois générations de grands prêtres; toutefois l'existence de Senousertankh empêche d'affirmer absolument que les trois générations se sont succédé sans interruption (voir aussi le chapitre sur l'unité probable du pontificat depuis la fin de l'Ancien Empire).

${ }^{8}$ Hori I, fils de Khamouas I; Rêhetep, fils de Pahemneter.

${ }^{9}$ Horsaïset I, son fils Ankhef-ny-sekhmet, son petit-fils Chedsounefertoum; avec Paoupaou, cela fait quatre générations de grands prêtres.

${ }^{10}$ Takelot, son fils Padiiset, ses deux petits-fils Peftjaouaouibastet et Horsaïset II; avec Chechanq, père de Takelot, cela fait quatre générations; comme c'était le cas au Moyen Empire, l'existence de Merenptah empêche d'affirmer absolument une succession ininterrompue.

${ }^{11}$ Neferibrêmen, fils de Hekairâa; le petit-fils de Neferibrêmen, Ahmèsmen est aussi grand prêtre.

${ }^{12}$ De père en fils: Anemher, Harmachis, Psenptaïs; nous n'avons pas de monument des deux générations suivantes; puis, Petobastis II, Psenptaïs II, Petobastis III-Imouthès; enfin, Teos, fils d'Anemher, qui n'a pas fait souche. En ajoutant à ces noms Nesqed, père d'Anemher, puis Psenamounis, cousin germain d'Imouthès, et en comptant les deux 
Aucun des grands prêtres de l'Ancien Empire ne nous dit sa filiation. Si plusieurs d'entre eux s'appellent Ptahchepses ou Sabou, l'exemple des quatre Ptahmès du Nouvel Empire, qui semblent bien n'avoir aucun lien de parenté entre eux, nous interdit d'affirmer que le pontificat de Ptah était héréditaire sous l'Ancien Empire ${ }^{1}$. Il ne faudrait pas oublier qu'il y avait deux grands des chefs des artisans à la fois en ce temps-là; un Ptahchepses III ou un Ptahchepses IV a pu nommer un de ses fils Sabou parce que c'était le nom de son collègue, et l'inverse se sera produit chez Sabou-Ibebi pour la même raison.

Le pontificat memphite n'a donc peut-être pas été du tout héréditaire au début; il paraît ne l'avoir été qu'exceptionnellement jusqu'à la fin du Nouvel Empire. Mais il a fortement tendu à le devenir à la Basse Epoque et l'a été complètement à l'époque ptolémaïque².

Les grands prêtres de Memphis sont avares de renseignements sur la partie de leur carrière antérieure au pontificat. Ptahchepses I a été élevé

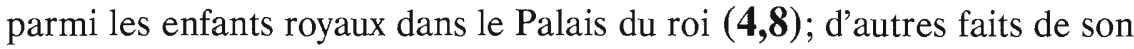
inscription biographique se sont peut-être aussi produits avant son arrivée au rang de grand des chefs des artisans, mais nous ne savons pas où situer cet événement dans sa vie. Khamouas a participé aux campagnes de son père en Asie (108) et en Nubie (107). Quelques membres de familles sacerdotales en disent un peu davantage. Ahmèsmen, sous les rois saïtes, a été "intronisé" père divin de Ptah, puis nommé directeur des artisans à la mort de son père, qui faisait partie du haut personnel de Ptah mais n'était pas grand prêtre (186,9-10). Sous les Ptolémées, Nesqed, sans doute une fois prêtre, a été nommé prophète de Philotéra, puis d'Arsinoé, et ensuite supérieur des secrets dans le temple de Ptah (190,10-12). Pétobastis II a été "intronisé" prêtre à 10 ans et l'est resté jusqu'à 28 ans $^{3}$. Psenptaïs II a passé treize années en présence de son père $(\mathbf{2 0 0 , 1 7 )}$; cela signifie, évidemment, qu'il a passé treize années du

générations encore vides de monuments, on obtient 11 grands prêtres de la même famille répartis en 9 générations.

${ }^{1}$ H. KEES le fait dans Memphis (PAULY-WISSOWA, Real-Encyclopädie, XXIX, 1931, p. 677-8).

${ }^{2}$ Ces conclusions rejoignent celles sur l'hérédité du sacerdoce, examinée en général, de W. OTTO, Priester und Tempel im hellenistischen Ägypten, I, 1905, p. 202.

${ }^{3}$ Voir au chapitre sur les grands prêtres ptolémaïques. 
vivant de son père, mais peut-être aussi qu'il a été instruit dans le sacerdoce par le grand prêtre Pétobastis II. Psenamounis s'est marié alors qu'il était prophète de $\operatorname{Ptah}^{1}$.

Il nous semble que les titulatures des grands prêtres de l'Ancien Empire peuvent compléter quelque peu ces notions. Ptahchepses I énumère une partie de ses titres sur son linteau (3); ce sont peut-être ceux qu'il estime les plus importants, étant donné la position du monument, au-dessus de la porte de sa chapelle funéraire. En tête, vient grand des chefs des artisans. Il est de nouveau question des artisans dans les titres directeur de la ouabet et chef des artisans ${ }^{2}$; or le second, en tout cas, de ces titres, qui sont donc gravés à la suite de grand des chefs des artisans, marque un degré inférieur au titre de tête. De même, ouab de Ptah, presqu'à la fin de l'inscription, désigne une classe de prêtres inférieure à celle de prophète de Ptah, indiquée auparavant. Ainsi, cette titulature paraît avoir été composée dans un ordre inversement chronologique. SANDMAN (Ptah, p. 25 et 52) écrit qu'il est possible que la fonction de chef de tous les artisans ait été remplie avant que son titulaire devînt grand chef des artisans. Nos remarques sur l'ordre de succession des titres des linteaux des grands prêtres de l'Ancien Empire nous permettent d'être plus affirmatif.

On retrouve des titulatures presque semblables sur les linteaux de Ptahchepses II (5) et de Sabou-Ibebi (17). Le texte de Ptahchepses II ne diffère de celui de Ptahchepses I que par des variantes graphiques et l'adjonction du mot "tous" après artisans dans chef de tous les artisans. Sabou-Ibebi a le même texte que Ptahchepses II et en reproduit la plupart des graphies. Y a-t-il copie d'un modèle chez ces derniers grands prêtres ? C'est peu probable à cause de la place de ces monuments.

Ptahchepses IV emploie à son tour la même énumération dans les deux dernières lignes de son linteau (21), mais en y intercalant, entre autres, ses titres de prophète des pyramides de Teti et d'Ounas. Le titre concernant Teti a été, pensons-nous, donné à Ptahchepses après celui qui se rapporte à Ounas et il est pourtant cité avant lui, de sorte que

1 idem.

${ }^{2}$ La ouabet est l'atelier, le lieu où, entre autres, on sculpte la pierre. 
l'adjonction de Ptahchepses IV est aussi composée dans un ordre inversement chronologique.

$\mathrm{Si}$ notre interprétation est juste, ces linteaux révèleraient, en commençant la lecture par la fin, les étapes principales que leurs possesseurs ont parcourues jusqu'au pontificat: prêtre-ouab de $\operatorname{Ptah}^{1}$, supérieur des secrets de son dieu $^{2}$, chef des artisans, directeur de la ouabet ${ }^{3}$, directeur du domaine de Sokar, confident du roi $^{4}$, chef des sem, prophète de Ptah et prophète de Sokar ${ }^{5}$. Comme il existe d'autres prophètes de Ptah que les grands des chefs des artisans, alors que les participants à la fête de Rê sont tous des grands des chefs des artisans, nous arrêtons à prophète de Ptah la carrière antérieure au pontificat et supposons que le titre de participant à la fête de Rê était décerné en même temps que celui de grand des chefs des artisans. Quant au titre "celui qui est dans la double maison", les possesseurs des linteaux l'ont acquis en même temps que le pontificat, mais Sabou-kem semble l'avoir acquis avant $(\mathbf{1 2})^{6}$. La carrière des grands prêtres dont nous venons de parler aurait été en partie civile et en partie sacerdotale ${ }^{7}$ et ils auraient suivi une filière bien établie ${ }^{8}$.

Chez les derniers grands prêtres de l'Ancien Empire, Sabou-Tjéti et Ptahchepses-Impy, l'ordre chronologique semble la règle: le titre de grand des chefs des artisans vient à la fin ou vers la fin des titulatures. Nous

1 "Celui qui est dans le coeur de son maître", à la fin de l'énumération, paraît plutôt une épithète qu'un titre véritable.

${ }^{2}$ C'est-à-dire: initié aux choses secrètes du dieu.

${ }^{3}$ Chef des artisans et directeur de la ouabet paraissent des titres d'égale importance qui ont pu être décernés en même temps à un personnage.

${ }^{4}$ Confident du roi semble plutôt un titre honorifique.

${ }^{5}$ Comme les deux autres titres sont la plupart du temps côte à côte et qu'ils se fondent même en "prophète de Ptah et de Sokar" (15,2; 24,7), il est probable que les grands prêtres ont accédé aux deux charges en même temps. de Ptah.

${ }^{6}$ Ce titre est aussi porté par de nombreux autres personnages que les grands prêtres

${ }^{7}$ Jusqu'à Ptahchepses IV, l'ordre inversement chronologique semble suivi aussi sur les autres monuments des grands prêtres que les linteaux.

${ }^{8}$ On pourrait se risquer à supposer que le temps nécessaire à parcourir cette filière était assez fixe pour qu'on eût là un argument contre l'hérédité du pontificat chez les Ptahchepses et les Sabou. 
pensons donc que Sabou-Tjéti a été en tout cas prêtre-lecteur en chef avant d'être grand prêtre. Nous n'oserions pas affirmer que les considérations chronologiques soient primordiales dans les titulatures postérieures à l'Ancien Empire ${ }^{1}$, mais l'existence de l'ordre chronologique est prouvée par la comparaison des titres et de la biographie sur la stèle ptolémaïque de Nesqed (190,1-9 et 10-12) 2 .

\section{§ 7. L'ACCESSION AU PONTIFICAT}

Nous ne pouvons rien affirmer de très catégorique sur la question de savoir s'il fallait avoir atteint un âge déterminé pour devenir grand prêtre. Lorsque le nouveau titulaire n'était pas le fils de son prédécesseur, il est vraisemblable que ce n'était plus un enfant. Par contre, aux temps où le pontificat est devenu héréditaire, un jeune homme, voire un jeune garçon a pu être grand prêtre ${ }^{3}$.

On sait que les grands prêtres d'Amon de Karnak, au Nouvel Empire, ont été nommés de diverses façons. Parmi les grands prêtres de Ptah que nous connaissons, quatre seulement donnent une indication sur ce sujet, en des termes d'ailleurs assez semblables. Ptahmès III déclare: "Le Dieu Bon a ordonné de me faire remplir la fonction excellente; il m'a nommé (littéralement: placé) grand des chefs des artisans" $(69,6)$. Nesqed raconte: "Mon maître redoubla la faveur dont j'étais l'objet en me faisant grand des chefs des artisans" $(\mathbf{1 9 0 , 1 2 )}$. Psenptaïs II affirme: "Le roi ... Ptolémée ordonna que la grande fonction de grand des chefs des artisans me fût (donnée)" (200,17-18). Psenamounis, enfin, dans la partie démotique de la stèle de Tanepherôs, dit que César l'a fait grand prêtre. Les trois derniers de ces grands prêtres appartiennent à la même famille et à une époque où le pontificat était héréditaire; leur nomination n'apparaît donc pas comme le résultat d'un choix du souverain et celui-ci n'a probablement rien fait d'autre que de signer l'ordre royal. Mais

\footnotetext{
${ }^{1}$ On peut se demander, par exemple, dans quelle mesure la chronologie est suivie chez Rêhetep $(\mathbf{1 0 3}, \mathbf{1 2 - 2 3})$.

${ }^{2}$ Sur le changement de méthode à la IVème dynastie, voir le Chapitre IV.

${ }^{3}$ Psenptaïs à 13 ans, Imouthès probablement à 7 ans. Les seuls grands prêtres dont nous connaissions l'âge lors de leur accession au pontificat sont ptolémaïques et appartiennent à la même famille.
} 
Ptahmès III, dont le père n'était pas grand prêtre de Ptah, a dû être désigné personnellement par Aménophis III. Si donc le rôle personnel des souverains ptolémaïques apparaît ici moins grand que celui d'un roi du Nouvel Empire ${ }^{1}$, il semble toutefois qu'on puisse généraliser ce que ces quatre nominations ont de commun et déclarer que chaque nouveau grand prêtre est créé par un ordre royal depuis le Nouvel Empire ${ }^{2}$. En reculant dans le passé, nous retrouvons l'intervention du roi dans la vie de Sabou-Tjéti, chez lequel on lit ceci: "Sa Majesté m'a nommé (littéralement: posé)...". L'énoncé de la fonction a, hélas, disparu, mais la conséquence de cette nomination nous est conservée; elle comportait un rapport du titulaire avec Ptah (26,5-6) ${ }^{3}$. Sabou-Tjéti a été l'objet d'une seconde nomination par le roi (26,9-10), sur laquelle nous n'en savons pas davantage. Il est donc possible de faire remonter la nomination du grand prêtre par ordre royal jusqu'à la fin de l'Ancien Empire.

Les documents antérieurs nous montrent encore à deux reprises le roi intervenant dans la vie des grands prêtres. Mais le caractère de ces interventions est très différent. Le roi accorde la main de sa fille à Ptahchepses I $(\mathbf{4 , 1 0})$ et un souverain probablement différent lui permet de baiser ses pieds au lieu du sol devant lui $\mathbf{( 4 , 1 3 )}$. Quant à Sabou-Ibebi, nous voyons le roi le faire entrer dans la "chambre privée". Ces renseignements sont tirés de textes appelés, un peu improprement, biographies car ils ne mentionnent qu'une sorte d'événement; Ptahchepses I, en se montrant élevé dans le palais, marié à une princesse, ayant accès au palais à toutes les fêtes où le roi se montre à son peuple, occupé aux travaux que Sa Majesté désire faire, ainsi que recevant les faveurs du roi, ne raconte que des relations qu'il a eues avec la cour (4,8-15). Sabou-Ibebi, dont une partie de la "biographie" n'est

${ }^{1}$ Cependant, comme Nesqed n'a peut-être pas eu pour père un grand prêtre (voir au chapitre sur les grands prêtres ptolémaïques), Ptolémée II pourrait l'avoir choisi et pas seulement nommé.

${ }^{2}$ C'est aussi le roi qui "intronise" Ahmèsmen et le nomme directeur des artisans (186, 9-10).

${ }^{3}$ Nous nous basons ici sur la copie du restant de l'inscription faite par A. Mariette. L'assertion de M. STOLK, Ptah, sur l'installation du grand prêtre Sabou-Tjéti (A. MARIETTE, Mastabas, E, 3) par le roi est basée sur les restitutions que K. Sethe a proposées dans les Urkunden. Nous examinons ces restitutions au Chapitre IV. 
qu'une titulature (20,16-18), fait comme Ptahchepses I (20,19-21). Si l'un et l'autre se sont tus sur leur arrivée à la dignité de grand des chefs des artisans, qu'ils prisaient par ailleurs tellement, c'est que, selon nous, cet événement ne dépendait vraisemblablement pas du roi. Ici, comme dans l'examen de la carrière des grands prêtres antérieurement au pontificat, nous sommes amenés à découvrir un changement au cours de la VIème dynastie ${ }^{1}$.

\section{§ 8. LA FIN DE LA CARRIERE DU GRAND PRETRE}

Le défaut complet de renseignements chronologiques précis jusqu'à l'époque ptolémaïque et le haut degré de la fonction de grand prêtre font supposer que la carrière d'un grand des chefs des artisans prenait fin à la mort du titulaire. Les dates fournies par des stèles ptolémaïques confirment la possibilité de cette opinion. Petobastis II est mort le 13 février 76 av. J.-C.; son fils, Psenptaïs II, né le 4 novembre 90 av. J.-C., avait alors eu ses treize ans; comme il dit qu'il a passé treize ans en présence de son père et qu'il a été nommé grand des chefs des artisans tandis qu'il était dans sa quatorzième année, il est infiniment probable que Petobastis II a été grand des chefs des artisans jusqu'à sa mort. Le fils de Psenptaïs II, Imouthès, est mort en 30 av. J.-C.; son cousin et successeur, Psenamounis, a probablement été nommé la même année, ce qui tendrait à prouver qu'Imouthès a été grand prêtre jusqu'à sa mort.

D'autres monuments de la même époque font envisager un autre moment que la mort pour la fin d'une carrière de grand des chefs des artisans. Les données des stèles d'Anemher et de son fils Teos permettent d'affirmer que Teos est mort avant son père. Comme en ce temps-là, il n'était plus question de deux grands des chefs des artisans depuis deux millénaires, Teos nous paraît avoir succédé à son père, qui aurait quitté la fonction de grand des chefs des artisans, mais vraisemblablement gardé celle de directeur des prophètes de tous les

${ }^{1}$ Voir au Chapitre IV l'ensemble des faits dans lequel ces constatations prennent place. 
dieux et déesses de Haute et Basse-Egypte ${ }^{1}$.

Aux époques antérieures, où les dates font complètement défaut, d'autres indications nous invitent à supposer que la carrière d'un grand prêtre pouvait prendre fin avant sa mort. Sabou-Ibebi, lorsqu'il a les traits d'un homme âgé sur sa stèle (20), ne tient plus le sceptre kherep que portent ses images d'un âge plus jeune; en second lieu, aucun des porteurs d'offrandes sur les parois de sa chapelle ${ }^{2}$ n'est directeur des artisans, tandis qu'il y en a chez Ptahchepses IV $^{3}$, son collègue pendant la vie et voisin dans la mort. Ne serait-ce pas que Sabou-Ibebi, à la fin de sa vie, n'exerçait plus la fonction de grand des chefs des artisans ? Nous l'admettons et, rapprochant alors Sabou-Ibebi d'Anemher, nous nous demandons s'il n'existait pas une limite d'âge pour les grands prêtres de Memphis.

S'il était possible à toutes les époques qu'un grand des chefs des artisans ne remplît pas sa fonction jusqu'à sa mort, on comprend qu'Hapidjefa qui finit ses jours loin dans le sud ait pu être grand prêtre de Memphis. Et l'on est en droit de se demander dans quelle partie de leur vie des personnages qui semblent avoir vécu ailleurs qu'à Memphis, tels Méryptah et Ptahmès IV à Thèbes ou Rêhetep à Pi-Ramsès et Abydos, ont été grands prêtres.

\section{§ 9. COSTUMES ET INSIGNES DES GRANDS PRETRES}

Les diverses pièces d'habillement et insignes qu'on rencontre sur les représentations en ronde-bosse ou en bas-relief se combinent, semble-t-il, de toutes les façons possibles. Cette variété provient évidemment, pour une bonne part, des monuments où le grand prêtre est figuré; l'un est assis devant une table d'offrandes qui lui est destinée; l'autre, au contraire, tient le rôle d'officiant, debout, derrière un guéridon supportant les offrandes faites à un dieu. Si chaque époque avait livré une image de grand prêtre célébrant le culte de Ptah, nous pourrions

${ }^{1}$ Voir au Chapitre IV, ainsi qu'au chapitre sur les grands prêtres ptolémaïques, dans la seconde partie.

${ }^{2}$ L. BORCHARDT, Denkmäler..., I, 1937, pl. 21.

${ }^{3}$ M.A. MURRAY, Saqqara Mastabas, I, 1904, pl. 29-31. 
définir le costume ainsi que le ou les insignes de cette fonction. Malheureusement, les ruines de Memphis ne fournissent que peu de documents pour cet examen.

Ceux dont nous avons connaissance datent de la XIXème dynastie, à une exception près. Sur un pilier à base carrée, Hori I (86) présente un pilier djed; son corps est vêtu et orné de la perruque, la tresse de cheveux bouclée, la peau de félin, le pagne, les sandales et la tête de quadrupède du pectoral ${ }^{1}$. Sur sa stèle, Hori I (87), debout et en compagnie de ses grand-père et père, lève les bras en adoration devant Ptah, qui est dans un naos posé sur un traîneau; les deux grands prêtres portent perruque, tresse de cheveux et pagne. Sur une colonne, Hori II (88), un genou en terre et les bras levés en adoration devant une statue assise de Ptah, a perruque, tresse de cheveux, peau de félin, pectoral ${ }^{2}$ et pagne. Sur son bassin à libations, Pahemneter (98), debout, les bras levés en adoration devant Ptah dans son naos ${ }^{3}$, porte perruque, tresse de cheveux, peau de félin et pagne. Khamouas, sur sa statue naophore avec Tatenen trouvée à Karnak $(119)^{4}$, n'a que perruque, tresse de cheveux et pagne. Ce prince apparaît deux fois sur des monuments provenant du temple de Ptah à Mitrahineh; sur une colonne (120) où il est debout les bras levés en adoration, il porte perruque, tresse de cheveux, peau de félin et pagne; sur un bas-relief de paroi $\mathbf{( 1 2 2 )}$, où il est intitulé "Pilier de sa mère et sem", il a perruque et tresse de cheveux. Ailleurs, ce personnage apparaît avec des éléments du pectoral, par exemple sur un demi-tambour de colonne provenant peut-être de son tombeau $(\mathbf{1 4 3})^{5}$. La tenue du grand prêtre sous la XIXème dynastie paraît ainsi comporter la tresse de cheveux attachée à la perruque et la peau de félin, qui sont tous deux les insignes du prêtre sem, ainsi que parfois le pectoral, d'ailleurs assez

${ }^{1}$ Pour ce dernier élément, voir Planche I, fig. 12.

2 Voir Planche I, fig. 13.

${ }^{3}$ La partie inférieure de la scène manque.

${ }^{4}$ Peut-être est-ce un hommage à Ptah de Karnak, qui est aussi "au sud de son mur", fait par Khamouas lors d'un de ses voyages pour annoncer les jubilés de son père.

${ }^{5}$ Voir Planche I, fig. 15. 
différent du modèle ancien ${ }^{1}$.

Deux linteaux du sanctuaire que le roi Siamon de la XXIème dynastie fit construire à Memphis pour le culte d'un "Amon du lapis-lazuli véritable" fournissent par leur comparaison les renseignements peut-être les plus intéressants sur les insignes du grand prêtre. Sur le linteau conservé au Musée du Caire (163), le roi présente une petite figure de Maât, posée sur une corbeille neb au dieu qui est précisément le "neb Maât", Ptah. L'image divine dans son naos posé sur un socle ma est probablement semblable à celle qui était dans le sanctuaire du grand temple de Ptah. Derrière le roi et à peine moins grand que lui, se tient le grand prêtre Paoupaou, vêtu d'une robe qui est serrée à la taille par une ceinture et dont les manches amples descendent jusqu'aux coudes; sa tête est coiffée d'une courte perruque avec la tresse de cheveux bouclée tombant sur l'épaule; il porte la peau de félin par-dessus sa robe et tient une laitue dans une main. L'autre linteau, conservé à Copenhague (164), a une scène semblable, mais, entre autres, la tenue de Paoupaou est différente: un serre-tête emprisonne ses cheveux, un disque avec trois pendeloques orne son oreille, la tête de "chacal" dépasse une de ses épaules'; il porte cependant une robe à manches et la peau de félin comme sur le premier linteau. Ces linteaux montrent que la tenue du grand prêtre de Ptah n'était pas uniforme.

Sans la peau de félin sur le linteau de Copenhague, on pourrait penser que Paoupaou, qui apparaît en sem sur l'autre linteau, a peut-être, en se coiffant d'un serre-tête et en faisant graver une portion du pectoral, voulu souligner sa qualité de grand des chefs des artisans. On trouverait des exemples appuyant cette opinion: la figure de Sabou-Tjéti qui est au milieu du fragment conservé de la stèle (26) a un serre-tête et le pectoral, ainsi d'ailleurs qu'une bande en sautoir ${ }^{3}$; une statue anonyme du Musée du Caire ${ }^{4}$ porte également serre-tête et pectoral ${ }^{5}$. Mais il y

${ }^{1}$ Rêhetep adorant Ptah (103) et Neferrenpet présentant un guéridon au dieu (150) ont la robe-fourreau du vizir. Rêhetep semble avoir été vizir après son pontificat. Il a pu en être de même pour Neferrenpet.

2 Comme sur la Planche I, fig. 12.

${ }^{3}$ Voir Planche I, fig. 3.

${ }^{4}$ Voir chapitre sur les grands prêtres du Moyen Empire.

${ }^{5}$ Voir Planche I, fig. 5. 
a d'autres exemples avec serre-tête, d'où le pectoral est absent: une des statues de Rênefer (9) ${ }^{1}$ et celle de Sabou-kem (13) sous l'Ancien Empire, et toutes les figures de grands prêtres ptolémaïques que nous connaissons: Nesqed, Harmachis, Psenptaïs II et Imouthès. Et il y a aussi des représentations de grands prêtres ornés du pectoral mais coiffés d'une perruque: Ptahmès II et III (38), un grand prêtre anonyme (71). Il nous paraît difficile d'établir une relation précise entre le serre-tête et le pectoral.

Une revue des divers éléments de la tenue des grands prêtres apporte quelques constatations.

Le pagne, retenu par une ceinture ornée d'un pendentif de perles enfilées $^{2}$, est employé régulièrement jusqu'à la fin de la XVIIIème dynastie. Ce vêtement est complété par une large écharpe au Moyen Empire et sous la XVIIIIème dynastie.

La robe apparaît à la XIXème dynastie $(\mathbf{8 2})$ et alterne dès lors avec le pagne.

Les sandales sont parfois portées depuis la XVIIIème dynastie (69).

La perruque, qui a diverses formes, est en usage de l'Ancien Empire à la Basse Epoque.

Le serre-tête, employé parfois dans l'Ancien Empire et toujours sous les Ptolémées, paraît presque délaissé au Moyen Empire et à la Basse Epoque et complètement négligé au Nouvel Empire.

La tresse de cheveux bouclée tombant sur l'épaule apparaît à la XVIIIème dynastie (36) et est très fréquente depuis ce moment.

${ }^{1}$ C'est l'autre statue de Rênefer (10), où il est coiffé d'une perruque, qui porte les traces d'un pectoral dessiné au trait rouge.

${ }^{2}$ L. BORCHARDT, Statuen... II, p. 139, à propos de Caire 584, décrit en détail la ceinture et le pendentif. 
Une petite barbe probablement postiche se trouve sur deux monuments de la XIXème dynastie: bas-relief de Hori II sur sa colonne $(\mathbf{8 8})^{1}$ et statue du prince Khamouas de Karnak $(\mathbf{1 1 9})^{2}$. On la voit déjà sous l'Ancien Empire $(\mathbf{1 1} ; \mathbf{2 1})$.

La peau de félin est employée fréquemment depuis la XVIIIème dynastie. Ainsi, tresse de cheveux et peau de félin, qui sont les insignes du sem, apparaissent à la XVIIIème dynastie. Toutefois, si nous pouvons affirmer leur absence complète de la toilette des grands prêtres de l'Ancien Empire, le petit nombre de monuments du Moyen Empire ne nous permet pas d'en dire autant sur cette époque.

Nous ne nous arrêterons pas aux objets tenus par les grands prêtres, serviettes, bâtons, sceptres ${ }^{3}$, ni aux colliers larges ou autres, à l'exception du pectoral.

Nous appelons pectoral uniquement l'ornement de Khabaouseker ${ }^{4}$ qui paraît rigide et se compose de trois barres ou lamelles découpées en zigzag attachées par leur extrémité supérieure à une pièce faisant le tour du cou et retenant par l'autre bout deux animaux fantastiques. Les douze cordons passés autour du cou de Khabaouseker et enfilés dans des pendeloques en forme de "ankh" et de disques sont un autre ornement ou insigne ${ }^{5}$.

${ }^{1}$ Voir Planche I, fig. 13.

2 Pour Khamouas, la barbe paraît bien postiche, car il y a de nombreuses représentations de ce prince sans barbe.

${ }^{3}$ Le bâton kherep est mis à plusieurs reprises dans la main d'un grand prêtre comme il convient à un our kherep hemout (par exemple: 24, 26, 61).

${ }^{4}$ Voir Planche I, fig. 1.

5 Pectoral et cordons à pendeloques de Khabaouseker ont été étudiés par M.A. MURRAY, Saqqara Mastabas, II, 1937, p. 1-2, qui voit dans les deux appendices partant de la poitrine vers les épaules les parties antérieure et postérieure d'une figure d'Anubis, sans qu'il soit possible de déterminer s'il y avait primitivement un ou deux chacals. Cet auteur, constatant que le pectoral est rare chez les grands prêtres et que Khabaouseker ne porte pas le titre complet du grand prêtre, bien qu'il soit "chef des artisans", conclut: "Il semble donc que l'ornement, au moins dans l'Ancien Empire, doit se rapporter à quelque autre prêtrise, et l'importance qu'y a le chacal suggère fortement une prêtrise d'Anubis". Nous examinerons cette hypothèse un peu plus loin. 
En effet, ces cordons à pendeloques n'accompagnent pas le pectoral dans l'Ancien et le Moyen Empires, Khabaouseker excepté. Ils apparaissent en même temps que le pectoral à la XVIIIème dynastie, mais sous ce dernier; de plus, ils ne font pas le tour du cou mais sont attachés aux barres du pectoral situées à hauteur des clavicules. A la XIXème dynastie, cordons et pectoral se combinent en un ensemble stylisé ${ }^{1}$, de sorte qu'on peut se demander si ces objets existaient encore. Leur absence complète à l'époque ptolémaïque tendrait à le confirmer.

Le pectoral semble avoir été fabriqué en une matière rigide d'après l'image de Khabaouseker et une autre du Moyen Empire ${ }^{2}$; cependant le dessin de Sabou-Tjéti peut signifier que les extrémités étaient posées sur les épaules, permettant ainsi de croire à une semi-rigidité ${ }^{3}$. Dès le Moyen Empire ${ }^{4}$, on relie les extrémités au support circulaire qui fait le

Aucun pectoral ne nous est parvenu. Voici la liste des représentations de pectoraux que nous connaissons, par ordre chronologique. Sauf trois d'entre eux, que nous mentionnons "d'après description", nous les avons vus en photographie, en dessin ou sur les monuments:

- Ancien Empire: (a) Khabaouseker; (b) Rênefer (10) (Caire); (c) Sabou-Tjéti (26) (Caire).

- Moyen Empire: (d) Sehetepibrê-ankh-nedjem et son fils (e) Nebpou (31) (Louvre); (f) anonyme (Caire, inédit).

- Nouvel Empire: (g) Ptahmès II et son frère; (h) Méryptah et (i) Ptahmès III (38) (Leyde); (j) Ptahmès III (69) (Florence, d'après description); (k) anonyme (MURRAY, Saqqara Mastabas, I, pl. 36,3); (l) anonyme (Caire 870, BORCHARDT, Statuen, III, p. 131); (m) anonyme (78) (Caire); (n) anonyme (Salle St-Ferriol, MORET, dans Revue Egyptologique, I, 1919, p. 173-174 et pl. V opp. p. 168); (o) anonyme (71) (Berlin 12410); (p) Hori I (86) (Caire); (q) Hori II (88) (Caire); (r) Pahemneter (101) (Louvre); (s) prince Khamouas (143) (Caire); (t) idem (Louvre, d'après description, BOREUX, Ant. ég., II, 1932, p. 481).

- Basse Epoque: (u) Paoupaou (164) (Copenhague); (v) Peftjaouaouibastet (181 a) (Berlin, d'après description; obligeamment signalé par G. Posener).

Il faut y ajouter le signe hiéroglyphique du pectoral au Nouvel Empire (71) (Berlin).

Plusieurs de ces représentations sont reproduites sur notre Planche I.

${ }^{1}$ Voir Planche I, fig. 13 et 14 (début de la XIXème dynastie). Cette combinaison des deux éléments ornementaux de Khabaouseker nous a fait attribuer à la XVIIIème dynastie les images de pectoraux ( $\mathrm{k}, \mathrm{l}, \mathrm{m}$ et $\mathrm{n}$ de la note précédente).

${ }^{2}$ Voir Planche I, fig. 1 et 5, Planche II, fig. 5. M.A. MURRAY, op. cit., 1937, a déjà conclu à la rigidité du pectoral.

${ }^{3}$ Voir Planche I, fig. 3a; même remarque sur fig. 11a et 13.

${ }^{4}$ Planche I, fig. 4. 
tour du cou. L'ensemble est ainsi consolidé, mais il faut remarquer que dans le plus ancien exemple que nous possédions du pectoral renforcé, une des attaches fait collier autour du cou du quadrupède.

Sauf dans deux cas du Moyen et du Nouvel Empires ${ }^{1}$, le support autour du cou est une simple couronne. Les trois attaches qui en descendent en éventail sur la poitrine sont toujours en zigzag et aboutissent à une barre rectiligne, sauf chez Khabaouseker. L'animal qui vient s'appuyer contre l'épaule droite de l'homme décoré du pectoral ${ }^{2}$ a une tête et un corps de quadrupède qu'on peut identifier aussi bien à un loup qu'à un chacal, et ses pattes antérieures sont des bras humains. L'appendice qui se dirige vers l'autre épaule représente nettement le corps, les deux paires de pattes et la queue d'un quadrupède sur quatre représentations appartenant aux Ancien, Moyen et Nouvel Empires ${ }^{3}$. Mais cet élément du pectoral a aussi été incompris dès l'Ancien Empire ${ }^{4}$; au Moyen Empire, on y a vu un oiseau ${ }^{5}$. La signification de ces animaux reste pour nous un problème ${ }^{6}$.

La plupart des pectoraux sont portés par des grands des chefs des artisans; quelques-uns le sont par des personnages qui sont plus ou moins probablement grands prêtres ${ }^{7}$; deux enfin ornent des dignitaires qui ne portent pas le titre de grand des chefs des artisans. Le plus récent de ceux-ci, le prophète et majordome du temple de Nebmaâtrê construit par Aménophis III à Memphis, Méryptah, est frère du grand prêtre de Ptah, Ptahmès II et fils du vizir Thoutmès. Est-ce en tant qu'administrateur d'un temple memphite qu'il porte un pectoral ? Nous ne voyons pas pour

\footnotetext{
${ }^{1}$ Planche I, fig. 5 et 10; Planche II, fig. 5.

${ }^{2}$ Parfois aussi contre l'épaule gauche (voir Planche I, fig. 1, 12 et 13) par une des conventions du dessin égyptien,

${ }^{3}$ Voir Planche I, fig. 1, 5, 6 et 10; Planche II, fig. 5 et 6.

${ }^{4}$ Voir Planche I, fig. 3 a.

${ }^{5}$ Planche I, fig. 4, qui a permis d'interpréter les deux animaux comme Horus et Seth.

${ }^{6}$ Comme le pectoral est un objet exclusivement memphite, la liaison de deux quadrupèdes, l'un sans queue et l'autre sans tête, pourrait-elle avoir un rapport quelconque avec la réunion des deux pays ? Dans un cas pareil, le pectoral ne serait pas antérieur à la Ière dynastie. Par contre, ces animaux qui ne sont pas seulement incomplets, mais aussi déformés, peuvent faire penser à ceux des palettes protohistoriques, qui sont également déformés.

${ }^{7}$ Ceux que nous avons signalés sous lettres f, k, 1, n, o dans la note 5, p. 27-28.
} 
quelle autre raison sa poitrine serait ornée de cet insigne. Toutefois, il est nécessaire de remarquer que le monument où apparaît le majordome Méryptah est incomplet (38); il y manque tout le texte du deuxième registre et la fin des proscynèmes verticaux ${ }^{1}$. Par contre le bon état de conservation du monument de Khabaouseker interdit de penser qu'il ait pu être grand des chefs des artisans.

Dans ces conditions, on peut se demander à quelle fonction le pectoral est lié. M.A. Murray y a vu l'insigne d'une prêtrise d'Anubis. Mais il y a deux objections à émettre contre cette hypothèse. Même s'il s'agit d'un chacal, c'est un animal dont deux membres sont humains, ce qui ne nous rapproche guère des représentations d'Anubis. En second lieu, Khabaouseker porte bien un titre où entre le nom d'Anubis, mais il n'en est pas ainsi pour les grands prêtres de l'Ancien Empire et des époques suivantes ${ }^{2}$. Il est donc difficile d'admettre que le pectoral est en relation avec Anubis. C'est pourquoi nous préférons aborder le problème d'une autre façon. Si Khabaouseker, qui n'est pas grand des chefs des artisans, et des grands des chefs des artisans portent le même insigne, on est en droit de se demander s'ils ont un autre point commun. Dans la négative, la signification du pectoral resterait une énigme. Mais Khabaouseker porte un titre qui peut être confronté avec celui du grand prêtre de Ptah. Il est kherep hemout iset ${ }^{3}$, "chef des artisans du Palais" ${ }^{\prime 4}$, et le grand prêtre de Ptah est our kherep hemout "grand des chefs des artisans".5. L'un et les autres commandent des artisans, et l'on peut supposer que le

${ }^{1}$ Si le majordome Méryptah se révélait grand prêtre, il y aurait lieu de voir s'il ne serait pas identique au grand prêtre de ce nom que nous connaissons $(\mathbf{7 9}=\mathbf{8 1})$.

2 Sauf erreur, il faut descendre à l'époque ptolémaïque pour rencontrer des grands prêtres exerçant une fonction pour Anubis (198,2; 200,13).

${ }^{3}$ Ou kherep hemout is, le dernier mot étant écrit au moyen d'un seul signe.

${ }^{4} \mathrm{Ou}$ "chef des artisans du tombeau". Il s'agirait ici du tombeau du roi: l'épithète que Khabaouseker se décerne après ce titre, "connaissant la pensée parfaite (litt.: ce qui est bon dans le coeur) de son maitre", le laisse entendre.

${ }^{5}$ Nous ne comparons pas le titre de Khabaouseker à "chef des artisans" ou "chef de tous les artisans", parce que nous ne connaissons pas de pectoral chez les "directeurs des artisans" et chez ce "chef de tous les artisans" déjà signalé au $\S 4$, qui ne sont pas grands des chefs des artisans. 
pectoral est l'insigne de cette fonction de chef des artisans ${ }^{1}$. Il n'y aurait alors plus rien d'étonnant au fait que le pectoral n'est pas toujours représenté sur les images des grands prêtres de Ptah qui, en dehors de cette fonction civile qu'était la direction des artisans, avaient d'autres charges à remplir².

Nous résumerons nos recherches sur la toilette et la parure des grands prêtres de Memphis en disant qu'elles ont varié. Bien qu'aucun détail vestimentaire ou insigne ne paraisse leur appartenir exclusivement, le pectoral, ornement spécifiquement memphite et peut-être lié originairement à la direction des artisans, est porté assez souvent par les grands prêtres jusqu'au Nouvel Empire.

\section{§ 10. REVENUS ET PERSONNEL DES GRANDS PRETRES}

Ptahmès III, au Nouvel Empire et Psenptaïs II sous les Ptolémées, donnent chacun une indication sur leurs revenus. Le premier, s'adressant à ses successeurs pour leur demander de réciter la formule d'offrandes en sa faveur, les assure qu'en échange Ptah les récompensera, qu'ils recevront ses aliments chaque jour, et qu'ils récolteront (?) ce qui est dans (ses ?) champs $(\mathbf{6 9 , 8})$. Ces paroles signifient que la nomination à la fonction de grand prêtre de Ptah entraînait un droit portant sur les aliments présentés chaque jour au dieu et sur les récoltes provenant des terrains appartenant au dieu.

Lorsque Psenptaïs II fut nommé prophète de Ptolémée Neos-Dionysos, des pains provenant des temples de Haute et Basse-Egypte lui furent donnés chaque année, par la suite $(\mathbf{2 0 0 , 2 0})$. D'autres grands prêtres qui se qualifient aussi de prophètes du roi, soit Nesqed (190,3-4) et Chedsounefertoum $(\mathbf{1 6 9 , 4 )}$ ont vraisemblablement joui du même

${ }^{1}$ Il serait alors plausible de faire remonter le pectoral au temps prédynastique qui vit naître le travail des vases en pierre, si l'on admet - à cause de l'outil représenté par le hiéroglyphe du mot artisan, soit une foreuse à main, que les hemout ont été d'abord les artisans qui fabriquaient les vases en pierre.

${ }^{2}$ Nous ne tenons pas compte, ici, de cette possibilité: si Khabaouseker n'est pas grand des chefs des artisans, serait-ce que ce titre n'existait pas à son époque ? Voir, à ce sujet, le Chapitre III. 
privilège. Et comme l'autorité des grands prêtres de Ptah s'est quelquefois étendue aux prophètes de tous les dieux et déesses de Haute et Basse-Egypte dès la XVIIIème dynastie, on peut admettre que certains grands prêtres de Memphis ont eu une part de leurs revenus qui provenait de toutes les régions de l'Egypte dès le Nouvel Empire.

Les déclarations de Ptahmès III et Psenptaïs II se complètent très probablement et Psenptaïs II a dû ajouter son revenu annuel de prophète du roi au revenu quotidien de grand des chefs des artisans. Comme les grands prêtres de Ptah cumulaient un certain nombre de fonctions, leurs revenus étaient, sans doute, importants et ont nécessité des entrepôts, attestés seulement à l'époque ptolémaïque, mais peut-être bien antérieurs (206) ${ }^{1}$.

A défaut d'autres renseignements sur la nature et le montant des revenus des grands prêtres de $\mathrm{Ptah}^{2}$, nous rencontrons parfois des signes de grande richesse chez certains d'entre eux. Sabou-Ibebi, regardant les boeufs amenés des bourgs de sa fondation funéraire, dénombre 435 têtes de bétail ${ }^{3}$. Ce grand prêtre de l'Ancien Empire possédait aussi deux bateaux ${ }^{4}$. Au Moyen Empire, Senousert-ankh a pu se faire bâtir une tombe imposante (33-35), ainsi, probablement, que le grand prêtre du Nouvel Empire dont le cortège funèbre est représenté sur un magnifique bas-relief (70). Psenptaïs II, sous Cléopâtre, raconte qu'il a été un riche en toutes sortes de biens et qu'il a possédé un beau harem $(\mathbf{2 0 0 , 2 2})$.

${ }^{1}$ Le "Magasin du grand des chefs des artisans" est, en effet, plus qu'un simple grenier, puisqu'il possède un sanctuaire dédié à Ptah et que son nom est déterminé par le hiéroglyphe de la ville. Comme nous n'avons aucun monument qui nous permette d'affirmer que les grands prêtres ptolémaïques ont exercé une activité régulière et prolongée dans une autre ville que Memphis, nous estimons que le lieu appelé "Magasin du grand des chefs des artisans" était situé à Memphis ou tout près de cette ville et que la réserve faite par SANDMAN, Ptah, p. 251, sur cette localisation est excessive.

${ }^{2}$ Le grand papyrus Harris nous apprend ce que le dieu Ptah, et non son grand prêtre, a reçu de Ramsès III. Une part de ces donations est revenue ensuite au grand prêtre, mais nous ignorons de quelle manière et dans quelles proportions.

${ }^{3}$ Caire 1419, 8ème registre $=$ L. BORCHARDT, Denkmäler des Alten Reiches, I, 1937, pl. 21.

${ }^{4}$ Ibidem, 7ème registre. 
Les fortunes en nature des grands des chefs des artisans réclamaient un personnel citadin et rural propre à chacun d'eux. En dehors de cette domesticité et du personnel de Ptah placé sous sa direction ${ }^{1}$, nous ne pouvons pas affirmer qu'il y ait eu un personnel attaché à la fonction du grand des chefs des artisans, en d'autres termes qu'il ait existé une maison du grand prêtre de Memphis analogue à la maison du grand prêtre d'Amon de Karnak. En effet, le supérieur des chanteurs du temple de Ptah, Ptahankh, est le serviteur d'un grand prêtre déterminé (59-61) et le jeune Neferrenpet du bas-relief de Berlin (70) peut tout aussi bien être le supérieur des suivants du grand des chefs des artisans enseveli dans le tombeau d'où vient le bas-relief qu'un supérieur des suivants qui aurait été attaché à la fonction de grand prêtre.

Au cours de ce chapitre, nous avons fait les constatations suivantes.

Le grand prêtre de Ptah de Memphis porte principalement le titre de "grand des chefs des artisans" ou, dans quelques cas exceptionnels, celui de sem, souvent aussi les deux. Il peut être recruté dans toutes les classes sociales, mais sa fonction tend à devenir héréditaire au cours des âges. Avant de parvenir au pontificat, le grand prêtre de la Vème et du début de la VIème dynastie semble suivre une filière et sa nomination ne paraît pas être faite par le roi. Depuis la VIème dynastie, c'est le roi qui nomme le grand prêtre. La carrière de ce dernier ne se termine pas toujours à sa mort.

La toilette des grands prêtres a varié. Le pectoral, porté parfois par eux jusqu'au Nouvel Empire, puis oublié par la suite, a pu être à l'origine un insigne en rapport avec la direction des artisans. Ce qu'il représente reste un problème.

Le revenu des grands prêtres a pu être considérable à toutes les époques, mais il ne se laisse pas évaluer avec précision.

${ }^{1}$ Ramsès III a donné à Ptah et mis sous la direction de son grand prêtre 841 personnes (162a). 



\section{H A P I T R E II}

\section{LES FONCTIONS DU GRAND PRETRE}

\section{$\S 11$. FONCTIONS CIVILES}

Sous l'Ancien Empire, le grand des chefs des artisans dirige, comme son nom l'indique, l'ensemble des artisans. Ceux-ci, dont une partie sculpte la pierre calcaire pour la décorer de textes et de figures en basrelief $(\mathbf{2})$, ou sculpte des statues $(\mathbf{1 , 1 1})$, ou peut-être travaille à aplanir les blocs de granit revêtant la pyramide de Mycérinus $(\mathbf{1 , 3})$, tandis que d'autres fabriquent de la faïence ${ }^{1}$, forment la ouabet ou atelier $(2,4)^{2}$. Comme la ouabet, qui est aussi appelée la ouabet du roi $(7,2)$, est un organisme civil, le "grand des chefs des artisans" est, en prenant ce terme dans son sens littéral, un haut fonctionnaire, au moins sous la IVème, la Vème et le début de la VIème dynasties. Nous pensons qu'il doit à cette qualité d'être "celui qui est dans la double maison", c'est à dire de faire partie de l'administration centrale de l'Egypte. D'autre part, la direction de la ouabet amène parfois le grand des chefs des artisans à être l'architecte du roi $^{3}$ ou, au moins, à participer aux projets architecturaux du souverain ${ }^{4}$.

${ }^{1}$ Ptahchepses I dirige, ou a dirigé dans la partie de sa carrière antérieure au pontificat, l'atelier où l'on fabriquait la faïence et il connaît la fabrication elle-même; il se déclare, en effet, "supérieur des secrets et chef de la maison de la faïnce" $(4,5)$.

2 Nous supposons, à cause de son titre, que le "chef de la maison de la faïence", est un des chefs ou directeurs des artisans formant cette espèce de conseil qu'aurait présidé plutôt que dirigé le grand des chefs des artisans (voir $\$ 4$ ci-dessus).

${ }^{3}$ Litt. "directeur de tous les travaux du roi" $(20,2 ; 29,2)$.

4 "Supérieur des secrets de tous les travaux que Sa Majesté désire faire" (4,12; 20,16). 
Au Moyen Empire, Senousert-ankh se fait construire un magnifique tombeau à Lisht (33), sans doute pour reposer plus tard auprès de son maître, mais aussi, pensons-nous, parce qu'il dirige les artisans travaillant à la tombe du roi.

Nous supposons que le grand des chefs des artisans des époques suivantes continue à commander aux corps de métier memphites puisqu'un grand prêtre saïte et un ptolémaïque parlent de leur relation avec l'atelier d'orfèvrerie ${ }^{1}$.

La direction des artisans est la seule fonction civile du grand prêtre de Ptah dont nous puissions affirmer l'existence à travers toutes les époques de l'Egypte ancienne. Nous ne pouvons pas, en effet, généraliser certaine fonction policière remplie par le prince Khamouas ${ }^{2}$.

Par contre, le grand des chefs des artisans porte des titres honorifiques dès la fin de l'Ancien Empire: compagnon unique $(\mathbf{2 6 , 2})$, trésorier du roi de Basse-Egypte $(\mathbf{2 7 , 2})$; depuis le Moyen Empire $(\mathbf{3 1}, \mathbf{2})$ il est presque toujours noble et gouverneur ${ }^{3}$.

\section{§ 12. SERVICE DE PTAH}

C'est en tant que "prophètes de Ptah" que les grands prêtres de l'Ancien Empire célèbrent régulièrement le culte du dieu, puisque leur

1 "Chef de la maison de l'or" $(\mathbf{1 8 8 , 3})$; au courant de la fabrication des objets en or (litt.: "connaissant les secrets de la maison de l'or") (193,5-6).

2 133; ce document est un rapport adressé par un préposé aux troupeaux à son maître Khamouas au sujet d'esclaves fugitifs qui appartenaient à un autre fils de Ramsès II. On y lit: "Mon maître m'a ordonné d'aller chercher six esclaves (litt. suivants) etc.".

${ }^{3}$ Des grands prêtres de l'Ancien Empire sont Imakhou. Puisqu'un Sabou-Tjéti n'a peut-être plus exercé sa fonction de grand des chefs des artisans à la fin de sa vie (voir $\S 8$ ), on peut se demander si sa qualité d'imakhou du roi $(\mathbf{1 9 , 1 )}$ ne lui valait pas des gratifications régulières équivalant en fait à une espèce de pension. La même question pourrait se poser au sujet de Ptahchepses I, qui se dit simplement imakhou (4,1) et a vécu sous une série de rois. Sur l'imakh royal, cf. J. SAINTE FARE GARNOT, L'imakh et les imakhous sous l'Ancien Empire, Melun, 1943, p. 25-26. 
fonction de "grand des chefs des artisans" a un caractère civil ${ }^{1}$. D'autre part, comme il n'y a pas de degrés différents, à cette époque, dans le plus haut personnel d'un dieu, soit chez les "serviteurs du dieu" ou "prophètes" ainsi qu'il y en aura par la suite, et comme il existe des "directeurs des artisans" qui sont aussi "prophètes de Ptah"2, il est vraisemblable que le grand des chefs des artisans partage alors avec ces autres prophètes de Ptah au moins le service régulier du dieu. Et comme les grands prophètes de Ptah contemporains des Ptolémées font connaître qu'ils appartenaient à l'une des tribus ou équipes qui se succédaient au service du dieu, nous estimons qu'une division analogue, à laquelle le grand prêtre lui-même n'échappait pas, a pu exister chez le personnel de Ptah de l'Ancien Empire.

Au Moyen Empire, le grand des chefs des artisans porte encore le titre de prophète de Ptah (35), qu'il échange au Nouvel Empire contre celui de "père divin aimé du dieu" (38,2; 68; etc.); il a alors auprès de lui des pères divins ${ }^{3}$ avec lesquels il continue à partager le service ordinaire de Ptah. Cette coopération continue dans les époques suivantes. Sous les Ptolémées, d'autres serviteurs de Ptah que le grand prêtre s'intitulent aussi pères divins aimés du dieu, mais le grand prêtre fait à son tour revivre le titre de prophète de $\mathrm{Ptah}^{4}$. Ainsi, tout au long de l'histoire d'Egypte, le grand prêtre de Memphis paraît avoir été ce primus inter

${ }^{1}$ Nous avons relevé que tous les grands des chefs des artisans de l'Ancien Empire dont nous possédons un monument complet de quelque importance sont prophètes de Ptah (voir § 3); des trois qui ne le sont pas, ou plus expressément, Neferef-rê-ankh est directeur du domaine de Sokar (8) et, comme tel, a aussi fait partie du personnel de Ptah (voir § 13); Sabou-Tjéti affirme ses relations avec le temple de Ptah $(\mathbf{2 6 , 6 )}$; quant à Ptahchepses-Impy (29), sa titulature ressemble singulièrement à celle de Sabou-Tjéti, de sorte qu'on est fondé à admettre à son sujet ce qu'on admet pour Sabou-Tjéti.

2 Voir Personnel de Ptah.

${ }^{3}$ Voir Personnel de Ptah. A.H. GARDINER, Ancient Egyptian Onomastica, Text, Vol. I, 1947, p. $47^{*}-53^{*}$, adopte la traduction "père du dieu" au lieu de père divin et émet à ce sujet l'hypothèse suivante: le père du dieu aurait été n'importe quel prêtre de condition et d'âge suffisant pour qu'il put s'attendre à ce que Pharaon adoptât une attitude filiale envers lui. Dans notre travail, nous nous sommes tenu à la traduction de G. LEFEBVRE, Grammaire, $\S 115$ b.

${ }^{4}$ Idem. Les grands prêtres ptolémaïques Psenptaïs II $(\mathbf{2 0 1 , 6 )}$, Imouthès $(\mathbf{2 0 3 , 5})$, Psenamounis $\mathbf{( 2 0 3 , 8 )}$ sont "prophètes de Ptah". 
pares que son principal titre avait laissé entrevoir ${ }^{1}$.

Le culte rendu à Ptah dans le saint des saints du temple de Memphis ${ }^{2}$ comprend la parure ${ }^{3}$ et l'alimentation du dieu ${ }^{4}$. Nous en déduisons que le rituel du culte divin journalier que nous connaissons par ailleurs est aussi celui de Ptah de Memphis.

En dehors de son culte journalier, Ptah de Memphis a sa fête, qui est conduite soit par le grand des chefs des artisans $(\mathbf{1 0 3 , 1 5})$, soit par d'autres membres importants du personnel religieux de Memphis ${ }^{5}$. C'est vraisemblablement cette solennité qui est brièvement décrite à l'époque de Ramsès III, auquel on a prêté, après sa mort, les paroles suivantes ${ }^{6}$ :

"I'ai construit ta barque auguste, appelée Seigneur d'éternité, qui mesure cent trente coudées sur le fleuve, avec de grands bois de cèdre neuf, du meilleur de la plantation. Sa cabine $e^{7}$ est en or et en pierres précieuses

1 Voir ci-dessus, $\$ 4$.

2 "Saint des saints" est employé par G. LEFEBVRE, Histoire, pour désigner la "grande place" des textes égyptiens. Celle-ci est mentionnée dans quelques épithètes de grands prêtres memphites, à partir du Nouvel Empire: "initié à ce qui est ou à ce qui se passe dans le saint des saints" (litt.: "supérieur des secrets de la grande place") $(\mathbf{3 7 , 3 ;} \mathbf{3 8 , 2}$; 188,2); "ayant accès au saint des saints" (litt.: "ayant accès aux secrets de la grande place") $\mathbf{( 6 9 , 2 - 3 ) ; ~ " m a i ̂ t r e ~ d e ~ s e s ~ a l l e ́ e s ~ e t ~ v e n u e s ~ d a n s ~ l e ~ s a i n t ~ d e s ~ s a i n t s " ~ ( 1 0 1 , 2 ; ~ 1 8 6 , 1 - 2 ) . ~}$

${ }^{3}$ Cf. l'épithète "rajeunissant la poitrine de Celui qui est au sud de son mur" $(\mathbf{7 9 , 2 )}$ et, avant le Nouvel Empire, "préposé au diadème quand Ptah est orné" (26,3; 27,2; 32,2; 186,1 est une exception apparente dans un texte archaïsant que nous examinerons dans le chapitre sur les grands prêtres de Basse Epoque).

${ }^{4} \mathrm{Cf}$. les épithètes "faisant en sorte que le dieu soit satisfait de ses repas" (69,5), "qui alimenterez ses (= de Ptah-Sokar) tables d'offrandes" (189,4); "faisant les libations à Ptah" $(\mathbf{1 8 8 , 2})$, et, avant le Nouvel Empire, "directeur de la place des aliments" $(\mathbf{4 , 4 )}$.

${ }^{5}$ Voir Personnel de Ptah, Nouvel Empire.

${ }^{6} \mathrm{~S}$. BIRCH, Facsimile of an Egyptian Hieratic Papyrus of the reign of Ramses III, 1876, pl. 48,11 - 49,3; transcription: W. ERICHSEN, Papyrus Harris I (Bibliotheca Aegyptiaca, V, 1933, p. 54-55). Traduction du second alinéa dans SANDMAN, Ptah, p. 192.

${ }^{7}$ Litt. "grande maison". Le même terme se trouve dans une épithète de grand prêtre, "purificateur de la Grande maison" (119,5; 124,4; 179,17; 166,4 ?), mais il semble plutôt désigner ici le naos contenant la statue de Ptah dans le saint des saints. Ce serait alors par extension de sens qu'il aurait désigné la cabine de la barque, qui servait de naos pendant le voyage de Ptah. 
véritables jusqu'à l'eau (?), et en or de tous ses côtés. Sa proue porte des faucons de bon or avec des incrustations de toute sorte de pierres précieuses, d'un travail aussi beau que la barque du matin. Sa poupe est en bon or; les rames-gouvernails qu'elle porte (?) et les figures de dieux (au bout de ces rames) sont de bon or.

Ptah au beau visage, qui est au sud de son mur, apparaît pour prendre place dans sa cabine comme Akhty (le dieu de l'horizon), le coeur satisfait et joyeux à sa vue, et pour accomplir son beau voyage sur les eaux de sa fille, la Maîtresse du Sycomore au sud du Mur (= Memphis). Tout le peuple se réjouit à sa vue et des cris de joie le précèdent jusqu'à sa maison auguste (c'est-à-dire jusqu'à son retour dans son temple)."

La visite de Ptah à Hathor rappelle la fête annuelle au cours de laquelle Amon de Karnak naviguait en grande pompe jusqu'à Louxor, et ce que nous savons sur celle-ci permet d'imaginer le faste de la solennité memphite lorsque le roi lui-même y participait. Car Ptah, bien qu'il ne fût pas aussi fabuleusement riche que son rival thébain au Nouvel Empire, n'en possédait pas moins une fortune considérable.

Nous ne connaissons pas de mention de grands des chefs des artisans en rapport avec une des autres fêtes de Ptah (sur ces fêtes, cf. A. RUSCH, Phthas, dans PAULY-WISSOWA, Real-Encyclopädie..., XXXIX, p. 943,1,47-63), ce qui ne signifie évidemment pas que les grands prêtres n'y ont pas participé.

Aussi, l'administration des biens de Ptah réclamait-elle certainement les soins du grand prêtre, secondé dans cette tâche par un "grand majordome du domaine de Ptah" au moins dès le Nouvel Empire. Il semble que le grand prêtre ne s'est jamais occupé personnellement que de cette partie de la richesse de Ptah qui consistait en réserves emmagasinées ${ }^{1}$. A notre connaissance, il n'a jamais été en rapport direct avec les biens-fonds du dieu. C'est pourquoi Ramsès III, donnant huit

1 Ptahchepses I est "directeur de la place des aliments" (4,4); Chedsounefertoum vérifie les livraisons faites au trésor et remplit les greniers de Ptah (169,9 et suiv.). Les magasins de Ptah ont même eu, sous les Ptolémées, leur statue du dieu servie par le grand prêtre: Anemher est "prophète de Ptah seigneur des aliments du temple de Memphis" (192,7). 
cent quarante et une personnes au domaine de Ptah pour le temple portant son nom, place ces gens non seulement sous la direction du grand des chefs des artisans mais en même temps des "principaux" (162a).

On peut supposer enfin que le grand prêtre de Ptah a pris une part active aux constructions de sanctuaires memphites, en tant que grand des chefs des artisans (123).

\section{§ 13. SERVICE DE SOKAR}

Le grand des chefs des artisans célèbre le culte de Sokar sous l'Ancien Empire en qualité de "prophète de Sokar"1. Comme pour le service de Ptah, il partage cette fonction religieuse avec d'autres prophètes de Sokar qui ne sont pas des grands des chefs des artisans, mais des directeurs ou chefs des artisans ${ }^{2}$. Par contre, le grand des chefs des artisans administre seul le domaine de Sokar, en tant que "directeur du domaine de Sokar" $\left(3\right.$ à 24) ${ }^{3}$.

A cette époque, Sokar n'est plus seulement le dieu de Ro-setau ${ }^{4}$, soit de cette partie du désert qui est en bordure de la plaine memphite, mais aussi d'autres lieux ${ }^{5}$, parmi lesquels il est plausible d'inclure le temple de Ptah puisque les titres de "prophète de Ptah" et de "prophète de Sokar" sont réunis $(3,4 ; 4,6 ; 5,4 ; 7,1,3 ;$ etc.) ou cités parallèlement $(\mathbf{1 4 , 1 - 2})$ la plupart du temps ${ }^{6}$.

${ }^{1}$ Nous ne contredisons pas le résultat acquis au $\$ 6$; l'accès à la fonction civile de grand des chefs des artisans ne nécessitait en effet pas l'abandon de la fonction religieuse de prophète.

${ }^{2}$ Voir Personnel de Ptah, Ancien Empire.

${ }^{3}$ La mention de directeur du domaine de Sokar chez les grands prêtres de l'Ancien Empire fait penser que l'absence d'un titre analogue concernant Ptah n'est pas fortuite et confirme ainsi que le grand prêtre de l'Ancien Empire n'administre pas directement le domaine de Ptah.

${ }^{4}$ Pyr. $\$ 445$.

${ }^{5}$ Voir "prophète de Sokar dans toutes ses places" $(4,6 ; 20,10)$; "directeur du château de Sokar en toutes places" $(\mathbf{1 8 , 6 )}$.

${ }^{6}$ La présence de Sokar dans le temple de Ptah explique que le terme géographique "Ro-setau" n'apparaisse qu'à la XIXème dynastie dans un titre porté par un grand prêtre 
Ce rapprochement dans l'espace a permis à Ptah de s'approprier peu à peu Sokar. Sous la Vème dynastie, Sokar a encore ses desservants particuliers, ses $\mathrm{sem}^{1}$ dirigés par un "chef des sem", mais ses prophètes sont les mêmes que les prophètes de Ptah. Au Moyen Empire, Sokar continue à avoir ses prophètes (35), mais il n'a plus ces sem que nous venons de mentionner ${ }^{2}$, ni son directeur de domaine. C'est donc que le domaine de Sokar avait passé à Ptah qui, en échange, assurait sans doute le nécessaire au culte de Sokar célébré dans le temple de Ptah.

Au Nouvel Empire, même les prophètes de Sokar ont disparu. L'assimilation de Sokar et de Ptah est alors complète ${ }^{3}$. Toutefois Sokar existe toujours à Memphis dans le temple de Ptah, sous la forme d'un

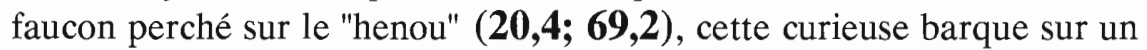
support magnifiquement représentée en $\mathrm{Abydos}^{4}$. Et la fête de Sokar, quoique d'un caractère différent de celle de Ptah, revêt peut-être encore plus d'éclat que cette dernière. Le grand prêtre en personne dirige la cérémonie initiale au cours de laquelle le henou est placé sur un traîneau $(159 ; 186,4)$ car, contrairement à ce qui se passe pour Ptah, qui embarque, Sokar ne quitte pas la terre ferme et la partie principale de sa fête consiste en une procession autour du Mur Blanc ${ }^{5}$. Le grand prêtre hale alors lui-même le henou $(69,2)$.

A défaut de bas-reliefs memphites, ceux d'Abydos contiennent un enseignement intéressant l'un des titres du grand prêtre de Ptah. Lorsque Séti I célèbre la fête de Ptah-Sokar, la présentation de l'offrande au dieu par le pharaon est illustrée par cette légende: "Apporter l'offrande à son

de Ptah (156,12).

${ }^{1}$ Voir ci-dessus, § 5.

2 186,1 n'est qu'une exception apparente dans un texte archaïsant déjà signalê au paragraphe précédent.

${ }^{3}$ Séti Ier en Abydos "célèbre la fête de Ptah-Sokar" (H. BRUGSCH, Etudes géographiques, dans Revue Egyptologique, I (1880), p. 45).

${ }^{4}$ A.M. CALVERLEY et M.F. BROOME, The Temple of King Sethos I at Abydos, IV, pl. 38,B; H. KEES, Götterglaube, 1941, pl. VII b, d'après J. CAPART, Le temple de Séti Ier, pl. 49.

5 A Dendérah, le roi halant le "henou" de Sokar a pour légende: "le setem fait le tour du Mur Blanc" (H. BRUGSCH, Etudes géographiques, dans Revue égyptologique, I (1880), p. 42-43. 
père Sokar par son fils, le sem, maître des Deux Pays, Menmaâtrê, etc. dans la maison du "henou"1. Le roi se présente en sem devant Sokar, ce qui nous fait envisager que le même titre, porté par les grands des chefs des artisans depuis la fin de l'Ancien Empire ${ }^{2}$, signifie au moins partiellement "le serviteur de Sokar"3. Ainsi la fréquence des mentions simultanées de "grand des chefs des artisans" et de sem s'expliquerait en partie par le rapport existant entre sem et "Sokar" d'une part et "grand des chefs des artisans" et "Ptah, patron des artisans" d'autre part, quoique cette fréquence signale peut-être au début le double caractère du grand prêtre de Memphis, civil chez le grand des chefs des artisans et religieux chez le sem $(26,4 ; 27,1)^{4}$.

\section{§ 14. SERVICE DU ROI}

Bien que ce ne soit qu'à partir du Nouvel Empire que nous ayons des témoignages du rôle de premier plan tenu par le grand prêtre de

1 Ibidem, p. 45.

2 Voir ci-dessus, $\$ \mathbf{5}$.

${ }^{3}$ Le passage du Livre des Morts, chapitre ler (159) où il est question du grand des chefs des artisans et du sem ne pourrait donc en tout cas pas être antérieur à la VIème dynastie, à cause du second de ces titres. Nous pensons même qu'il date d'une époque plus récente dans laquelle "grand des chefs des artisans" signifiait déjà pour des noninitiés "le grand prêtre au service des dieux memphites". Ce serait la raison pour laquelle le texte en question relie la mise du "henou" sur un traîneau au grand des chefs des artisans. Cette interprétation semble confirmée par la suite du passage: après avoir attribué au grand des chefs des artisans un rite qui, croyons-nous, était dirigé par le sem, le rédacteur s'est trouvé pris de court pour caractériser la fonction du sem et a recouru à une formule générale.

${ }^{4}$ Il n'y a rien d'étonnant à ce que le serviteur d'un dieu à caractère funéraire comme Sokar et le prêtre accomplissant les rites qui redonnent vie au cadavre portent le même titre, d'autant que Sokar et Osiris se sont amalgamés en Sokar-Osiris. A.H. GARDINER, Ancient Egyptian Onomastica, 1947, Text, Vol. I, p. 41*, constate que "l'association particulièrement étroite qui s'est développée" entre le prêtre sem et Ptah est difficile à expliquer - à moins cependant que Ptah, lui-même un joaillier, attachât plus de prix à la parure personnelle que d'autres dieux. Dans l'unique cas de titre d'un grand prêtre de Ptah de l'Ancien Empire cité ci-dessus (= Sabou-Tjéti, 26,4), il est appelé "ami unique qui embellit la tête en ornant Ptah." La raison invoquée par Gardiner ne nous semble pas suffisante pour expliquer que le grand prêtre de Memphis fût régulièrement appelé sem depuis la VIème dynastie. 
Memphis lors de la fête Sed $(122 ; 162 b ; 169,4 ; 200,18)$ ou plus simplement de sa participation à cet ensemble de cérémonies $(\mathbf{1 0 3 , 1 5 -}$ 16; $\mathbf{1 7 2 a})^{1}$, il est très vraisemblable qu'il en a été ainsi aux époques antérieures puisque nous savons que la fête Sed s'y célébrait. Mais il ne s'agit ici que d'une fonction occasionnelle.

Le culte rendu à la statue du roi, dès son vivant, dans son temple funéraire était, au contraire, régulier, et c'est pourquoi sans doute SabouIbébi (18; 19) et Ptahchepses IV (21) parlent de leur service aux pyramides d'Ounas et de Téti.

On peut supposer qu'à toutes les époques, le roi a fait placer sa statue dans le temple de Ptah pour qu'elle y fût l'objet d'un culte, mais ce n'est que sous les Ptolémées que nous rencontrons un grand prêtre prophète d'une statue de Nectanébo $(\mathbf{1 9 2 , 6 )}$. Aussi pensons-nous que le service de la statue royale n'était pas effectué ordinairement par le grand prêtre ${ }^{2}$.

Sous les Ptolémées également, le grand prêtre de Memphis devient le prophète des cultes dynastiques: c'est ainsi qu'Harmachis est le serviteur ${ }^{3}$ des dieux Adelphes, des dieux Evergètes, des dieux Philopators et des dieux Epiphanes $(\mathbf{1 9 7 , 1 1 )}$ et que plusieurs grands prêtres sont chargés du culte d'Arsinoé (190,5-6; 192,5-6; 197,12).

La personne même du roi a trouvé un prophète dans le grand prêtre de Ptah, en tout cas depuis la Basse Epoque: Chedsounefertoum est "prophète du roi" (169,4); Nesqed est "prophète de l'Horus" (190,3-4); Psenptaïs II est "premier prophète du maître des Deux Pays" (200,14) et Petobastis III-Imouthès est prophète de Cléopâtre (203a,2).

${ }^{1}$ Il semble que Khamouas doit à sa qualité de fils du roi d'annoncer les jubilés de son père $(110 ; 111 ; 112)$.

2 Nous en voyons confirmation indirecte dans le fait que le "temple de Ramsèsrégent d'Héliopolis" bâti par Ramsès III dans le domaine de Ptah (162a) - ce sanctuaire dont le personnel était en partie sous les ordres du grand prêtre de Ptah - n'apparaît dans aucun titre ou épithète de grand des chefs des artisans.

3 "Serviteur" est souvent l'équivalent hiéroglyphique ptolémaique de "prophète", peut-être une simple abréviation. 
Une épithète des grands prêtres de l'Ancien Empire semble préparer la voie à ce culte de la personne du roi: celle de "supérieur des secrets de son dieu" $(3,4 ; 5,4 ; 17,4 ; 21,5 ; 24,11)^{1}$.

On peut remarquer un développement analogue au sujet des "apparitions" du roi. Sous l'Ancien Empire, Ptahchepses I est admis ou invité à toutes les fêtes de l'apparition (du roi) (4,11 et 14); il ne jouit donc que d'un simple privilège. Mais sous les Ptolémées, le grand prêtre de Memphis est "prophète de l'Horus de la Fenêtre de l'apparition", et même "prophète de la Fenêtre de l'apparition", sans parler des "dieux de la Fenêtre de l'apparition" $(192,9 ; 193,8-9 ; 194,5-6 ; 203,6)^{2}$. Le privilège du courtisan devient, avec le temps, une fonction religieuse. C'est, à notre avis, une manifestation de cette tendance à tout envisager sous l'angle de la religion qui a fait passer les Egyptiens pour les plus religieux de tous les hommes aux yeux d'un étranger du Vème siècle av. J.-C. ${ }^{3}$.

\section{§ 15. SERVICE DE RE}

Deux grands prêtres de l'Ancien Empire, Ptahchepses I et Sabou-Ibebi, ont été prophètes de Rê dans trois des temples solaires construits par les rois de la Vème dynastie ${ }^{4}$ : ceux d'Ouserkaf, de Neferirkarê et de Nyouserrê $\mathbf{( 4 , 4 - 5 ; ~ 2 0 , 1 1 - 1 2 ) . ~ P l u s ~ e x a c t e m e n t , ~ i l s ~ o n t ~ e ́ t e ́ ~ p r o p h e ̀ t e s ~ d e ~}$ Rê dans le premier et le troisième de ces monuments, tandis qu'ils étaient prophètes de Rê-Horakhti dans le deuxième: différence qui est

1 "Son dieu" semble ne désigner que le roi dans les inscriptions des grands prêtres de l'Ancien Empire; cf. l'épithète "supérieur des secrets du roi dans toutes ses places" $(\mathbf{2 0 , 1 7 )}$ citée une fois au lieu de "supérieur des secrets de son dieu".

${ }^{2}$ Le temple de Ptah à Memphis devait avoir, à cette époque, un balcon semblable à celui de la première cour du temple de Ramsès III à Medinet-Habou.

${ }^{3}$ Hérodote, II, 37.

${ }^{4}$ K. SETHE, Die Heiligtümer des Re im alten Reich, dans Ä.Z. XXVII (1889), p. 111. Le fait que ces temples paraissent n'avoir plus été en service après Menkaouhor n'oblige pas à considérer les inscriptions de Sabou-Ibebi comme des copies de Ptahchepses I (SETHE, p. 117, texte et note 5). Puisque Sabou-Ibebi a atteint la vieillesse, peut-être même une sorte de retraite (voir ci-dessus, $\S 8$ et 11, p. 32, note 3 ). Il peut avoir été un jeune homme sous Menkaouhor et avoir encore connu les premières années de Téti, les deux règnes intermédiaires de Djedkarê-Isesi et Ounas comptant environ 58 ans. 
marquée par la place des trois titres sur les stèles. Ptahchepses I et Sabou-Ibebi partagent ce service de Rê avec d'autres prophètes des mêmes sanctuaires ${ }^{1}$. D'autre part, s'il est possible que d'autres grands prêtres de Ptah connus seulement par de courtes inscriptions aient été également prophètes de temples solaires, il est certain que tous ne l'étaient pas; nous possédons, en effet, une trop grande partie du tombeau de Ptahchepses IV pour supposer qu'il a pu revêtir une fonction semblable.

L'épithète "participant à la fête de Rê" est d'un emploi différent. Premièrement, il est vraisemblable qu'elle a été portée par tous les grands des chefs des artisans de l'Ancien Empire; des deux grands prêtres qui en paraissent privés, l'un, Neferef-rê-ankh n'est connu que par une brève mention sur un monument qui ne lui appartient pas, et l'autre, Setjou, par un petit guéridon et un groupe que sa femme a fait sculpter pour lui $(8 ; 14$ et 15$)$.

En second lieu, seuls des grands des chefs des artisans sont qualifiés de participants à la fête de $\mathrm{Re}^{3}$. Enfin, cette épithète disparaît après l'Ancien Empire ${ }^{4}$.

Ces trois caractères invitent à penser que la fête de Rê mentionnée dans "participant à la fête de Rê" se célébrait dans les temples solaires de la Vème dynastie. Quant à la nature de la "participation" du grand prêtre de Memphis à la fête de Rê, nous ne pouvons pas la définir. Tout au plus savons-nous que cette participation était importante aux yeux des grands des chefs des artisans puisqu'il en est fréquemment question dans leurs titulatures abrégées $(6,2 ; 9,4 ; 10,1 ; 20,1 ; 24,2)$.

${ }^{1}$ K. SETHE, op. cit., p. 113. Ptahchepses I et Sabou-Ibebi sont aussi prophètes de Maât et d'Hathor dans les mêmes temples solaires $(4,6-7 ; 20,12-13)$. Dans ces quatre passages, les grands prêtres portent le titre de prophète d'Hathor, ou de Maât, "dans ces places". Il nous semble assez évident que les mots "ces places" ne désignent pas d'autres lieux que les temples solaires, et non les "centres de cultes" de Maât et d'Hathor, selon l'interprétation de SANDMAN, Ptah, p. 148, inscription 184 ("hem-priest of Maat at all her cult centres").

${ }^{2}$ Litt.: "appartenant à la fête de Rê".

${ }^{3}$ M.A. MURRAY, Index, p. 26.

${ }^{4} 186,1$ est ici également une exception apparente. 
Aux époques postérieures, des grands prêtres memphites ont pu individuellement être au service de Rê en tant que grands prêtres d'Héliopolis. Nous en connaissons deux, Rêhetep et Chedsounefertoum, contemporains l'un de Ramsès II et l'autre de Chechanq Ier (103,14; 169,6).

\section{§ 16. SERVICE D'APIS}

Les grands prêtres Khamouas $(\mathbf{1 1 5 , 2 )}$ et Padiiset $\mathbf{( 1 7 8 , 1 4 - 1 6 )}$ ont introduit le taureau sacré à Memphis. Nous pouvons penser qu'il en a toujours été ainsi, de même qu'il est permis de supposer que, dès l'Ancien Empire, le grand prêtre dirigeait la "fête d'Apis" $(\mathbf{1 , 1 4}=$ Urk. I, 20), qui était peut-être la même cérémonie que la course d'Apis de la pierre de Palerme ${ }^{1}$. Mais il est aussi possible que le grand prêtre ait borné là son activité en faveur du taureau vivant à Memphis².

Depuis le Nouvel Empire, quelques grands prêtres sont en relation avec des Apis morts ${ }^{3}$. Sous la XVIIIème dynastie, le prince Thoutmôsis présente une offrande au dieu Apis représenté sur les murs du plus ancien caveau d'Apis connu (63). Sous la XIXème dynastie, cet autre grand prêtre et prince qu'est Khamouas agit de la même façon dans une tombe d'Apis séparée (109), où trois momies de taureaux ${ }^{4}$ reposaient dans deux caveaux lorsque Mariette l'a ouverte ${ }^{5}$. Khamouas a formé et exécuté un dessein plus grand. Il a ouvert cette tombe commune que

${ }^{1}$ A. ERMAN, La religion des Egyptiens, trad. H. WILD, 1937, p. 46, note 2.

2 Les titres "prophète d'Apis vivant" et "protecteur magique d'Apis vivant", qui apparaissent seulement chez des grands prêtres ptolémaïques pourraient aussi bien se rapporter à la statue d'Apis vivant placée dans le temple "funéraire" du Sérapéum qu'au taureau lui-même. Voir, à ce sujet, la suite du texte.

${ }^{3} \mathrm{La}$ pratique de l'ensevelissement des Apis dans la nécropole de Saqqara est attestée depuis la XVIIIème dynastie. A quand remonte-t-elle ? Sur Apis, cf. W. OTTO, Beiträge zur Geschichte der Stierkulte in Aegypten, Leipzig, 1938 = Untersuchungen..., XIII, et l'excellent résumé de J. VANDIER, La religion égyptienne, 1944, p. 221-225.

${ }^{4}$ Dont une de Mnévis, d'après la stèle du Louvre (CHASSINAT, dans Rec. Trav. XXI (1899), p. 70-72, No 34).

5 A. MARIETTE, Renseignements sur les soixante-quatre Apis (Bulletin arch. de l'Athénaeum français, I, 1855, p. 66). 
Mariette a appelée les "petits souterrains" et qui a été en usage jusqu'à l'époque saïte, où un éboulement fit commencer les "grands souterrains", ceux que l'on visite aujourd'hui.

Les petits souterrains, comme d'ailleurs les grands, ont été creusés au fur et à mesure des besoins, mais, dès le début de l'entreprise, ils ont été accompagnés au-dessus du sol par un temple dédié à Apis vivant, dont la statue était peut-être à tête de taureau sur corps humain. Une stèle de Khamouas (115) nous renseigne sur ce temple, qui possédait des prophètes et des prêtres-lecteurs et dans l'enceinte duquel Khamouas fit placer sa statue (117) et peut-être aussi un groupe le représentant sous la protection d'Apis (145).

A la XXIIème dynastie, Chedsounefertoum a été chargé de l'atelier d'embaumement que le roi Chechanq a fait construire pour Apis à la lisière des cultures (166). Un peu plus tard, Padiiset et ses fils ont honoré Apis comme l'avaient fait Thoutmôsis et Khamouas (177 179) ${ }^{1}$.

Sous les Ptolémées, Anemher est "scribe royal décomptant toutes choses du temple d'Osiris-Apis" $(\mathbf{1 9 2 , 6 )}$ et Psenptaïs II est "scribe d'Osiris-Apis et d'Osiris dans Ro-qedit" $(\mathbf{2 0 0 , 1 3 )}$. Il semblerait donc que le temple d'Apis vivant de la XIXème dynastie était devenu, sous les Ptolémées, le temple d'Osiris-Apis, ou Sérapéum. On continuait à y adorer l'Apis vivant, qui paissait dans son enclos à Memphis tandis qu'on creusait son futur caveau ${ }^{2}$. C'est pourquoi nous estimons que les titres ptolémaiques de "prophète d'Apis vivant" $(192,9-10 ; 193,10 ; 198,3)$ et de "protecteur magique d'Apis vivant" $(\mathbf{1 9 3 , 6} ; \mathbf{2 0 3 , 6})^{3}$ ne concernent pas nécessairement le seul taureau à Memphis.

${ }^{1}$ Nous ne savons pas si l'ensevelissement d'Apis a pu être conduit par le grand prêtre, mais il l'a été en tout cas par d'autres personnages (cf. CHASSINAT, Textes..., dans Rec. Trav., XXI et suiv.).

${ }^{2}$ W. SPIEGELBERG, dans $\ddot{A} . Z, 50$ (1912), p. 32 et 51, (1913), p. 137-138.

3 Litt.: "garde de place". Nous empruntons l'expression "protecteur magique" à A.H. GARDINER, The House of Life dans J.E.A., 24 (1938), p. 179. 
D'autre part, comme les souterrains devaient rester ouverts au moins pendant le temps où l'on préparait un nouveau tombeau, la vénération pour les Apis-Osiris, ou Apis ensevelis, pouvait se manifester par des dépôts de stèles postérieurs à un ensevelissement. De plus, Apis-Osiris était vraisemblablement adoré dans le même temple qu'Apis vivant ${ }^{1}$.

Nous savons enfin par les documents grecs que le Sérapéum était dirigé au second siècle avant notre ère par un archiprêtre et un "épistate" qui dépendaient d'un "épistate des temples" siégeant vraisemblablement à Memphis ${ }^{2}$. On peut donc supposer, avec quelque chance d'être dans le vrai, que le temple érigé par Khamouas était dans des conditions analogues, c'est-à-dire jouissant d'une certaine autonomie, mais dépendant de Memphis.

\section{§ 17. SERVICE D'AUTRES DIVINITES}

Quelques-unes des divinités dont le grand prêtre a été prophète à certaines époques sont, dans les temps historiques, assimilées à $\mathrm{Ptah}^{3}$. Mekhent-our $(\mathbf{4 , 3} ; \mathbf{2 0}, \mathbf{1 4})$, Khenty-iaoutef $(\mathbf{4 , 3} ; \mathbf{2 0 , 1 4})$, Khenty-medefet $(4,7 ; 20,13)$, Khenty-tenenet $(4,6 ; 20,10)$, Khery-bakef $(4,7 ; 20,13)$ ont leur culte sous l'Ancien Empire en tout cas. L'un ou l'autre de ces dieux réapparaît dans la titulature d'un grand prêtre postérieur: Khnemibrê-sa-ptah-Ahmèsmen est père divin et prophète de Ptah-kherybakef $(\mathbf{1 8 7}, \mathbf{2 4 - 2 5})$ et Psenptaïs II est scribe de Ptah-khenty-tenenet $(\mathbf{2 0 0}, \mathbf{1 3})$. Il en est de même pour le pilier Djed: Ptahchepses I et SabouIbebi sont prophètes du pilier Djed sacré $(\mathbf{4 , 6} ; \mathbf{2 0 , 1 0})$, qu'on rencontre

${ }^{1}$ Cf. 200,4 où Psenptaïs II adore Apis-Osiris en même temps que plusieurs dieux qui avaient un culte au Sérapéum: Osiris, seigneur de Ro-Setaou, qualifié de "dieu grand dans Ro-qedit" (Ro-qedit = Sérapéum, d'après 200,13); Harendotes dans Ro-Qedit; Imhotep (culte d'Asklepios au Sérapéum: U. WILKEN, Urkunden der Ptolemäerzeit, I, 1927, p. 38-41).

${ }^{2}$ U. WILKEN, Urkunden der Ptolemäerzeit, I, 1927, p. 44-45.

${ }^{3}$ H. KEES, Eine Liste memphitischer Götter im Tempel von Abydos, dans Rec. Trav., XXXVII (1915), p. 57-76. 
plus tard sous la même forme ${ }^{1}$ et qui réapparaît dans l'épithète bien connue "second du roi lors de l'érection du pilier Djed", attestée aux époques saïte et ptolémaïque $(\mathbf{1 8 6 , 4 - 5} ; \mathbf{2 0 0 , 1 4}=\mathbf{2 0 1 , 6})$.

Osiris, qui a supplanté Sokar à Ro-setaou, a aussi pour prophète l'un ou l'autre grand prêtre: à la XIXème dynastie, Iyry est premier prophète d'Osiris seigneur de Ro-setaou (156,12); les contemporains des Ptolémées Psenptaïs II et Imouthès sont prophètes d'Osiris seigneur de Ro-setaou $(200,12=201,14 ; 203,6)$.

Les titulatures de grands prêtres que nous connaissons ne mentionnent jamais Sekhmet, Nefertoum ou Hathor du Sycomore du sud avant les Ptolémées. Ce silence pourrait paraître bizarre si nous ne pouvions supposer que ces divinités, comme le Sérapéum, avaient leur personnel propre ne relevant qu'indirectement de l'autorité du grand prêtre de Memphis. Il en allait évidemment ainsi pour des dieux ne résidant pas dans Memphis même: Anubis et Imhotep, par exemple.

Les remarques que nous avons faites sur les fonctions du grand prêtre de Memphis peuvent se résumer ainsi.

Jusqu'à la Basse Epoque, le grand prêtre de Ptah n'assume personnellement qu'un nombre restreint de cultes réguliers: celui de Ptah qu'il partage avec d'autres hauts serviteurs de ce dieu, celui de Sokar, celui de Rê sous l'Ancien Empire.

Il officie aux fêtes Sed et aussi à toutes les fêtes principales de Memphis, entre autres la visite de Ptah à Hathor du Sycomore du sud, la fête de Sokar au cours de laquelle le henou est halé autour du Mur Blanc, la procession d'Apis.

Il dirige lui-même le personnel du temple de Ptah à Memphis et, par personne interposée, le personnel des autres sanctuaires memphites.

1 "Ptah-Pilier Djed sacré, seigneur d'Ankh-Taoui" est invoqué dans une formule d'offrandes du Nouvel Empire (Ch. NICHOLSON, On some funeral hieroglyphic inscriptions found at Memphis, dans Transactions of the Royal Society of Literature, Londres 1866, série 2, vol. 8, p. 308-325, ou dans Aegyptiaca, Londres 1891, pl. III,4. 
Le pouvoir du grand prêtre de Ptah s'exerce sur le nome memphite jusqu'au Nouvel Empire. A partir de cette époque, quelques grands prêtres de Memphis sont appelés à diriger l'ensemble des prophètes des sanctuaires de Haute et Basse-Egypte ${ }^{1}$.

${ }^{1}$ Le titre "directeur des prophètes de Haute et Basse- Egypte" (et variantes) est porté par Ptahmès II, Thoutmôsis, Neferrenpet, Chedsounefertoum, Anemher et Psenptaïs II. 


\section{H A P I T R E I I I}

\section{LA DUALITE DU PONTIFICAT DE LA IVème \\ A LA VIème DYNASTIE}

\section{$\S$ 18. LES MENTIONS DES DEUX GRANDS PRETRES}

Les cinq mentions connues de l'expression "les deux grands des chefs des artisans" se trouvent dans trois inscriptions de l'Ancien Empire: au tombeau de Debehen, sur la stèle de Ny-ankh-sekhmet et sur le fragment de stèle de Sabou-Tjéti.

Le tombeau de Debehen date de la IVème dynastie, du règne de Mycérinus. On y rencontre trois fois les "deux grands des chefs des artisans" $(\mathbf{1}, \mathbf{2 , 8 , 1 0})$; la première nous apprend qu'ils sont sur le chantier de la pyramide au moment où le roi le visite; la seconde paraît établir une relation entre les deux grands des chefs des artisans et la pierre apportée de Toura pour revêtir le temple funéraire du roi; la troisième paraît également relier les grands des chefs des artisans et la fabrication d'une statue.

La stèle de Ny-ankh-Sekhmet date de la Vème dynastie, du règne de Sahourê. On y lit (2,3-4) que les deux grands des chefs des artisans de la ouabet et les artisans ont été "mis" à deux fausses-portes en calcaire de Toura. Le travail exécuté sur ces monuments a donc pu consister en polissage et décoration de la pierre au moyen de bas-reliefs comprenant figures et textes. Sans doute faut-il comprendre que ces sculptures ont été exécutées par les artisans sous la direction des deux grands des chefs des artisans.

Le fragment de stèle de Sabou-Tjéti ne porte aucune indication chronologique précise, mais il peut être approximativement daté d'autre 
manière. Le mastaba de Sabou-Tjéti est, en effet, postérieur à celui de Sabou-Ibebi et Ptahchepses IV puisqu'il a un angle rentrant correspondant à un angle du premier monument ${ }^{1}$. Ce détail architectural laisse aussi supposer qu'il n'y a pas eu un long intervalle entre les deux constructions. Or Sabou-Ibebi et Ptahchepses IV ont orné leur double mastaba $^{2}$ sous le règne de Téti $(20,16$ et 19$)$. Sabou-Tjéti a donc bâti son mastaba assez probablement sous Pépi Ier, mais comme la longueur du règne de ce roi prête à discussion, il ne nous paraît pas exclu, quoique moins probable, que le monument funéraire de Sabou-Tjéti date de Merenrê, ou même de Pépi II.

Dans la partie de son inscription qui nous est parvenue, Sabou-Tjéti, après avoir parlé de quelque fonction que le roi lui a octroyée et qui concerne le temple de Ptah (26,5-6), puis de Sokar dans la chetyt, mentionne "toutes les choses divines, tous les devoirs que les deux grands des chefs des artisans remplissaient habituellement" $(\mathbf{2 6 , 7 )}$. Dans ce passage, les "choses divines" ne nous semblent pas être liées aux grands des chefs des artisans, mais être un des termes d'une énumération qui commençait dans la partie disparue de la même ligne. Le sens général aurait été: il n'y a eu personne jusqu'ici à qui le roi ait confié le service de Ptah et le service de Sokar, en résumé toutes les choses divines, ainsi que les devoirs remplis habituellement par les deux grands des chefs des $\operatorname{artisans}^{3}$; ces devoirs nous sont connus par l'inscription de Debehen et la stèle de Ny-ankh-sekhmet.

SANDMAN (Ptah, p. 54) interprète autrement que nous le passage de Sabou-Tjéti sur les devoirs des grands prêtres (26,7-8). Elle traduit "... Sokaris dans Chetit. Chaque rite divin et chaque devoir accompli par les deux chefs suprêmes des métiers..." et déclare plus loin: "... le texte montre clairement que les deux chefs suprêmes des métiers, en cette qualité, accomplissaient certains rites divins...". Nous avons peine à admettre que les mots "chaque rite divin" forment le début d'une phrase, mais ce point est sans importance ici. Dans son état actuel, hélas bien mutilé, le texte

${ }^{1}$ D'après le plan de MARIETTE, Mastabas, p. 391, E 3.

${ }^{2}$ Voir ci-dessous, § 19.

${ }^{3}$ Nous ne tenons compte que du texte ancien, et non des restitutions proposées par K. SETHE, Urk. I; nous les examinerons au chapitre suivant. 
de Sabou-Tjéti autorise aussi bien l'interprétation de Mme SandmanHolmberg que la nôtre. Toutefois, notre traduction s'appuie sur l'enseignement des textes de Debehen et de Ny-ankh-sekhmet, tandis que l'Ancien Empire ne fournit pas d'exemple d'un grand des chefs des artisans accomplissant quelque rite. Nous maintenons donc notre interprétation.

On est donc certain qu'il y a eu deux grands des chefs des artisans au moins depuis le règne de Mycérinus sous la IVème dynastie jusqu'à celui de Pépi Ier sous la VIème. Pour toutes les autres périodes de l'histoire de l'Egypte ancienne, on ne peut que faire des conjectures à ce sujet, aussi vraisemblables par ailleurs que puissent être ces dernières.

\section{§ 19. LES RAPPORTS DES DEUX GRANDS PRETRES}

Les inscriptions où il est question des deux grands des chefs des artisans n'établissent aucune distinction entre l'un et l'autre de ces personnages. Elles laissent au contraire supposer que c'étaient des collègues aux droits égaux, exerçant leur fonction en commun et le plus souvent ensemble.

Les monuments appartenant à des grands prêtres individuels confirment cette manière de voir tout en révélant qu'il y avait certaines limites à cette fraternité.

Nous avons relevé ${ }^{1}$ l'étroite ressemblance des linteaux de Ptahchepses I, Ptahchepses II, Sabou-Ibebi et Ptahchepses IV (3; 5; 17; 21). Les autres grands prêtres que nous connaissons antérieurement à Sabou-Tjéti, Neferef-rê-ankh (8), Rênefer (9; 10), Ptahchepses III (11), Sabou-kem $(12 ; 13)$ et Setjou $(14 ; 15)$ laissent deviner qu'ils ont revêtu les mêmes charges que les possesseurs des linteaux.

Le double mastaba de Sabou-Ibebi et de Ptahchepses IV est un témoignage éclatant des liens qui pouvaient exister entre les deux grands des chefs des artisans. Le premier était un homme âgé lorsqu'il a fait

\footnotetext{
${ }^{1}$ Voir ci-dessus, § 6.
} 
exécuter les parois et la fausse-porte de sa chapelle, puisqu'une de ses parois montre ses deux petits-fils Ptahchepses et Khenoul ${ }^{1}$ et que deux des figures de sa fausse-porte le représentent en vieillard ${ }^{2}$. Quant à Ptahchepses IV, aucune de ses figures ne lui donne les traits d'un homme âgé et les parois de sa tombe ${ }^{3}$ le montrent en compagnie de son épouse et de ses fils; mais on n'y trouve pas de petits-fils. Il y avait donc une différence d'âge assez grande, peut-être même l'espace d'une génération, entre Sabou-Ibebi et Ptahchepses IV. Comme il ne parait pas qu'il y ait eu un lien de parenté entre eux, c'est à leur qualité commune de grand des chefs des artisans que nous attribuons les deux faits suivants: SabouIbebi a donné le nom de Ptahchepses à son fils préféré ${ }^{4}$ et Ptahchepses IV celui de Sabou au sien ${ }^{5}$; d'autre part, l'un et l'autre ont fait construire un mastaba commun dont les chapelles sont contemporaines ${ }^{6}$. C'est pourquoi il ne nous semble pas exagéré de parler d'une entente profonde entre ces collègues.

Toutefois, bien que les titres des linteaux qui surmontaient les portes de leurs chapelles et qui étaient donc bien en vue des passants soient les mêmes, Sabou-Ibebi a été prophète d'un certain nombre de divinités dont on ne trouve pas l'équivalent chez Ptahchepses IV: il a été prophète de Rê dans trois des temples solaires de la Vème dynastie et prophète d'autres divinités telles que Khenty-medefet, Khenty-iaoutef, Khery-bakef, etc. Si les temples solaires ont cessé d'être en service après Menkaouhor $^{7}$, la différence d'âge entre Sabou-Ibebi et Ptahchepses IV rendrait compte de l'absence de titres se rapportant à ces sanctuaires chez Ptahchepses IV, car celui-ci n'avait probablement pas encore vu le jour sous Menkaouhor. Pour les divinités telles que Khery-bakef, on peut

${ }^{1}$ L. BORCHARDT, Denkmäler, pl. 21.

2 A. MARIETTE, Mastabas, p. 414-415.

${ }^{3}$ M.A. MURRAY, Saqqara Mastabas, I, 1904, pl. 29-31.

${ }^{4}$ L. BORCHARDT, Denkmäler..., 1937, pl. 21.

${ }^{5}$ M.A. MURRAY, op. cit., pl. 31,3.

${ }^{6}$ A. MARIETTE, Mastabas, 1889, p. 373: "Quelque soin que nous y ayons mis, nous n'avons découvert dans la construction aucune retouche, aucun remaniement. On n'a pas taillé de force dans la masse du mastaba un compartiment pour y introduire après coup une des deux chambres. Le mastaba a été fait tout entier d'un seul jet, les chambres comprises".

${ }^{7}$ K. SETHE, Die Heiligtümer des Re im alten Reich, dans Ä.Z., 27 (1889), p. 117. 
supposer que leur service était attribué au plus ancien des deux grands des chefs des artisans et qu'il passait à son collègue chaque fois que le plus âgé des deux mourait le premier. En effet, les deux grands prêtres qui, à notre connaissance, ont été prophètes des divinités dont nous venons de parler ont atteint tous deux la vieillesse: nous en avons vu les témoignages pour Sabou-Ibebi; l'autre grand prêtre dans le même cas est Ptahchepses I, qui se qualifie d'ancien du temple de Ptah $(\mathbf{4 , 6 )}$.

Enfin, en rappelant nos remarques touchant la filière suivie antérieurement à leur pontificat par les grands prêtres de l'Ancien Empire $^{1}$, nous estimons que la différence d'âge entre Sabou-Ibebi et Ptahchepses IV entraîne la possibilité pour les deux sièges de grands des chefs des artisans d'être repourvus à des dates différentes, donc indépendamment l'un de l'autre.

\section{§ 20. LA RAISON DE L'EXISTENCE SIMULTANEE DE DEUX GRANDS PRETRES ${ }^{2}$}

On peut s'étonner qu'il y ait eu deux grands prêtres de Ptah à la fois puisque l'un et l'autre avaient la même importance. Cette dualité du pontificat s'explique cependant si on la définit clairement. Nous avons vu qu'elle n'est indiscutable que du règne de Mycérinus à celui de Pépi Ier, soit de la IVème à la VIème dynastie. En second lieu, elle ne concerne qu'une des fonctions des grands prêtres de Ptah, celle de grand des chefs des artisans. Or cette fonction a un caractère civil nettement exprimé par les termes mêmes du titre qui la désigne et par les textes qui l'évoquent. Aussi sommes-nous fondés à la rapprocher de cet autre titre, également civil, des grands des chefs des artisans de l'Ancien Empire: "celui qui est dans la double maison". La double maison, ce sont les deux moitiés de l'administration centrale qui apparaît divisée ainsi dans tous ses départements $^{3}$. Cette division correspond à l'ancienne division

${ }^{1}$ Voir ci-dessus, $\S 6$.

${ }^{2}$ SANDMAN, Ptah, p. 52, se borne à constater qu'il y a eu deux grands des chefs des artisans dans l'Ancien Empire jusqu'à Sabou-Tjéti, mais ne recherche pas la raison de cette dualité.

${ }^{3}$ Cf. la liste abondante de "ceux qui sont dans la double maison" dans M.A. MURRAY, Index, p. 17. Cf. aussi J. PIRENNE, Histoire des Institutions, II, p. 151. 
territoriale ${ }^{1}$, comme l'indique le titre "celui qui est dans la double maison du Nord et du Sud"2, mais elle n'est qu'un rappel du temps qui a duré jusqu'à la IIIème dynastie et pendant lequel les institutions du Nord et du Sud étaient différentes ${ }^{3}$. En effet, tous ceux qui sont dans la double maison participent aux deux moitiés de l'administration centrale, c'est-àdire à celle-ci tout entière.

Etant donné cette division de l'administration, nous pouvons admettre l'existence de deux moitiés de ouabet ${ }^{4}$ et de deux jeux d'équipes d'artisans dont chacun aurait été dirigé par un des grands des chefs des artisans. L'un et l'autre de ceux-ci auraient cependant eu le droit de commander aux équipes des deux à cause de leur qualité de "celui qui est dans la double maison".

Il n'y avait donc pas, à proprement parler, deux grands prêtres de Ptah de la IVème à la VIème dynastie. Un collège de prophètes de Ptah dirigeait vraisemblablement les affaires du dieu. La plupart des membres de ce collège exerçaient la fonction civile de directeur des artisans. Deux des prophètes de Ptah avaient une position civile supérieure à celle de leurs collègues et portaient à ce propos le titre de grand des chefs des artisans $^{5}$.

${ }^{1}$ Ce sont les termes mêmes de J. PIRENNE, op. cit., II, p. 152.

${ }^{2}$ M.A. MURRAY, op. cit.

${ }^{3}$ Au sujet de ces différences, cf. J. PIRENNE, op. cit., I, p. 157.

${ }^{4}$ Il semble que ouabet, sous la Vème dynastie, désignait exactement une des deux ouabet; c'est ainsi que chaque grand prêtre aurait été directeur d'une ouabet avant d'être admis dans les deux moitiés de l'administration des artisans en tant que "celui qui est dans la double maison" (cf. la place respective des deux titres dans les inscriptions des grands prêtres). Toutefois, en dehors de cette signification technique précise, ouabet a pu désigner de façon abrégée les deux ouabet; il semble du moins que ce soit le cas dans l'inscription du médecin Ny-ankh-sekhmet $(\mathbf{2}, \mathbf{4})$. L'existence de deux ouabet au début de la VIème dynastie est confirmée par la stèle de Sabou-Tjéti $(\mathbf{2 6 , 2 )}$.

5 Il serait intéressant d'étudier la documentation des grands prêtres d'Héliopolis pour elle-même et de confronter ensuite les conclusions qu'elle donnerait sur la dualité du pontificat héliopolitain avec les nôtres. 


\section{§ 21. HYPOTHESE SUR L'ORIGINE DES GRANDS DES CHEFS DES ARTISANS}

Le grand des chefs des artisans occupe une position plus élevée que les directeurs des artisans. Mais, en tant que prophète de Ptah, il est sur pied d'égalité avec ces mêmes directeurs, qui sont prophètes de Ptah comme lui. Il y a donc un désaccord entre la fonction civile du grand des chefs des artisans et la fonction religieuse de prophète de Ptah exercée par le même personnage. Ce désaccord ne nous paraît pas pouvoir signifier autre chose qu'une différence d'origine des deux fonctions.

SANDMAN, Ptah, p. 54-55, est déjà parvenue à la même conclusion, mais par une autre voie. Elle considère, d'une part, que les plus anciens textes qui mentionnent des grands des chefs des artisans nous les montrent fonctionnant comme de vrais chefs d'artisans (Debehen, Nyankh-sekhmet) et, d'autre part, que les grands des chefs des artisans n'apparaissent que plus tard en relation avec le culte de Ptah (SabouTjéti, d'après l'interprétation ci-dessus, p. 40, note 4). Constatant alors que rien n'indique que le développement de la fonction de grand des chefs des artisans a suivi un cours inverse, elle écrit ceci: "A mon avis, l'explication la plus probable est que le titre wr kherep hemet ne comprenait à l'origine rien de plus que ce que le terme signifie, qu'ainsi c'était la désignation de l'inspecteur principal des métiers et que les maîtres-artisans qui portaient ce titre eurent secondairement le droit de participer au culte de Ptah et de Sokaris". Ces derniers mots nous ont fait constater une lacune au premier alinéa de notre $\$ 21$ : nous avons omis de comparer la fonction civile du grand des chefs des artisans et la fonction religieuse de prophète de Sokar exercée par le même personnage. Nous pouvons combler ce défaut en ajoutant après chaque mention de "prophète(s) de Ptah" les mots "ou prophète(s) de Sokar", et conclure que la direction des artisans et la prêtrise de Sokar ont aussi des origines différentes.

Mme Sandman-Holmberg propose ensuite quatre explications possibles du développement de la fonction de grand chef des artisans:

1/ le travail du calcaire à Memphis et l'importance du rôle joué par les artisans dans la capitale seraient à l'origine de l'attribution à Ptah de sa qualité de patron des artisans. Il aurait été alors naturel que ceux-ci prissent dès lors soin de son culte; 
2/ Ptah aurait été de bonne heure regardé comme un maître-artisan, et consécutivement, comme le patron des artisans, qui auraient, à leur tour, pris soin de son culte;

3/ les artisans de la nécropole memphite auraient adopté Sokar pour patron à cause de leurs contacts fréquents avec cette divinité. Ils seraient ensuite devenus prêtres de Sokar, puis, à cause des relations qui s'établirent entre Sokar et Ptah, auraient aussi pris soin du culte de Ptah dont le caractère se serait modifié en conséquence;

4/ Sokar aurait possédé l'attribut de maître-artisan, et serait en conséquence, devenu patron des artisans.

En concluant qu'on ne peut pas dépasser le stade des hypothèses, Mme Sandman-Holmberg déclare qu'on doit aussi considérer que Ptah a pu recevoir de Sokar son caractère de maître-artisan, et ne pas tenir compte seulement du développement inverse, comme on l'a fait jusqu'ici.

La documentation dont on dispose pour résoudre le problème abordé par Mme Sandman-Holmberg est insuffisante, comme elle le remarque elle-même; c'est pourquoi nous avons laissé cette question de côté. Cependant, il n'est peut-être pas inutile que nous signalions une autre solution que celles qui ont été suggérées par l'égyptologue suédoise. $\mathrm{Ne}$ se pourrait-il pas que Ptah et Sokar fussent tous deux devenus, à la même époque, des patrons des artisans ? A l'appui de cette hypothèse, on pourrait citer deux petits textes de tombeaux de l'Ancien Empire situés dans la région memphite. Dans l'un, le préparateur d'une onction invoque l'aide de Sokar au moment où il va appliquer cette onction sur un patient: "Sokar rende ton odeur agréable" (cité par SANDMAN, Ptah, p. 49, inscription 68). Dans l'autre, un nain s'adresse à un autre nain qui l'aide à fabriquer un collier: "Aussi vrai que Ptah t'aime, je voudrais le finir aujourd'hui" (Selim HASAN, Excavations at Giza 1930-1931, 1936, p. 198 et fig. 219). C'est seulement aux époques suivantes que le patronage des artisans aurait été peu à peu attribué presque entièrement à Ptah.

La prêtrise de Ptah est évidemment aussi ancienne que le dieu luimême, mais nous ne connaissons pas de prophète de Ptah antérieur au temps des grands des chefs des artisans. 
Il en va autrement pour la direction des artisans. L'étude du pectoral ${ }^{1}$ nous a conduit à rechercher un point commun à la titulature de Khabaouseker d'une part et à celle des grands des chefs des artisans d'autre part. Nous avons constaté que Khabaouseker est "chef des artisans du palais, ou du tombeau (royal)" et c'est peut-être à cette fonction qu'il faut rattacher le pectoral.

Or, Khabaouseker appartient à la IIIème dynastie qui vit naître l'emploi généralisé de la pierre, mais d'une pierre découpée en blocs de petites dimensions et pour laquelle on n'avait pas encore trouvé de formule entièrement adaptée à sa nature. Les grands des chefs des artisans apparaissent à l'époque des grandes pyramides. Ils sont attestés sous Mycérinus mais on peut faire remonter leur existence aux débuts de la IVème dynastie. S'ils ont hérité de la fonction que Khabaouseker exerçait en tant que chef des artisans du palais, ou du tombeau ${ }^{2}$, il n'y a qu'une hypothèse à envisager: les grands des chefs des artisans auraient été créés au début de la IVème dynastie, au moment où l'extension du travail de la pierre réclamait un personnel plus nombreux ${ }^{3}$. Comme d'autre part, Khabaouseker n'a aucune attache avec Ptah, ni avec Sokar (excepté son nom !), ce serait aussi postérieurement à la IIIème dynastie que les prophètes de Ptah et de Sokar auraient été appelés à diriger des artisans soit comme directeurs des artisans soit comme grands des chefs des artisans, ou inversement que les directeurs des artisans et les grands des chefs des artisans nouvellement créés se seraient vu confier les services de Ptah et de Sokar.

${ }^{1}$ Voir ci-dessus, $\$ 9$.

${ }^{2}$ La transformation de l'administration à l'avènement de la IVème dynastie explique suffisamment qu'on ne retrouve plus les titres de Khabaouseker chez les grands des chefs des artisans du reste de l'Ancien Empire. Mais ces titres ne sont pas tous tombés dans l'oubli: le grand des chefs des artisans Senousert-ankh au Moyen Empire semble être "chef des frontaliers" $(\mathbf{3 5}, \mathbf{x}+\mathbf{3})$; il est "gardien du troupeau $(?)^{\prime \prime}(\mathbf{3 5}, \mathbf{x}+\mathbf{4})$ et "chef du grand palais" $(\mathbf{3 5}, \mathbf{x}+\mathbf{5})$, trois titres qu'on peut rapprocher de "chef des frontaliers du Sud et du Nord", "gardien du troupeau (?)" et "chef des artisans du palais", portés par Khabaouseker (M.A. MURRAY, Saqqara Mastabas, II, p. 11).

${ }^{3}$ Snefrou, qui s'est fait bâtir deux pyramides de pierre, l'une à Meïdoum, l'autre à Dahchour, aurait peut-être alors créé le double poste de grand des chefs des artisans. Si ce même roi s'est fait construire non pas deux, mais trois pyramides (cf. A. VARILLE, A propos des pyramides de Snefrou, Le Caire, imprimerie Schindler, 1947, p. 11), il aura eu d'autant plus besoin de développer la corporation des artisans ! 
En résumé, la dualité du pontificat de la IVème à la VIème dynastie s'explique par le caractère civil de la fonction de grand des chefs des artisans, qui la place dans le cadre de la double administration de l'époque. Nous avons d'autre part supposé que la direction des artisans et le service de Ptah n'ont été mis dans les mêmes mains qu'au début de la IVème dynastie. 


\section{H A P I T R E I V}

\section{LA REFORME DU PONTIFICAT SOUS LA VIème DYNASTIE}

\section{§ 22. LE PONTIFICAT ANTERIEUREMENT A PEPI Ier}

$\mathrm{Au}$ cours des chapitres précédents, nous avons défini quelques caractères du pontificat dans les temps qui ont précédé Pépi Ier. Il nous paraît nécessaire de grouper ces données pour y gagner une vision d'ensemble.

Il y a alors deux grands des chefs des artisans. Avant d'accéder au pontificat, ils semblent être dans l'obligation de suivre une filière administrative bien définie ${ }^{1}$. A ce sujet, nous avons remarqué que les grands des chefs des artisans antérieurs à Pépi Ier énumèrent leurs titres dans un ordre inversement chronologique, en commençant par le plus récent et le plus élevé et en terminant par le plus ancien et le plus bas².

L'accession à la fonction de grand des chefs des artisans ne dépend vraisemblablement pas du roi ${ }^{3}$. Il y a là un complément qu'on pourrait qualifier de naturel à l'obligation de passer par les divers degrés d'une hiérarchie fixe.

Le service de Ptah et le service de Sokar appartiennent en commun à tous les prophètes de ces dieux. Ces prophètes ne comptent pas seulement les grands des chefs des artisans, mais aussi des directeurs des

\footnotetext{
${ }^{1}$ Voir ci-dessus $\$ 6$. C'est par commodité que nous employons le terme de pontificat, mais en ayant en mémoire ce que nous avons écrit à la fin du $§ \mathbf{2 0}$.

${ }^{2}$ Voir ci-dessus, $\$ 6$.

${ }^{3}$ Voir ci-dessus, $§ 7$.
} 
$\operatorname{artisans}^{1}$.

Par contre, le domaine de Sokar n'est administré que par les grands des chefs des artisans. Les serviteurs particuliers que sont les sem de Sokar $^{2}$ ne sont dirigés, également, que par les grands des chefs des artisans ${ }^{3}$.

\section{§ 23. LA STELE DE SABOU-TJETI}

En cherchant à déterminer la date de ce monument, nous l'avons attribué au règne de Pépi Ier, sans exclure les deux rois suivants ${ }^{4}$. Lorsque A. Mariette l'a découvert, il n'en restait que le quart environ, soit la partie inférieure droite, conservant une petite fraction de la fausseporte et, à droite de celle-ci, l'extrémité inférieure de trois bandes verticales finissant chacune par une figure du possesseur, debout et tourné vers la fausse-porte. Ces trois figures sont nommées respectivement Tjéti, Sabou et Tjéti ${ }^{5}$. Celle de gauche, contre la fausseporte, est surmontée d'une titulature en trois colonnes et une ligne horizontale $\mathbf{( 2 6 , 2 - 4 )}$ entièrement conservée. Les deux autres figures sont surmontées respectivement des fins de trois $\mathbf{( 2 6 , 5 - 7 )}$ et de quatre (26,811) colonnes de texte. On peut admettre que les quatre colonnes $\mathbf{( 2 6 , 8 -}$ 11) se lisaient à la suite des trois (26,5-7). Cependant, d'après la disposition des textes sur la stèle de Sabou-Ibebi (20), on peut aussi supposer que le texte biographique commençait à gauche de la fausseporte, dans les trois colonnes symétriques à 26,5-7, aujourd'hui

${ }^{1}$ Voir ci-dessus, $\$ 12$ et $\mathbf{1 3 .}$

${ }^{2}$ Voir ci-dessus, $§ 5$.

${ }^{3}$ Voir ci-dessus, $\S \mathbf{1 3}$ et $\mathbf{5}$.

${ }^{4}$ Voir ci-dessus, $\S 18$.

${ }^{5}$ Malgré l'état fragmentaire du monument, on ne saurait y voir autre chose qu'un personnage portant deux noms, comme Sabou-Ibebi ou Ptahchepses-Impy. Dans le cas de Sabou-Ibebi, sa stèle nous apprend qu'Ibebi est son "beau nom" (20,6). Dans celui de Ptahchepses-Impy, le cercueil d'un autre Ptahchepses, peut-être contemporain mais différent du grand prêtre, révèle qu'Impy était un "beau nom" de Ptahchepses (cf. Ahmed Bey KAMAL, Rapport sur les fouilles de Saïd Bey Khachaba au Déîr-el-Gebraouî, dans Annales du Service des Antiquités, 13, (1914), p. 175, cité par H. RANKE, Personennamen, 326, 19). 
disparues, continuait par 26,5-7, puis par les quatre colonnes symétriques à 26,8-11, et finissait par 26,8-11 ${ }^{1}$. On peut supposer avec vraisemblance que les colonnes disparues de la moitié gauche de la stèle racontaient, comme celles qui sont conservées et comme les biographies de Ptahchepses I (4,8-15) et de Sabou-Ibebi (20,16-21), des événements au cours desquels Sabou-Tjéti avait été en rapport avec le roi. Quant à la partie supérieure de la stèle, elle était probablement remplie par un tableau central représentant Sabou-Tjéti assis à une table d'offrandes et bordé de chaque côté par la partie supérieure des colonnes de texte de la biographie.

\section{§ 24. LA CARRIERE DE SABOU-TJETI}

La titulature conservée dans les trois colonnes les plus proches de la fausse-porte est la seule de Sabou-Tjéti sur ce qui reste de sa stèle (26,2-4). Comparée aux séries de titres des stèles de Ptahchepses I (4,17) et de Sabou-Ibebi (20,8-15), elle apparaît formée de titres entièrement nouveaux, à l'exception de "grand des chefs des artisans", "celui qui est dans la double maison" et "participant à la fête de Rê". De plus, dans les deux énumérations dont elle paraît composée (26,2 et 26,3-4), "grand des chefs des artisans" n'est donné que vers la fin: une fois, ce titre n'est suivi que d'imakhou qui peut se rapporter à une qualité acquise après le pontificat; l'autre fois, "grand des chefs des artisans" est suivi de "celui qui est dans la double maison" et de "participant à la fête de Rê", deux titres dont le second nous a paru n'être décerné aux grands prêtres qu'en même temps que leur fonction de grand des chefs des artisans et le premier la plupart du temps à ce moment ${ }^{2}$. Ces remarques nous font considérer que les titulatures de Sabou-Tjéti sont établies selon un ordre chronologique, ou qu'elles ne citent que les fonctions réelles et honorifiques que Sabou-Tjéti a exercées ensemble à partir de son accession au pontificat ${ }^{3}$. En tout cas, ces titulatures ont été rédigées sur

\footnotetext{
${ }^{1}$ Voir le croquis de nos "Inscriptions". On peut envisager encore d'autres successions des diverses parties disparues et conservées du texte biographique.

2 Voir ci-dessus, $\$ 6$.

${ }^{3}$ Il semble que la majorité des titulatures de grands prêtres postérieurs à SabouTjéti soit établie selon l'un ou l'autre des modes que nous envisageons pour celui-ci.
} 
une autre base que celles des prédécesseurs de Sabou-Tjéti.

Que ce soit donc avant ou en même temps que sa fonction de grand des chefs des artisans, Sabou-Tjéti a été "gouverneur placé à la tête de Nekheb, compagnon unique, directeur des deux ouabet $\mathbf{( 2 6 , 2 )}$ et "prêtrelecteur en chef, compagnon unique, préposé au diadème quand Ptah est orné, prêtre-sem, chef de toute la garde-robe" (26,3-4), toutes fonctions qui n'avaient pas été attribuées aux grands prêtres antérieurs.

La biographie de Sabou-Tjéti, si fragmentaire soit-elle, confirme l'enseignement des titulatures. Sabou-Tjéti a été deux fois l'objet d'une nomination décrétée par le roi lui-même (26,5 et 9). On a fait pour lui ce qui n'avait encore été fait pour aucun grand des chefs des artisans (26,8): il a été chargé de "tous les devoirs que les deux grands des chefs des artisans remplissent habituellement" $(\mathbf{2 6 , 7 )}$, semble-t-il.

La nature de ces renseignements nous fait admettre les restitutions proposées par K. Sethe ${ }^{1}$ pour le début des deuxième et troisième colonnes conservées de la biographie (26,6 et $\mathbf{7})$. Le texte ainsi obtenu est le suivant: "(26,5) ... Aujourd'hui auprès de Sa Majesté ! Sa Majesté m'a nommé $(\mathbf{2 6 , 6 )}$ (unique grand des chefs des artisans)... temple de Ptah au sud de son mur en toutes ses places sous ma surveillance, alors qu'il n'y avait pas eu $(\mathbf{2 6 , 7 )}$ (d'unique grand des chefs des artisans auparavant)... Sokar dans la chetyt, toutes les choses divines et tous les devoirs que les deux grands des chefs des artisans remplissaient habituellement ${ }^{\prime 2}$.

Sabou-Tjéti n'aurait donc pas eu de collègue grand des chefs des artisans, ou tout au moins pas depuis un certain moment de sa carrière.

La participation d'un grand des chefs des artisans Tjéti, sans doute Sabou-Tjéti, au mastaba d'une "véritablement connue du roi Inti" (28) que

\footnotetext{
${ }^{1} \mathrm{~K}$. SETHE, Urkunden, I, 84, 15 et 17.

${ }^{2}$ Le verbe est à la forme relative imperfective; bien que celle-ci se réfère le plus souvent au présent, elle peut exceptionnellement se rapporter au passé: G. LEFEBVRE, Grammaire de l'égyptien classique, 1940, § 479, cite un exemple.
} 
nous proposons d'identifier à l'épouse de Ptahchepses $I^{1}$ tend à confirmer ce que la proximité des tombeaux de Sabou-Tjéti et de SabouIbebi et Ptahchepses IV nous a appris, à savoir que Sabou-Tjéti a succédé à ces grands prêtres ${ }^{2}$, peut-être d'abord au premier, puis aux deux à la mort de Ptahchepses IV.

\section{§ 25. LA REFORME, OEUVRE DU ROI}

Les bribes de la biographie de Sabou-Tjéti mentionnent cinq fois "Sa Majesté". La première est sans importance parce qu'elle ne sert qu'à fixer un moment de la carrière du grand prêtre. Deux des autres mentions concernent des nominations par le roi. Les termes "Sa Majesté" apparaissent aussi dans l'expression "selon le désir de Sa Majesté" $(\mathbf{2 6 , 9})^{3}$, qui vient après une phrase sur la maison de l'administration (?), et enfin dans la dernière proposition "car le coeur de Sa Majesté est plus fort que n'importe quelle chose faite habituellement là"; nous ne croyons pas trahir le sens de ces mots en les rendant par "la volonté de Sa Majesté est plus forte que toute tradition administrative". Le roi a donc transformé le pontificat memphite.

Il est très possible que cette réforme soit l'oeuvre de Pépi Ier puisque Sabou-Tjéti n'a pas pu être seul grand des chefs des artisans avant la mort de Ptahchepses IV et que cet événement coïncide approximativement avec la fin du règne de Téti ${ }^{4}$.

La transformation du pontificat n'a pas consisté seulement dans la suppression d'un des deux postes de grands des chefs des artisans. En s'arrogeant le droit de nommer lui-même le grand prêtre, le roi a probablement supprimé l'obligation, pour ce dernier, de gravir d'abord les échelons d'une hiérarchie rigide. Si le monument de Sabou-Tjéti, trop détérioré, ne permet pas de prononcer sur ce point, les inscriptions des

${ }^{1}$ On voit celle-ci à côté de son mari dans M.A. MURRAY, Saqqara Mastabas, I, 1904, pl. 31,1, avec la légende: "La connue du roi, prophétesse d'Hathor, prophétesse de Néit, Inti".

\footnotetext{
${ }^{2}$ Voir ci-dessus, § 18.

${ }^{3}$ Litt. "selon le désir (venu) de Sa Majesté".

${ }^{4}$ Voir ci-dessous, § 41.
} 
grands prêtres postérieurs le prouvent.

Il est possible que le roi ait modifié le service de Ptah et que l'apparition de l'épithète "préposé au diadème quand Ptah est orné"1 en soit un indice.

Le service de Sokar a certainement été remanié. Les "sem de Sokar" dirigés jusqu'alors par les futurs grands prêtres ${ }^{2}$ ont été supprimés. Le domaine de Sokar a passé à Ptah qui, en échange, assure vraisemblablement le nécessaire au culte de Sokar ${ }^{3}$. Enfin, le grand prêtre de Ptah porte désormais le titre de prêtre-sem, et nous pouvons penser qu'il célèbre la fête de $\operatorname{Sokar}^{4}$ en cette qualité depuis Pépi $\mathrm{Ier}^{5}$.

A ces modifications réelles, le roi a ajouté l'octroi de titres honorifiques tels que "compagnon unique" et "trésorier du roi de BasseEgypte" qui se substituent à l'ancien "confident du roi".

C'est donc un remaniement total du pontificat que le roi a effectué.

\section{§ 26. L'UNITE PROBABLE DU PONTIFICAT DEPUIS PEPI Ier}

La réforme du pontificat, qui fut l'oeuvre d'un des derniers grands souverains de l'Ancien Empire a fait sentir ses effets jusque sous la domination romaine. Nous avons en effet des témoignages de nomination de grands des chefs des artisans par le roi au Nouvel Empire et aux époques ptolémaïque et romaine ${ }^{6}$. D'autre part, le titre de sem, en relation avec Sokar, a été porté par tous les grands prêtres des époques

${ }^{1}$ Cette épithète est peut-être encore employée au Moyen Empire $(\mathbf{3 2}, \mathbf{2})$.

2 Voir ci-dessus, § 5 .

3 Voir ci-dessus, § 13.

${ }^{4}$ Ce n'est peut-être pas un hasard si la fête de Sokar apparaît parmi d'autres fêtes dans les formules d'offrandes des tombeaux memphites seulement à la fin de l'Ancien Empire; le fait a été noté par H. KEES, Götterglaube, 1941, p. 295, note 4, qui cite en référence: H. JUNKER, Giza II, p. 61 et IV, p. 21. Rappelons que la fête de Sokar apparaît au début de l'Egypte unifiée sur la pierre de Palerme.

5 Voir ci-dessus, § 13.

${ }^{6}$ Voir ci-dessus, § 7. 
suivantes.

Nous sommes malheureusement renseignés d'une façon moins catégorique sur l'unité du pontificat depuis Pépi Ier. Si, après SabouTjéti, nous ne connaissons plus de mention de "deux grands des chefs des artisans", il n'y a, non plus, pas de texte affirmant qu'il n'y eut plus qu'un grand des chefs des artisans. Comme nous ne basons par ailleurs la suppression de la dualité du pontificat que sur une restitution de texte, très probable, nous estimons nécessaire de passer en revue les documents postérieurs à l'Ancien Empire qui peuvent donner une indication à ce sujet.

Lorsque Hormin, sous Séti Ier, sollicite l'aide du sem pour lui faire l'ouverture de la bouche et celle du grand des chefs des artisans pour exalter son ka (93), il s'adresse, selon une tournure de style fréquente en égyptien, à un personnage exerçant deux fonctions. Celui-ci est évidemment le grand prêtre de Memphis. S'il y avait eu alors deux grands prêtres, on peut penser que Hormin se serait adressé, sinon aux deux sem, aux deux grands des chefs des artisans dont l'action aurait été plus efficace que celle d'un seul.

Lorsqu'Osorkon célèbre la fête Sed représentée à Bubastis, il fait appel aux grands prêtres de Memphis et d'Héliopolis (172 a). Dans deux scènes auxquelles ces personnages participent, il n'y a chaque fois qu'un grand des chefs des artisans ${ }^{1}$ et ce n'est pas un grand prêtre donné qui est représenté puisque la légende n'accompagne le titre d'aucun nom propre. On est encore plus en droit de penser qu'Osorkon aurait fait représenter deux grands des chefs des artisans s'ils avaient été deux à cette époque.

Le même raisonnement s'applique au passage du papyrus Harris I où Ramsès III déclare qu'il a placé huit cent quarante et une personnes attribuées au temple memphite portant son nom sous la direction du grand des chefs des artisans et des "principaux" (162 a).

\footnotetext{
${ }^{1}$ Et aussi un seul grand prêtre d'Héliopolis !
} 
Le papyrus Hood (184) et la liste géographique du temple d'Edfou (205) ne mentionnent aussi qu'un grand des chefs des artisans. Dans le dernier de ces documents, le déterminatif de grand des chefs des artisans indique clairement qu'il n'y a qu'un seul personnage exerçant cette fonction.

Un document figuré de la fin de la XVIIIème dynastie confirme les inscriptions que nous venons de parcourir. C'est le cortège funèbre d'un grand prêtre memphite sur un bas-relief du Musée de Berlin (70) ${ }^{1}$. Le traîneau chargé du corbillard y est suivi des deux fils du défunt, puis d'une série de dignitaires: un chef d'armée ${ }^{2}$, les deux vizirs, un groupe de trois personnages à perruque dont le premier est scribe royal et majordome, le second scribe royal et directeur du sceau, le troisième directeur d'un édifice administratif ${ }^{3}$; puis vient un groupe de cinq hommes dont les deux premiers et le dernier ont des perruques, tandis que les troisième et quatrième ne montrent que le sommet de leur tête, rase ou coiffée d'un serre-tête, au second plan derrière le second et le cinquième; trois légendes seulement surmontent ce groupe et nous penșons qu'elles désignent le premier, le second et le cinquième personnages qui sont respectivement officier, chef de cabinet et directeur de la maison du trésor; un dernier ${ }^{4}$ groupe de deux hommes à serre-tête et d'un à perruque comprend un grand prêtre d'Héliopolis ${ }^{5}$, un sem et un gouverneur. Il n'est guère plausible de supposer qu'un des deux personnages sans légende dont on ne voit que le sommet de la tête soit un grand des chefs des artisans accompagnant la dépouille de celui qui aurait été son collègue; si tel avait été le cas, on l'aurait, semble-t-il, représenté d'une façon moins discrète ! On peut au contraire affirmer qu'il n'y a pas de grand des chefs des artisans dans ce cortège funèbre de grand prêtre. Comme les bas-reliefs d'un tombeau n'étaient pas exécutés entre la mort et l'ensevelissement du défunt mais du vivant du personnage, on ne peut pas expliquer l'absence d'un grand des chefs des

1 Pour l'attribution certaine de ce bas-relief à un tombeau de grand prêtre de Memphis, voir ci-dessous au chapitre sur les grands prêtres du Nouvel Empire, $\S \mathbf{5 4}$.

${ }^{2}$ C'est le futur roi Horemheb.

${ }^{3}$ rwy.t (?); cf. Wörterbuch, II, p. 407, 14.

${ }^{4}$ Il y a encore plusieurs prêtres sculptés à une échelle plus petite derrière ce dernier groupe.

${ }^{5}$ Ici, comme à la fête Sed de Bubastis, le grand prêtre d'Héliopolis est seul. 
artisans dans le cortège de Berlin par un éventuel décès simultané de deux grands des chefs des artisans. La seule autre raison possible est qu'il n'y avait qu'un grand prêtre en fonction à la fois.

Les arguments à invoquer contre cette conclusion n'ont qu'une valeur relative. Le fait qu'Anemher, ce grand prêtre ptolémaïque, a survécu à son fils Téos, qui fut aussi grand prêtre, ne prouve pas qu'ils ont exercé leur fonction ensemble, car Anemher, qui a vécu soixante-douze ans, s'est retiré, pensons-nous, de la vie active avant sa mort et Téos a été grand prêtre du vivant de son père alors que celui-ci n'était plus en fonction.

La généalogie d'Ankhef-ny-sekhmet (182), où les trois-quarts des grands prêtres memphites ne sont pas confirmés par un autre monument a fait défendre par son éditeur la thèse de la dualité du pontificat à travers l'histoire de l'Egypte ancienne ${ }^{1}$. Mais cette généalogie, que nous examinerons en détail plus $\operatorname{loin}^{2}$, contient de telles invraisemblances touchant la durée des diverses générations qu'elle ne peut pas être prise à la lettre. Serait-elle même en tout point conforme à la réalité qu'on pourrait encore supposer qu'elle nomme les grands prêtres par un "beau nom" ou un surnom et qu'elle ne prouve nullement l'existence simultanée de deux grands prêtres de Ptah après l'Ancien Empire.

Les seuls arguments à retenir contre l'unité du pontificat depuis la fin de l'Ancien Empire sont les mentions de deux grands des chefs des artisans $^{3}$ et l'absence de témoignage catégorique de l'existence d'un seul grand des chefs des artisans. C'est pourquoi nous n'affirmons pas que l'unité du pontificat est absolument certaine depuis Pépi Ier, mais qu'elle est hautement probable du fait qu'elle se serait produite à un moment où le pontificat memphite a subi d'autres transformations profondes ${ }^{4}$.

Un roi de la VIème dynastie, peut-être Pépi Ier, a donc transformé le pontificat memphite, remaniant entre autres le service de Sokar, attribuant au souverain la nomination du grand des chefs des artisans, et supprimant très probablement l'un des titulaires de cette fonction.

\footnotetext{
${ }^{1}$ L. BORCHARDT, Die Mittel zur zeitlichen Festlegung..., p. 105.

${ }^{2}$ Voir ci-dessous Chapitre VI, $\$ 33$.

${ }^{3}$ Voir ci-dessus, § 18.

${ }^{4}$ Voir ci-dessus, § 25.
} 
. 
C H A P I T R E V

\section{LES MODIFICATIONS DU PONTIFICAT AU NOUVEL EMPIRE}

\section{§ 27. LE PONTIFICAT AU COURS DES DEUX PERIODES INTERMEDIAIRES}

Entre le grand prêtre Sabou-Tjéti, contemporain de Pépi Ier sous la VIème dynastie et Sehetepibrê-ankh-nedjem ou Hapidjefa, contemporains de Sésostris Ier sous la XIIème dynastie, il y a un intervalle de quatre siècles environ qui ne nous a livré jusqu'à présent aucun monument de grand prêtre memphite. La seule possibilité d'émettre une hypothèse sur le pontificat pendant les temps troublés de la première période intermédiaire consiste à comparer les monuments antérieurs et postérieurs à cette époque. En fait de monuments antérieurs, nous ne pouvons faire appel qu'à ceux de Sabou-Tjéti, qui ont suivi la réforme de Pépi Ier. Pour le Moyen Empire, notre récolte de documents n'a pas été abondante. Le caractère limité de ces sources n'empêche cependant pas d'en tirer quelque renseignement.

La majeure partie des titres portés par Sabou-Tjéti se retrouvent chez les grands prêtres du Moyen Empire. Ceux-ci sont, sauf Senousert-ankh, gouverneurs $^{1}$ et trésoriers du roi de Basse-Egypte ${ }^{2}$ comme Sabou-Tjéti. Sehetepibrê-ankh-nedjem et Senousert-ankh sont $\mathrm{sem}^{3}$. Hapidjefa, Sehetepibrê-ankh-nedjem et Senousert-ankh sont dans la double maison ${ }^{4}$. Senousert-ankh est placé à la tête de Nekheb $^{5}$ et prêtre-lecteur en chef ${ }^{6}$.

\footnotetext{
1 "haty-â": 26,2; 30; 31,1-4; 32,1-3; Senousert-ankh était peut-être gouverneur: 34,1.

2 "sedjaouty-bity": 27,$2 ; 30,31,1-4 ; 32,1$ et 3 .

3 26,4; 27,1; 31,1-2; 32,1-3; 33,1.

${ }^{4} 26,3 ; 28 ; 30 ; 32,3 ; 35, x+4$.

526,$2 ; 33,4$.
} 
Sehetepibrê-ankh-nedjem est probablement préposé au diadème quand Ptah est orné ${ }^{1}$ Enfin, Hapidjefa est compagnon unique ${ }^{2}$.

En plus des fonctions ci-dessus, honorifiques ou réelles, Sabou-Tjéti a été imakhou, chef de toute la garde-robe, participant à la fête de Rê et directeur des deux ouabet. On ne retrouve pas ces désignations au Moyen Empire. Mais la première, qui est d'ailleurs une "qualité", réapparaît plus $\operatorname{tard}^{3}$, ainsi que la seconde ${ }^{4}$, d'un emploi sporadique 5 . Seuls les titres participant à la fête de Rê et directeur des deux ouabet, mentionné uniquement par Sabou-Tjéti, disparaissent complètement après l'Ancien Empire $^{6}$.

Avec Senousert-ankh, on rencontre des titres qui ne sont pas chez Sabou-Tjéti, mais quelques-uns nous semblent comparables à des appellations de Khabaouseker ${ }^{7}$, tandis que prophète de Ptah et prophète de Sokar $(\mathbf{3 5}, \mathbf{x}+\mathbf{5})$ sont d'un emploi régulier avant Sabou-Tjéti. Le seul titre dont on puisse dire qu'il apparaît au Moyen Empire est celui de "noble", porté par Hapidjefa, Sehetepibrê-ankh-nedjem et Nebpou ${ }^{8}$.

Au total, il y a disparition de deux fonctions et apparition d'une fonction honorifique entre l'Ancien et le Moyen Empire. La transformation politique du pays, l'effondrement du pouvoir central entraînant avec lui celui de l'administration royale ont causé la disparition du titre directeur des deux ouabet chez les grands prêtres de Memphis. Quant au titre "participant à la fête de Rê", s'il se rapportait à une fête célébrée dans les temples solaires, l'abandon de ceux-ci aurait

${ }^{6} 26,3 ; 27$ et 29,1 (seulement prêtre-lecteur); 33,3; 34,1; 35,x +1 (?).

126,3 et $27,2(?) ; 32,2$.

2 26,2 à $4 ; 27 ; 29,1-2 ; 30$.

3140.

426,$4 ; 27,1 ; 89,7$; etc.

${ }^{5}$ Voir ci-dessous, § 30.

${ }^{6}$ Pour la présence de "participant à la fête de Rê" sur un monument saite archaïsant, voir ci-dessous au chapitre sur les grands prêtres de la Basse Epoque, § 71.

${ }^{7}$ Voir ci-dessus, § 21, p. 47, note 2 .

${ }^{8} 30 ; 31,2 ; 32,1-2$; il est très fréquent jusqu'à la fin de l'époque ptolémaïque. 
causé l'abandon du titre'; si, par contre, participant à la fête de Rê ne concerne pas les temples solaires de la Vème dynastie, il faudrait d'abord savoir le sens de cette expression avant de chercher le motif qui l'a fait abandonner.

On peut donc conclure que la première période intermédiaire n'a apporté aucune modification importante au pontificat memphite.

Un second intervalle où les documents de grands prêtres de Memphis font défaut ${ }^{2}$ sépare Sehetepibrê-cheri, petit-fils de Sehetepibrê-ankhnedjem qui n'est lui-même pas antérieur à Sésostris Ier, de Ptahmès Ier, contemporain de Thoutmosis III, soit de nouveau environ quatre siècles, même peut-être quatre siècles et demi. En comparant les grands prêtres de la XVIIIème dynastie ${ }^{3}$ à ceux du Moyen Empire, on constate les faits suivants: les six grands prêtres de la XVIIIème dynastie sont prêtres-sem, comme Sehetepibrê-ankh-nedjem et Senousert-ankh, et, plus anciennement, Sabou-Tjéti; cinq d'entre eux portent les titres honorifiques de noble, gouverneur et trésorier du roi de Basse-Egypte; Thoutmôsis seul n'en est pas doté, sans doute à cause de sa qualité princière; Ptahmès II, Ptahmès III et Ptahemhat-Ty sont compagnons uniques. Ces titres sont employés ordinairement au Moyen Empire et, sauf noble, sont portés par Sabou-Tjéti sous l'Ancien Empire.

La XVIIIème dynastie marque la disparition des titres et épithètes suivants: placé à la tête de Nekheb, prêtre-lecteur en chef et préposé au diadème quand Ptah est orné.

1 Cependant, comme les temples solaires n'ont plus étê en service depuis Menkaouhor (K. SETHE, dans Ä.Z., 27 (1889), p. 111), il faudrait admettre que les grands prêtres de l'Ancien Empire postérieurs à ce roi auraient conservé le titre sans remplir la fonction.

${ }^{2}$ Nous ne tenons pas compte ici, pour les raisons qui sont exposées au chapitre suivant, de la généalogie d'Ankhef-ny-sekhmet au Musée de Berlin.

${ }^{3}$ Ceux de la XIXème dynastie ne peuvent être rangés aux côtés de ceux de la XVIIIème; voir ci-dessous, $\S 30$ à 32. 
En revanche, elle fait apparaître le titre de père divin aimé du dieu ${ }^{1}$, porté par Ptahmès II et Ptahmès III, et fréquemment aux époques postérieures. Ansi que l'antique "prophète de Ptah", il sert à désigner la fonction religieuse du grand des chefs des artisans ${ }^{2}$, mais il ne semble pas qu'il y ait de relation véritable entre l'un et l'autre titre ${ }^{3}$.

Nous sommes ainsi amenés à faire, pour la seconde période intermédiaire, la même constatation que pour la première. Elle n'a apporté aucune modification importante au pontificat memphite. Une comparaison du groupe de Sehetepibrê-ankh-nedjem du Moyen Empire (31) avec le naos de Ptahmès I du Nouvel Empire (36) illustre bien la continuité de l'institution: l'un et l'autre monument ont exactement les mêmes titres.

${ }^{1}$ Au Nouvel Empire, ce titre semble réservé au grand prêtre, alors que d'autres membres du clergé memphite sont simplement pères divins; voir l'aperçu sur le personnel de Ptah.

${ }^{2}$ Voir ci-dessus, $\$ 12$.

${ }^{3}$ S'il y a eu échange entre prophète de Ptah et père divin aimé du dieu, on pourrait penser que cela s'est passé entre le Moyen et le Nouvel Empire puisque prophète de Ptah apparaît, une fois, au Moyen Empire, chez Senousert-ankh $(35, \mathbf{x}+\mathbf{5})$.

Mais, comme le titre sem, porté pour la première fois par Sabou-Tjéti, nous paraît désigner le service de Sokar (voir ci-dessus, § 13), nous pensons que "prophète de Sokar" pourrait n'être chez Senousert-ankh que l'expression d'un certain penchant à l'archaïsme, qui se serait aussi manifesté dans l'emploi de titres de la IIIème dynastie (voir ci-dessus, $\$ 21$, p. 59, note 2 ) et d'une version des textes des pyramides aussi ancienne que celle des rois de l'Ancien Empire pour son caveau (inscription 33 pour la référence); il en serait de même pour le titre prophète de Ptah. L'un et l'autre auraient peut-être alors disparu de la titulature réelle des grands prêtres avec la réforme de Pépi Ier. D'autre part, comme il est peu vraisemblable que père divin aimé du dieu ait été attribué aux grands prêtres de la fin de l'Ancien Empire, car son absence sur le groupe du Moyen Empire (31) s'expliquerait difficilement, il est probable qu'il n'y a pas de relation directe entre les deux titres, mais que le premier a cessé d'être employé longtemps avant l'apparition du second. 


\section{§ 28. LE PONTIFICAT MEMPHITE ET LE PONTIFICAT THEBAIN ${ }^{1}$}

Ptah et Sokar ont atteint de bonne heure la région thébaine, puisqu'ils sont adorés, sous la forme de Ptah-Sokar, dans une tombe de la VIème dynastie $^{2}$. Au Moyen Empire, Ptah a son sanctuaire à Karnak, qui sera rebâti par Thoutmôsis III et complété par des rois postérieurs. Sokar possède aussi son sanctuaire, sur la rive gauche du $\mathrm{Nil}^{3}$. Ces cultes thébains sont administrés par le grand prêtre d'Amon.

A partir de la XIXème dynasttie, l'un ou l'autre grands prêtres portent des titres qui les relient à ces cultes originaires de Memphis: Nebneterou, premier prophète d'Amon sous Horemheb, est "sem dans le temple de Ptah"4; Bakenkhonsou, grand prêtre d'Amon pendant vingt-sept ans sous Ramsès II, est "sem et grand des chefs des artisans de Ptah"5. Ce fait n'a rien d'étonnant, mais on peut se demander s'il n'est pas une conséquence indirecte des attaques dont Amon a été l'objet sous Akhenaton; par la suite, des grands prêtres de Karnak auraient jugé bon de se montrer moins exclusivement amoniens et se seraient intéressés ou auraient feint de s'intéresser aux cultes originaires de Memphis ${ }^{6}$.

${ }^{1}$ L'examen des rapports entre les pontificats memphite et thébain est possible grâce à l'ouvrage de G. LEFEBVRE, Histoire... Pour aborder une recherche du même genre sur les pontificats héliopolitain et memphite, il faudrait disposer d'une étude sur les grands prêtres d'Héliopolis. On trouvera quelque indication sur les rapports de ces pontificats au $\S 15$ ci-dessus. Nous ignorons si Rêhetep (103), sous Ramsès II, a exercé simultanément les fonctions de grands prêtres héliopolitain et memphite, parce que sa carrière a été itinérante. Par contre Chedsounefertoum, personnage manifestement memphite (voir les rapports de sa famille avec Ptah: 167), a vraisemblablement été à la fois grand prêtre de Memphis et d'Héliopolis au début de la XXIIème dynastie.

${ }^{2}$ P.E. NEWBERRY, A sixth dynasty tomb at Thebes, dans Annales du Service des Antiquités, 4 (1903), p. 97.

3 A. VARILLE, Nouvelles listes géographiques..., dans Annales du Service des Antiquités, 36 (1936), p. 213.

${ }^{4}$ G. LEFEBVRE, Histoire, p. 247.

${ }^{5}$ ibidem, p. 254-255. D'autres personnages thébains sont aussi grands des chefs des artisans ou sem de Ptah thébain; voir ci-dessus, § 5, p. 15, note 3 .

${ }^{6}$ Le titre de grand prêtre de Rê (thébain) porté par des grands prêtres de Karnak s'explique peut-être différemment; on peut penser qu'il est l'équivalent, sur le plan des institutions religieuses, de la synthèse de Rê et d'Amon en Amon-Rê sur le plan théologique. 
De son côté, Amon a été adoré à Memphis dans un sanctuaire qui lui était consacré; au cours de la XXIème dynastie, le roi Siamon a fait bâtir un temple pour Amon du lapis-lazuli véritable $(163 ; 164)$, et on peut supposer qu'Amon a eu son culte memphite dès la XVIIIème dynastie ${ }^{1}$. Mais aucun grand prêtre memphite n'a, à notre connaissance, jamais porté le titre de premier prophète d'Amon (memphite); nous n'avons même rencontré qu'une fois le nom du dieu de Karnak dans la titulature d'un grand prêtre memphite: Neferrenpet est "conducteur (de la fête ?) d'Amon" $(\mathbf{1 5 0 , 3})$, mais, comme il se déclare "directeur des prophètes de tous les dieux de Haute et Basse-Egypte" (149,6-7,17-18) sur un autre monument où il s'adresse à Amon-Rê (149,3 et 5) autant qu'à Ptah $(\mathbf{1 4 9 , 4}$ et 9), nous pensons que l'épithète "conducteur de la fête d'Amon" pourrait se rapporter à Amon de Karnak. Les grands prêtres de Ptah de Memphis, conscients de l'ancienneté de leur dieu, estimaient peut-être qu'ils auraient dérogé en portant le titre du grand prêtre d'Amon de Karnak !

Mais ni Amon memphite ni Ptah thébain n'ont établi une relation quelconque entre les institutions religieuses de Thèbes et de Memphis comme l'a fait au contraire la direction des prophètes de Haute et BasseEgypte, sorte de ministère des cultes.

L'importance grandissante du clergé d'Amon sous la XVIIIème dynastie a eu, avant Akhenaton, une conséquence avantageuse pour le grand prêtre memphite. Car c'est sous Aménophis III qu'on rencontre pour la première fois un grand prêtre de Ptah, Ptahmès (II), fils du vizir Thoutmôsis, chargé de la direction administrative de tous les prophètes égyptiens $(39,3 ; 40 ; 41,2)$. Cette haute fonction ${ }^{2}$ avait été jusqu'alors remplie par des grands prêtres d'Amon de Karnak ${ }^{3}$ : Hapouseneb sous

${ }^{1}$ Ce culte a fait nommer Memphis "Thèbes du nord" à l'époque saïte: le vizir Bakenrenef (A. WEIL, Veziere, p. 145, n 19) est "sem, prophète de Ptah et prophète d'Amon de Thèbes du Nord". Nous ne pensons pas que ce Bakenrenef soit le grand prêtre memphite du même nom (Berlin, Ausführliches Verzeichnis, 1894, p. 288), si tant est que celui-ci soit grand des chefs des artisans.

${ }^{2}$ La liste des directeurs des prophètes de Haute et Basse-Egypte a été dressée par R. ANTHES, Der Wesier Paser..., dans Ä.Z., 67 (1931), p. 8 et Die hohen Beamten namens Ptahmose..., dans $\ddot{A} . Z, 72$ (1936), p. 60.

${ }^{3}$ G. LEFEBVRE, Histoire, p. 98. 
Hatchepsout, Menkheperrêseneb sous Thoutmôsis III et Aménophis II, Méry sous Aménophis II, Amenemhat sous Aménophis II et Thoutmôsis IV, ainsi que par un personnage thébain administrant les terrains d'Amon, Horemheb, sous Thoutmôsis IV et Aménophis III. Le grand prêtre d'Amon et vizir Ptahmès est encore ministre des cultes sous ce pharaon. G. Lefebvre a placé ce pontife, par hypothèse, après le grand prêtre d'Amon Méryptah, c'est-à-dire dans la dernière décade du règne d'Aménophis III $^{1}$. R. Anthes préfère le placer avant Méryptah en s'appuyant sur ce qu'aucun vizir appartenant à la Haute-Egypte n'est connu au cours des deux premières décades d'Aménophis III $^{2}$. Comme les grands prêtres d'Amon n'ont plus été ministres des cultes sous la XVIIIème dynastie après ce Ptahmès, il $\mathrm{y}$ a là un argument pour le situer au début du règne d'Aménophis III. On peut supposer que le roi, lorsqu'il a fallu pourvoir à la succession du grand prêtre d'Amon, vizir du sud et ministre des cultes Ptahmès, a enlevé au clergé thébain ce qui était devenu une de ses prérogatives et fait passer la fonction de ministre des cultes au grand prêtre de Ptah à Memphis ${ }^{3}$.

Cette supposition est étayée par l'existence d'un autre ministre des cultes memphite sous Aménophis III: c'est le grand des chefs des artisans Thoutmôsis, propre fils du roi ${ }^{4}$. Son pontificat confirme le dessein arrêté

${ }^{1}$ G. LEFEBVRE, Histoire, p. 97 et 99.

${ }^{2}$ R. ANTHES, Die hohen Beamten, dans Ä.Z., 72 (1936), p. 62, n 6, N.B. !

${ }^{3}$ En suivant au contraire l'hypothèse de G. Lefebvre, le ministère des cultes aurait passé au grand prêtre de Ptah de Memphis peu après le début du règne d'Aménophis III, mais aurait été repris par le grand prêtre d'Amon dans le courant du règne, pour passer avant la fin de celui-ci au vizir Ramôse.

${ }^{4}$ R. Anthes, dans sa liste de ministres des cultes (Ä.Z., 67 (1931), p. 8) fait suivre le numéro d'ordre qu'il attribue à Thoutmôsis et le règne (Aménophis III) pendant lequel il aurait été ministre des cultes de deux points d'interrogation. Plus tard (̈̈.Z., 72 (1936), p. 68), il a exclu Thoutmôsis des ministres des cultes sous Aménophis III. C'est donc qu'il hésite à identifier le grand prêtre memphite et ministre des cultes Thoutmôsis (62) avec le prince Thoutmôsis d'une chapelle d'Apis (63). Il y a cependant de bonnes raisons en faveur de cette identification; voir ci-dessous au chapitre sur les grands prêtres du Nouvel Empire. Peut-être R. Anthes estime-t-il accessoirement qu'il y a assez de ministres des cultes sous Aménophis III (quatre, sans compter Thoutmôsis) pour ne pas leur en adjoindre un dont la date lui parait douteuse. Mais Thoutmôsis peut n'avoir eu qu'une carrière assez courte (voir aussi ci-dessous). D'autre part, comme le titre du ministre des cultes a varié, sa forme particulière chez Thoutmôsis (directeur des prophètes en Haute et Basse-Egypte) ne peut pas être un motif d'exclure ce grand prêtre des 
qu'eût Aménophis III de réagir contre la puissance des pontifes de Karnak en leur enlevant le ministère des cultes pour le confier à des grands prêtres de Memphis. De plus, en nommant son fils grand des chefs des artisans, le roi a pu poursuivre un double but consistant à accorder un pouvoir plus étendu aux grands prêtres de Memphis, pour contenir ceux de Karnak, et à s'assurer en même temps la fidélité complète du grand prêtre de Ptah, pour ne pas courir les mêmes risques qu'avec ceuxlà.

Les grands prêtres de Memphis n'ont cependant pas conservé le ministère des cultes, qui n'est pas davantage retourné dans les mains du grand prêtre d'Amon mais est échu, à la fin du règne d'Aménophis III et sous Akhenaton, au vizir Ramôse.

Au début de la XIXème dynastie, Nebneterou, grand prêtre d'Amon d'Hermonthis et peut-être d'Amon de Karnak ${ }^{1}$, est ministre des cultes et a pour successeur son fils, le vizir et grand prêtre d'Amon d'Hermonthis, Paser.

C'est seulement depuis la fin du règne de Ramsès II que des grands prêtres de Karnak et de Memphis sont de nouveau certainement ministres des cultes. A Memphis il y a le vizir et grand des chefs des artisans Neferrenpet (149,6-7,17-18). A Karnak, sous Meneptah, Romê-Roy ${ }^{2}$ fait revenir le ministère des cultes au grand prêtre d'Amon pour un temps assez long.

Aux époques postérieures au Nouvel Empire, trois grands prêtres de Memphis ont été ministres des cultes; Chedsounefertoum au début de la XXIIème dynastie $(\mathbf{1 6 9 , 6 )}$ puis, sous les Ptolémées, Anemher au troisième siècle avant $\mathrm{J}$.-C. et Psenptaïs II au premier, tandis que le fils d'Anemher et grand prêtre memphite Téos, mort avant son père, porte le titre de "qui est à la tête de tous les ouab et prophètes dans les temples de haute et Basse-Egypte" $(\mathbf{1 9 3 , 7 )}$ et a, de ce fait, peut-être aidé son père.

\footnotetext{
ministres des cultes.

${ }^{1}$ G. LEFEBVRE, Histoire, p. 116.

2 ibidem, p. 146 et 258.
} 
Le ministère des cultes semble donc être venu à Memphis sous la XVIIIème dynastie par volonté royale. Par contre, nous croyons que les grands prêtres de Ptah eux-mêmes ont créé l'appellation "grand des chefs des artisans de Ptah". Elle apparaît pour la première fois au début de la XIXème dynastie, chez Khamouas I (85), et est, dès lors, employée fréquemment jusque vers la fin de la XXIIème dynastie (Peftjaouaouibastet, 181) puis disparaît complètement. A quel motif le promoteur de cette nouvelle désignation a-t-il obéi ? Ce n'était pas pour éviter une ambiguïté ou une confusion quelconque, car personne autre que le grand prêtre de Ptah n'a jamais porté le titre de grand des chefs des artisans. Nous estimons que ce pourrait être simplement pour bien marquer l'indépendance du clergé memphite par rapport au clergé thébain, et plus particulièrement de son chef par rapport à celui du sacerdoce thébain. Ce dernier, en effet, faisait nommer son dieu chaque fois qu'on parlait de lui puisqu'il s'intitulait premier prophète d'Amon. En regard, le titre grand des chefs des artisans, bien que beaucoup plus ancien, paraissait peut-être insuffisant, d'autant qu'il ne désignait pas, en le prenant à la lettre, une fonction religieuse ${ }^{1}$, tandis que grand des chefs des artisans de Ptah rétablissait une sorte d'équilibre entre les pontifes memphite et thébain. La nouvelle appellation pourrait être, en partie du moins ${ }^{2}$, l'indice d'une réaction du clergé memphite contre la puissance que le clergé thébain avait reconquise après le temps d'El-Amarna.

Le titre "sem de Ptah" qui n'apparaît chez les grands prêtres memphites que postérieurement à grand des chefs des artisans de Ptah, a évidemment été créé sur le modèle de ce dernier, mais peut-être pour d'autres motifs ${ }^{3}$. Il est resté en usage jusque sous les Ptolémées $(\mathbf{1 9 7}, \mathbf{2}, 12,19,26)$. C'est pourquoi nous estimons que la disparition de grand des chefs des artisans de Ptah à partir de l'époque saïte a été causée non seulement par la recherche d'une forme plus ancienne mais aussi par l'évanouissement du motif qui avait fait ajouter "de Ptah" à grand des chefs des artisans, soit l'importance d'Amon de Thèbes, excessive aux yeux d'un Memphite du Nouvel Empire et de l'époque suivante.

${ }^{1}$ Voir ci-dessus, $\S \mathbf{1 1}$ et $\mathbf{1 2}$.

${ }^{2}$ Voir au $\$ 30$.

${ }^{3}$ Voir ci-dessous, § 32. 


\section{§ 29. LE PONTIFICAT PENDANT LE REGNE D'AKHENATON}

Nous ne savons pas de grand prêtre memphite qui ait été indubitablement en fonction sous Akhenaton. Aussi sommes-nous obligés d'avoir de nouveau recours à la méthode par laquelle nous nous sommes informés sur le pontificat pendant les périodes intermédiaires ${ }^{1}$.

Entre Ptahmès III, sous Aménophis III, et Ptahemhat-Ty, sous Toutankhamon ${ }^{2}$, il y a les ressemblances suivantes: tous deux sont nobles, gouverneurs, compagnons uniques, trésoriers du roi de Basse-Egypte et sem (69,4-5 et 72). Si Ptahmès III est père divin aimé du dieu, l'absence de ce titre sur les monuments connus de Ptahemhat-Ty n'a pas de signification ${ }^{3}$.

Il y a donc continuité entre les titulatures du grand prêtre avant et après Akhenaton. Cela ne prouve d'ailleurs pas que Ptah memphite n'a été l'objet d'aucune attaque sous Akhenaton, mais invite à le supposer.

D'autre part, nous ne connaissons pas de martelage du nom de Ptah sur des monuments memphites et même le nom d'Amon a été, au moins parfois, respecté à Memphis ${ }^{4}$. Enfin, un frère d'Akhenaton avait été grand prêtre de Ptah pendant le règne d'Aménophis III $^{5}$.

Il est ainsi très vraisemblable qu'Akhenaton n'a jamais inquiété le grand prêtre de Ptah. Le nouveau roi a fait construire à Memphis un "temple d'Aton"6 qui était sans doute sous la dépendance administrative

${ }^{1}$ Voir ci-dessus, $§ 27$.

${ }^{2}$ Voir au chapitre sur les grands prêtres du Nouvel Empire les motifs d'attribuer le cortège funèbre d'un grand prêtre au Musée de Berlin, sous Toutankhamon, au grand des chefs des artisans Ptahemhat-Ty.

${ }^{3}$ Voir ci-dessus, § 27.

4 La statue du scribe royal Amenhotep portant un texte de fondation du temple construit par Aménophis III à Memphis était probablement encore à sa place dans le temple en question sous le règne d'Akhenaton. Or le nom d'Amon n'y est martelé ni dans le nom du possesseur de la stèle ni dans la mention du dieu "Amon dans la Ville du sud" à la ligne 21 du texte (A.H. GARDINER, dans Fl. PETRIE, Memphis V, p. 33-36).

${ }^{5}$ Voir au chapitre sur les grands prêtres du Nouvel Empire: Thoutmôsis.

${ }^{6}$ B. PORTER ET R. MOSS, Topographical bibliography, III, Memphis, p. 188, 192, $220 s q$. 
du temple de Ptah, comme le "temple de Nebmaâtrê" bâti par Aménophis III ${ }^{1}$.

Ces diverses considérations autorisent à envisager que Méryptah (79 81), dont nous ne savons au juste s'il a précédé ou suivi Ptahemhat-Ty, aurait été dans le premier cas grand prêtre de Memphis sous Akhenaton.

\section{§ 30. L'EVOLUTION DU PONTIFICAT AU DEBUT DE LA XIXème DYNASTIE}

La titulature des grands prêtres a été modifiée à deux reprises sous la XIXème dynastie, une première fois au début et une autre fois sous Ramsès $\mathrm{II}^{2}$. Les changements de ce temps ne sont pas du tout comparables aux transformations effectuées sous la VIème dynastie. En effet, les grands prêtres de la XIXème dynastie continuent à être $\mathrm{sem}^{3}$ et "pères divins aimés du dieu ${ }^{4}$, ainsi que "nobles et gouverneurs 5 .

Mais aucun d'eux n'est "compagnon unique". Cet honneur, échu à des grands prêtres depuis Sabou-Tjéti jusqu'à la fin de la XVIIIème dynastie, n'apparaît plus ensuite que chez Chedsounefertoum de la XXIIème dynastie $(169,4)$.

Un autre titre honorifique, "trésorier du roi de Basse-Egypte", porté également, et fréquemment, de Sabou-Tjéti à la fin de la XVIIIème dynastie, disparaît presque avec la XIXème dynastie. On ne le rencontre plus que chez Pahemneter $(\mathbf{8 9} ; \mathbf{9 8 , 1 , 5 )}$ de la XIXème dynastie, Chedsounefertoum $(\mathbf{1 6 9 , 4 )}$ de la XXIIème, et Psenptaïs II $(\mathbf{2 0 0 , 2 , 1 2 )}$

\footnotetext{
1 A.H. GARDINER, dans Fl. PETRIE, Memphis V, p. 33-36.

${ }^{2}$ Voir ci-dessous, $\$ 32$.

3 Dedia seul n'est pas intitulé sem mais il n'est connu que par une mention $(\mathbf{1 0 2 , 2})$.

${ }^{4}$ Hori I $(\mathbf{8 7 , 4 )}$, Hori II (88), Pahemneter $(\mathbf{8 9 , 6 ; 9 5 , 2 ; ~ 1 0 1 , 2 ) , ~ R e ̂ h e t e p ~}(\mathbf{1 0 3 , 3 1 , 4 1 , 4 3 -}$ 46), Neferrenpet (147 - 151), et Iyry (156,15-17).

5 Seuls ceux qui ne sont connus que par une ou deux petites inscriptions, Nebmehyt $(82)$, Sennefer $(83 ; 84 ; 98,6)$, Dedia $(102,2)$ et Hy $(\mathbf{1 0 6})$, ne mentionnent pas les titres de nobles et gouverneurs, qu'ils ont vraisemblablement portés.
} 
sous les Ptolémées ${ }^{1}$.

En revanche, la XIXème dynastie complète souvent ${ }^{2}$ le titre "noble" par l'épithète "placé à la tête des deux pays", qui apparaît et disparaît peut-être ${ }^{3}$ avec cette seule époque.

Enfin, ce temps fait revivre le titre "chef de toute la garde-robe", porté par Sabou-Tjéti mais abandonné au Moyen Empire et sous la XVIIIème dynastie. On ne le retrouve, ensuite, que chez Horsaïset II $(179,17)$ à la XXIIème (?) dynastie et chez Anemher $(192,11 ; 193,10$ et 198,3). "Chef de toute la garde-robe" accompagne la plupart du temps sem, sur lequel Ramsès II semble avoir mis l'accent $t^{5}$.

Ces changements dont les trois premiers n'affectent que des titres ou épithètes honorifiques nous paraissent un reflet, très pâle, des transformations de toute nature qui marquent le passage de la XVIIIème dynastie à la XIXème dynastie.

\section{§ 31. L'IMPORTANCE RESPECTIVE DES DEUX TITRES DU GRAND PRETRE}

Au moment où ils deviennent sem, les grands prêtres de Ptah commencent à énumérer leurs titres dans l'ordre chronologique ${ }^{6}$ et ils

${ }^{1}$ Chez Psenptaïs II, la mention de "trésorier du roi de Basse-Egypte" semble n'être que le rappel d'une fonction, d'ailleurs honorifique, portée autrefois par le grand prêtre de Ptah. Il est plus difficile d'en juger chez Chedsounefertoum et chez Pahemneter. Toutefois ce dernier a pu songer à se donner ce titre, désuet à son époque, en le lisant sur les monuments de ses devanciers, auxquels il s'est intéressé $(\mathbf{6 9 , 1 1}$ et 89, 6-8).

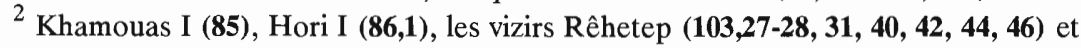
Neferrenpet (150 à 152), Iyry $(\mathbf{1 5 6 , 1 0})$.

${ }^{3}$ Cette épithète ne se trouve chez aucun grand prêtre postérieur à Iyry de la fin de la XIXème dynastie, mais notre liste des grands prêtres memphites présente à nouveau une période vide d'environ un siècle et demi entre Iyry et Paoupaou sous Siamon.

4 Hori I ou II $(\mathbf{9 0 , 4 )}$, Pahemneter $(\mathbf{8 9 , 7 )}$, Rêhetep $(\mathbf{1 0 3 , 1 4})$, le prince Khamouas (143), et Neferrenpet $(149,17)$.

5 Voir ci-dessous, $\$ 32$.

${ }^{6}$ Voir ci-dessus, $\$ 24$. 
ne semblent pas avoir changé de manière par la suite ${ }^{1}$. C'est pourquoi il est intéressant d'examiner l'ordre dans lequel les deux titres du grand prêtre memphite, sem et "grand des chefs des artisans", se succèdent, car cet ordre a varié. Aussi remonterons-nous à son origine.

Quand les titulatures étaient dans un ordre inversement chronologique, les grands prêtres se disaient "prophète de Ptah et prophète de Sokar", ce qui, à notre avis, ne veut pas dire qu'ils devenaient prophètes de Ptah plus tard que prophètes de Sokar, mais que, parvenant en même temps aux deux fonctions, ils mettaient l'accent sur le service de Ptah. Lorsque ces titres n'ont plus été portés effectivement par les grands prêtres ${ }^{2}$, sem a désigné chez eux le service de Sokar $^{3}$ et "grand des chefs des artisans" a commencé, ou poursuivi le chemin à la fin duquel ce titre, d'origine tout à fait civile, a signifié grand prêtre de Ptah. Dans ces conditions, il est normal qu'on ait écrit d'abord "sem et grand des chefs des artisans" parce que cette expression donne plus de poids au second élément dans une titulature ordonnée chronologiquement.

La prédilection des grands prêtres pour "grand des chefs des artisans" provient peut-être non seulement du fait que ce titre a été peu à peu l'équivalent de Grand prêtre de Ptah, le dieu qui était chez lui dans la cité du Mur Blanc tandis que Sokar y est venu des bords du désert, mais aussi de ce que sem s'appliquait à d'autres prêtres ${ }^{4}$.

Chez Sabou-Tjéti, où sem est encore séparé de l'autre titre, celui-là précède celui-ci. Par la suite, on réunit les deux titres sous la forme "sem et grand des chefs des artisans". C'est l'expression habituelle jusqu'au cours de la XIXème dynastie, et elle déborde le cercle des grands prêtres puisqu'au Chapitre I du Livre des Morts (159) le défunt s'identifie d'abord au sem, puis au grand des chefs des artisans ${ }^{5}$ et que sur la stèle dite des colliers, au temps de Séti Ier, le lecteur appelle à l'aide de

1 Voir ci-dessus, § 6.

2 Voir ci-dessus, $\$ 27$.

3 Voir ci-dessus, \$ 13.

${ }^{4}$ On peut aussi noter que plusieurs grands prêtres mentionnent leur nomination à la fonction de grand des chefs des artisans tandis qu'aucun d'eux ne dit comment et quand il est devenu sem. Mais la stèle de Sabou-Tjéti y faisait peut-être allusion.

${ }^{5}$ Sur les actions attribuées à l'un et à l'autre, voir ci-dessus, $\S 13$ p. 42 , note 3. 
Hormin d'abord le sem, puis le grand des chefs des artisans (93).

Le grand prêtre Thoutmôsis de la XVIIIème dynastie fait seul véritablement exception à l'ordre habituel ${ }^{1}$ : les deux fois où il s'intitule "grand des chefs des artisans", il se donne ensuite le titre de sem (62,12). Sur ses autres monuments, Toutmôsis est "fils de roi et sem" (63-65, peut-être 66). Nous avons déjà noté la préférence de Thoutmôsis pour le titre $\mathrm{sem}^{2}$ et supposé qu'elle est due, comme chez Khamouas, à sa qualité princière. En effet, chez Khamouas, sem ne désigne pas seulement le service de Sokar comme chez les autres grands prêtres ${ }^{3}$, mais aussi le service de son royal et divin père, dont il annonce les jubilés $(110$ - 112). De même, Thoutmôsis, "fils de roi et sem", signifie probablement par ce second titre qu'il est à la fois sem de Sokar et sem de son père.

Au début de la XIXème dynastie, les grands prêtres s'intitulent encore "sem et grand des chefs des artisans" jusqu'à Pahemneter compris ${ }^{4}$. Mais Rêhetep, Hy, le prince Khamouas, Neferrenpet, dont les pontificats datent du règne de Ramsès II, et Iyry, à la fin de la dynastie, sont au contraire toujours "grand des chefs des artisans et sem"5; c'est la raison pour laquelle nous attribuons la palette de Ptahmès au Musée du Louvre

${ }^{1}$ Nous ne connaissons qu'un autre cas, avant la XIXème dynastie, où sem paraisse avant grand des chefs des artisans: Ptahmès (II ?) se nomme une fois, selon l'ordre habituel, "sem et grand des chefs des artisans" $(\mathbf{3 7 , 3})$ et une autre fois "grand des chefs des artisans, celui qui est dans la double maison, $\operatorname{sem}^{\prime \prime}(37,2)$. La présence du second titre, qui n'avait plus sa raison d'être à la XVIIIème dynastie, a peut-être fait intervertir l'ordre habituel des autres titres, qui sont d'ailleurs séparés. Cette exception a donc un caractère très accidentel.

${ }^{2}$ Voir ci-dessus, $\S \mathbf{5}$.

3 Voir ci-dessus, § 13.

${ }^{4}$ Sennefer fait une fois exception, d'une façon encore plus curieuse que Ptahmès sous la XVIIIème dynastie. Alors qu'il est "sem et grand des chefs des artisans" sur son vase canope (84), il est "grand des chefs des artisans et sem" sur une table d'offrandes de Pahemneter $(\mathbf{9 8 , 6 )}$ qui, lui, est "sem et grand des chefs des artisans" sur le même monument $\mathbf{( 9 8 , 5 )}$. Comme nous ne savons pas si Sennefer a précédé Pahemneter ou s'il l'a suivi (dans ce cas, il aurait été grand prêtre entre Pahemneter et Rêhetep, ce qui est possible), il est prudent de ne pas échafauder des hypothèses pour expliquer le fait cidessus.

${ }^{5}$ Parfois sem est complété par "de Ptah"; voir le paragraphe suivant. 
(155) à ce temps. L'onomasticon d'Amenemope, composé probablement vers la fin de la XXème dynastie, mentionne aussi le grand des chefs des artisans avant le setem (185).

Il apparaît donc que les grands prêtres ont mis l'accent sur leur titre de sem, de Ramsès II à la fin de l'époque ramesside.

Nous ignorons l'expression préférée au début de la XXIème dynastie. Puis, Paoupaou fait retour à "sem et grand des chefs des artisans". Horsaïset I, que nous considérons comme fils et successeur de Paoupaou, met sem tantôt avant, tantôt après grand des chefs des artisans. A la XXIIème dynastie, Chedsounefertoum est toujours "grand des chefs des artisans et sem" et ses successeurs le sont la plupart du temps ${ }^{1}$.

A l'époque saïte, comme on peut s'y attendre, on revient à l'expression primitive, sem précédant grand des chefs des artisans $(\mathbf{1 8 6 , 5} ; \mathbf{1 8 9 , 3})$ et les contemporains des Ptolémées font de même $(192,11 ; 193,9)$, sauf Anemher, qui a une prédilection marquée pour sem $(\mathbf{1 9 2 , 1 , 1 2})^{2}$.

Ainsi le titre du grand prêtre memphite dans l'inscription du temple d'Edfou, "grand des chefs des artisans et sem" (205) remonte à une tradition qui n'est ni antérieure à Ramsès II, ni postérieure au temps qui a précédé la dynastie saïte.

\section{§ 32. RAMSES ET LES GRANDS PRETRES DE MEMPHIS}

Ramsès II a régné assez longtemps et avec assez d'énergie pour s'occuper activement de l'administration de l'Egypte et les grands prêtres de Ptah de Memphis ont eu leur part de cette attention. Pharaon les a favorisés de plusieurs façons, non parce qu'il se serait fait le champion du

1 Takelot (176) et Horsaïset II $(\mathbf{1 7 9 , 4 )}$ ont l'ordre inverse.

${ }^{2}$ Comme les mentions d'Anemher sont toutes postérieures au pontificat de son fils Téos, on peut supposer qu'après avoir abandonné sa fonction de grand des chefs des artisans en faveur de ce dernier, Anemher n'en a pas moins continué à être sem, puisque l'acquisition de ce titre semble avoir eu d'autres bases que celle de grand des chefs des artisans. Voir note 4, p. 83. 
dieu memphite, mais pour contenir la puissance des prêtres d'Amon. C'est pour cette raison, pensons-nous, qu'il a remis le ministère des cultes au grand prêtre memphite Neferrenpet, car le ministère en question était sinon dans les mains du grand prêtre d'Amon, au moins sous son influence au début du règne. Il a nommé Minmès grand prêtre d'Amon; or, Minmès, fils d'un grand prêtre memphite, a vraisemblablement été élevé à Memphis. Enfin, il a fait conduire la fête d'Amonrêsonter par des personnages memphites dont un grand prêtre de Ptah $^{1}$; même si l'on préfère penser qu'il s'agit d'une fête d'Amonrêsonter à $\mathrm{Memphis}^{2}$, la mention exceptionnelle d'une fête en l'honneur d'Amon chez un grand prêtre de Ptah indique que Ramsès II aurait donné à cette fête un éclat particulier qui rejaillissait forcément sur Memphis et son clergé.

Ramsès II a peut-être suivi l'exemple d'Aménophis III en plaçant son propre fils, Khamouas, à la tête du clergé memphite. Il nous a paru que le pharaon de la XVIIIème dynastie avait poursuivi un double but en nommant Thoutmôsis grand prêtre de Memphis ${ }^{3}$. Ramsès II, sous lequel les aspirations des grands prêtres d'Amon n'étaient pas moins grandes, a pu chercher les mêmes fins.

Ces témoignages des soins que Ramsès II a voués aux grands prêtres de Ptah font supposer qu'il n'est pas étranger à la mise en évidence du titre sem.

Nous venons de constater que l'ordre de sem et "grand des chefs des artisans" a changé avec Rêhetep, ou Sennefer et que la nouvelle expression mettait l'accent sur sem. Ce point de vue est confirmé par le titre "sem de Ptah", qui apparaît au même moment et dont l'emploi coïncide généralement, par la suite, avec "grand des chefs des artisans et sem". On trouve sem de Ptah chez Rêhetep, le prince Khamouas, Neferrenpet et Iyry de la XIXème dynastie, puis de Horsaïset I à la fin

${ }^{1}$ Le vizir Hori, sur le groupe de Pahemneter au Louvre (101), est "conducteur des fêtes d'Amonrêsonter" et Neferrenpet, ministre des cultes et grand prêtre memphite, est "conducteur (de la fête) d'Amon" (150,3).

${ }^{2}$ Nous estimons (voir ci-dessus, § 28) que Neferrenpet a pu conduire une fête d'Amon à Thèbes en qualité de ministre des cultes. Il ne serait donc pas étonnant que le vizir memphite Hori (101) ait été appelé à cet honneur, d'ailleurs antérieurement à Neferrenpet.

${ }^{3}$ Voir ci-dessus, $§ 28$. 
de la XXIème à Horsaïset II à la fin de la XXIIème dynastie, enfin chez Anemher sous les Ptolémées.

"Sem de Ptah" semble avoir été formé sur le modèle de "grand des chefs des artisans de Ptah" et peut, dans une certaine mesure, avoir servi au même but ${ }^{1}$. Mais le second titre, malgré son caractère un peu étonnant, ne fait qu'assembler deux termes qui s'accordent: au début de la XIXème dynastie, Ptah est depuis "toujours"2 le patron des artisans. "Sem de Ptah" a pour premier élément un titre que nous avons vu au contraire lié au service de Sokar ${ }^{3}$; pour passer à celui de Ptah, il a fallu que Ptah et Sokar, même Sokar-Osiris, s'amalgament en une divinité. On notera, à ce propos, le long temps qui s'est écoulé entre ce dernier fait qu'on peut retracer jusqu'à l'Ancien Empire ${ }^{4}$ et son expression dans l'institution du pontificat.

Nous ferons aussi remarquer qu'au point de vue théologique, "sem de Ptah" signifie un triomphe, d'ailleurs temporaire, de Sokar sur Ptah. Car si Ptah se voit attribuer un sem, c'est que sa nature tient passablement de celle de Sokar-Osiris ${ }^{5}$. On comprend dès lors le véritable sens du renversement de l'ordre des titres sem et "grand des chefs des artisans": le grand prêtre de Memphis qui est "grand des chefs des artisans et sem" reste bien ce que le premier de ces titres signifie mais il est surtout le "sem de Ptah".

Cette modification est la plus importante de celles que nous avons constatées sous le Nouvel Empire. C'est pourquoi, en tenant compte de ce que Ramsès II a fait en faveur des grands prêtres de Ptah et des constructions qu'il a édifiées à Memphis ${ }^{6}$, nous supposons qu'il a pu

${ }^{1}$ Voir ci-dessus, § 28, fin.

2 Voir ci-dessus, § 21.

${ }^{3}$ Voir ci-dessus, § 13.

${ }^{4}$ H. KEES, Götterglaube, p. 298, note 4.

${ }^{5}$ L'ordre des trois dieux de "Ptah-Sokar-Osiris" a la même signification. Cf. aussi le caractère héliopolitain d'Amon-Rê, et sur ce sujet en général l'étude de H. JUNKER, dans Götterlehre. Cf. enfin, plus récemment, SANDMAN, Ptah, p. 123-147 et spécialement p. 137: "... il semble que Ptah-Sokaris a été ordinairement un nom donné aux images de Sokaris", et p. 144-145: " ... Ptah a joué un très petit rôle dans la formation du concept Ptah-Sokar-Osiris".

${ }^{6}$ H. KEES, Memphis, dans PAULY-WISSOWA, p. 678 sq. 
créer lui-même ou au moins donner droit de cité au titre sem de Ptah.

Nous nous permettrons une suggestion pour finir: l'expression "grand des chefs des artisans et sem" n'apparaît plus qu'une fois après la XXIIème dynastie; on peut donc dire qu'elle n'a la faveur ni des saïtes ni des ptolémaïques. Le titre sem de Ptah suit le même chemin. Les grands prêtres de Memphis sont donc revenus à une conception moins "sokarienne", si l'on nous passe cette expression, ou osirienne de Ptah en un temps que nous ne pouvons fixer avec précision mais qui se place entre la fin de la XXIIème dynastie et l'époque saïte. Dans ces conditions, ne se pourrait-il pas que le vieux texte recopié sur l'ordre de Chabaka eût attiré l'attention parce qu'il révélait un Ptah plus "orthodoxe" que le Ptah ramesside, qui pouvait avoir un sem ? Nous le pensons ${ }^{1}$.

$\mathrm{Au}$ cours de ce chapitre, nous avons d'abord constaté que ni la première période intermédiaire ni la seconde n'ont apporté de modification importante au pontificat memphite. Le clergé de Ptah n'a vraisemblablement pas été malmené par les Hyksôs. Il ne paraît pas non plus avoir été inquiété par Akhenaton.

Les grands prêtres memphites ont bénéficié des efforts d'Aménophis III et de Ramsès II pour contenir le pouvoir grandissant des prêtres de Karnak. Le titre "grand des chefs des artisans de Ptah", employé du début de la XIXème dynastie à la fin de la XXIIème, est une prise de position des grands prêtres memphites en face des "premiers prophètes d'Amon" thébains.

Depuis le premier tiers environ du règne de Ramsès II, les grands prêtres de Memphis, qui avaient jusqu'alors insisté sur leur fonction de grand des chefs des artisans, donnent le pas à celle de sem, tant par le renversement de l'ordre habituel des deux titres que par l'emploi d'un nouveau titre, "sem de Ptah". Celui-ci correspond, dans l'institution du

${ }^{1}$ La date la moins ancienne attribuée à la composition du document de théologie memphite est, à notre connaissance, celle de la première période intermédiaire: $\mathrm{A}$. RUSCH, Phthas, dans PAULY-WISSOWA, Real Encyclopädie der klassischen Altertumswissenschaft, XXXIX, p. 940,2,36 sq. 
pontificat, à la synthèse théologique beaucoup plus ancienne de PtahSokar-Osiris ${ }^{1}$.

Sous la dynastie saïte, si ce n'est déjà sous Chabaka, les grands prêtres memphites reviennent à une conception moins osirienne de Ptah. Sous les Ptolémées, ils continuent dans la même voie. A l'une et l'autre époques, ils ont parfois tendance à faire revivre d'anciennes appellations, mais ce penchant à l'archaïsme n'affecte que des formes ${ }^{2}$.

${ }^{1}$ H. KEES, Götterglaube, p. 379-380, a noté l'importance de l'époque ramesside à Memphis, dans le domaine religieux. Sur le zèle des pharaons de la XIXème dynastie pour Ptah, cf. aussi A. RUSCH, op. cit., p. 946,1,56 sq., qui mentionne les noms royaux de cette époque composés avec le nom "Ptah".

${ }^{2}$ La comparaison des titres des grands prêtres saïtes et ptolémaïques avec ceux de leurs devanciers ne révèle plus de moment aussi important que la XIXème dynastie pour le pontificat memphite. Les renseignements que nous avons pu extraire des grands prêtres de la XXVIème dynastie et de l'époque gréco-romaine sont utilisés ci-dessus dans les Chapitres I à V. 

SECONDE PARTIE

LES GRA N D S PRETRES

M E M P H I T E S 

C H A P I T R E VI

\section{LES GENEALOGIES}

\section{§ 33. LA GENEALOGIE D'ANKHEF-NY-SEKHMET}

Le père divin de Ptah et prophète de Sekhmet Ankhef-ny-sekhmet, qui vivait au temps du dernier roi Chechanq ${ }^{1}$, a fait graver sur une pierre plate et à petite échelle soixante personnages surmontés chacun d'une courte légende. Ils sont répartis en quatre registres et portent tous une courte perruque, un pagne, la peau de félin et un fouet qu'ils tiennent à la main. En face d'eux, on lit la légende: "Il dit: Les grands des chefs des artisans ont été rassemblés pour le roi Chechanq $(\mathbf{1 8 2 , 6 1 )}$. En réalité, ce n'est pas une liste de grands prêtres de Memphis, mais une généalogie de la famille d'Ankhef-ny-sekhmet. Sur ses soixante membres, elle ne compte en effet, dans son état actuel, que vingt "grands des chefs des artisans". En admettant que les cinq ancêtres d'Ankhef-ny-sekhmet dont le nom et la titulature ont disparu aient exercé la même fonction, on atteindrait au maximum le chiffre de vingt-cinq grands des chefs des artisans.

Voici, en partant du plus ancien, les vingt grands des chefs des artisans $(182,58$ à 182,13):

3ème génération : Ptahemheb

11ème génération: Ankhsehetepibrê

12ème génération: Ankhkhakarê

13ème génération : Ankhnoubkaourê sous Nebkherourê

sous Sésostris Ier

sous Amenemhat II

sous Sésostris II

1 Le dernier pharaon nommé dans la généalogie est Psousennès II (984-950; cf. DRIOTON-VANDIER, L'Egypte, p. 539); 11 générations le séparent de Chechanq, qui ne peut alors être que Chechanq V (767-730; ibidem, p. 540). 
15ème génération : Ou(?)khet

18ème génération: Ser-met(?)

27ème génération : Mentou

29ème génération : Paiimy-(khe)red (?) sous Aménophis Ier

34ème génération: Penpanebes (?)

35ème génération: Méryour (?)

38ème génération: Ty

39ème génération: Sekeremsaef

40ème génération: Neterouy-hetep

42ème génération : (...)enchenet (?)

43ème génération: Ptahemakhet

44ème génération: Neferrenpet

45ème génération: Ptahemakhet

46ème génération: Achakhet

47ème génération: Papa

48ème génération : Saïset sous Neferhotep II

sous Iby

sous Ahmès

sous Aménophis III

sous Horemheb

sous Séti Ier

sous Ramsès II

sous (détruit)

sous Amenophthis

sous Psousennès II

Comme nous n'examinons la généalogie d'Ankhef-ny-sekhmet qu'au sujet des grands prêtres de Memphis, nous n'avons pas fait entrer dans la liste que nous venons de dresser les personnages intitulés "sem de Ptah", conformément au principe que nous avons établi ${ }^{1}$. Les ancêtres d'Ankhef-ny-sekhmet qui sont "sem de Ptah", sont, en commençant par le plus ancien:

19ème génération: Paser

25ème génération: Ourhetep

32ème génération: Nebrề sous Aaken(en)

sous Chareq

sous Thoutmôsis III.

Avant de confronter la généalogie avec d'autres monuments, il sied de la considérer seule. De Ptahemheb, sous Nebkherourê-Mentouhotep III qui a commencé à régner vers $2060^{3}$, à Ankhsehetepibrê, sous Sésostris Ier dont le règne a débuté en 1970, il y a quatre-vingt-dix ans pour huit générations, soit onze ans et une fraction d'année en moyenne

${ }^{1}$ Voir ci-dessus, $\$$ 5, fin.

${ }^{2}$ Nebrê porte aussi le titre du grand prêtre d'Héliopolis.

${ }^{3}$ DRIOTON-VANDIER, L'Egypte, p. 599 (liste chronologique des rois d'Egypte, que nous utilisons pour d'autres dates). 
par génération !

De Ankhnoubkaourê, sous Sésostris II qui commence son règne en 1906, à Ou(?)khet, sous Neferhotep II qui, n'étant pas du début de la période s'étendant entre la XIIème dynastie et les Hyksôs, peut avoir régné vers 1750 , il y a environ 156 ans pour deux générations, soit près de quatre-vingts ans par génération.

Au contraire, de Ou(?)khet, sous Neferhotep II vers 1750, à Mentou, sous Ahmès vers 1580 , il y a cent soixante-dix ans pour douze générations, ou une moyenne de quatorze à quinze ans par génération!

De la XVIIIème à la XIXème dynastie, la durée moyenne des générations est parfaitement possible: il y a en effet deux cent soixantedeux ans pour douze générations, soit vingt et un à vingt-deux ans par génération, de Mentou, sous Ahmès vers 1580, à Sekeremsaef, sous Séti Ier, vers 1318.

On retombe ensuite dans l'invraisemblable: entre Neferrenpet, sous Ramsès II mort en 1232, et Achakhet, sous Amenophthis qui a régné de 1009 à 1000 , il y a plus de deux siècles pour un intervalle de deux générations !

La généalogie d'Ankhef-ny-sekhmet contient donc, au point de vue de la durée moyenne des générations calculée sur les seuls grands prêtres deux impossibilités et des invraisemblances à côté de données acceptables.

Une comparaison de la généalogie avec les monuments de grands des chefs de artisans que nous avons réunis d'autre part entraîne des conclusions analogues sur la valeur documentaire de l'inscription d'Ankhef-ny-sekhmet, comme nous allons le voir.

Si nous excluons de la généalogie les grands prêtres qu'elle place aux époques vides de monuments touchant les grands des chefs des artisans, il reste treize grands prêtres de la généalogie à mettre en regard de ceux qui sont connus autrement. Sur ces treize, cinq seulement sont attestés ailleurs que dans la généalogie. Ankhsehetepibrê, sous Sésostris Ier, peut être Sehetepibrê-ankh-nedjem $(\mathbf{3 1} ; \mathbf{3 2})$ à condition d'admettre que son 
nom a été estropié dans la généalogie. Ty, sous Horemheb, est vraisemblablement Ptahemhat-Ty (72 à 75) dans lequel nous voyons le possesseur du cortège funèbre conservé à Berlin (70); celui-ci a eu affaire avec Horemheb alors généralissime sous Toutankhamon mais pas encore roi, et il y aurait aussi une inexactitude au sujet de Ty dans la généalogie. Neferrenpet, sous Ramsès II, est certainement le vizir et ministre des cultes (147 à 154) dont nous avons déjà parlé ${ }^{1}$. Papa, sous Psousennès II, est probablement Paoupaou $(163 ; 164)$ et Saïset, son fils, est probablement aussi, Horsaïset I (165), mais leurs noms auraient été simplifié pour l'un et estropié pour l'autre dans la généalogie. Là où la confrontation est directement possible, il faut reconnaître qu'elle n'est guère favorable à la généalogie.

Pour les huit grands prêtres nommés par la généalogie à des époques où nous pouvons nous croire bien documentés d'autre façon, il n'est, croyons-nous, pas exclu qu'il s'agisse de "beaux noms" ou de surnoms de grands des chefs des artisans connus depuis longtemps. Dès l'Ancien Empire en effet, des grands prêtres ont eu deux noms. Un des Sabou a pour beau nom ${ }^{2}$ Ibebi; un des Ptahchepses, celui d'Impy; un autre Sabou se nomme aussi Tjéti; à la XVIIIème dynastie, Ptahemhat est appelé Ty (73), et à la XXIème dynastie, il y a Neterkheperrê-Méryptah appelé Paoupaou $(\mathbf{1 6 4 , 2 5})$. Il serait donc possible que Ankhkhakarê de la généalogie fût Nebpou (31) et que Ankhnoubkaourê fût Sehetepibrêcheri (31) de la XIIème dynastie; de même, Penpanebes (?) et Méryour (?) sous Aménophis III pourraient être l'un et l'autre des Ptahmès. Ce n'est cependant qu'une hypothèse.

${ }^{1}$ Voir ci-dessus, $\S 28,31$ et 32.

2 Au terme d'une étude de la racine nefer, G. JEQUIER, Considérations sur les religions égyptiennes, 1946, p. 53, écrit :" Il faudrait donc admettre que la racine nefer a non seulement les sens ... de "beau, bon, agréable", mais aussi celui de "jeune" dont on doit tenir compte, tout spécialement dans le domaine religieux" et il ajoute plus loin: "Il n'y aurait même rien d'extraordinaire à ce que le "beau nom" des grands personnages de l'Ancien Empire soit plutôt le nom qu'ils doivent porter en tant que régénérés dans leur nouvelle vie." 

La généalogie d'Achaout-akh comprend huit grands des chefs des artisans. Ce sont, en partant du plus ancien:

1ère génération: Papa

2ème génération : Horsaïset

3ème génération: Dempedjet (?)

4ème génération: Achaout-akhou

5ème génération: Ankh-en-ef-sḩmt

6ème génération: Chedsounefertoum

7ème génération: Chechaqen (sic)

8ème génération: Osorkon.

Ces indications ne présentent en elles-mêmes aucune invraisemblance et peuvent ainsi être immédiatement comparées à celles d'autres monuments.

Papa peut être identifié à Paoupaou, contemporain de Siamon (163; 164), et son fils Horsaïset à Horsaïset I (165). A la cinquième génération, Ankh-en-ef-shmt est certainement le grand prêtre Ankhef-nysekhmet $(166 ; 167 ; 169 ; 170)$ dont le nom aura été estropié, puisque celui-ci a pour fils le grand prêtre Chedsounefertoum. Les deux grands des chefs des artisans les plus récents de la généalogie ne sont pas attestés ailleurs, mais Chedsounefertoum mentionne sur un monument qu'il a fait graver de son vivant, à en juger d'après l'inscription dorsale (167,18-25), "son fils, le sem de Ptah, Chechanq" (167,9-11). D'autre part, nous ne connaissons pas, en dehors de la généalogie, de grand prêtre entre Chedsounefertoum, contemporain de Chechanq Ier, et le prince Chechanq, en fonction au temps de son père Osorkon II; or une soixantaine d'années se sont écoulées entre les deux règnes ${ }^{1}$. Nous pouvons ainsi supposer que Chechanq, le sem $(\mathbf{1 6 7 , 9 )}$ serait devenu grand des chefs des artisans à la mort de Chedsounefertoum. Avec Osorkon, cité seulement par la généalogie, nous arrivons au trisaïeul d'Achaout-akh; à ce degré de parenté, on peut admettre que le rédacteur de la stèle a connu assez exactement les titre et nom de son ancêtre. Il est donc possible que Chedsounefertoum ait eu pour successeurs deux

1 DRIOTON-VANDIER, L'Egypte, p. 541; le règne de Chechanq Ier finit en 929 et celui d'Osorkon II commence en 870 . 
grands prêtres nommés Chechanq et Osorkon, qui auraient été ses fils et petit-fils.

Entre Papa, ou Paoupaou, grand prêtre sous Siamon, qui a régné de 1000 à 984, et Chedsounefertoum, grand prêtre sous Chechanq Ier, qui a régné de 950 à $920^{1}$, il y a un écart de cinquante années, au maximum soixante-dix. Selon la généalogie d'Achaout-akh, il y aurait cinq générations entre les deux grands prêtres, ce qui donne une durée moyenne de dix, ou au plus de quatorze ans par génération. Sans accorder à ce résultat plus de valeur qu'il en mérite, car on peut supposer que Paoupaou était un vieillard sous Siamon ${ }^{2}$ et son petit-fils Dempedjet (?) un adulte, nous inclinons à croire que les deux grands prêtres Dempedjet ${ }^{3}$ et Achaout-akhou, aux troisième et quatrième générations de la généalogie, n'ont pas existé et que leurs noms ont été inventés ou sont les surnoms d'autres grands prêtres que le rédacteur aurait dédoublés.

Dans l'ensemble, la généalogie d'Achaout-akh paraît mieux fondée au sujet des grands prêtres que celle d'Ankhef-ny-sekhmet, parce qu'elle ne remonte pas au-delà de la douzième génération tandis que celle-ci va jusqu'à la soixantième.

Les deux généalogies ont été rédigées à peu près à la même époque puisqu'un intervalle de treize générations dans celle d'Ankhef-ny-sekhmet et de onze dans celle d'Achaout-akh sépare le grand prêtre Papa du rédacteur. Comme l'un et l'autre monument font du grand des chefs des artisans Papa le père du grand des chefs des artisans Saïset ou Horsaïset, cette concordance semble témoigner d'une tradition à l'époque où les rédacteurs des généalogies ont vécu, quelque deux siècles après Paoupaou. Nous adhérons à cette tradition faute de documentation

1 ibidem, p. 601.

2 Paoupaou a toutefois les traits d'un homme jeune sur les linteaux du sanctuaire dédié par Siamon $(163 ; 164)$.

${ }^{3} \mathrm{La}$ lecture de ce nom bizarre est incertaine. Nous préférons la laisser inexpliquée plutôt que nous arrêter à l'hypothèse de L. BORCHARDT, Die Mittel ..., p. 102, note 3, qui y voit un cryptogramme de "p-p" dans son désir de faire concorder les généalogies d'Achaout-akh et d'Ankhef-ny-sekhmet (voir la note suivante). 
meilleure et considérons Horsaïset I comme le fils de Paoupaou ${ }^{1}$.

\section{§ 35. LA GENEALOGIE DE PETOBASTIS}

Le grand prêtre memphite Petobastis, qui vivait aux second et premier siècles avant J.-C., a laissé une stèle démotique ${ }^{2}$. Il y raconte sa vie et son récit lui fournit l'occasion d'énumérer les noms et dignités de ses ancêtres ${ }^{3}$. Ce sont, avec la date approximative que leur éditeur leur a assignée en se basant sur le fait que Petobastis est contemporain de Ptolémée Alexandre Ier:

\begin{tabular}{|c|c|c|}
\hline & & \\
\hline & & \\
\hline & & $\mathrm{Ev}$ \\
\hline & & $t$ \\
\hline & Pse & Ptolémée Epiphane) \\
\hline & Pet & S Ptolémée Philomêtor) \\
\hline & Psenpta & mée Evergète II) \\
\hline
\end{tabular}

la huitième génération est celle de Petobastis, le rédacteur de la stèle.

Les ancêtres de Petobastis sont tous intitulés "scribe, prophète, supérieur des secrets dans les temple de Ptah, surveillant ${ }^{4}$ d'Osiris et de

${ }^{1}$ Nous ne suivons pas L. BORCHARDT, Die Mittel zur zeitlichen Festlegung..., p. 102, qui identifie non seulement les deux Papa ainsi que Saïset et Horsaïset mais encore les quatre générations suivantes des deux généalogies, parce que, dans celle d'Ankhefny-sekhmet, ces quatre générations, dont deux ont, en plus, d'autres noms que chez Achaout-akh, ne comprennent aucun grand des chefs des artisans. On n'y trouve que des prophètes et des supérieurs des secrets du saint des saints (litt.: grande place), soit des personnages moins haut placés que les grands des chefs des artisans. Or, si les généalogies contiennent des titres ne correspondant pas à la réalité, c'est pour élever et non pour abaisser ceux qui en sont dotés.

2 Décrite et traduite par J. KRALL, Studien zur Geschichte des alten Ägypten, dans Sitzungsberichte der Wiener Akademie der Wissenschaften, phil.-hist. Klasse, vol. 105 (1883), p. 373-379: Le texte démotique n'a pas été publié, à notre connaissance.

${ }^{3}$ Lignes 12 à 19; J. KRALL (op. cit., p. 376) dit que l'écriture en est très effacée.

4 J. KRALL donne ici entre parenthèses le mot hiéroglyphique $r s$ (= Wörterbuch, II, 451, réf. 16-17). 
Serapis, grand setem". Petobastis est aussi "grand setem". Ni lui ni ses ancêtres ne sont "grand des artisans", qui est l'équivalent démotique de "grand des chefs des artisans"1. Cette absence nous empêcherait de considérer ces personnages comme des grands prêtres ${ }^{2}$, si nous ne pouvions tenir pour certain que Petobastis est identique au père du grand prêtre Psenptaïs de la stèle Harris (200), qui est explicitement nommé "le grand des chefs des artisans Petobastis" (200,15). Nous nous basons pour cela sur la comparaison des dates et des noms de la stèle démotique de Petobastis et de la stèle Harris: d'après la première, Petobastis, qui avait pour père un Psenptaïs, est né en 121 av. J.-C., et d'après la seconde Psenptaïs est né en 90 avant $\mathrm{J}$.-C. et avait pour père Petobastis. On peut donc estimer que les mots démotiques que J. Krall a traduits "grand setem" ont le sens de notre "grand prêtre memphite".

La généalogie de Petobastis ne contient pas de contradiction interne et peut ainsi, comme celle d'Achaout-akh, être immédiatement confrontée à d'autres monuments.

Nous ne connaissons qu'un grand prêtre nommé Anemher. Né sous Sôter et mort sous Philopator, il pourrait aussi bien être celui de la première que de la troisième génération de la généalogie $e^{3}$. Dans le premier cas, on peut se demander pourquoi le rédacteur de la généalogie ne serait pas remonté jusqu'au père d'Anemher, le grand prêtre NesqedPetobastis (190), qui semble avoir été le premier grand prêtre de la famille. Dans l'autre cas, Nesqed aurait été le nom détruit de la deuxième génération et aurait eu un grand prêtre Anemher pour père. Mais Nesqed ne mentionne sur sa stèle que le nom de sa mère $(\mathbf{1 9 0 , 7 )}$ et semble indiquer ainsi que son père n'a revêtu aucune charge importante. On en trouve confirmation sur les statues de son arrière

1 Voir ci-dessus, § 4, fin.

${ }^{2}$ Voir ci-dessus, $\$ \mathbf{5}$, fin.

3 J. KRALL, op. cit., p. 377, opte pour la première, et W. OTTO, Priester und Tempel im hellenistischen Ägypten, I, 1905, p. 206, note 1, pour la troisième. Les dates de naissance d'Anemher, en 289 av. J.-C., et de Petobastis, en 121, ne peuvent servir qu'à confirmer la possibilité du nombre de générations de la généalogie: les 168 ans d'intervalle se répartissent en cinq générations, soit une moyenne de 33 ans par génération. Si l'on préfère identifier Anemher à celui de la première génération, on obtient 24 ans par génération. 
petit-fils, qui énumère ses père, aïeul et bisaïeul, tous grands prêtres de Ptah (198,3); malgré la destruction du nom du bisaïeul, il ne peut y avoir de doute que c'était Nesqed si l'on compare le monument en question $(\mathbf{1 9 8 , 3})$ avec ceux des petit-fils et fils de Nesqed $(\mathbf{1 9 4 , 1 0}$ et 192,10-11). Le père de Nesqed n'était pas grand prêtre et le rédacteur de la généalogie aura inventé la fonction du premier Anemher. Les indications fournies par d'autres généalogies nous font préférer cette seconde possibilité car on sait que des généalogies attribuent faussement des fonctions importantes tandis qu'on imagine moins aisément le rédacteur d'une généalogie passant sous silence un ancêtre haut placé. De plus, il nous a paru, dans la généalogie d'Achaout-akh, que son auteur avait inventé ses ancêtres aux huitième et neuvième générations en remontant le temps. Or le premier Anemher est à sept ou huit générations du rédacteur de la stèle, qui a été soit le fils de Petobastis, soit Petobastis; si c'est ce dernier, il aurait laissé en blanc la date de sa mort, comme c'est le cas sur un monument de son ancêtre Harmachis (195). Nous tenons donc pour acquis que le premier Anemher de la généalogie n'a pas été grand prêtre et que son nom seul peut être réel.

L'aîné des deux fils d'Anemher, le grand prêtre Teos, est mort avant son père et sans enfant masculin, puisque son frère Harmachis lui a succédé. Comme Harmachis a eu des fils, il était sans doute nommé à la quatrième génération de la généalogie.

L'aîné des deux fils d'Harmachis s'appelait Nesqed (195) et ne paraît pas avoir été grand prêtre. L'autre s'appelait Psenptaïs. On le connaît par deux statues trouvées dans le Sérapéum d'Alexandrie (198). Ces monuments lui donnent une série de titres, mais pas celui de grand prêtre. Nous nous trouvons donc ici en présence d'un cas semblable à celui de Chechanq dans la généalogie d'Achaout-akh, soit d'un personnage de généalogie dont l'existence est confirmée par un monument contemporain tandis que sa titulature ne l'est pas. Pour Chechanq, que cinq générations séparent du rédacteur de la généalogie, nous avons conclu à la possibilité de son pontificat. Psenptaïs est à trois générations seulement de Petobastis, qui pouvait être mieux renseigné sur son bisaïeul qu'Achaout-akh sur l'aïeul de son bisaïeul. C'est pourquoi l'existence d'un grand prêtre Psenptaïs, fils du grand prêtre Harmachis, nous paraît probable; ce serait celui de la cinquième génération dans la généalogie. 
Petobastis, à la sixième, et Psenptaïs, à la septième générations, ne sont jusqu'à présent confirmés par aucun monument ${ }^{1}$. Comme ce sont cependant l'aïeul et le père du Petobastis pour lequel la généalogie a été faite, le rédacteur pouvait être encore plus facilement et exactement instruit à leur sujet que sur le bisaïeul. Cette circonstance nous fait admettre la réalité de ces deux grands prêtres.

En résumé, la généalogie de Petobastis, dans l'état où nous la connaissons, est fausse à la première génération; ailleurs, nous pouvons nous y fier à des degrés divers: entièrement à la troisième génération, probablement à la cinquième et vraisemblablement aux sixième et septième. L'ensemble paraît donc un document assez digne de foi.

Cette conclusion complète la leçon que nous avons tirée des deux autres généalogies de grands prêtres: il ne sied pas de rejeter les données généalogiques concernant des générations proches du rédacteur sans de sérieuses raisons. Lorsqu'on remonte un peu dans le temps, il faut, au contraire se souvenir que le fictif et le réel peuvent alors s'entremêler jusque fort loin dans le passé.

Les données que nous retenons des trois généalogies pour les grands prêtres de Memphis sont les suivantes:

1/ la filiation Paoupaou - Horsaïset;

2/ la possibilité du pontificat du Chechanq mentionné sur le groupe de son père, et de celui du fils de ce Chechanq, Osorkon;

3/ l'appartenance à une seule famille des deux séries de grands prêtres ptolémaïques dont l'une comprend Nesqed, Anemher, Teos et Harmachis et l'autre Petobastis II, Psenptaïs II, Imouthès et Psenamounis;

${ }^{1}$ C'est la raison pour laquelle nous n'en avons pas tenu compte dans la numérotation des derniers Petobastis et Psenptaïs de nos "Inscriptions" (199; 200; 203). 
4/ la probabilité du pontificat de Psenptaïs (I), le possesseur des statues trouvées à Alexandrie;

5/ deux noms de grands prêtres du second siècle avant J.-C., un Petobastis et un Psenptaiis, pour lesquels nous ne connaissons pas encore de monument. 


\section{H A P I T R E VII}

\section{LES GRANDS PRETRES DE L'ANCIEN EMPIRE}

\section{§ 36. PTAHCHEPSES I}

Parmi les grands des chefs des artisans que nous connaissons dans l'Ancien Empire, il y en a peut-être qui ont rempli leur fonction sous les rois de la IVème dynastie ${ }^{1}$, mais Ptahchepses $\mathrm{I}^{2}$ est le plus ancien qui se laisse dater certainement.

Les phrases de sa stèle qu'on a pompeusement appelées biographie $\left(\mathbf{4 , 1 - 7 )}\right.$ nous renseignent sur ses relations avec la cour $^{3}$, tandis que les séries de titres de son linteau $(3)$ et de sa stèle $(\mathbf{4 , 1 - 7 )}$ permettent de tracer les grandes lignes de sa carrière ${ }^{4}$.

Nous ne savons presque rien de l'origine de ce grand prêtre. Elle n'était sans doute pas humble puisqu'il a été élevé parmi les enfants royaux sous Menkaourê, pendant le règne duquel il est vraisemblablement né $e^{5}$, et sous Chepseskaf, soit à la fin de la IVème dynastie. Ce dernier roi, ou l'un de ses successeurs, le choisit pour gendre, ce qui valut en partie à Ptahchepses I de poursuivre sa carrière de

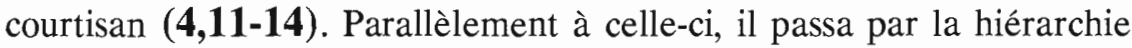
qui le mena de prêtre-ouab à prophète de Ptah et de Sokar, et de

${ }^{1}$ On peut faire remonter l'existence des grands des chefs des artisans avec certitude jusqu'au début de la IVème dynastie, mais peut-être pas au-delà (voir ci-dessus, § 21).

${ }^{2}$ Nos Ptahchepses I et II = MARIETTE, Mastabas, C 1 et C 9; nos Ptahchepses III et IV = MURRAY, Saqqara Mastabas II, où ils sont numérotés I et II.

${ }^{3}$ Voir ci-dessus, $\$ 7$.

4 Voir ci-dessus, § 6.

${ }^{5}$ En acceptant la restitution proposée par Sethe $(4,8)$. 
directeur de la ouabet et chef des artisans à grand des chefs des artisans.

Vers le début de sa carrière pontificale, Ptahchepses I fut probablement l'un des deux grands des chefs des artisans qui eurent à diriger le polissage et la décoration de la stèle du médecin de Sahourê, Ny-ankh-sekhmet $(\mathbf{2 , 3})$; on sait que les artisans eurent l'insigne honneur de travailler à ce monument en présence du roi lui-même $(\mathbf{2 , 4})$.

Ptahchepses I a été en fonction jusque sous Nyouserrê $\hat{e}^{1}$, dans le temple solaire duquel il a été prophète de Rê $(\mathbf{4 , 4 )}$. Sa longue carrière l'a fait survivre au grand des chefs des artisans qui avait été son premier collègue; du moins, supposons-nous cette circonstance puisque Ptahchepses I a rempli une série de fonctions religieuses qui paraissent avoir été l'apanage du plus ancien des deux grands prêtres, telles celles de prophète de Rê dans trois temples solaires et prophète de divinités assimilables à $\mathrm{Ptah}^{2}$.

Ptahchepses I aura bénéficié de diverses donations que la pierre de Palerme indique pour les trois premiers règnes de la Vème dynastie: donations d'Ouserkaf aux divers dieux de son temple solaire dans lequel Ptachepses I a été prophète de Rê $(\mathbf{4 , 4 )}$; donations de Sahourê à Rê dans des sanctuaires dont le nom est détruit mais dont l'un était probablement le temple solaire d'Ouserkaf, ainsi qu'à Khenty-iaoutef dont Ptahchepses I a été prophète $(\mathbf{4 , 3})$; donations de Neferirkarê à Rê dans Setibrê, pour la barque du soir et pour celle du matin; or Ptahchepses I a été prophète de Rê-Horakhti dans le temple solaire de Neferirkarê $\mathbf{( 4 , 5 )}$; enfin, donations du même roi à Ptah.

Nous ignorons la date même approximative de la mort de Ptahchepses I; nous croirions volontiers que, parvenu à un certain âge, il a abandonné sa fonction de grand prêtre, puis vécu encore pendant une partie du règne de Nyouserrê de ses propres revenus et des libéralités que le roi lui aurait accordées ${ }^{3}$.

1 D'après les durées de règnes de G. REISNER, Mykerinos, p. 242 et 246, Ptahchepses I a pu avoir environ 60 ans au début du règne de Nyouserrê.

${ }^{2}$ Voir ci-dessus, § 19.

${ }^{3}$ Voir ci-dessus, $\S 11$, p. 36 , note 3 . 


\section{§ 37. SETJOU}

On sait bien peu de choses sur Setjou: il a été grand des chefs des artisans, prophète de Ptah et de Sokar, et il est décédé avant sa femme, une "connue du roi" dont le nom se terminait par ...ka (14).

L'épouse de Setjou a fait sculpter un groupe asymétrique où son mari est assis à l'un des bouts d'un banc à deux places; l'autre place est inoccupée et la femme, représentée à une échelle plus petite que Setjou, est accroupie devant cette place vide, à côté du mollet de son mari; le sommet de sa tête n'atteint pas la hauteur du siège (14).

Un guéridon (15) appartient sans doute au même Setjou'. Il s'y nomme "prophète de Ptah et de Sokar"2 et cette expression peut servir à fixer deux dates possible pour Setjou. En remarquant que Ptahchepses I et Sabou-Ibebi séparent toujours les deux sacerdoces et s'appellent "prophète de Ptah et prophète de Sokar", nous estimons que l'expression "prophète de Ptah et de Sokar" n'a peut-être été employée qu'avant Ptahchepses I et après Sabou-Ibebi. En fait, on la trouve chez Ptahchepses IV, le jeune collègue de Sabou-Ibebi $(\mathbf{2 4 , 7 )}$ au début de la VIème dynastie. Mais Ptachepses IV part de l'expression traditionnelle "prophète de Ptah et prophète de Sokar" (21,5) pour aboutir à l'abréviation "prophète de Ptah et de Sokar" $(\mathbf{2 4 , 7 )}$ et il étend l'emploi des termes "Ptah et Sokar"3 jusqu'à en faire les compléments

${ }^{1}$ L. BORCHARDT, Statuen ..., I, p. 130, suivi par PORTER ET MOSS, Memphis, p. 104, indique le mastaba de Mariette B 6 comme lieu possible de provenance. Mais Mariette ne signale ni statue ni guéridon dans B 6. Dans B 7, par contre, il mentionne deux guéridons, mais pas de groupe. Ce mastaba serait-il celui du grand prêtre Setjou ?

${ }^{2}$ Nous ne retenons pas la traduction, grammaticalement possible, de "prophète de Ptah-Sokar", parce que les grands prêtres de l'Ancien Empire distinguent les deux dieux, comme on le voit d'après le titre "directeur du domaine de Sokar", porté par les grands prêtres jusqu'au début de la VIème dynastie.

3 Quelques-unes des séries de titres de Ptahchepses IV ont été traduites par SANDMAN, Ptah, p. 125, et les textes en sont cités sous les numéros 156 et 157 (qui n'est pas une stèle, mais un linteau !):

156 a de SANDMAN = notre 24,12;

$156 \mathrm{~b} " \quad "=\quad 24,2,4,5,3$ ( $\mathrm{sic})$;

$156 \mathrm{c} "$ " $=$ " 24,7;

$157 " \quad$ = " 21,4-5 (début). 
d'imakhou $(21,4 ; 24,3)$. Setjou abrège aussi "prophète de Ptah" et "prophète de Sokar" en "prophète de Ptah et de Sokar" (15,2), mais il use de "prophète de Ptah" et de "prophète de Sokar" dans des titulatures parallèles $(\mathbf{1 4 , 1 - 2})$ au lieu de citer ces titres l'un après l'autre. On peut ainsi se risquer à supposer que Setjou n'appartient pas au temps de Ptahchepses IV; il aurait vécu au début de la Vème dynastie, soit antérieurement à Ptahchepses I soit en même temps, comme son collègue plus âgé.

Une stèle du Musée de Naples ( $\mathrm{N}^{\circ}$ 1017), de forme rectangulaire et bordée de deux traits, représente, au registre supérieur, "le connu du roi Setjou, prophète de Ptah et prophète de Sokar" et sa femme "l'imakhet Khentetka" assis devant une table d'offrandes. Au-dessous, un rectangle allant presque d'un bord latéral de la stèle à l'autre et dessinant peutêtre un linteau surmonte une porte étroite avec tambour demi-cylindrique qui va jusqu'au bas de la stèle; sept hommes et une femme, groupés deux par deux en deux registres superposés de chaque côté de la fausse-porte, sont les enfants du couple. On peut se demander si cette stèle est un monument du grand prêtre Setjou antérieur à son pontificat. Il y a des éléments pour l'identification, et d'autres contre, de sorte que nous renonçons à trancher cette question.

Mme Sandman Holmberg incline à lire plutôt "Ptah-Sokaris" que "Ptah et Sokaris" chaque fois que les deux noms divins sont réunis. Toutefois, elle constate (p. 126), d'après L.D., II, pl. 59 a, où il est question d'un prophète de Rê et d'Hathor, que "Ptah + Sokar + hem-nțr" pourrait bien signifier "prophète de Ptah et de Sokar"; mais elle préfère la traduction "ímakhou auprès de Ptah-Sokar" à "ỉmakhou auprès de Ptah et de Sokar", parce que, déclare-t-elle, ce serait une manière peu usuellle d'écrire cette expression.

Mme Sandman Holmberg s'appuie sur son inscription 157 (= notre 21,4-5) pour affirmer (p. 126): "Quand une inscription a pour énoncé "celui qui est vénérable avec Ptah-Sokar en tous ses lieux de culte", cela doit impliquer que Ptah et Sokar étaient regardés comme des êtres identiques qui pouvaient être mentionnés au singulier".

Nous maintenons, en nous basant sur la comparaison (que nous avons faite cidessus), des titres portés par Ptahchepses IV (21,5), "prophète de Ptah et prophète de Sokar", qui s'abrège en "prophète de Ptah et de Sokar" (24,7), que l'Ancien Empire n'a connu que des prophètes de Ptah et des prophètes de Sokar. Par contre, Mme Sandman Holmberg a peut-être raison au sujet de l'expression "ímakhou auprès de Ptah-Sokar"; il ne s'agit plus ici d'un titre exprimant une fonction exercée réellement sur terre, mais d'une qualité qu'on souhaite à un mort pour sa vie dans l'au-delà. 


\section{§ 38. PTAHCHEPSES II, RENEFER ET NEFEREFRE-ANKH}

Ces trois grands prêtres appartiennent à la Vème dynastie, au milieu de laquelle on peut situer approximativement leurs pontificats parce qu'ils semblent antérieurs à Sabou-kem et Ptahchepses III qui sont probablement proches de Sabou-Ibebi, c'est-à-dire de la fin de la dynastie.

Ptahchepses II avait un mastaba ${ }^{1}$ dont la chapelle avait la même forme et presque les mêmes dimensions que celle de Ptahchepses I, mais elle en différait par l'ornementation. Tandis que la plus grande partie de la paroi du fond était occupée chez Ptahchepses I par la stèle que nous lui connaissons, chez Ptahchepses II la paroi correspondante était "décorée d'un élégant système de rainures prismatiques terminées par des bouquets de fleurs de lotus épanouies"2. Cette description fait songer à la partie centrale des scènes de chasse et de pêche, à laquelle la Vème dynastie accorde déjà une grande importance ${ }^{3}$. La porte donnant accès à la chapelle de Ptahchepses II était surmontée d'un linteau énumérant une série de titres presque identique à celle du linteau de Ptahchepses I (5 et 3). Nous y voyons les étapes principales de leur carrière ${ }^{4}$. Bien que ces linteaux, ainsi que ceux de Sabou-Ibebi et Ptahchepses IV, aient été rédigés évidemment sur le même modèle, leur emplacement d'autrefois garantit leur véracité, comme nous l'avons remarqués. Ptahchepses II a suivi la même filière que Ptahchepses I; nous le plaçons après ce dernier seulement à cause de la décoration de sa chapelle.

Nous ne pouvons pas déterminer le possesseur de chacune des deux statues au nom d'un grand des chefs des artisans Ptahchepses (6 et 7).

Le tombeau du grand prêtre Rênefer comprenait une chambre construite à l'extérieur d'un mastaba massiff. Mariette y a trouvé trois

\footnotetext{
${ }^{1}$ MARIETTE, Mastabas, C 9.

2 ibidem, p. 130.

${ }^{3}$ E. de KEYSER, Scènes de chasse et de pêche, dans Chronique d'Egypte, 43, janvier 1947, p. 44.

${ }^{4}$ Voir ci-dessus, § 6.

5 ibidem.

${ }^{6}$ Plan dans MARIETTE, Mastabas, p. 122, C 5.
} 
statues: une de la femme de Rênefer, la "connue du roi Hekenou"1 et deux de Rênefer. Ces beaux exemples de la production des ateliers memphites sous la Vème dynastie ont été très souvent publiés². Contrairement à ce qu'on a pensé ${ }^{3}$, ils représentent Rênefer au même âge $^{4}$, mais coiffé différemment, ici d'un serre-tête, là d'une perruque. Nous avons noté que la tenue du grand prêtre memphite en fonction n'est pas uniforme ${ }^{5}$; on peut y voir la raison des deux aspects de Rênefer. Par contre, il ne nous paraît pas possible de mettre une des coiffures en rapport avec une activité de Rênefer plutôt qu'une autre, à cause du pectoral inachevé. Le dessinateur qui préparait le travail du peintre sur la statue déjà sculptée avait tracé en rouge le contour d'un pectoral sur la statue à perruque; on ne peut dire si le dessin était complet car on ne voit aujourd'hui qu'une partie du collier proprement dit et le début des trois supports en zigzag. Le reste, s'il a jamais existé, a été recouvert par la teinte ocre adoptée pour les chairs de Rênefer, et la statue finie porte un collier large. Que s'est-il passé ? Une erreur du dessinateur ne nous paraît pas très probable parce que l'exécution d'une statue de grand prêtre de Ptah devait être particulièrement soignée dans les ateliers memphites. Nous croirions davantage à un changement décidé pendant la décoration.

Les inscriptions des deux statues contiennent les mêmes titres sauf "directeur du domaine de Sokar", mentionné seulement par la statue au serre-tête $(\mathbf{9 , 3})$. Nous n'estimons cependant pas qu'on puisse établir un lien entre le serre-tête et le titre en question, puisque nous avons toutes raisons de croire que Sabou-Tjéti, qui porte le serre-tête, n'était plus "directeur du domaine de Sokar"7; de plus, le serre-tête est porté parfois

1 Caire 53, reproduit dans J. CAPART, L'Art égyptien, 1912, pl. 115; L. BORCHARDT, Statuen... I, pl. 14.

2 Bibliographie dans PORTER ET MOSS, Memphis, p. 110-111.

${ }^{3}$ W. SPIEGELBERG, Die Darstellung des Alters in der älteren ägyptischen Kunst, dans $\ddot{A} . Z$., 54 (1918), p. 70-71.

${ }^{4}$ R. ENGELBACH, The portraits of Ranufer, dans Mélanges Maspero I, Orient ancien, 1er fascicule (= Mémoires de l'Institut Français d'Archéologie Orientale, tome 66, 1934), p. 101-103.

5 Voir ci-dessus, § 9.

${ }^{6}$ Voir Planche I, fig. 2.

${ }^{7}$ Voir ci-dessus, $\$ 13$ et 25. 
en même temps que le pectoral ${ }^{1}$ et celui-ci est en rapport avec la direction des artisans, donc indirectement avec Ptah et non avec Sokar.

Tous les titres de Rênefer ont été portés par Ptahchepses I et II, et bien que leur liste ne soit pas aussi complète que celle des linteaux des Ptahchepses, sans doute parce que ces listes se trouvent sur des monuments de nature différente, Rênefer a suivi la même filière.

Le grand des chefs des artisans et directeur du domaine de Sokar, Neferefrê-ankh a fait cadeau d'une stèle au chef de tente Senimen. Si la mention du second titre du grand prêtre paraît bizarre dans une titulature aussi abrégée (8), elle s'expliquerait cependant au cas où Senimen aurait fait partie du domaine de Sokar. Le nom de Neferefrê-ankh fixe un terminus post quem pour ce grand prêtre, qui ne peut pas être antérieur au premier tiers de la Vème dynastie; d'autre part, la transformation du service de Sokar sous la VIème dynastie interdit de placer Neferefrê-ankh après Sabou-Tjéti. C'est pourquoi nous supposons qu'il a suivi la même voie que les Ptahchepses I et II et que Sabou-Ibebi.

L'un et l'autre des trois grands prêtres que nous venons d'étudier pourrait avoir été prophète de Rê dans des temples solaires et prophète du Pilier sacré, de Khenty-iaoutef, Khenty-medefet et autres divinités analogues. En effet, Ptahchepses I et Sabou-Ibebi mentionnent les fonctions ci-dessus sur leur stèle; or, ni Ptahchepses II, ni Rênefer, ni Neferefrê-ankh ne nous sont connus par un monument pareil.

\section{§ 39. PTAHCHEPSES III ET SABOU-KEM}

Ptahchepses III $^{2}$ et Sabou-le-noir, que son teint a vraisemblablement fait surnommer ainsi, portent tous deux le titre de "celui qui scelle" (11; 12,1). On ne le trouve pas chez Ptahchepses I mais chez Sabou-Ibebi et Ptahchepses IV $(\mathbf{1 8 , 4 ; 2 4 , 9 )}$ qui sont de la fin de la Vème et du début de la VIème dynastie. Cette circonstance permet d'attribuer à Ptahchepses III et à Sabou-kem une date moins hypothétique qu'aux trois

\footnotetext{
1 Voir ci-dessus, § 9.

${ }^{2}$ Notre Ptahchepses III est le Ptahchepses I de M.A. MURRAY, Saqqara Mastabas.
} 
grands prêtres dont nous avons parlé au paragraphe précédent.

Le tombeau de Ptahchepses III ${ }^{1}$ comprenait une chambre rectangulaire dont le fond était occupé par une stèle pauvre en inscription (11) ; on $\mathrm{y}$ voit Ptahchepses III assis à une table d'offrandes, et il est aussi représenté quatre fois debout au bas de la stèle. Sa tenue n'offre qu'une particularité: il porte une petite barbe postiche ${ }^{2}$. Les titres ne sont qu'un abrégé de ceux des grands prêtres de son temps.

D'autres fragments de décoration mentionnent deux enfants de ce grand prêtre: "son fils Sabou" et "son (fils) Ptahchepses"3. L'identification de l'un ou l'autre avec l'un des deux grands des chefs des artisans qui ont vécu sous Ounas et Téti serait une supposition gratuite que nous ne retenons $\mathrm{pas}^{4}$.

Sabou-kem avait fait orner sa chapelle de peintures sur stuc. Lors de leur découverte, Mariette y a vu encore, malgré leur effacement, le grand prêtre devant une table d'offrandes, des serviteurs apportant des produits divers et une scène d'agriculture sans légende ${ }^{5}$. A l'un des bouts de la paroi du fond, il y avait une stèle en calcaire siliceux, peinte en rouge piqueté de brun pour imiter le granit et mentionnant les noms et titres du défunt (12). Ceux-ci, comme chez Ptahchepses III, sont un résumé de la titulature ordinaire à cette époque.

Il est vraisemblable que la statue de Sabou-kem (13) provient de son tombeau. Elle le représente coiffé d'un serre-tête et orné d'un collier large. Sabou y est appelé une fois Sabou-kem et une fois Sabou, comme sur sa stèle.

D'après la grandeur et la décoration des monuments de Ptahchepses III et de Sabou-kem, on peut penser que ces grands prêtres ont été moins riches que Sabou-Ibebi et Ptahchepses IV.

1 Plan dans M.A. MURRAY, Saqqara Mastabas, II, pl. 34. Cet auteur signale (ibidem, p. 24, § 51) que cette petite tombe n'a pas été notée par Mariette.

2 ibidem, pl. 26.

3 ibidem, pl. 27.

${ }^{4}$ Voir ci-dessus, § 6.

${ }^{5}$ MARIETTE, Mastabas, p. 156-157, C 23. 


\section{$\S 40$. SABOU-IBEBI}

Grâce au bon état de conservation du tombeau construit par SabouIbebi et par son collègue Ptahchepses IV, nous sommes bien renseignés sur ces deux grands prêtres.

Ibebi est le beau nom ${ }^{1}$ de Sabou, qui est né vers le second tiers de la Vème dynastie, sous le règne de Nyouserrê s'il a déjà rempli des fonctions religieuses sous Menkaouhor ${ }^{2}$.

Les étapes principales de sa carrière le font passer, comme les autres grands prêtres de la même époque, de la fonction de prêtre-ouab de Ptah à celles de chef de tous les artisans et directeur de la ouabet, puis à celle de directeur du domaine de Sokar et des sem particuliers au service de ce dieu sous l'Ancien Empire; les titres de prophète de Ptah et prophète de Sokar le classent parmi le haut personnel religieux memphite; une dernière ascension en fait un grand des chefs des artisans ${ }^{3}$.

$\mathrm{Au}$ cours de sa longue vie, Sabou-Ibebi remplit de nombreuses fonctions religieuses dont la plupart ont déjà été exercées par Ptahchepses I: il a la charge et reçoit le bénéfice du service de Rê dans des temples solaires ${ }^{4}$ et des divinités probablement anciennes qui se nomment Khery-bakef, Khenty-medefet, Khenty-iaoutef, Mekhenty-our. Mais il revêt aussi d'autres titres religieux et civils dont l'équivalent n'existe pas chez Ptahchepses I. Il participe, en effet, aux cultes d'Ounas

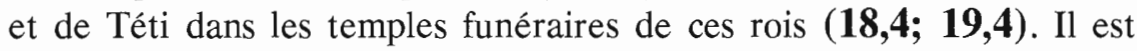
"celui qui scelle" $(\mathbf{2 0 , 8})$. Enfin, le roi ne le met pas seulement au courant ou le charge de dresser les plans des travaux architecturaux qu'il se propose d'entreprendre, comme on l'a fait pour Ptahchepses I puisque tous deux sont "supérieurs des secrets de tous les travaux que Sa Majesté désire faire" $((\mathbf{4 , 1 2 ;} \mathbf{2 0 , 1 6 )}$, mais il confie à Sabou-Ibebi l'exécution

${ }^{1}$ Sur la formation du "beau nom", cf. H. JUNKER, Die Stele des Hofarztes Iry, dans A.Z., 63 (1928), p. 59-64, et, pour sa signification, G. JEQUIER, dans l'ouvrage cité cidessus, $\S 33$, p. 96 , note 2 .

${ }^{2}$ Voir ci-dessus, $\$ 15$, p. 44 , note 4 .

${ }^{3}$ Inscription 17; voir ci-dessus, § 6.

${ }^{4}$ Contre l'hypothèse de K. Sethe, qui voit dans la stèle de Sabou-Ibebi une copie, vide de sens, des inscriptions de Ptahchepses I, voir ci-dessus, § 15. 
même de ses monuments, ce qui vaut à ce dernier le titre de "directeur de tous les travaux du roi" $(\mathbf{2 0 , 2})$, que nous retrouverons chez un autre grand prêtre, Ptahchepses-Impy $(\mathbf{2 9 , 2})$.

Le propos des entreprises royales nous fournit l'occasion d'une remarque. On sait que les grands des chefs des artisans dirigeaient une partie du travail effectué sur les chantiers des pyramides royales de l'Ancien Empire et que, d'autre part, ils commandaient les artisans qui sculptaient des inscriptions pour le compte du roi ${ }^{1}$. En rapprochant ces deux activités, nous pensons que les grands des chefs des artisans avaient la haute main sur l'exécution des bas-reliefs et inscriptions des temples funéraires et qu'ils l'eurent aussi, le moment venu, sur la décoration des chambres funéraires des pyramides royales. Nous ne nous aventurons donc pas en déclarant que Sabou-Ibebi et son collègue ${ }^{2}$ ont conduit personnellement les artisans qui ont gravé la plus ancienne copie que nous possédions des textes des pyramides, celle d'Ounas.

Bien qu'il n'eût, semble-t-il, pas été élevé à la cour ni épousé une princesse, Sabou-Ibebi a été aussi bon courtisan que Ptahchepses I, et non en vain, puisqu'il a pu se dire "ỉmakhou auprès de chaque souverain" $(\mathbf{2 0 , 1 8 )}$ et proclamer que le roi l'a comblé de faveurs "parce que, écrit-il, Sa Majesté m'aimait plus qu'aucun de ses serviteurs, parce que je faisais chaque jour ce qu'elle récompense, parce que mon attachement ${ }^{3}$ était dans son coeur"4.

Enfin, les images et le texte de ses bas-reliefs nous permettent d'imaginer Sabou-Ibebi vers la fin de ses jours. Comblé d'honneurs et de richesses $^{5}$, il dépose le sceptre du commandement et n'exerce vraisemblablement plus la fonction de grand des chefs des artisans ${ }^{6}$, mais

1 Voir ci-dessus, § 11.

2 Le collègue de Sabou-Ibebi était peut-être alors le prédécesseur de Ptahchepses IV; voir au paragraphe suivant.

${ }^{3}$ Mon attachement $=$ le sentiment de mon attachement.

4 20,21; traduction de la dernière proposition et note s'y rapportant d'après $\mathbf{J}$. SAINTE FARE GARNOT, L'imakh et les imakhous sous l'Ancien Empire, Melun, 1943, p. 17 , note 3 .

\footnotetext{
5 Voir ci-dessus, § 10.

${ }^{6}$ Voir ci-dessus, $\S 19$ et 8.
} 
vit du revenu des biens qu'il a amassés pendant sa carrière et que des libéralités royales accroissent peut-être ${ }^{1}$. Dans cette sorte de retraite, il a régulièrement accès à la cour et au conseil du roi $(\mathbf{2 0 , 2 0})$; chez lui, il est entouré par ses enfants et petits-enfants. Nous supposons qu'il avait perdu sa femme puisque, contrairement à Ptahchepses IV, il ne l'a pas fait représenter sur ses bas-reliefs. Mais nous connaissons plusieurs de ses enfants: son fils "qu'il aime", Ptahchepses, lui-même père d'un Ptahchepses; son autre fils, Sabou, père de Khenou; ses fils Hemioun, Ptahchepses-le-noir, Chepesipouptah ${ }^{2}$. Sabou-Ibebi voue encore ses soins au mastaba qu'il construit en commun avec son collègue Ptahchepses IV et dirige peut-être la décoration de sa chapelle funéraire, sous le règne de Téti. A-t-il survécu à ce roi ? Nous n'en savons rien.

\section{§ 41. PTAHCHEPSES IV}

En comparant les chapelles de Sabou-Ibebi et de Ptahchepses IV, nous avons fixé à une génération environ la différence d'âge entre ces grands prêtres $^{3}$. Comme Sabou-Ibebi est probablement né sous Nyouserrê, une génération plus tard nous fait descendre, par-dessus le règne de Menkaouhor, jusqu'à Djedkarê-Isesi. Ptahchepses IV est probablement né sous ce roi. Son port et ses traits, qui sont ceux d'un homme mûr sous Téti $\mathrm{I}$, et sa descendance, qui ne comprend alors que des enfants ${ }^{4}$, confirment cette date.

Ptahchepses IV a donc été revêtu de sa première charge soit à la fin du règne d'Isesi, soit, plus vraisemblablement encore comme nous allons le voir, sous Ounas. Ce fait vient appuyer notre interprétation des

\footnotetext{
1 Voir ci-dessus, § 11, p. 36, note 3.
}

2 L. BORCHARDT, Denkmäler, pl. 21. Nous ne prétendons pas dénombrer exactement la descendance de Sabou-Ibebi, parce qu'elle est répartie en plusieurs registres, avec possibilité mais nullement certitude de représentations multiples d'un seul personnage, et aussi parce que des noms propres sont détruits. Si Sabou-Ibebi a eu des filles, il ne leur a pas même fait l'aumône d'une mention, comme le fera au moins l'un de ses très lointains successeurs, Psenptaïs II $(\mathbf{2 0 1 , 8 )}$.

3 Voir ci-dessus, $§ 19$.

4 Voir ci-dessus, § 19. 
linteaux de grands prêtres sous la Vème dynastie ${ }^{1}$, d'après laquelle la première fonction de Ptahchepses IV aurait été celle de ouab, et la seconde prophète du culte rendu à Ounas dans le temple de sa pyramide $(\mathbf{2 1 , 5})$. Il avait été admis auparavant, ou il l'a été alors, à connaître toutes les choses divines scellées $(\mathbf{2 5 , 2})$; nous pensons qu'il s'agit ici du culte d'Ounas et non d'un autre dieu. Ptahchepses IV est devenu ensuite prophète du culte rendu à Téti $(\mathbf{2 1 , 6 )}$ et, comme la majeure partie de son ascension date de ce roi, nous pouvons préciser qu'il a commencé sa carrière sous Ounas.

Il avait femme et enfants lorsqu'il a fait orner sa chapelle funéraire. Son épouse, Inti, qui a elle-même revêtu des fonctions religieuses ${ }^{2}$, a peut-être été ensevelie par Sabou-Tjéti ${ }^{3}$; elle aurait alors survécu à son mari. Parmi les enfants de Ptahchepses IV, citons: son fils aimé, imy-khet des artisans, Sabou; un Ptahchepses qui porte le même titre; un autre Ptahchepses qui est khenty-che de Pharaon; un Chepesipouptah ${ }^{4}$.

Deux "directeur des artisans", Sebekhotep et Iry, prennent rang parmi les .porteurs d'offrandes du tombeau de Ptahchepses IV $^{5}$. Nous en déduisons que celui-ci était en fonction quand il a fait décorer sa chapelle. D'autre part, comme il n'a peut-être pas survécu au règne de Téti, puisqu'il ne mentionne pas de roi postérieur, il sera décédé peu après l'achèvement de sa tombe.

\section{§ 42. SABOU-TJETI ET PTAHCHEPSES-IMPY}

Avec Ptahchepses IV, le pontificat memphite achève une partie de son histoire. Le roi, dont la volonté est plus forte que l'esprit traditionaliste

1 Voir ci-dessus, § 6.

${ }^{2}$ Elle est prophétesse de divinités de son sexe, Hathor et Néit.

${ }^{3}$ Voir ci-dessus, § 24.

${ }^{4}$ M.A. MURRAY, Saqqara Mastabas, I, pl. 29-31. La remarque faite au paragraphe précédent sur la descendance de Sabou-Ibebi s'applique aussi aux enfants de Ptahchepses IV.

5 ibidem, pl. 29, paroi nord, 3ème registre. Nous avons mentionné l'existence de ces personnages, ci-dessus, $\S 8$. 
de ses bureaucrates, procède à une refonte complète de cette institution ${ }^{1}$ et Sabou-Tjéti apparaît comme le premier grand prêtre nommé selon le nouveau statut. Nous avons déjà fait l'étude de sa carrière, si importante pour ce sujet ${ }^{2}$; il suffit d'en rappeler les résultats principaux.

Il est extrêmement probable que Sabou-Tjéti a été le premier grand prêtre qui n'ait pas partagé sa fonction avec un collègue, au moins à partir d'un certain moment de sa carrière. Il a été par deux fois l'objet d'une nomination par le roi. C'est aussi le plus ancien grand des chefs des artisans qui ait servi Sokar en qualité de $\mathrm{sem}^{3}$.

Ces faits si intéressants, et peut-être d'autres encore, Sabou-Tjéti les avait gravés sur une grande stèle qui occupait une partie de la paroi du fond dans sa chapelle funéraire. Un quart seulement de la stèle était encore debout lors de sa découverte. La façade du mastaba avait des proportions monumentales dont témoignaient des fragments sur lesquels des hiéroglyphes ne mesuraient pas moins de quarante centimètres de haut ${ }^{4}$. La sépulture de Sabou-Tjéti était bien digne de l'évènement dont elle perpétuait le souvenir, et nous faisons entièrement nôtre la note de A. Mariette: "Les fouilles faites pour mettre à jour ce tombeau ont donné des débris d'inscriptions et de sculptures qui en font vivement regretter la perte" .

Un grand des chefs des artisans nommé Tjéti apparaît sur deux monuments indépendants du mastaba ci-dessus. Dans un mastaba ruiné qui ne comportait pas de chapelle mais seulement une niche en calcaire, A. Mariette a trouvé une stèle dont toute la partie supérieure avait disparu; le bas seul des lignes subsistait et a permis de lire que le tombeau avait été fait pour la "véritablement connue du roi Inti"; à gauche de la stèle, un personnage dont la titulature finissait par "grand des chefs des artisans, celui qui est dans la double maison" et qui était nommé Tjéti était représenté (28). Il est possible que ce tombeau ait été celui de l'épouse de Ptahchepses IV, qui aurait été ensevelie par

\footnotetext{
${ }^{1}$ Voir ci-dessus, § 25.

${ }^{2}$ Voir ci-dessus, $\$ 24$.

${ }^{3}$ Voir ci-dessus, $\S 25$ et $\mathbf{5}$.

${ }^{4}$ MARIETTE, Mastabas, p. 391, E 3.

5 ibidem.
} 
Sabou-Tjéti. La place respective des mastabas de Sabou-Tjéti et de Ptahchepses IV rend plausible la double identification que nous avons déjà proposée ${ }^{1}$.

Un "compagnon unique et prêtre-lecteur" figuré parmi d'autres sur les bas-reliefs du temple funéraire de Pépi II a reçu après coup la légende suivante gravée, d'ailleurs moins soigneusement, devant ses jambes: "Le sem, chef de toute la garde-robe, trésorier du roi, préposé au diadème, grand des chefs des artisans (?), Tjé(t)i" (27). L'identification de ce grand prêtre avec Sabou-Tjéti est également possible, mais la part d'hypothèse est ici plus grande que dans le cas précédent.

Sabou-Tjéti a les traits d'un homme dans la force de l'âge sur les trois représentations conservées de sa stèle ${ }^{2}$. Mais comme nous ignorons tant la date de son tombeau que celles de sa naissance et de sa mort, et que nous n'avons aucun renseignements sur sa parenté, nous ne pouvons que le placer dans la première moitié de la VIème dynastie.

Nous avons rencontré jusqu'ici quatre grands prêtres nommés Ptahchepses, que nous avons numérotés pour les distinguer. Un cinquième porte le même nom, mais aussi celui d'Impy (29); c'était son beau nom ${ }^{3}$. Ptahchepses-Impy, connu seulement par une statue, énumère ses titres dans l'ordre chronologique $\mathbf{2 9 , 1}$ ); on peut donc le dater après la réforme du pontificat sous la VIème dynastie. De plus, il est "compagnon unique" et "prêtre-lecteur", comme Sabou-Tjéti. Or celui-ci a été le premier grand prêtre après la réforme. Ptahchepses-Impy vient donc à sa suite, vraisemblablement sous Pépi $\mathrm{II}^{4}$. En tant que "directeur des travaux du roi" $(\mathbf{2 9 , 2})^{5}$, il a peut-être eu sous ses ordres les artisans qui ont décoré le temple funéraire du roi et le monument similaire de l'une ou l'autres des épouses de Pépi II.

${ }^{1}$ Voir ci-dessus, § 24.

2 Pour ses costume et pectoral, voir ci-dessus, § 9.

${ }^{3}$ Voir ci-dessus, $\$ 23$, p. 62 , note 5 .

4 CH. BOREUX, Antiquités égyptiennes, II, 1932 (Catalogue-guide du Louvre), p. 451, date la statue de Ptahchepses-Impy de la "fin de la IVème ou commencement de la Vème dynastie"; l'examen des inscriptions nous empêche de le suivre.

${ }^{5}$ Sabou-Ibebi avait porté le même titre; voir ci-dessus, $\S \mathbf{4 0 .}$ 
Les grands prêtres de l'Ancien Empire, tant après qu'avant la transformation du pontificat sous la VIème dynastie, ont tous fait carrière seulement à Memphis et leurs champs d'activité ont toujours été les mêmes; d'une part, ils ont dirigé les artisans qui travaillaient la belle pierre calcaire venue de l'autre côté du Nil; d'autre part, il ont servi Ptah et les diverses divinités memphites. Il n'ont pas exercé d'autre fonction civile que celle de grand des chefs des artisans.

\section{§ 43. APPENDICE AU CHAPITRE VII}

Khabaouseker, qui était "chef des artisans du Palais (ou: du tombeau royal)", a peut-être rempli la charge qui a été à l'origine de la fonction civile du grand prêtre désignée par le titre "grand des chefs des artisans"1; ce serait la raison pour laquelle l'un et les autres porteraient le pectoral. Mais Khabaouseker n'a eu aucune attache avec Ptah et ne peut pas être classé parmi les grands prêtres de $\mathrm{Ptah}^{2}$.

Le "chef de tous les artisans" Iny-ankh a passé à tort pour un grand des chefs des artisans ${ }^{3}$. Il en a été de même pour Nebou ${ }^{4}$ dont le titre, quoi qu'il puisse signifier ${ }^{5}$, n'est en tout cas pas celui de grand des chefs des artisans. Nymaâtrế ${ }^{6}$ a été aussi classé parmi les grands prêtres de Memphis $^{7}$, mais il n'est que "sem, chef de la garde-robe et compagnon unique"8.

${ }^{1}$ Voir ci-dessus, § 9 et 21.

2 E. SCHIAPARELLI, Museo Archeologico di Firenze, 1887, p. 201, est, à notre connaissance, le premier à avoir commis cette erreur.

3 Voir ci-dessus, § 4.

${ }^{4}$ M.A. MURRAY, Index, p. 19.

${ }^{5}$ LEPSIUS, Denkmäler, II, 93 b.

${ }^{6}$ MARIETTE, Mastabas, p. 224, D 17; PORTER ET MOSS, Memphis, p. 123, "VIth dyn. (?)".

\footnotetext{
7 E. SCHIAPARELLI, op. cit.

${ }^{8}$ Voir à ce sujet ci-dessus, $\S \mathbf{5}$, fin.
} 



\section{H A P I T R E VIII}

\section{LES GRANDS PRETRES DU MOYEN EMPIRE}

\section{$\S$ 44. HAPIDJEFA}

Bien que nous ne connaissions aucun grand prêtre de la Première Période Intermédiaire, il nous est apparu que le pontificat est resté à peu près sans changement de la fin de l'Ancien Empire jusqu'au Moyen Empire, et au-delà ${ }^{1}$.

La XIIème dynastie nous fournit quelques noms de grands prêtres dont le plus ancien est peut-être Hapidjefa. Pendant longtemps on n'a connu ce nomarque que par le tombeau qu'il s'est fait creuser au-dessus d'Assiout. On supposait sans doute qu'il avait passé sa vie dans cette ville. Il êtait alors difficile de voir en lui un grand prêtre memphite ${ }^{2}$ bien qu'il en portât deux titres, dont le principal: "grand des chefs des artisans" et "celui qui est dans la double maison" (30). Depuis les découvertes faites à Kerma, on sait qu'Hapidjefa a passé la fin de sa vie bien loin d'Assiout, dans le sud, près de la troisième cataracte. Il s'y est même fait préparer un tombeau sous le règne de Sésostris Ier $^{3}$. A la lumière de ces données, il est moins difficile d'admettre qu'Hapidjefa a vécu également à Memphis et y a été grand prêtre dans le cours de sa carrière, peut-être encore sous le règne d'Amenemhat ${ }^{4}$.

1 Voir ci-dessus, § 27.

2 M. MULLER, Über einige Hieroglyphenzeichen, dans Rec. Trav., IX (1887), p. 167.

${ }^{3}$ G.A. REISNER, The tomb of Hepzefa, nomarch of Siut, dans Journal of Egyptian Archaeology, 5 (1918), p. 79-98.

4 Voir ci-dessus, $\$ 8$. 
On pourrait tirer argument de la graphie particulière du mot "artisans" dans le titre d'Hapidjefa (30) pour lui refuser la qualité de grand prêtre memphite. Mais cette graphie insolite peut provenir de l'ignorance où les scribes d'Assiout pouvaient être de l'orthographe memphite du titre porté par le grand prêtre de Ptah.

\section{§ 45. SENOUSERT-ANKH}

L'imposant mastaba de Senousert-ankh à Lisht (33 - 35) nous assure que ce grand prêtre a été en fonction sous Sésostris Ier et qu'il a dirigé au moins une partie du chantier de la pyramide royale. Ce travail, ainsi d'ailleurs que la construction du mastaba, a réclamé la présence régulière, si ce n'est continuelle, de Senousert-ankh à Lisht. D'autre part, le service de Ptah à Memphis demandait aussi qu'il ne fût pas toujours absent de ce lieu. Comment a-t-il partagé son temps entre les deux endroits ? C'est là un problème dont nous ne pouvons pas donner la solution, mais qui se pose clairement pour ce personnage.

La situation du tombeau de Senousert-ankh nous semble comporter un autre enseignement, celui-là touchant les grands prêtres de la Vème et de la VIème dynasties dont on a retrouvé les sépultures. Ils se sont fait enterrer dans la nécropole memphite non seulement parce que c'était l'emplacement le plus naturel, mais aussi pour que leurs mastabas fussent proches des pyramides royales de leur temps.

Un torse de statue anépigraphe représente très probablement Senousert-ankh ${ }^{1}$ : c'est un homme très jeune. Et l'importance de son mastaba donne une idée de sa richesse; d'ailleurs plusieurs de ses contemporains, qui ont vraisemblablement appartenu à sa maison et parmi lesquels il y a deux majordomes, se sont fait ensevelir dans l'enceinte de son mastaba ${ }^{2}$. Ajoutons à ces rares indications un goût possible pour l'ancien ${ }^{3}$ et nous aurons énuméré nos connaissances peu nombreuses de ce grand prêtre !

${ }^{1}$ A. LANSING, The Egyptian Expedition. The Excavations at Lisht, dans Section II of the Bulletin of the Metropolitan Museum, nov. 1933, p. 11 et fig. 14, p. 14.

2 ibidem, p. 23.

${ }^{3}$ Voir ci-dessuis, § 27 , note. 


\section{§ 46. SEHETEPIBRE-ANKH-NEDJEM, NEBPOU ET SEHETEPIBRE-CHERI}

Les monuments de ces trois grands prêtres sont pauvres en inscriptions et ne livrent probablement qu'un abrégé de leur titulature $(31 ; 32)$. Le groupe du Louvre (31) donne toutefois une image de Sehetepibrê-ankhnedjem et de Nebpou, ornés du pectoral; on y lit que Nebpou était le fils de Sehetepibrê-ankh-nedjem et le père de Sehetepibrê-cheri, dont le nom signifie Sehetepibrê le Jeune.

Sehetepibrê-ankh-nedjem n'est, d'après son nom, pas antérieur à Amenemhat Ier, mais ce terminus post quem n'est pas certain parce qu'il y a eu d'autres rois qui se sont appelés Sehetepibrê, après la XIIème dynastie. Faute d'autre donnée, on peut admettre la date fournie par la généalogie d'Ankhef-ny-sekhmet et placer provisoirement le pontificat de Sehetepibrê-ankh-nedjem sous Sésostris Ier $^{1}$, et, pour ne pas supposer que les trois générations ne se sont pas succédé directement, supposer que Sehetepibrê-ankh-nedjem a été grand prêtre après Senousert-ankh.

Nebpou et Sehetepibrê le Jeune ont donc été grands prêtres sous la XIIème dynastie ou au début de la seconde période intermédiaire.

Les grands prêtres de l'Ancien Empire faisaient carrière à Memphis. Avec le Moyen Empire, un pharaon énergique fait appel, à notre connaissance pour la première fois, à un homme d'une autre ville pour diriger le clergé memphite. Plus tard, cependant, sans d'ailleurs que nous puissions préciser la date, une petite "dynastie" de grands des chefs des artisans apparaît à Memphis, révélant peut-être chez les rois de ce temps un souci moins grand de maintenir leur pleine autorité sur les grands prêtres de Ptah.

\section{§ 47. APPENDICE AU CHAPITRE VIII}

Un personnage anonyme, dont le pectoral a déjà retenu notre attention $^{2}$, a pu être grand prêtre de Memphis. Sa statue ${ }^{3}$ le représente

\footnotetext{
1 182,50; voir ci-dessus, $\S 33$.

2 Sous "f" dans notre liste en note 5, p. 27-28. Voir Planche I, fig. 5 et II, fig. 5.
} 
accroupi, assis seulement sur son talon droit, la jambe gauche étant pliée verticalement. Il n'est vêtu que d'un pagne. Un serre-tête le coiffe et un pectoral orne sa poitrine. Des plis graisseux barrent son abdomen. Le socle, qui fait corps avec la statue, est carré devant et arrondi derrière. Le nom "Noubrê", sans cartouche, est gravé sur l'épaule droite; s'agit-il d'un roitelet postérieur à la XIIème dynastie ? A part ce nom, deux fragments d'inscriptions apparaissent sur le bord inférieur du pagne et sur le socle. Le premier est formé de deux sections de colonnes où l'on peut lire: "...pour (?) le noble, gouverneur, trésorier du roi, ... (2ème colonne) ..... place ... vie, santé, force." Sur le socle, une ligne débute contre l'intérieur du pied gauche: "le trésorier du roi, compagnon unique aimé ${ }^{1}$......". Le mot "roi" du titre "trésorier du roi" est écrit avec l'abeille sur le pagne et avec la couronne rouge sur le socle; il semble bien qu'on ait affaire ici à un "remplacement sans raison d'être sérieuse"2.

On ne peut donc savoir si ce contemporain de Noubrê a porté ou non le titre de grand des chefs des artisans et le pectoral n'est pas un indice suffisant pour le classer parmi les grands prêtres memphites.

Il est par contre certain que le gouverneur d'Eléphantine Sarenpout ${ }^{3}$ n'a pas été grand prêtre de Memphis, bien qu'il porte un titre composé avec les mêmes éléments que "grand des chefs des artisans", mais ordonnés différemment ${ }^{4}$. Sarenpout est en effet kherep hemou our, que

${ }^{3}$ Caire 14-6-24-12. Nous remercions M. Leibovitch d'avoir attiré notre attention sur ce monument.

${ }^{1} n(y) m r(w) t$.

2 Cf. P. LACAU, Suppressions et modifications de signes dans les textes funéraires, dans $\ddot{A} . Z$., 51 (1914), p. 57, qui signale "trésorier du roi" écrit avec la couronne rouge et note expressément que cette graphie ne se rencontre pas exclusivement dans les inscriptions à l'usage d'un mort.

${ }^{3}$ Les inscriptions de son tombeau: K. SETHE, Urkunden des mittleren Reiches (VII), p. 9. Sur un bâtiment construit par Sarenpout à Eléphantine, cf. Chronique d'Egypte, 42, juillet 1946, p. 200.

${ }^{4}$ A.H. GARDINER, Inscriptions from the tomb of Sirenpowet I, dans Ä.Z., 45 (1908), p. 140 , tendait à identifier les deux titres. 
nous traduisons "chef des grands artisans" parce que deux inscriptions de l'île de Sehel mentionnent un "supérieur des travaux dans le domaine d'Amon, supérieur des sculpteurs dans le domaine de Ptah, grand artisan (hemou our) dans le Château de l'Ancien, Amenemope"1.

${ }^{1}$ MARIETTE, Monuments divers, pl. 71, n 35; pl. 73, n 69. 


\section{H A P I T R E I X}

\section{LES GRANDS PRETRES DU NOUVEL EMPIRE}

\section{$\S$ 48. LES PTAHMES}

La comparaison des grands prêtres du Moyen Empire avec ceux de la XVIIIème dynastie nous a fait constater que le pontificat memphite n'avait pas subi de modification importante pendant la seconde période intermédiaire. Les envahisseurs Hyksôs, si honnis par la suite, n'ont vraisemblablement malmené ni Ptah ni son clergé ${ }^{1}$. Si l'on voulait se baser sur l'absence de monument au nom d'un grand prêtre à cette époque, il faudrait appliquer la conclusion qu'on en tirerait non seulement aux Hyksôs mais aux premiers rois et reine de la XVIIIème dynastie. Nous ignorons, en effet, les noms des grands prêtres sous Ahmès, Aménophis Ier, Thoutmôsis Ier et II et Hatchepsout. Sous le règne de Thoutmôsis III, un Ptahmès a été grand prêtre memphite. Comme d'autres grands prêtres de Ptah ont porté le même nom au Nouvel Empire, il nous sera utile de les avoir dénombrés ${ }^{2}$.

Nous connaissons trois monuments qui, tout en mentionnant un grand des chefs des artisans Ptahmès, sont datés en toute certitude:

- un naos conservé au Caire et fabriqué sous le règne de Thoutmôsis III (36);

- une statue fragmentaire, conservée aussi au Caire et portant le cartouche de Thoutmôsis IV (37);

1 Voir ci-dessus, $\$ 27$.

2 Nous sommes d'accord sur plusieurs points avec R. ANTHES, Die hohen Beamten namens Ptahmose in der 18. Dynastie, dans Ä.Z., 72 (1936), p. 60-68; mais nous ne le suivons pas partout, d'où la nécessité de notre exposé. 
- une statue conservée à Florence et portant le cartouche d'Aménophis III (69).

La statue de Florence appartient à un grand des chefs des artisans Ptahmès qui est fils de Menkheper $(\mathbf{6 9 , 5})$. Ce même Ptahmès apparaît sur un monument $(\mathbf{3 8 , 8})$ qu'on pourrait par lui seul dater avec probabilité du règne d'Aménophis III: c'est la stèle fragmentaire conservée à Leyde et dédiée par le majordome d'un temple de Nebmaâtrê. Cette stèle mentionne un autre grand prêtre memphite Ptahmès, qui a pour père un vizir Thoutmôsis $(\mathbf{3 8 , 6 )}$.

La stèle de Leyde établit donc l'existence de deux grands prêtres de Ptah nommés Ptahmès. On pourrait à la rigueur attribuer à l'un d'eux le naos du Caire sculpté sous Thoutmôsis III et en conséquence la statue datée de Thoutmôsis IV. Cependant l'intervalle séparant les règnes de Thoutmôsis III et d'Aménophis III nous invite à voir sur le naos un Ptahmès différent de ses homonymes de la stèle de Leyde.

Les autres monuments au nom d'un grand des chefs des artisans Ptahmès semblent appartenir à l'un ou l'autre des trois personnages que nous venons de déterminer, à l'exception d'une palette du Louvre (155) que nous datons de la XIXème dynastie ${ }^{1}$. Nous admettons, en fin de compte, l'existence de quatre grands prêtres memphites appelés Ptahmès.

\section{§ 49. PTAHMES I}

Il est possible que le naos déposé par Ptahmès I en Abydos ait été taillé et gravé à Memphis, dans les ateliers du temple de Ptah, puisque ce monument monolithe ne mesure pas plus de quatre-vingts centimètres de hauteur. Le grand prêtre y est représenté en haut-relief sur la face antérieure, avec les cartouches de Thoutmôsis III sur le bras et la poitrine $(\mathbf{3 6 , 1})$. Ces inscriptions indiquent assez sûrement que Ptahmès a été grand prêtre sous le règne de Thoutmôsis III. D'ailleurs Thoutmôsis III est assimilé à Horus fils d'Osiris dans un texte gravé sur une des faces latérales. C'est un hymne à Osiris à la fin duquel Ptahmès

${ }^{1}$ Voir ci-dessus, § 31. 
s'écrie: "Tel es-tu, Osiris ${ }^{1}$, fils de Nout ! Le roi Menkheperrê doué de vie est ton fils Horus $^{2}$; il bat tes ennemis pour toi, etc..." ${ }^{\prime 3}$. C'est donc pendant le règne de Thoutmôsis III que le naos a été taillé.

La statue fragmentaire portant le cartouche de Thoutmôsis IV (37), dont nous avons parlé au paragraphe précédent, peut appartenir à Ptahmès I, ou à celui des Ptahmès de la stèle de Leyde que nous appelons Ptahmès II, ou encore à un Ptahmès différent et situé chronologiquement entre Ptahmès I et II. A priori, nous estimons qu'il est inutile d'attribuer deux monuments portant mêmes noms et titulatures à deux personnages distincts, à moins que les données de ces monuments n'exigent le contraire. Ce n'est pas le cas pour la statue fragmentaire. Il nous reste donc à l'attribuer à l'un des Ptahmès I ou II, et, pour choisir, nous ne disposons que de deux arguments assez fragiles. Le premier nous est fourni par les successions de règnes; il est plus facile d'attribuer un monument daté de Thoutmôsis IV à un contemporain d'Aménophis III que de Thoutmôsis III. Le second argument est à puiser dans la comparaison des titulatures. Ptahmès I semble tenir au titre de "trésorier du roi" qu'il place souvent en tête des autres titres $(\mathbf{3 6 , 2 , 4 , 5 )}$ et même une fois seul devant "grand des chefs des artisans" $(\mathbf{3 6 , 2 )}$. Sur la statue fragmentaire, Ptahmès n'est qu'une fois "trésorier du roi", et encore cette mention se trouve-t-elle dans une énumération de titres $(37,2)$; l'abrégé des titres de ce Ptahmès ne comprend que "grand des chefs des artisans" $(\mathbf{3 7 , 4 )}$. Comme le naos est antérieur à la statue, il serait bizarre que Ptahmès I, s'il était le possesseur de cette dernière, n'y eût pas accordé plus d'importance à "trésorier du roi". Les deux arguments concordent

${ }^{1}$ Litt. "Tel est Osiris"; cf. A.H. GARDINER, Egyptian Grammar, § 130.

${ }^{2}$ Sujet et prédicat sont simplement juxtaposés, ce qui ferait prendre le prédicat pour une apposition si le texte parallèle de Maï ne portait: "Je suis ton fils Horus" (je = pronom indépendant). Pour l'hymne à Osiris, conservé en plusieurs exemplaires, cf. L. SPEELERS, La stèle de Maï au Musée de Bruxelles, dans Rec. Trav., XXXIX (1921), p. 113-144 (pour le passage noté ici, p. 123).

${ }^{3}$ R. ANTHES, Die hohen Beamten..., dans Ä.Z., 72 (1936), p. 60-61, suppose que le naos a été fait après la mort de Thoutmôsis III par Ptahmès qui aurait survécu au roi. Il se base sur les inscriptions qui, écrit-il, se rapportent à Thoutmôsis III en tant qu'Osiris. Comme Ptahmès, sur l'autre face latérale du naos, implore vie, stabilité et prospérité pour Thoutmôsis, le passage auquel R. Anthes fait allusion ne peut être que celui que nous traduisons et dans lequel Thoutmôsis est assimilé à Horus, fils d'Osiris et non à Osiris. Nous ne retenons donc pas la supposition d'Anthes. 
donc et permettent d'attribuer la statue à Ptahmès II plutôt qu'à Ptahmès I.

\section{§ 50. PTAHMES II}

La stèle de Leyde qui représente deux grands prêtres memphites nommés Ptahmès et contemporains d'Aménophis III $^{1}$ est aussi le seul monument d'après lequel on puisse définir le rapport de l'un et l'autre. La recherche a été faite et nous en adoptons la conclusion: Ptahmès fils du vizir Thoutmôsis est antérieur à Ptahmès fils de Menkheper ${ }^{2}$. Comme R. Anthes l'a relevé, Ptahmès fils de Menkheper est le seul des cinq personnages qui n'est pas "juste de voix" (34,4-8).

Ptahmès II est donc issu d'une famille très haut placée. Son père et sa mère, Thoutmôsis et Taoui $(\mathbf{3 8 , 7}$ et 4 ) lui donnèrent sans doute, ainsi qu'à son frère Méryptah $(\mathbf{3 8 , 5})$ l'éducation qui convenait à des fils de vizir. Il ne serait pas étonnant que Ptahmès et Méryptah aient connu dès leur jeunesse le futur pharaon Aménophis $\mathrm{III}^{3}$ car le vizir Thoutmôsis approchait certainement le souverain d'alors, Thoutmôsis IV, chaque fois que celui-ci passait à Memphis.

Ptahmès II a peut-être été déjà grand prêtre sous Thoutmôsis IV, sous le règne duquel il se serait fait une statue, qui fut déposée dans un sanctuaire de la Vallée Sacrée $(\mathbf{3 7 , 4})$, donc dans la nécropole memphite. On peut se demander si c'est à cause de cette destination qu'une des deux inscriptions de cette statue $(\mathbf{3 7 , 2 )}$ énumère les titres "grand des chefs des artisans" et sem dans un ordre exceptionnel à l'époque ${ }^{4}$, c'està-dire en accentuant sem.

De toute façon, la partie la plus importante du pontificat de Ptahmès II date du règne d'Aménophis III. Lorsque ce roi, dans son désir probable

1 Voir ci-dessus, $\S 48$.

2 R. ANTHES, op. cit., p. 66 et 61 , note 2.

${ }^{3}$ Plus tard, Méryptah fut choisi par le roi pour administrer le "temple de Nebmaâtrê" bâti à Memphis.

${ }^{4}$ Voir ci-dessus, § 31. 
de contenir l'ambition des grands prêtres d'Amon, leur enleva la direction administrative des prophètes de Haute et Basse-Egypte pour la confier au grand prêtre memphite ${ }^{1}$, il put d'autant mieux le faire qu'il connaissait Ptahmès II de longue date. En cette période de sa vie, Ptahmès II perdit son père, pour lequel il fit sculpter une stèle fausse-porte à la manière de l'Ancien Empire (39); il est assez curieux qu'il s'y intitule lui-même à la fois "fils qui fait vivre le nom de son père" et de "juste de voix" $(\mathbf{3 9}, 3)$. Aurait-il suivi de près son père au tombeau ou pensait-il au temps où il ne serait plus ? On ne peut le savoir. Il faut cependant noter que le pyramidion découvert il y a plus d'un siècle par Passalacqua dans la nécropole memphite ${ }^{2}$ est de la même époque (41); Ptahmès II érigeait donc son propre tombeau, dont la chapelle funéraire était aménagée dans une petite pyramide, quand il était ministre des cultes.

Ptahmès II est le seul grand prêtre memphite de ce nom dont le tombeau nous ait certainement livré quelque chose. C'est la raison pour laquelle nous n'hésitons pas à lui attribuer de nombreux objets au nom d'un sem et grand des chefs des artisans Ptahmès. Dans quelques cas, cette attribution est confirmée par la filiation de Ptahmès ou par la présence du titre "directeur des prophètes de Haute et Basse-Egypte". La plupart des objets en question ont été utilisés ou portés par Ptahmès II de son vivant: coudée $(\mathbf{4 0})$, vases $(49$ - $54 ; 47 ; 45)$, mortier et pilon $(46)^{3}$, molette $(\mathbf{5 5})$, double boîte à fard $(\mathbf{4 8})$, scarabée $(\mathbf{4 3})$, bague en or (58), pommeau de canne (56); d'autres ont été destinés au tombeau dès leur fabrication: palette en pierre $(\mathbf{4 2})$, couteau en calcaire (57), enfin statuette funéraire (44).

En admettant que le ministère des cultes a été dans les mains d'un grand prêtre d'Amon au début du règne d'Aménophis III $^{4}$, Ptahmès II aura occupé ce poste dans le second quart environ du règne, même dans le second tiers.

${ }^{1}$ Voir ci-dessus, § 28.

2 R. ANTHES, op. cit, p. 64.

${ }^{3}$ Sur le pilon, le nom est "Mes": diminutif porté réellement ou employé seulement ici par manque de place.

${ }^{4}$ Voir ci-dessus, $\$ 28$. 


\section{$\S$ 51. LE PRINCE THOUTMOSIS}

Si Ptahmès II aimait à se faire suivre par un singe ${ }^{1}$ qu'il tenait en laisse, Thoutmôsis eut un autre animal préféré. C'était une chatte, appelée simplement Ta-miat; elle mourut avant son maître. Celui-ci, touché par ce trépas, fit momifier sa bête favorite et donna ordre aux artisans qu'il commandait de sculpter pour elle un sarcophage en calcaire. La chatte y est représentée deux fois, ruban au cou, devant une table d'offrandes. Dans l'une de ces images, elle est assise sur un coussin et s'appelle l'"imakhyt auprès de La Chatte". Cette "déesse", à tête féline sur corps humain, se tient debout derrière la chatte attablée. La majeure partie des inscriptions sont des formules à l'usage de la chatte, mais deux lignes gravées sur le couvercle nous renseignent sur l'identité de celui qui a fait faire ce singulier monument. Thoutmôsis y est appelé une fois "le fils aîné du roi, son aimé, le grand des chefs des artisans, sem, Thoutmôsis" et sur l'autre ligne "le fils du roi, directeur des prophètes en Haute et BasseEgypte, grand des chefs des artisans, sem, Thoutmôsis" (62).

Nous n'employons pas la traduction "fils du roi" au lieu de "fils royal " sans raison. Alors que des personnages qui ne sont pas à proprement parler des enfants du roi portent le titre de "fils royal", tels les gouverneurs de Nubie, Thoutmôsis était réellement le fils d'un roi. Son origine princière est confirmée par le tombeau d'un Apis enseveli dans un caveau particulier, comme cela se faisait à l'époque. Sur les bas-reliefs de la chapelle funéraire, on voyait Aménophis III, accompagné du "fils $d u$ roi, sem, Thoutmôsis" offrant l'encens au taureau sacré .

A considérer seulement le cercueil de chatte et le bas-relief d'Apis, l'identité des Thoutmôsis qui y sont mentionnés ne serait que possible. Mais elle devient certaine lorsqu'on compare ces monuments avec ceux d'un autre grand prêtre memphite d'origine princière. Khamouas, le fils de Ramsès II, est "le fils du roi, sem, Khamouas" sur le bas-relief d'un tombeau d'Apis où il est représenté officiant aux côtés de son père

${ }^{1}$ Statue fragmentaire du règne de Thoutmôsis IV (37) et stèle de Leyde (38). Sur les singes domestiqués, cf. J. VANDIER D'ABBADIE, Catalogue des ostraca figurés de Deir el-Médineh = I.F.A.O., Documents de fouilles, Tome II, 3ème fascicule, Le Caire 1946 , p. 17.

2 Inscription 63; description dans MARIETTE, Sérapéum, 1857, p. 8. 
(109). Il use très fréquemment de cette titulature, qui n'est qu'un abrégé comme le montre clairement son inscription sur la pyramide d'Ounas: celle-ci mentionne au début le "grand des chefs des artisans, sem, fils $d u$ roi, Khamouas", puis, dans le cours du texte, simplement "le sem, fils du roi, Khamouas" (114, 1-2). Le prince Thoutmôsis, bien qu'il fût "grand des chefs des artisans", se sera servi de l'expression "fils du roi, sem" et son exemple, si même il était le premier à faire ainsi, aura été suivi par Khamouas.

La comparaison des deux princes nous a fait supposer que Thoutmôsis insiste sur sa fonction de sem non seulement en qualité de grand prêtre memphite, mais aussi parce qu'il joue ce rôle vis-à-vis de son père ${ }^{1}$. Cette préférence peut également provenir du fait que sem ne marque pas un degré de l'administration, si élevé soit-il, comme "grand des chefs des artisans", titre conféré par nomination.

Enfin, de même que plus tard Khamouas prendra part aux campagnes de son père, Thoutmôsis a peut-être commencé par le métier des armes, si le fouet portant l'inscription "le fils $d u$ roi, chef de troupe, Thoutmôsis" lui a appartenu. Il faudrait admettre que cet objet, découvert dans la tombe de Toutankhamon ${ }^{2}$ aurait survécu à la tourmente thébaine du règne d'Akhenaton dans quelque magasin royal, ou fait partie des objets déménagés à El-Amarna.

Thoutmôsis n'a pas succédé à son père, bien qu'il fût son fils aîné. Il a donc exercé son pontificat sous le règne d'Aménophis III. D'autre part, c'est un des deux grands prêtres memphites de la XVIIIème dynastie qui ont été ministres des cultes ${ }^{3}$. C'est pourquoi nous rapprochons, par hypothèse, son pontificat de celui de Ptahmès II. Le petit nombre de monuments de Thoutmôsis permet, en plus, de supposer que sa carrière pontificale a été assez courte. On peut ainsi le classer après Ptahmès $\mathrm{II}^{4}$ et avant Ptahmès III, qui ne peuvent pas être très éloignés l'un de l'autre comme nous le verrons à propos de ce dernier.

${ }^{1}$ Voir ci-dessus, $§ 31$.

${ }^{2}$ H. CARTER, The tomb of Tut-ankh-amen, III, 1933, p. 97; cf. H.W. HELCK, Der Einfluss der Militärführer in der 18. äg. Dynastie, 1939, p. 31 et note 4.

3 Voir ci-dessus, § 28.

${ }^{4}$ Ainsi fait H. KEES, Götterglaube, 1941, p. 368, note 6. 
Un seul objet provient peut-être du tombeau de Thoutmôsis. C'est une statuette funéraire broyant du grain¹.

\section{§ 52. PTAHMES III}

Après le pontificat court, mais important, de Thoutmôsis, Aménophis III choisit comme grand prêtre de Memphis $(\mathbf{6 9 , 6 )}$ un personnage d'origine moins illustre que ses deux prédécesseurs. Ptahmès III avait en effet pour père un simple prophète, appelé Menkheper, dont le titre n'est accompagné d'aucun nom de divinité $\mathbf{( 3 8 , 8 )}$; par la suite, ce Menkheper devint premier prophète $(\mathbf{6 9 , 5 )}$, toujours sans mention du dieu qu'il servait.

Il n'y avait pas longtemps que Ptahmès III avait été nommé grand des chefs des artisans lorsque Méryptah, le frère de Ptahmès II et majordome du temple de Nebmaâtrê fit fabriquer la stèle dont la moitié supérieure seule est conservée à Leyde (38). A part les inscriptions, que nous donnons d'autre part, on y voit cinq personnages, debout, sculptés en haut-relief. En commençant à la gauche de celui qui regarde la stèle, ce sont: la dame Taoui, ses fils Méryptah et Ptahmès II, leur père qui est le vizir Thoutmôsis, enfin Ptahmès III. La main de Ptahmès III est posée sur l'épaule du vizir, qui pose la sienne sur l'épaule de Ptahmès II; d'autre part, Taoui pose également sa main sur l'épaule de Méryptah. Les cinq personnages apparaissent ainsi sur trois plans: au premier, Méryptah et Ptahmès II; au second, Taoui et son mari le vizir, tous deux encadrant leurs fils; au troisième, Ptahmès III, qui s'efface derrière le vizir.

${ }^{1}$ 64; cf. A.H. GARDINER, A statuette of the High Priest of Memphis, Ptahmose, dans A.Z., 43 (1906), p. 55. D'autres monuments appartiennent certainement (65) ou peut-être (66) à Thoutmôsis, mais n'apprennent rien sur ce grand prêtre. D'après la ressemblance de 64 et 44, A.H. GARDINER, op. cit., a conclu que Thoutmôsis de 64 semble être le père de Ptahmès de 44 . Ptahmès II, auquel nous attribuons la statuette 44 , avait bien pour père un Thoutmôsis, mais celui-ci était vizir et non fils de roi. L'identification proposée par A.H. Gardiner est tenue, à tort, pour certaine par A. VOLTEN, Demotische Traumdeutung, p. 28, note 3. 
On a déjà relevé que le dédicant de ce monument est Méryptah qui adresse l'appel aux vivants inscrit tout au haut de la stèle $(\mathbf{3 8 , 1})$ et, ainsi que nous l'avons noté ${ }^{1}$, que tous les membres de la famille du vizir sont justes de voix ${ }^{2}$, tandis que Ptahmès III ne l'est pas. Cette particularité suggère l'explication suivante: Ptahmès II était mort lorsque son frère Méryptah fit faire ce monument destiné à la célébration, future ou immédiate, du culte de toute sa famille, y compris lui-même. Ce culte devait être célébré par le grand prêtre Ptahmès III, vivant et en fonction lors de la fabrication du monument. Au lieu de quatre personnages assis à une table d'offrandes et d'un cinquième, officiant debout, il y aurait sur la stèle de Leyde quatre personnages "justes de voix" sur deux rangs et un cinquième en retrait ${ }^{3}$.

L'inscription de Ptahmès III sur cette stèle de Leyde est aussi courte que possible $(\mathbf{3 8 , 8})$. Il était nécessaire d'indiquer sa filiation pour éviter une confusion avec son homonyme. D'autre part, son titre est le plus abrégé qui soit pour un grand prêtre memphite: "grand des chefs des artisans" ! Or ce n'est pas la place qui manquait pour graver le mot sem et ce titre n'aurait guère allongé l'inscription. Nous sommes ainsi amenés à supposer que l'omission de sem a été voulue, et cette circonstance pourrait justifier notre attribution à Ptahmès III d'une stèle conservée en Avignon ${ }^{4}$. Ptahmès y est assis seul devant une table d'offrandes et le texte qui surmonte ce repas est ainsi conçu: "Tout ce qui est déposé $e^{5}$ sur l'autel d'Horakhty-Toum, seigneur des Deux Pays, l'Héliopolitain, au commencement de chaque saison et qui apparaît $t^{6}$ dans son temple, au ka $d u$ noble, gouverneur, trésorier du roi, compagnon unique, grand des chefs

${ }^{1}$ Voir ci-dessus, $\$ \mathbf{5 0}$.

${ }^{2}$ R. ANTHES, op. cit., p. 65-66 et 61 , note 2.

3 A propos du pectoral porté par Méryptah, nous avons émis l'hypothèse (voir cidessus, $\S 9$, p. 30, note 1) que ce personnage pourrait être le grand des chefs des artisans du même nom (ci-dessous, $\S 53$ ). Si cette hypothèse se confirmait un jour, il y aurait lieu de placer le pontificat de Méryptah entre ceux du prince Thoutmôsis et de Ptahmès III. Dans l'état actuel de nos connaissances, l'identité du majordome Méryptah et du grand prêtre du même nom nous paraît trop problématique pour être retenue.

4 67; son éditeur, A. MORET, dans Rec. Trav., XXXII (1910), p. 160, a déjà proposé de l'attribuer à Ptahmès fils de Menkheper.

5 Litt. : "tout ce qui sort".

${ }^{6}$ Litt. : "qui devient". 
des artisans, Ptahmès, juste de voix et possesseur d'imakh". Ici, comme sur la stèle de Leyde, l'absence de sem paraît voulue parce qu'elle ne se justifie pas par un manque de place.

Avant de proposer une explication de ce fait, nous avons à parler du "supérieur des chanteurs du temple de Ptah, Ptahankh", qui était "serviteur du grand des chefs des artisans Ptahmès"1. Sur une stèle triangulaire (61), Ptahankh offre l'eau et l'encens au grand prêtre assis à une table d'offrandes et implore Horakhty-Toum pour que le dieu donne un millier de toutes choses bonnes et pures à son maître (61). Ce monument a été déposé dans le temple d'Horakhty-Toum, comme la stèle de Ptahmès conservée en Avignon. Nous nous basons sur cette constatation pour considérer Ptahankh comme le serviteur dévoué du grand prêtre Ptahmès qui est sur la stèle d'Avignon et que nous identifions à Ptahmès III $^{2}$.

Le dépôt de deux stèles, l'une du grand prêtre, l'autre de son serviteur, dans le temple d'Horakhty-Toum ne pourrait-il pas être consécutif à une visite d'Aménophis III à ce sanctuaire memphite ? On sait l'intérêt que le roi prit aux anciennes formes du culte solaire ${ }^{3}$. Les deux stèles en sont peut-être un reflet modeste. On peut enfin se demander si ce n'est pas dans cette voie qu'il faudrait rechercher la raison pour laquelle Ptahmès III ne mentionne pas le titre sem sur les stèles de Leyde et d'Avignon. En effet, sem se rapporte, chez les grands prêtres memphites, au service de Sokar, dieu funéraire, tandis que le soleil est un dieu de vie.

Nous n'attachons cependant pas à cette suggestion plus d'importance qu'elle en mérite ${ }^{4}$ parce que, sur la statue de Ptahmès III au Musée de

159 - 61; "serviteur" est rendu par "celui qui entend l'appel" sur 59 et 60 , et par "échanson" sur 61.

${ }^{2}$ On pourrait cependant voir dans Ptahankh un serviteur de Ptahmès II en laissant inexpliquée la raison pour laquelle il a déposé une stèle dans le temple d'HorakhtyToum.

${ }^{3}$ Références dans H. KEES, Götterglaube, p. 368.

4 On pourrait aussi supposer que l'absence de sem sur les stèles de Leyde et d'Avignon n'est qu'une flatterie de courtisan ou une marque de tact à l'égard du roi: Ptahmès III aurait évité de s'intituler comme le faisait régulièrement le défunt fils du 
Florence (69), trois des six mentions de "grand des chefs des artisans" sont accompagnées de $\operatorname{sem}^{1}$. Nous avons cité à plusieurs reprises ce monument bien connu ${ }^{2}$, que Ptahmès fit placer dans le temple de Ptah et de Sokar pour qu'il y fût l'objet de soins rituels de la part des grands prêtres futurs (69,7-10). Cette tâche fut rapidement négligée malgré l'exhortation gravée au dos de la statue, puisqu'un grand prêtre de la XIXème dynastie, Pahemneter, s'est vanté de l'avoir remplie à nouveau $(69,11)$.

Une coudée conservée au Musée de Leyde comme celle de Ptahmès II, mais provenant d'une autre collection ${ }^{3}$, a peut-être appartenu à Ptahmès III puisqu'on n'y relève pas sem mais seulement "grand des chefs des artisans" à la fin d'une série de titres (68). Cette coudée porte aussi un proscynème à Ptah, seigneur de vérité, en faveur du "grand des chefs des artisans Ptahmès, renouvelant sa vie, possesseur d'imakh (dans le temple de Ptah) ${ }^{\mathrm{m}}$; on y constate aussi l'absence de sem. Deux des fonctions exercées par Ptahmès méritent d'être remarquées sur ce monument. Il est "préfet de la ville du pays entier" (sic) et "directeur de tous les travaux des temples de Ptah" (68). R. Anthes suppose que la lacune qui suit malheureusement le premier titre a pu contenir le mot "vizir"; ; la longueur du mot cadrerait avec celle de la lacune, mais nous doutons que Ptahmès III eût indiqué cette fonction si importante au milieu de la titulature s'il l'avait exercée. Quoi qu'il en soit, la mention "du pays entier" et celle "des temples de Ptah" font penser que l'activité de Ptahmès a débordé les limites du nome memphite. Comme il n'est pas impossible que son pontificat ait duré jusqu'au début du règne d'Aménophis IV, la coudée pourrait être de ce temps.

roi, le prince Thoutmôsis. On pourrait encore supposer que Ptahmès III, vu sa modeste origine, a voulu "s'affirmer" chez les Memphites en s'intitulant aussi différemment que possible de son prédécesseur, ou encore, qu'ayant acquis le titre grand des chefs des artisans par décret royal, mais non le titre sem $(\mathbf{6 9 , 6 )}$, il méprisait un peu celui-ci.

${ }^{1}$ 69,3,5,6; sans sem: $69,6,7,10$.

${ }^{2}$ Voir ci-dessus, $\$ \mathbf{7 , 9}, \mathbf{1 0}, \mathbf{1 2}$ (notes), 13.

${ }^{3}$ R. ANTHES, Die hohen Beamten namens Ptahmose, dans Ä.Z., 72 (1936), p. 63 , $\mathrm{n}^{\circ} 8 \mathrm{~b}$, nota bene.

4 E. SCHIAPARELLI, Museo Archaeologico di Firenze, p. 205, III b.

${ }^{5}$ R. ANTHES, Die hohen Beamten namens Ptahmose, dans Ä.Z., 72 (1936), p. 63 , $\mathrm{n}^{\circ} 8 \mathrm{~b}$, nota bene. 


\section{§ 53. MERYPTAH}

Le grand des chefs des artisans Méryptah, dont on possède la partie inférieure d'une statue (79), une table d'offrandes $(\mathbf{8 0})$ et un bas-relief

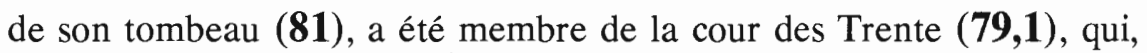
si nous en croyons Diodore ${ }^{1}$, comprenait dix juges choisis à Héliopolis, dix à Thèbes et dix à Memphis, tous réunis en un seul collège. Un témoignage plus ancien nous apprend que les Trente pouvaient être appelés à accompagner le souverain ${ }^{2}$.

Méryptah a donc eu l'occasion de voyager et il n'est pas impossible que le cône funéraire du "sem dans le temple de Ptah et grand des chefs des artisans dans Héliopolis de Haute-Egypte" ${ }^{13}$ lui ait appartenu; nous estimons que cette identification est très problématique.

On peut en dire autant au sujet du majordome du temple de Nebmaâtrê, ce Méryptah qui était le frère du grand prêtre Ptahmès II. A-t-il été grand prêtre lui-même ? Dans ce cas, est-ce le même que le grand prêtre que nous connaissons (79) ? Autant de questions qu'on ne peut que poser.

Le titre "trésorier du roi" $(\mathbf{7 9 , 1 )}$ permet d'attribuer certainement Méryptah à la XVIIIème dynastie ${ }^{4}$. Il y a peut-être exercé une partie de son pontificat sous Akhenaton ${ }^{5}$.

\section{§ 54. PTAHEMHAT-TY}

Sous le règne de Toutankhamon, alors qu'Horemheb était généralissime des armées de Pharaon, un grand prêtre de Memphis faisait décorer son tombeau d'un cortège funèbre dont une partie nous est parvenue et est conservée au Musée de Berlin (70). La date de ce

\footnotetext{
${ }^{1}$ Diodore, I, 76.

2 Inscription de Nebounnef, cf. G. LEFEBVRE, Histoire, p. 120.

3 Voir ci-dessus, § 5, p. 15 , note 3.

4 Voir ci-dessus, $\$ 30$.

5 Voir ci-dessus, $\$ 29$.
} 
monument, qui avait été fixée par son premier éditeur ${ }^{1}$, a été précisée depuis lors ${ }^{2}$. Comme le nom et la qualité du défunt n'apparaissent pas sur ce bas-relief, il est nécessaire que nous examinions à notre tour ce sujet.

Dans le registre supérieur, parmi des berceaux de verdure fabriqués, un officiant, encore enfant, est désigné par ces mots, gravés postérieurement à l'image: "Le supérieur des suivants du grand des chefs des artisans, Neferrenpet" (70,1). Si Neferrenpet n'appartenait pas au personnel du grand prêtre dont le cortège funèbre est représenté au registre inférieur, il se serait nommé probablement "Le supérieur des suivants $d u$ grand des chefs des artisans $X$., Neferrenpet", comme Ptahankh, qui s'intitule "le serviteur du grand des chefs des artisans Ptahmès, Ptahankh" (59 - 61), entre autres sur une stèle déposée dans le temple d'Horakhty-Toum ${ }^{3}$. Quant à la traduction "le supérieur des suivants du grand des chefs des artisans Neferrenpet", elle n'est guère plausible; l'absence du nom du supérieur serait alors difficile à expliquer, d'autant que l'inscription a été gravée après coup.

Au registre inférieur, le corbillard est suivi par les deux fils du défunt, par quinze dignitaires que nous avons étudiés d'autre part ${ }^{4}$, puis par des prêtres dont les noms sont partiellement conservés. Ceux-ci psalmodient: "Vers l'Occident, vers l'Occident, le pays de la justification, grand"; le texte, écrit sur deux lignes horizontales, continuait sur une autre pierre que nous n'avons pas, de sorte que nous ne possédons pas la fin de la première ligne; la seconde ligne commençait par: "Seigneur de vérité. Tu es notre père" (70,3-4) sont à l'adresse du défunt. Prononcés par des prêtres qui parlent de Ptah, ils s'adaptent parfaitement au grand prêtre de Memphis.

Enfin, la composition du cortège funèbre, avec le généralissime, les deux vizirs, le grand prêtre d'Héliopolis et d'autres hauts personnages,

1 A. ERMAN, Aus dem Grabe eines Hohenpriesters von Memphis, dans Ä.Z., 33 (1895), p. 18-24.

${ }^{2}$ W. SPIEGELBERG, Die Datierung des Berliner Trauerreliefs, dans Ä.Z., 60 (1925), p. 56-58; K. PFLÜGER, Haremhab und die Amarna-Zeit, p. 14.

${ }^{3}$ Voir ci-dessus, §52.

${ }^{4}$ Voir ci-dessus, § 26. 
mais pas de grand des chefs des artisans, fournit une troisième raison, et non la moindre, de considérer le bas-relief comme un fragment du tombeau d'un grand prêtre.

Cette attribution permet d'expliquer la différence d'appellation des divers personnages: dans la partie supérieure, titres et noms désignent complètement chacun de ceux qui s'affairent aux préparatifs des berceaux de verdure; dans la partie inférieure où se déroule le cortège, les deux fils du défunt et les prêtres qui psalmodient sont aussi indiqués par leurs titres et noms. Par contre les dignitaires du cortège ne sont signalés que par leurs titres. Or les dignitaires sont indépendants du grand prêtre qu'ils accompagnent tandis que tous les autres personnages lui appartiennent ou dépendent de lui, qu'ils soient ses fils, ses serviteurs ou les prêtres de Ptah.

Le grand prêtre si pompeusement escorté n'est pas nommé, mais nous savons la qualité du premier des personnages qui suivent sa dépouille. C'est "son fils, le père divin dans le temple de Bastet, Say" (70,2); le titre et le nom du second personnage, que son maintien désigne comme un frère de Say, étaient gravés sur une pierre rapportée qui a disparu. Il y a d'autre part au British Museum une stèle d'un grand prêtre de la XVIIIème dynastie nommé Ty, dont le fils, Say, est prêtre-lecteur dans le temple de Bastet (72). Nous proposons d'identifier les deux Say, bien qu'ils officient à des titres différents dans le temple de Bastet, et de reconnaître dans Ty le possesseur du bas-relief de Berlin. Ce faisant, nous rejoignons la généalogie d'Ankhef-ny-sekhmet qui mentionne un grand des chefs des artisans Ty sous Horemheb $(\mathbf{1 8 2 , 2 3})$.

Ty s'appelait aussi Ptahemhat (73); il était fils d'un père divin de Ptah, nommé Hori (73); la stèle d'où nous extrayons ce renseignement est en contradiction avec la généalogie d'Ankhef-ny-sekhmet, qui donne à Ty, pour père, un "père-divin d'Amon-Rê", nommé Sekeremsaef. 
Deux bas-reliefs conservés à Copenhague ${ }^{1}$ et à Berlin ${ }^{2}$ proviennent peut-être du tombeau de Ptahemhat-Ty. Le premier offre quelque analogie avec le cortège funèbre que nous avons étudié. Il pourrait même venir de la même assise, car il est découpé en deux registres dont le supérieur représente des pavillons de verdure pour offrandes, comme le morceau du cortège funèbre. Au registre inférieur, le bas-relief de Copenhague montre le sarcophage déposé dans une île; des dignitaires sont arrêtés au bord du lac et regardent vers l'île. Le bas-relief de Berlin représente une procession funéraire.

Un troisième bas-relief conservé à Berlin (71) provient probablement d'un tombeau de grand prêtre puisqu'il y est question du pectoral, avec lequel le défunt se vante d'être équipé, mais, contrairement à son premier éditeur ${ }^{3}$, nous hésitons à l'attribuer à Ty parce que les deux fils du défunt qu'il mentionne (71,1-2) sont différents de ceux de Ty.

\section{§ 55. NEBMEHYT, KHAMOUAS I, HORI I ET II}

On ne possède qu'une statuette funéraire du grand prêtre Nebmehyt (82). Le style du monument le date de la XIXème dynastie, tandis que l'ordonnance des titres sem et "grand des chefs des artisans" permet de préciser que Nebmehyt a été en fonction au début de la dynastie.

Khamouas I était le fils d'un vizir (87,2); il a été lui-même vizir (85), très vraisemblablement de Basse-Egypte, bien qu'il ne le dise pas expressément ${ }^{4}$. C'est aussi un des premiers grands prêtres qui se sont intitulés "grand des chefs des artisans de Ptah" $(\mathbf{8 5} ; \mathbf{8 6})^{5}$, s'il n'a luimême inventé cette appellation.

${ }^{1}$ Ny Carlsberg E 92: H. MADSEN, Aus dem Hohenpriestergrabe zu Memphis, dans A.Z., 41 (1904), p. 110-113 et pl. 1; M. MOGENSEN, La collection égyptienne, p. 95-96 et pl. 103 (A 704).

2 Berlin 13.297: Ägyptische und Vorderasiatische Alterthümer, pl. 111.

3 A. ERMAN, Aus dem Grabe..., dans Ä.Z., 33 (1895), p. 21.

${ }^{4}$ Nous ne suivons pas A. WEIL, Die Veziere, p. 117, qui identifie Khamouas I du groupe d'Aix en Provence (85) avec un "vizir Khamouas de Haute Egypte" mentionné sur des papyri dont deux sont datés des années 16 et 17 de Ramsès IX.

5 Voir ci-dessus, $\$ 28$. 
Le fils de Khamouas I, Hori I, a également été grand prêtre de Memphis; son tombeau s'est peut-être conservé assez longtemps, puisqu'un fragment de colonne de la chapelle funéraire a été utilisé beaucoup plus tard dans les fondations de l'église d'Apa Jérémie (86). Une stèle (87) appartenant à Hori I nomme non seulement son père et son aïeul, mais aussi sa femme, qui était "chanteuse d'Hathor, maîtresse du Sycomore du sud" et, à part ses deux filles, son fils, qui était "prophète de Baal".

Le grand prêtre Hori II avait pour père un Ptahmès dont le nom n'est accompagné d'aucun titre (88,5-6); ce Ptahmès n'était donc pas l'un des grands prêtres de ce nom que nous avons rencontrés. Il semble qu'en mentionnant le nom de son père qui n'était revêtu d'aucune dignité, Hori II cherchait à n'être pas confondu avec son homonyme Hori I et que les pontificats de l'un et de l'autre sont assez proches, exactement comme pour Ptahmès II et III ${ }^{1}$.

Quelques monuments, un sarcophage au nom du "grand des chefs des artisans de Ptah, Hori" (90), une statuette funéraire transportée plus tard au Sérapéum (92), une statue naophore (91), appartiennent à l'un ou à l'autre des deux grands prêtres Hori. Il n'est pas davantage possible de déterminer celui des deux qui a peut-être fait exécuter un travail au sanctuaire d'Anubis, dans la nécropole memphite (89). Le bas-relief où un grand prêtre Hori adorait Anubis a été l'objet de soins que nous ignorons de la part du grand prêtre Pahemneter, en fonction au début du règne de Ramsès II. Nous avons déjà rencontré sur la statue de Ptahmès III un témoignage de l'intérêt que Pahemneter portait à ses prédécesseurs ${ }^{2}$.

\section{§ 56. SENNEFER, DEDIA ET PAHEMNETER}

Le grand prêtre Sennefer $(83 ; \mathbf{8 4})$ paraît avoir été en fonction à une date rapprochée de Pahemneter, puisque celui-ci l'a fait figurer sur le

\footnotetext{
${ }^{1}$ Hori II porte un pectoral; voir Planche I, fig. 13.

${ }^{2}$ Voir ci-dessus, § 52; cf. inscription 69,11.
} 
bord d'une table d'offrandes $\left(\mathbf{9 8 , 6 )}\right.$, en renversant l'ordre de ses titres ${ }^{1}$.

Dedia a été "grand des chefs des artisans de Ptah" $\mathbf{( 1 0 2 , 2 )}$ vers le même temps que Pahemneter.

Pahemneter lui-même, dont le fils a été ambassadeur extraordinaire au pays des Hittites $\mathbf{( 1 0 4 , 2 )}$ "à une date voisine de l'an 21" de Ramsès II $^{2}$, a vraisemblablement exercé une partie de son pontificat sous Séti Ier et une autre sous Ramsès II. Il semble être, en effet, d'après une description, "grand des chefs des artisans et sem" sur un monument que nous datons de Ramsès II à cause de l'ordre des deux titres ${ }^{3}$, tandis qu'il est "sem et grand des chefs des artisans" sur plusieurs autres.

Pahemneter a pu naître vers la fin de la XVIIIème dynastie. Son père,

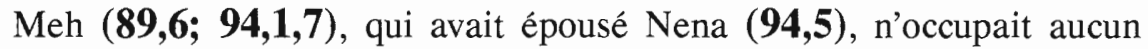
poste officiel, ce qui n'exclut pas qu'il ait été dans une situation assez aisée pour faire instruire son ou peut-être ses fils; on peut supposer que le vizir Hori, qui eut l'honneur de conduire des fêtes d'Amonrêsonter et qui forme avec Pahemneter un groupe conservé au Louvre (101), était aussi un fils de Meh.

La vie de Pahemneter s'est écoulée à Memphis, mais la destruction probable de sa chapelle funéraire nous prive, pour lui comme pour les autres grands prêtres du Nouvel Empire, de renseignements sur le temps qui précéda son pontificat. Il fut sans doute nommé grand des chefs des artisans par Séti ler sous le règne duquel il a fait préparer son tombeau; un beau pilier (94), un fragment (97), un sarcophage (100) de facture semblable à celui du grand prêtre Hori ${ }^{4}$ proviennent de ce monument.

Pahemneter a pris soin de se faire représenter au côté du vizir Hori dans le groupe que nous venons de mentionner et qui était peut-être placé dans l'enceinte du temple de Ptah. Au dos de ce groupe, on lit un

${ }^{1}$ Nous avons noté ci-dessus, au $\S 31$, ce fait dont nous proposons une explication à la fin du présent paragraphe.

${ }^{2}$ G. LEFEBVRE, Histoire, p. 124.

3 Voir ci-dessus, \$ 31.

${ }^{4}$ Musée de Berlin, Ausführliches Verzeichnis, 1894, p. 154. 
proscynème à Ptah et à Sokar-Osiris en faveur de Hori et un autre à Horakhty-Toum, à Hathor maîtresse de Hetepet, à Hathor maîtresse du Sycomore du sud et au Désert occidental en faveur de Pahemneter.

Ce grand prêtre s'est intéressé aux services d'offrandes des statues de ses devanciers également déposées dans le temple de Ptah ainsi qu'à des monuments élevés par ses prédécesseurs dans la nécropole. On en a le témoignage sur une statue de Ptahmès III $(\mathbf{6 9 , 1 1 )}$ et sur un bas-relief d'un des Hori $(\mathbf{8 9 , 6 )}$. L'intérêt de Pahemneter pour les anciens grands prêtres lui a peut-être fait adopter les titres désuets de trésorier du roi $(\mathbf{8 9 , 7 ; 9 8 , 1 , 5})^{1}$ et de celui qui est dans la double maison $\mathbf{( 9 8 , 3 )}$, ainsi que chef de toute la garde-robe $\mathbf{( 8 9 , 7 )}$ remis en honneur sous la XIXème dynastie ${ }^{2}$.

Une statue que Méry, petit-fils de Pahemneter et fils du vizir Rêhetep, fit sculpter pour son père sous Ramsès II (104) nous apprend que Pahemneter épousa la "grande des concubines d'Onouris, Khatnesout"; il en eut deux fils qui parvinrent plus tard aux plus hautes dignités: Rêhetep devint vizir et Minmès ${ }^{3}$ fut mis par Ramsès II à la tête du clergé d'Amon de Karnak.

Il nous a paru que Ramsès II a fait mettre l'accent sur le titre sem des grands prêtres de Memphis ${ }^{4}$. Le premier d'entre eux pourrait avoir été Pahemneter; on expliquerait ainsi qu'il se soit fait faire un second sarcophage de pierre sur lequel il est, d'après description, "grand des chefs des artisans et sem" (99) ${ }^{5}$. Et l'on pourrait aussi supposer que la table d'offrandes sur laquelle Pahemneter, principal personnage, est "sem et grand des chefs des artisans" $\mathbf{( 9 8 , 5 )}$ tandis que Sennefer, représenté sur le plat du rebord, est "grand des chefs des artisans et sem" $(\mathbf{9 8 , 6 )}$ était en cours de fabrication lors de la nouvelle orientation donnée par le roi aux grands prêtres memphites.

\footnotetext{
${ }^{1}$ Voir ci-dessus, $§ 30$.

2 ibidem.

${ }^{3}$ G. LEFEBVRE, Histoire, p. 124.

${ }^{4}$ Voir ci-dessus, \$ 32.

${ }^{5}$ Rien n'interdirait, cependant, de supposer l'existence de deux grands des chefs des artisans nommés Pahemneter à cause des deux sarcophages.
} 
Comme les monuments de Pahemneter que nous pourrions dater de Ramsès II sont très peu nombreux, nous estimons que la majeure partie de son pontificat date de Séti Ier.

\section{§ 57. REHETEP ET HY}

Le principal monument du grand prêtre Rêhetep est une stèle monolithe en syénite, mesurant quatre-vingt-un centimètres de largeur, vingt-sept d'épaisseur et cent cinquante-neuf de hauteur (103). Elle est conservée au Musée du Caire et le "Journal" indique qu'elle y est entrée du temps de Mariette et donne comme provenance Memphis. Le contenu de la stèle, gravée sur les deux grandes faces verticales, sur le sommet et les faces latérales permet de préciser le lieu et l'orientation originels.

Dans un appel aux vivants, Rêhetep s'adresse aux prêtres-lecteurs et aux pleureuses d'Apis pour leur demander de faire attention à lui chaque fois qu'ils viendront au tombeau d'Apis $(\mathbf{1 0 3 , 2 0 - 2 2})$. La stèle était donc dressée près de la chapelle funéraire d'un Apis, à moins que ce ne fût dans l'enceinte du sanctuaire des Apis bâti par Khamouas. La stèle nous parait, en effet, comme nous allons le voir, postérieure au pontificat de Rêhetep et pourrait être contemporaine de celui de Khamouas.

Sur une des grandes faces, Rêhetep adore Ptah et Anubis. Ptah, représenté à gauche pour le spectateur, est tourné vers la droite et intitulé "Ptah dont le visage est vers Memphis" (103,1-4). Cette face était donc orientée au sud; c'est aussi celle où il énumère ses titres et fait appel aux vivants. Sur l'autre grande face, tournée vers le nord, Rêhetep adore Osiris et Apis (103,24-30) et décrit ses qualités et son savoir. Il semble qu'il le fait partiellement à l'usage de l'autre monde, puisqu'il se présente comme "Rêhetep de Memphis" (103, 33-34); les Memphites devaient connaître ce fils d'un de leur grand prêtre, qui avait été luimême grand prêtre !

Nous estimons que le possesseur de cette grande stèle est ce Rêhetep envoyé du roi au pays des Hittites $\mathbf{( 1 0 4 , 2 )}$ et fils de Pahemneter, parce que Rêhetep de la stèle s'intitule nettement "porte-parole du roi dans tous les pays étrangers" $(\mathbf{1 0 3 , 4 4 )}$. 
Rêhetep a ainsi été élevé sous Séti Ier, à l'ombre du temple de Ptah, et Ramsès II n'aura pas craint de le faire succéder à son père, dont il avait peut-être éprouvé la fidélité; n'a-t-il pas dépêché à Karnak le propre frère de Rêhetep ?

Celui-ci fut non seulement grand prêtre de Memphis mais aussi "grand des voyants de Rê-Toum" $(\mathbf{1 0 3 , 1 4})$, probablement vers la même époque, ou en même temps. Nous pensons que son pontificat memphite dura jusqu'au moment où Pharaon fit de Rêhetep un vizir, et le vizirat de ce personnage a pu durer beaucoup plus longtemps que son temps de grand prêtre, car tous les monuments concernant Rêhetep lui décernent le titre de vizir. D'autre part, le prince Khamouas est devenu grand des chefs des artisans au plus tard en l'an 16 puisqu'il a officié aux funérailles d'un Apis à ce moment et il semble que $\mathrm{Hy}$ ait été grand prêtre entre Rêhetep et Khamouas.

Un des grands moments de la vie de Rêhetep, postérieur à son pontificat, fut son ambassade au pays des Hittites vers l'an $21(\mathbf{1 0 4 , 2})$ de Ramsès II.

Rêhetep eut pour épouse Houel, qui était "grande des concubines de Herchefy" $(\mathbf{1 0 4 , 5})$. Le titre porté par sa femme nous permet de lui attribuer une stèle trouvée en Abydos, où apparaissent plusieurs serviteurs de Herchefy (105, 3-4,9-10) tandis qu'il est nommé "le préfet de la ville et vizir Rêhetep, juste de voix, de Pi-Ramsès" $(\mathbf{1 0 5}, \mathbf{1})$. Cette dernière épithète indique que Rêhetep, au temps de son vizirat, vécut souvent ou peut-être même s'installa à Pi-Ramsès d'où il assista le roi dans l'administration du pays.

La renommée de Rêhetep était encore vivante près de huit siècles plus tard, sous le règne de Darius. Un directeur des travaux, Khnemibrê, l'a fait figurer parmi ses ancêtres, en l'intitulant "prophète d'Amonrêsonter, chef des mystères du temple de Rê, directeur des travaux du sud et du nord, préfet et vizir Rêhetep, dont la renommée est plus grande que (celle de ?) ...Imhotep"1. Rêhetep a été effectivement directeur des travaux $(\mathbf{1 0 3 , 1 6 )}$, préfet et vizir. On peut penser que, comme "grand des voyants de Rê-

${ }^{1}$ G. POSENER, La première domination perse en Egypte, 1936, inscription $n^{\circ} 14$. 
Toum", il a été chef des mystères du temple de Rê, quoiqu'il ne s'intitule pas ainsi. Par contre, il n'a pas été prophète d'Amonrêsonter. La généalogie l'a peut-être confondu en ce point avec son frère Minmès. Quant aux relations de parenté attribuées à Rêhetep par Khnemibrê, elles sont inexactes ${ }^{1}$.

L'existence du grand prêtre Hy nous est signalée par une statuette funéraire au nom de "l'Osiris, grand des chefs des artisans et sem, Hy" (106), découverte au Sérapéum parmi les ossements d'un taureau enseveli dans le premier caveau utilisé sous Ramsès II, en l'an 16. L'ordre des titres certifie qu'il ne s'agit pas d'un grand prêtre antérieur à Ramsès II. Ces données classent Hy entre Rêhetep et Khamouas.

\section{$\S$ 58. LE PRINCE KHAMOUAS}

Les mentions du prince Khamouas sont si nombreuses que nous avons presque la certitude d'en ignorer plusieurs. Celles que nous avons recueillies (107 à 146) permettent cependant de fixer quelques événements de sa carrière ${ }^{2}$.

Ce fils de Ramsès II $(107 ; 111 ; 119,3 ; 130 ; 145,4)$ et de la reine Isetnefert (130) a pris part, dans son jeune âge, aux campagnes de son père en Nubie et en Syrie. On le voit sur un bas-relief du temple nubien de Bet el-Walli, qui le nomme "le fils du roi, de son corps, son aimé, essence divine issue de Ka-nekht ${ }^{3}$, Khamouas, juste de voix" (107), et le "fils du roi Khamouas" se trouve également avec ses frères à la bataille de Qadech en l'an 5 de Pharaon (108).

${ }^{1}$ Un tambour de colonne au Musée de Carlsruhe (A. WIEDEMANN, Varia, dans Sphinx, 14 (1910-11), p. 35, porte un fragment d'inscription où est nommée "Sekhmet la grande, aimée de Ptah" et une mention du "premier prophète de Ptah Rêhetep juste de voix". L'ignorance où nous sommes du lieu de provenance nous empêche d'attribuer ce monument au grand prêtre memphite Rêhetep, d'autant que le sanctuaire thébain de Ptah, avec chapelle de Sekhmet, a eu des "premiers prophètes de Ptah" (G. LEGRAIN, Le temple de Ptah Rîs-anbou-f dans Thèbes, dans Annales du Service des Antiquités, 3 (1902), p. 100).

${ }^{2}$ Le prince Khamouas est le second grand prêtre de ce nom.

3 "Taureau puissant" = Ramsès II. 
C'est sans doute après ces événements et au plus tard en l'an 16 qu'il commença sa carrière memphite. Cette année-là, il officia aux côtés de son père lors de l'ensevelissement d'un Apis. Le tombeau du taureau représentait Khamouas devant son père, à une échelle plus petite que ce dernier; le "fils du roi et sem Khamouas" était coiffé de la tresse et revêtu de la peau de félin (109).

Les titres portés par Khamouas dans ce caveau d'Apis ne sont qu'un abrégé de "grand des chefs des artisans, sem, fils du roi", comme on peut le constater sur un fragment de statue au Caire (113), dans l'inscription de la pyramide d'Ounas (114,2 et $\mathbf{1})$, sur un fragment de statue d'Achmounéïn $(\mathbf{1 1 8 , 4})$, une statue extraite de la "favissa" de Karnak (119,7 et 9), des colonnes du temple de Ptah à Memphis (121 et 120), enfin une base de statue trouvée à Saqqara $(145,5$ et 145, 1-3). De plus, certains de ces monuments datent vraisemblablement de l'un ou l'autre des voyages que Khamouas fit dans le sud pour annoncer les jubilés de son père; le premier eut lieu en l'an $30(\mathbf{1 1 0})$ et le dernier en l'an 41 (111). Or, en l'an 41, Khamouas s'intitule "fils du roi et sem de $P t a \dot{h}^{\prime 1}$ et ne se donne pas le titre de grand des chefs des artisans qu'il portait sûrement à cette époque. Enfin le "sem et fils de roi" $\mathbf{( 1 1 5 , 1 , 3 , 8 )}$ qui a inauguré les "petits souterrains" du Sérapéum et bâti un temple pour les Apis ne pouvait être qu'un grand des chefs des artisans. Ainsi, bien qu'aucun des monuments où Khamouas est "grand des chefs des artisans" (113; 114; 116; 118; 119,9; 120; 124,4; 127; 136; 145,1-3) ne soit daté d'une année de Ramsès II, il n'est pas douteux que Khamouas a été nommé grand prêtre au plus tard en l'an 16. En plaçant son propre fils à la tête du clergé de Ptah, le roi a peut-être suivi l'exemple d'Aménophis III et cherché les mêmes fins ${ }^{2}$.

Faute de pouvoir classer chronologiquement la plupart des monuments

${ }^{1}$ Comme Rêhetep s'intitule aussi "setem de Ptah" $(\mathbf{1 0 3 , 1 4})$, on peut se demander si sa stèle n'a pas été dressée à une époque proche de l'an 41. "Sem de Ptah" ne serait alors apparu que dans la seconde moitié du règne de Ramsès II.

${ }^{2}$ Voir ci-dessus, $\S 32$ et $\mathbf{2 8 .}$ 
de Khamouas ${ }^{1}$, nous nous occuperons de son activité dans les différents endroits où elle s'est exercée.

Khamouas a participé aux constructions de son père à Memphis ainsi qu'en témoignent un dépôt de fondation (123), des fragments de colonnes $(120 ; 121)$ et de bas-relief $(122)$ du temple de Ptah.

A Saqqara, Khamouas a enseveli en l'an 16 un Apis pour lequel on avait préparé, comme à la dynastie précédente, une tombe particulière comprenant caveau et chapelle. Après cette date, le prince fit creuser un second caveau voisin du premier ${ }^{2}$, de sorte que la chapelle correspondit désormais à deux caveaux. C'était un premier pas vers l'établissement d'une tombe commune, dont Khamouas entreprit le creusement après l'an 30, car à ce moment il ensevelit un $\mathrm{Apis}^{3}$ dans le second caveau dont nous venons de parler. Le plan de cette tombe commune a vraisemblablement été élaboré sous la direction de Khamouas, tandis que l'exécution des caveaux disposés de chaque côté d'une galerie centrale a dû se faire au fur et à mesure des besoins, comme on sait par des textes démotiques que cela s'est pratiqué pour des Apis bien postérieurs. Un temple correspondant à la tombe commune et dédié à Apis vivant fut bâti au-dessus du sol', et Khamouas fit graver le souvenir de sa création sur une stèle composée à l'occasion de l'enterrement d'un Apis (115). La première partie du texte y concerne la vie du taureau à Memphis. Dans la seconde partie qui a forme d'un appel aux vivants, Khamouas s'adresse au haut clergé du temple de Ptah à Memphis, "sem, grands des chefs des artisans ${ }^{5}$ et dignitaires du temple de Ptah", puis aux "pères divins et ouab supérieurs" de temples que, malgré une lacune, nous

${ }^{1}$ Les différentes graphies du nom Khamouas nous avaient paru susceptibles de fournir un critère chronologique, mais l'enquête que nous avons faite à ce sujet n'a pas donné de résultat probant.

2 A. MARIETTE, Renseignements sur les soixante-quatre Apis, dans Bulletin archéologique de l'Athénaeum français, l, (1855), p. 66.

${ }^{3}$ E. CHASSINAT, Textes provenant du Sérapéum de Memphis, dans Rec. Trav. XXI (1899), $\mathrm{n}^{\circ} 34$.

${ }^{4}$ Voir ci-dessus, $\$ 16$, les monuments au nom de Khamouas provenant probablement de ce temple.

${ }^{5}$ La double indication du pluriel dans ce titre indique, comme nous l'avons noté au $\S 4$, que Khamouas l'interprétait comme "grands des chefs des artisans" et pas autrement. 
pensons être memphites, et au personnel supérieur du nouveau temple d'Apis, c'est-à-dire aux "prophètes et prêtres-lecteur de ce temple". L'expression "ce temple"1 pourrait être le terme administratif appliqué au "temenos" d'Apis à Saqqara, tandis que la "demeure divine d'Apis" mentionnée dans la suite du texte se rapporte tant au nouveau souterrain qu'au sanctuaire construit au-dessus du sol. En effet, lorsque les "petits souterrains" devinrent inutilisables par suite d'un effondrement plusieurs siècles après leur inauguration, une stèle datée de l'an 52 de Psammétique, reçut le texte suivant: "On vint dire à Sa Majesté: la demeure divine de ton père Osiris-Apis et les choses qui y sont vont à l'oubli. On voit les chairs divines dans son sanctuaire. La décrépitude s'est emparée de ses sarcophages"2.

En même temps qu'il s'occupait de la construction du nouvel ensemble funéraire d'Apis, Khamouas organisait le personnel (115,5) et instituait un nouveau service d'offrandes pour Apis $(\mathbf{1 1 5}, \mathbf{5})$. Ce service s'ajoutait aux offrandes qu'Apis recevait déjà du temple de Ptah. Ainsi Khamouas n'exagérait-il pas en qualifiant de "petit" le travail de ses prédécesseurs en faveur d'Apis $\mathbf{( 1 1 5 , 7 ) . ~}$

La tombe d'Apis n'a pas accaparé toute l'attention que Khamouas a vouée à la nécropole memphite. On connaît les inscriptions qu'il a fait graver sur les parements en beau calcaire de monuments de la IVème et de la Vème dynastie: Mastabat Faraoun, temple solaire de Nyouserrê, pyramides de Sahourê et d'Ounas (114). Ces édifices étaient alors en bon état comme l'emplacement des inscriptions l'indique. C'est pourquoi, tout en nous tenant à la traduction qui en a été donnée, nous proposons

${ }^{1} r-p r$.

2 E. CHASSINAT, Textes provenant du Sérapéum de Memphis, dans Rec. Trav., XXII (1900), p. 166. En contradiction avec ce que nous disons des mots "temple" et "demeure divine", A. ROWE, Discovery, dans Supplément aux Annales du Service des Antiquités, Cahier $\mathrm{N}^{\circ} 2,1946$, p. 8-9, se basant sur un document bilingue du règne de Ptolémée III, croit pouvoir traduire $p r$ par "temple" et $h t-n t r$ par "enclos sacré". Mais la version grecque n'est pas une traduction de la version hiéroglyphique, ni vice-versa. D'autre part, on peut même prétendre que l'une des deux énumère les termes en question à l'inverse de l'autre afin que toutes deux citent la "demeure divine" (respectivement le "naos") à côté du nom divin "Sérapis". Nous ne retenons donc pas les interprétations d'A. Rowe. 
d'interpréter le mot $m n w$, monuments, non seulement dans son sens strictement architectural, mais aussi dans le sens plus complet de "fondation (pieuse)"1. Les inscriptions commémoreraient alors la restauration, sous une forme ou une autre, d'un service religieux attaché à ces monuments de l'Ancien Empire. En somme, Khamouas aurait agi à l'égard des rois des IVème et Vème dynasties comme le grand prêtre Pahemneter l'avait fait pour ses prédécesseurs dont il faisait "revivre le nom", encore bien gravé, sur les statues déposées dans le temple de $\operatorname{Ptah}^{2}$.

D'ailleurs l'activité de Khamouas n'a peut-être pas été seulement constructive à Saqqara. S'il n'a pas ordonné lui-même l'exploitation du calcaire de la pyramide de Khendjer, il n'a pas pu ignorer le travail de démolition systématique qui y était accompli sous la surveillance d'un scribe. Celui-ci nous en informe par un long graffito daté de "l'an 34 d'un roi non nommé, mais qui ne peut être que Ramsès II" $^{\text {"3 }}$. L'année de règne donne à penser que les pierres de la pyramide de Khendjer ont pu servir à la construction du temple d'Apis.

En dehors de Memphis, on trouve des témoins du passage de Khamouas en divers endroits, même jusqu'aux confins méridionaux de l'Egypte. Il s'est déplacé à plusieurs reprises pour "annoncer les fêtes Sed" de son père, comme il le dit à El Kab sur le temple d'Aménophis III (111) et au Guébel Silsileh, où il s'est rendu plus d'une fois (110; 112; 128; 129; 130). Comme il est aussi allé sur la route d'Assouan à Philae (131), il n'a donc pas remonté la vallée du Nil seulement en qualité de sem à l'occasion des jubilés de son père, mais aussi visité, sans doute en "grand des chefs des artisans" deux importantes carrières de Haute-Egypte. C'est au cours de l'un ou l'autre de ses voyages dans le sud qu'il aura dédié une statue le représentant à Hathor d'Aphroditopolis (118), qu'il aura laissé une autre de ses images à Assiout (126), et une troisième au temple de Ptah à Karnak (119). Il n'y a que la statue

${ }^{1}$ La proposition "dont la solidité menaçait ruine" n'interdit pas notre interprétation, car le mot rwd peut qualifier des offrandes; cf. Wörterbuch II, p. 411, réf. 25 , et Belegstellen, ibidem.

${ }^{2}$ Voir ci-dessus, § 56.

${ }^{3}$ G. JEQUIER, Douze ans de fouilles dans la nécropole memphite, Neuchâtel, 1940, p. 161, et Deux pyramides du Moyen Empire, p. 13-14. 
dédiée à Hathor pour laquelle nous pourrions proposer une date, parce que Khamouas s'y intitule "sem de Ptah" $(\mathbf{1 1 8 , 1 0})$ comme il le fait en l'an 41 au Guébel Silsileh (111).

Khamouas s'est aussi rendu en Basse-Egypte; il n'y aurait rien d'étonnant que ce fût lors d'un voyage à Pi-Ramsès qu'il laissa un gobelet à Tell Horbéït (127) ${ }^{1}$.

Il a eu au moins un fils auquel il donna le nom de son père, Ramsès (124,1-2), comme une statue de Khamouas, vraisemblablement déposée dans le temple de Ptah, nous l'apprend. Quant à l'épouse de Khamouas, elle se serait appelée Mehousekht, si nous pouvons en croire un conte démotique $^{2}$. Signalons aussi son ami, le père divin Ity, auquel il fit cadeau d'un vase canope (125), comme son lointain prédécesseur Neferefrê-ankh avait fait cadeau d'une stèle à Senimen (8).

Comme le grand'oeuvre de Khamouas dans la nécropole memphite a été, ainsi que nous l'avons dit, la création d'un temple pour le dieu Apis et des "petits souterrains" pour les momies des taureaux, il est naturel que Khamouas ait songé à établir son monument funéraire dans le voisinage. En effet, il demande à "reposer devant les dieux dans la chapelle ${ }^{3} d u$ domaine funéraire" $(\mathbf{1 4 5 , 1 )}$. Or le socle de statue portant cette inscription et trouvé dans les fondations de l'église du monastère de l'Apa Jérémie provient certainement du temple d'Apis ${ }^{4}$ aux desservants duquel Khamouas s'adresse $\mathbf{( 1 4 5 , 5 )}$ tandis qu'il rappelle "ce qu'il a fait", par quoi nous entendons les monuments d'Apis, dans un appel général aux vivants $(\mathbf{1 4 5 , 2 )}$.

1 Il y avait d'ailleurs un sanctuaire de Ptah à Tell Horbéit; cf. SANDMAN, Ptah, p. 240-241.

${ }^{2}$ F.LL. GRIFFITH, Stories of the high priests of Memphis, p. 42, note 1.

${ }^{3}$ Litt. "demeure de $k a$ "; cf. C. ROBICHON ET A. VARILLE, Le temple du scribe royal Amenhotep fils de Hapou, 1936, p. 7, d'après lesquels le sens "chapelle" est dérivé du sens originel de "demeure de ka".

4 J.E. QUIBELL, Excavations at Saqqara, IV (1912), p. 46 note, suggère que ce socle, dont la forme indique qu'il servait de base à une statue du taureau sacré, a pu supporter un groupe formé par Khamouas et Apis "en quelque façon dans le style du groupe bien connu du Musée du Caire" (Vache Hathor et Thoutmôsis III). 
Si, sur ce socle, il n'est pas question du possesseur de la "chapelle", c'est qu'elle appartient à Khamouas. D'autre part, si elle n'est pas localisée, c'est qu'elle était proche du socle. Il se pourrait bien que le pylône d'accès au temenos d'Apis, bâti près de neuf siècles plus tard, ait été édifié sur les ruines de la chapelle de Khamouas ${ }^{1}$, d'où proviennent vraisemblablement un fragment de paroi $(\mathbf{1 4 2})$ et un demi-tambour de colonne mesurant à peu près cinquante centimètres de diamètre $(\mathbf{1 4 3})$, tous deux conservés au Caire et inédits. On voit sur le premier la tête et le haut des épaules de Khamouas, orné de la tresse et d'un collier large; ses titres sont gravés au-dessus, et un fragment de formule d'offrande lui fait face. Sur le tambour, où la scène est surmontée du signe du ciel, Khamouas est assis devant une table d'offrandes. Il est orné de la tresse et les extrémités du pectoral dépassent ses épaules².

Le caveau qui devait compléter le monument funéraire de Khamouas pourrait être celui dans lequel il ensevelit un Apis en l'an 16. En effet, une niche ménagée dans une des parois contenait deux statuettes funéraires du prince mesurant respectivement trente-quatre et trente-huit centimètres $^{3}$. Ou bien, si l'on admet que le caveau a été creusé pour un taureau, la niche avec les statuettes indiquerait que Khamouas aurait songé dès le début de sa carrière memphite à se faire ensevelir dans un tombeau d'Apis. Cependant le corps de Khamouas n'a pas été déposé dans ce caveau, mais dans une chambre des "petits souterrains". Mariette

${ }^{1}$ H. BRUGSCH dans Bericht über die ... Verhandlungen der klg. preuss. Akademie der Wissenschaften zu Berlin, 1853, p. 728, écrit entre autres au sujet des fouilles de Mariette (nous traduisons): "A une courte distance de la chapelle dans laquelle M. Mariette trouva le taureau Apis sculpté en calcaire et grandeur nature, il y a les premières assises (überreste) encore distinctes d'un pylône qui servait d'entrée au temenos des tombeaux d'Apis et qui provient de l'époque d'Amyrtée. Toute cette construction repose, comme sur un fondement (Fundament), sur une base (Grundlage) plus ancienne qui contient des représentations de personnes qui, d'après les inscriptions, apportent leurs hommages au fils du roi Khamouas .... Du même fondement dont je viens de parler est sortie une pierre avec son portrait, exécuté proprement en bas-relief, qui le représente en bel adolescent avec une petite barbe au menton et avec la tresse princière de la jeunesse. Sa pose est celle de quelqu'un qui fait une offrande. Ces fondements ont appartenu à une ancienne petite chapelle (plus de quatre pas en longueur et largeur)". Le portrait de Khamouas décrit par Brugsch n'est-il pas celui qui est au Louvre (141)?

2 Voir Planche I, fig. 15.

${ }^{3}$ A. MARIETTE, Sérapéum, 1857, pl. 8 et CH. BOREUX, Antiquités égyptiennes (Catalogue-guide du Musée du Louvre) II, 1932, p. 323. 
raconte ainsi sa découverte: "Précisément à l'endroit où la voûte s'est effondrée, on a trouvé un sarcophage de bois et une momie humaine. Le sarcophage, encastré assez profondément dans le sol, avait eu toute la partie supérieure broyée; mais la momie, avec tous les objets qui composaient sa parure funéraire, n'avait pas été touchée. La seule détérioration qu'elle avait subie provenait de l'humidité du lieu" ${ }^{\prime \prime}$. Dans la première édition de son ouvrage, le grand fouilleur avait écrit: "Qu'on se figure une momie de forme humaine, détruite dans toute sa partie inférieure à partir de la poitrine. Un épais masque d'or, aujourd'hui au Louvre, couvrait le visage. Au cou étaient passées deux chaînes également en or à l'une desquelles trois amulettes étaient suspendues. Quant à l'intérieur, il ne présentait plus qu'une masse de bitume odorant, mêlée d'ossements sans forme au milieu desquels furent trouvés deux ou trois bijoux à cloisons d'or, emplies de plaquettes de verre ${ }^{2}$. Enfin, auprès de ce singulier monument, je ramassai un gros scarabée en stéaschiste grisâtre, une colonnette en feldspath vert et une vingtaine de statuettes funéraires de forme humaine". Mariette ajoute ici en note que ces objets sont conservés au Musée du Louvre, et il écrit plus loin: "Estce là la momie de Scha-em-Djom ${ }^{3}$ ?"4 $^{\text {. }}$. Si nous interprétons correctement les récits de Mariette, il n'a jamais douté que la momie fût celle d'un être humain, mais seulement de l'identité de celui-ci dans la première édition du Sérapéum 5 . Or, le nom inscrit sur plusieurs des bijoux (136) décorant la momie indique assez clairement que c'était celle du prince Khamouas ${ }^{6}$.

1 A. MARIETTE, Sérapéum, 1882, p. 58.

2 Dans l'édition de 1882, la description est plus complète: "Un admirable bijou, épervier d'or à mosaïques cloisonnées, les ailes étendues, était posé sur la poitrine".

3 Ancienne lecture de Khamouas.

4 A. MARIETTE, Sérapéum, 1857, p. 15-16.

${ }^{5}$ Nous ne suivons pas A. WIEDEMANN, Ägyptische Geschichte, p. 466, lorsqu'il écrit au sujet de la momie qu'elle "n'a pas été examinée d'assez près pour qu'on sût si elle appartenait à un être humain ou au taureau sacré".

${ }^{6}$ D'après G. Maspero, Khamouas aurait été enterré près de la grande pyramide de Gizeh, à Kafr el-Batran; son tombeau y aurait été découvert vers 1870 (PORTER ET MOSS, Memphis, p. 240, sans doute d'après G. MASPERO, dans A. MARIETTE, Monuments divers, 1889, p. 10: "...son (= de Khamouas) tombeau, découvert, il y a environ vingt ans, par Vassali-Bey"). Nous regrettons que G. Maspero n'ait donné ni description ni référence d'aucune sorte sur ce tombeau, chaque fois qu'il en parle (dans A. MARIETTE, Monuments divers, cité ci-dessus; dans Histoire ancienne, Premières 
La chambre des petits souterrains renfermant la momie de Khamouas contenait aussi celle d'un Apis enseveli alors que le prince Merenptah

mêlées, p. 426, note 1: "les débris de son tombeau"; idem dans Histoire ancienne des peuples de l'Orient, 4ème édition, 1886, p. 255, note 2).

Ce regret est d'autant plus vif qu'il y a eu à Kafr el-Batran, un tombeau appartenant à un "chef des coiffures du roi, Khamouas". Ce monument fut détruit par les gens du pays et des fragments de bas-reliefs qui en provenaient furent acquis en 1883 pour le Musée, alors à Boulaq (G. MASPERO, Guide du visiteur au Musée de Boulaq, 1883, p. 427-429, sous numéros 6050,6053 et 6056 ; nous avons reconnu trois fragments du chef des coiffures du roi, Khamouas, sous les numéros 8380, 27-6-24-9 et 1-7-24-3 (provisoires) dans la salle 8 du Musée du Caire; cf. L. BORCHARDT, Bilder der Zerbrechens der Krüge, dans $\ddot{A} . Z$. 64 (1929), p. 15).

G. Maspero date la tombe de ce Khamouas de la fin du règne d'Aménophis III, peut-être du commencement du règne d'Aménophis IV (Guide du visiteur, op. cit.). Il ne nous parait pas du tout impossible qu'il y ait eu confusion entre les deux Khamouas, à cause de leurs noms et de la présence du mot "roi" dans leurs titres.

Fl. Petrie a bien trouvé des statuettes funéraires de Khamouas à Gizeh, mais il se garde d'en tirer conclusion; voici ce qu'il en dit (Gizeh, p. 24, 1ère colonne): "Ce sol (à environ un quart de mille au sud de la tombe de la Ière dynastie) a été retourné par nous et nous avons trouvé quelques blocs calcaires de fondations d'un bâtiment, quelques puits d'environ dix pieds de profondeur comblés avec du sable et des décombres, et beaucoup d'ouchebtis. La plupart de ceux-ci étaient pour des personnes privées, quelques-uns étaient pour Khamouas, le fils de Ramsès II, et un de la reine Nefertarimery-mut ..... On ne doit pas conclure, parce que des ouchebtis, et l'on dit aussi un vase canope, ont été trouvés ici, que Khamouas fut enseveli ici pour cette raison, comme cela a été supposé (MASPERO, ...): des ouchebtis semblables et un vase fait par Khamouas ont été trouvés avec les sépultures d'Apis au Sérapéum".

Le premier vase canope auquel Petrie fait allusion a été trouvé dans la tombe d'Aménophis III à la Vallée des Rois ! G. Maspero a évidemment raison de dire qu'il "fut transporté à Louxor par quelque fellah voyageur, comme le sont chaque année nombre d'objets en Egypte" (dans A. MARIETTE, Monuments divers, 1889, p. 10), mais nous ne le suivons pas lorsqu'il laisse entendre, sans le déclarer expressément (et Petrie s'y est laissé prendre), que ce canope vient de Gizeh (ibidem), car il pourrait fort bien provenir des fouilles de Mariette au Sérapéum; G. REISNER, The dated canopic jars of the Gizeh Museum, dans Ä.Z., 37 (1899), p. 66, signale en effet: "Deux vases, sans couvercle, calcaire blanc. Nom de Khamouas. Bab el-Mulûk, 1859 (acquisition ?). Inv. 4085" (cf. notre inscription 140). Quant au second canope dont parle Petrie, il n'est d'aucune utilité pour son argumentation parce qu'il n'a pas été fait par Khamouas pour lui-même (A. MARIETTE, Sérapéum, 1857, pl. 10).

Nous concluons: l'hésitation de Mariette à reconnaitre un prince et fils du grand Ramsès dans la momie humaine qu'il a découverte dans les petits souterrains et l'affirmation de Maspero, que nous n'avons pas réussi à étayer par un document, ne pèsent pas assez en face du témoignage des bijoux pour nous faire douter que Khamouas a été enseveli dans le tombeau d'Apis. 
avait remplacé son frère Khamouas dans le gouvernement de Memphis. Mariette y a également relevé la date de l'an 55, au sujet de laquelle il écrit: "Par la position de la momie (= celle de l'Apis), je ne pense pas que ce soit à cet Apis que se rapporte la date"1. Khamouas serait donc mort en l'an 55 de Ramsès II ou, si la date concernait malgré tout l'ensevelissement de l'Apis, avant ce moment.

Le pontificat de Khamouas a donc été très long, sans doute même un des plus longs que Memphis ait jamais vus. C'est peut-être en partie pour cette raison que la renommée du grand prêtre a subsisté jusqu'à la fin de la civilisation pharaonique, bien qu'on ne l'ait pas divinisé comme d'autres grands de l'Egypte. On n'a guère vu en lui que le chercheur intéressé par les vieux textes et emporté par cette passion jusqu'à les rechercher dans les tombeaux; ne lui a-t-on pas attribué la découverte de chapitres du Livre des Morts "à la tête d'une momie à l'Occident de Memphis" (146) ? Par la suite, on a fait de lui le héros d'une histoire fantastique, ou encore le père d'un enfant prodige qui aurait possédé l'âme réincarnée d'un vieux sage ${ }^{2}$.

\section{§ 59. NEFERRENPET}

Nous donnons comme successeur à Khamouas le grand prêtre et vizir Neferrenpet, qui a été investi de la fonction importante de ministre des cultes $^{3}$, car nous estimons que Ramsès II aurait, semble-t-il, confié la même charge à son fils Khamouas si celui-ci avait succédé à Neferrenpet. D'autre part, Neferrenpet s'intitule fréquemment "sem de Ptah" $(147 ; 148 ; 150,4 ; 151,5 ; 153,2 ; 154,2)$ comme Khamouas l'a fait dans la seconde moitié de sa carrière. L'époque que nous attribuons à Neferrenpet correspond ainsi à la généalogie d'Ankhef-ny-sekhmet, qui

1 A. MARIETTE, Renseignements sur les soixante-quatre Apis, dans Bulletin de l'Athénaeum français, I, 1855, p. 86.

${ }^{2}$ F.LL. GRIFFITH, Stories of the high priests of Memphis, the Sethon of Herodotus and the demotic tales of Khamuas, 1900.

3 Voir ci-dessus, § 28. 
en fait le dernier des trois grands prêtres du règne de Ramsès II $^{1}$.

Neferrenpet était peut-être fils d'un juge $(\mathbf{1 5 2 , 1 )}$; il a fait carrière à Memphis, où son fils Bakenptah est devenu père divin de Ptah (152,1011). Si c'est le même Neferrenpet qui est mentionné sur une statuette funéraire du Musée de Berlin², il aurait été "père divin de Ptah et prophète" avant d'être nommé grand des chefs des artisans, vers l'an 55 de Ramsès II.

Neferrenpet paraît être devenu vizir après avoir été mis à la tête du clergé memphite puisqu'il est toujours vêtu de la robe-fourreau particulière au plus haut fonctionnaire. Il se pourrait fort bien qu'il eût cumulé non seulement les fonctions de grand des chefs des artisans et de vizir $^{3}$, contrairement à Rêhetep, mais encore celle de directeur des prophètes de tous les dieux de Haute et Basse-Egypte, de telle sorte que nous ne pouvons dire à quel titre Ramsès II lui aura fait conduire une fête d'Amon $(\mathbf{1 5 0 , 3})$. Neferrenpet a pu faire sculpter à cette occasion le petit monument en forme de naos (149); comme il s'y adresse à Amonrêsonter $(\mathbf{1 4 9 , 3})$ autant qu'à Ptah-Tatenen $(\mathbf{1 4 9 , 4 )}$ et qu'on connaît d'autre part une plaquette dédiée par lui à Khonsou-dansThèbes-Neferhotep (154), nous tenons en fin de compte pour certain ce que nous avons supposé ${ }^{4}$ : c'est Amon de Karnak dont le grand prêtre memphite, ministre des cultes et vizir Neferrenpet aura conduit une fête.

Le successeur de Neferrenpet au ministère des cultes a vraisemblablement été le grand prêtre d'Amon de Karnak Romê-Roy, qui fut chargé de cette fonction administrative sous le règne de

${ }^{1}$ Voir ci-dessus, § 33. La tentative de R. ANTHES (dans $\ddot{A} . Z$., 74 (1938), p. 111 et 112) de dater Neferrenpet au moyen d'une graphie particulière de la copule du génitif $n$ dans les titres composés avec "de Ptah" ne donne pas de résultat assez précis, puisque cette graphie remonte en tout cas à l'an 30 de Ramsès II (idem, dans Ä.Z., 77 (1941), p. 55-56). Elle ne peut pas davantage servir à classer Khamouas et Neferrenpet l'un par rapport à l'autre, car un monument de Khamouas a aussi la même graphie, mais pour le $n$ du datif (cf. 161,1-2).

2 Ägyptische Inschriften aus ... Berlin, II, p. 589. = LEPSIUS, Denkmäler, Text, V, p. 396 (statuette de Malte).

3 Voir ci-dessus, $\S 9$, p. 25, note 1.

${ }^{4}$ Voir ci-dessus, $\S 28$ et 32. 
Meneptah ${ }^{1}$. Nous en déduisons que Neferrenpet a survécu à Ramsès II et est décédé sous Meneptah. Sa tombe, sans doute bâtie à Memphis, comprenait une chapelle en forme de pyramide dont le sommet nous est parvenu $(\mathbf{1 5 2})^{2}$.

\section{$\S$ 60. PTAHMES IV ET IYRY}

Nous avons déjà signalé deux fois la raison pour laquelle nous admettons l'existence d'un grand prêtre memphite du nom de Ptahmès

${ }^{1}$ G. LEFEBVRE, Histoire, p. 146.

2 Deux monuments ont peut-être encore appartenu au grand prêtre Neferrenpet, quoique leur attribution reste douteuse:

a) une statuette funéraire à Florence (ouchebti Florence $n^{\circ} 63$ d'après la fiche $d u$ Wörterbuch citée par A. WEIL, Die Veziere, p. 94-95, d) au nom du "noble, gouverneur, père divin aimé du dieu, celui du Rideau, juge, porte-parole de l'Egypte, prophète de Maât, préfet de la ville et vizir Neferrenpet" (cf. les titres de 149,15);

b) un monument du "noble, gouverneur, père divin aimé du dieu, supérieur des secrets ....., préfet de la ville et vizir Neferrenpet" (A. WEIL, Die Veziere, p. 95, i).

Une porte du Musée de Turin (Turin 129 = FABRETTI, Regio Museo di Torino, 2, 1882, p. $125, \mathrm{n}^{\circ}$ 1464; LIEBLEIN, Dict., $\mathrm{n}^{\circ} 929$ et Supplément; G. MASPERO, Rapport ..... sur une mission en Italie, dans Rec. Trav., II (1880), p. 181; A. WEIL, Die Veziere, p. 94, c) où un vizir Neferrenpet apparaît au-dessus d'un chef d'équipe des artisans de Deir el-Médineh, n'appartient certainement pas au grand prêtre memphite mais à un vizir thébain du même nom. Que ce vizir soit contemporain de Ramsès II dont les cartouches sont gravés sur la face du monument, ou qu'il soit du règne de Ramsès IV dont les cartouches, non signalés dans le catalogue du Musée de Turin, sont sur les faces latérales internes des montants (d'après une copie que M. G. Nagel m'a obligeamment communiquée), c'est ce qu'un examen du monument permettrait peut-être d'établir. Nous notons à ce propos qu'il y a eu un vizir Neferrenpet sous Ramsès IV (A. WEIL, op. cit., p. 115, $\mathrm{n}^{\circ} 40$ ).

Enfin, la stèle $127 \mathrm{c}$ du Vatican (MARUCCHI, Il Museo egizio di Vaticano, p. 124), au nom d'un "premier père divin dans le temple de Ptah Neferrenpet", appelé aussi "grand père divin de Ptah et premier prophète Neferrenpet" ne peut également pas appartenir au grand prêtre memphite, mais à un homonyme thébain, puisque le temple thébain de Ptah a eu ses "premiers prophètes de Ptah" (voir ci-dessus, § 57, p. 147, note 1). Sans aller aussi loin que nous, A.H. GARDINER, Ancient Egyptian Onomastica, Text, Vol. I, p. 48*, estime cependant qu'"il semble très incertain que Neferrenpet du Vatican soit identique au vizir et grand prêtre memphite de ce nom". 
postérieur à la XVIIIème dynastie ${ }^{1}$ et même à Séti Ier $^{2}$. Nous sommes certains que l'ordre des titres "grand des chefs des artisans - sem" a été en usage de Ramsès II à la fin de la XIXème dynastie, et nous ne manquons pas de noms de grands prêtres pour le règne de Ramsès II; c'est pourquoi nous plaçons Ptahmès IV par hypothèse entre Neferrenpet et Iyry. Mais nous ne connaissons pas de grand prêtre contemporain de la XXème dynastie et Ptahmès IV pourrait ainsi être postérieur à Iyry.

Ptahmès IV a été "directeur des Six Grandes Maisons" $(\mathbf{1 5 5 , 2 )}$. Cette haute fonction juridique ${ }^{3}$ paraît avoir été essentiellement thébaine au Nouvel Empire: sur les sept vizirs qui l'ont remplie à cette époque à part Ptahmès IV, six sont certainement connus par des monuments thébains et le septième probablement ${ }^{4}$. On en peut conclure que Ptahmès IV a exercé une partie de sa carrière à Thèbes et qu'il y a été vizir. Si nous admettons qu'il n'a pas porté en même temps tous les titres énumérés sur sa palette et qu'ils sont classés chronologiquement ${ }^{5}$, il aurait été ensuite grand prêtre memphite. Ou bien, après avoir été directeur des Six Grandes Maisons à Thèbes, il aurait passé vizir à Memphis dont il serait devenu grand prêtre plus tard, tout en conservant sa fonction de vizir; nous expliquerions ainsi que Ptahmès IV soit représenté en tenue de vizir sur sa palette, comme d'ailleurs Rêhetep et Neferrenpet, qui furent aussi grands prêtres memphites et vizirs, le sont sur leurs monuments.

A la fin de la XIXème dynastie, le chef du clergé memphite s'appelait Iyry. Comme il s'est fait sculpter une statue sous Séti II (156), il a peutêtre succédé à Neferrenpet et précédé Ptahmès IV. On sait bien peu de chose sur le grand prêtre Iyry; son fils a été père divin de Ptah $(\mathbf{1 5 6 , 1 4 )}$

${ }^{1}$ R. ANTHES, Die hohen Beamten namens Ptahmose in der XVIII. Dyn., dans Ä.Z., 72 (1936), p. 66-67 et 68, tableau, classe Ptahmès de la palette du Louvre (= Ptahmès IV) à la XVIIIème dynastie et cherche à l'identifier avec l'un ou l'autre des Ptahmès de ce temps.

${ }^{2}$ Voir ci-dessus, $\$ 31$ et 48.

${ }^{3}$ J. PIRENNE, Histoire des institutions, tome 2, p. 114.

${ }^{4}$ A. WEIL, Die Veziere, Nouvel Empire; les $n^{\circ} 3,4,5,7,9$ et 18 sont certainement thébains; 6 , probablement.

${ }^{5}$ Voir ci-dessus, $\$ 6$, fin. 
et lui-même avait une prédilection marquée pour le titre "sem de Ptah"1, témoin du triomphe temporaire de Sokar et d'Osiris sur Ptah $^{2}$. Nous noterons à ce propos que l'image divine dans le naos qu'Iyry tient devant lui n'a rien d'un Ptah mais tout d'un Osiris, tant par sa couronne et par la position des bras pliés, dont les coudes dépassent la ligne du corps à droite et à gauche, que par le fouet et le sceptre qu'elle tient dans ses mains. De plus, Iyry s'adresse au dieu Ptah-Sokar-Osiris (156,5). Enfin, il est le seul grand prêtre memphite, à notre connaissance, qui soit "premier prophète d'Osiris, seigneur de Ro-Setaou" $(\mathbf{1 5 6 , 1 2})^{3}$. Ce dernier titre permet de supposer qu'Iyry a réorganisé et peut-être développé le culte d'Osiris dans la nécropole memphite.

Iyry est le dernier grand prêtre du Nouvel Empire que nous pouvons dater avec précision. Nous ne connaissons pas de grand prêtre contemporain des Ramessides. Car, bien que l'admiration professée par Ramsès III pour son illustre homonyme l'ait sans doute amené à nommer l'un de ses fils Khamouas et à en faire un "sem de Ptah" ou "sem de Ptah le Grand au sud de son mur" $^{\prime 4}$, nous pensons que ces titres concernent Ptah thébain puisque le prince Khamouas, fils de Ramsès III a eu son tombeau à la Vallée des Reines.

Le grand des chefs des artisans qui contrôlait les richesses de Ptah que rapporte le grand papyrus Harris reste ainsi anonyme (162a).

En opposition à leurs devanciers qui, lorsqu'ils étaient grands prêtres, n'avaient pas affaire à une grande distance de Memphis, les grands prêtres du Nouvel Empire ont vu le cadre de leur activité s'élargir dès le règne d'Aménophis III. Ils adjoignent depuis lors à leurs fonctions religieuses et civiles de chef des sacerdoces memphites et de grand des chefs des artisans parfois le ministère des cultes de toute l'Egypte, parfois le vizirat, parfois même les deux ensemble. Leur pouvoir temporel s'accroît, sans qu'on puisse cependant le comparer à celui des grands prêtres d'Amon.

${ }^{1}$ Voir ci-dessus, $\S \mathbf{5}$.

${ }^{2}$ Voir ci-dessus, § 32.

${ }^{3}$ Des grands prêtres ptolémaïques sont "prophètes d'Osiris, seigneur de Ro-Setaou"; voir ci-dessus, § 17.

${ }^{4}$ H. GAUTHIER, Le livre des rois, 3, p. 177, ${ }^{\circ} 8$, A, B et C. 
En même temps, ils laissent les croyances dont ils sont les plus hauts représentants dévier de leur origine à partir de Ramsès II et de plus en plus après ce pharaon.

\section{§ 61. APPENDICE AU CHAPITRE IX}

Nous rayons sans hésitation de la liste des grands prêtres memphites un certain Ankhty cité par Schiaparelli, parce que le monument de ce personnage appartient au grand prêtre Ty (74).

Nos conclusions sur l'ordre des titres du grand prêtre nous permettent de classer quelques monuments dépourvus de noms propres: un fragment de paroi (76) et un autre de statue (78) sont antérieurs à Ramsès II. Par contre, des fragments d'une stèle relative à un temple de Sekhmet dans le temple funéraire de Sahourê et mentionnant les "grands des chefs des artisans, sem, directeurs des prophètes" $(\mathbf{1 5 8 , 2 )}$ ne peut pas être antérieure à Ramsès II; un des fragments $(\mathbf{1 5 8 , 4 )}$ confirme qu'elle lui est contemporaine. De même, la stèle sur laquelle le père divin de Ptah Ptahmès demande aux grands prêtres de ne pas déplacer sa statue, sous prétexte qu'elle les empêcherait de contempler leur dieu, ne peut pas être antérieure à Ramsès II (157).

Signalons enfin que l'expression "grand des chefs des artisans" a servi de nom propre au Nouvel Empire (160 - 162). 



\section{H A P I T R E X}

\section{LES GRANDS PRETRES DE LA BASSE EPOQUE}

\section{§ 62. PAOUPAOU, HORSAISET I ET ANKHEF-NY-SEKHMET}

Nous manquons de documents sur les grands des chefs des artisans pendant une partie de la XXIème dynastie. Cette pénurie s'ajoute malheureusement à celle de la fin du Nouvel Empire, de sorte que notre liste des pontifes memphites présente une lacune qui s'étend sur environ deux siècles, peut-être un peu moins si Ptahmès IV a succédé à Iyry.

Le roi Siamon, qui régnait vers l'an 1000, a fait bâtir à Memphis un sanctuaire dédié à Amon du lapis-lazuli véritable. Six linteaux de ce monument nous sont parvenus. Sur les deux plus grands, composés de la même façon, les cartouches du roi, gravés verticalement, partagent la surface en deux tableaux. Ceux de gauche des deux linteaux font voir Siamon et le grand prêtre Paoupaou ${ }^{1}$ adorant respectivement Ptah et Sekhmet, et Ptah et Hathor. Dans les tableaux de droite, Amon et Mout sur un linteau, et Ptah et Sekhmet sur l'autre sont adorés par Siamon et un personnage porteur d'éventail nommé Ankhef-ny-mout. Celui-ci est "père divin, supérieur des secrets de Ptah, hiérogrammate du temple de Ptah, comptable des troupeaux dans le temple de Ptah, prophète"; il est aussi qualifié de "fils du gouverneur Iouy" et d"appartenant à Amon du lapis-lazuli véritable" (164,26-27).

Ankhef-ny-mout, toujours porteur d'éventail, est seul représenté sur les quatre autres linteaux, où il est agenouillé de part et d'autre des cartouches du roi.

\footnotetext{
${ }^{1}$ Sur la tenue de Paoupaou, voir ci-dessus, § 9.
} 
On peut déduire de ces bas-reliefs que le temple d'Amon du lapislazuli renfermait, à part une image d'Amon peut-être ornée de lapislazuli, des statues de Mout, ainsi que de Ptah et des déesses Sekhmet et Hathor. Comme Paoupaou n'adore pas Amon, nous pensons que le roi aura confié le service de ce dieu au père divin Ankhef-ny-mout. Le grand prêtre Paoupaou figurerait sur ces monuments comme officiant du culte de Ptah et aussi comme chef de tous les cultes rendus à d'autres divinités dans Memphis.

Paoupaou portait aussi un nom forgé avec la première partie du nom de son souverain. Il est le "sem, grand des chefs des artisans de Ptah, Neterkheper-rê-Méryptah, appelé Paoupaou" (164,24-25). Toutefois, comme Paoupaou est le nom courant de ce grand prêtre, puisque c'est ainsi qu'il se nomme sur l'autre linteau $(\mathbf{1 6 3 , 1 0})$ et que des généalogies ont conservé ce nom ${ }^{1}$, nous nous demandons s'il ne faudrait pas plutôt traduire de la façon suivante: "le sem et grand des chefs des artisans, celui qui est appelé Neter-kheper-rê-Méryptah ${ }^{2}$, Paoupaou"; la place occupée par la forme verbale, bizarre à première vue, correspondrait alors à celle du mot "fils" dans l'expression "N. fils de N.", quand le nom du père vient le premier.

D'après sa titulature, Paoupaou n'a exercé aucune activité en dehors de Memphis. Ses fils et petit-fils qui lui ont succédé donnent lieu à la même constatation. Nous pouvons supposer que, dès le début de la XXIème dynastie, peut-être même plus tôt, les grands prêtres memphites n'ont pas eu un pouvoir plus étendu que sous l'Ancien Empire.

Les données concordantes des généalogies permettent de considérer Paoupaou comme le père du grand prêtre Horsaïset $\mathrm{I}^{3}$. Celui-ci, si nous admettons que la statue du grand prêtre Horsaïset derrière un naos lui appartient ${ }^{4}$, a épousé une "grande des concubines" $(\mathbf{1 6 5 , 1 , 3 )}$ d'un dieu qui n'est pas nommé, probablement parce que c'était Ptah. Il a eu pour fils Ankhef-ny-sekhmet. Ce dernier, devenu grand prêtre, a fait sculpter

\footnotetext{
${ }^{1}$ Voir ci-dessus, $\S 33$ et $34(\mathbf{1 8 2}, 14$ et $\mathbf{1 8 3 , 1 1 )}$.

2 Litt. "Neter-kheper-rê-Méryptah est dit à lui".

${ }^{3}$ Voir ci-dessus, $\S 33$ et $\mathbf{3 4}$.

${ }^{4}$ Voir l'appendice au présent chapitre, ci-dessous, $\S 72$.
} 
une statue qui représentait son père debout derrière un naos arrivant à hauteur de poitrine (165). Ce monument est aujourd'hui privé du torse et de la tête, ce qui non seulement gêne l'interprétation des restes de l'inscription dorsale $\mathbf{( 1 6 5 , 6 - 7 )}$ mais empêche de dire si la statue était destinée au temple d'Hathor maîtresse du Sycomore du sud $(\mathbf{1 6 5 , 7 )}$, comme la formule d'offrandes gravée sur la face du naos $(\mathbf{1 6 5 , 2 )}$ et la figure de la déesse sculptée à l'intérieur de ce dernier le suggèrent.

\section{§ 63. CHEDSOUNEFERTOUM}

On peut supposer qu'Ankhef-ny-sekhmet a élevé son fils Chedsounefertoum dans le temple de Ptah. Le futur grand prêtre y aura eu l'occasion de se familiariser avec les monuments des anciens grands prêtres, ce qui l'aura amené à se décerner des titres tout à fait désuets chez les grands des chefs des artisans de la Basse Epoque: compagnon unique, et trésorier du roi de Basse-Egypte $(\mathbf{1 6 9 , 4})^{1}$.

Chedsounefertoum a été grand des chefs des artisans sous Chechanq Ier $(\mathbf{1 6 6 , 2})$ qui lui a confié le soin de bâtir un atelier d'embaumement pour les momies d'Apis, en bordure du désert (166,1213).

Un groupe que Chedsounefertoum s'est approprié $(167)^{2}$ montre le grand prêtre assis à côté d'une femme intitulée "la première grande du harem de Ptah et prophétesse de Mout, Tachepeniset" $(\mathbf{1 6 7 , 2 3 )}$. Nous considérons celle-ci comme l'épouse de Chedsounefertoum parce que les faces latérales du siège énumèrent des enfants $(\mathbf{1 6 7 , 1 - 1 7 )}$ dont la présence s'explique aisément sur un monument de leurs parents. Ces enfants, comme il sied aux rejetons d'une lignée de grands prêtres de Ptah, remplissent tous des fonctions sacerdotales à Memphis: l'un est un "père divin de Ptah" attaché au domaine memphite d'un Amon-rê (167,13); un autre est "sem de Ptah" (167, 9-11) et nous estimons qu'il a

1 Voir ci-dessus, $\$ 30$.

${ }^{2}$ L. BORCHARDT, Statuen..., III (1930), p. 67-69, en attribue la création à la XVIIIème dynastie. 
succédé à son père ${ }^{1}$; un troisième, "père divin de Ptah et directeur des troupeaux", ainsi que "prophète" d'une image particulière de Ptah, est attaché au "temple de Sekhmet de la Vallée" $(\mathbf{1 6 7 , 1 2 - 1 6 )}$; un quatrième, aussi "père divin de Ptah", est attaché au temple du "Seigneur de Rosetaou" (167,16-17); il s'agit sans doute ici du temple d'Osiris dont Iyry avait été premier prophète ${ }^{2}$; enfin, deux filles sont "chanteuse du choeur de l'Aimée de Ptah" (167,4-7).

Tachepeniset était fille du "grand chef des $M a$ " dont le nom n'est pas cité parce qu'il était assez illustre et de "la première grande du harem de Ptah et prophétesse d'Hathor Mehousekh" (167,23-24). Ce sont les proches parents de Nemrod et les aïeux de Chechanq Ier. Tachepeniset était donc la tante du fondateur de la XXIIème dynastie ${ }^{3}$, et ce degré de parenté permet de penser que Chedsounefertoum était plus âgé que le roi qu'il a servi. De ce fait, le mariage de Chedsounefertoum a peutêtre précédé l'avènement de Chechanq Ier et le grand prêtre aura usé de son influence pour faire accepter le nouveau roi dans Memphis.

C'est ainsi, pour une part, en récompense des services rendus au roi que Chedsounefertoum a été nommé "directeur des prophètes de tous les dieux de Haute et Basse-Egypte" et, à la même époque d'après la position des titres, "grand des voyants de Rê-Toum" (169,6). Par cette double nomination, Chechanq Ier ne reconnaissait pas seulement le zèle de son oncle par alliance. On sait qu'il a placé son fils Aoupout à la tête du clergé d'Amon, suivant l'exemple des rois de la XXIème dynastie ${ }^{4}$; ceuxci s'étaient eux-mêmes inspirés de la conduite des deux rois de la XVIIIème et de la XIXème dynastie qui avaient fait de leurs fils des grands prêtres memphites. En faisant de son parent le chef des clergés de Memphis et d'Héliopolis, et un ministre des cultes, Chechanq Ier a repris énergiquement à son compte la politique d'Aménophis III et de Ramsès II.

${ }^{1}$ Voir ci-dessus, $\$ 33$.

2 Voir ci-dessus, $\$ \mathbf{6 0 .}$

3 DARESSY, dans Rec. Trav., XVIII, p. 48 (d'après le groupe de Chedsounefertoum), suivi par GAUTHIER, Rois, 3, p. 318.

${ }^{4}$ G. LEFEBVRE, Histoire, p. 219-220. 
Le renom de Chedsounefertoum était encore assez grand plus d'un siècle après son pontificat pour qu'un de ses descendants, Nesptah, se réclamât de lui sur une stèle du Sérapéum (171) et peut-être sur un fragment de monument (170) connu par sa généalogie de prophètes de Baal de Memphis et de prophètes du temple d'Apis ${ }^{1}$.

\section{§ 64. CHECHANQ ET OSORKON}

Il est possible que Chedsounefertoum ait eu pour successeur son fils Chechanq, que nous ne connaissons que comme "sem de Ptah" (167,911) et que Chechanq ait été suivi par son propre fils Osorkon ${ }^{2}$. Ces données de la généalogie d'Achaout-akh sont vraisemblables non seulement à cause du temps qui s'est écoulé entre Chedsounefertoum et le prince Chechanq, mais aussi à cause de la parenté des grands prêtres avec les souverains d'alors: le grand des chefs des artisans Chechanq aurait été le cousin du roi Chechanq Ier.

\section{§ 65. LE PRINCE CHECHANQ}

La longue lignée de grands prêtres dont on suit l'histoire pendant près d'un siècle et demi prend fin à Osorkon. Ce dernier n'a pourtant pas manqué d'héritier masculin, puisqu'il a eu un fils, Takelot, qui a été "sem et prophète de Ptah" $(\mathbf{1 8 3 , 5 - 6 )}$. Nous sommes certains que ce Takelot n'a pas été choisi par le roi pour succéder à son père, car, dans le cas contraire, Achaout-akh n'eût pas manqué d'intituler son bisaïeul "grand des chefs des artisans" dans sa généalogie. On peut supposer que Takelot montrait peu de zèle pour la dynastie régnante et que cette circonstance a engagé le roi Osorkon II à imiter quelques-uns de ses prédécesseurs en plaçant son fils, le prince Chechanq, à la tête du clergé de Ptah (173).

\footnotetext{
${ }^{1}$ Un fragment de colonne daté par le cartouche de Chechanq Ier pourrait appartenir à Chedsounefertoum si les titres du personnage dont le nom est détruit comprennent vraiment "grand des chefs des artisans" (172).

2 Voir ci-dessus, § 34.
} 
Le prince Chechanq est peut-être le grand des chefs des artisans qui prit part à la fête Sed célébrée par son père à Bubastis (182a). Nous ne savons rien d'autre de la carrière de ce grand prêtre, qui est mort avant son père, comme ses prédécesseurs princiers de la XVIIIème et de la XIXème dynasties. Il est en effet encore intitulé"grand premier noble ${ }^{1}$ de Sa Majesté" et "fils $d u$ roi" sur un monument de l'an 28 de Chechanq III, donc postérieur d'un demi-siècle à la mort d'Osorkon II $(\mathbf{1 7 7 , 5 - 7 , 1 3})^{2}$.

\section{§ 66. MERENPTAH ET TAKELOT}

Le fils du prince Chechanq, Takelot $(177,3-7,12-13)$ a été grand des chefs des artisans $(\mathbf{1 7 6})^{3}$ sous un roi Chechanq $(\mathbf{1 7 6 , 1})$ qui ne peut être que Chechanq $\mathrm{III}^{4}$ dont le règne s'étend de 823 à $772^{5}$. Mais Takelot n'a pas succédé directement à son père, car il y a eu un grand des chefs des artisans nommé Merenptah sous Takelot II, qui a régné avant Chechanq III. Nous ne pouvons que proposer deux hypothèses pour le motif qui a fait nommer Merenptah: ou bien c'était aussi un fils du prince Chechanq, donc un frère aîné de Takelot, qui serait mort sans descendant masculin ${ }^{6}$, ou, plutôt, Takelot aurait été jugé trop jeune pour être grand prêtre à la mort du prince Chechanq, et Merenptah n'aurait alors eu aucun lien direct de parenté avec ses prédécesseur et successeur.

1 Il peut sembler étrange qu'un prince soit qualifié de "noble", mais ce titre avait été déjà porté par Khamouas, fils de Ramsès II (137).

${ }^{2}$ On a fait autrefois du prince Chechanq un roi, Chechanq II, mais on ne le considère plus comme tel; cf. GAUTHIER, Rois, 3, p. 344, qui nomme Chechanq II celui qui a régné plus de cinquante ans, et DRIOTON-VANDIER, L'Egypte, p. 541, qui conservent l'ancienne numérotation, tout en n'attribuant aucune année de règne à Chechanq II.

${ }^{3}$ Comme Padiiset, qui a été "grand des chefs des artisans" et "grand chef des $M a^{\prime \prime}$ (178,15-16), s'intitule la plupart du temps seulement "grand chef des Ma" (même monument: 178,1-2; aussi 177,2-3,8,10; 179,1), nous identifions le "grand chef des $M a$ " fils du prince Chechanq (177,3-7,12-13) Takelot au "grand des chefs des artisans et grand chef des Ma Takelot" (176).

${ }^{4}$ Chechanq II selon H. Gauthier.

5 DRIOTON-VANDIER, L'Egypte, p. 541.

${ }^{6}$ C'est le cas de Teos sous les Ptolémées. 
Merenptah a vraisemblablement été nommé par Osorkon II, et son pontificat a continué sous Takelot II, d'où date la seule inscription qui nous apprenne son existence (175).

A sa mort, survenue vers la fin du règne de Takelot II ou le début de celui de Chechanq III, Takelot fils du prince Chechanq est devenu grand prêtre. Son ascendance lui valut sans doute de porter le titre de "grand chef des $M a^{\prime \prime}(176,3 ; 177,3-4 ; 177,12 ; 178,3-5)$, qu'on retrouve chez son fils Padiiset, mais qui n'est plus porté par ses petits-fils Peftjaouaouibastet et Horsaïset II. Ce titre peut donc aussi bien être honorifique que correspondre à une fonction réelle chez Takelot et Padiiset.

Takelot a épousé une demi-soeur de son père, la "fille charnelle $d u$ roi, celle qu'il aime, Tesbastetperet" $(\mathbf{1 7 8 , 1 7 )}$, qu'Osorkon II avait eue de sa concubine Isetemkheb ${ }^{1}$ probablement à la fin de son règne ${ }^{2}$.

Une grande partie de son pontificat, si ce n'est la totalité, est contemporaine du règne de Chechanq III $(\mathbf{1 7 6 , 1 )}$. On peut être certain qu'il est mort au plus tard en l'an 27 puisque son fils a introduit un Apis à Memphis le 1er jour du 2ème mois d'Akhet de l'an 28 sous Chechanq III (178).

\section{$\S$ 67. PADIISET}

Le grand prêtre Padiiset était donc petit-fils du roi Osorkon II par sa mère et arrière petit-fils par son père. Nous le connaissons par quatre monuments (177 - 180) qui nous apprennent qu'il a eu plusieurs épouses. Chacune lui a donné au moins un enfant. De Tairy, il a eu un fils, Peftjaouaouibastet, qui a été grand des chefs des artisans (177,8-9). D'une seconde épouse, dont le nom n'est conservé qu'en partie, il a eu un fils, Takelot, qui a été "sem de Ptah" (177,9-10). D'une troisième

${ }^{1}$ GAUTHIER, Rois, III, p. 347 et note 1.

2 Takelot a peut-être été aussi le mari de la "grande du harem de Ptah le grand ..., prophétesse de Nefertoum ..., prophétesse de Mout ..., Djedbastetiouesankh", nommée sur un bloc qui "semble provenir du même édifice" que 176; cf. réf. ibidem. 
enfin, qui était "grande des concubines de Memphis", il a eu un fils, Horsaïset, qui a été grand des chefs des artisans (179,4-9,17-20). Padiiset a eu aussi une fille, Taperet (180).

Nous venons de rappeler que Padiiset a introduit à Memphis un Apis, qui est le quatrième de la XXIIème dynastie, le premier jour du deuxième mois d'Akhet de l'an 28 de Chechanq III. Ce renseignement nous est fourni par une stèle du Sérapéum que le grand prêtre Horsaïset II a fait graver vingt-six ans plus tard, en l'an 2 de Pamy $(\mathbf{1 7 8})^{1}$ et qui est le procès-verbal de l'ensevelissement du quatrième Apis.

La recherche de cet Apis avait pris trois mois (178,13-14), au cours desquels on avait eu le temps d'ensevelir le troisième Apis, alors que Padiiset était grand prêtre, puisque ses fils, quelques mois plus tard, l'ont représenté en adoration devant un Apis avec la date de l'an 28 de Chechanq III (177). Le monument qui nous procure ces renseignements est différent du procès-verbal de l'an 2 de Pamy. La date n'y est exprimée qu'en années; on n'y trouve aucune indication sur l'Apis, mais seulement les titres et filiations des trois personnages en adoration devant Apis. Or un monument de ce genre n'est pas nécessairement tout à fait contemporain de l'ensevelissement de l'Apis auquel il se rapporte ${ }^{2}$. Dans le cas présent, il se serait écoulé moins d'un an entre la mise au tombeau de l'Apis et la stèle d'adoration. Lorsque celle-ci a été sculptée, Padiiset était mort et son fils Peftjaouaouibastet avait été nommé grand des chefs des artisans $(\mathbf{1 7 7 , 7 - 8})^{3}$. Il semble donc que le pontificat de Padiiset a été assez court.

${ }^{1}$ Horsaïset II est seulement "sem de Ptah" sur ce monument (178,6), mais une autre stèle, qui est peut-être exactement de la même date, l'intitule "sem de (sic) et grand des chefs des artisans de Ptah" (179, 4-5).

${ }^{2} \mathrm{~A}$ la XXVIème dynastie, où le quatrième Apis est mort en l'an $12 \mathrm{~d}$ 'Apriès et son successeur en l'an 23 d'Amasis, il y a des stèles datées de l'an 14 d'Apriès: E. CHASSINAT, Textes..., dans Rec. Trav. XXII, p. 20, n 63 et p. 167, n 90; XXV, p. 58, $n^{\circ} 175$.

${ }^{3}$ En fixant la mort de Padiiset à un moment de l'an 28 postérieur à l'intronisation du quatrième Apis, nous n'excluons pas que son père Takelot soit décédé au plus tard en l'an 27 (voir au § précédent). 


\section{§ 68. PEFTJAOUAOUIBASTET ET HORSAISET II}

Le "grand des chefs des artisans de Ptah Peftjaouaouibastet" (177,79,13-14) est entré en fonction peu après l'intronisation du quatrième Apis de la XXIIème dynastie. Son demi-frère, le "sem de Ptah Takelot" $(\mathbf{1 7 7 , 9 - 1 0 , 1 5})^{1}$, et lui se sont fait représenter derrière leur père, qui venait de mourir, sur la stèle du Sérapéum de l'an 28 de Chechanq III.

Des statuettes funéraires $(\mathbf{1 8 1} ; \mathbf{1 8 1 a})$ confirment l'existence du grand prêtre Peftjaouaouibastet, dont la mort est antérieure à l'enterrement du quatrième Apis.

Le procès-verbal de l'ensevelissement de cet Apis est en effet surmonté de l'image du dieu adoré par Padiiset et Horsaïset II (178,1-7). Comme Padiiset avait introduit le taureau sacré à Memphis, Horsaïset II aura présidé à ses funérailles. Horsaïset II était à ce moment non seulement "sem de Ptah" $(\mathbf{1 7 8 , 6 )}$ mais aussi "grand des chefs des artisans de Ptah" $(\mathbf{1 7 9}, \mathbf{4 - 5})$, ainsi qu'il le dit sur une autre stèle qui est, en partie, une copie du procès-verbal. L'image est semblable et comprend Padiiset et Horsaïset II en adoration devant Apis (179,1-9). La moitié du texte, malgré les lacunes du début et de la fin de chaque ligne, est un résumé du procès-verbal $(\mathbf{1 7 9}, \mathbf{1 0 - 1 6})$. L'autre moitié seule du texte diffère du procès-verbal. Nous y apprenons que la stèle a été faite sur l'ordre d'Horsaïset II, dont le nom est précédé de plusieurs titres, et que ce grand prêtre a eu deux fils de deux femmes différentes (179,16-21). Les noms des enfants d'Horsaïset ne sont accompagnés d'aucun titre.

La titulature d'Horsaïset II permet d'affirmer que son pouvoir n'a pas dépassé Memphis.

${ }^{1}$ Il n'est pas impossible que ce "sem de Ptah" ait été aussi par la suite "grand des chefs des artisans" comme ses deux demi-frères. Mais nous ne connaissons pas de monument qui lui donne ce dernier titre. En conséquence (voir ci-dessus, $\S \mathbf{5}$, fin) nous ne le considérons pas comme un grand prêtre. 


\section{§ 69. BAKENRENEF}

Nous sommes pauvrement renseignés sur les grands prêtres des cent cinquante années postérieures au pontificat d'Horsaïset II. L'un d'eux, dont le souvenir est conservé par deux statuettes funéraires $(\mathbf{1 8 3 a})^{1}$, s'appelait Bakenrenef'.

Nous n'avons rien à puiser dans l'information d'Hérodote qui raconte que "le prêtre d'Héphaistos, dont le nom est sethôn", a été roi après Chabaka ${ }^{3}$ puisque "sethôn" correspond au titre égyptien "setem" ${ }^{\prime \prime}$, souvent employé à la place de sem depuis Rêhetep sous Ramsès II. Chabataka, le successeur de Chabaka, a pu être sem de Ptah, comme le roi Tefnakht, qui a régné environ trois décades plus tôt, est sem de Ptah d'après la stèle de Piankhi: ce titre n'est pas suffisant pour que nous les regardions comme des grands prêtres memphites ${ }^{5}$.

Nous avons remarqué d'une part que la titulature des grands prêtres avait reflété l'emprise d'Osiris sur Ptah depuis Ramsès II $^{6}$ mais était revenue à la forme plus ancienne qui fait passer Ptah avant Sokar-Osiris précisément entre la fin de la XXIIème dynastie et l'époque saïte et que, d'autre part, l'intérêt porté au vieux texte recopié sous Chabaka est peut-

1 Nous ne connaissons ces monuments que par la description de l'Ausführliches Verzeichnis der ägyptischen Altertümer ... du Musée de Berlin qui signale Bakenrenef comme "Hoherpriester von Memphis". D'après cette expression, nous admettons que les statuettes portent effectivement le titre "grand des chefs des artisans".

${ }^{2}$ Nous n'identifions pas ce Bakenrenef avec le vizir saïte du même nom; celui-ci est bien "sem" et "prophète, ou père divin, de Ptah" (A. WEIL, Die Veziere, p. 145), mais il n'est pas grand des chefs des artisans.

${ }^{3}$ Hérodote, II, 141.

${ }^{4}$ F.LL. GRIFFITH, Stories of the high priests of Memphis, 1900, p. 7-11, suivant la voie ouverte par A. WIEDEMANN, Herodot's zweites Buch, 1890, p. 501, qui voit dans "sethôn" un indéclinable, et par J. KRALL, Ein neuer historischer Roman, 1897, p. 1, note, qui identifie Sethôn avec Setne du conte de Khamouas sans en donner la raison, écrit: "La forme stme fournie par la nouvelle histoire suggère fortement que stne est le titre sacerdotal stm" et traduit en conséquence le passage d'Hérodote: "ce prêtre d'Héphaïstos qui était appelé (le) sethôn".

${ }^{5}$ Voir ci-dessus, § 5, fin.

${ }^{6}$ Voir ci-dessus, $\S 32$; le triomphe le plus complet de cette tendance apparaît chez le grand prêtre Iyry, sous le règne de Séti II $(\$ 60)$. 
être en relation avec le retour à l'ancienne titulature. On peut donc penser que le document de théologie memphite a requis les soins du grand prêtre en fonction sous Chabaka.

\section{§ 70. HEKAIRAA, NEFERIBREMEN ET NEKAOMEN}

Sur les quatre grands prêtres saïtes que nous connaissons, trois appartiennent en tout cas à la même famille. Hekairâa a eu pour fils Neferibrêmen, qui a été le grand-père d'Ahmèsmen (186). Nous ne savons rien de la famille de Nekaomen.

Les dates de ces grands prêtres peuvent être fixées approximativement grâce à une stèle de l'an 34 de Darius ${ }^{1}$, sur laquelle il est question du fils d'Ahmèsmen, le père divin et prophète d'Arsaphès Semataouitefnakht (187,2-3). A ce moment, soit en 489 av. J.-C., Semataouitefnakht était adulte puisqu'il était père divin. En prenant provisoirement une durée de vingt-cinq ans par génération, nous situons Hekairâa adulte vers 589, et son fils Neferibrêmen adulte vers 564 . Nous pouvons admettre cette date parce qu'elle correspond à l'enseignement chronologique du nom propre. Neferibrêmen, dont le nom est composé avec celui de Psammétique II, est né au plus tôt pendant le règne de ce roi (594-588).

Hekairâa est donc né vraisemblablement sous Psammétique Ier et son pontificat peut dater des règnes de Nekao et Psammétique II. Ses descendants et lui-même portent les titres de "grand des chefs des artisans et prophète de Ptah" (186,7-8), qu'on retrouve chez les grands prêtres ptolémaïques.

Le fils de Hekairâa, Neferibrêmen (186,7), a pu être grand prêtre environ sous le règne d'Apriès.

Son fils, Nekao-méryptah $(\mathbf{1 8 6 , 6 )}$, a été père divin mais n'a pas accédé au pontificat. C'est pourquoi nous plaçons par hypothèse le grand

${ }^{1}$ E. CHASSINAT, Textes ..., dans Rec. Trav., XXI (1899), p. 66, n 24. 
prêtre Nekaomen (188a) ${ }^{1}$ entre Neferibrêmen et Ahmèsmen. Nekaomen, dont nous ignorons complètement les attaches familiales de sorte qu'il a pu aussi bien être apparenté à la famille de Hekairâa que le contraire, aurait rempli sa fonction sous Amasis.

\section{§ 71. AHMESMEN}

Le fils de Nekao-méryptah est né probablement vers le début du règne d'Amasis (568-525). Il a été instruit dans le temple de Ptah, comme Chedsounefertoum, et y a acquis bien du savoir. Une stèle du Sérapéum prouve qu'il était fort au courant des titres portés par les grands prêtres d'autrefois; il s'y dit en effet "chef des sem" (186,1), de ces "sem" particuliers au culte de Sokar sous l'Ancien Empire et supprimés lors de la réforme du pontificat sous la VIème dynastie; il s'intitule aussi "participant à la fête de $\hat{R}^{\prime}(\mathbf{1 8 6 , 1 )}$, comme les grands prêtres de l'Ancien Empire; il se qualifie de "préposé au diadème quand Ptah est orné" $(\mathbf{1 8 6 , 2})$ comme ses prédécesseurs de la VIème dynastie et du Moyen Empire. Il est aussi "sem et grand des chefs des artisans" (186,5), ainsi que "prophète de Ptah" $(\mathbf{1 8 6 , 1 )}$, de sorte que l'absence de l'ancien titre "prophète de Sokar" pourrait ne pas être fortuite dans cette titulature.

Le roi, qui était sans doute Amasis à ce moment, a intronisé Ahmèsmen "père divin de Ptah" et l'a nommé "directeur des artisans" (186,8-9); les deux verbes différents confirment nos conclusions sur le caractère civil de la fonction et du titre "grand des chefs des artisans"2.

Une stèle du Sérapéum qui est l'oeuvre d'un fils d'Ahmèsmen, le "père divin Hory" $(\mathbf{1 8 6 , 6 , 1 6 )}$, révèle un second nom du grand prêtre, Khnemibrê-saptah $\mathbf{( 1 8 7 , 2 5 )}$, formé comme le premier au moyen d'un nom du roi Amasis. Cette stèle, ainsi qu'une autre sur laquelle seul le nom Khnemibrê-saptah apparaît, fait connaître la famille d'Ahmèsmen.

${ }^{1}$ Nous ne connaissons qu'une statuette funéraire du "Hoherpriester von Memphis" Nekaomen conservée à Berlin. Comme pour Bakenrenef (ci-dessus, § 69), nous admettons que Nekaomen est intitulé "grand des chefs des artisans".

2 Voir ci-dessus, $\$ \mathbf{4}, \mathbf{1 1}$, et 20. 
Sekhmet-nefer, qui était fille d'un père divin et prophète d'Arsaphès (187,4-5), lui a donné trois fils: Semataouitefnakht, Hory et Ahmès $(\mathbf{1 8 7 , 1 - 8})$. Une autre femme, Setairetbint, lui a donné deux fils, Padihorresne et Pacherenmout (187,9-27). Une troisième enfin, Isetrechet a mis au monde son fils Psammétique. Les six fils d'Ahmèsmen sont tous pères divins.

Ahmèsmen était peut-être en fonction lorsque Cambyse conquit l'Egypte en 525 et que ses mercenaires pillèrent les temples, n'épargnant sans doute pas celui de Ptah à Memphis.

Nous ne savons malheureusement rien des grands des chefs des artisans depuis la fin de la dynastie saïte jusqu'à l'époque ptolémaïque ${ }^{1}$.

Les grands prêtres de la Basse Epoque se sont presque tous acquittés seulement des mêmes tâches memphites que leurs prédécesseurs de l'Ancien et du Moyen Empires. Aucun d'eux n'a été vizir. Seul, Chedsounefertoum, sous le règne énergique du fondateur de la XXIIème dynastie, a joué un rôle comparable à celui de quelques grands prêtres de Ptah au Nouvel Empire en assumant la direction de tous les clergés égytiens.

Au cours du siècle qui a précédé l'avènement de Psammétique Ier, les grands prêtres ont réagi contre la conception trop osirienne de Ptah qui avait prévalu aux siècles antérieurs et sont revenus à une notion plus ancienne de leur dieu.

\section{$\S$ APPENDICE AU CHAPITRE $X$}

Si nous nous fiions entièrement à la généalogie d'Achaout-akh², nous devrions attribuer la statue d'Horsaïset derrière un naos (165) à

${ }^{1}$ L'anecdote d'Hérodote, qui raconte que le prêtre d'Héphaïstos s'est opposé à ce que Darius élevât sa statue devant celle de Sésostris parce que le souverain perse aurait conquis une nation de moins que l'égyptien (Hérodote, II, 110), est sans doute une invention égyptienne; cf. H. KEES, Memphis, p. 664, 21-26 dans PAULY-WISSOWA, Real-Encyclopädie, XXIX.

2 Voir ci-dessus, $\$ 34$. 
Horsaïset $\mathrm{II}^{1}$ puisque le grand prêtre Horsaïset de la généalogie a pour fils et successeur un Dempedjet (?) tandis qu'Horsaïset de la statue est le père du grand prêtre Ankhef-ny-sekhmet. Nous ferions ainsi d'Horsaïset II le père d'un second grand des chefs des artisans Ankhefny-sekhmet.

Cette attribution n'est pas impossible parce que nous ne connaissons pas le successeur d'Horsaïset $\mathrm{II}^{2}$. On serait alors conduit à admettre que le mariage d'Horsaïset II et d'une "grande des concubines" $(\mathbf{1 6 5 , 1 , 3 )}$ et que la naissance de leur fils Ankhef-ny-sekhmet seraient postérieurs à la seconde stèle du Sérapéum de l'an 2 de Pamy (179), qui nomme deux femmes et deux fils d'Horsaïset II; les deux fils Takelot et Ankhpadiiset, n'ont aucun titre sur ce monument.

Mais une généalogie n'est pas toujours une source digne de foi. Dans le cas présent, nous avons déjà constaté la difficulté de faire entrer toutes les générations de la généalogie comprises entre Paoupaou et Chedsounefertoum dans le temps écoulé entre les règnes de Siamon et Chechanq $\mathrm{Ier}^{3}$. En admettant que la généalogie contient des indications inexactes entre Paoupaou et Horsaïset I, nous pouvons attribuer la statue d'Horsaïset derrière un naos à Horsaïset I.

L'existence d'un second grand prêtre nommé Ankhef-ny-sekhmet, qui aurait succédé à Horsaïset II et serait contemporain du règne de Chechanq $\mathrm{V}$, dernier roi de la XXIIème dynastie, nous paraît donc discutable.

${ }^{1}$ Voir ci-dessus, § 68.

2 La liste des grands prêtres des XXIème et XXIIème dynasties établie par E. BRUGSCH sur ces bases (Thesaurus, p. 1255-1256) donne à Horsaiset II un fils et successeur en la personne d'Ankhef-ny-sekhmet. Nous ne voyons pas pourquoi l'auteur accompagne cette filiation d'un point d'interrogation puisqu'il admet sans réserve les données de la généalogie d'Achaout-akh.

${ }^{3}$ Voir ci-dessus, § 34. 
H. Brugsch inclut aussi dans sa liste le fils d'Osorkon, Takelot. Or celui-ci est "setem et prophète de Ptah" (183,5-6), mais ne porte pas le titre de grand des chefs des artisans ${ }^{1}$. Nous ne le considérons donc pas comme un grand prêtre ${ }^{2}$.

${ }^{1}$ Voir au sujet de ce Takelot, ci-dessus, $\$$ 65. Il ne faut pas le confondre avec cet autre sem de Ptah Takelot qui est fils de Padiiset et demi-frère de Peftjaouaouibastet et Horsaïset II (§ 68).

${ }^{2}$ Voir ci-dessus, $§ 5$, fin. 



\section{H A P I T R E XI}

\section{LES GRANDS PRETRES DE L'EPOQUE PTOLEMAIQUE}

\section{§ 73. NESQED-PETOBASTIS}

Une tradition postérieure rapporte qu'Alexandre se serait fait couronner roi dans le temple de Ptah à Memphis ${ }^{1}$. S'il s'agit d'un événement réel, le récit en aura été fait par le grand prêtre d'alors ${ }^{2}$, mais nous ignorons tout de ce personnage, de même que de ses successeurs jusqu'à Nesqed, contemporain de Ptolémée II et grand prêtre dans le courant du troisième siècle avant $\mathrm{J}$.-C.

La date de naissance de ce grand des chefs des artisans, appelé aussi Petobastis $(\mathbf{1 9 0 , 6 )}$ peut être fixée au plus tard vers 310 , soit une vingtaine d'années avant la venue au monde de son fils Anemher. Le père de Nesqed, qui s'appelait peut-être Anemher, ne semble pas avoir rempli une fonction quelconque ${ }^{3}$; sa mère se nommait Renpet-nefer $(\mathbf{1 9 0 , 7 )}$.

$\mathrm{Au}$ commencement de sa carrière, Nesqed a occupé le poste secondaire de prophète du sanctuaire de Ramsès II dans le temple de Memphis $(\mathbf{1 9 0 , 2 )}$. La comparaison de sa titulature $(\mathbf{1 9 0 , 2 - 7 )}$ et de sa biographie (190,10-13) laisse entendre qu'il est resté dans cette fonction jusqu'en l'an 23 d'un roi dont le nom n'est pas conservé ou n'a jamais

${ }^{1}$ H. KEES, Memphis, p. 665, 42-57, dans PAULY-WISSOWA, Real-Encyclopädie, XXIX.

2 Le roman d'Alexandre l'appelle "l'archiprophète du temple dans Memphis" (Pseudo-Callisthène, III, 34).

${ }^{3}$ Voir ci-dessus, $\$ 35$. 
figuré dans sa biographie. Toutefois, comme Nesqed a été nommé prophète d'Arsinoé $(\mathbf{1 9 0 , 1 0 - 1 2})$ après cette date et que le culte d'Arsinoé ne paraît pas avoir été créé avant Ptolémée II, c'est à ce roi que se rapporte la mention de l'an 23 , soit 264-263 av. J.-C. 1 .

En 290, ou plus tôt, Nesqed a épousé Nefersebek, dont il eut un fils, Anemher, en 289. Plus tard, il épousa Renpet-nefer, qui lui donna un autre fils, Khonsouiou. Nous ignorons la date de naissance de Khonsouiou, mais comme il est mort après son père, qualifié de juste de voix sur la stèle funéraire de son fils $(\mathbf{1 9 1 , 9 )}$, et sans avoir été grand prêtre, il était donc le cadet d'Anemher.

Nesqed avait en tout cas plus de quarante-cinq ans et était déjà grandpère, puisque son petit-fils Teos était né trois ou quatre ans auparavant, lorsque Ptolémée II jeta les yeux sur lui $(\mathbf{1 9 0 , 1 0 )}$ et le tira de la situation qu'il occupait depuis de nombreuses années. Dès ce moment, l'ascension du prophète du sanctuaire de Ramsès II fut rapide. Le roi le chargea successivement de plusieurs fonctions importantes, entre autres des .cultes memphites de Philotéra et d'Arsinoé; puis il le mit à la tête des clergés memphites en le faisant "grand des chefs des artisans" $(190,10-13)$.

Il est probable qu'au début de l'époque ptolémaïque les prêtres égyptiens ne montrèrent pas une attitude conciliante aux souverains étrangers, qui vivaient à Alexandrie d'une manière au moins différente des indigènes, si ce n'est plus tout à fait la même que dans leur pays d'origine, et qui parlaient grec. Ptolémée II aurait alors choisi Nesqed parce qu'il n'appartenait pas à une famille de prêtres. Il s'assurait ainsi le zèle du prêtre memphite d'autant plus sûrement que celui-ci était resté plus longtemps dans la même situation. Cependant, avant de le nommer grand prêtre, il l'a favorisé en améliorant peu à peu sa position et ses revenus afin de se l'attacher complètement.

Nous pouvons conjecturer que le pontificat de Nesqed n'a pas duré de nombreuses années. D’une part, en effet, le récit de ses funérailles suit

${ }^{1}$ H. GAUTHIER, Rois, 4, p. 229, n 22: "En l'an 23 (de Ptolémée II ?)". L'auteur ne commente pas son point d'interrogation. 
immédiatement celui de sa nomination (190,13 sq.); d'autre part, Nesqed est mort avant son fils Khonsouiou, qui est lui-même décédé en l'an 37 de Ptolémée $\mathrm{II}^{1}$, soit en $249-248^{2}$.

\section{§ 74. ANEMHER}

A la mort de Nesqed, Ptolémée II n'avait plus les mêmes raisons qu'autrefois de faire appel à un prêtre issu d'un milieu qui ne fût pas ecclésiastique puisqu'il avait désormais une famille memphite à sa dévotion. C'est pourquoi il nomma simplement le fils aîné de Nesqed.

Anemher était né le 3 du troisième mois de Peret, ou Phamenoth, en l'an 16 de Ptolémée Ier, soit en mai $289^{3}$. A l'âge de vingt ou vingt et un ans, il épousa Harunchis, que les monuments de ses enfants qualifient de "musicienne accomplie de Ptah" $(\mathbf{1 9 3 , 1 1 )}$, de "grande musicienne de Ptah" $(\mathbf{1 9 4 , 1 2 )}$ ou de "musicienne accomplie de Sekhmet la grande et l'aimée de Ptah" $(\mathbf{1 9 7 , 2 , 1 3 )}$, et aussi de "belle de forme" $(\mathbf{1 9 7 , 1 3 , 2 0})$, qui est le titre ptolémaïque de l'épouse du grand prêtre placée à la tête du clergé féminin de Ptah (205). En 267, Harunchis mit au monde un fils, Teos, et plus tard, à une date que nous ne pouvons fixer, elle eut un autre fils, Harmachis.

Auparavant Anemher avait été intronisé père divin, commençant ainsi une carrière longue et remplie dont la première partie s'est déroulée pendant la vie de son père. Il est fort probable qu'Anemher recueillit sa part de faveur de Ptolémée II dès que celui-ci eut distingué Nesqed. L'une ou l'autre des fonctions mentionnées sur la stèle d'Anemher lui aura été attribuée dès l'an 23 du roi, mais il nous paraît impossible de la déterminer, car Anemher n'a pas énuméré tous ses titres dans l'ordre

${ }^{1}$ Le texte démotique de la stèle de Khonsouiou (191) commence par la date de sa mort, "L'an 37, 27 Thot, du roi Ptolémée, fils de Ptolémée, du dieu Sôter, toujours vivant" et finit par la mention du seul jour de sa naissance, "14 Phamenoth" (E. BRUGSCH, Thesaurus, p. 907). Sur l'année de mort, cf. H. GAUTHIER, Rois, 4 (1916), p. 232 et note 1.

2 En calculant d'après H. GAUTHIER, Rois, 4, p. 222, note 4, selon lequel Ptolémée II compte ses années à partir de l'abdication de son père, vers fin 285.

${ }^{3}$ H. GAUTHIER, Rois, 4 (1916), p. 216, note 1. 
où il les a reçus. Il y a en effet au début de sa titulature ceux de prophète des Evergètes et de prophète des Philopators $(192,3)^{1}$; ce sont des charges dont il n'a pas pu être investi avant 246 et 221 , dates respectives d'avènement de Ptolémée III et Ptolémée IV, et Anemher avait soixante-huit ans en 221 ! D'autre part, sa titulature n'est pas établie selon l'ordre inversement chronologique, puisque ses plus hautes fonctions, soit celles de grand des chefs des artisans et de ministre des cultes, ne sont citées qu'à la fin $(\mathbf{1 9 2 , 1 0})$.

Anemher a succédé à son père comme grand des chefs des artisans entre 260 et 250 environ, avant le décès de son frère Khonsouiou.

En 245, au cours de la seconde année de règne de Ptolémée III Evergète, un soulèvement éclata en Egypte et força le roi à abandonner sa campagne contre Seleucus. Il ne serait pas étonnant que la révolte n'ait pas été étrangère à la décision que le roi prit de confier le ministère des cultes à Anemher. Mais, comme on ne connaît pas la date à laquelle le grand prêtre devint "directeur des prophètes de tous les dieux et dẹesses de Haute et Basse-Egypte" $(192,10 ; 193,10)$, on pourrait aussi supposer que la nomination d'Anemher eut lieu à une date proche du synode de Canope.

Au début de 238, Anemher, accompagné sans doute de ses fils Teos et Harmachis, se rendit à Canope. En qualité de grand prêtre de Memphis, et peut-être de ministre des cultes, il prit part aux délibérations qui aboutirent, entre autres résultats, non seulement à la réforme du calendrier mais aussi à la création d'une cinquième classe sacerdotale, ou "phylé", nommée d'après Evergète.

Plus tard, Anemher perdit sa femme, qui est qualifiée de "juste de voix" sur la stèle de son fils Teos $(\mathbf{1 9 3 , 1 1})$, et résolut de mettre fin à sa carrière de grand des chefs des artisans. Nous ne savons pas du tout si le chagrin d'Anemher a joué un rôle dans sa décision; il est par contre très probable que sa tâche de ministre des cultes lui parut suffisante.

${ }^{1}$ Litt:: "serviteur des dieux Evergètes, serviteur des dieux Philopators"; depuis Nesqed $\mathbf{( 1 9 0 , 6 )}$, les grands prêtres ptolémaïques emploient souvent le terme "serviteur" au lieu de "serviteur du dieu" (= prophète) devant un nom de divinité, jugeant sans doute que le mot "du dieu" est alors superflu. 
Ptolémée III nomma alors Teos grand des chefs des artisans. Cela se passait avant 223, année de la mort de Teos. Anemher avait ainsi soixante-cinq ans au plus lorsqu'il cessa son activité de grand prêtre. Il conserva le ministère des cultes en tout cas jusqu'à la mort de son fils, comme l'indique une épithète de Teos. Ce dernier, qui était "à la tête de tous les ouab et prophètes dans les temples de Haute et Basse-Egypte" $(\mathbf{1 9 3 , 7})$, a aidé son père dans sa tâche administrative ${ }^{1}$, mais n'a pas été lui-même ministre des cultes. Comme il est décédé quelques années avant son père, Anemher n'a pas été qualifié de juste de voix sur la stèle de Teos, mais a été gratifié du souhait fort insolite pour un autre que le roi de "vie, santé, force" $(\mathbf{1 9 3 , 1 1 )}$

Anemher ne s'est pas chargé à nouveau du pontificat après le décès prématuré de Teos, mais il l'a laissé aux mains de son second fils, Harmachis. C'est ainsi du moins que nous expliquons le titre le plus fréquemment porté par Anemher, soit celui de sem. Nous avons déjà remarqué à ce propos $^{2}$ que tous les monuments qui mentionnent Anemher sont postérieurs au pontificat de Teos: stèles funéraires de Teos (193), d'Anemher (192) et d'Harmachis (194; 195), sarcophage d'Harmachis (197), statues de Psenptaïs fils du "grand des chefs des artisans Harmachis" (198); après la nomination de son fils, Anemher n'était plus "grand des chefs des artisans" mais il est demeuré "père divin et prophète" ainsi que sem, comme il est intitulé au premier registre de sa stèle $(\mathbf{1 9 2 , 1 )}$. Puisque l'état de "père divin" s'acquérait par "intronisation"3, on peut supposer qu'il en était de même pour celui de sem. L'absence complète d'indication sur la façon dont les grands prêtres devenaient sem permet en tout cas cette conjecture.

Sous le pontificat d'Harmachis, après l'avènement de Ptolémée IV Philopator, Anemher a encore été chargé du culte des dieux Philopators $(\mathbf{1 9 2 , 3 )}$. C'est sans aucun doute la dernière fonction qui lui a été attribuée.

\footnotetext{
${ }^{1}$ Voir ci-dessus, $\$ 28$ et $\mathbf{8}$, p. 78 et p. 23 , note 1.

2 Voir ci-dessus, $\$ 31$, p. 85, note 3.

${ }^{3}$ Voir ci-dessus, $\$ 71$.
} 
Le père divin et sem, ancien grand des chefs des artisans et ministre des cultes Anemher est mort âgé de soixante-douze ans, un mois et vingttrois jours en date du 26 du quatrième mois de Peret, ou Pharmouthi, de l'an 5 de Ptolémée IV Philopator (192,13-14), soit au début de l'été 217 av. J.-C. .

\section{$\S 75$. TEOS}

Le texte hiéroglyphique de la stèle de Teos n'indique que la durée exacte de sa vie: 43 ans, 6 mois, 29 jours $(\mathbf{1 9 3 , 1 1 )}$. Les trois lignes démotiques écrites au bas du monument sont plus explicites. Nous y apprenons que le "grand des artisans"2 Teos, fils du setem Anemher et d'Harunchis, est né le 29 du troisième mois de Chemou, ou Epiphi, en l'an 18 (sic !), qu'il a "quitté sa maison" le 24 du deuxième mois de Peret, ou Mechir, en l'an 24 (sic !), enfin qu'il a "reposé dans Ro-setaou" le 6 du premier mois de Chemou, ou Pachons, en l'an 24 (sic !), après avoir vécu 43 ans, 6 mois et 29 jours $^{3}$. Remarquons en passant que soixante-douze jours se sont écoulés de la mort à l'ensevelissement de Teos.

Bien que les noms des rois ne soient pas cités après les mentions des années, nous pouvons les déterminer grâce à la stèle d'Anemher et aux durées de règnes des premiers Ptolémées. La stèle d'Anemher dit que ce grand prêtre est né en l'an 16 de Ptolémée Ier $(\mathbf{1 9 2 , 1 2 - 1 3 )}$ et ses données chronologiques prouvent que les durées de règnes étaient évaluées en années pleines ${ }^{4}$.

Teos n'a pu venir au monde que sous Ptolémée II Philadelphe qui a régné 38 ans ou sous Ptolémée III Evergète qui a régné 25 ans. Ptolémée IV, avec son règne de 17 ans, ne peut pas être pris en

${ }^{1}$ Une ligne et demie en démotique, au bas de la stèle d'Anemher, se traduit: "Le prêtre sem, Anemho, que Na-nefer-sebek a enfanté. Sa naissance: 4 (sic !) Phamenoth. Il a quitté sa maison le 26 Pharmouthi. Durée de vie: 72 ans, 1 mois, 23 jours" (d'après W. SPIEGELBERG, Demotische Miscellen, dans Rec. Trav., XXX (1908), p. 147-148).

2 "Grand des artisans" est le correspondant démotique de "grand des chefs des artisans"; voir ci-dessus, § 4.

${ }^{3}$ W. SPIEGELBERG, Demotische Miscellen, dans Rec. Trav., XXX (1908), p. 149.

${ }^{4}$ M.L. STRACK, Die Dynastie der Ptolemäer, 1897, p. 159 sq. 
considération, d'autant qu'Anemher aurait alors eu quatre-vingt-cinq ans passés ! Comme Teos est né le 29 du 11ème mois de l'an 18, si nous supposons que c'est sous Ptolémée Philadelphe, il aura vécu sous ce roi la différence entre 38 années pleines et 17 ans, 10 mois et 29 jours, soit 20 ans, 1 mois et 1 jour. Comme il est mort le 24 du 6ème mois de l'an 24 , ce ne peut être que sous le roi suivant et il aura alors vécu 23 ans, 5 mois et 23 jours sous Ptolémée Evergète. En ajoutant les deux périodes de vie, on obtient 43 ans, 6 mois et 24 jours. La différence de 5 jours avec les données de la stèle provient de la façon dont nous avons effectué notre calcul. Si nous procédions comme Anemher a dû le faire après la mort de son fils ${ }^{1}$, nous compterions 43 ans de la naissance au 29ème jour du 11ème mois de l'an 23 de Ptolémée III Evergète et, en supputant les mois et jours qui séparent cette date de celle de la mort, nous ferions entrer dans notre total les 5 jours épagomènes de l'an 23.

Il est inutile que nous tentions le calcul de la durée de vie de Teos en supposant qu'il est né sous Evergète: la durée de son règne n'étant pas la même que celle de Philadelphe, nous arriverions à un résultat différent des 43 ans indiqués par la stèle.

Teos est donc né à la fin du 11ème mois de l'an 18 de Ptolémée II, soit au commencement de l'automne 267 . Son père avait alors vingt-deux ans passés ${ }^{2}$. Teos était encore un enfant lorsque le roi prêta attention à son aïeul Nesqed, et un adolescent quand son père fut nommé grand des chefs des artisans. Elevé dans le temple de Ptah, il y étudia si bien qu'il sut "combler les lacunes dans les livres sacrés" $(\mathbf{1 9 3 , 5 )}$; il "connaissait les secrets de l'atelier d'orfèvrerie" (193,5-6); il n'y avait "rien dont le nom lui fût caché dans tout ce qui est sacré dans le temple de Ptah" $(\mathbf{1 9 3 , 6 )}$.

${ }^{1}$ Ch. MAYSTRE, Le compte des épagomènes dans les chronologies individuelles, dans $R d E 7$ (1950), p. 85-88.

2 En enlevant aux 20 années que le canon donne à Ptolémée Ier le temps écoulé jusqu'à la naissance d'Anemher le 3ème jour du 7ème mois de l'an 16, nous trouvons qu'Anemher a vécu 4 ans, 5 mois et 27 jours sous Ptolémée Ier; en ajoutant à cette durée le temps de Ptolémée II écoulé jusqu'à la naissance de Teos, soit 17 ans, 10 mois et 29 jours, nous obtenons l'âge d'Anemher à la naissance de son fils: exactement 22 ans, 4 mois et 26 jours. 
Anemher a sans doute aucun formé son fils avec la pensée qu'il lui succéderait un jour. Il l'aura associé de bonne heure aux joies et soucis de son existence. Lorsque le moment vint où il décida d'abandonner son poste de grand des chefs des artisans, il put faire nommer Teos à sa place.

Teos a secondé son père dans sa tâche de ministre des cultes, mais il n'a pas été lui-même investi de cette fonction. La mort est venue interrompre sa carrière au printemps 223 .

Il nous semble difficile d'identifier le fils d'Anemher et le Teos divinisé et assimilé à Thot pour lequel Ptolémée VII Evergète II construisit un sanctuaire au Kasr el-Agouz ${ }^{1}$, à l'ouest de Thèbes, dans la seconde moitié du deuxième siècle av. J.-C. parce que le fils d'Anemher n'a vécu qu'un siècle avant la construction du temple et aussi parce qu'il n'est pas du tout certain que le Thot-Teos ou Thos-Setem du Kasr el-Agouz soit une combinaison de Thot et d'un grand prêtre de Ptah appelé Teos, comme K. Sethe l'a pensé $e^{2}$ En effet, rien ne nous dit que le "Setem" Teos du Kasr el-Agouz a été "grand des chefs des artisans"'. On pourrait même se demander si ce Teos divinisé n'est pas identique au roi de la XXXème dynastie qui a porté ce nom, puisque Tefnakht a aussi été "sem de Ptah"4, mais pas grand prêtre.

\section{§ 76. HARMACHIS}

Les monuments d'Harmachis ne donnent ni la durée de vie du grand prêtre, ni ses dates de naissance et de mort. Mais celles-ci se laissent fixer d'autre manière.

${ }^{1}$ D. MALlET, Le Kasr el-Agoûz, 1909 (Mém. I.F.A.O, tome 11).

2 K. SETHE, Imhotep, der Asklepios der Ägypter, p. 8-10, dans Untersuchungen ..., 2 (1902), p. 100-102.

${ }^{3}$ De même, l'histoire démotique du "setme Ptahhotep" n'est peut-être pas celle d'un grand prêtre, comme l'a pensé W. SPIEGELBERG, Die demotischen Papyrus (Catal. Caire) 1908, p. 145.

${ }^{4}$ Stèle de Pianchi, 1. 20. 
Le père d'Harmachis, Anemher, avait été "scribe de Ptah de toutes les troisièmes phylai du temple de Memphis" (192,4-5). Selon la coutume et même peut-être la règle ${ }^{1}$, Harmachis aurait dû appartenir aussi aux troisièmes phylai. Il est fort possible qu'il en ait fait partie avant 238 av. J.-C. Cette année-là, le décret de Canope créa, en addition aux quatre phylai qui existaient dans les temples égyptiens, une cinquième phylê, formée de tous ceux qui étaient devenus prêtres depuis l'avènement de Ptolémée III Evergète en 246 ainsi que de ceux qui le devenaient au cours de l'année du décret. Harmachis est donc entré dans la carrière religieuse au cours des années 246 à 238 , comme W. Otto l'a remarqué ${ }^{2}$. En supposant qu'il avait une vingtaine d'années à ce moment, sa naissance se placerait entre 266 et 258 approximativement.

D'autre part, le fils aîné d'Harmachis a été prophète des Philopators (195), ainsi d'ailleurs que des Evergètes. L'avènement de Ptolémée IV Philopator date de 221. Si Harmachis avait un fils adulte à ce moment, il était lui-même né une quarantaine d'années auparavant. Il est ainsi très probable qu'Harmachis est né un petit nombre d'années après Teos.

Harmachis a épousé Nefertiti dont il a eu d'abord un fils, Nesqed (195), puis une fille (196) et probablement son second fils, Psenptaïs (198). Bien que nous n'ayons pas de témoignage sur la date de son accession au pontificat, nous pensons que cet événement s'est passé après la mort de Teos, en 223, sous Ptolémée III Evergète, puisqu'Anemher, en rédigeant alors la stèle de Teos, donne plus d'importance à sa qualité de sem qu'à son titre de grand des chefs des artisans $(\mathbf{1 9 3 , 1 0 )}$. La nomination d'Harmachis lui aura valu, comme à son frère, les charges et bénéfices du service de la "Fenêtre de l'apparition" $(194,4-6 ; 193,8-9)$ qui avaient appartenu déjà à leur père $(192,9)^{3}$.

Lorsque Teos était devenu grand prêtre, Anemher avait conservé sa fonction de prophète d'Arsinoé-Philadelphe, que son père avait exercée avant lui. Aucun titre de Teos ne se rapporte en effet à ce culte

\footnotetext{
${ }^{1}$ W. OTTO, Priester und Tempel im hellenistischen Ägypten, 1905, p. 203 sq.

2 ibidem, p. 31.

${ }^{3}$ Voir ci-dessus, § 14.
} 
dynastique. C'est pourquoi il est possible qu'Harmachis n'ait commencé à servir la déesse qu'après la mort d'Anemher, quoique ce ne fût pas comme prophète $(\mathbf{1 9 7 , 1 1 - 1 2})$.

Au cours de son pontificat, Harmachis a perdu sa fille (196) et peutêtre son fils aîné, Nesqed, qui n'a pas été grand prêtre à notre connaissance. Harmachis l'avait fait représenter en officiant sur une stèle funéraire qui a pu être fabriquée entre la mort de Teos et celle d'Anemher puisque ce dernier y fait une libation à Osiris et qu'Harmachis y est grand prêtre (195). Le texte de ce monument n'a pas été complété à la mort d'Harmachis par les données chronologiques pour lesquelles on avait laissé des espaces en blanc.

Le sarcophage d'Harmachis a été fait aussi pendant la vie du grand prêtre; celui-ci y a, en effet, laissé un espace "en blanc" pour ses titres de prophète des cultes dynastiques (197,11,note). Comme Harmachis y mentionne qu'il est au service d'Arsinoé, le monument pourrait être postérieur à la mort d'Anemher. Enfin, dans l'inscription symétrique à celle qui contient un espace en blanc, Harmachis est prophète des dieux Adelphes, des Evergètes, des Philopators et des Epiphanes $(\mathbf{1 9 7 , 1 1 )}$. Cette indication nous permet de dater à peu près la mort d'Harmachis. Comme Ptolémée V Epiphane a été fiancé à Cléopâtre Ière en 196-195 et qu'il l'a épousée trois ans plus tard ${ }^{1}$, Harmachis aura vécu au moins encore pendant la première décade du second siècle av. J.-C.

\section{$\S$ 77. PSENPTAIS I}

En étudiant la généalogie de Petobastis ${ }^{2}$, nous avons conclu que l'existence d'un grand prêtre Psenptaïs, fils du grand prêtre Harmachis, nous paraît probable.

Le fils cadet d'Harmachis était représenté par deux statues dont plusieurs fragments ont été découverts à Alexandrie (198), d'ailleurs

\footnotetext{
${ }^{1}$ H. GAUTHIER, Rois, 4 (1916), p. 286 et note 2.

${ }^{2}$ Voir ci-dessus, $§ 35$.
} 
pêle-mêle avec d'autres objets ${ }^{1}$.

Dans l'inscription dorsale de ses monuments, Psenptaïs énumère ses aïeux qui ont été grands prêtres: son père, Harmachis, son aïeul, Anemher, enfin son bisaïeul; une partie des titres et le nom de Nesqed ont disparu. Cette perte nous empêche de contrôler la supposition suivante: comme ni Harmachis ni Anemher ne sont qualifiés de justes de voix, les statues ont peut-être été sculptées entre les décès de Teos et d'Anemher. Dans ce cas, Psenptaïs aurait eu peut-être une vingtaine d'années ${ }^{2}$ au plus tard en 217 et serait né très approximativement vers 237 .

Or le Sérapéum d'Alexandrie a été fondé dans la seconde moitié du IIIème siècle av. J.-C. par Ptolémée III Evergète ${ }^{3}$, dont le règne a pris fin en 222. Nous pouvons donc tenir pour assuré que les statues ont bien été destinées au temple alexandrin et qu'elles rappelaient un séjour de Psenptaïs à Alexandrie ${ }^{4}$. On peut alors se demander si le titre "supérieur des secrets du temple de Ptah de Ro-setau, d'Osorapis de Ro-qedit et du domaine du coffre d'Anubis qui est sur sa montagne" $(\mathbf{1 9 8 , 2})^{5}$ ne se rapporte pas aux cultes alexandrins de Ptah, Sérapis et Anubis. Il est vrai que la stèle Harris distingue nettement la région de la nécropole

${ }^{1}$ E. BRECCIA, Les fouilles dans le Sérapéum d'Alexandrie, dans Annales du Service des Antiquités, 8 (1907), p. 63.

${ }^{2}$ E. BRECCIA, op. cit., p. 64, écrit que le morceau de tête d'une des statues "montre un homme âgé, coiffé d'une sorte de bonnet collant à la tête chauve, mais pourvu d'une grosse et longue natte ...". Nous n'admettons pas sans réserve l'âge ainsi attribué à Psenptaïs et pensons qu'E. Breccia a peut-être pu être trompé par le serre-tête comme d'autres l'ont été par la statue de Rênefer coiffé de la même manière (voir ci-dessus, $\S 38)$.

${ }^{3}$ P. JOUGUET, dans Comptes rendus de l'Académie des Inscriptions et Belles-Lettres, Paris 1946, d'après Chronique d'Egypte, 44 (juillet 1947), p. 297; A. ROWE, Discovery of the famous temple and enclosure of Serapis at Alexandria, dans Supplément aux Annales du Service des Antiquités, Cahier n², 1946, p. 9.

${ }^{4}$ Les statues ne fournissent aucun renseignement qui permette de préciser la date de fondation du Sérapéum d'Alexandrie.

${ }^{5}$ Les titres précédents, jusqu'à "prophète de Ptah seigneur des aliments du temple de Ptah qui est au sud de son mur" $(\mathbf{1 9 8 , 1 - 2 )}$ sont, au moins en partie, bien memphites; Anemher avait déjà été "serviteur de Ptah seigneur des aliments du temple de Memphis" $(192,7)$. 
memphite appelée Ro-qedit $(\mathbf{2 0 0 , 3 , 7 )}$ de Râ-qedit $\mathbf{( 2 0 0 , 1 9 ) , ~ s o i t ~ l a ~}$ Râkôtis des Grecs, mais comme il s'agit d'un monument memphite, nous inclinons à admettre que Ro-qedit des statues alexandrines de Psenptaïs I doit être lue Râ-qedit. Nous adoptons donc la traduction de MASPERO': "...chef du secret du temple d'Osor-hapi de Râkôtis, c'est-àdire du Sérapéum d'Alexandrie..."

Il nous paraît également difficile d'accepter et de repousser l'identification de Psenptaïs II et du personnage appelé Psintaès ${ }^{2}$ dont les papyri grecs du Sérapéum de Memphis nous apprennent l'existence en l'an 19 de Ptolémée VI Philomêtor, soit 163-162 av. J.-C. Ce Psintaès portait le titre d'épistate des temples" et résidait probablement à Memphis. L'épistate et l'archiereus du Sérapéum lui étaient subordonnés ${ }^{3}$.

A propos de Psintaès, nous pouvons nous demander s'il y a eu un terme grec correspondant à "grand des chefs des artisans" ou, plutôt, à sa forme usuelle sous les Ptolémées de "grand des artisans". Comme ce titre ne se rapportait alors qu'à une fonction secondaire du grand prêtre, car celui-ci, en fait d'artisans, ne dirigeait sans doute plus guère que ceux du temple de Ptah, nous penchons vers une réponse négative. Il est donc possible que le grand prêtre de Memphis ait été désigné en grec par le titre d'épistate des temples.

\section{§ 78. PETOBASTIS ET PSENPTAIS}

La généalogie de Petobastis ${ }^{4}$ est notre seule source sur les deux successeurs de Psenptaïs I, son fils Petobastis et son petit-fils Psenptaïs. Leurs degrés de parenté avec le rédacteur de la généalogie garantissent l'existence de ces deux grands prêtres, dont le premier a pu naître dans le premier quart, et le second vers le milieu du second siècle av. J.-C.

${ }^{1}$ Dans E. BRECCIA, Les fouilles dans le Sérapéum d'Alexandrie, dans Annales du Service des Antiquités, 8 (1907), p. 66.

${ }^{2}$ H. RANKE, Die ägyptischen Personennamen, 1935, p. 118,18, ne donne que deux formes grecques du nom hiéroglyphique $\mathrm{P}(\mathrm{a})$-cher-en-ptah: Psenephthas et Psenptaïs.

${ }^{3}$ U. WILCKEN, Urkunden der Ptolemäerzeit, I, 1927, p. 44-45.

${ }^{4}$ Voir ci-dessus, § 35. 


\section{§ 79. PETOBASTIS II ${ }^{1}$}

Il y a plus de soixante ans que $\mathbf{J}$. Krall a étudié la stèle démotique de Petobastis II et qu'il a publié ce qu'il avait pu déchiffrer sur ce monument dont les vingt-six lignes écrites à l'encre noire étaient déjà très effacées ${ }^{2}$. La stèle était dédiée à Petobastis, fils de Psenptaïs et de Bérénice. On y lisait une date, que J. Krall n'a pas reproduite mais qui est peut-être la première de celles que J.F. Champollion avait remarquées sur ce monument ${ }^{3}$, puis les titres du défunt qui occupaient huit lignes. Ici, nous traduisons ce que J. Krall a lu sur la stèle: "Petubastis était entre autres - je ne donne que ce qui se laisse lire sûrement - scribe de la double maison ${ }^{4}$, scribe des comptes du roi, scribe de Ptah et d'Arsinoé-Philadelphe dans les quatrième et cinquième phylê tout entières, scribe des décrets et des rouleaux de papyrus, scribe de la double maison, prophète (?) des archives. A la ligne 8 il s'appelle prophète d'Horus de la niche de fête ${ }^{5}$, prophète des dieux de la niche de fête, premier seschet ${ }^{6}$ dans la Maison de Ptah, premier seschet dans Ta-tekhen ${ }^{7}$, celui qui connaît les secrets dans ... ${ }^{8}$, premier seschet d'Osiris, grand Setem."

Le texte continue par des données sur la vie de Petobastis: "(lignes 9 et 10) Le roi Ptolémée, ... Alexandre, le dieu Philomêtor, le fit entrer dans

${ }^{1}$ Le premier Petobastis est Nesqed-Petobastis; nous ne numérotons pas le père et l'aieul de Petobastis II parce que nous ne connaissons pas de monument qui leur ait appartenu.

2 J. KRALL, Studien zur Geschichte des alten Ägypten, dans Sitzungsberichte der Wiener Akad., philosophische-historische Klasse, vol. 105 (1883), p. 373-379.

3 ibidem, p. 373, note 1: Champollion a lu: l'an 14, le 18 du Mechir du roi Ptolémée et de la reine Cléopâtre, Dieux Evergètes. Cette date, qui correspond à l'an 156, indiquerait-elle la naissance du père de Petobastis ??

4 En note, les mots hiéroglyphiques "scribe de Pharaon".

${ }^{5}$ En note, J. Krall indique le titre hiéroglyphique d'Anemher "prophète de l'Horus de la Fenêtre de l'apparition".

${ }^{6}$ C'est-à-dire "supérieur des secrets".

${ }^{7}$ J. Krall remarque que Ta-tekhen, la "terre cachée" a pour correspondant Ro-setaou chez Anemher.

${ }^{8} \mathrm{~J}$. Krall indique ici le titre hiéroglyphique correspondant d'Anemher, "qui connâ̂t les secrets de la Chambre cachée". 
le temple ${ }^{1}$. Il but devant le roi. Il (= le roi) lui donna ... d'or, les bandes et la peau (ligne 11) (en tant que) prêtre de Ptah à la fête (?) ... Il mit son ornement d'or sur sa tête comme à ses pères dans (?) sa dixième année jusqu'à (?) (sa) vingt-huitième année". Les signes suivants étaient illisibles et en partie complètement effacés.

Les lignes 12 à 19 contiennent la généalogie que nous avons examinée d'autre part ${ }^{2}$. Les lignes 20 à 26 comprennent d'abord des indications chronologiques: "An 5 du roi (Ptole)mai(os) ..... Trupaïn", puis nous apprenons que Petobastis "est entré dans sa demeure en l'an 5, Mechir, jour 5 du roi Ptolémée .... (et de la) reine ...... des dieux Philopators et Philadelphes ${ }^{5}$ (lignes 21-22), qu'il était né en l'an 50, Athyr, jour 11 du roi ......, des dieux Evergètes ${ }^{6}$, enfin que sa durée de vie sur terre a été de 44 ans, 2 mois, ... jours". Des remarques presque illisibles sur l'embaumement et l'ensevelissement terminent la stèle.

En se servant des données du canon des rois, soit cinquante-trois ans pour le règne d'Evergète II et trente-six pour ceux de Soter II et Alexandre Ier, J. Krall a non seulement vérifié le bien-fondé du canon mais établi la durée exacte de la vie de Petobastis: 44 ans, 2 mois et 24 jours ${ }^{7}$. Comme la date de la mort de Petobastis correspond au 13 février 76 av. J.-C. ${ }^{8}$, ce grand prêtre était né à fin novembre 121 . Telles sont les données chronologiques claires de la stèle.

Mais les autres indications du même genre sont plutôt obscures. A qui appartiennent la dixième année et la vingt-huitième mentionnées à propos de "l'entrée dans le temple" de Petobastis ? J. Krall y voit des âges de Petobastis: dans ce cas, celui-ci serait "entré dans le temple" à neuf ans et resté dans les mêmes conditions jusqu'à vingt-sept ans; aurait-

1 J. Krall ajoute: c.à.d. il l'intronisa prêtre.

2 Voir ci-dessus, $\$ 35$.

3 Trupaïn est le nom de l'épouse de Ptolémée Neos Dionysos. Quant à la mention de l'an 5, sans indication de mois ni de jour, J. Krall y voit la date de la stèle.

4 En note, J. Krall indique: euphémisme pour "il mourut".

${ }^{5}$ Neos Dionysos et son épouse.

${ }^{6}$ Ptolémée Evergète II et son épouse.

7 J. KRALL, op. cit., p. 379.

${ }^{8}$ H. GAUTHIER, Rois, 4 (1916), p. 394, note 2. 
il été alors nommé grand prêtre ? En rapportant au contraire les chiffres à Ptolémée-Alexandre dont les années de règne étaient comptées depuis 114 av. J.-C. ${ }^{1}$, Petobastis serait "entré dans le temple" en 105-104 à l'âge de seize ans. Dans la vingt-huitième année d'Alexandre, soit 87-86, Petobastis était âgé de trente-quatre ans. Nous préférons cette interprétation et admettons provisoirement que l'année 87-86 est celle de la mort de Psenptaïs, le père de Petobastis II, et de la nomination de son fils.

Petobastis II a été non seulement "grand setem", ainsi que J. Krall l'écrit ${ }^{2}$, mais aussi "grand des chefs des artisans" comme il est intitulé dans le texte hiéroglyphique des stèles de son fils $(\mathbf{2 0 0 , 1 5})$ et de la fille de ce dernier $(\mathbf{2 0 2 , 4})$. Il a exercé les fonctions attachées au pontificat telles que le service de la Fenêtre de l'apparition et de ses dieux. Mais il a aussi célébré, comme ses ancêtres, le culte d'Arsinoé-Philadelphe. Si nous ajoutons que Ptolémée-Alexandre l'a lui-même intronisé prêtre, nous concluerons que la faveur des Lagides restait toujours acquise aux descendants de Nesqed.

Petobastis II a épousé la "musicienne accomplie de Ptah, Harunchis" $\mathbf{( 2 0 0 , 1 5 )}$, qui lui a donné un fils en novembre 90. Petobastis achevait alors sa trente et unième année.

\section{§ 80. PSENPTAIS II}

Psentaïs II est né exactement le 21 du 2ème mois d'Akhet ou Paophi en l'an 25 de Ptolémée Alexandre Ier $^{3}$, soit le 4 novembre 90 av. J.-C. ${ }^{4}$

1 ibidem, p. 366, notes 1 et 2.

2 Nous ne connaissons pas d'autre monument que la stèle de Petobastis où des grands prêtres soient intitulés "grand setem". Comme le correspondant démotique de "grand des chefs des artisans" est "grand des artisans" (voir ci-dessus, § 4), nous nous demandons si l'état d'effacement de la stèle n'a pas pu tromper J. Krall. Sa lecture est antérieure aux études de Müller et de Brugsch (références $\S 4$, p. 12, note 5) sur le titre démotique $w r h m$.

${ }^{3}$ La "stèle Harris", rédigée pour Psenptaïs après sa mort soit un demi-siècle après sa naissance, date cet événement de Ptolémée Sôter II (200,17). Mais Psenptaïs est mort le 15 Phamenoth de l'an 11 de Cléopâtre (200,23), donc en l'an 41 av. J.-C., et sa durée

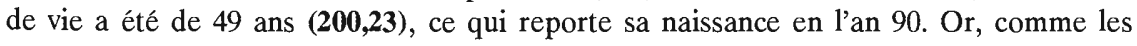


Son aïeul, dont il portait le nom, était peut-être encore en vie à ce moment.

Psenptaïs "passa treize années en présence de son père" $(\mathbf{2 0 0 , 1 8 )}$. Celuici est décédé, comme nous l'avons vu, le 13 février 76 . Un décret de Ptolémée Philopator Philadelphe Neos Dionysos nomma alors Psenptaïs "grand des chefs des artisans alors qu'il était dans sa quatorzième année" $(\mathbf{2 0 0 , 1 7 - 1 8})^{1}$. Le grand prêtre raconte ensuite qu'il a tenu le principal rôle lorsque le roi "réunit les deux Pays et accomplit toutes les cérémonies dans les demeures $d u$ Sed" $(\mathbf{2 0 0 , 1 8})$. Ce couronnement à l'égyptienne du roi eut lieu "quand le dieu naît", c'est-à-dire à l'équinoxe de printemps ${ }^{2}$. L'absence de toute mention d'année à ce propos indique que la cérémonie a eu lieu l'année de la nomination de Psenptaïs, soit le 23 mars $76^{3}$. Le rescrit royal nommant Psenptaïs "grand des chefs des

années de Ptolémée Sôter II étaient comptées depuis 117, l'an 90 correspondrait à la 28ème année de ce roi, tandis que les années de Ptolémée Alexandre Ier étaient comptées à partir de 114. Nous nous rangeons donc à l'avis de H. GAUTHIER, Rois, 4 (1916), p. 384, note 2: "le scribe qui rédigea cette stèle en l'an 41 av. J.-C., n'était plus très au courant des surnoms donnés aux divers Ptolémées et il a confondu Alexandre Ier avec Sôter II".

${ }^{4}$ H. GAUTHIER, op. cit.

${ }^{1}$ H. BRUGSCH, Dict. géogr., (1879), p. 654-655 et H. GAUTHIER, Rois, 4 (1916), p. 394, note 2 rapportent la proposition temporelle "alors que j'étais dans ma quatorzième année" $(\mathbf{2 0 0 , 1 8 )}$ aux cérémonies égyptiennes du couronnement de Neos Dionysos, tandis que H. BRUGSCH, Thesaurus, V (1891), p. VIII (texte imprimé) et E. BEVAN, Histoire des Lagides (trad. Lévy, Payot, 1934), p. 385 (d'après l'interprétation de la stèle par Glanville) la rapportent à la nomination de Psenptaïs. Nous suivons ces derniers parce que la construction "pronom indépendant + participe" au début du récit du couronnement nous paraît être plus à sa place au commencement d'une phrase qu'après une circonstancielle.

${ }^{2}$ H. BRUGSCH, Dict. géogr., (1879), p. 655.

3 Neos Dionysos était roi en fait depuis l'été 80 (H. GAUTHIER, Rois, 4 (1916), p. 394, note 2). Les "anaklétéria" (désignant les cérémonies qui accompagnent la proclamation d'un roi) avaient certainement eu lieu bien avant le couronnement selon le rite égyptien. On ignore la raison de la date tardive de cet événement (H. GAUTHIER, op. cit.; E. BEVAN, op. cit., p. 384) qui eut lieu, bien que Psenptaïs ne le précise pas, à Memphis (et non à Alexandrie, comme on le lit dans E. BEVAN, op. cit.).

D'autres Ptolémées s'étaient en effet déjà fait sacrer rois à Memphis selon les rites égyptiens: peut-être Ptolémée IV Philopator (d'après U. WILCKEN, réf. dans E. BEVAN, op. cit., p. 292, note 1); sûrement Ptolémée V Epiphane en 197 (E. BEVAN, p. 292) et Ptolémée VII Evergète II (ibidem, p. 347). Le fils de ce dernier naquit à 
artisans" a donc été émis dès que la mort de Petobastis II fut connue à Alexandrie'.

Nous savons par la stèle de la jeune Kheredouankh (202) que Psenptaïs a eu une fille en octobre 65, lorsqu'il finissait lui-même sa vingt-cinquième année. Comme il a "eu de belles concubines" $(\mathbf{2 0 0}, \mathbf{2 2})^{2}$, la mère de Kheredouankh a vraisemblablement été l'une d'elles.

Lorsque Psenptaïs s'est marié en été 58, il était "directeur des prophètes des dieux et déesses de Haute et Basse-Egypte" $(\mathbf{2 0 1 , 6 )}$ ou, comme il le dit plus emphatiquement sur sa stèle, "directeur des prophètes de tous les dieux et connaissant ... dans les temples de Haute et Basse-Egypte" $(\mathbf{2 0 0 , 1 4 )}$. Le ministère des cultes lui aura donc été confié entre 76 et 58.

Psenptaiis approchait de ses trente-deux ans quand le père divin Khahapi lui donna sa fille en mariage. Taimouthès, qui était née le $9 \mathrm{du}$ 1er mois d'Akhet ou Thot de l'an 9 de Neos Dionysos, soit en septembre 73, n'avait pas tout à fait atteint ses quinze ans. A cette occasion, Psenptaïs a dévoilé ses sentiments, d'ailleurs d'une façon laconique, par

Memphis pendant les fêtes du couronnement et fut appelé Memphitès.

${ }^{1}$ Une statue du fils de Psenptaïs (203a,2) permet de supposer que celui-ci a peut-être officié dans le temple alexandrin de Ptah avant d'être grand prêtre de Memphis.

${ }^{2}$ D'après le texte donné par E. BRUGSCH, Thesaurus. Rien n'indique que ces "concubines" ne se sont pas succédé. Peut-être même le terme employé par Psenptaïs fait-il allusion à la position de l'épouse du grand prêtre vis-à-vis de Ptah: Tachepeniset, femme de Chedsounefertoum, est "première grande du harem de Ptah" (voir ci-dessus, $\S$ 63). Psenptaïs serait alors à rapprocher de Padiiset, Horsaïset II, Ahmèsmen et Nesqed-Petobastis (voir ci-dessus, $\S 67,68,71,73$ ) qui ont eu chacun deux ou trois épouses différentes; nous ignorons si celles-ci ont été successives ou simultanées.

En suivant au contraire le texte donné par H. BRUGSCH, Dict. géogr., nous traduirions: "j'ai eu un beau harem". On pourrait alors admettre le jugement d'E. BEVAN, Hist. des Lagides, p. 387: "... Pshéréni-Ptah se vante de son "nombreux harem". On n'a rien trouvé de semblable dans tous les documents qui nous sont parvenus sur le clergé égyptien et cela nous montre singulièrement bien ce que le primat de l'Eglise égyptienne était devenu à l'époque de Ptolémée Aulète. Le jeune homme doit avoir été pour son souverain un digne compagnon de plaisirs". Remarquons que, sur la stèle, le harem est qualifié de "possesseur de beau = beau" ! 
cette phrase, mise plus tard dans la bouche de Taimouthès qu'il venait de perdre: "Le grand des chefs des artisans en fut extrêmement heureux" (201,8).

A ce moment, Psenptaïs n'était pas encore "prophète du roi" (201,6-7), tandis qu'à la mort de son épouse, sous Cléopâtre, il s'intitule clairement "prophète du roi de Haute et Basse-Egypte, maître des deux Pays, Ptolémée juste de voix" $(\mathbf{2 0 1}, \mathbf{1 4})$. Nous en concluons que cette fonction lui fut attribuée entre 58 , date de son mariage, et 51 , où finit le règne de Neos Dionysos. La stèle de Psenptaïs passe donc sous silence une vingtaine d'années de la vie du grand prêtre.

Psenptaïs, qui fut sans doute mandé à Alexandrie, nous a laissé un récit assez évocateur de son passage dans la "résidence des rois grecs"; Neos Dionysos, "étant sorti de son palais vivant et florissant, arriva au temple d'Isis, maîtresse de Iat-Oudjat; il lui fit des offrandes nombreuses et grandes. Le roi, sorti du temple d'Isis sur son char, arrêta lui-même son char. Il coiffa ma tête d'une belle couronne d'or et de toutes sortes de pierres précieuses véritables ... Je fus nommé son prophète. Il adressa un rescrit royal aux villes et aux nomes, disant: J'ai fait du grand des chefs des artisans Psenptaïs juste de voix ${ }^{1}$ mon prophète". Psenptaïs ne manque pas d'ajouter à son récit: "et des pains provenant des temples de Haute et Basse-Egypte m'ont été donnés chaque année" $(\mathbf{2 0 0 , 1 9 - 2 0 )}$. Le titre de prophète du roi et le revenu attaché à cette fonction et touché sans doute par Psenptaïs jusqu'à la fin de ses jours valaient bien le voyage de Memphis à Alexandrie et une station dans la poussière au bord de la route en attendant le passage du roi !

Le ministère des cultes n'était plus dans les mains de Psenptaïs à la mort de Taimouthès, en hiver $42 \mathbf{( 2 0 1 , 1 4 )}$. Il avait passé à un autre membre du clergé égyptien entre 58 et 42 , sans que nous puissions préciser si ce fut sous le règne de Neos Dionysos ou sous Cléopâtre. A propos de ce ministère, on pourrait s'étonner que Psenptaïs n'ait pas parlé de cette fonction importante dans sa biographie; nous supposons qu'il n'a pas vu le roi à cette occasion. Par contre, il a mentionné le voyage que Neos Dionysos fit vers la fin de son règne à Memphis et au

\footnotetext{
${ }^{1}$ Le récit est sur la stèle "funéraire" de Psenptais.
} 
cours duquel le souverain célébra une fête égyptienne et daigna s'asseoir dans la barque du grand prêtre (200,21-22).

$\mathrm{Au}$ cours des onze premières années de leur union, Psenptaïs et

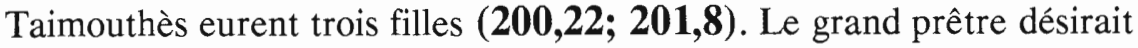
vivement un garçon qui pût lui succéder un jour. C'est pourquoi il s'adressa à l'Esculape égyptien. Taimouthès, que son nom portait à requérir le secours d'Imhotep en cette circonstance raconte ce qui advint: "J'implorai avec le grand des chefs des artisans la majesté de ce dieu auguste, grand par ses miracles, ..., qui donne un fils à celui qui n'en a pas, Imhotep fils de Ptah. Il entendit notre prière, il écouta les supplications à son adresse. La Majesté de ce dieu vint ... ce grand des chefs des artisans en songe et lui dit: Fais faire le grand travail dans le Lieu Sacré d'Ankh-taoui, le lieu où mon corps est caché, et je t'en récompenserai par un enfant mâle. Là-dessus, il (le grand prêtre) s'éveilla. Il se prosterna pour ce dieu auguste. Il fit part de ces choses aux prophètes, aux supérieurs des secrets, aux ouabs et aux sculpteurs de l'atelier d'orfèvrerie à la fois. Il leur ordonna d'accomplir le travail parfait dans le Lieu Sacré, et ils firent selon tout ce qu'il avait dit. Il accomplit l'ouverture de la bouche pour ce dieu auguste. Il lui fit une grande offrande de toutes sortes de bonnes choses. Il récompensa les sculpteurs de la part de ce dieu et réjouit leur coeur avec toutes sortes de choses. En contre-partie de cela, il me rendit enceinte d'un enfant mâle. Il naquit en l'an 6, troisième mois de Chemou (Epiphi), quinzième jour, sous la majesté de la Régente et mâtresse des deux Pays Cléopâtre, vie, santé, force! C'est la fête des Offrandes-sur-l'autel de ce dieu auguste Imhotep fils de Ptah ... On lui donna le nom d'Imouthès appelé Petobastis et tout le monde se réjouit à son sujet" (201,8-14).

Le Lieu Sacré d'Ankh-taoui où Psenptaïs reçut l'ordre de faire exécuter certains travaux était vraisemblablement l'Asklépiéion, construit à proximité du tombeau de l'architecte divinisé de Djeser ${ }^{1}$.

${ }^{1}$ K. SETHE, Imhotep, der Asklepios der Ägypter, p. 7 = Untersuchungen ..., 2 (1902), p. 99. D'après U. WILCKEN, Urkunden der Ptolemäerzeit, 1 (1927), p. 38-41, l'Asklépiéion devait se trouver exactement à l'est du Sérapéum. Le tombeau d'Imhotep et l'Asklépiéion étaient vraisemblablement peu éloignés de la bordure du désert, à un kilomètre approximativement au nord de l'endroit où la route conduisant de Mit-Rahineh à la nécropole antique de Saqqara aborde le désert. Il serait étonnant que plus rien ne subsistât aujourd'hui de la tombe et du sanctuaire d'Imhotep. 
Le bonheur de Psenptaïs après la naissance de son fils, en juillet 46, ne dura pas bien longtemps et la fin de l'existence du grand prêtre fut assombrie par deux chagrins. En décembre 44, il perdit sa fille, âgée seulement de vingt et un ans. Kheredouankh fut ensevelie dans le tombeau de Psenptaïs, auprès des père et mère du grand prêtre, le $15 \mathrm{du}$ 2ème mois de Peret ou Mechir de l'an 9 de Cléopâtre et son fils César, soit le 14 février 43 (202,5-6).

Un an et un jour plus tard, le 16 du 2ème mois de Peret ou Mechir de l'an 10, Taimouthès mourait, prématurément aussi, puisqu'elle n'avait vécu que trente années et quelques mois. Son mari l'enterra également dans "son tombeau derrière Ro-qedit" $(\mathbf{2 0 1 , 1 5 ) .}$

Sur la stèle de Taimouthès, Psenptaïs, développant à son tour d'anciens thèmes, a mis dans la bouche de sa femme une exhortation à jouir de la vie et un tableau de l'activité de la mort (201,15-21). Faudrait-il ne voir dans celui-ci qu'un morceau de littérature et dans celle-là qu'une manifestation égoïste d'un veuf vite consolé ? Le double deuil de Psenptaïs pourrait contredire cette interprétation.

Le grand prêtre n'eut d'ailleurs pas le loisir "de boire, de manger, de s'enivrer, de pratiquer l'amour, de passer heureusement le jour et de suivre son désir chaque jour" (201,15-16) pendant de longues années. En l'an 11 de Cléopâtre, soit en 41 av. J.-C. ${ }^{1}$ la mort l'appelait, elle dont il avait écrit un an auparavant que "personne n'ose la regarder parmi les dieux et les hommes. Les grands sont pour elle comme les petits et il n'y a de résistance contre elle chez aucun de ceux qu'elle désire. Elle enlève l'enfant à sa mère, aussi bien que le vieillard, en parcourant son chemin de terreur"

${ }^{1}$ Dans le texte édité par H. BRUGSCH, Thesaurus, la mort de Psenptaïs est datée du 15 du 3ème mois de Peret ou Phamenoth de l'an $11(\mathbf{2 0 0 , 2 3 )}$. Cette date est également donnée par E. BEVAN, Histoire des Lagides, p. 386, d'après une étude de l'original égyptien par S.R.K. GLANVILLE (ibidem, p. 385). Deux cents jours sépareraient alors le décès de l'ensevelissement.

Par contre, H. GAUTHIER, Rois 4 (1916), p. 384, note 2, lit sur la stèle Harris (200), la date du 15 Epiphi (3ème mois de Chemou) de l'an 11, correspondant au 14 juillet 41 . Se serait-il basé sur la traduction du texte démotique de la stèle d'Imouthès faite par E. Revillout (voir au § suivant) ? Si la stèle Harris porte Chemou au lieu de Peret, il y aurait eu quatre-vingts jours entre le décès et l'ensevelissement. 
$(\mathbf{2 0 1}, \mathbf{2 0})$. Psenptaïs était alors dans sa quarante-neuvième année. On l'ensevelit dans le tombeau familial le 30 du 1er mois d'Akhet, ou Thot, de l'an 12 , soit le 2 octobre $41^{1}$.

\section{§ 81. IMOUTHES-PETOBASTIS}

Les stèles de Psenptaïs et de Taimouthès nous ont déjà renseignés sur les circonstances qui entourèrent la venue au monde d'Imouthès. Sa propre stèle contient, en plus d'une formule d'offrandes hiéroglyphique où apparaissent ses titres (203,5-6), une biographie en démotique. Nous en connaissons deux traductions, déjà anciennes ${ }^{2}$. La première est d'E. Revillout $^{3}$ :

"L'an 6 de la reine Cléopâtre, Epiphi 13, panégyrie de Bast la grande déesse, fut le jour de naissance de l'ami de Bast, bien aimé de Bast, prophète de Ptah, prêtre des dieux de la région du Mur Blanc, écrivain des divines paroles de Ptah, écrivain de Ptah, écrivain de Neb-Nehit, prophète d'Osiris, seigneur de Rosta, oreille du roi, prophète (d'Imouth-se-Ptah), hiérogrammate d'Apis vivant, grand chef de l'oeuvre, Peti-Bast, surnommé Imouth fils de Pa-ptah-ni, surnommé Psé-Ptah, enfanté par la dame de maison Ta(t)imouth.

Il eut de vie 16 ans, 25 jours. Il fut grand chef de l'oeuvre, en l'an 19 de la reine Cléopâtre, pour 4 années. Mais il ne les accomplit pas. L'apothéose du divin père, prophète de Ptah, grand chef de l'oeuvre, Psé-ptah, son père, eut lieu en l'an 11 de la reine Cléopâtre, le 15 Epiphi; et, en l'an 12, on lui fit son teb de bonne demeure en 70 jours. On fit son kesau (=

\footnotetext{
${ }^{1}$ H. GAUTHIER, Rois, 4 (1916), p. 384, note 2.
}

${ }^{2}$ M. Michel Malinine a bien voulu, sur notre demande, se livrer au travail ingrat de comparer entre elles les éditions modernes des textes démotiques des stèles d'ImouthèsPetobastis et de Tanepherôs. Ce travail n'est, hélas, que provisoire, car il est bien évident qu'il faudrait qu'un démotisant comparât les éditions en question aux monuments euxmêmes. C'est pourquoi, nous avons reculé le plus possible le moment où nous prierions M. Malinine de nous aider, parce que nous espérions qu'entre temps les circonstances, nées de la guerre, qui empêchent la consultation des textes originaux prendraient fin. En attendant, les remarques de M. Malinine, que nous donnons plus loin, nous permettent de corriger certaines de nos interprétations, et nous lui en sommes très reconnaissants.

${ }^{3}$ Un prophète d'Auguste, dans Revue égyptologique, 2 (1882), p. 101-102.

4 = la Maîtresse du Sycomore. 
ensevelissement). On le fit conduire en ses résidences. On le plaça dans la catacombe de Memphis. En l'an 22 de la reine Cléopâtre, le 8 Mesorê, fut l'apothéose du prophète de Ptah, grand chef de l'oeuvre, Peti-Bast surnommé Imouth. On lui fit son teb en 70 (jours). On le pleura. Il resta enfermé dans la bonne demeure, en ses lieux de repos, jusqu'à l'an 7 du dieu, fils de dieu, le dieu grand, étranger, l'autocrate César, Pharmouthi 14, c'est-à-dire 6 années, 8 mois et 10 jours. Réunion de Psé-amen, frère par le père, prophète de Ptah, grand chef de l'oeuvre, prophète d'Hor-nebsekhem, prophète de César. Lorsqu'on fit la réunion à la bonne demeure, on lui apporta des kel. On lui mit des talismans en or, en argent, en pierres précieuses. On lui fit tous les rites d'un grand prêtre ..... (La suite de la stèle est en trop mauvais état pour être rétablie)".

H. BRUGSCH a accompagné l'édition du texte démotique d'une traduction en allemand, et mot à mot. Nous en interprétons ainsi la partie biographique ${ }^{2}$ :

"L'an 6 de la reine Cléopâtre, le 13 Phamenoth, à la panégyrie de Bast la grande déesse, fut le jour de naissance du prince héréditaire, père divin, prophète de Ptah, prêtre des dieux des temples du Mur Blanc, scribe du stylus de Ptah, scribe de Ptah, scribe du Seigneur de Nohe, prophète d'Osiris, seigneur de la nécropole, prophète du roi, prophète du nom du roi, scribe de la maison des hiérogrammates, scribe de l'Apis vivant, grand chef des artisans Peta-Bast, surnommé Imhotp, fils de celui qui a les mêmes titres Psêre-n-Ptah, enfant de la maîtresse de maison Taïmhotpe. Il vécut 16 ans et 21 jours. Il fut grand prêtre en l'an 13 de la reine Cléopâtre pendant 7 ans et 10 jours. La toilette fut faite au père divin, prophète de Ptah, grand prêtre Psêre-n-Ptah, son père, en l'an 11 de la reine Cléopâtre, le 16 Pharmouthi. En l'an 22, ils lui firent toutes les purifications pour la bonne maison en 70 jours. Ils l'ensevelirent. Ils le rendirent beau pour son lieu de repos dans la nécropole de Memphis. En l'an 22 de la reine Cléopâtre, le

${ }^{1}$ La traduction porte "Phamenoth". Nous corrigeons d'après E. REVILLOUT, Le comput de Ptolémée Denys et le canon des rois, dans Revue égyptologique, 5 (1888), p. 130 , note 2 .

${ }^{2}$ Thesaurus, 5, p. 929-933. 
6 Mesori, ils procédèrent à la toilette du père divin, sem $^{1}$, prophète de Ptah, grand prêtre Peta-Bast, surnommé Imhotp; ils lui firent toutes les purifications selon les instructions. On l'emmaillotta dans la bonne demeure pour ses lieux de repos jusqu'à l'an $7 \mathrm{du}$ dieu, du fils du dieu, du grand dieu (?) Autocrator César, le 14 Pharmouti, en 6 ans, 8 mois et 10 jours. Ensevelissement du fils du frère de sa mère, du père divin, sem, prophète de Ptah, grand prêtre, prophète d'Horus seigneur de Létopolis, prophète de César. Ils donnèrent à son ensevelissement de bons onguents, de la myrrhe, des résines; il fit ses talismans en or, en argent, en toutes sortes de véritables pierres précieuses. Il exécuta pour lui toutes les prescriptions pour entretenir sa vénération: ses vêtements, ses onguents selon (le désir de) son coeur".

Dans la suite, $\mathrm{H}$. Brugsch a reconnu un passage qui est la transcription de la fin de la ligne 5 et du début de la ligne 6 du texte hiéroglyphique, soit du commencement de la titulature d'Imouthès. Ensuite, il est dit que la biographie de ce personnage est racontée "sur le couvercle de son

1 A propos du signe que Brugsch transcrit "sem", M. Malinine nous écrit: "L'interprétation de Brugsch me paraît de la plus haute fantaisie. Pour moi, il est absolument clair que ce signe appartient à la graphie du mot précédent qui est à transcrire $\& \quad \square P$. Je confesse ne point pouvoir vous citer un autre exemple de ce titre "it-ntr" écrit avec le déterminatif. Notez qu'il existe en démotique deux graphies du titre

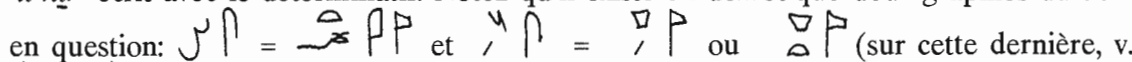
ČERNÝ, dans Griffith Studies, p. 50, note 4). Il n'y a rien de surprenant ni d'anormal, en soi-même, qu'à cette dernière graphie, on adjoigne le déterminatif $\frac{q}{6}$. En ce qui concerne le titre $s m$, je ne connais que les graphies normales Thesaurus, p. 891, 1. 1; stm; ibid., 915, dernière ligne, 912, 3ème ligne de la fin et 906, 1 . 1. Sur ces orthographes, voir GRIFFITH, Stories of the High Priests of Memphis, p. 3-4, $\S 4$ et SETHE, Bürgschafts Urkunden, p. 162. Il me semble que Brugsch a été influencé en ce qui concerne la lecture de sem par des passages comme p. ex. Thesaurus, p. 913,

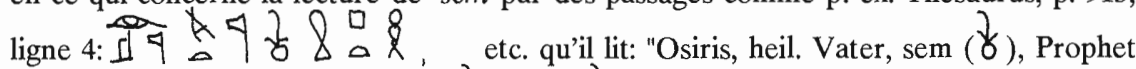
des Ptah"; si je ne me trompe pas, $\gamma$, var. 8 représente le titre $r h$-nswt".

Cette note de M. Malinine nous permet d'affirmer qu'Anemher est le seul grand prêtre ptolémaïque de Memphis qui soit intitulé "setem" dans les textes démotiques (la traduction de Krall, "grand setem", sur la stèle de Petobastis II nous paraît très douteuse). Or Anemher a abandonné sa fonction de grand des artisans avant la fin de sa vie. Le démotique ne connaît donc qu'un titre pour désigner le grand prêtre de Memphis, et c'est "wr hm" (transcription du titre démotique de BRUGSCH, Thesaurus, p. 930, 1. 5-6 par M. MALININE :

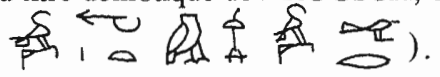


soit du commencement de la titulature d'Imouthès. Ensuite, il est dit que la biographie de ce personnage est racontée "sur le couvercle de son sarcophage" que nous ne possédons malheureusement pas, et qu'il "est un Osiris" et le "Soleil du couchant pour l'éternité".

Nous considérons que le texte démotique contient véritablement les données sur lesquelles les interprétations d'E. Revillout et de $\mathrm{H}$. Brugsch concordent. Ce sont:

- la naissance d'Imouthès en l'an 6 de Cléopâtre;

- la durée de vie: 16 ans et une vingtaine de jours;

- la nomination d'Imouthès comme grand prêtre;

- le décès et l'ensevelissement de son père;

- son propre décès au début de Mesorê de l'an 22 de Cléopâtre;

- son ensevelissement, 6 ans, 8 mois et 10 jours plus $\operatorname{tard}^{1}$, soit le 14 Pharmouthi de l'an 7 de César Auguste.

Nous pouvons préciser quelques points de cette chronologie. En remontant de 6 ans, 8 mois et 10 jours à partir du 14 Pharmouthi de l'an 7 de César Auguste, nous aboutissons au 9 Mesorê de l'an 22 de Cléopâtre ${ }^{2}$, correspondant au 4 août 30 av. J.-C. ${ }^{3}$. Tenant compte d'une possibilité d'erreur dans la reproduction du texte démotique, nous pouvons affirmer qu'Imouthès est mort au début du mois d'août 30 . Comme il a vécu 16 ans et une vingtaine de jours, il est né au mois

${ }^{1}$ Sur ce délai, voir ci-dessous, $\$ \mathbf{8 3}$.

${ }^{2}$ La durée du règne de Cléopâtre est de 22 ans d'après le canon. Du 9 Mesorê an 22 de la reine au 9 Mesorê an 6 de César Auguste, il y a 6 ans; du 9 Mesorê an 6 au 9 Pharmouthi an 7, il y a 8 mois et les 5 jours épagomènes de l'an 6; enfin du 9 au 14 Pharmouthi an 7, il y a 5 jours; au total, 6 ans, 8 mois et 10 jours.

H. Brugsch a lu le 6 Mesorê pour la mort d'Imouthès.

E. Revillout a lu le 8 Mesorê. Constatant plus tard qu'avec cette date il y a 6 ans, 8 mois et 11 jours entre la mort et l'ensevelissement d'Imouthès, il a alors écrit ( $L e$ comput de Ptolémée Denys et le canon des Rois, dans Revue Egyptologique, 5 (1888), p. 131): "D'après la copie de Young, le 10 seul est visible pour les jours. Mais cela tient à ce que l'unité complétant le chiffre de 11 jours formait un des bouts de ligne que n'a pas copiés Young".

M. Malinine: "6 Mesore me paraît juste d'après les deux copies." Il y aurait donc désaccord entre les dates et l'intervalle qui les sépare dans le texte démotique.

3 D'après H. GAUTHIER, Rois, 4 (1916), p. 416, note 2, qui donne la correspondance pour la lecture de H. Brugsch: 6 Mesorê an $22=1$ er août 30 av. J.-C. 
d'Epiphi de l'an 6 de Cléopâtre ${ }^{1}$. La stèle de Taimouthès, sa mère, date en effet l'événement du 15 Epiphi de l'an $6 \mathbf{( 2 0 1 , 1 2 ) . ~ D ' a p r e ̀ s ~ u n ~}$ calendrier des six fêtes célébrées chaque année en l'honneur du dieu Imhotep, l'anniversaire de sa naissance tombait le 16 du 3ème mois de Chemou, ou Epiphi (H. GAUTHIER, Un nouveau monument du dieu Imhotep, dans Bulletin de l'I.F.A.O., 14 (1918), p. 37-38). Le surnom d'Imouthès donné à Petobastis III nous permet de supposer que celui-ci est né exactement le jour où l'on célébrait l'anniversaire de la naissance du dieu, et qu'une erreur d'un jour s'est glissée sur l'un des deux monuments. Nous nous tenons à cette indication parce qu'elle est donnée par un monument presque contemporain ${ }^{2}$. Enfin, entre le 15 Epiphi de l'an 6 et le 9 Mesorê de l'an 22 de Cléopâtre, il y a 16 ans et 24 jours.

La stèle d'Imouthès contient encore quelques données chronologiques. Les unes concernent la mort et l'ensevelissement de son père, Psenptaïs ${ }^{3}$. Les autres sont relatives au pontificat d'Imouthès. La comparaison des copies des textes démotiques de Young et de Brugsch faite par M. Malinine nous permet d'écarter l'interprétation de Revillout.

${ }^{1}$ M. Malinine: "Epiphi" de Revillout est à rejeter avec certitude. D'après Brugsch, le passage est à transcrire $三 \supset \odot \gg \square \overparen{11}$. Cependant la copie de Young, en ce qui concerne le nombre des jours, permet la lecture $=\equiv \subset$. Seule une confrontation avec l'original permettra de résoudre cette question définitivement."

Ainsi, le texte démotique de la stèle d'Imouthès date la naissance de ce dernier du troisième mois de Peret, soit Phamenoth. Il est en contradiction non seulement avec la stèle de Taimouthès $(\mathbf{2 0 1 , 1 2})$, beaucoup plus proche de la naissance d'Imouthès, et confirmée par le calendrier des fêtes du dieu Imhotep, mais encore avec lui-même: entre le 13 (ou 15) Phamenoth an 6 et le 6 Mesore an 22, il y a 16 ans, 4 mois et une vingtaine de jours, et non les 16 ans et 21 jours indiqués par la même stèle. Il s'agit donc manifestement d'une erreur du graveur ancien.

${ }^{2}$ E. Revillout et $\mathbf{H}$. Brugsch lisent tous deux sur la stèle d'Imouthès que celui-ci est né le 13 du mois. Nous considérons donc que c'est bien le quantième indiqué par le texte démotique. Mais celui-ci a été écrit en l'an 7 de César Auguste. Il est postérieur de 19 ans à la stèle de Taimouthès, qui est de l'an 10 de Cléopâtre.

${ }^{3}$ Nous ne nous y arrêtons pas parce que les interprétations d'E. Revillout et de $\mathrm{H}$. Brugsch diffèrent à leur sujet (avec un lapsus calami chez H. Brugsch: "l'an 22" au lieu de "l'an 12". M. Malinine nous signale que "la copie de Young (et non pas celle de Brugsch) permet la lecture "an $12^{\prime \prime}$.) et ne nous permettent pas, en conséquence, de trancher la difficulté que nous avons soulevée (p. 197, note 1), à savoir en quelle saison Psenptaïs est décédé. 
M. Malinine nous écrit au sujet de l'"an 13": "D'après les deux copies, la lecture "an 19" me paraît impossible. Je dois toutefois attirer votre attention sur ce que la graphie en question n'est pas très catholique pour le nombre 111. D'après moi, on peut la lire aussi 1111 , c'est-à-dire, "an $14 " . "$

Deux ou trois années se seraient donc écoulées entre la mort de Psenptaïs, en l'an 11, et la nomination d'Imouthès.

Au sujet des mots qui suivent, traduits par Brugsch "während Jahre 7, Tage 10", M. Malinine nous donne l'explication suivante: "Le passage doit être transcrit: $r m p(t)$ 7.t $h(r) w$ 10. Le $r$ initial ne représente pas ici la préposition $r$ "vers" etc. Il s'agit ici d'une graphie dégénérée de

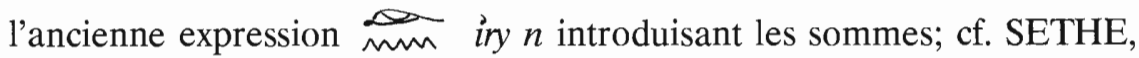
Bürgschafts Urkunden, p. 21: "macht an". Il faut donc traduire: "(ce qui) fait 7 ans, 10 jours".

Si donc Imouthès a été grand prêtre de Memphis pendant 7 ans et 10 jours à partir de l'an 13 ou 14, ce ne serait pas sa mort, survenue en l'an 22, qui aurait mis fin à son pontificat. On ne saurait toutefois se prononcer à ce sujet avant qu'un démotisant ait établi sur la stèle du British Museum la lecture certaine de la date de la nomination d'Imouthès.

Bien qu'Imouthès-Petobastis n'ait pas revêtu de nombreuses fonctions, sans doute parce que son pontificat n'a pas duré longtemps, il a été cependant premier prophète de la reine et ministre des cultes (203a). L'inscription qui lui donne ces titres offre deux particularités. La plus frappante est l'absence de "grand des artisans-chefs" dans la titulature du père d'Imouthès, Psenptaïs II. En second lieu, les titres portés par Imouthès-Petobastis et par Psenptaïs II peuvent être comparés à ceux que leur ancêtre Psenptaïs I a sur ses statues (198). On se souvient que celles-ci ont été découvertes à Alexandrie. Comme la statue acéphale d'Imouthès dont l'inscription nous occupe (203a) a été extraite, il y a une quarantaine d'années, du sol de Cherchel en Algérie, il est possible qu'elle y ait été amenée, peut-être déjà à l'époque romaine, d'Alexandrie plutôt que de Memphis. Cette circonstance permettrait d'expliquer non seulement les points communs des inscriptions gravées 
sur les statues d'Imouthès et de Psenptaïs $\mathrm{I}^{1}$, mais aussi l'absence de "grand des artisans-chefs" parmi les titres de Psenptaïs II, soit que cette appellation n'eût plus de sens à Alexandrie, soit plutôt, puisqu'Imouthès la mentionne pour lui-même, que Psenptaïs II, comme son ancêtre homonyme, eût servi au temple alexandrin de Ptah dans son enfance, puisqu'il fut nommé grand prêtre à l'âge de 13 ans. Quant à Imouthès, il aurait peut-être été déjà grand prêtre lorsqu'il officia à Alexandrie.

En résumé, Imouthès-Petobastis est né au mois d'Epiphi de l'an 6 de Cléopâtre, en juillet 46 avant J.-C. Il a perdu sa mère à trois ans et demi et son père à cinq ans. Ce n'est que deux ou trois ans plus tard qu'il fut nommé grand prêtre, et son pontificat ne dura que sept années.

Cet enfant, tant désiré par ses parents, mourut déjà à l'âge de seize ans, en août 30 av. J.-C. ${ }^{2}$. Octave venait alors de s'emparer d'Alexandrie et de mettre fin à la dynastie des Lagides.

De Ptolémée II Philadelphe à Cléopâtre VII, pendant plus de deux siècles, le pontificat memphite a été dans les mains d'une seule famille. Deux de ses membres ont été ministres des cultes. Bien que le pouvoir dont ils disposèrent s'étendît jusqu'aux confins de l'Egypte, il ne s'exerçait que sur la classe des prêtres. C'est pourquoi les souverains lagides n'eurent jamais à craindre que cette "dynastie" de grands des chefs des artisans devînt dangereuse pour eux.

Cependant, les Ptolémées ont peut-être, dès la seconde moitié du troisième siècle, fait venir à Alexandrie l'un ou l'autre des futurs grands prêtres de Ptah Is Psenptaïs I ou même Psenptaïs II. Nous n'avons pas les moyens de vérifier si cette politique, analogue à celle que les grands pharaons de la XVIIIème dynastie ont pratiquée à l'égard des fils des princes palestiniens et syriens, a été suivie régulièrement. D'ailleurs, comme on a pu supposer que le temple de Ptah à Alexandrie dépendait

${ }^{1}$ La statue d'Imouthès (203a) nous autorise à restituer "celui qui connaît les secrets et qui voit les secrets (de Ro-setaou de) Ro-qedit" (= Râqedit, voir § 77) sur les statues de Psenptaïs I (198,2). Le sanctuaire alexandrin de Ptah se serait alors appelé "le temple de Ptah de Ro-setaou de Roqedit (=Râqedit)" (203a). A l'appui de cette interprétation, on peut relever que les inscriptions memphites des grands prêtres de Ptah ne mentionnent ni "Ptah de Ro-setaou" ni "Ro-setaou de Ro-qedit".

${ }^{2}$ Sur son ensevelissement, plus de six ans après sa mort, voir ci-dessous, § 83. 
du temple de Memphis (références dans SANDMAN, Ptah, p. 250, note 26), cette circonstance peut aussi expliquer en partie les séjours que plusieurs descendants du grand prêtre Harmachis ont faits à Alexandrie.

Les grands des chefs des artisans ptolémaïques se montrent toujours respectueux de l'autorité royale dans leurs inscriptions. Il en fut ainsi. Mais nous croyons qu'on se tromperait en concluant que les sentiments personnels des grands prêtres à l'égard des divers rois n'ont pas varié. Lorsque Nesqed fut élevé au pontificat après des années au cours desquelles sa position n'avait pas changé, il éprouva sans doute une véritable reconnaissance et peut-être même du respect pour le souverain capable qu'était Philadelphe. Mais quand, deux siècles plus tard, le "Joueur de flûte" convoqua Psenptaïs à Alexandrie pour en faire son prophète, le grand prêtre ressentit peut-être bien de la colère, voire un peu de haine et de mépris pour le roi lorsqu'il dut attendre au bord de la route.

\section{§ 82. APPENDICE AU CHAPITRE XI}

Nous ne rangeons pas le "prophète de Ptah" Amasis parmi les grands prêtres ptolémaïques, parce qu'il ne porte pas le titre de "grand des chefs des artisans". H. BRUGSCH a donné un texte de ce personnage dans ses inscriptions des grands prêtres memphites ${ }^{1}$, ce qui a conduit W. OTTO à considérer Amasis comme un grand prêtre ${ }^{2}$, même ${ }^{3}$ après que W. SPIEGELBERG eût montré que c'était un grand prêtre de Létopolis ${ }^{4}$.

Un autre personnage, Pahemneter-Padihor (voir Appendice, Aperçu du Personnel de Ptah, époque ptolémaïque, p. 442, note 4) considéré par Spiegelberg comme un grand prêtre de Ptah, ne porte pas davantage le titre de grand des chefs des artisans, et nous ne le rangeons pas parmi les grands prêtres memphites.

\footnotetext{
1 Thesaurus, p. 909 sq., 945-946.

2 Priester und Tempel im hellenistischen Ägypten, 1 (1905), p. 204, note 2.

3 ibidem, Compléments, p. 415.

${ }^{4}$ Varia, dans Rec. Trav., XXVI (1904), p. 42.
} 


\section{H A P I T R E XII}

\section{UN GRAND PRETRE DE L'EPOQUE ROMAINE}

\section{$\S$ 83. PSENAMOUNIS}

Nous connaissons Psenamounis par les monuments de son cousin Imouthès ${ }^{1}$ et de sa femme Tanepherôs. La stèle de cette dernière ressemble à celle d'Imouthès. Elle comporte une scène d'adoration, une formule hiéroglyphique d'offrandes partiellement détruite (204) et un texte démotique qu'E. REVILLOUT a traduit ainsi²:

"L'an 19 du roi, dieu neuvième, nouvel Osiris (Neos Dionysos), Ptolémée, le 13 Tybi - fête de Seklet - fut le jour de naissance de la chanteuse ... de Ptah, Nofré-ho, fille du divin père de Ptah seigneur de $\mathrm{Nen}^{3}$, prêtre des dieux de la région (du Mur Blanc), écrivain de Ptah, écrivain d'(Hathor)-neb-Nehi, hiérogrammate d'Apis vivant, oeil du roi, écrivain des divines paroles des dieux, seigneur de Bési (Bubastis ?), écrivain des divines paroles, écrivain des livres, écrivain ministre divin du sanctuaire de Memphis, prophète d'Horus, seigneur de Sekhem (Létopolis), prophète de Chnoum seigneur de Smenhor (Héracléopolis), Kha-hapi-i, dont la mère est Her-anch (ou Ho-anch).

Elle fit années de vie $38^{4}$, mois 9, jours 5. On l'intronisa chanteuse de Ptah en l'an 15 à perpétuité. . On la fit prendre pour femme au prophète

${ }^{1}$ Voir ci-dessus, § 81.

${ }^{2}$ Un prophète d'Auguste, dans Revue égyptologique, 2 (1882), p. 100-101.

3 = "Khenty-tenenet".

4 Le texte imprimé porte 37. Nous corrigeons d'après l'indication qu'E. REVILLOUT a donnée lui-même (Le comput de Ptolémée Denys et le canon des Rois, dans Revue Egyptologique, 5 (1888), p. 130, note 2.

5 E. REVILLOUT indique en note: "mot à mot: pour année quelconque". 
de Ptah, Psé-amen, en l'an 20 de la reine Cléopâtre, unie à Amon. Elle conçut pour lui pendant de nombreuses années. Elle lui enfanta une fille sage, belle d'aspect. Elle l'appela Ho(anch), comme nom. On l'intronisa grande femme de Ptah pour 9 ans. Elle lui enfanta aussi un fils mâle, beau et fort. Elle l'appela Psé-Ptah, comme nom. On le fit inscrire dans la double maison de vie.

En l'an ler du dieu, le fils de dieu, le dieu grand, étranger, l'autocrate César, il fit prendre le prophète de Ptah, Psé-amen, le mari de Nofré-ho cidessus, comme prophète de César et grand chef de l'oeuvre. Il lui donna une couronne d'or, des présents en quantité. Fut l'apothéose de ce grand personnage, dans le sanctuaire de Memphis, avec ses pères (?). On le fit amener en dieu dans ses appartements d'Osiris, Seigneur d'éternité.

En l'an 7 de César, le 18 Paophi, fut l'apothéose de la grande dame. On fit son teb depuis le jour ler jusqu'au jour 70ème. On la conduisit à la bonne demeure.

En l'an 7, le 14 Pharmouthi, on fit un complément de teb et de kesau à cause du prophète de Ptah, grand chef de l'oeuvre, prophète de César, Pséamen, son mari. Réunion à la bonne demeure. On lui donna des kel, des talismans, en argent, en or, en pierres précieuses. On fit venir pour elle des étoffes de divin service de chanteuse, depuis les sanctuaires et les temples de Syrie jusqu'en Egypte. On l'ensevelit. On la fit conduire à la bonne demeure. Elle y reste éternellement à jamais !"

H. BRUGSCH a traduit le même texte en allemand et mot à $\operatorname{mot}^{1}$. Sa version, mise en français, est la suivante:

"En l'an 19 du roi ... du dieu nouveau et jeune, Ptolémée, Tybi, jour 13, à la fête de Sokar, le jour de naissance de la bonne musicienne et joueuse de sistre de Ptah Ta-nfr-ho, la fille du père divin, sem, prophète de Ptah Seigneur de la force, prêtre des dieux des châteaux du Mur Blanc, scribe de Ptah Seigneur de Nuhi, scribe de la maison des hiérogrammates de l'Apis vivant, yeux du roi, scribe des écrits sacrés des dieux seigneur de Bki, scribe des écrits sacrés, scribe des décrets et scribe des sceaux sacrés des châteaux de Won-khem, prophète d'Horus seigneur de Sekhem, prophète de Khnoum

${ }^{1}$ Thesaurus, p. 935-940. 
seigneur de Smen-hor, Kha-hapi, le nom de sa mère étant Ta-ho-onkh.

Elle fit années de vie: 38 ans, 9 mois, 10 jours.

Ils firent son élévation au rang de musicienne de Ptah en l'an 191, en (? $)^{2} 3$ ans. Le prophète de Ptah Pchêre-en-Amon la nomma sa fermme en l'an 3 de la reine Cléopâtre qui aime son père. Elle lui enfanta beaucoup de fois. Elle lui enfanta six filles ... Bast, nommée Ho... de son nom. Ils firent son élévation au rang de grande épouse de Ptah en l'an 9. Elle lui enfanta aussi un fils ... nommé Pchêre-en-Amon de son nom.

Ils firent pour lui un décret comme voyant du dieu (en) l'an 3 du dieu, fils de dieu, dieu grand ... (?) d'Autokrator César, il était comme père divin, sem, prophète de Ptah, Pchêre-en-Amon (et) la joueuse de sistre Ta-nfr-ho, qui est ci-dessus, comme prophétesse de César, (le ?) grand prêtre; il lui donna une ... en or, beaucoup de cadeaux. Il fit une maison de toilette ${ }^{3}$

1 M. MALININE: "La lecture "an 15" comme Revillout est possible seulement d'après la copie de Young. Elle me paraît plus probable que 1'an 19" de Brugsch (bien que sa copie donne nettement ce nombre) puisque, dans le même document, le nombre 9 est écrit autrement."

A propos des mots suivants "en 3 ans", M. MALININE nous écrit: "Ces trois derniers mots se rapportent à ce qui précède; $r m p(t) 3 . t$ "(ce qui) fait 3 ans"; cf. ce que je dis plus haut (voir p. 203). En d'autres termes, Tanepherôs a été "musicienne de Ptah" pendant 3 années. A propos de "en l'an 3 de la reine Cléopâtre" à la ligne suivante, $M$. MALININE écrit: "L'an 3 de la reine Cléopâtre" est certain seulement d'après la copie de Brugsch. La graphie donnée par Young permet la lecture "an 20" comme Revillout, ou plutôt laisse hésiter entre les deux lectures proposées."

A propos de "grande épouse de Ptah en l'an 9", à la fin du même alinéa, M. MALININE écrit: "Passage mal traduit par Brugsch, qui reproduit mal le texte original. Il faut lire $i r . w$ shny-s $r t 3 h m(t) c 3(t) n$ Pth r mpt (clairement dans la copie de Young et non pas $h$-sp, comme Brugsch) 9 (le nombre 9 est certain d'après les deux copies); "on la fit introduire à (la charge de) grande épouse de Ptah, (ce qui) fait 9 ans." Cf. plus haut, p. 203)." A propos du nom "Pchêre-en-amon" M. MALININE écrit: "La lecture du nom propre proposée par Revillout me paraît correcte P3-šry-(n)-Ptah (= Pa-cher-en-ptah = Psenptaiis). Dans le même texte, le nom "Amon" est écrit d'une façon nettement différente.

2 En allemand: "bei Jahren 3".

3 En allemand: "Waschungshaus". 
pour la grande purification des châteaux sacrés de Memphis avec leurs temples ... (?) sa superstructure pour la demeure du Seigneur de la nécropole ${ }^{1}$.

En l'an 7 de César, 18 Paophi, survint la toilette de la grande dame. Ils lui firent la purification depuis le ler jour jusqu'au 70ème jour. Ils firent qu'elle fût belle pour la belle demeure.

En l'an 7, le 14 Pharmouthi, ils firent la purification, l'ensevelissement ... (? $)^{2}$ le père divin, sem, prophète de Ptah, grand prêtre, prophète d'Horus Seigneur de Sekhem, prophète de César, Pchêre-en-Amon pour la joueuse de sistre par un bel ensevelissement avec onguent, myrrhe, résine, or, toute sorte de pierres précieuses véritables. Ils apportèrent pour elle des tissus des dieux de Haute-Egypte ${ }^{3}$. (Le reste, un peu plus d'une ligne, est à peine lisible; l'ensevelissement a eu lieu en grande pompe et à la manière des "très grands d'Egypte")".

Le texte hiéroglyphique et les deux interprétations de la partie démotique proclament Tanepherôs fille du père divin et prophète de Ptah Khahapi et de la musicienne accomplie Harunchis (204,6-7). Comme E. Revillout l'a déjà remarqué ${ }^{4}$, les noms des parents sont identiques à ceux des parents de Taimouthès, la femme de Psenptaïs (201,3-4), et les titres des deux Khahapi correspondent en partie. Tanepherôs est née dix ans après Taimouthès. On peut admettre qu'elles

${ }^{1}$ M. MALININE nous écrit au sujet de cet alinéa: "Brugsch, "Sie machten ihm ein Dekret als Seher des Gottes" termine la phrase. La traduction me paraît bonne. La phrase suivante commence "(En) l'an 3 (la lecture "an 1" me parait difficilement défendable) du dieu, le fils de dieu, etc." L'interprétation de tout le passage qui suit me parait plus correcte chez Revillout que chez Brugsch. Il faut bien lire $p 3$ hy (n) N3-nfr-ḥr "le mari de Nofré-ho" et non pas "(und) die Sistrumschlägerin Ta-nfr-ho". Le mot chy "Sistrumschlägerin" est, d'après moi, une invention de Brugsch. Ce titre devrait se lire ihy, avec $h$ et $i$ initial. La graphie que Brugsch donne à la page 935 , ligne 4 est incontrôlable puisque, dans la copie de Young, ce mot est en partie dans la lacune et ce qui en reste ne ressemble point à la graphie que donne Brugsch.

${ }^{2}$ M. MALININE écrit: "C'est Psenamounis qui a fait ensevelir son épouse. Lisez le mot laissé sans traduction par Brugsch: $m$ - $\underline{d} r=\mathrm{NT} \in \mathrm{N}$-, et traduisez: "durch", "par"; la tournure est passive; cf. SPIEGELBERG, Demot. Gram., paragraphe 380."

3 En allemand: "des Südlandes".

${ }^{4}$ Un prophète d'Auguste, dans Revue égyptologique, 2 (1882), p. 100, note 12. 
étaient soeurs. Les différences entre les titulatures du père de Taimouthès et du père de Tanepherôs proviendraient alors du temps écoulé entre la rédaction des stèles des deux femmes ${ }^{1}$.

D'autre part, la stèle d'Imouthès apprend que Psenamounis était le "fils du frère de la mère" d'Imouthès (204,7). Le père de Psenamounis était donc le "frère" de Taimouthès. En interprétant ce terme comme un équivalent de "mari"2, nous faisons de Psenamounis un "frère par le père" d'Imouthès ${ }^{3}$.

Tanepherôs est née le 13 Tybi de l'an 19 de Neos Dionysos et elle est morte le 18 Paophi de l'an 7 de César Auguste, après avoir vécu 38 ans, 9 mois et 10 jours ${ }^{4}$.

E. Revillout et $\mathrm{H}$. Brugsch lisent encore tous deux la date du 14 Pharmouthi de l'an 7 de César. Or la stèle d'Imouthès raconte que Psenamounis a enseveli son parent précisément ce 14 Pharmouthi de l'an 7 de César ${ }^{5}$. Sur la stèle de Tanepherôs, cette date ne peut se rapporter

${ }^{1}$ Khahapi est peut-être devenu "hiérogrammate de tous les dieux de l'Egypte" $(\mathbf{2 0 4 , 7 )}$ entre les décès de ses filles. Ce titre paraît aussi dans le texte démotique; E. Revillout l'a rendu par "écrivain des divines paroles des dieux, seigneur de Bési (Bubastis?)" et $\mathbf{H}$. Brugsch par "scribe des écrits sacrés des dieux seigneurs de $B \mathrm{ki}^{3}$.

2 Cf. par exemple le début de l'exhortation adressée par Taimouthès à son mari, qu'elle qualifie de "frère, mari, ami" $(\mathbf{2 0 1 , 1 5 )}$.

${ }^{3}$ Cf. la traduction du texte démotique de la stèle d'Imouthès par E. Revillout (cidessus, § 81). Comme la mère de Psenamounis n'était pas Taimouthès, E. Revillout la considère comme une "concubine du harem de Psé-Ptah" (Un prophète d'Auguste, dans Revue égyptologique, 2 (1882), p. 100, note 12). Après avoir constaté, dans la même note, que Tanepherôs est soeur de Taimouthès, E. Revillout se trompe en affirmant: "Nous pouvons être certains que Psé-Amen avait épousé la propre soeur de Psé-Ptah, son père". Tanepherôs était belle-soeur de Psenptaïs.

${ }^{4}$ Le règne de Neos Dionysos compte 29 ans selon le canon et celui de Cléopâtre 22 ans. Du 13 Tybi an 19 de Neos Dionysos au 13 Tybi an 29, il y a 10 ans; du 13 Tybi an 29 du roi au 13 Tybi an 22 de Cléopâtre, il y a 22 ans; du 13 Tybi an 22 de Cléopâtre au 13 Tybi an 6 de César, il y a 6 ans; du 13 Tybi an 6 de César au 13 Paophi an 7, il y a 9 mois et cinq jours (les épagomènes de l'an 6); enfin, du 13 Paophi an 7 de César au 18 Paophi an 7, il y a 5 jours; total: 38 ans, 9 mois, 10 jours, comme H. Brugsch l'a lu. E. Revillout a lu " 5 jours", mais a constaté plus tard qu'il y a bien 10 jours (Le comput ..., dans Revue égyptologique, 5 (1888), p. 130, note 2).

${ }^{5} \mathrm{Cf}$. le texte démotique de la stèle d'Imouthès, ci-dessus, $\$ \mathbf{8 1}$. 
qu'à l'ensevelissement de Tanepherôs elle-même, et pas à celui de Psenamounis comme E. Revillout l'a compris ${ }^{1}$. Plusieurs mois, exactement 176 jours, se sont écoulés entre le décès et l'ensevelissement de Tanepherôs. Une partie de ce temps a été employée à la momification, "du 1er au 70ème jour", comme le texte l'affirme. Le reste a peut-être passé dans l'attente des tissus nécessaires aux funérailles d'une grande épouse de Ptah. Ces étoffes venaient de sanctuaires éloignés ${ }^{2}$ et nous pouvons supposer qu'ils sont arrivés à Memphis avec un certain retard. Pour Imouthès, le temps entre sa mort et son ensevelissement a été si long que Psenamounis en a mentionné la durée: 6 ans, 8 mois et 10 jours $^{3}$. Comme Imouthès-Petobastis a peut-être officié à Alexandrie alors qu'il était déjà grand prêtre (voir supra), nous pouvons supposer qu'il est mort à Alexandrie et que sa momie y est restée jusqu'à la mort de Tanepherôs. C'est alors que Psenamounis aurait décidé de faire transporter le corps d'Imouthès à Memphis pour l'y ensevelir définitivement, et ce transfert aurait été la cause du retard apporté à l'ensevelissement de Tanepherôs.

En comparant les interprétations de la biographie de Tanepherôs, nous pouvons établir ceci: Tanepherôs a été faite musicienne de Ptah. Elle a épousé Psenamounis et lui a enfanté une ou plusieurs filles. Elle a été promue grande épouse de Ptah. Elle a donné le jour à un fils. Ces événements paraissent tous contemporains du règne de Cléopâtre. L'un d'eux est même daté de cette reine: c'est le mariage de Psenamounis, alors prophète de Ptah, et de Tanepherôs. ${ }^{4}$. Psenamounis a donc été déjà adulte sous Cléopâtre; il est né avant Imouthès, qui n'atteignit ses seize ans qu'à la fin du règne. Nous en déduisons que Psenamounis a pu servir de tuteur à Imouthès et en assumer les charges de grand des chefs des artisans entre la mort de Psenptaïs et la nomination d'Imouthès.

${ }^{1}$ Un prophète d'Auguste, dans Revue égyptologique, 2 (1882), p. 101, notes 3 et 5.

${ }^{2}$ E. Revillout: "On fit venir pour elle des étoffes de divin service de chanteuse, depuis les sanctuaires et les temples de Syrie jusqu'en Egypte". H. Brugsch: "Ils apportèrent pour elle des tissus des dieux de Haute-Egypte".

${ }^{3}$ Ci-dessus, § 81.

${ }^{4}$ En l'an 20 d'après E. Revillout, en l'an 3 d'après H. Brugsch. Tanepherôs aurait eu alors respectivement 30 ou 13 ans. 
La suite de la biographie de Tanepherôs présente, d'une traduction à l'autre, des différences assez grandes entre lesquelles les remarques de M. Malinine permettent de faire un choix. Nous croyons pouvoir dire que le fils de Psenamounis et de Tanepherôs, auquel on donna le nom de Psenptaïs (c'est là une raison de plus de considérer Psenamounis comme un fils de Psenptaïs II), fut nommé "voyant du dieu" antérieurement à l'an 3 de César. C'est à cette dernière date que Psenamounis fut alors nommé grand prêtre de Memphis et prophète de César. Les lignes suivantes racontent probablement les cérémonies qui accompagnèrent la double nomination de Psenamounis; ce dernier, en rédigeant la stèle de son épouse, s'est un peu étendu sur le rappel de son élévation au pontificat.

Nous pouvons maintenant recueillir ce que nous savons de Psenamounis. Il est né à une date qui pourrait être voisine de l'an 19 de Neos Dionysos puisque son épouse a vu le jour en cette année. C'était un neveu de Taimouthès, c'est-à-dire un petit-fils du prophète Khahapi, et peut-être un fils de Psenptaïs né avant le mariage de ce dernier et de Taimouthès; c'est alors pour cette raison qu'il n'aurait pas été nommé grand prêtre à la mort de son père mais seulement lorsqu'Imouthès mourut sans héritier.

Psenamounis a pu être tuteur d'Imouthès et suppléer à l'absence de grand des chefs des artisans de l'an 11 à l'an 13 de Cléopâtre. Sous le même règne, il épousa, quand il n'était encore que prophète de Ptah, la musicienne de Ptah Tanepherôs. Celle-ci lui donna une ou plusieurs filles et un fils, dont la naissance date aussi de Cléopâtre, de même que la promotion de Tanepherôs au rang de grande épouse de Ptah.

Psenamounis pouvait avoir une trentaine d'années ou davantage à la mort d'Imouthès, en $30 \mathrm{av}$. J.-C. Octave, qui venait de mettre le pied en Egypte, ne chercha pas à innover lorsqu'il fut question de nommer un nouveau grand des chefs des artisans. Il suivit la coutume des Ptolémées. Comme Imouthès n'avait pas d'enfant, Octave nomma le plus proche parent du grand prêtre défunt et fit en même temps de Psenamounis le prophète de César. En outre, il lui donna une couronne et d'autres présents, ainsi que Neos Dionysos l'avait fait à son prophète Psenptaïs. Toutefois, il s'écoula un certain temps entre le décès d'Imouthès et l'accession de Psenamounis au pontificat, puisque Psenamounis ne fut probablement nommé qu'en l'an 3 de César. 
Quelques années plus tard, Psenamounis perdit sa femme. Quand tout fut prêt pour les funérailles, il l'ensevelit, ainsi qu'Imouthès, probablement dans le tombeau familial où Psenptaïs, sa fille Kheredouankh et Taimouthès reposaient avec les ancêtres de Psenptaïs.

Nous ignorons à quelle date la carrière de Psenamounis prit fin.

Les stèles d'Imouthès et de Tanepherôs sont les derniers documents que nous connaissions sur les "grands des artisans-chefs" ${ }^{\text {"1 }}$ et nous sommes réduits à supposer l'existence de cette fonction sous la domination romaine.

Le fils de Tanepherôs ${ }^{2}$, né vers la fin du règne de Cléopâtre, a pu succéder à Psenamounis. Après lui, le titre "grand des artisans" ${ }^{13}$ a pu être porté pendant longtemps, même jusqu'au quatrième siècle de notre ère puisqu'une procession d'Apis s'est encore déroulée à Memphis en 398 , sous le règne d'Arcadius ${ }^{4}$. Toutefois, d'après ce qu'on sait des temples égyptiens au cours de cette période ${ }^{5}$, il est fort possible que les grands des artisans aient perdu peu à peu toute importance. Ils ont disparu au plus tard lorsqu'on cessa de célébrer les cultes païens à Memphis.

${ }^{1}$ C'est la forme hiéroglyphique prise au cours de l'époque ptolémaïque par le titre original "grand des chefs des artisans"; voir ci-dessus, $§ 4$.

${ }^{2}$ Il s'appelait Psenptaïs et était peut-être le petit-fils de Psenptaïs II.

${ }^{3}$ Equivalent démotique de grand des chefs des artisans.

${ }^{4}$ H. KEES, Memphis, dans PAULY-WISSOWA, Real-Encyclopädie, XXIX (1931), p. 666-667.

${ }^{5}$ A.M. BLACKMAN, Priest, Priesthood (Egyptian), dans HASTINGS, Encyclopaedia of Religion and Ethics, vol. 10 (1918), p. 298, 1ère col.: "Dans la période romaine. Quoiqu'il y eût encore des surintendants des temples (épistates), l'administration d'un temple était généralement dans les mains du collège des anciens qui, comme les prêtres conseillers, changeaient chaque année. Après 202 ap. J.-C., les temples perdirent tout ce qui subsistait encore de leur position spécialement privilégiée jadis et furent placés sous l'administration des sénats municipaux." 


\section{O N C L U S I O N}

Nous avons déjà essayé de dégager, chapitre après chapitre, les résultats de nos recherches. Quelques-uns d'entre eux nous semblent nouveaux et méritent à ce titre que nous les exposions encore une fois. De plus, en les réunissant, nous définirons les enseignements apportés par l'étude des grands prêtres de Ptah de Memphis.

Nous avons constaté que le grand prêtre memphite exerce une double fonction dès le plus ancien temps atteint par notre documentation, soit la IVème dynastie. D'une part, il célèbre le culte divin; il officie alors en "prophète de Ptah", ou plus tard en "père divin aimé du dieu". D'autre part il dirige en qualité de "grand des chefs des artisans" les corps de métier memphites qui dépendent de l'administration royale; c'est alors un haut fonctionnaire. Le grand prêtre memphite de l'Ancien Empire est ainsi revêtu d'une double dignité. Ses successeurs l'ont d'ailleurs conservée en tout cas jusqu'à la fin des Ptolémées, puisqu'à cette époque ils sont "intronisés" prêtres tandis qu'ils sont "faits" ou "nommés" grands des chefs des artisans. Nous avons donc là un trait essentiel du haut sacerdoce memphite.

Toutefois ce trait n'existait peut-être pas à l'origine du pontificat; il se pourrait même qu'il eût été acquis seulement au début de la IVème dynastie. L'Ancien Empire a en effet connu des "prophètes de Ptah" qui n'étaient que "directeurs des artisans" et non grands des chefs des artisans, de sorte que les premiers grands prêtres que nous connaissons ont eu des égaux sur le plan ecclésiastique tandis qu'ils ont été des primi inter pares sur le plan civil. En considérant cette différence, et aussi le fait qu'un chef d'artisans de la IIIème dynastie, orné de l'insigne pectoral qu'on retrouve sur des grands prêtres, n'avait pas été au service de Ptah, nous pouvons supposer que le culte rendu au dieu memphite et le travail effectué par les artisans n'ont peut-être été associés étroitement qu'au début de la IVème dynastie. 
En plus des grands prêtres, nous notons que les autres membres du clergé de Ptah ont également exercé une double fonction, ecclésiastique et civile, depuis l'Ancien Empire.

Si nous comparons maintenant entre eux les grands prêtres des diverses époques, nous constatons que les deux éléments qui composent leur dignité, le civil et le religieux, ont évolué au cours des âges. Le premier a subi une transformation vers la fin de l'Ancien Empire. Le second a été modifié trois fois: vers la fin de l'Ancien Empire, sous Ramsès II et à la veille de l'époque saïte.

De la IVème dynastie à la VIème il y a en même temps deux grands prêtres de Ptah parce que leur fonction civile de grand des chefs des artisans participe du dédoublement administratif de l'époque. Cette dualité disparaît lorsqu'un roi de la VIème dynastie, peut-être Pépi Ier, réforme le pontificat en unifiant le rouage des artisans. Comme il s'arroge aussi le droit de nommer le grand des chefs des artisans désormais unique, nous estimons qu'il a essayé de raffermir le pouvoir royal dangereusement affaibli par de nombreuses donations aux temples.

Sous la VIème dynastie également, les grands prêtres de Ptah commencent à s'appeler "prêtre-sem". Ce titre religieux paraît se rapporter chez eux au culte de Sokar, qui n'est plus alors le dieu memphite primitif mais une divinité transformée par assimilation à Osiris. Nous en concluons que l'apparition de "prêtre-sem" parmi les titres des grands prêtres de Ptah concrétise dans le domaine ecclésiastique la synthèse théologique de Sokar-Osiris.

Beaucoup plus tard, sous Ramsès II, les grands prêtres deviennent des "prêtres-sem de Ptah". En même temps ils font passer ce nouveau titre, ainsi que prêtre-sem qu'ils continuent à employer, au premier rang dans l'énoncé de leurs diverses fonctions et relèguent grand des chefs des artisans à la seconde place. La nouvelle appellation et l'importance accordée au titre de prêtre-sem correspondent à une autre synthèse théologique plus complexe que la précédente: nous voulons parler de Ptah-Sokar-Osiris, divinité qui tient davantage d'Osiris que de Sokar ou de Ptah.

Notons que le premier emploi de prêtre-sem signale vraisemblablement un changement dans le culte de Sokar sous la VIème dynastie; de même, 
prêtre-sem de Ptah indique probablement une modification du culte de Ptah sous Ramsès II.

Enfin, au cours de la période pré-saïte, les grands prêtres redonnent la préférence à leur ancien titre de grand des chefs des artisans. Or, c'est sous Chabaka qu'on recopie le vieux document de théologie memphite dans lequel Ptah apparaît en dieu créateur et non comme une divinité assimilée à Osiris. Ces deux faits témoignent d'une réaction contre les syncrétismes légués par les époques précédentes et d'un retour partiel à la tradition de l'Ancien Empire.

Aux temps suivants, jusqu'au début de la domination romaine, on ne constate plus de modifications comparables à celles que nous venons de voir.

Ainsi les transformations successives des principaux titres des grands prêtres de Ptah marquent les étapes d'une histoire du pontificat memphite. On y relève pendant un temps assz long une emprise grandissante de la religion osirienne sur le dogme memphite, puis une opposition au triomphe d'Osiris. Mais cette histoire montre aussi que les grands prêtres avaient déjà lutté, antérieurement à l'époque pré-saiite, contre l'assimilation de Sokar et ensuite de Ptah à Osiris puisqu'il y eut chaque fois décalage entre l'apparition des synthèses théologiques que nous avons dites et les transformations correspondantes du pontificat. L'origine de ces synthèses paraît dès lors étrangère à l'école théologique de Memphis.

En résumé, l'étude des grands prêtres memphites aide à mieux connaître les clergés égyptiens. Elle permet aussi de mieux saisir les rapports de la théologie et des institutions religieuses. Ces résultats nous semblent de nature à encourager des recherches sur d'autres pontificats de l'ancienne Egypte ! 



\section{TROISIEME PARTIE}

L E S I N S C R I P T I O N S 



\section{N T R O D U C T I O N}

Comme la plupart des inscriptions concernant les grands prêtres memphites sont dispersées dans les musées du monde entier, que plusieurs sont inédites, que d'autres n'ont été publiées qu'imparfaitement, qu'elles n'ont, également, pas toutes été traduites ou que leur traduction n'est plus à la mesure des connaissances grammaticales et lexicographiques actuelles, nous estimons qu'il est utile de les publier toutes ensemble et d'en donner une traduction.

Nous groupons ainsi des documents qui, tout en ne provenant presque tous que d'un seul endroit et ne se rapportant qu'à un des prêtres égyptiens les plus importants, ont l'avantage de couvrir plus de deux millénaires. Leur seule confrontation permet déjà de résoudre bien des difficultés qu'ils présentent isolément.

Cette Troisième Partie contient les inscriptions relatives aux "grands des chefs des artisans", car tel est le titre qui nous paraît désigner indubitablement les grands prêtres de Memphis. La majeure partie des textes originaux sont hiéroglyphiques. Les hiératiques sont rares. Quant aux démotiques, pour lesquels nous n'aurions pu que calquer les publications et les traductions, parfois peu concordantes, d'autres auteurs, nous nous sommes bornés à indiquer leur bibliographie.

Nous avons signalé dans chaque cas la base de notre édition: copie personnelle, collation de publications antérieures avec les originaux, ou à défaut leur photographie, parfois aussi publication. Chacune de nos inscriptions n'a qu'une numérotation interne, qui tient cependant compte de la disposition originale du texte. Les flèches, verticales et horizontales, donnent le sens de la lecture des hiéroglyphes.

Genève, juin 1947 



\section{LES INSCRIPTIONS DE L'ANCIEN EMPIRE}

\section{LE TOMBEAU DE DEBEHEN}

Paroi d'entrée, côté droit

Bibliographie:

LEPSIUS, Denkmäler, II, 37 b (texte);

SETHE, Urkunden I, p. 18-21 (texte);

BREASTED, Ancient Records, I, § 210-212 (traduction);

$\mathrm{PM} \mathrm{III}^{2} / 1$, p. 235-236, LG 90;

ma collation:

\& 1 店

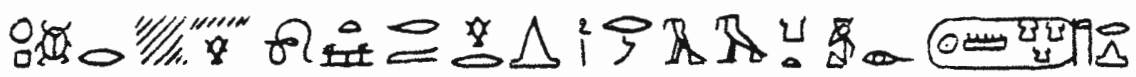

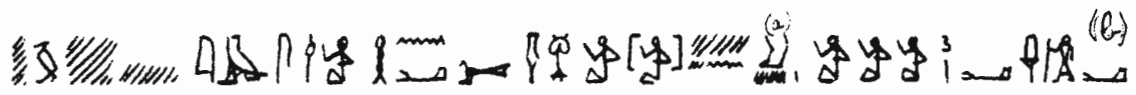

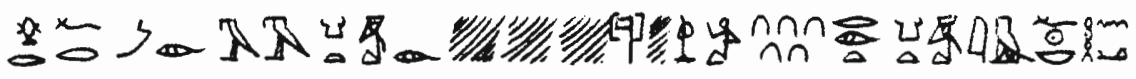

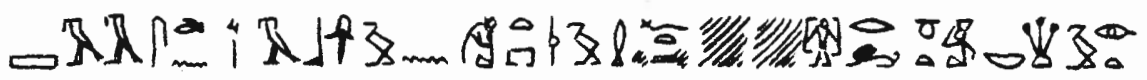

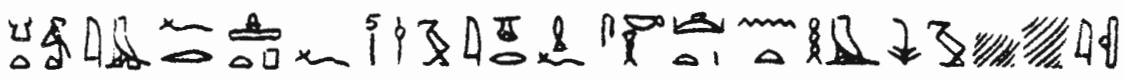

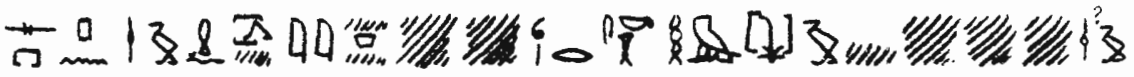
W/W/// 


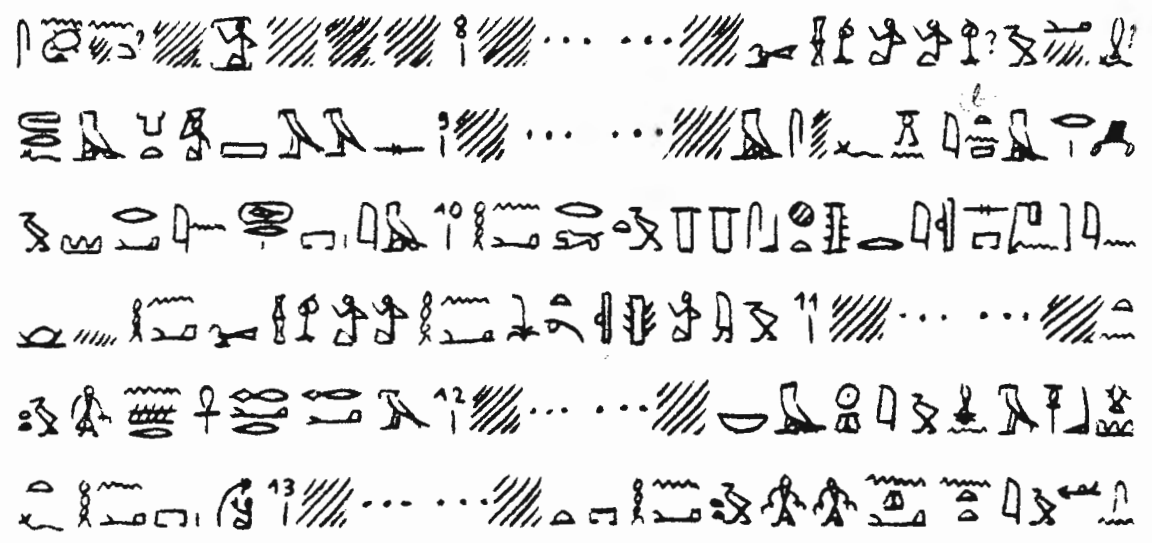

(a) Signe que je n'ai pas réussi à identifier.

(b) Métathèse apparente due à la disposition du texte en colonnes.

"Il dit : Ce tombeau c'est le roi de Haute et Basse-Egypte Men(kaou)rê (vivant à jamais) (a) qui m'a donné son emplacement.

Or il arriva que ( $\mathrm{Sa}$ Majesté) (a) était en route, près du plateau des pyramides, [2] pour voir travailler (b) à la pyramide Menkaourê-est-divin .... là le surveillant et les deux grands des chefs des artisans, tandis que les ...[3] s'y tenaient pour voir travailler (à la pyramide Menkaourê)-est-divin, et cinquante artisans pour travailler là chaque jour et .... [4] ..... de (?) la ouabet. Sa Majesté ordonna .... à (?) chaque équipe horaire (en ?) plus afin que le travail y fût fait à sa satisfaction. [5] Sa Majesté ordonna aussi de nettoyer la butte de Hemsou ... ce tombeau. Sa Majesté ordonna ..... [6] pour nettoyer Hemsou ....... Les deux trésoriers divins vinrent et ils dirent ..... [7] ........ à cause de cela plus que toute chose .... (?) .... [8] ...... les deux grands des chefs des artisans ... (?) quand il parcourait (?) le travail ... [9] rapporter de la pierre de Toura pour revêtir le temple là [10] ainsi que les deux montants (?) d'une porte pour ce tombeau. Ce sont le ... (?) et les deux grands des chefs des artisans et le charpentier (?) - constructeur du roi qui sont venus [11] ...... la statue plus que grandement travaillée d'après le modèle vivant [12] ........ tout ... en besoin journalier. C'est aujourd'hui la halte (?) sur cette montagne et la demeure pure (?) [13] ........ et les deux 
statues à l'assistant, dont l'une est [14] ......... la fête d'Apis dans le palais du dieu [15] ........ son imakh sera auprès de son maître [16] ........ la ... de (?) (mon) père et de (ma) mère; ils ont gardé (c) [17] ........ du fard vert et du fard noir ... (?) [18] à l'extérieur de la place. Or donc ... (?) ... (?) en BasseEgypte, dans le nome du Taureau, dans la butte dont le nom est Menkh. [19] Un rescrit royal fut délivré au directeur .... les ... jusqu'à ce qu'il ait fait un tombeau de cent coudées dans sa longueur [20] sur cinquante coudées de largeur et sur neuf coudées de (hauteur ?) ..... plus qu'avait fait mon père de son vivant. Sa Majesté fit aussi que (Fin)."

(a) Restitution de Sethe.

(b) Deux traductions littérales sont possibles, en considérant la proposition dont le verbe est "faire", comme une complétive ou une temporelie: 1) voir le travail (et voir qu')on fait (ou: est faite) la pyramide; 2) voir le travail tandis qu'on fait (ou: est faite) la pyramide.

(c) Ou: gardant (pseudo-participe à la troisième personne du pluriel) pour eux.

\section{LA STELE DE NY-ANKH-SEKHMET}

\section{Caire 1482}

Bibliographie:

MARIETTE, Mastabas, p. 204-205 (texte);

MASPERO, dans P.S.B.A., XI (1889), p. 306 (texte, trad.);

BORCHARDT, Denkmäler des alten Reiches, I, 1937, p. 172 (texte) et pl. 39 (photo);

SETHE, Urkunden I, p. 38 (texte).

BREASTED, Ancient Records, I, § 238-239 (trad.);

ma collation:

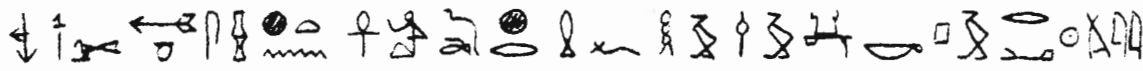

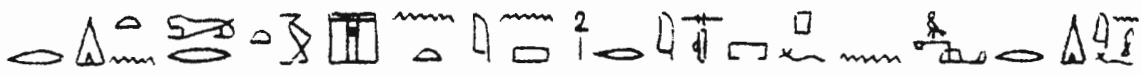

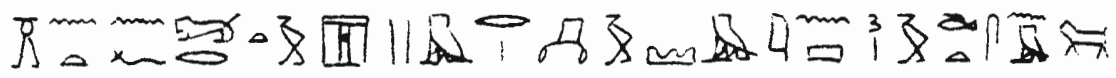

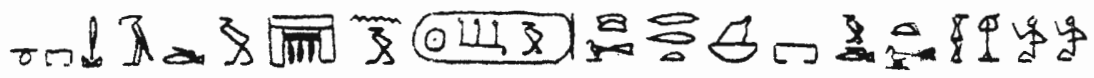

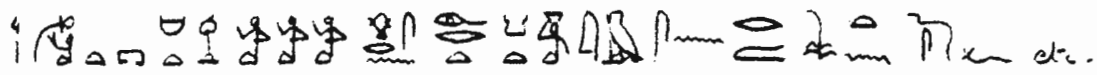


"[1] Le grand des médecins Ny-ankh-sekhmet dit à Sa Majesté: Puisse ton ka aimé de Rê commander qu'une porte de pierre me soit donnée [2] pour ce tombeau de la nécropole! Sa Majesté fit rapporter pour lui deux portes en pierre de Toura. [3] Elles furent placées dans la salle "La couronne de Sahourê apparait". Les deux grands des chefs des artisans [4] de la ouabet et des artisans y furent mis. On y travailla en présence du roi lui-même, etc...".

\section{3-4. LE TOMBEAU DE PTACHEPSES I}

Bibliographie:

$\mathrm{PM} \mathrm{III}^{2} / 2$, p. $464, \mathrm{n}^{\circ} 48$.

\section{Linteau British Museum 32 (682)}

Bibliographie:

d'après Hieroglyphic Texts from Egyptian Stelae, part I, pl. 10:

republié dans T.G.H. JAMES, Hieroglyphic Texts from Egyptian Stelae, Part I, 2nd edition, 1961, p. 17, pl. XVII;

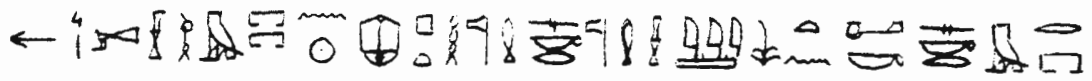

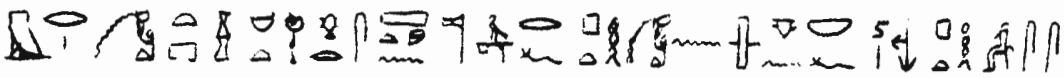

(Proscynème en faveur du) "[4] grand des chefs des artisans, celui qui est dans la Double Maison, le participant à la fête de Rê, prophète de Ptah et prophète de Sokar, chef des sem, confident du roi, directeur du domaine de Sokar, directeur de la ouabet, chef des artisans, supérieur des secrets de son dieu, ouab de Ptah, celui qui est dans le coeur de son maitre, [5] Ptahchepses."

4. Stèle British Museum 32 (682)

Disposition du texte:

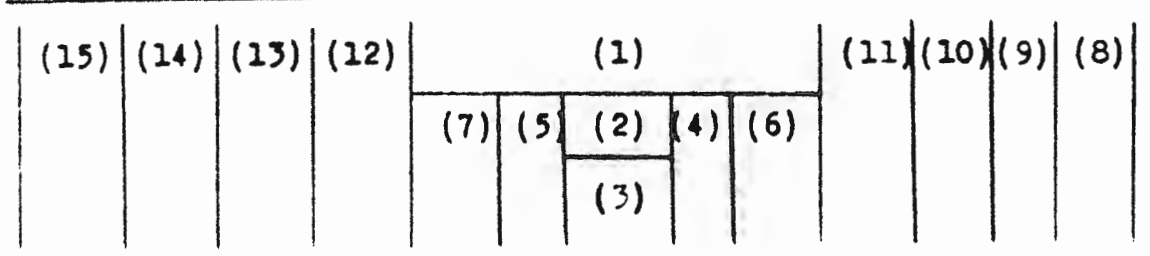


Titulature : [1] - [7]

Bibliographie:

MARIETTE, Mastabas, p. 112, 113;

Hieroglyphic Texts from Egyptian Stelae, Part I, pl. 12;

d'après Hierogl. Texts:

republié dans T.G.H. JAMES, Hieroglyphic Texts from Egyptian Stelae, Part 1, 2nd Edition, 1961, p. 17, pl. XVII;

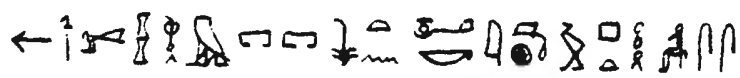

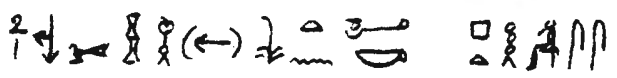

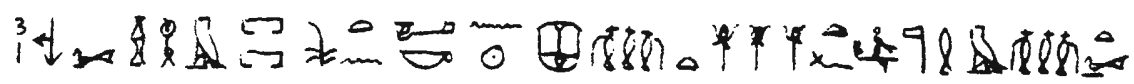
4 $78: 5: \operatorname{sip}$

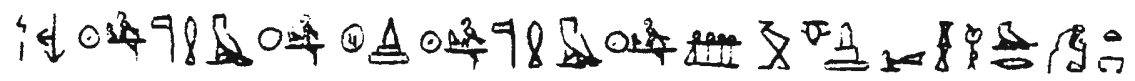

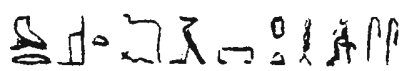

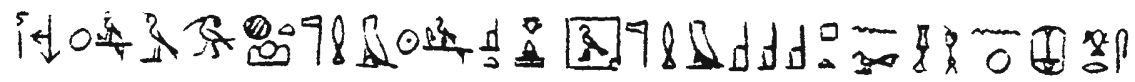

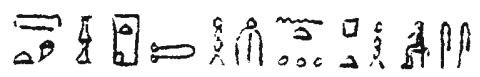

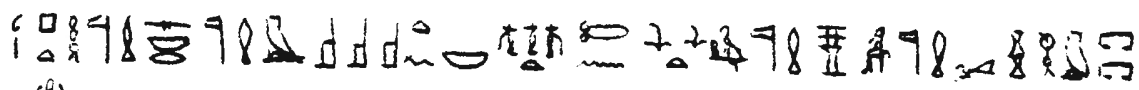

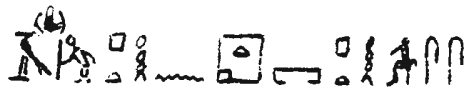

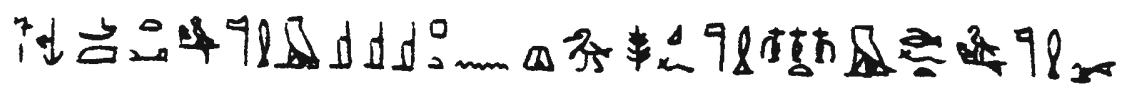

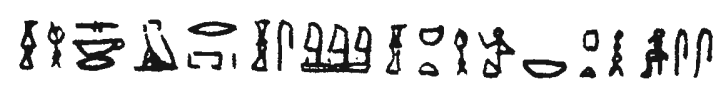

(a) Mariette: $4 \Omega$.

"[1] Le grand des chefs des artisans, celui qui est dans la Double Maison, confident du roi, imakhou, Ptahchepses". 
"[2] Le grand des chefs des artisans, confident du roi, Ptahchepses". "[3] Le grand des chefs des artisans, celui qui est dans la Double Maison, confident du roi, participant à la fête de Rê, prophète de Khenty-iaout-ef, prophète de Mekhent-our, Ptahchepses".

"[4] Le prophète de Rê dans Nekhen-Rê (a), prophète de Rê dans Chesepou-ib-Rê (b), grand des chefs des artisans, directeur de la ouabet, directeur de la place des aliments, Ptahchepses".

"[5] Le prophète de Rê-Horakhti dans Set-îb-Rê (c), prophète d'Hathor dans ces places, grand des chefs des artisans, participant à la fête de Rê, supérieur des secrets et chef de la maison de la faience, Ptahchepses".

"[6] Le prophète de Ptah et prophète de Sokar dans toutes ses places, prophète de Khenty-tenenet, prophète du Djed sacré, grand des chefs des artisans, celui qui est dans la Double Maison, l'ancien de la maison de Ptah, Ptahchepses".

"[7] Le prophète de Maât dans ces places, prophète de Khery-bak-ef, prophète de Khenty-medefet, grand des chefs des artisans, directeur du domaine du Sokar, chef des sem, chef de tous les artisans, Ptahchepses".

(a) Temple solaire d'Ouserkaf, cf. K. SETHE, Die Heiligtümer des Re im alten Reich, dans $\ddot{A} . Z$., 27 (1889), p. 111 sq.

(b) Temple solaire de Nyouserrê.

(c) Temple solaire de Neferirkarê.

Biographie: [8] - [15]

Bibliographie:

MARIETTE, Mastabas, p. 112-113 (texte);

SETHE, Urkunden I, p. $51-53$ (texte, avec restitutions de l'auteur);

Hieroglyphic Texts from Egyptian Stelae, Part I, pl. 11 et 13 (texte); republié dans T.G.H. JAMES, Hieroglyphic Texts from Egyptian Stelae, Part I, 2nd Edition, 1961, p. 17, pl. XVII;

BREASTED, Ancient Records, I, § 256-262 (trad.);

d'après Hierogl. Texts, 1ère édition: 


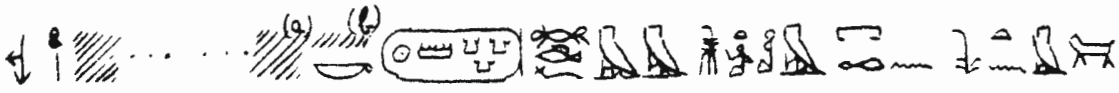

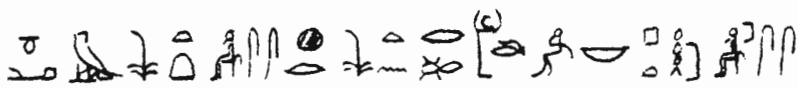

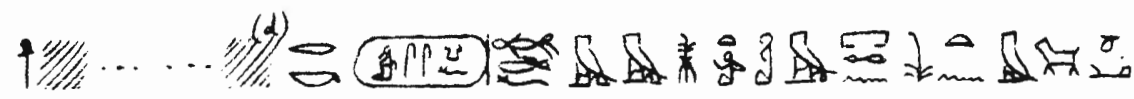

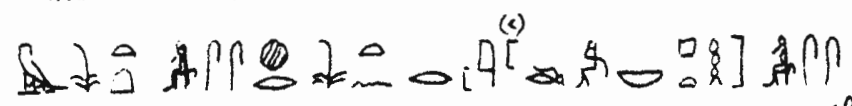

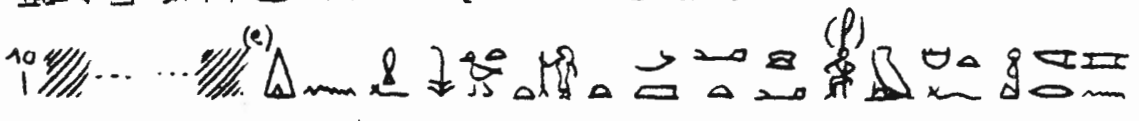
\&

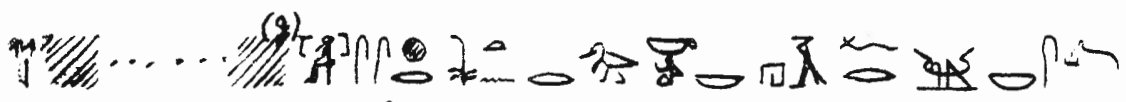
क दे 1"

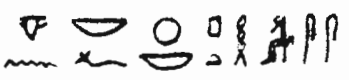

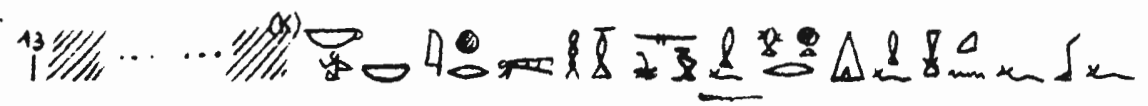

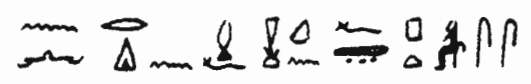

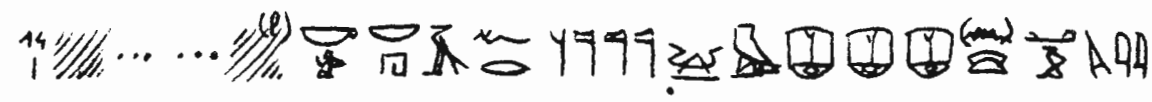
豆路仰

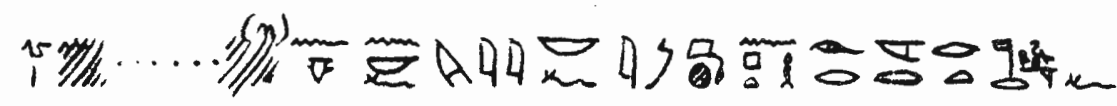

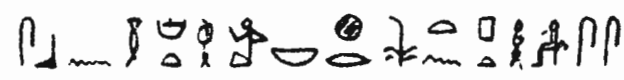




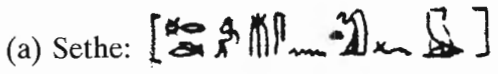
(b) Mariette, Sethe: $\mathbb{\Xi}$
(c) Restitué d'après Mariette.

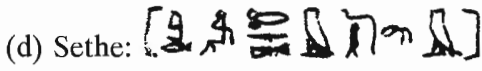
(e) Sethe: $\left[40 \% 98-238 l^{2} \sigma\right]$
(f) Femme sur un trône.

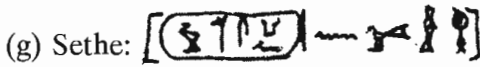
(h) Mariette, Sethe: $\Delta \frac{\Delta}{d}$

(h') Sethe: 은

(i) Mariette, Sethe: $18=$

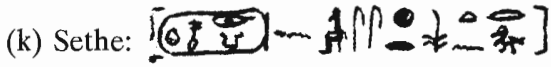

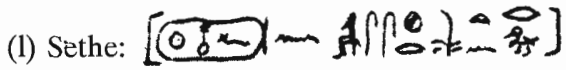

(m) Mariette, Sethe: 웁

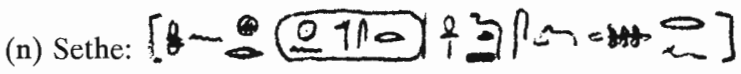

"[8] ..... temps de Menkaourê; il est élevé parmi les enfants royaux dans le palais du roi, dans la chambre privée, dans le harem du roi, plus honoré auprès du roi qu'aucun enfant, Ptahchepses !"

"[9] ..... temps de Chepseskaf; il est élevé parmi les enfants royaux dans le palais du roi, dans la chambre privée, dans le harem du roi, plus honoré auprès du roi qu'aucun jeune homme, Ptahchepses !"

"[10] ..... Sa Majesté lui donna sa fille aînée Khamaât pour femme, car Sa Majesté désirait qu'elle fût auprès de lui plus qu'auprès d'aucun homme, Ptahchepses !"

"[11] ..... honoré auprès du roi plus qu'aucun serviteur; il descend dans tout bateau; protégé, il entre sur les chemins du palais à toutes les fêtes de l'apparition, Ptahchepses !"

"[12] ..... tout serviteur, comme supérieur des secrets de tous les travaux que Sa Majesté désire faire, faisant du bien au coeur de son maître chaque jour, 
Ptahchepses!"

"[13] ..... tout serviteur; quand Sa Majesté le récompense pour quelque chose, Sa Majesté lui fait baiser son pied; Sa Majesté ne permet pas qu'il baise le sol, Ptahchepses!"

"[14] .... tout serviteur; il descend dans la barque "Porteuse de dieux" à toutes les fêtes de l'apparition, l'aimé de son maître, Ptahchepses !"

"[15] ..... qui a part au coeur de son maître, aimé de son mâtre, imakhou de Ptah, accomplissant ce que son dieu désire et faisant que chaque artisan soit en faveur auprès du roi, Ptahchepses!"

\section{LE LINTEAU DE PTAHCHEPSES II}

Bibliographie:

$\mathrm{PM} \mathrm{III}^{2} / 2$, p. $464-465, \mathrm{~N}^{\circ} 50$

MARIETTE, Mastabas, C 9, p. 130 (texte);

d'après Mariette:

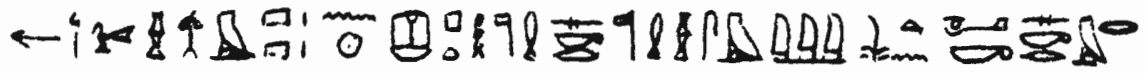

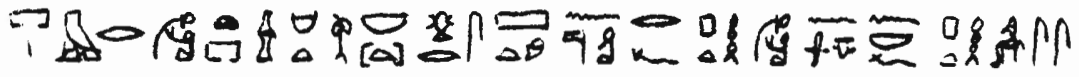

(Proscynème en faveur du) "[4] grand des chefs des artisans, celui qui est dans la Double Maison, le participant à la fête de Rê, prophète de Ptah et prophète de Sokar, chef des sem, confident du roi, directeur du domaine de Sokar, directeur de la ouabet, chef de tous les artisans, supérieur des secrets de son dieu, ouab de Ptah, celui qui est dans le coeur de son maître, [5] Ptahchepses !"

\section{6-7. LES DEUX STATUES DE PTAHCHEPSES I OU II}

\section{Caire 93}

Bibliographie:

$\mathrm{PM} \mathrm{III}^{2} / 2$, p. $464-465, \mathrm{~N}^{\circ} 50$

BORCHARDT, Statuen ..., I, p. 73 (texte) et pl. 21 (photo);

d'après Borchardt: 


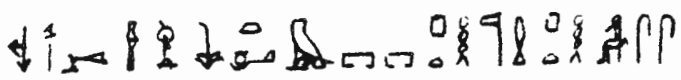

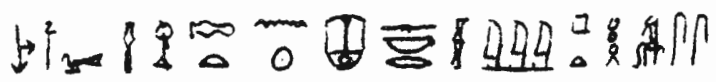

"[1] Le grand des chefs des artisans, confident du roi, celui qui est dans la Double Maison, prophète de Ptah, Ptahchepses".

"[2] Le grand des chefs des artisans ... (?) le participant à la fête de Rê, chef des sem de Sokar, Ptahchepses".

\section{Caire 368}

Bibliographie:

$\mathrm{PM} \mathrm{III}^{2} / 2$, p. $464-465, \mathrm{~N}^{\circ} 50$

BORCHARDT, Statuen ..., I, p. 193-194 (texte);

d'après Borchardt:

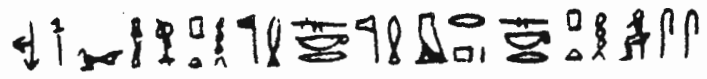

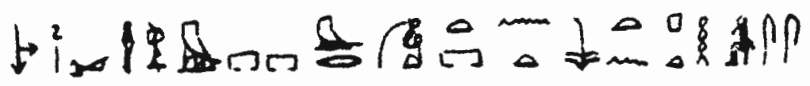

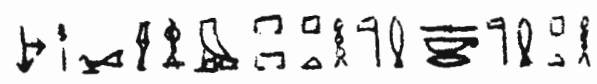

"[1] Le grand des chefs des artisans, prophète de Ptah et prophète de Sokar, directeur du domaine de Sokar, Ptahchepses".

"[2] Le grand des chefs des artisans, celui qui est dans la Double Maison, directeur de la ouabet du roi, Ptahchepses".

"[3] Le grand des chefs des artisans, celui qui est dans la Double Maison, prophète de Ptah et prophète de Sokar, Ptah(chepses ?)".

\section{LA STELE DE SENIMEN}

Caire 1410

Bibliographie:

BORCHARDT, Denkmäler des alten Reiches im Museum von Kairo, Teil I, 1937, p. 74-75;

$\mathrm{PM} \mathrm{III}^{2} / 2$, p. 698;

ma copie: 
(au haut de la stèle, en une ligne horizontale):

$\leftarrow 1 \times 1 \%$

"[1] Le grand des chefs des artisans, directeur du domaine de Sokar, Neferefrê-ankh."

\section{9-10. LES DEUX STATUES DE RENEFER}

9. Caire 18

Bibliographie:

BORCHARDT, Statuen ..., I, p. 19 (texte) et pl. 5 (photo);

Tombeau: $\mathrm{PM} \mathrm{III}^{2} / 2$, p. 461-462, $\mathrm{N}^{\circ} 40$;

ma collation:

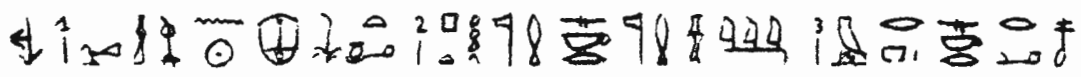

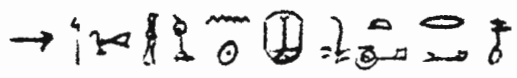

"[1] Le grand des chefs des artisans, le participant à la fête de Rê, confident du roi, [2] prophète de Ptah et prophète de Sokar, chef des sem, [3] et directeur du domaine de Sokar, Rênefer".

"[4] Le grand des chefs des artisans, participant à la fête de Rê, confident du roi, Rênefer."

10. Caire 19

$\mathrm{PM} \mathrm{III}^{2} / 2$, p. 461-462, $\mathrm{N}^{\circ} 40$;

BORCHARDT, Statuen ..., I., p. 20 (texte) et pl. 5 (photo);

ma collation:

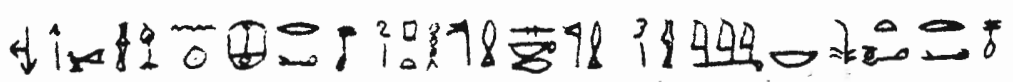

"[1] Le grand des chefs des artisans, participant à la fête de Rê, Rênefer; [2] le prophète de Ptah et prophète de Sokar, [3] chef de tous les sem et confident du roi, Rênefer." 


\section{LA STELE DE PTAHCHEPSES III}

Bibliographie:

M.A. MURRAY, Saqqara mastabas, part II (British School of Archaeology, 11th year), 1937, p. 24-26 et pl. 26-27 (texte);

SETHE, dans l'ouvrage ci-dessus, p. 21 (traduction);

$\mathrm{PM} \mathrm{III}^{2} / 2$, p. 499;

d'après M. Murray:

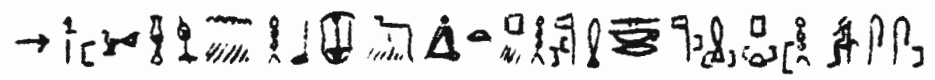

et quatre fois: 5

"[1] Le grand des chefs des artisans, participant à la fête de Rê, celui qui scelle, le prophète de Ptah et prophète de Sokar, Ptahchepses".

"[2] Le grand des chefs des artisans, Ptahchepses".

\section{2-13. LE TOMBEAU DE SABOU-KEM}

Bibliographie:

$\mathrm{PM} \mathrm{III}^{2} / 2$, p. 463.

\section{Stèle}

Bibliographie:

MARIETTE, Mastabas, p. 157;

d'après Mariette:

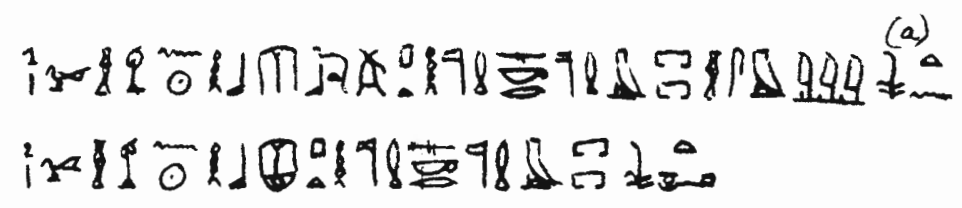

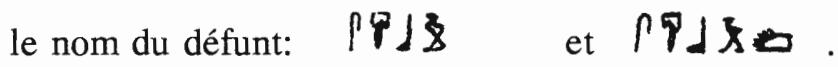

(a) à corriger sans doute en 
"[1] Le grand des chefs des artisans, participant à la fête de Rê, celui qui scelle, le prophète de Ptah et prophète de Sokar, celui qui est dans la Double Maison, chef des sem et confident (?) du roi, (Sabou)".

"[2] Le grand des chefs des artisans, participant à la fête de Rê, prophète de Ptah et prophète de Sokar, celui qui est dans la Double Maison, confident du roi, (Sabou)".

\section{Statue Caire 143}

Bibliographie:

BORCHARDT, Statuen ..., I, p. 105 (texte) et pl. 32 (photo);

d'après Borchardt:

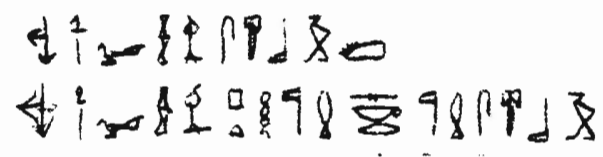

"[1] Le grand des chefs des artisans, Sabou-Kem".

"[2] Le grand des chefs des artisans, prophète de Ptah et prophète de Sokar, Sabou".

\section{4-15. LE TOMBEAU DE SETJOU}

Bibliographie:

PM III ${ }^{2} / 2$, p. 698.

\section{Groupe Caire 190}

Bibliographie:

BORCHARDT, Statuen ..., I, p. 130 (texte) et pl. 40 (photo);

ma collation:

(devant le siège) His

(sur le siège) $t^{2}-1590=18 \%$

(sur le socle, devant la femme) ${ }^{3} \leftarrow \neq \delta$ \&

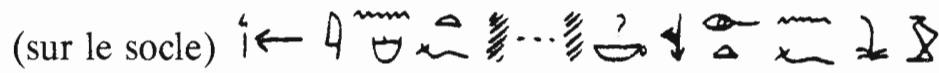


"[1] Le grand des chefs des artisans, prophète de Ptah, Setjou".

"[2] Le grand des chefs des artisans, prophète de Sokar, Setjou".

"[3] La connue du roi ... $k(a)$ ".

"[4] C'est sa femme, ... k(a), qui l'a fait pour lui".

15. Guéridon Caire 1300

Bibliographie:

BORCHARDT, Denkmäler ..., p. 3 (texte) et pl. 1 (photo);

ma collation:

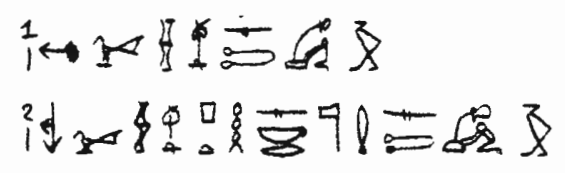

"[1] Le grand des chefs des artisans, Setjou".

"[2] Le grand des chefs des artisans, prophète de Ptah et de Sokar, Setjou".

\section{6-20. LE TOMBEAU DE SABOU-IBEBI}

Bibliographie:

PM III ${ }^{2} / 2$, p. 460-461, N॰37-38

\section{Au-dessus du linteau}

Bibliographie:

MARIETTE, Mariette, Mastabas, p. 375;

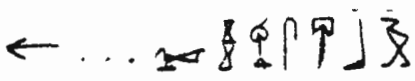

"Le grand des chefs des artisans, Sabou".

\section{Linteau}

Bibliographie:

ibidem:

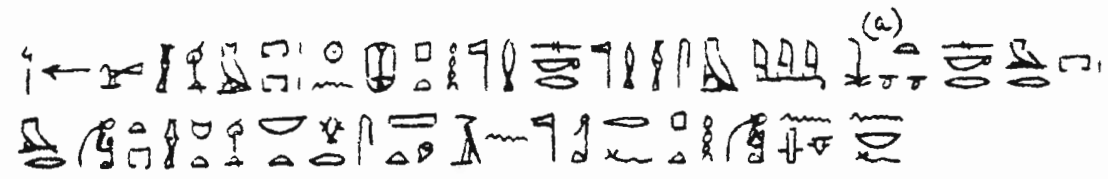


(a) à corriger probablement en

(Proscynème en faveur du) "[4] grand des chefs des artisans, celui qui est dans la Double Maison, participant à la fête de Rê, prophète de Ptah et prophète de Sokar, chefs des sem, confident (?) du roi, directeur du domaine de Sokar, directeur de la ouabet, chef de tous les artisans, supérieur des secrets de son dieu, ouab de Ptah, celui qui est dans le coeur de son maittre, ([5] Sabou)".

\section{Montant droit de la porte}

Bibliographie:

MARIETTE, Mastabas, p. 375:

(face extérieure) (aussi SETHE, Urk., I, p. 81, 1. 5-10)

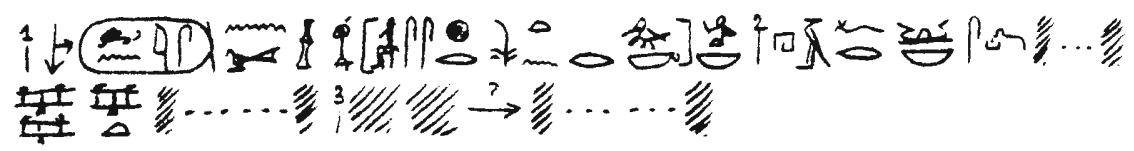

(dans l'épaisseur de la porte)

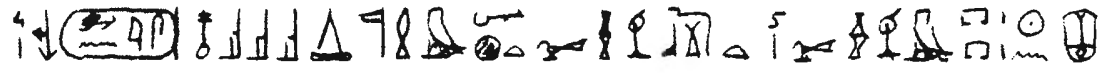

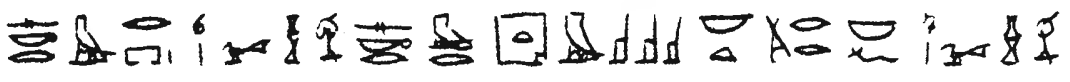

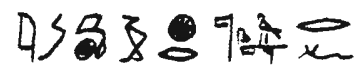

"[1] Attaché à Ounas, grand des chefs des artisans; plus honoré auprès du roi qu'aucun serviteur, [2] il descend dans toute barque; protégé, ..... chemins ..... [3] .....".

"[4] Prophète de Nefer-sout-Ounas et imy-khet, grand des chefs des artisans, - celui qui scelle; [5] grand des chefs des artisans, celui qui est dans la Double Maison, participant à la fête de Rê, directeur du domaine de Sokar; [6] grand des chefs des artisans, directeur du château de Sokar en toutes places, aimé de son maître; [7] grand des chefs des artisans, ìmakhou auprès de son dieu". 


\section{Montant gauche de la porte}

Bibliographie:

MARIETTE, Mastabas, p. 375:

(face extérieure) (aussi SETHE, Urk., I, p. 81, 1. 13-17)

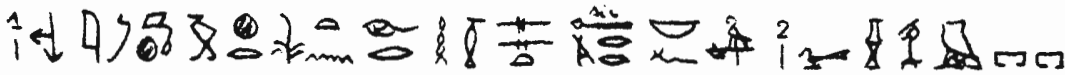

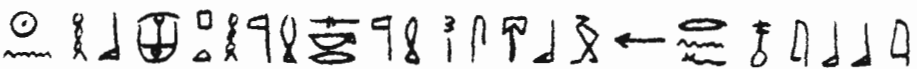

(dans l'épaisseur de la porte)

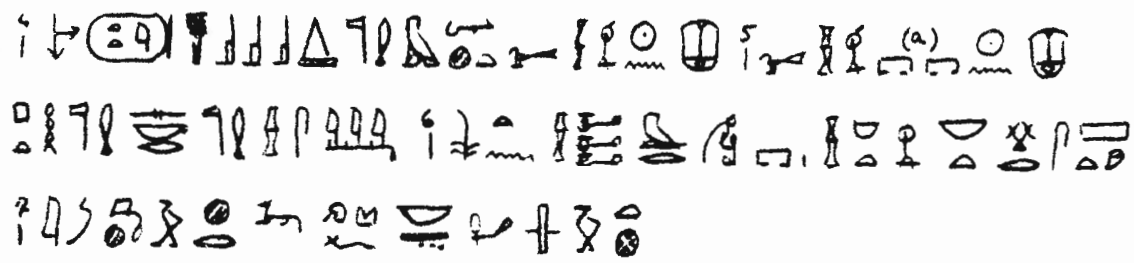

(a) Mariette a-t-il omis un $S$ ?

"[1]'Imakhou auprès du roi, accomplissant ce qu'il (a) loue, aimé de son Maitre; [2] grand des chefs des artisans, celui qui est dans la Double Maison, participant à la fête de Rê, prophète de Ptah et prophète de Sokar, [3] Sabou, de son beau nom (b) Ibebi".

"[4] Prophète de Djed-sout-Teti et imy-khet, grand des chefs des artisans, participant à la fête de Rê; [5] grand des chefs des artisans de la Double Maison (c), participant à la fête de Rê, prophète de Ptah et prophète de Sokar, chef des sem; [6] chef des confidents du roi, directeur de la ouabet, chef de tous les artisans, supérieur des secrets; [7] ìmakhou auprès d'Anubis qui est sur sa montagne, le seigneur de To-djeser, imy-out".

(a) D'après la correction proposée par SETHE, op. cit.

(b) Sur le "beau nom", cf. JUNKER, Die Stele des Hofarztes Iry, dans Ä.Z., 63 (1928), p. 59-64.

(c) Ou: Grand des chefs des artisans, celui qui est dans la Double Maison, en supposant une omission dans MARIETTE, Mastabas, p. 375. 
20. Stèle Caire 1565

Disposition du texte:

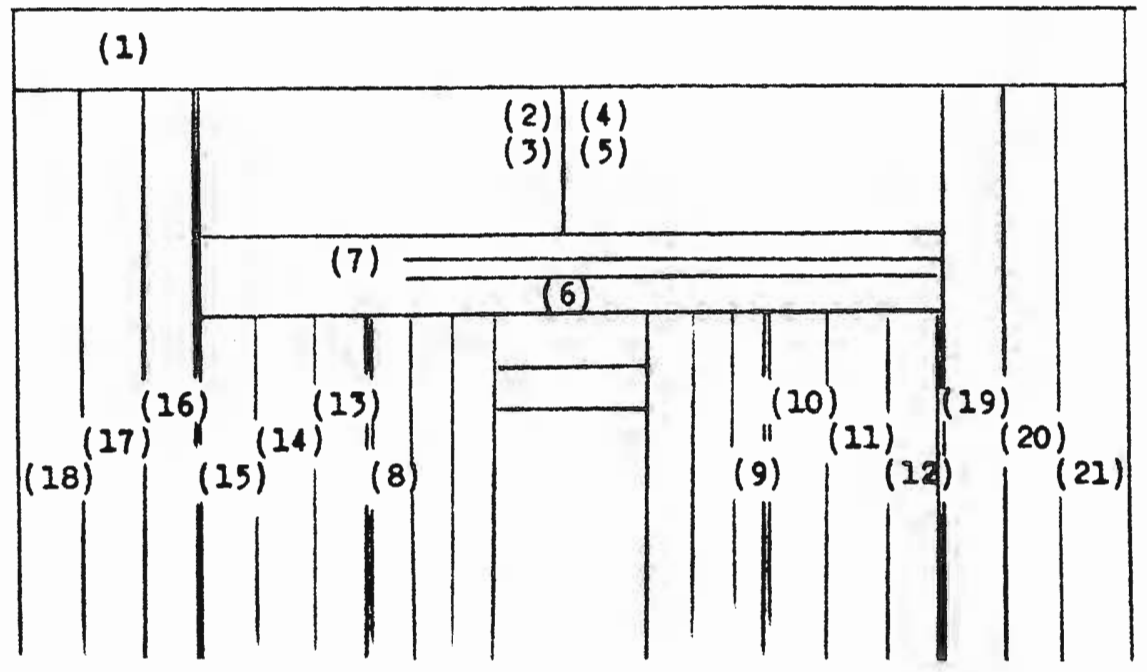

Légendes et proscynèmes [1] - [9]

Bibliographie:

MARIETTE, Mastabas, p. 413-415;

ma collation:

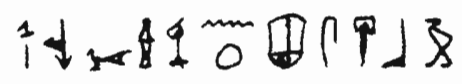

(Légende) "[1] Le Grand des chefs des artisans, participant à la fête de Rê, Sabou".

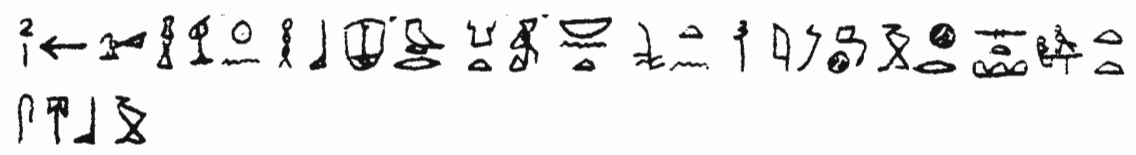

(Légende) "[2] Le grand des chefs des artisans, participant à la fête de Rê, directeur de tous les travaux du roi, [3] imakhou auprès du Désert Occidental, Sabou".

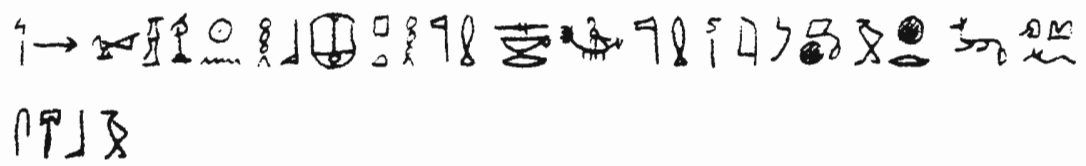


(Légende) "[4] Le grand des chefs des artisans, participant à la fête de Rê, prophète de Ptah et prophète de Sokar, [5] ìmakhou auprès d'Anubis qui est sur sa montagne, Sabou".

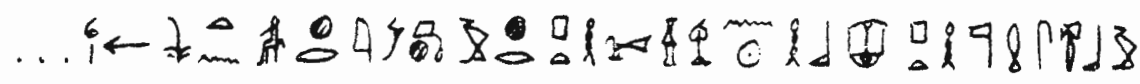

$$
\begin{aligned}
& \text { 各万प」dQ }
\end{aligned}
$$

(Proscynème en faveur de) "[6] l'honoré auprès du roi, imakhou auprès de Ptah, grand des chefs des artisans, participant à la fête de Rê, prophète de Ptah, Sabou, de son beau nom Ibebi".

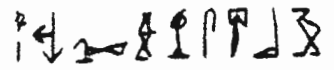

(Légende) "[7] Le grand des chefs des artisans Sabou".

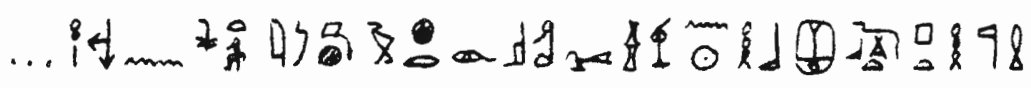

$$
\begin{aligned}
& \text {-斤甲]及 }
\end{aligned}
$$

(Proscynème en faveur de) "[8] l'honoré du roi, imakhou auprès d'Osiris, grand des chefs des artisans, participant à la fête de Rê, celui qui scelle, prophète de Ptah, Sabou".

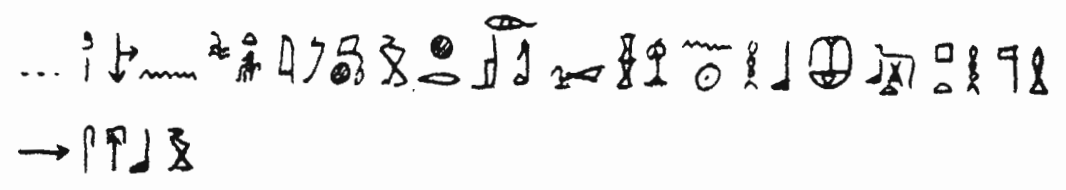

(Proscynème en faveur de) "[9] l'honoré du roi, imakhou auprès d'Osiris, grand des chefs des artisans, participant à la fête de Rê, celui qui scelle, prophète de Ptah, Sabou".

Titres [10] - [15]

MARIETTE, Mastabas, p. 413-415 (texte);

ma collation: 


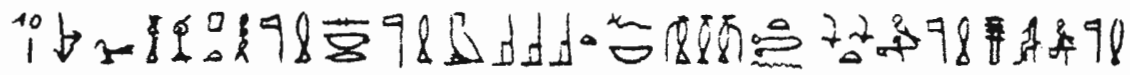 $<190$

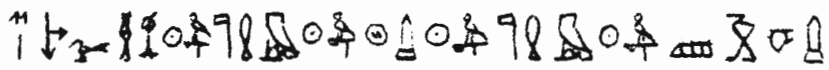

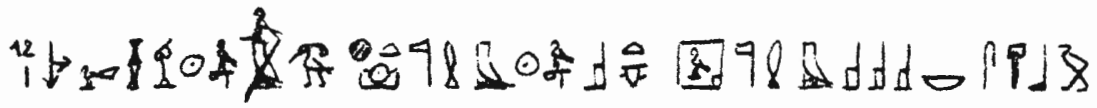

"[10] Le grand des chefs des artisans, prophète de Ptah, prophète de Sokar en toutes ses places, prophète de Khenty-Tjenenet, prophète du Djed sacré, grand des chefs des artisans, participant à la fête de Rê;

"[11] Le grand des chefs des artisans, prophète de Rê dans Nekhen-Rê (a), prophète de Rê dans Chesepou-ìb-Rê (b)";

"[12] Le grand des chefs des artisans, prophète de Rê-Horakhti dans Set-ibRê (c), prophète d'Hathor en toutes places, Sabou".

(a) temple solaire d'Ouserkaf.

(b) temple solaire de Nyouserrê.

(c) temple solaire de Neferirkarê.

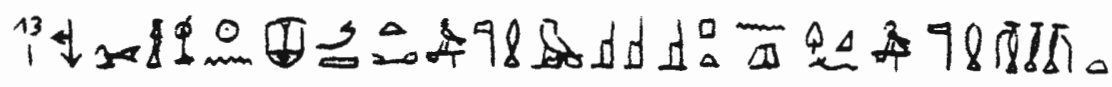
a.p.

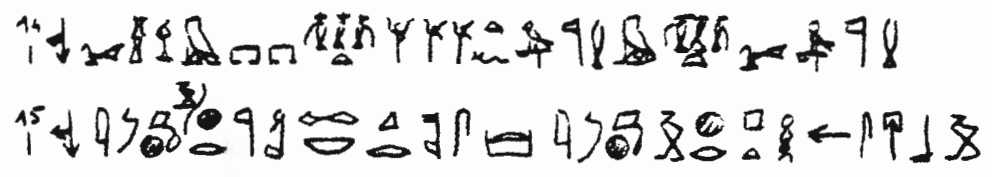

"[13] Le grand des chefs des artisans, participant à la fête de Rê, prophète de Maât dans ces places (a), prophète de Khery-bakef, prophète de Khentymedef(et) (b)";

"[14] Le grand des chefs des artisans, celui qui est dans la Double Maison, prophète de Khenty-iaoutef, prophète de Mekhent-our"; 
"[15] L'imakhou auprès du dieu grand, possesseur d'un tombeau, ỉmakhou auprès de Ptah, Sabou".

(a) Il me semble que "ces races" (au pluriel !) ne peut se rapporter qu'aux temples solaires désignés dans [11] et : 2], d'autant que Maât est la "fille de Rê". L'ordre que j'ai adopté pour la lecture des cu.unnes verticales de la stèle de Sabou-Ibebi provient de cette identification.

(b) Lecture complétée d'après la stèle de Ptahchepses, 4, ligne [7].

Biographie [16] - [21]

MARIETTE, Mastabas, p. $412-415$ (texte);

SETHE, Urk., I, p. 82-84 (texte);

BREASTED, Ancient Records, I, § 285-286 (traduction); ma collation:

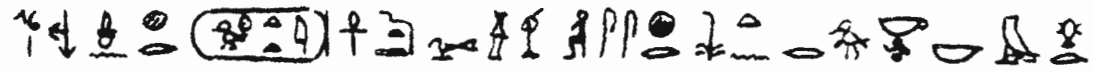

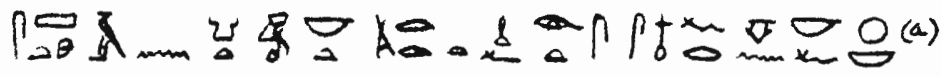

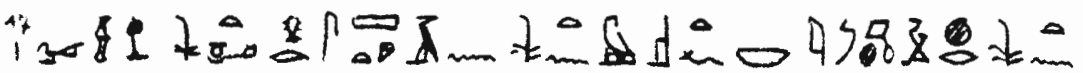

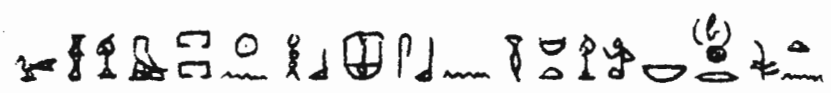

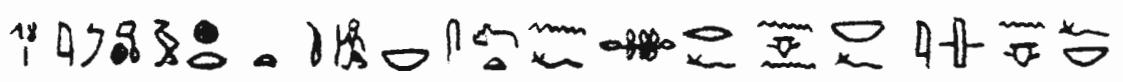

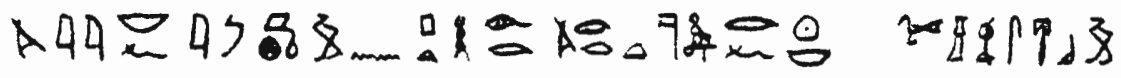

(a) Le trait de séparation entre les colonnes [16] et [17] s'arrête à la hauteur de $\stackrel{\odot}{\rightleftharpoons}$; les mots suivants "Le grand des chefs des artisans Sabou" n'appartiennent pas à la colonne [16] comme Mariette et Sethe l'indiquent, mais servent de légende à la figure de Sabou.

(b) Le trait de séparation entre les colonnes [17] et [18] s'arrête à la hauteur de P 1 mom de sorte que les mots $\neq$, bien que placés sous la colonne [18], doivent être lus avec la colonne [17], contrairement à ce qu'indiquent Mariette et Sethe. 
"[16] Aujourd'hui auprès du Fils de Rê Téti vivant éternellement, le grand des chefs des artisans, plus honoré auprès du roi qu'aucun serviteur en tant que supérieur des secrets de tous les travaux que Sa Majesté désire faire, procurant du plaisir au coeur de son maître chaque jour; [17] le grand des chefs des artisans, confident du roi, supérieur des secrets du roi en toutes ses places, imakhou auprès du roi, grand des chefs des artisans, celui qui est dans la Double Maison, participant à la fête de Rê, faisant que chaque artisan soit en faveur auprès du roi; [18] imakhou auprès de chaque souverain, leur protégé (a), celui qui a part au coeur de son maitre, celui qui est dans tout ce qui a part à son (= du maître) coeur, celui qui est aimé de son maître, l'imakhou de Ptah, accomplissant chaque jour ce que son dieu désire, (légende de la figure:) le grand des chefs des artisans Sabou!"

(a) litt.: il (le roi) le (Sabou) protège.

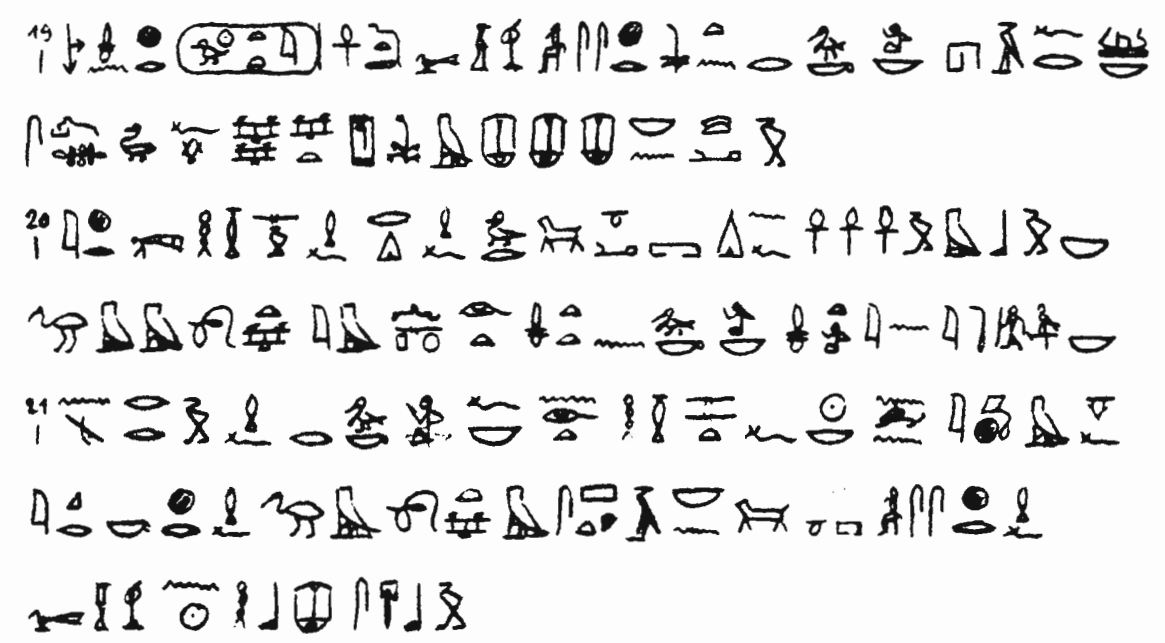

"[19] Aujourd'hui auprès du Fils de Rê Téti vivant éternellement, grand des chefs des artisans plus honoré auprès du roi qu'aucun serviteur, il descend dans toute barque; protégé, il entre sur les chemins du Palais du sud à toutes les fêtes de l'apparition!

[20] Or Sa Majesté m'a favorisé, car Sa Majesté m'a fait entrer dans la chambre privée, placer pour elle les gens dans chaque place et en trouver le 
chemin; jamais pareille chose n'avait été faite à aucun serviteur qui fût mon égal par aucun souverain, [21] parce que Sa Majesté m'aimait plus qu'aucun de ses serviteurs, parce que je faisais chaque jour ce qu'elle récompense, parce que mon attachement était dans son coeur (a); j'étais excellent auprès de Sa Majesté, trouvant un chemin en tout secret de la Résidence, étant honoré auprès de Sa Majesté ! (Légende de la figure:) Le grand des chefs des artisans, participant à la fête de Rê, Sabou".

(a) Cf. J. SAINTE FARE GARNOT, L'imakh et les imakhous sous l'Ancien Empire, 1943, p. 17 , note 3 .

\section{1-25. LE TOMBEAU DE PTAHCHEPSES IV}

Bibliographie:

$\mathrm{PM} \mathrm{III}^{2} / 2$, p. $460-461$

\section{Linteau}

Bibliographie:

MARIETTE, Mastabas, p. 377 B (texte);

DE ROUGE, Inscriptions hiérogl., p. 92 (texte);

d'après Mariette:

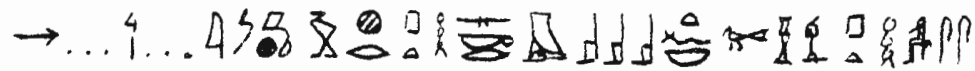

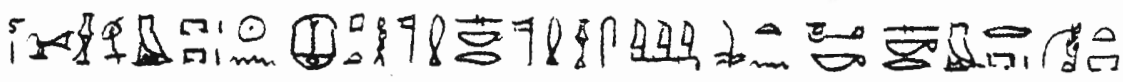

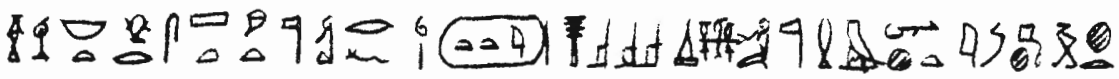

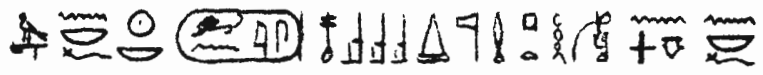

(Proscynème en faveur de) "[4] l'ìmakhou auprès de Ptah et de Sokar en toutes ses places (a), le grand des chefs des artisans Ptahchepses! [5] Le grand des chefs des artisans, celui qui est dans la Double Maison, participant à la fête de Rê, prophète de Ptah et prophète de Sokar, confident du roi, directeur du domaine de Sokar et de la ouabet (b), chef de tous les artisans, supérieur des secrets de son dieu, [6] prophète et imy-khet de Djed-souFTeti, imakhou auprès de son Maître chaque jour, prophète de Nefer-sout-Ounis, ouab de Ptah, participant à ce qui est dans le coeur de son maître". 
(a) Cf. inscription 4,6: "prophète de Ptah et prophète de Sokar en toutes ses places".

(b) Cf. inscription 5: "directeur du domaine de Sokar, directeur de la ouabet".

\section{MONTANTS DE PORTE}

MARIETTE, Mastabas, p. 377-378 (texte):

Ptahchepses n'y porte que le titre de "grand des chefs des artisans"

\section{TAMBOUR DE PORTE}

MARIETTE, Mastabas, p. 378 (texte):

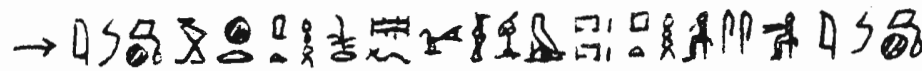

"L'imakhou auprès de Ptah qui est au sud de son mur, le grand des chefs des artisans, celui qui est dans la Double Maison, Ptahchepses, possesseur (?) de l'état d'imakh !"

\section{STELE}

MURRAY, Saqqara Mastabas, I, pl. 28 (texte)

SETHE, dans MURRAY, Saqqara Mastabas, I, § 24 (traduction des titres);

d'après Murray:

Disposition du texte:

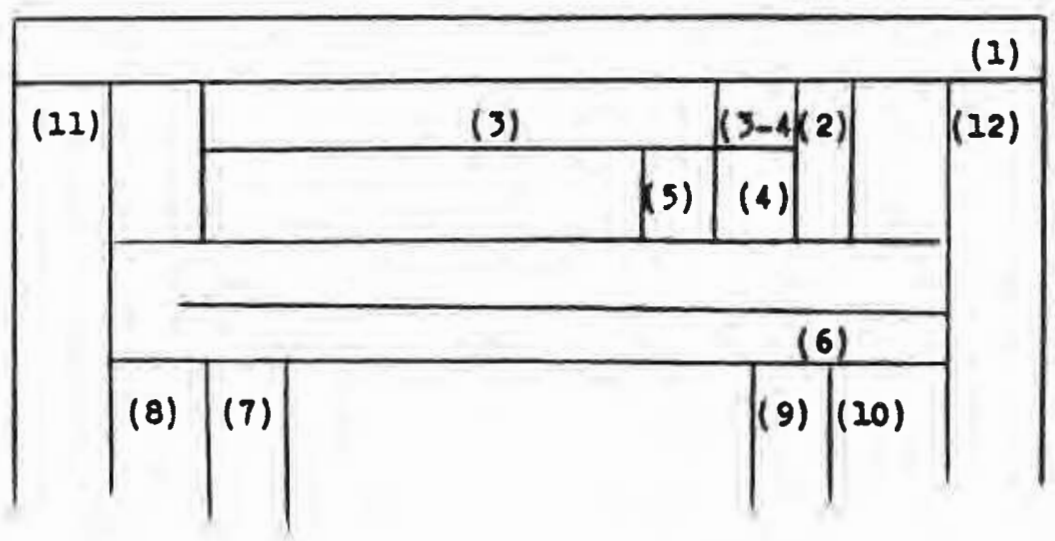




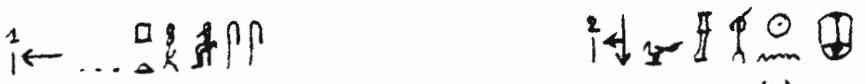

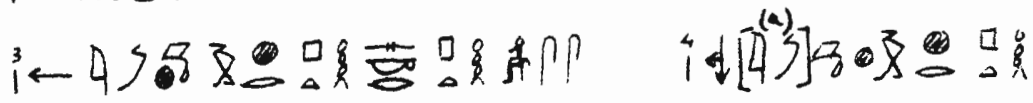

$$
\begin{aligned}
& \text { it } 453098 \text { i. }
\end{aligned}
$$

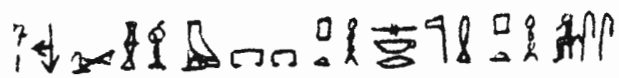

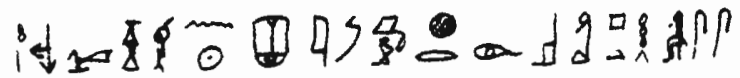

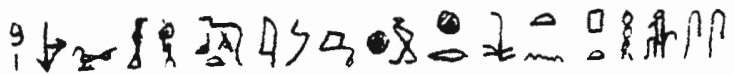

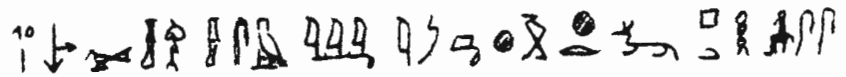

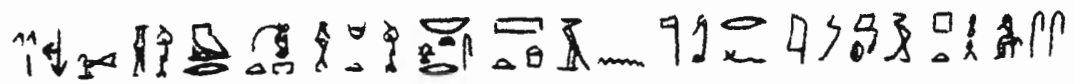

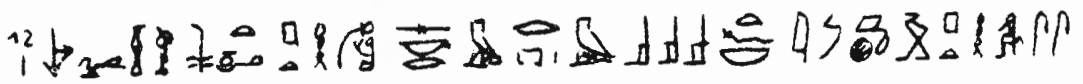

(a) les signes 43 appartiennent à la fois à [3] et [4].

"[1] (Proscynème en faveur de) Ptahchepses".

[2] - [5] (Légendes de Ptahchepses devant la table d'offrandes):

"[2] Le grand des chefs des artisans, participant à la fête de Rê, [3] l'imakhou auprès de Ptah et de Sokar, Ptahchepses; [4] l'ỉmakhou auprès de Ptah; [5] l'ìmakhou auprès du dieu grand".

"[6] (Proscynème en faveur du) prophète et ỉmy-khet de Dejd-Sout-Téti, Ptahchepses".

"[7] Le grand des chefs des artisans, celui qui est dans la Double Maison, prophète de Ptah et de Sokar, Ptahchepses".

"[8] Le grand des chefs des artisans, participant à la fête de Rê, ìmakhou auprès d'Osiris, Ptahchepses".

"[9] Le grand des chefs des artisans, celui qui scelle, ìmakhou auprès du roi, Ptahchepses.

"[10] Le grand des chefs des artisans, chef des sem, imakhou auprès 
d'Anubis, Ptahchepses".

"[11] Le grand des chefs des artisans, directeur de la ouabet, chef de tous les artisans, supérieur des secrets de son dieu, imakhou, Ptachepses".

"[12] Le grand des chefs des artisans, confident du roi, ouab de Ptah, directeur du domaine de Sokar en toutes ses places, imakhou, Ptachepses".

\section{CHAPELLE}

Sur les parois nord et sud, Ptahchepses est intitulé les deux fois de la même façon.

MURRAY, Saqqara Mastabas, pl. 29 et 30 (texte):

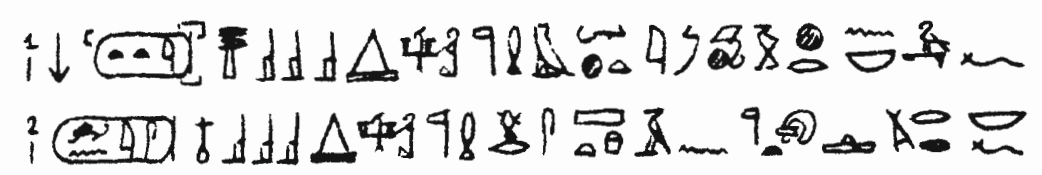

"[1] Prophète et ìmy-khet de Dejd-sout-Teti, ìmakhou auprès de son maitre, [2] prophète de Nefer-sout-Ounis, supérieur des secrets du trésor divin, aimé de son maître".

\section{LA STELE DE SABOU-TJETI}

Bibliographie du tombeau:

$\mathrm{PM} \mathrm{III}^{2} / 2$, p. $463, \mathrm{~N} \circ 47$.

Caire 1709 et 1756

Bibliographie:

MARIETTE, Mastabas, p. 390 (texte);

SETHE, Urk., I, p. 84-85 (texte de la biographie);

BREASTED, Ancient Records, I, § 288 (traduction de la biographie);

Stèle 1756 = BORCHARDT, Denkmäler, II, p. 148, 177-178.

ma collation (de Caire 1756; je n'ai pas retrouvé Caire 1709):

Disposition du texte (un quart seulement de la stèle - la partie inférieure droite - a été connu de Mariette): 


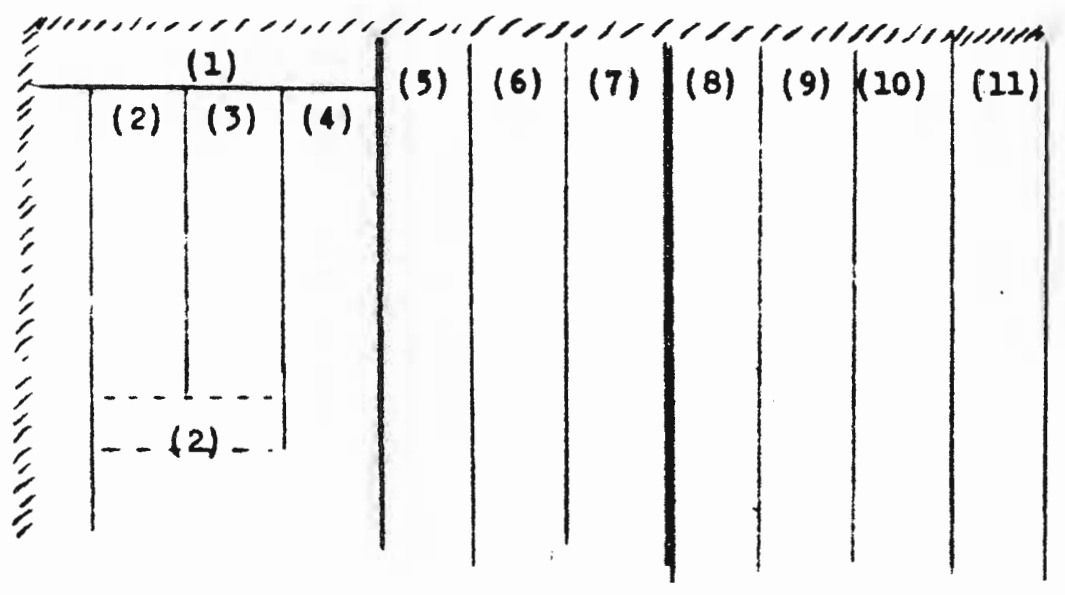

$1 \leftarrow 2 e^{3}+\cdots$

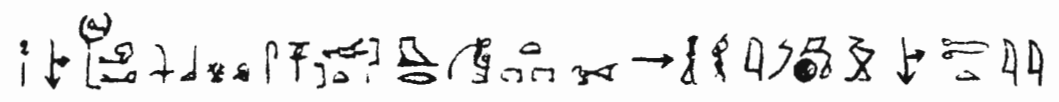

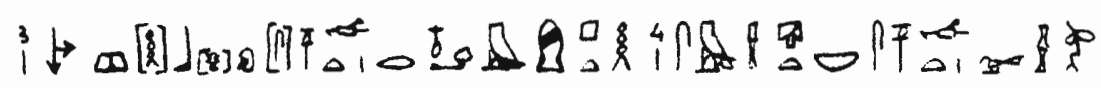
的舟 i b

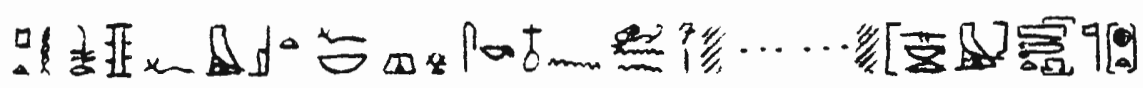

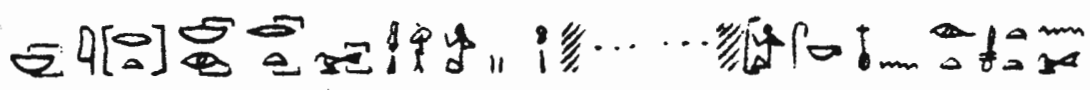

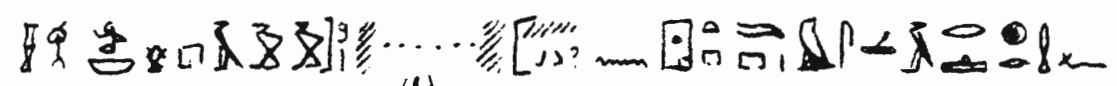

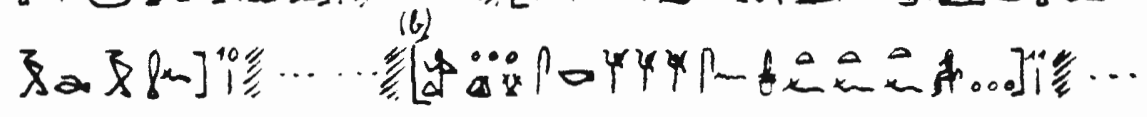

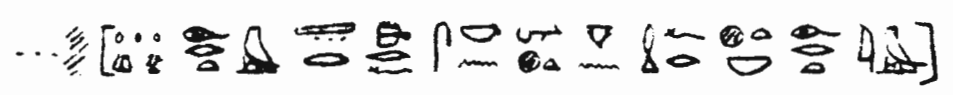


(a) Les passages entre crochets sont restitués d'après Mariette, sauf la colonne [10].

(b) La colonne [10], omise par Mariette, est restituée d'après Sethe.

"[1] Proscynème ...".

"[2] Le gouverneur placé à la tête de Nekheb, compagnon unique, directeur des deux ouabet, grand des chefs des artisans, imakhou Tjéti; [3] le prêtrelecteur en chef, compagnon unique, préposé au diadème quand Ptah est orné; [4] le sem, chef de toute la garde-robe, compagnon unique, grand des chefs des artisans, celui qui est dans la Double Maison, participant à la fête de Rê". [5] ..... auparavant. Aujourd'hui auprès de Sa Majesté ! Sa Majesté m'a nommé [6] ..... temple de Ptah au sud de son mur en toutes ses places sous ma surveillance, alors qu'il n'y avait pas eu [7] ..... Sokar dans le sanctuaire, toutes les choses divines, tous les devoirs que les deux grands des chefs des artisans remplissaient habituellement; [8] ..... alors que pareille chose n'avait pas été faite à aucun grand des chefs des artisans au temps [9] ..... (?) de la maison de l'administration (?) selon le désir de Sa Majesté. Sa Majesté m'a nommé [10] ..... les ... sous ma surveillance, leurs fonctions étant comme (celles de leurs) pères; [11] ..... les ... sous ma surveillance, ce qui est fait (?) dans le pays entier, car le coeur de Sa Majesté est plus fort que n'importe quelle chose faite là".

Les trois représentations de Sabou-Tjéti en bas-relief placées sous les colonnes [1]-[4], [5]-[7] et [8]-[11] sont accompagnées respectivement des légendes: $\mathfrak{t}: 44$ Tjéti, déjà reproduit à la fin de la colonne [2] cidessus; $\downarrow$ îj Sabou; $\Vdash 244$ Tjéti.

\section{LE TEMPLE DE PEPI II}

Bibliographie:

G. JEQUIER, Le monument funéraire de Pépi II, tome II, le Temple, 1938, p. 60 et pl. LXXIV:

Un personnage, dont le bas-relief est surmonté de l'inscription originale

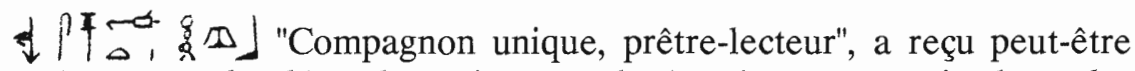
après coup la légende suivante placée devant ses jambes; les 
hiéroglyphes en sont beaucoup moins bien dessinés:

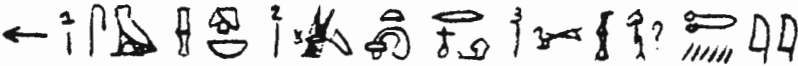

"[1] Le sem, chef de toute la garde-robe, [2] trésorier du roi, préposé au diadème, [3] grand des chefs des artisans Tjé(t)i".

Ce personnage est un simple porteur d'offrandes, avec perruque et pagne, semblable à ceux qui le précèdent et le suivent.

\section{LE TOMBEAU D'INTI}

Bibliographie:

MARIETTE, Mastabas, p. 148: "A gauche de la stèle, un personnage à quel titre il intervient ici".

Traduction: "... le grand des chefs des artisans, celui qui est dans la Double Maison, Tjéti".

\section{LA STATUE DE PTAHCHEPSES-IMPY}

\section{Louvre A 108}

Bibliographie:

BOREUX, Catalogue, p. 451;

ma copie:

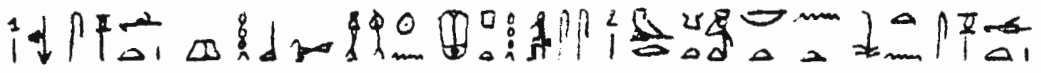

$$
\begin{aligned}
& \operatorname{sef} 42 \div 04
\end{aligned}
$$

"[1] Le compagnon unique, prêtre-lecteur, grand des chefs des artisans, participant à la fête de Rê, Ptahchepses;

"[2] Le directeur de tous les travaux du roi, compagnon unique, grand des chefs des artisans, Impy". 


\section{CHAPITRE XIV}

\section{LES INSCRIPTIONS DU MOYEN EMPIRE}

\section{LE TOMBEAU DE HAPIDJEFA}

Bibliographie:

GRIFFITH, Siut, pl. 9;

MONTET, Les tombeaux de Siout et de Deir Rifeh, Kêmi, III (1930-35), p. 74:

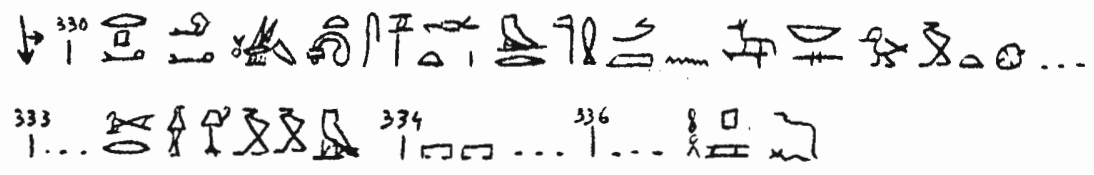

"[330] Le noble, gouverneur, trésorier du roi, compagnon unique, véritable directeur des prophètes d'Oupouat seigneur d'Assiout ... [333] ... le grand des chefs des artisans, celui qui est dans [334] la Double Maison ... [336] Hapidjefa".

\section{LE GROUPE DE SEHETEPIBRE-ANKH-NEDJEM ET DE SON FILS NEBPOU}

\section{Louvre A 47}

Bibliographie:

BOREUX, Catalogue I, p. 52 (description);

BRUGSCH, Thesaurus, p. 1234 (partie du texte);

Musée du Louvre, E. DELANGE, Catalogue des statues égyptiennes du Moyen-Empire, Paris, 1987, p. 81-83;

ma copie: 
Sur le personnage de droite du groupe:

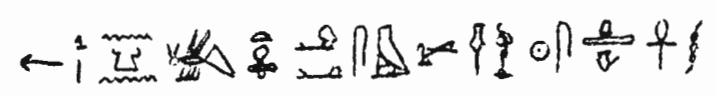

"(Proscynème) [1] pour le ka du trésorier du roi, gouverneur, sem, grand des chefs des artisans, Sehetepibrê-ankh-nedjem".

Entre les deux personnages:

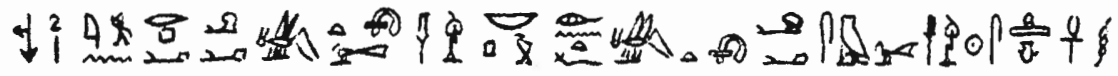

"[2] C'est le noble, gouverneur, trésorier du roi, grand des chefs des artisans Nepbou qui (l') a fait pour son père, le trésorier du roi, gouverneur, sem, grand des chefs des artisans, Sehetepibrê-ankh-nedjem".

Sur le personnage de gauche du groupe:

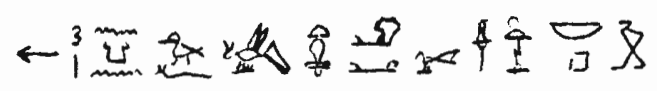

"(Proscynème) [3] pour le ka de son fils, le trésorier du roi, gouverneur, grand des chefs des artisans, Nebpou".

A gauche du personnage de gauche du groupe:

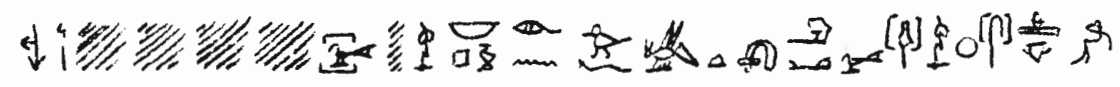

"[4] ..... le grand des chefs des artisans Nebpou (l') a fait pour son fils (a), le trésorier du roi, gouverneur, grand des chefs des artisans, Sehetepibrê-cheri (Sehetepibrê-le-Jeune)".

(a) La destruction du haut de la colonne permet aussi de traduire: ".... Nebpou, qu'a fait son fils, ....".

\section{LA TABLE D'OFFRANDES DE SEHETEPIBRE-ANKH-(NEDJEM ?)}

\section{Berlin 1189}

Bibliographie:

Ägyptische Inschriften aus den kgl. Museen zu Berlin, I, p. 208 (texte); Ägyptischen und Vorderasiatische Altertümer, pl. LVII, haut, 
gauche (photo);

d'après Äg. Inschr.:

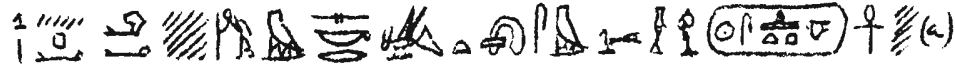

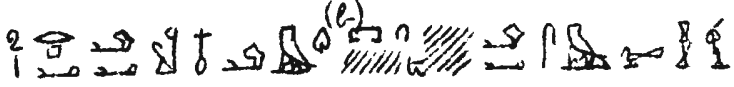

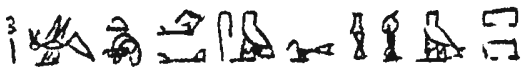

(a) Äg. Inschr. donne $d$ recouvert par

(b) Ne faudrait-il pas lire \& 80.4 comme dans 26,3

"[1] Le noble, gouverneur, ancien dans la barque (?) de Sokar, trésorier du roi, sem, grand des chefs des artisans, Sehetepibrê-ankh-(nedjem ?)".

"[2] Le noble, gouverneur, préposé au diadème quand Ptah (?) est orné (?), noble (?), gouverneur, sem, grand des chefs des artisans".

"[3] Le trésorier du roi, gouverneur, sem, grand des chefs des artisans, celui qui est dans la Double Maison".

\section{3-35 LE TOMBEAU DE SENOUSERT-ANKH}

\section{Linteau et tambour}

Bibliographie:

D'après LANSING, The Egyptian expedition 1932-1933, dans Section II of the Bulletin of the Metropolitan Museum of Art, New York, November 1933, fig. 12, p. 12;

W.C. HAYES, The Texts in the Mastabeh of Se'n-Wosret- ${ }^{c}$ Ankh at Lisht, New York 1937;

$$
\text { i }
$$

"[1] Sem Senousert-ankh, ..." [2] Imy-is Senousert-ankh, ...".

"[3] Le prêtre-lecteur en chef Senousert-ankh".

"[4] Celui qui est placé à la tête de Nekheb, Senousert-ankh". 


\section{Fragment de paroi}

d'après LANSING, op. cit., fig. 16, p. 15 (photo):

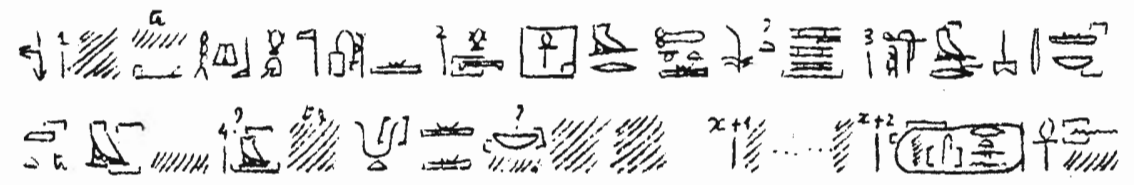

"[1] ... gouverneur (?), prêtre-lecteur en chef, scribe des archives du dieu, [2] supérieur de la Maison de Vie, directeur du recensement des pacages royaux (?), [3] suivant ... (?), directeur de tout jugement de vérité (?) dans ... [4] ... les deux (?) missions ..... [x + 1] ..... [x + 2] Sen (ou)sert-ankh".

\section{Fragment de paroi}

d'après LANSING, op. cit., fig. 15, p. 15 (photo):

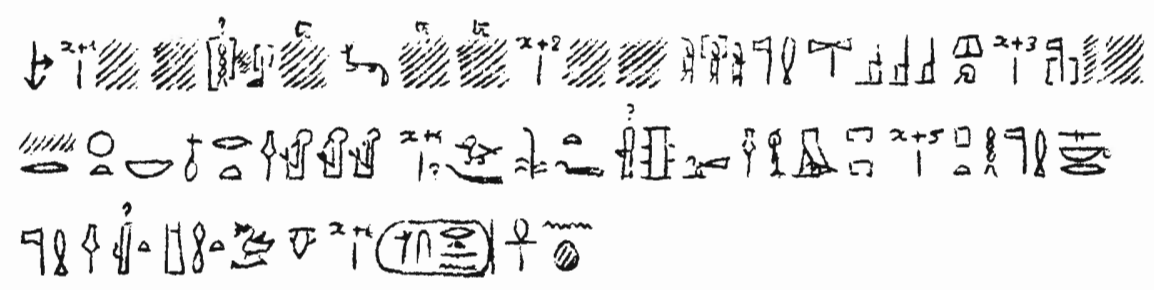

"[x +1$] \ldots$ prêtre-lecteur $(?)$... supérieur $(?) \ldots[\mathrm{x}+2] \ldots$ prophète des $(?)$ suivants ..... les places de ... (a) étant sous (ma ?) tête (?), $[\mathrm{x}+3]$ prophète (?) ... toutes bonnes choses, chefs des frontaliers (?) (b) $[\mathrm{x}+4]$ gardien du troupeau (?) (b), sculpteur et constructeur du roi (c), grand des chefs des artisans, celui qui est dans la Double Maison, $[\mathrm{x}+5]$ prophète de Ptah et prophète de Sokar, chef du grand palais, ... (?) de coeur (d), [x + 6] Senousert-ankh".

(a) DITTMANN, Zum Titel T , dans A.Z., 77 (1941), p. 7-12, rapproche marques de poterie et conclut que le titre ras trois fois sous la Vème dynastie, pourrait se traduire librement "Aufsichtsrat des - Betriebes". Les questions de la lecture et de la signification du signe restent ouvertes.

(b) Cf. les titres de Khabaouseker (K. SETHE, dans M.A. MURRAY, Saqqara Mastabas, II (1937), p. 11). 
(c) D'après LANSING, op. cit., p. 12: "Royal Sculptor and Builder".

(d) Wb. V, p. 342, 9: 



\section{LES INSCRIPTIONS DU NOUVEL EMPIRE}

\section{LE NAOS DE PTAHMES I}

\section{Caire 70038}

Bibliographie:

G. ROEDER, Naos (Catalogue gén. du Musée du Caire), p. 126-129 (texte) et pl. (photo):

Face antérieure:

sur la poitrine et sur le bras de Ptahmès:

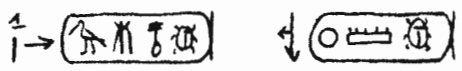

"[1] Thotmès-nefer-kheper. Men-kheper-rê (Thoutmôsis III)".

sur Ptahmès:

\section{${ }_{1}^{2} \rightarrow x$ 皮}

"[2] Le trésorier du roi, grand des chefs des artisans, Ptahmès".

sur le naos: (à droite et à gauche)

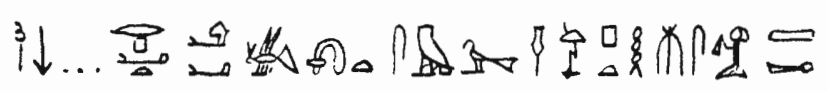

"[3] (Proscynème en faveur du) noble, gouverneur, trésorier du roi, sem, grand des chefs des artisans, Ptahmès, juste de voix". 
Face extérieure droite du naos:

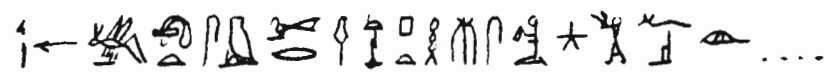

"[4] Le trésorier du roi, sem, grand des chefs des artisans, Ptahmès, adore Osiris ...".

Face extérieure gauche du naos:

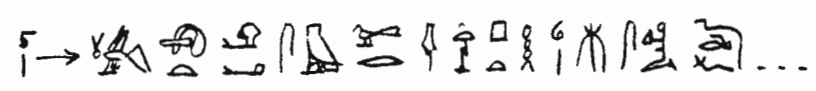

"[5] Le trésorier du roi, gouverneur, sem, grand des chefs des artisans, Ptah[6]mès, dit ...".

\section{LA STATUE DE PTAHMES II}

\section{Caire 584}

Bibliographie:

BORCHARDT, Statuen ..., II, p. 139-140 (texte) et pl. (photo); ma collation:

Sur l'estomac et sous le noeud de la ceinture:

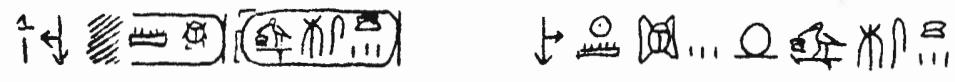

"[1] Men-kheperou-(rê)-Thotmes-kha-khaou".

Sur le pagne:

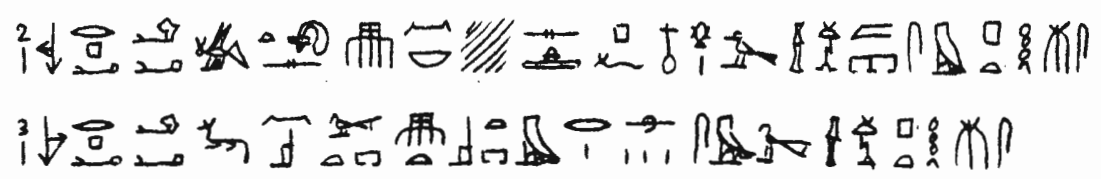

"[2] Le noble, gouverneur, trésorier du roi, celui qui a été mis à la première place par (son) maître (a) - il contente Celui dont le visage est beau -, le grand des chefs des artisans, celui qui est dans la Double Maison, sem, 
Ptahmès".

"[3] Le noble, gouverneur, supérieur des secrets de la Grande Place, celui qui est à la tête de la place dans Ro-Setaou, sem, grand des chefs des artisans, Ptahmès".

(a) litt.: le "placé devant" de son maître.

Sur le socle:

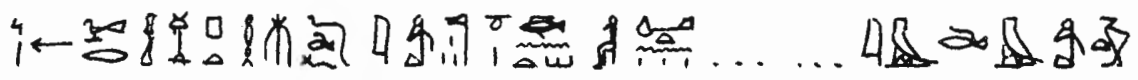

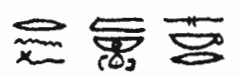

"[4] Le grand des chefs des artisans Ptahmès dit: $O$ dieux de la Vallée Sacrée, faites ..... et faites que son (sic) nom soit prononcé à la fête de Sokar !"

\section{LA STELE DE MERYPTAH ET DES PTAHMES II ET III}

\section{Leyde V 14}

Bibliographie:

Beschreibung der ägyptischen Sammlung des niederländischen Reichsmuseums der Altertümer in Leiden, t. VI = BOESER, Die Denkmäler des Neuen Reiches, Abt. 3, pl. 15 (photo):

Au-dessus de la corniche:

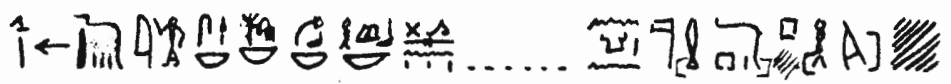

"[1] Paroles à réciter: $O$ tous surveillants, tous scribes, tous ouab, tous prêtres-lecteurs qui passerez ... (appel aux vivants pour les engager à dire le proscynème) pour le ka du prophète et majordome Mér(y)ptah".

Sous la corniche, deux lignes horizontales: 


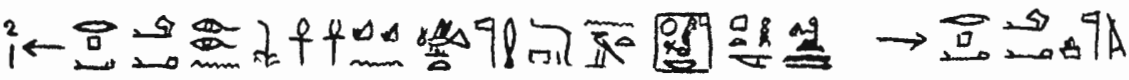

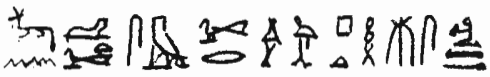

"[2] Le noble, gouverneur, yeux du roi de Haute-Egypte et oreilles du roi de Basse-Egypte, prophète et majordome du temple de Neb-maât-rê, Méryptah, juste de voix. Le noble, gouverneur, père divin aimé (du dieu), supérieur des secrets de la Grande Place, sem, grand des chefs des artisans, Ptahmès, juste de voix".

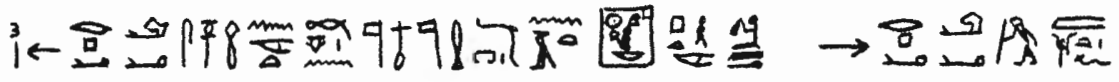

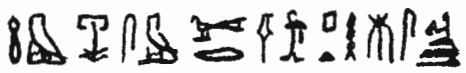

"[3] Le noble, gouverneur, compagnon très aimé, homme de confiance du dieu bon, prophète et majordome du temple de Neb-maât-rê, Méryptah, juste de voix. Le noble, gouverneur, grand dans sa fonction et grand dans la maison du roi (a), sem, grand des chefs des artisans, Ptahmès, juste de voix".

(a) C'est-à-dire: grand dans la fonction qu'il remplit dans l'administration royale.

Sur chacune des cinq figures, en partant de la gauche (du lecteur de la stèle):

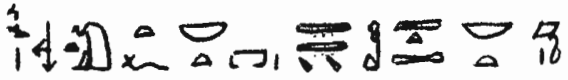

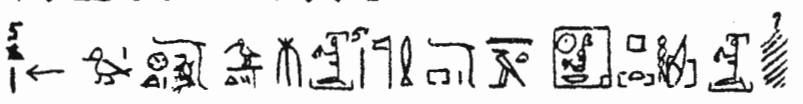

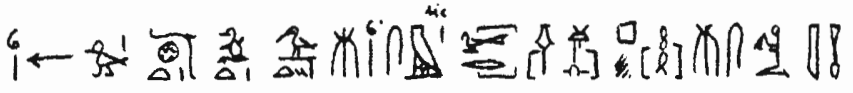

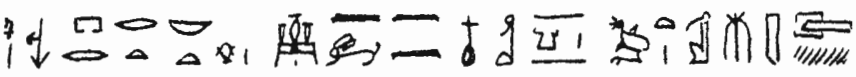

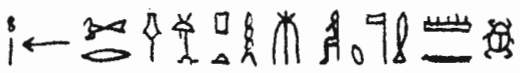


"[4] Sa mère, celle qui possède une maison, Taouï, juste de voix, qui possède l'état d'imakh".

"[5] Le fils du préfet de la ville et vizir Thoutmôsis, prophète et majordome du temple de Neb-maât-rê, Méryptah, (juste de voix ?)".

"[6] Le fils du préfet de la ville et vizir Thoutmôsis, sem, grand des chefs des artisans, Ptahmès, juste de voix".

"[7] Tout ce qui paraît sur la table d'offrandes d'Ounennefer pour le ka du vizir Thoutmôsis, juste (de voix)".

"[8] Le grand des chefs des artisans Ptahmès, fils du prophète Menkheper".

\section{LA STELE DU VIZIR THOUTMOSIS}

\section{Florence 2565}

Bibliographie:

SCHIAPARELLI, Museo archaeologico di Firenze (Catalogo generale dei Musei di Antichità, VIe série, Vol. I), n 1570, p. 292-295 (texte);

MINTO, Il regio museo archaeologico di Firenze, p. 25, bas, droite (petite photo);

Museo Archeologico di Firenze, S. BOSTICCO, Le stele egiziane del Nuovo Regno, p. 39-41, N॰33, pl. 33.

d'après Schiaparelli:

Légende du défunt assis devant une table d'offrandes:

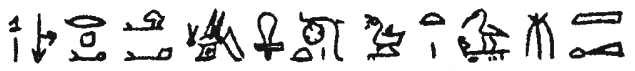

"[1] Le noble, gouverneur, trésorier du roi, préfet de la ville et vizir, Thoutmôsis, juste de voix".

Légende du personnage présentant les offrandes:

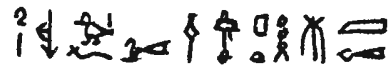

"[2] Son fils, le grand des chefs des artisans Ptahmès, juste de voix". 
Sous l'officiant:

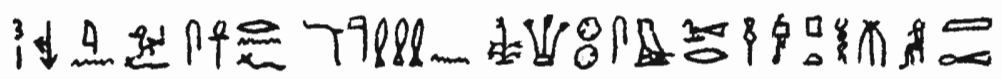

"[3] C'est son fils qui fait vivre son nom, le directeur des prophètes de Haute et Basse-Egypte, sem, grand des chefs des artisans, Ptahmès, juste de voix".

\section{LA COUDEE DE PTAHMES II}

\section{Leyde 635}

Bibliographie:

LEEMANS, Monuments égyptiens du Musée de Leide, II, pl. 248, $\mathrm{n}^{\circ} 635$ :

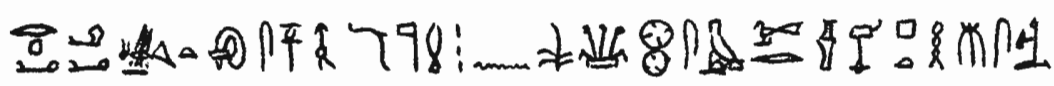

"Le noble, gouverneur, trésorier du roi, compagnon unique, directeur des prophètes de Haute et Basse-Egypte, sem, grand des chefs des artisans, Ptahimès".

\section{LE PYRAMIDION DE PTAHMES II}

\section{Berlin 2276}

Bibliographie:

Ägyptische Inschriften ..., II, p. 230-231 (texte);

ANTHES, Die hohen Beamten namens Ptahmose, dans Ä.Z., 72 (1936), pl. III, opp. p. 64 (photo);

d'après Äg. Inschr.:

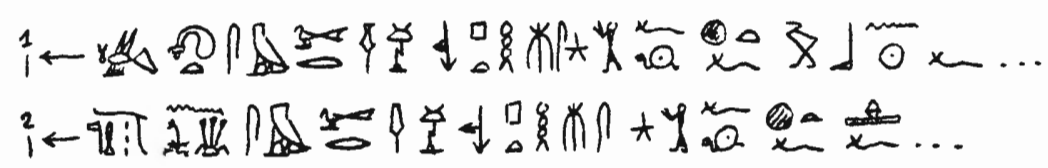

"[1] Le trésorier du roi, sem, grand des chefs des artisans Ptahmès adore Rê quand il se lève ...".

"[2] Le directeur des prophètes de Haute et Basse-Egypte, sem, grand des chefs des artisans Ptahmès adore Rê quand il se couche ...". 


\section{LA PALETTE EN PIERRE DE PTAHMES II}

\section{Berlin 3427}

Bibliographie:

Ägyptische Inschriften ..., II, p. 304 (texte);

ANTHES, Die hohen Beamten namens Ptahmose, dans Ä.Z., 72 (1936), pl. III, opp. p. 64 (photo) et p. 64 (traduction);

Colonne médiane:

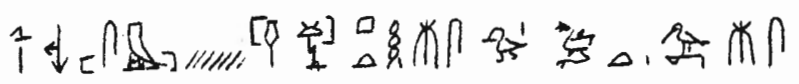

"[1] Le sem, (grand) des chefs des artisans Ptahmès, fils du vizir Thoutmôsis".

Colonne de gauche:

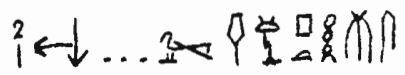

"[2] (Proscynème en faveur du) grand des chefs des artisans Ptahmès".

Colonne de droite:

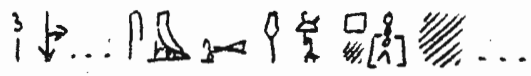

"[3] (Proscynème pour le) sem, grand des chefs des artisans, Ptah(mès) ...".

\section{LE SCARABEE DE PTAHMES II}

\section{Berlin 20661}

Bibliographie:

Ägyptische Inschriften ..., II, p. 519 (texte):

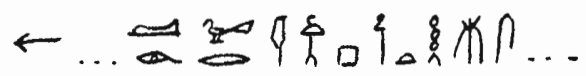

"L'Osiris, grand des chefs des artisans Ptahmès ...". 


\section{LA STATUETTE FUNERAIRE DE PTAHMES II}

Bibliographie:

GARDINER, $A$ statuette of the High Priest of Memphis, Ptahmose, dans Ä.Z., 43 (1906), p. 55:

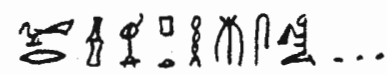

"Le grand des chefs des artisans Ptahmès".

\section{UN FRAGMENT DE VASE DE PTAHMES II}

Bibliographie:

BIRCH, Catalogue of the collection of Egyptian antiquities at Alnwickcastle, 1880 , p. $176, \mathrm{n}^{\circ} 1379$ :

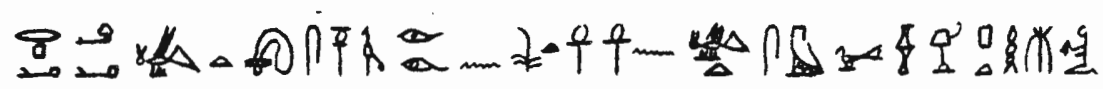

"Le noble, gouverneur, trésorier du roi, compagnon unique, yeux du roi de haute Egypte et oreilles du roi de Basse Egypte, sem, grand des chefs des artisans Ptahmès".

\section{MORTIER ET PILON DE PTAHMES II}

\section{Brit. Mus. 56863}

Bibliographie:

WEIGALL, Egyptian notes, dans P.S.B.A., 23 (1901), p. 12 (texte et dessin);

HALL, Objects belonging to the memphite highpriest Ptahmase, dans J.E.A., 17 (1931) p. 48-49 (photo):

Mortier:

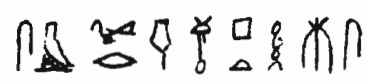

"[1] Le sem, grand des chefs des artisans, Ptahmès". 
Pilon:

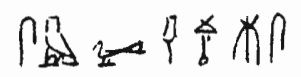

"[2] Le sem, grand des chefs des artisans, Mes".

\section{COUVERCLE DE VASE DE PTAHMES II}

Bibliographie:

J. LEE, Catalogue of the Egyptian antiquities in the Museum of Hartwell House, 1858, p. 69, n 495:

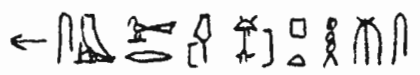

"Le sem, grand des chefs des artisans, Ptahmès".

\section{DOUBLE BOITE A FARD DE PTAHMES II}

Bibliographie:

S. BIRCH, Catalogue of the collection of Egyptian antiquities at Alnwick Castle, 1880, p. 100, n० 761 ;

WILKINSON, Manners and customs, III, p. 383, $\mathrm{n}^{\circ} 411,6$ :

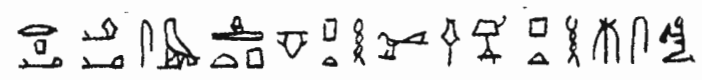

"Le noble, gouvemeur, sem, celui qui satisfait le coeur de Ptah, grand des chefs des artisans, Ptahmès".

\section{9-54 VASES EN ALBATRE DE PTAHMES II}

\section{British Museum 4640}

Bibliographie:

HALL, Objects belonging to the memphite high-priest Ptahmase, dans J.E.A., 17 (1931), pl. VII, n 2 (photo):

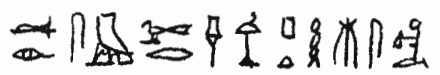


"L'Osiris, sem, grand des chefs des artisans, Ptahmès".

\section{Musée Thorwaldsen 267}

Bibliographie:

Sphinx, 13 (1910), p. 59:

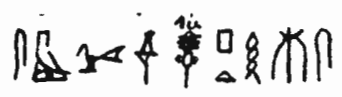

"Le sem, grand des chefs des artisans, Ptahmès".

\section{Leyde}

Bibliographie:

LEEMANS, Monuments égyptiens du Musée de Leide, II, pl. LX, $\mathrm{n}^{\circ} 299$;

cf. aussi NEWBERRY, dans P.S.B.A., 21, p. 306, et HALL, op. cit.:

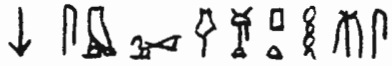

"Le sem, grand des chefs des artisans, Ptahmès".

\section{Leyde}

Bibliographie:

LEEMANS, Monuments égyptiens du Musée de Leide, II, pl. LX, $n^{\circ} 305$ a et $b$ :

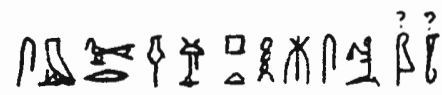

"Le sem, grand des chefs des artisans, Ptahmès, juste de voix (?)".

\section{Leyde}

Bibliographie:

LEEMANS, Monuments égyptiens du Musée de Leide, II, pl. LXI, $\mathrm{n}^{\circ} 309 \mathrm{a}$ et $\mathrm{b}$ :

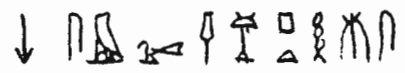


"Le sem, grand des chefs des artisans, Ptahmès".

\section{Louvre I 1099}

Bibliographie:

(L'existence de ce vase avec couvercle inscrit m'a été signalée par Madame Noblecourt);

ma copie:

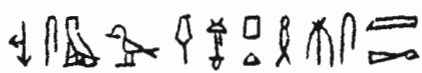

"Le sem, grand des chefs des artisans, Ptahmès, juste de voix".

\section{MOLETTE DE PTAHMES II}

\section{Leyde}

Bibliographie:

LEEMANS, Monuments égyptiens du Musée de Leide, II, p. 58 et pl. 94, $\mathrm{n}^{\circ} 219$ :

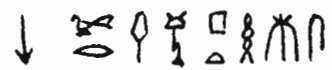

"Le grand des chefs des artisans, Ptahmès".

\section{POMMEAU DE CANNE DE PTAHMES II}

Brit. Mus. 54989

Bibliographie:

HALL, Objects belonging to the memphite high-priest Ptahmase, dans J.E.A., 17 (1931), n 2 (photo);

NEWBERRY, Extracts from my notebooks, dans P.S.B.A., 21 (1899), p. 305 (photo):

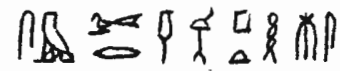

"Le sem, grand des chefs des artisans, Ptahmès". 


\section{COUTEAU EN CALCAIRE DE PTAHMES II}

Brit. Mus. 5472

Bibliographie:

HALL, Objects belonging to the memphite high-priest Ptahmase, dans J.E.A., 17 (1931), $\mathrm{n}^{\circ} 4$ (photo):

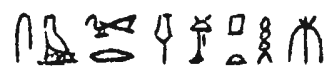

"Le sem, grand des chefs des artisans, Ptahmès".

\section{BAGUE DE PTAHMES II}

Brit. Mus. 2939

Bibliographie:

HALL, Objects belonging to the memphite high-priest Ptahmase, dans J.E.A., 17 (1931), $\mathrm{n}^{\circ}$ 5, (photo):

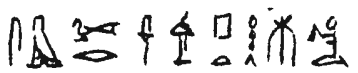

"Le sem, grand des chefs des artisans, Ptahmès".

\section{STATUE DE PTAHANKH}

\section{Budapest}

Bibliographie:

M. CRAMER, Ägyptische Denkmäler im Kestner-Museum zu Hanover, dans Ä.Z., 72 (1936), p. 91, note 3:

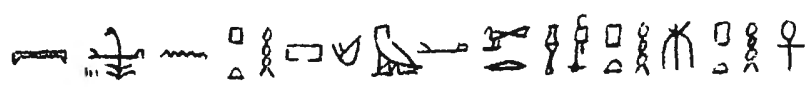

"Le supérieur des chanteurs du temple de Ptah et serviteur du grand des chefs des artisans Ptahmès, Ptahankh". 


\section{STATUE DE PTAHANKH}

\section{Vienne}

Bibliographie:

BERGMANN, Inschriftliche Denkmäler der Sammlung ägyptischer Altertümer des österr. Kaiserhauses, dans Rec. Trav., VII (1886), p. 195-196; MADSEN, Aus dem Hohenpriestergrabe zu Memphis, dans Ä.Z., 41 (1904), p. 115;

d'après Bergmann:

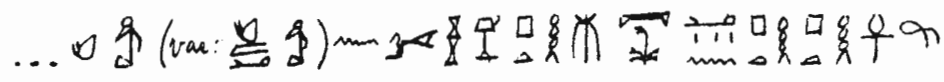

"(Proscynème en faveur $\mathrm{du})$ serviteur du grand des chefs des artisans Ptahmès et supérieur des chanteurs de Ptah, Ptahankh".

\section{STELE TRIANGULAIRE DE PTAHANKH}

\section{Florence 2537}

Bibliographie:

SCHIAPARELLI, Museo archaeologico di Firenze (Catalogo generale dei Musei di Antichità, VIe série, Vol. I), n 1571, p. 295-297 (texte);

A. MINTO, Il regio museo arch. di Firenze, p. 38 haut, droite (petite photo);

Museo Archeologico di Firenze, S. BOSTICCO, Le stele egiziane del Nuovo Regno, p. 41-42, N॰34, 2 pl. N॰34.

Texte entourant la niche:

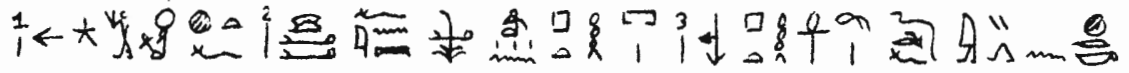

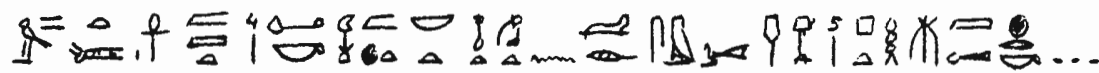

"[1] Adorer Rê quand [2] il apparaît par le supérieur des chanteurs du temple de Ptah, [3] Ptahankh; il dit: Je viens à toi Hor-akhty-Toum qui vis de vérité [4] afin que tu donnes un millier de toutes les choses bonnes et pures à l'Osiris, sem, grand des chefs des artisans [5] Ptahmès, justifié auprès de toi, ..." 
Légende du personnage dans la niche:

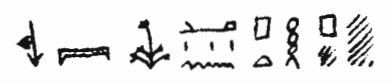

"Le supérieur des chanteurs de Ptah, P(tahankh)".

Légende du personnage offrant l'eau et l'encens à Ptahmès:

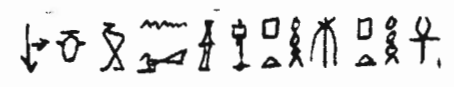

"Le serviteur (a) du grand des chefs des artisans Ptahmès, Ptahankh".

(a) le mot est différent de celui de $\mathbf{5 9}$ et $\mathbf{6 0}$.

\section{SARCOPHAGE DE LA CHATTE DE THOUTMOSIS}

\section{Caire 5003}

Bibliographie:

DARESSY, Notes et Remarques, dans Rec. Trav., XIV (1893), p. 174-175 (texte);

BORCHARDT, Ein Katzensarg aus dem neuen Reich, dans Ä.Z., 44 (1907), p. 97 (photo);

GAUTHIER, Rois, II, p. 336;

ma copie:

Sur le couvercle, près de chaque bord:

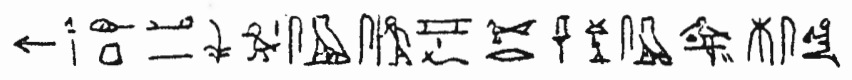

"[1] Fait sous la conduite du fils aîné du roi, son aimé, le grand des chefs des artisans, sem, Thoutmôsis".

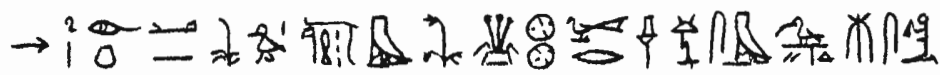

"[2] Fait sous la conduite du fils du roi, directeur de prophètes en Haute et Basse-Egypte, grand des chefs des artisans et sem, Thoutmôsis". 


\section{CHAPELLE D'APIS}

Bibliographie.

MARIETTE, Sérapéum (1857), p. 8, et (1882), p. 124-125:

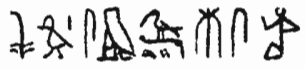

"Le fils du roi et sem, Thoutmôsis".

\section{STATUETTE FUNERAIRE DE THOUTMOSIS}

\section{Louvre}

Bibliographie:

PIERRET, Catalogue de la Salle historique, p. 11, n 10 (inv. 792);

LEPSIUS, Denkmäler, Text, I, p. 11;

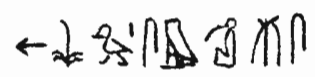

"Le fils du roi et sem, Thoutmôsis".

\section{VASE EN ALBATRE DE THOUTMOSIS}

Louvre (Salle hist. $n^{\circ} 364$, inv. $n^{\circ} 482$ )

Bibliographie:

GAUTHIER, Rois, II, p. 336, n²:

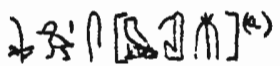

"Le fils du roi et $\mathrm{s}(\mathrm{em}$, Thoutmôsis)".

(a) Restitution proposée par Loret, sans doute parce que le vase provient (GAUTHIER, op. cit.) de la chapelle de l'Apis enseveli sous Aménophis III; cf. inscription 63 ci-dessus. 


\section{STELE DU FILS DU ROI TH(OUTMOSIS)}

\section{Caire 34041}

Bibliographie:

LACAU, Stèles du Nouvel Empire (Catal. gén.), p. 75:

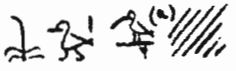

"Le fils du roi Th(outmôsis) (?)".

(a) Note de LACAU, op. cit.: "on voit seulement la tête d'un oiseau qui semble bien être l'ibis".

\section{STELE DE PTAHMES III}

\section{Avignon}

Bibliographie:

MORET, Monuments égyptiens du Musée Calvet à Avignon, dans Rec. Trav., XXXII (1910), p. 159-160 (texte) et pl. I,4 (photo):

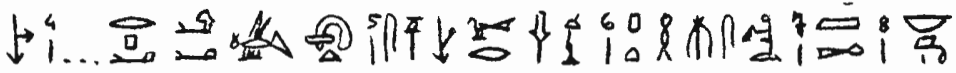

"([4] pour le ka du) noble, gouverneur, trésorier du roi, [5] compagnon unique, grand des chefs des artisans, [6] Ptahmès, [7] juste de voix, [8] possesseur de l'état d'imakh".

\section{COUDEE DE PTAHMES III}

\section{Leyde}

Bibliographie:

LEEMANS, Monuments égyptiens du Musée de Leide, II, pl. 248, n 634 (texte);

SCHIAPARELLI, Museo archaeologico di Firenze, p. 205 (texte);

ANTHES, Die hohen Beamten namens Ptahmose, dans Ä.Z., 72 (1936), p. $63, \mathrm{n}^{\circ} 8 \mathrm{~b}$ (texte);

d'après Anthes: 


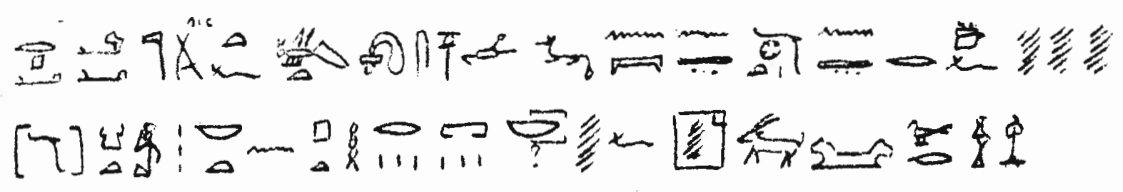

"Le noble, gouverneur, père divin aimé (du dieu), trésorier du roi, compagnon unique, supérieur des secrets du ciel, de la terre, préfet de la ville du (sic) pays entier, ......., directeur de tous les travaux des temples de Ptah, possesseur (?)

(?), grand des chefs des artisans, (Ptahmès)".

\section{STATUE DE PTAHMES III}

\section{Florence 1790}

Bibliographie:

SCHIAPARELLI, Museo archaeologico di Firenze, p. 197, n 1505 (texte);

ma collation, sur photographie du Musée de Florence prêtée par Monsieur G. Nagel, des faces antérieure et droite de la statue:

sur l'épaule droite: $\quad \hat{i} 0$ "[1] Neb-maât-rê".

sur le devanteau du pagne:

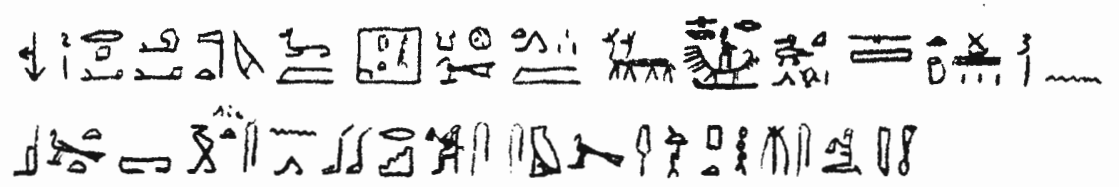

"[2] Le noble, gouverneur, père divin aimé du dieu, supérieur des secrets dans Het-ka-ptah, l'homme aux grandes enjambées lors du halage de Sokar, qui a accès aux secrets [3] de la Grande Place, qui marche librement (a) vers l'escalier auguste, le sem, grand des chefs des artisans, Ptahmès, juste de voix".

(a) wstn rdwy, Wb. I, 367,20. 


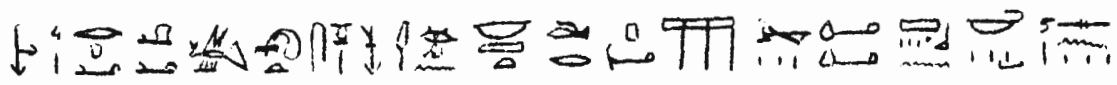

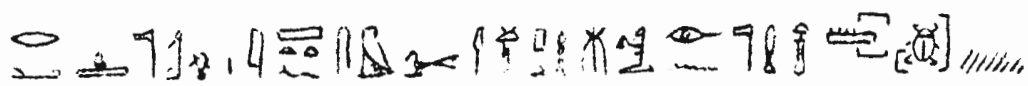

"[4] Le noble, gouverneur, trésorier du roi, compagnon unique, conducteur vigilant du Seigneur de la Vérité, celui qui nourrit les dieux grands, qui donne les aliments à Ceux auxquels ils appartiennent, [5] qui fait en sorte que le dieu soit satisfait de ses repas, le sem, grand des chefs des artisans, Ptahmès, né du premier prophète Menkhe(per)".

autour de la base: une ligne horizontale commençant au milieu de la face antérieure, se dirigeant vers l'angle droit (de la statue, non du spectateur), continuant sur les faces droite, postérieure et gauche, et finissant au milieu de la face antérieure, contre son début:

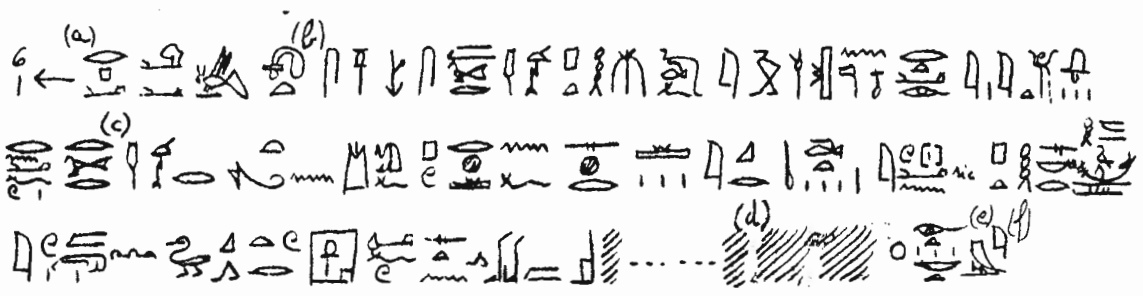

(a) - (b) Sur la face antérieure.

(b) - (c) Sur la face droite.

(c) - (d) Sur les faces postérieure et gauche; cette partie de l'inscription n'était pas visible sur la photo, je l'ai reproduite d'après Schiaparelli.

(d) - (f) Sur la face antérieure.

(e) L'inscription est probablement complète, contrairement à l'affirmation de Schiaparelli (op. cit. , p. 200, note 2). Le graveur n'a manqué de place que pour écrire l'adverbe im de la façon habituelle. Ailleurs aussi, il a mal calculé la disposition de son texte: en [2],

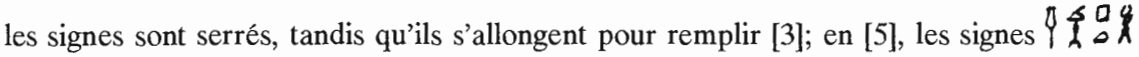
ne forment qu'un cadrat.

"[6] Le noble, gouverneur, trésorier du roi, compagnon unique, sem, grand des chefs des artisans Ptahmès dit: Le Dieu Bon a donné l'ordre de me faire 
remplir l'office excellent; il m'a nommé grand des chefs des artisans ...... (a) de ce Pilier de Sa Mère (b) car il connaissait mes pensées, et que mes paroles sont excellentes. J'ai ... (c) Ptah-Sokar; j'étais en face (de lui) et on n'entrait pas dans la Maison de Vie. Il (= le roi) a fait de moi quelqu'un qui a le droit de marcher librement dans la (Grande Place) .......... toutes les choses faites là (d)".

(a) th.t, Wörterbuch, V, p. 239, 1: signe d'écriture; ibid., 3: ti.t $r$ ti.t, signe à signe. Faut-il traduire $r$ tl.t conformément au signe?

(b) Ce Pilier de Sa Mère me paraît désigner ici le roi; s'agit-il d'Aménophis III ou de celui qui a institué la fonction de grand des chefs des artisans?

(c) D'après la copie de Schiaparelli, on aurait ici le verbe $r d l$, mais alors il manquerait un complément de lieu après Ptah-Sokar.

(d) cf. une fin de biographie analogue sous $\mathbf{2 6 , 1 1}$.

sur le pilier dorsal, d'après Schiaparelli:

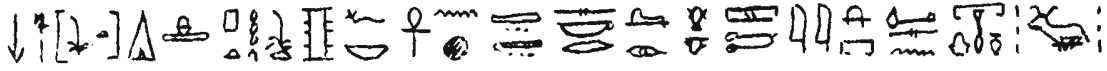

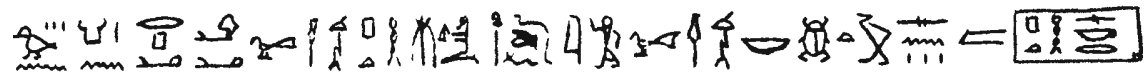

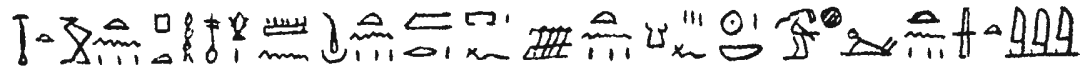

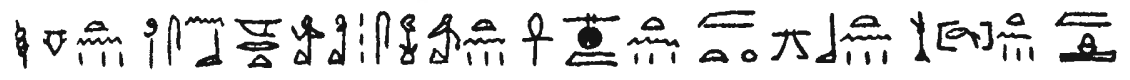

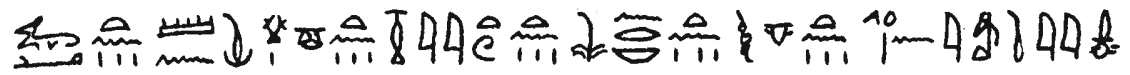

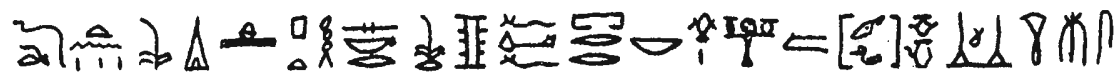

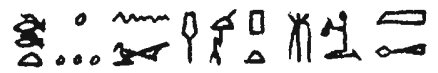

"[7] Proscynème à Ptah qui est au sud de son mur, le Seigneur d'Ankhtaoui, et à Sokar-Osiris qui est dans le "sanctuaire" pour qu'ils donnent l'offrande - pain, bière, boeufs et volaille au ka du noble, gouverneur, grand des chefs des artisans, Ptahmès ! [8] Il dit: $O$ tous grands des chefs des 
artisans futurs dans le temple de Ptah-Sokar, Ptah au beau visage vous récompensera, vous durerez dans son temple, vous recevrez ses aliments chaque jour, vous récolterez (?) ce qui est dans (ses ?) champs, vos coeurs seront joyeux [9] et (vos ?) gens seront sains, vous penserez à la vie et vous oublierez la mort, vous passerez votre pontificat (a) en paix, vos héritiers resteront sur vos sièges, le roi vous louera auprès de votre seigneur et votre coeur sera joyeux à cause du souverain [10] dans la mesure où vous direz un proscynème à Ptah-Sokar qui est au sud de son mur afin qu'il donne tous ce qui paraît sur (son) autel - (boeufs, volaille), vin, lait, tissus, fards vert et noir - au grand des chefs des artisans, Ptahmès, juste de voix".

(a) Litt.: votre ordre, c'est-à-dire l'ordre que le roi vous donnera d'être grands prêtres, et, par extension, tout le temps soumis à cet ordre.

sur la face antérieure de la base, sous la ligne 6:

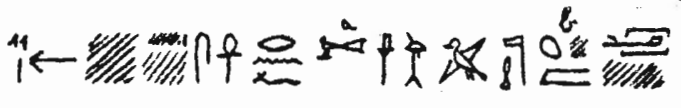

(a) il sous 34 .

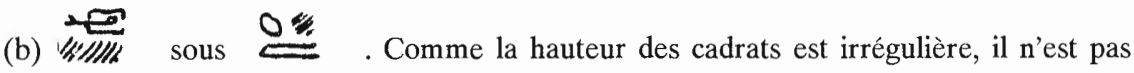
possible de dire si était placé au-dessus d'un ou de deux signes "horizontaux". FARINA, Minima, dans Sphinx, XXI (1924), p. 24-25, a lu

(c) L'écriture de cette ligne [11] présente des différences avec les autres inscriptions de la statue de Ptahmès III: dans [11], la hauteur des signes est très irrégulière: $\mathbf{X}$ descend moins bas que 1 , qui descend moins bas que $\nmid$ (placés sous 3 ), tandis que dans la ligne 6, l'espace compris entre les signes et les traits qui bordent l'inscription est toutjours le même; dans [11], la seconde branche de $\bigcap$ s'arrête à mihauteur de la première, et dans les autres inscriptions elle descend aux trois-quarts; dans [11], $\bigcirc$ est court et enflé, ailleurs il est long et mince; [11] a $\quad$, ailleurs on ne trouve que . Il est donc certain que [11] a été gravé par une autre main que les autres inscriptions. 
"[11] ...... qui fait vivre son nom, le grand des chefs des artisans Pahemneter, fils de $M(e h)$ (a)".

(a) Je complète le nom propre d'après la lecture, ou plutôt la restitution de Farina. Un estampage que M. G. Botti a eu l'amabilité de prendre pour moi ne montre en effet que ce que je donne ci-dessus.

\section{BAS-RELIEF D'UN TOMBEAU DE GRAND PRETRE}

\section{Berlin 12.412}

Bibliographie:

ERMAN, Aus dem Grabe eines Hohenpriesters von Memphis, dans Ä.Z., 33 (1895), p. 19-21 (texte et traduction) et pl. I et II a (photo); pour les publications ultérieures, cf. PORTER-MOSS, Memphis, p. 197; d'après Erman:

au registre supérieur, fabrication de berceaux de verdure par plusieurs personages, dont un garçon qui a reçu auprès coup la légende suivante:

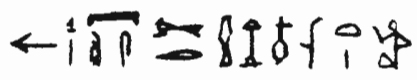

"[1] Le supérieur des suivants du grand des chefs des artisans, Neferrenpet".

au registre inférieur, cortège funèbre; le traîneau est suivi d'abord par les deux fils du défunt:

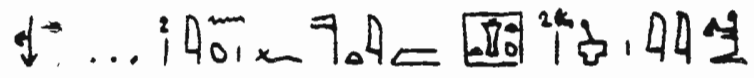

"[2] ... dit son fils, le père divin dans le temple de Bastet, Say".

La légende du second a été découpée.

Puis viennent des dignitaires dont la légende n'indique que les titres, enfin des prêtres, nommés individuellement, qui psalmodient:

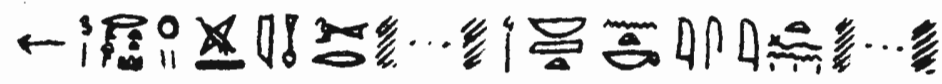


"[3] Vers l'Occident, vers l'Occident, le pays de la justification, $(\hat{o})$ grand ...... [4] du (?) Seigneur de la Vérité. Tu es notre père ......".

\section{BAS-RELIEF D'UN TOMBEAU DE GRAND PRETRE}

\section{Berlin 12.410}

Bibliographie:

ERMAN, Aus dem Grabe eines Hohenpriesters von Memphis, dans $\ddot{A} . Z$., 33 (1895), p. 21-22 (texte et traduction) et pl. II b (photo):

dans la partie gauche, les deux fils du défunt:

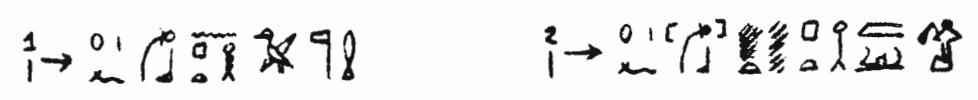

"[1] Son fils, le ouab de Ptah, Pahemneter".

"[2] Son fils, le ouab (de P)t(ah), Ptahemouïa".

dans la partie droite:

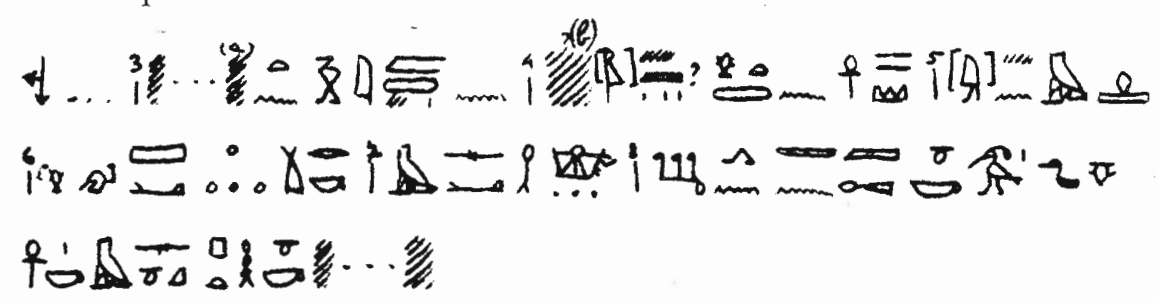

(a) Peut-être n'y a-t-il qu'un demi-cadrat manquant au haut de cette colonne, qui était précédée d'une ou de plusieurs autres sur un autre bloc.

(b) Peut-être aussi seulement un demi-cadrat. En effet, comme [5]-[8] peuvent se lire sans qu'il soit nécessaire de supposer une lacune au haut de chacune d'elles, le trait horizontal limitant la partie supérieure de ce texte devait se trouver tout près du bord supérieur du présent bas-relief.

"[3] (Puissiez-)vous me .... dans l'Ile de [4] (la ?) Vérité, la nécropole d'Ankh-taoui ! [5] Je suis venu en paix [6] en haut des sables, pourvu [7] de 
l'insigne pectoral (a); [8] je suis arrivé à la terre de justification, (car) je suis un esprit akh au coeur ... (b), vivant des gâteaux de Ptah, je suis ....".

(a) Litt.: pourvu de la "dignité", ce mot étant déterminé par l'insigne pectoral.

(b) $p 3 k$; le Wörterbuch, I, p. 499, 7, ne donne que $p 3 k$ ib $r$, désirer ardemment quelque chose, l'autre sens de $p 3 k$ étant "mince, fin", en parlant d'or travaillé.

\section{STELE DE TY}

Bibliographie:

A.-P. ZIVIE, La localisation de la tombe du grand-prêtre de Ptah Ptaemhat-Ty, RdE 35 (1984), p. 200-203.

\section{British Museum 972}

Bibliographie:

British Museum, A Guide to the Egyptian Galleries (Sculpture), 1909, p. 239, n० 876 ;

M.L. BIERBRIER, The British Museum Hieroglyphic Texts from Egyptian Stelae, Part 10, 1982, p. 10, pl. 8;

cf. Ch. MAYSTRE, Le grand prêtre de Ptah du bas-relief Berlin 12411, Mélanges Hintze, p. 303-307.

d'après la description du Guide:

nom du défunt: $\ 99$ Ty;

ses titres: "Noble, gouverneur, trésorier du roi, compagnon unique, sem, grand des chefs des artisans" (je suppose que c'est ce titre que le Guide rend par "high priest of Ptah of Memphis"); Ty est aussi appelé "yeux du roi du sud et oreilles du roi du nord".

L'offrant est son fils 预

Le Guide attribue ce monument à la XXVIème dynastie; cette date doit être corrigée d'après BORCHARDT, Die Mittel zur zeitlichen Festlegung ..., 1935, p. 104, note 8 ("an der Stelle der dort gegebenen Zeitbestimmung "26. Dyn." has WB. "18. Dyn."). 


\section{STELE DE PTAHEMHAT DIT TY}

Bibliographie:

LIEBLEIN, Dict. de noms hiéroglyphiques, II, n²070:

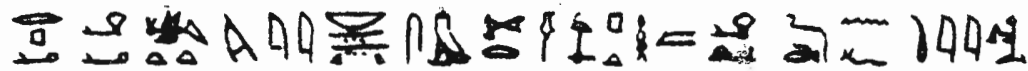

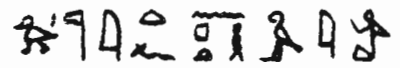

"Le noble, gouverneur, trésorier du roi, aimé du Maître des Deux Pays, sem, grand des chefs des artisans, Ptahemhat, surnommé Ty, fils du père divin de Ptah, Hori".

\section{POMMEAU DE CANNE DE TY}

\section{Leyde}

Bibliographie:

LEEMANS, Monuments égyptiens ..., p. 55 et pl. 85, n 89 , a et b (dessin):

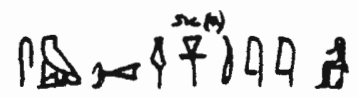

(a) La forme de ce signe a induit Schiaparelli en erreur et lui a fait nommer, dans sa liste de grands prêtres de Memphis (SCHIAPARELLI, Museo archaeologico di Firenze, p. 202), un "Ankhty" dont je n'ai trouvé trace nulle part ailleurs.

"Le sem, grand des chefs des artisans Ty".

\section{AUTRE MONUMENT DE TY}

\section{Louvre E 8420}

Bibliographie:

BORCHARDT, Die Mittel zur zeitlichen Festlegung ..., p. 104, note 8, ne fait que donner le signalement ci-dessus.

Ch. MAYSTRE, Un objet du grand-prêtre memphite Ty (Louvre E 8420), RdE 27 (1975), p. 175-179, pl. 14. 


\section{FRAGMENT DE PAROI D'UN GRAND PRETRE}

\section{Stockholm 37}

Bibliographie:

MOGENSEN, Stèles égyptiennes au Musée de Stockholm, 1919, p. $27-$ 28:

fragment de discours tenu par

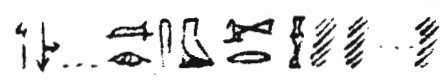

"... l'Osiris, le sem, grand des chefs [des artisans] ...."

\section{PETITE STATUE D'UN GRAND PRETRE}

lieu de conservation ?

Bibliographie:

FISCHER, The Eckley B. Coxe Jr. Egyptian Expedition, dans The Museum Journal (University of Pennsylvania), vol. VIII, n 4, décembre 1917, p. 228 (description) et p. 226 (photographie).

L'inscription n'est malheureusement pas lisible sur la photographie.

\section{FRAGMENT DE STATUE D'UN GRAND PRETRE}

\section{Caire 852}

Bibliographie:

BORCHARDT, Statuen ..., III, 1930, p. 122:

à gauche (de la statue):

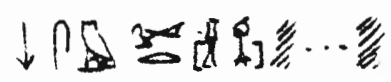

"Le sem et grand des chefs des artisans .....".

\section{9-81. OBJETS DE MERYPTAH PROVENANT DE SA TOMBE}

Bibliographie:

I. GAMER-WALLERT, Das Grab des Hohenpriesters des Ptah, Mrj-Pth, 
in Saqqara, dans Welt des Orients, p. 99-129.

$\mathrm{PM} \mathrm{III}^{2} / 2$, p. 706

\section{Statue de Méryptah}

\section{Louvre A 60}

Bibliographie:

BOREUX, Antiquités égyptiennes I, 1932, p. 54 (description);

REVILLOUT, Le tribunal égyptien de Thèbes, dans Revue égyptologique, III (1883), p. 12-13 (texte et trad.);

d'après Revillout:

sur le socle:

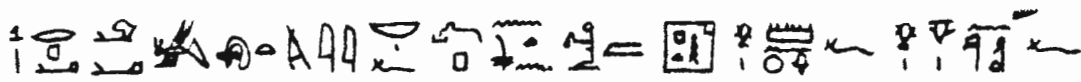

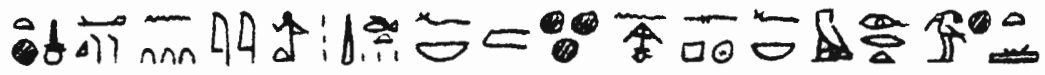

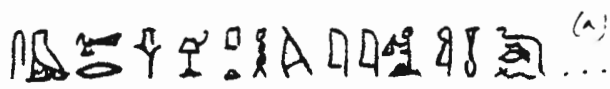

(a) La suite de ce texte a été publiée et traduite par GRAPOW, Ägyptische Jenseitswünsche in Sprüchen ungewöhnlicher Fassung aus dem neuen Reich, dans Ä.Z., 77 (1942), p. 64.

"[1] Le noble, gouverneur, trésorier du roi, l'aimé de son maître, choisi par le roi dans le temple de Ptah à cause de sa perfection, résidant dans le coeur de son dieu, peson exact des Trente dont toutes les paroles sont à la manière de la balance et dont tout le caractère est dans l'accomplissement de ce qui est utile, le sem et grand des chefs des artisans, Méryptah, juste de voix, dit: (a)".

(a) La suite du texte est composée de souhaits concernant l'au-delà.

D'après $\ddot{A} . Z ., 72$ (1936), p. 92, fin de la note 4, Méryptah est aussi appelé sur cette même statue: 


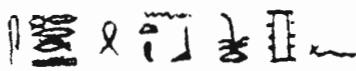

"[2] Celui qui rajeunit la poitrine de Celui qui est au sud de son mur", ce qui me semble signifier: celui qui renouvelle la parure de la statue de Ptah.

\section{Table d'offrandes de Méryptah}

\section{Berlin 2273}

Bibliographie:

Ägyptische Inschriften ..., II, p. 318 (texte);

Ägyptische und Vorderasiatische Altertümer, pl. LVII, bas, droite (photo);

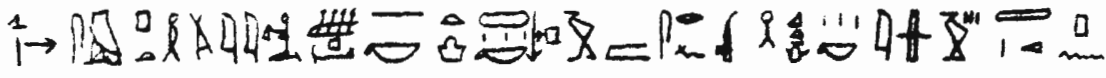

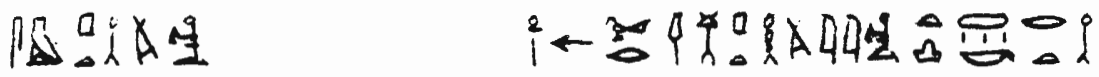

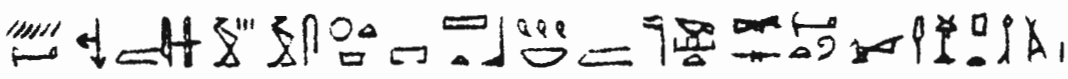

"[1] Sem Méryptah, prends pour toi ce pain encore chaud et ta bière qui sont dans ce pays, sem Méryptah !"

"[2] Grand des chefs des artisans Méryptah, ton pain reteh (vient) de ceux qui sont dans la Salle Large et tous les morceaux de viande (proviennent) de l'abattoir du dieu mis en état (?) (a), grand des chefs des artisans Méryptah!"

(a) "Mis en état" d'après Wörterbuch, V, p. 398, 8: $t s$, Gebäude im Stand setzen. Pour la phrase entière, cf. Pyr. § 2194:

$$
\ldots \circlearrowright \text { … }
$$

"Les deux côtelettes (viennent) de l'abattoir du dieu, le grand pain reteh (vient) de la Salle Large". 


\section{BAS-RELIEF DU TOMBEAU DE MERYPTAH}

\section{Karlsruhe H 1046}

Bibliographie:

WIEDEMANN et PORTNER, Ägyptische Grabreliefs ... Karlsruhe, p. 32 et pl. VII (photo);

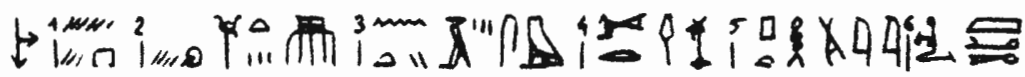

"[1] ... [2] ... les fonctions des [3] premiers, le sem, [4] grand des chefs des artisans, [5] Méryptah, [6] juste de voix".

\section{STATUETTE FUNERAIRE DE NEBMEHYT}

\section{Turin 2666}

Bibliographie:

FABRETTI etc..., Regio Museo di Torino, I, p. 376, et BRUGSCH, Thesaurus, p. 1234 (texte);

d'après une photo du Musée de Turin que m'a communiquée M. Grdseloff:

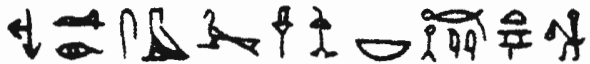

"L'Osiris, sem, grand des chefs des artisans, Nebmehyt".

\section{CANOPE DE SENNEFER}

\section{Leyde R 53}

Bibliographie:

BOESER, Ägyptische Sammlung, XIII, p. 2 et $n^{\circ} 7$ (texte):

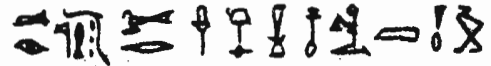

"L'Osiris, directeur des prophètes, grand des chefs des artisans, Sennefer, juste de voix". 


\section{AUTRE CANOPE DE SENNEFER}

\section{Bruxelles E 5895}

Bibliographie:

SPEELERS, Recueil des inscriptions ..., p. 80, n²97:

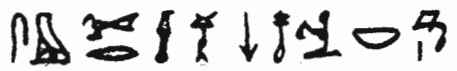

"Le sem, grand des chefs des artisans, Sennefer, possesseur d'imakh".

\section{GROUPE DE KHAMOUAS}

\section{Aix-en-Provence 15}

Bibliographie:

DEVERIA, Catalogue des monuments égyptiens du Musée d'Aix, dans Mémoires et Fragments, 1896, tome I, p. 237-238, n 15 (description et traduction);

WEIL, Die Veziere, p. 117, f (texte de la titulature):

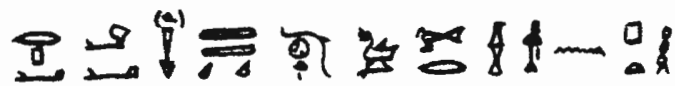

(a) Sans doute 8 mal gravé.

"Le noble, gouverneur placé à la tête (?) des Deux Pays, préfet de la ville et vizir, grand des chefs des artisans de Ptah (a)".

(a) D'après Devéria, l'inscription se lit: "L'Osiris, noble chef, commandant de la terre, toparque, fonctionnaire, grand chef des artisans (du temple) de Ptah, S'âsou, proclamé juste". Bien que je ne voie pas comment le nom "Khamouas" a pu être transcrit S'âsou, il me semble impossible que la notice de Devéria et la référence de Weil ne s'appliquent pas au même monument. Le second personnage du groupe n'a aucune légende. 


\section{PILIER DE HORI FILS DE KHAMOUAS}

Caire JE 43271

Bibliographie:

QUIBELL, Saqqara, IV, p. 144 et pl. LXX (dessin):

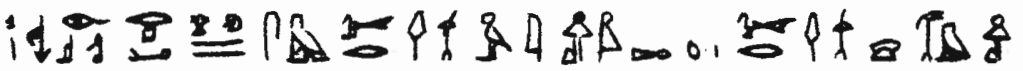

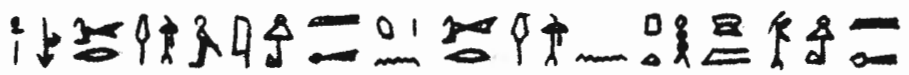

"[1] L'Osiris, le noble placé à la tête des Deux pays, le sem, grand des chefs des artisans Hori, juste de voix, fils du grand des chefs des artisans Khamouas ".

"[2] Le grand des chefs des artisans Hori, juste de voix, fils du grand des chefs des artisans de Ptah Khamouas, juste de voix".

\section{STELE DE HORI FILS DE KHAMOUAS}

Caire, collection Michaelidès

Bibliographie:

Ch. MAYSTRE, Une stèle d'un grand prêtre memphite, Annales du Service des Antiquités 48 (1948), p. 449-455., pl. I.

K.A. KITCHEN, Ramesside Inscriptions, IV, p. 292-293 (48).

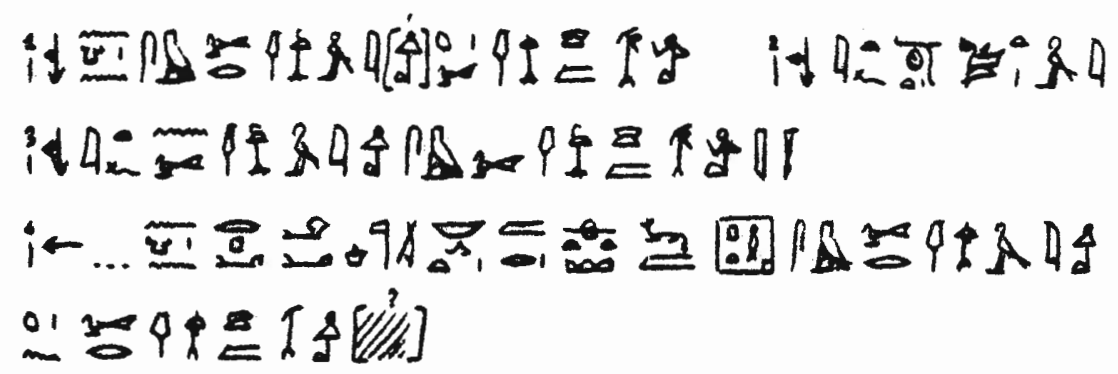

"[1] Pour le ka du sem, grand des chefs des artisans Hori, fils du grand des chefs des artisans, Khamouas!"

"[2] Son aïeul, le préfet de la ville et vizir Hori".

"[3] Le père du grand des chefs des artisans Hori, le sem et grand des chefs des artisans Khamouas juste de voix".

"[4] (Proscynème) pour le ka du noble, gouverneur, père divin aimé du dieu, 
maître de ses allées et venues dans Ro-Setaou, supérieur des secrets dans le temple de Ptah, sem, grand des chefs des artisans Hori, fils du grand des chefs des artisans Khamouas (juste de voix ?)".

\section{COLONNE DE HORI FILS DE PTAHMES}

Caire 20-1-25-4

Bibliographie:

DARESSY, Remarques et notes, dans Rec. Trav., XI (1889), p. 81, $\mathrm{n}^{\circ} \mathrm{XXIV}$ (texte);

ma copie:

au-dessus de Hori agenouillé devant Ptah:

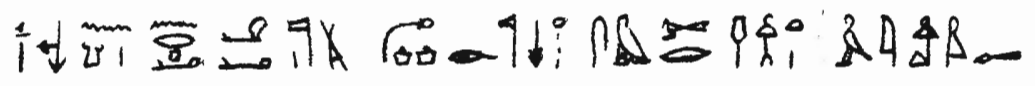

au-dessus de Hori agenouillé devant Mout ... temple de Ptah:

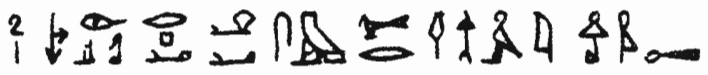

en colonnes séparées sous les bas-reliefs:

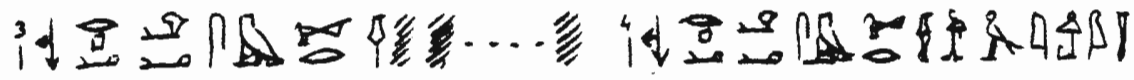

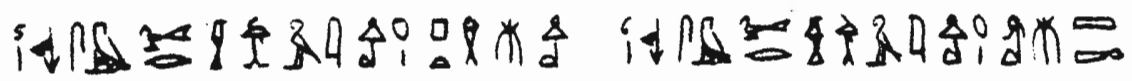

"[1] Pour le ka du noble, gouverneur, père divin aimé du dieu, le pur des deux mains (?), celui qui accomplit les fumigations, le sem, grand des chefs des artisans, Hori, juste de voix".

"[2] L'Osiris, noble, gouverneur, sem, grand des chefs des artisans Hori, juste de voix".

"[3] Le noble, gouverneur, sem, grand des chefs ......".

"[4] Le noble, gouverneur, sem, grand des chefs des artisans Hori, juste de voix".

"[5] Le sem, grand des chefs des artisans Hori, fils de Ptahmès".

"[6] Le sem, grand des chefs des artisans Hori, fils de Ptahmès juste de voix". 


\section{BAS-RELIEF DE HORI}

Ce fragment de bas-relief a été vu en 1944 chez un marchand du Caire par M. Grdseloff, qui m'a obligeamment communiqué la copie qu'il a prise des inscriptions:

à droite Anubis debout;

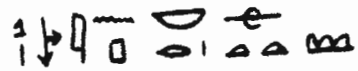

"[1] Anubis, seigneur de Ro-Setaou".

à gauche, un homme en adoration devant Anubis; il porte la tresse de cheveux tombant sur l'épaule droite et la peau de félin; au-dessus de lui:

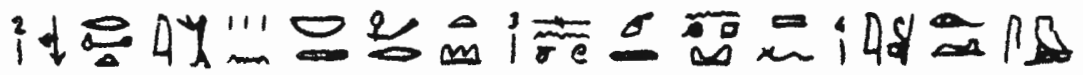

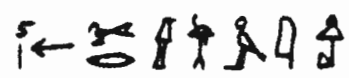

"[2] Louanges au seigneur de To-djeser [3] et prosternement devant Celui qui est sur sa montagne [4] par l'Osiris, sem, [5] grand des chefs des artisans, Hori".

Derrière l'homme en adoration:

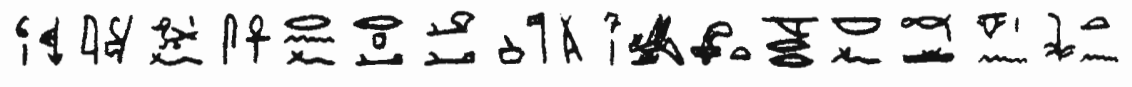

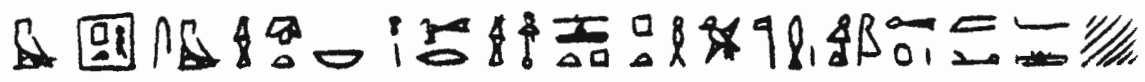

"[6] C'est son descendant (a) qui fait vivre son nom, le noble, gouverneur, père divin aimé du dieu, [7] trésorier du roi, l'aimé de son maître, l'homme de confiance du roi (b) dans le temple de Ptah, le sem, chef de toute la garde robe, [8] grand des chefs des artisans qui contente Ptah, Pahemneter, juste de voix, fils de Meh (juste de voix ?)".

(a) Pahemneter, étant fils de Meh, ne peut être fils de Hori. "s3.f" est donc à prendre dans le sens élargi de descendant, si ce n'est dans le sens encore plus large de "un des successeurs".

(b) Litt. remplissant le coeur du roi. 


\section{SARCOPHAGE DE HORI}

\section{Berlin 57}

Ägyptische Inschriften, II, p. 361-367 (texte):

p. 362:

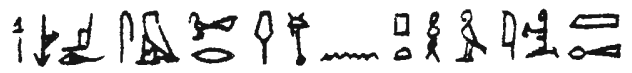

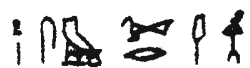

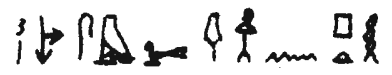

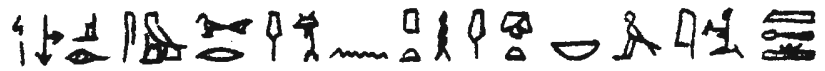

"[1] L'Osiris, sem, grand des chefs des artisans de Ptah, Hori, juste de voix".

"[2] Le sem et grand des chefs des artisans".

"[3] Le sem et grand des chefs des artisans de Ptah".

"[4] L'Osiris, sem, grand des chefs des artisans de Ptah, chef de toute la garde-robe, Hori, juste de voix (a)".

(a) "Chef de toute la garde-robe" ne se trouve qu'une fois sur ce monument.

\section{STATUE NAOPHORE DE HORI}

\section{British Museum 845}

Bibliographie:

A Guide to the Egyptian Galleries (Sculpture), 1909, p. 240, n 879 (qui attribue ce monument à la XXVIème dynastie);

M.L. BIERBRIER, The British Museum Hieroglyphic Texts from Egyptian Stelae, Part 10, 1982, p. 18, pl. 36-39;

K.A. KITCHEN, Ramesside Inscriptions, IV, p. 293;

d'après le Guide:

\section{\&}

"Le noble, gouverneur, grand des chefs des artisans de Ptah (?) Hori". 


\section{STATUETTE FUNERAIRE DE HORI (?)}

\section{Louvre Sérapéum 1738 (N 768)}

Bibliographie:

MARIETTE, Sérapéum, 1857, pl. 10;

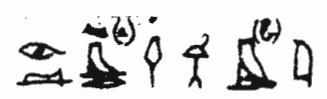

(a) Je n'hésite pas à corriger en $3-$ parce que j'ai rencontré des exemples de ce signe dessiné presque comme un

(b) Tenant compte de la présence de 4 , ainsi que de la correction évidente du

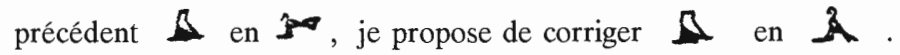

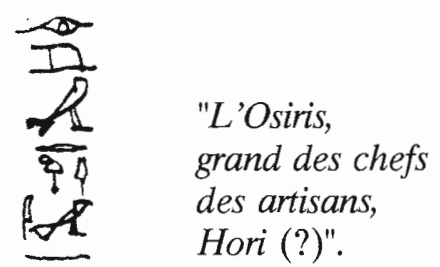

\section{STELE DE HORMIN DITE DES COLLIERS}

\section{Louvre C 213}

Bibliographie:

PIERRET, Recueil d'inscriptions ... du Louvre, 2ème partie, 1878 (Etudes égyptologiques, 8ème livraison), p. 10 (texte);

BOREUX, Antiquités égyptiennes (Catalogue-guide); I, 1932, p. 80 et pl. VIII, opp. p. 85 (photo);

A. VIGNEAU, Encyclopédie photographique de l'art, I, pl. 90; date: Séti Ier.

au second registre:

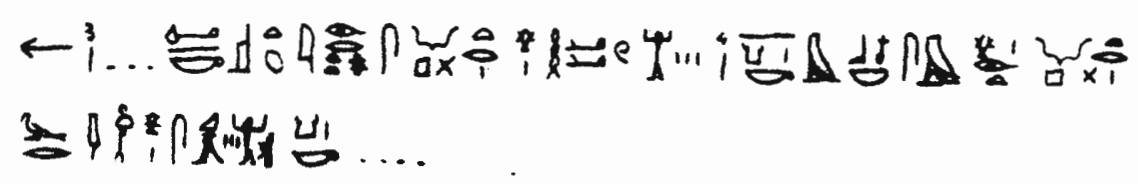


(Proscynème ...) "[3] ...; qu'Isis te donne son lait et l'Ouverture de la bouche, en joie [4] pour ton ka dans tout bon lieu, le sem faisant l'ouverture de la bouche et le grand des chefs des artisans exaltant ton ka ...".

\section{PILIER DE PAHEMNETER}

Florence 2607

Bibliographie:

SCHIAPARELLI, Museo ... Firenze, p. 424-427, $\mathrm{n}^{\circ} 1679$ (texte);

HERMANN, Eine ungewöhnliche Gesichtsdarstellung des neuen Reiches, dans $\ddot{A} . Z$., 75 (1939), pl. VIII, opp. p. 60 (photo des faces II et III):

face I (d'après Schiaparelli):

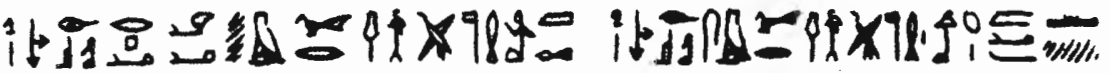

"[1] L'Osiris, noble, gouverneur, (s)em, grand des chefs des artisans Pahemneter juste de voix"."[2] L'Osiris, sem, grand des chefs des artisans, Pahemneter, fils de Meh (justifié) (a)".

(a) Restitution d'après face III.

face II (d'après photo):

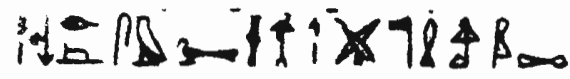

"[3] L'Osiris, sem, grand des chefs des artisans, [4] Pahemneter, juste de voix".

face III (d'après photo):

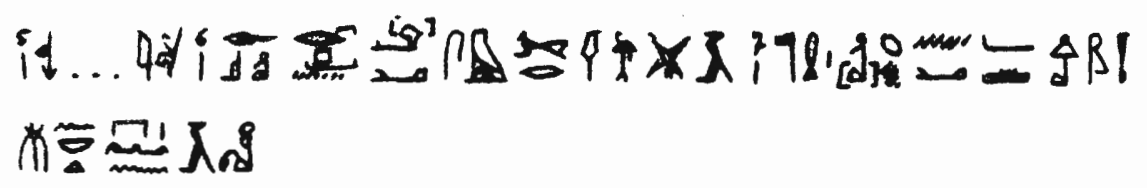

"[5] ... par [6] l'Osiris, noble, gouverneur, sem, grand des chefs des artisans, Pa[7]hemneter, fils de (M)eh, juste de voix, et de la propriétaire de maison Nena". 
face IV (d'après Schiaparelli):

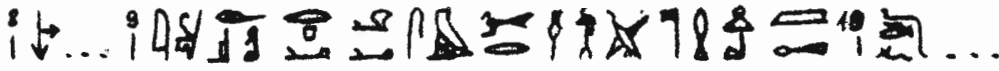

"[8] (Adoration de Sekhmet) [9] par l'Osiris, noble, gouverneur, sem, grand des chefs des artisans, Pahemneter, juste de voix. [10] Il dit: ...".

\section{SOCLE DE STATUE DE PAHEMNETER}

\section{Caire 1087}

Bibliographie:

BORCHARDT, Statuen ..., IV, 1934, p. 50:

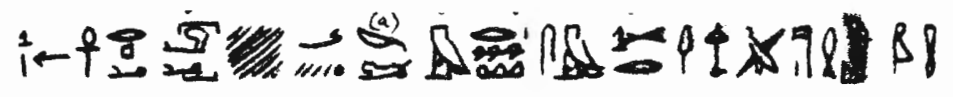

(a) $d s r$; graphie originale du ptolémaïque 45 ?

"[1] Que vive le noble, gou(verneur), ... celui qui voit (?) ce qui est sacré dans Ro-setaou, le sem, grand des chefs des artisans Pahemneter juste de voix".

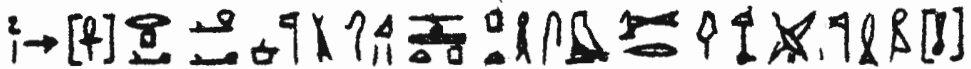

"[2] (Que vive) le noble, gouverneur, père divin aimé (du dieu) régent des Deux Pays (a), celui qui satisfait Ptah, le sem, grand des chefs des artisans Pahemneter juste (de voix)".

(a) $=$ Ptah.

\section{STELE DE PAHEMNETER}

\section{Stockholm 54}

Bibliographie:

MOGENSEN, Stèles de Stockholm, p. 64: 
trois fins de colonnes où se lit:

\section{$\ldots 9 \$ \times 981$}

"... grand des chefs des artisans Pahemneter".

\section{FRAGMENT DU TOMBEAU DE PAHEMNETER}

lieu de conservation?

Bibliographie:

QUIBELL, Saqqara, IV, 1908-1910. p. 30:

un bloc avec mention d'un "High priest of Memphis $/ 97$ Pahemneter".

\section{BASSIN A LIBATION DE PAHEMNETER}

\section{Caire 17-3-25-5}

DARESSY, Remarques et Notes, XIII, dans Rec. Trav., X (1888), p. 150 (texte);

ma copie: il s'agit d'un fragment de bassin rectangulaire très peu profond; il est brisé parallèlement à l'un des petits côtés et c'est la partie inférieure des inscriptions qui manque.

I. Dans le creux: Ptah adoré par un homme orné de la tresse de cheveux pendant sur l'épaule et vêtu de la peau de félin: au-dessus de lui:

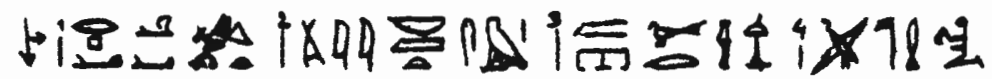

"[1] Le noble, gouverneur, trésorier du roi, [2] l'aimé du Seigneur de la Vérité (a), le sem, [3] celui qui est dans la Double Maison, le grand des chefs des artisans, [4] Pahemneter".

(a) $=$ Ptah

II. Sur le plat du rebord, au-dessus de la scène ci-dessus: 


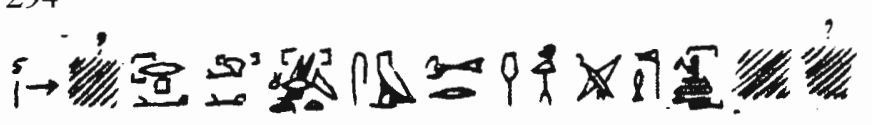

"[5] ..., le noble, gouverneur, trésorier du roi, sem, grand des chefs des artisans, Pahemneter, juste de voix ..." (a).

(a) Comme le coin supérieur gauche de la table est cassé, je ne peux pas affirmer qu'il y ait eu un mot devant $\Phi_{0}$; par contre, à la fin de l'inscription, la ligne bordant l'inscription atteste qu'elle ne se terminait pas par

III. Sur le plat du rebord gauche, il y a deux personnages l'un au-dessous de l'autre, tous deux accroupis; le supérieur porte la tresse de cheveux pendant sur l'épaule; l'inférieur est détruit au-dessous des hanches; ce sont:

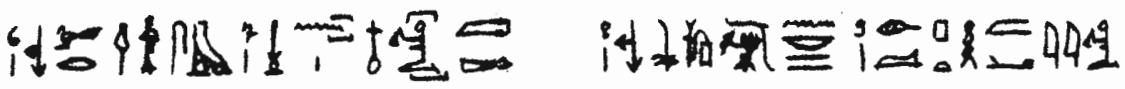

"[6] Le grand des chefs des artisans, sem, [7] Sennefer, juste de voix". "[8] Le scribe royal, général du mâtre des Deux Pays, [9] l'Osiris Ptahmy".

IV. Sur le plat du rebord droit, deux personnages comme en III; ce sont:

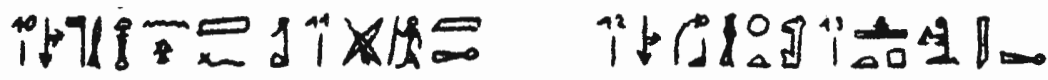

"[10] Le premier prophète d'Arsaphès, [11] Paser, juste de voix". "[12] Le ouab de Sekhmet, [13] Hetep, juste de voix".

\section{SARCOPHAGE DE PAHEMNETER}

\section{British Museum 18}

Bibliographie:

British Museum, A guide to the Egyptian galleries (Sculpture), 1909, p. $182, n^{\circ} 654$;

T.G.H. JAMES, The British Museum Hieroglyphic Texts from Egyptian Stelae, Part 9, 1970, p. 21-22, pl. XVII;

D'après le Guide, c'est un monument de 
" X78요 $\approx$, a high-priest $\approx$, of Memphis, and Sem priest.

... The coffin appears to be unfinished".

D'après la description, on peut traduire:

"Le grand des chefs des artisans, sem, Pahemneter, juste de voix".

\section{AUTRE SARCOPHAGE DE PAHEMNETER}

\section{Berlin 33}

Bibliographie:

Ägyptische Inschriften ..., II, p. 374-381 (texte);

(p. 381):

\section{1}

"[1] L'Osiris, grand des chefs des artisans de Ptah, Pahemneter".

Ailleurs, Pahemneter est intitulé:

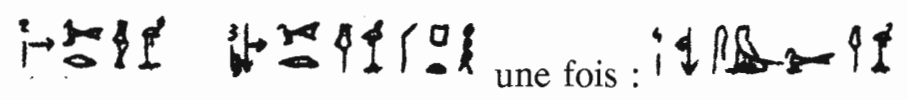

"[2] Grand des chefs des artisans". "[3] Grand des chefs des artisans de Ptah". "[4] Sem, grand des chefs des artisans".

\section{GROUPE DE PAHEMNETER}

\section{Louvre A 72}

Bibliographie:

BOREUX, Antiquités égyptiennes (Catalogue), I, 1932, p. 55 et pl. IV, opp. p. 53 (photo);

CAPART, Recueil de monuments égyptiens, I, 1902, pl. 42 (photo);

K.A. KITCHEN, Ramesside Inscriptions, IV, p. 294.

ma copie:

I. sur le devanteau du pagne: 


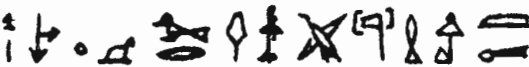

"[1] L'Osiris, grand des chefs des artisans Pahemneter, juste de voix".

II. sur le côté de la chaise:

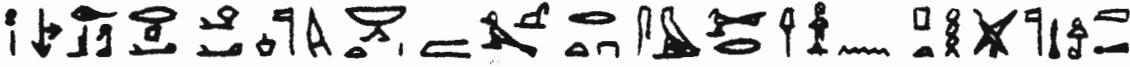

"[2] L'Osiris, noble, gouverneur, père divin aimé du dieu, maître de ses allées et venues dans la Grande Place, sem, grand des chefs des artisans de Ptah, Pahemneter, juste de voix".

III. au dos:

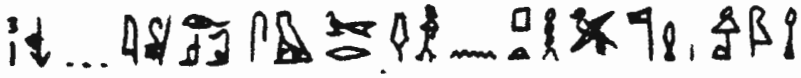

"[3] (Proscynème ...) ... par l'Osiris, sem, grand des chefs des artisans de Ptah, Pahemneter, juste de voix".

L'autre personnage du groupe est (au dos):

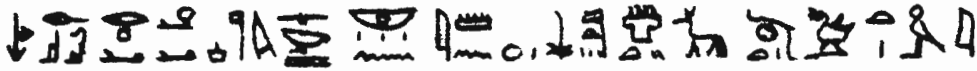

"L'Osiris, noble, gouverneur, père divin aimé du dieu, conducteur des fêtes d'Amon-Rê roi des dieux, celui du Rideau, le Juge, préfet de la ville et vizir Hori".

\section{STELE DE PAHEMNETER}

\section{British Museum 183}

Bibliographie:

A Guide to the Egyptian galleries (Sculpture), 1909, p. 203, n 738;

T.G.H. JAMES, The British Museum Hieroglyphic Texts from Egyptian Stelae, Part 9, p. 19-20, pl. XV;

K.A. KITCHEN, Ramesside Inscriptions, III, p. 56-57;

D'après le Guide: 
"Limestone sepulchral stele, with pyramidal top, of Pa-neter-hen, 298 high-priest of Memphis, Tata-âa, $204 \mathbf{2} 2$ high priest of Memphis, Meriti, 44.2 , the chief inspector of the cattle of Amen, Pai $\times 942$, the chief bowman, and the five princesses Nera-ra, Theti, Mut-nefert, Setmenthi and Hunrui. On the stele are sculptured a figure of Anpu and a scene representing the above mentioned adoring Osiris and Hathor".

Je suppose que c'est le même monument qui est dans LIEBLEIN, Dictionnaire de noms, $n^{\circ}$ 997, p. 325, avec l'indication: London Tablet 796:

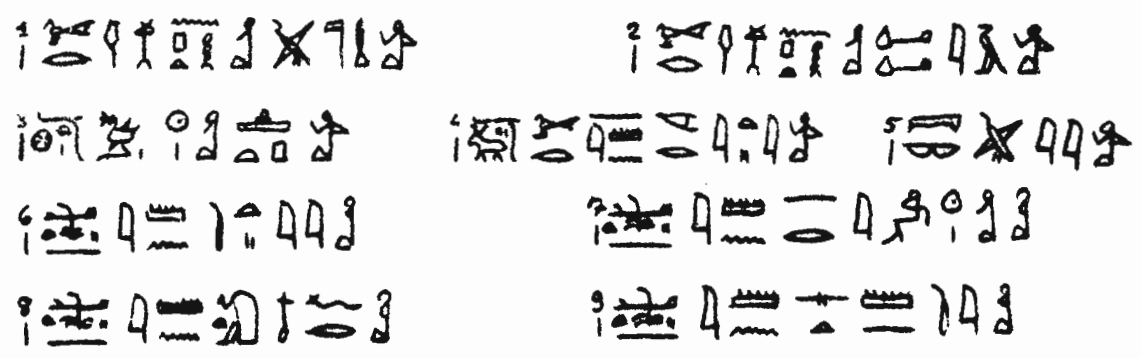

"[1] Le grand des chefs des artisans de Ptah, Pahemneter".

"[2] Le grand des chefs des artisans de Ptah, Dedia".

"[3] Le préfet de la ville et vizir Rêhetep".

"[4] Le grand directeur des troupeaux d'Amon (a), Meryty".

"[5] Le supérieur des archers, Pay".

"[6] La chanteuse d'Amon (a), Tety. [7] La chanteuse d'Amon (a), Nerirê". "[8] La chanteuse d'Amon (a), Moutnefert. [9] La chanteuse d'Amon (a), Setmenti".

(a) Comme dans 101, il s'agit sans doute d'Amon de Memphis.

\section{STELE DE REHETEP}

Caire JE 48845

Bibliographie:

BRUGSCH, Thesaurus, V, p. 950-951 (textes de la face I et du côté III 
publiés à l'envers, c'est-à-dire d'après des estampages);

PIEHL, Inscriptions hiéroglyphiques, IIIème série, Planches, 1895, pl. LXXXI-LXXXII (texte de la face II et du côté IV), et Texte, 1903, p. $52-53$ (traduction de la face II);

M. MOURSI, Die Stele des Veziers Re-hotep (Kairo JE 48845), MDAIK 37 (1981), p. 321-329, 2 pl.;

K.A. KITCHEN, Ramesside Inscriptions, III, p. 53-54 (les inscriptions de Rêhetep figurent aux pages 52-66, sous la rubrique "(P)rahotep B, vizir"); ma copie et mes photographies:

I. 1er registre: adoration de Ptah et Anubis par Rêhetep:

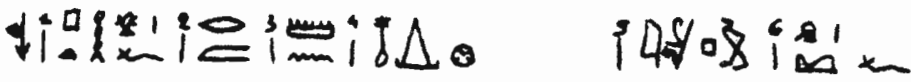

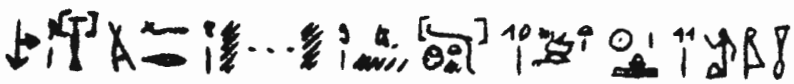

"[1] Ptah dont le visage [2] est vers [3] Mem[4]phis". [5] Anubis [6] qui est sur sa montagne. [7] Son loué et aimé plus que [8] ... [9] préfet de la ville [10] et vizir Rêhetep [11] juste de voix".

I. 2ème registre:

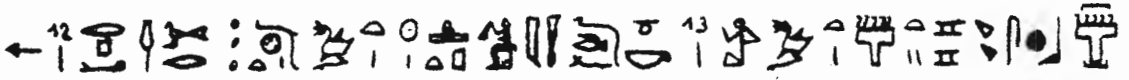

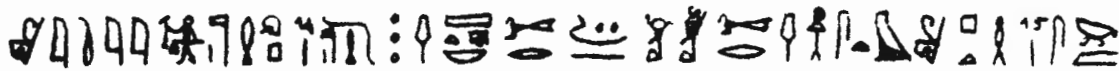

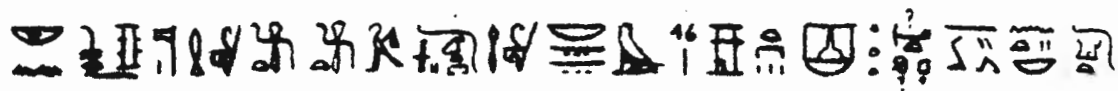

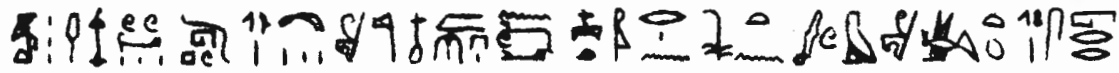

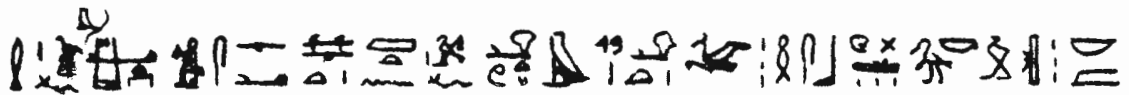

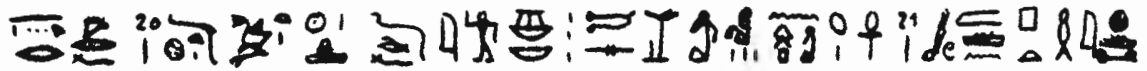

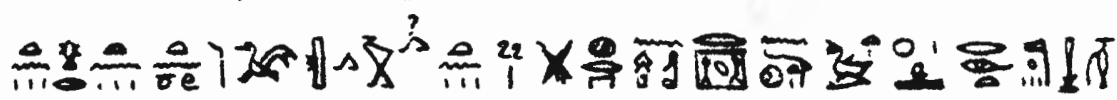

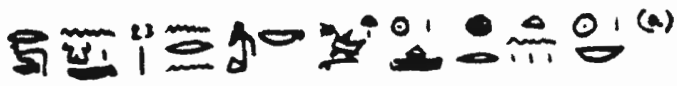


(a) Le texte ne remplit qu'une partie de la ligne 22, dont toute la fin est en blanc.

"[12] Le noble, chef des grands, préfet de la ville et vizir Rêhetep juste de voix dit: J'ai été [13] vizir, un du Rideau des deux rives (?), un de la Porte du souverain, premier prophète, [14] directeur des prophètes, chef de toute la garde-robe, grand des voyants de Rê-Atoum, grand des chefs des artisans et setem de Ptah, [15] conducteur de la fête de Celui qui est au sud de son mur, prophète des deux Uraei de l'Horus, directeur du grand ... (a) du maitre des Deux Pays dans [16] les demeures des fêtes Sed, ... quiconque, directeur des travaux, chef des artisans, directeur des lois [17] du dieu bon dans la salle du juste jugement, porte-parole du roi du nord, héraut du roi du sud, réjouissant Sa Majesté dans le palais magnifique, faisant parvenir (b) la vérité à Sa Majesté, le premier à [19] la tête du peuple, faisant le compte de tous les travaux dans le pays entier. [20] Le préfet de la ville et vizir dit: $O$ tous les prêtres-lecteurs et les pleureuses d'Apis, le fils vivant [21] et l'intermédiaire de Ptah, puissiez-vous faire attention - chaque fois que vous viendrez au (c) [22] tombeau d'Apis, à la demeure d'éternité - (d) au préfet de la ville et vizir Rêhetep, en (e) faisant des encensements et des libations, et en disant: pour ton ka, [23] pour ton nom, vizir Rêhetep ! direz-vous chaque jour".

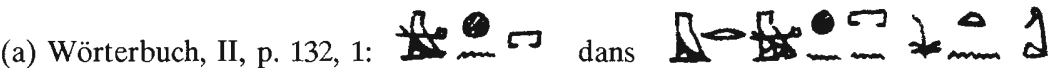
(Papyrus Hood); ibidem, III, p. 307, 10: \II Aufenthaltsort des Königs"; s'agit-il ici d'un de ces mots ?

(b)

(noter les signes

) est à lire, me semble-t-il,

(c) Noter l'absence de préposition entre le verbe et son complément.

(d) En admettant que le m qui suit [0요 peut se lire $n(t)$, l'expression on

serait suivie de trois compléments introduits par $\boldsymbol{D}$, et le passage entier signifierait: "... puissiez-vous faire attention - chaque fois que vous viendrez (au) tombeau d'Apis - à la demeure d'éternité du préfet de la ville et vizir Rêhetep et (puissiez-vous faire attention) de faire des encensements ...".

(f) $\supset$ devant les infinitifs $\triangle$ et 2 dépend de 0 \%

II. 1er registre: adoration d'Osiris et d'Apis par Rêhetep: 


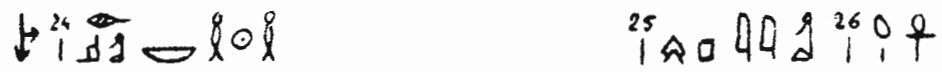

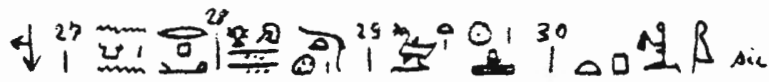

"[24] Osiris, seigneur d'éternité".

"[25] Apis, [26] le fils vivant (sic)".

"[27] Pour le ka du noble [28] placé à la tête des Deux Pays, préfet de la ville [29] et vizir Rêhetep [30] juste".

II. 2ème registre:

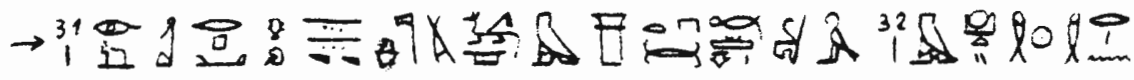

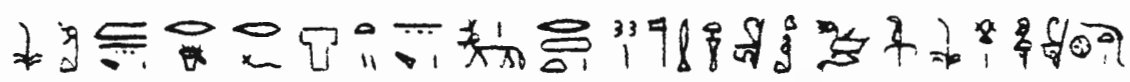

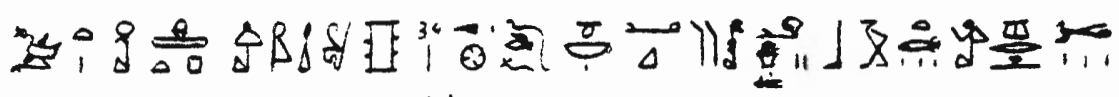

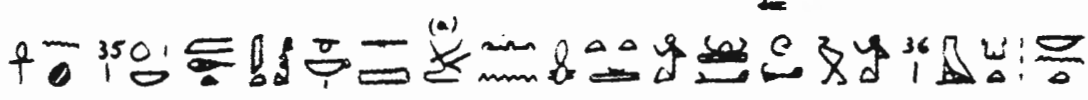

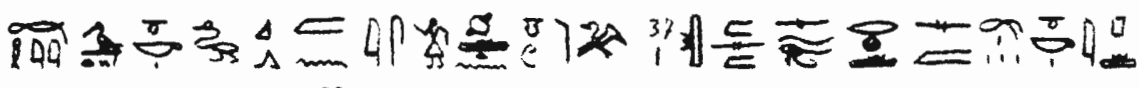

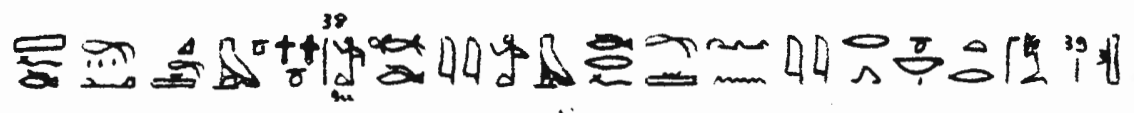

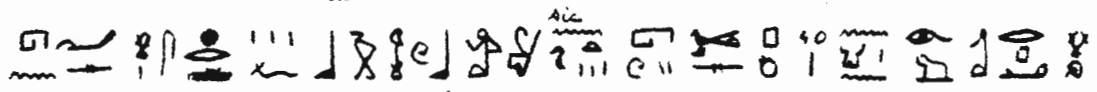

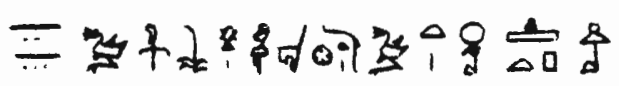

$$
\begin{aligned}
& \text { (a) }=\sum \text {. }
\end{aligned}
$$

"[31] L'Osiris, le noble placé à la tête des Deux Pays, père divin aimé du dieu, supérieur des secrets dans le temple de Néit, le confident de l'Horus [32] dans l'Horizon d'éternité, porte-parole du roi dans le pays entier, celui du Rideau du pays, juge et porte-parole de Nekhen, [33] premier prophète de Maât, porteur de flabellum à la droite du roi, préfet de la ville et vizir Rêhetep juste de voix, de Mem[34]phis (a) dit: J'ai été intègre et juste (b), 
j'ai haï le mensonge, vivant [35] chaque jour de l'accomplissement de la vérité. J'ai été un savant sans pareil, un homme qui dénoue (sous-entendu: les difficultés) [36] dans tous les travaux du Remplisseur (c). J'entrais (sousentendu: vers le roi) en homme qui était appelé; je comptais chaque [37] mise en ordre de l'Oudjat (d); je la (?) (e) connaissais par les écrits (f). Je tenais le compte des papyri exactement ... (g) [38] et je lisais dans les livres sans ... (h). J'étais respecté [39] et l'on se fiait à mes plans; je ne me courbais pas devant des gens manquant (i) de caractère. [40] Pour le ka de l'Osiris, le noble placé à la tête des Deux Pays, porteur de flabellum à la droite du roi, préfet de la ville et vizir Rêhetep".

(a) Litt.: du Mur.

(b) Litt.: exact et vrai de coeur.

(c) Thot, patron des scribes.

(d) Oudjat signifie-t-il ici "lune" ou "boisseau" ? L'expression gsgs wd $3 t$ s'emploie avec les deux acceptions (Wörterbuch, V, p. 207, 2 et 3).

(e) $¥=\vec{a}$ se rapportant à Oudjat ?

(f) PIEHL, op. cit., p. 52, traduit ainsi toute la phrase: "Je suis celui qui entre à l'appel, qui compte les quantités, qui mesure les phases de la lune, qui comprend les dispositions (des fêtes?)".

(g) ndnd: Wörterbuch, II, p. 382, 1-9, "interroger"; 382, 10, "en parallèle avec šs sfs.t "considération", dans un texte du M.E."

(h) D'après le déterminatif: "sans m'écarter (du texte)" ou quelque mot analogue.

(i) whì, Wörterbuch, I, p. 339, "être déficient, échouer"; c'est peut-être ce dernier sens qu'il faudrait retenir pour l'expression whi $s p$.

III.

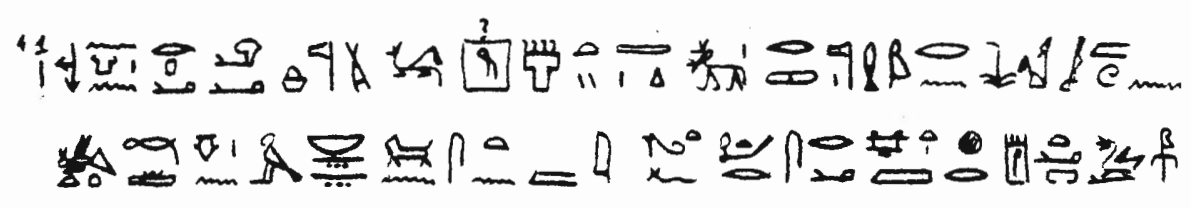




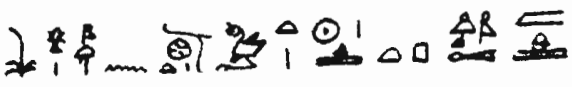

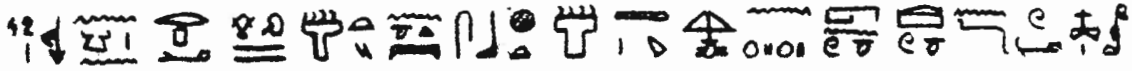

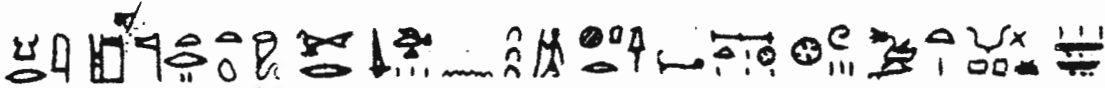

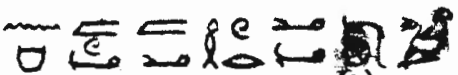 \\ (a)

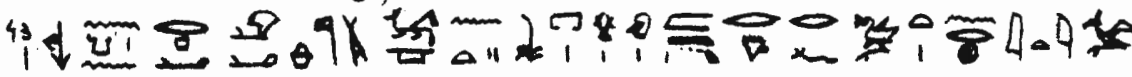

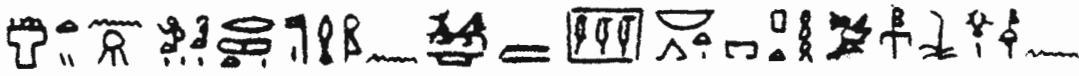

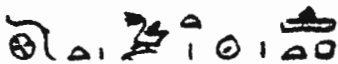

(a) $\overrightarrow{1}$, étant donné la disposition du texte en colonnes, doit se lire à la fois avec

"[41] Pour le ka du noble, gouverneur, père divin aimé du dieu, supérieur des secrets du temple de ... (?), celui du Rideau du pays, juge et porte-parole de Nekhen, prophète de Maât, porte-parole du roi du sud, héraut du roi du nord, confident de l'Horus maitre des Deux Pays, approchant le roi (a) dans sa forme magnifique, faisant parvenir la vérité (b) au Palais, porteur de flabellum à la droite du roi, préfet de la ville et vizir Rêhetep, juste de voix, en paix".

"[42] Pour le ka du noble placé à la tête des Deux Pays, celui du Rideau de Nout, celui de la Porte du pays, balance d'égalisation (?) (c), coffre (d) de jugement juste, chapelle de la Déesse (e), grand de paroles des Trente, chef des déserts et des villes, vizir départageant les Deux Pays et protégeant du pillage, préfet de la ville et vizir" (sic).

"[43] Pour le ka du noble, gouverneur, père divin aimé du dieu, supérieur des secrets de la Maison du roi, celui qui est à la tête dans le pays entier, vizir du peuple, celui du Rideau des petites gens, porte-parole de Nekhen, prophète de Maât, supérieur des secrets dans le temple des Chefs, maître de 
ses allées et venues dans le temple de Ptah, porteur de flabellum à la droite du roi, préfet de la ville et vizir Rêhetep".

(a) $\underline{h} n n$ nswt.

(b) Voir ci-dessus, 1. 17.

(c) $s b 3 n$ hh; Wörterbuch, III, p. 331, 12 "von der Tätigkeit mit der Setzwage. Kopt.: y wwy gleichmachen".

(d) Wörterbuch, II, p. 492, 1, cite un exemple où le mot coffre sert d'épithète à un haut fonctionnaire de la XVIIIème dynastie dans l'expression: coffre du roi avec les plans pour les Deux Pays. En me référant aux lignes 16-17 ci-dessus où Rêhetep est qualifié de directeur des lois du dieu bon dans la salle du jugement juste, je crois reconnaitre dans l'épithète coffre du jugement juste une tournure elliptique signifiant: coffre contenant les papyri sur lesquels les lois sont écrites, ces lois qui permettent de juger justement.

(e) La Déesse désigne ici sans doute Maât. Je ne connais pas d'exemple du mot chapelle employé comme épithète d'un homme.

IV.

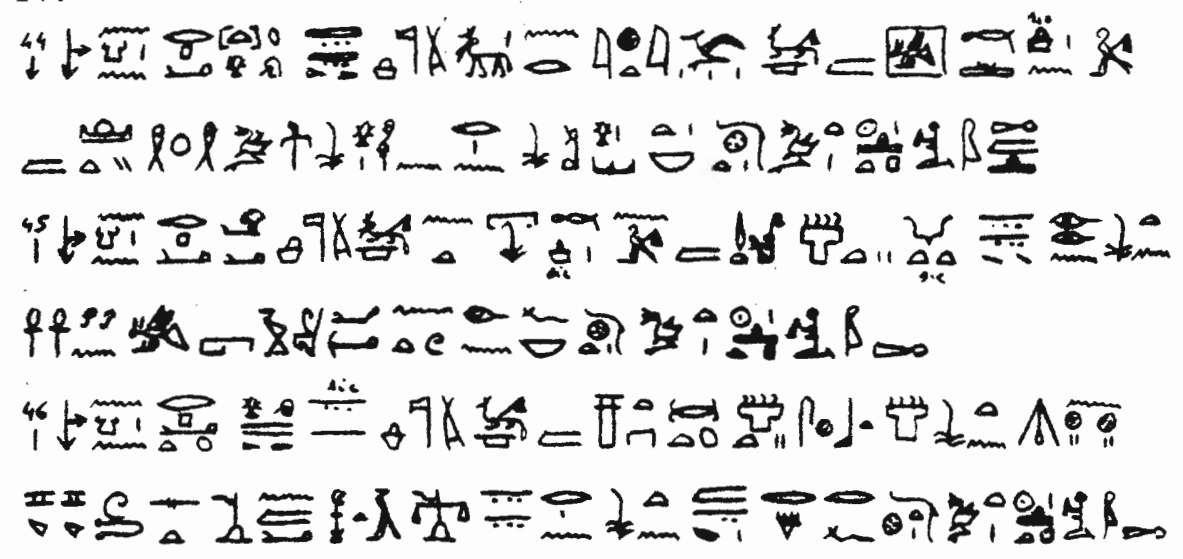

"[44] Pour le ka du noble placé à la tête des Deux Pays, père divin aimé du dieu, juge du peuple, supérieur des secrets dans la Demeure du roi du nord, confident de l'Horus dans l'Horizon d'éternité, porteur de flabellum à la droite du roi, porte-parole du roi dans tous les pays étrangers, préfet de la ville et vizir Rêhetep juste de voix, en paix".

"[45] Pour le ka du noble, gouverneur, père divin aimé du dieu, supérieur 
des secrets de la Maison du roi du sud, confident de l'Horus dans les affaires de justice (?), celui du Rideau, qui départage (?) (a) les Deux Pays, yeux du roi du sud et oreilles du roi du nord, celui à toute l'activité duquel on se fie (b), préfet de la ville et vizir Rêhetep juste de voix".

"[46] Pour le ka du noble placé à la tête des Deux Pays, père divin aimé du dieu, supérieur des secrets dans le temple de Néit, celui du Rideau, celui de la Porte du roi, balance d'égalisation des deux régions, pied de la Balance des Deux Pays, porte-parole du roi dans le pays entier, préfet de la ville et vizir Rêhetep juste de voix".

(a) Ou: "envoyé (des Deux Pays)" ?

(b) $h n(w) . n . t w$, forme relative. S'il s'agit bien du verbe $h n n$, celui-ci aurait ici un complément direct au lieu d'un complément introduit par $h r$ ou $n$ (Wörterbuch, II, p. 494, 15-16).

\section{STATUE DE REHETEP}

\section{British Museum 712}

Bibliographie:

LE PAGE RENOUF, An ambassador royal of Rameses the great, dans P.S.B.A., XIV (1892), p. 163-165 (description et texte avec traduction); LIEBLEIN, Dict. de noms, $\mathrm{n}^{\circ} 2562$ (texte)

T.G.H. JAMES, The British Museum Hieroglyphic Texts from Egyptian Stelae, Part 9, p. 18-19, pl. XIV;

M. VALLOGGIA, Recherche sur les "messagers", p. 131, Doc. n 78; H. DE MEULENAERE, Deux vizirs de Ramsès II, CdE 41, (1966), p. 225-232;

d'après Lieblein:

Sur les genoux, entre les mains:

$$
\sin +\sin x
$$

"[1] Ramsès Méry-Imen". 
Au-dessous:

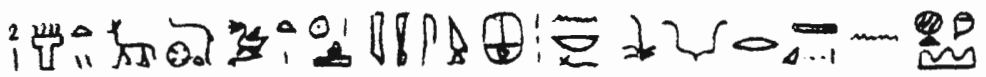

"[2] Celui du Rideau, le juge, préfet de la ville et vizir Rêhetep juste de voix, conducteur des fêtes de son Maître, envoyé du roi au pays de Kheta".

Sur une colonnette:

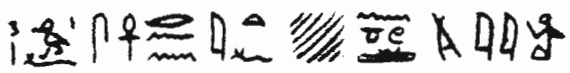

"[3] Son fils, qui fait vivre le nom de son père, le ... (a), Méry".

(a) Etant donné le déterminatif a d e semble appartenir plutôt à un titre qu'à un nom divin entrant dans la composition du nom propre.

Sur le côté droit du siège:

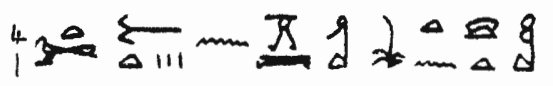

"[4] (Sa mère) (a), la grande des concubines d'Onouris, Khatnesout".

(a) Le Page Renouf ne donne que la traduction de ce mot.

Sur le côté gauche du siège:

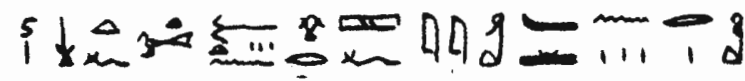

"[5] Sa soeur, la grande des concubines de Herchefy, Houel".

Sur le dos de la statue, agenouillés devant une table d'offrandes placée entre eux:

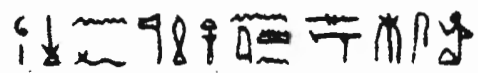

"[6] Son frère, le premier prophète d'Amon, Minmès". 


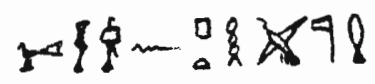

"[7] (Son père), le grand des chefs des artisans de Ptah, Pahemneter".

Le Page Renouf ajoute que tous les personnages de ce monument sont $\approx$, justes de voix; de plus, Pahemneter l'est $\Longleftarrow$, "en paix".

\section{STELE DE REHETEP}

Musée du Caire, sans numéro

Bibliographie:

d'après Porter et Moss, V, p. 26;

MARIETTE, Catalogue général des monuments d'Abydos, 1880, p. 424$425, \mathrm{n}^{\circ} 1138$ (description et texte):

(la partie inférieure manque)

En haut, Isis et Osiris adorés par quatre personnages:

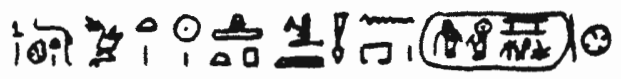

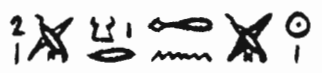

[3] (Son frère) 94 絭08

[4] (Le fils du précédent) 94

"[1] Le préfet de la ville et vizir Rêhetep juste de voix, de Pi-Ramsès-MéryImen".

"[2] Le grand ... (?) de ... Parê...".

"[3] Son frère, le prophète ... juste de voix, de la ... dans le temple de Herchefy, Parêhetep".

"[4] Son fils, père divin dans le temple de Herchefy, Rêhetep juste de voix". 
Au registre inférieur:

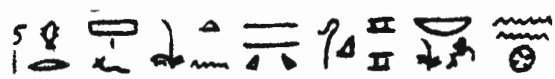

"[5] Herchefy, roi des Deux Pays, régent des deux régions, seigneur d'Héracléopolis".

En face de lui:

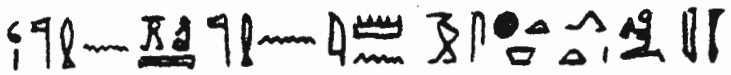

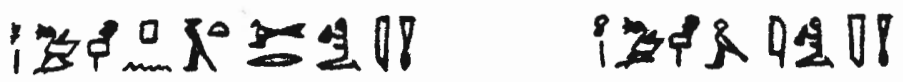

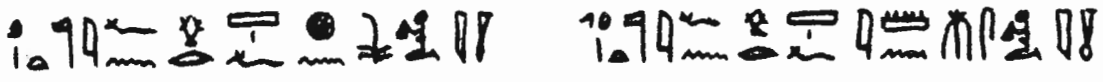

"[6] Le prophète d'Onouris et prophète d'Amon Ousekh-nemtet juste de voix".

"[7] Le porteur de flabellum Pentaour juste de voix".

"[8] Le porteur de flabellum Hori juste de voix".

"[9] Le père divin de Herchefy Khonsou juste de voix".

"[10] Le père divin de Herchefy Imenmès juste de voix".

Les monuments de Rêhetep sont cités dans KITCHEN, Ramesside Inscriptions, III, p. 52-66, sous "(P)rahotep B, vizir".

\section{STATUETTE FUNERAIRE DE HY}

\section{Louvre S 1207}

Bibliographie:

MARIETTE, Sérapéum, 1857, pl. 10, haut, droite (dessin);

K.A. KITCHEN, Ramesside Inscriptions, II, p. 369, F.

J.F. et L. AUBERT, Statuettes égyptiennes, Paris, 1974, p. 91

d'après Mariette:

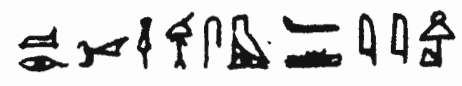

"L'Osiris, grand des chefs des artisans et sem, $H y "$. 
(Cette statuette a été trouvée parmi les ossements de l'Apis mort en l'an 26 de Ramsès II).

\section{7-112. BAS-RELIEFS DE KHAMOUAS}

Sur le prince Khamouas, voir maintenant

F. GOMAA, Chaemwese, Sohn Ramses' II und Hoherpriester von Memphis, $\ddot{A g}$. Abh. 27 (1973).

Dans KITCHEN, Ramesside Inscriptions, les monuments appartenant à Khamouas sont édités dans le Vol. II, p. 871-928.

\section{Bas-relief de Bet el Walli}

Bibliographie:

LEPSIUS, Denkmäler, II, 176 b:

K.A. KITCHEN, Ramesside Inscriptions, II, p. 198, 38 (iv)

d'après Lepsius:

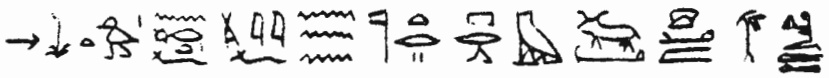

"Le fils du roi, de son corps, son aimé, essence divine issue de Taureau Puissant (a), Khamouas, juste de voix".

(a) Un des noms de Ramsès II.

\section{Bas-relief de Bet el Walli}

Bibliographie:

Ch. KUENTZ, La bataille de Qadech (Mém. I.F.A.O., tome LV, 1928), p. $51, n^{\circ} 10$;

K.A. KITCHEN, Ramesside Inscriptions, II, p. 144, § 73. d'après Kuentz:

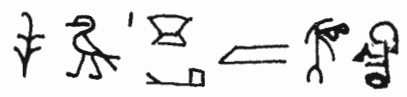

"Le fils du roi, Khamouas". 
109. Tombeau d'Apis

Bibliographie:

MARIETTE, Sérapéum, 1857, pl. VIII (dessin);

K.A. KITCHEN, Ramesside Inscriptions, II, p. 367, n 131 A; d'après Mariette:

devant Ramsès II:

Widops is

"Le fils du roi, sem, Khamouas".

\section{Guebel Silsileh}

Bibliographie:

LEPSIUS, Denkmäler, III, 175, f (texte);

K.A. KITCHEN, Ramesside Inscriptions, II, p. 377, n 134 A; d'après Lepsius:

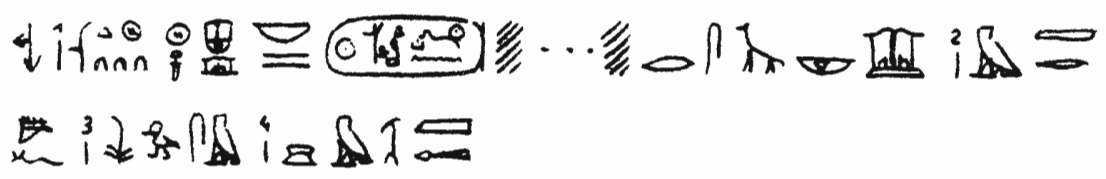

"[1] L'an 30, première fois de la fête Sed du mâtre des Deux Pays Ousermaât-rê-Setep-en-rê ...... pour annoncer la fête Sed [2] dans le pays entier, [3] le fils du roi, sem [4] Khamouas, juste de voix".

\section{El Kab}

Bibliographie:

LEPSIUS, Denkmäler, III, 174 d (texte);

K.A. KITCHEN, Ramesside Inscriptions, II, p. 393-394, n 138 A;

d'après Lepsius:

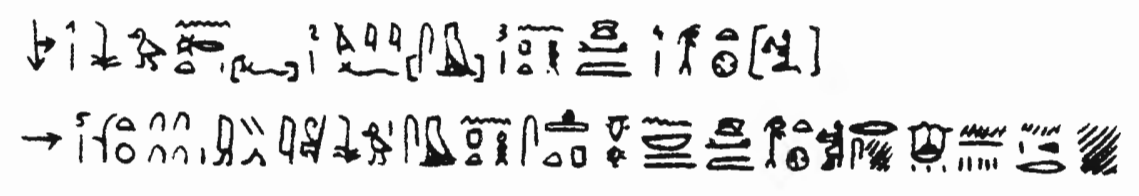


"[1] Le fils du roi, de son corps, [2] son aimé, le sem [3] de Ptah, Kham[4]ouas".

"[5] L'an 41, le fils du roi, sem de Ptah, qui satisfait le coeur et qui est dans le coeur (?) du mâtre des Deux Pays Khamouas est venu pour annoncer la fête Sed de la cinquième (fois) (dans) le pays en(tier)".

\section{Guebel Silsileh}

Bibliographie:

CHAMPOLLION, Monuments ..., II, 115 (texte);

K.A. KITCHEN, Ramesside Inscriptions, II, p. 390, n 137 A; d'après Champollion:

Derrière Ramsès II offrant "Maât" à Ptah assisté d'Amon:

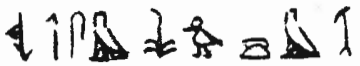

"[1] Le sem, fils du roi, Khamouas".

Au-dessous, mention des quatre premiers jubilés, suivie de:

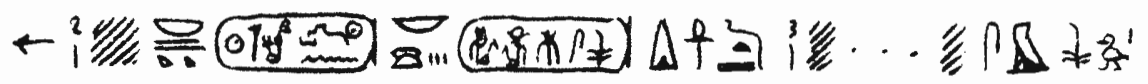

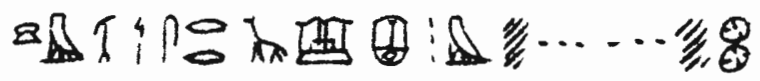

"[2] ... mâtre des Deux Pays Ouser-maât-rê-Setep-en-rê, maître des levers, Ramsès-Méry-Imen, doué de vie éternellement, [3] ...... sem, fils du roi Khamouas [4] pour annoncer les fêtes Sed dans ......".

\section{FRAGMENT DE STATUE DE KHAMOUAS}

\section{Caire 1205}

Bibliographie:

BORCHARDT, Statuen ..., IV, 1934, p. 107 (texte);

K.A. KITCHEN, Ramesside Inscriptions, II, p. 892, n 348;

d'après Borchardt: 
sur le socle,

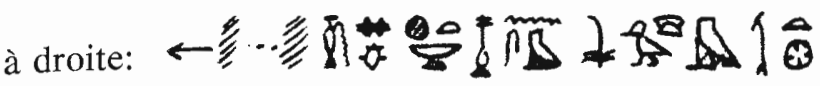

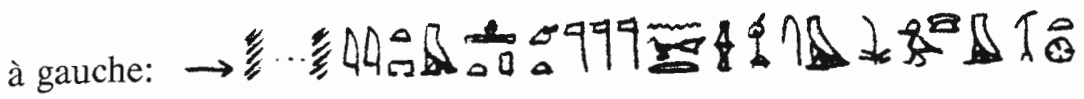

"[1] ... l'eau fraîche, le vin, le lait, toutes les bonnes choses pour le sem, fils du roi Khamouas".

"[2] ... reposer devant les dieux, pour le grand des chefs des artisans, sem, fils du roi Khamouas".

\section{INSCRIPTION DE KHAMOUAS SUR LA PYRAMIDE D'OUNAS}

Bibliographie:

DRIOTON-LAUER, Une inscription de Khamouas sur la face sud de la pyramide d'Ounas à Saqqarah, dans Annales du Service des Antiquités, 37 (1937), p. 201-211 (texte et traduction) et pl. II (photo);

K.A. KITCHEN, Ramesside Inscriptions, II, p. 873, n 333 A; d'après Drioton-Lauer:

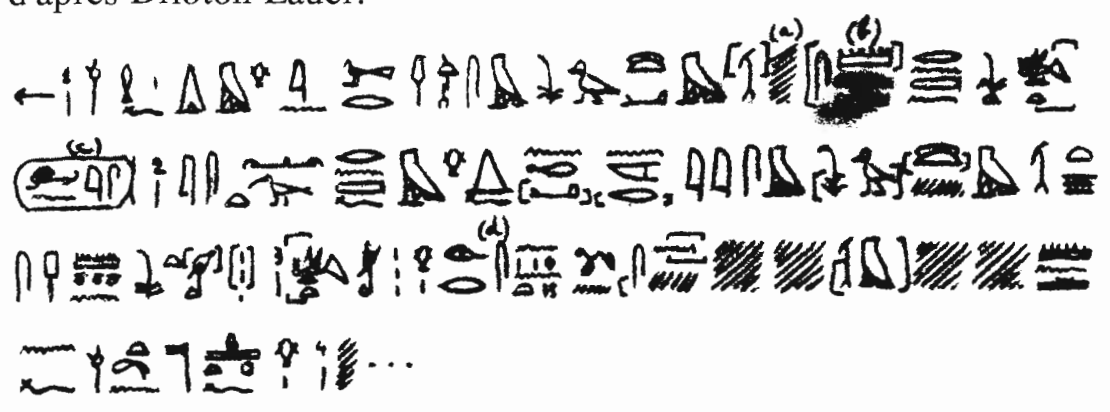

(a) Drioton restitue $\left[\begin{array}{l}0 \\ 0\end{array}\right]$ (cf. ligne 2).

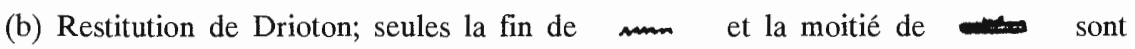
conservées. Dans les textes parallèles, ce passage est entièrement détruit.

(c) Tous les signes contenus dans le cartouche sont endommagés, mais leur lecture est absolument certaine. 
(d) Un texte parallèle, gravé autrefois sur le Mastabat Faraoun, donne la version suivante:

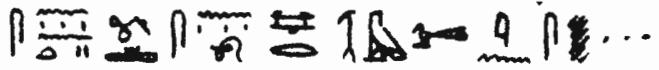

Traduction de Drioton:

"[1] Sa Majesté a ordonné de publier: C'est le Chef des maîtres des artistes (a), le sem, le Fils royal Khamouas, qui a inscrit le nom du Roi de Haute et Basse-Egypte Ounas, [2] alors qu'on ne trouvait pas son nom, sur une face de sa pyramide, parce que le sem, le Fils royal Khamouas aimait beaucoup restaurer les monuments des rois de Haute [3] et Basse-Egypte dans ce qu'ils avaient fait dont la solidité menaçait ruine ... il a inscrit la charte de son revenu sacré sur [4] ... (b)".

(a) = grand des chefs des artisans.

(b) Version du Mastabat Faraoun: "... ce qu'ils avaient fait dont la solidité menaçait ruine. C'est le sem ... (la suite est détruite)".

\section{STELE DE KHAMOUAS}

BRUGSCH, Thesaurus, p. 957-958 (texte);

K.A. KITCHEN, Ramesside Inscriptions, II, p. 878-879;

d'après Brugsch:

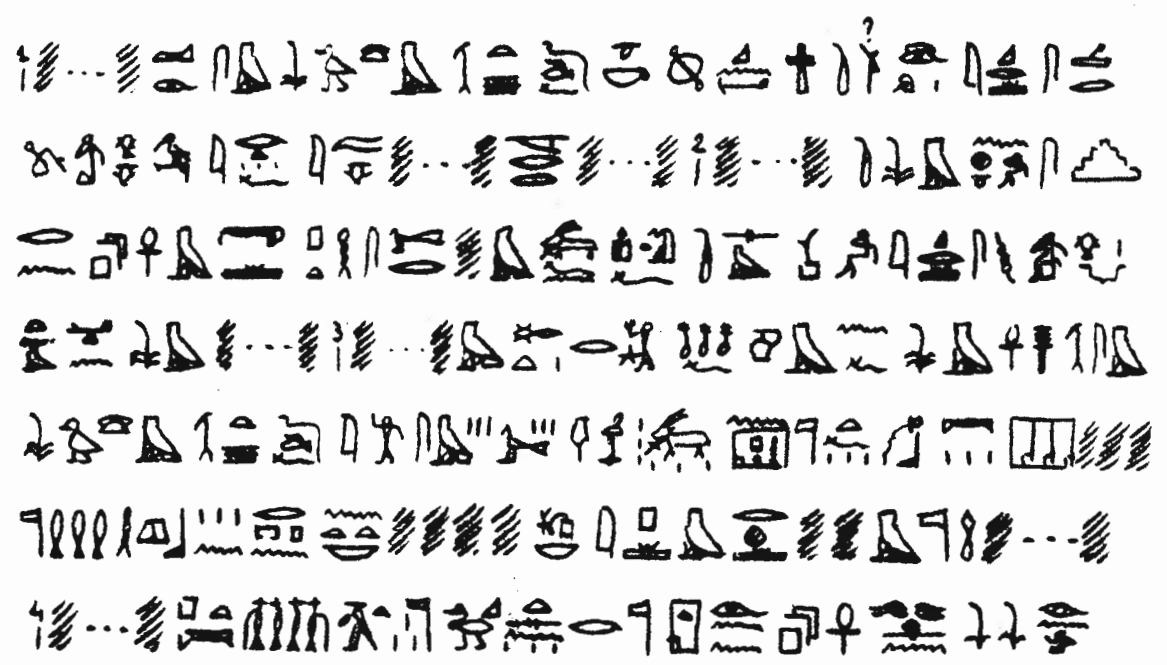




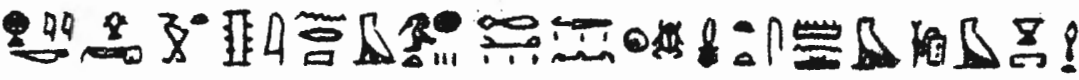

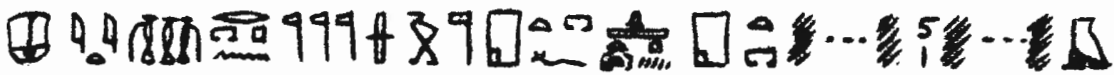

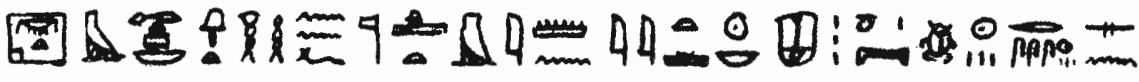
Pfomه

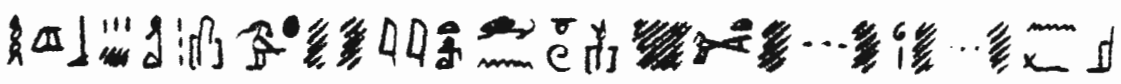

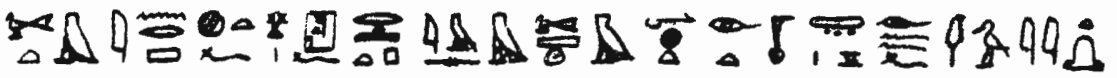

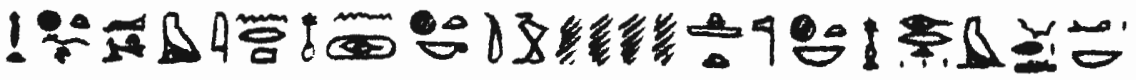
及落

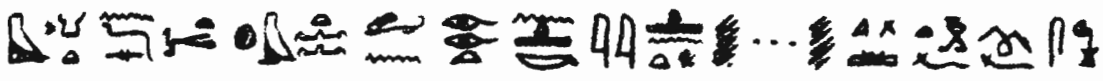

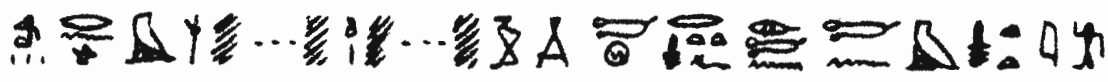

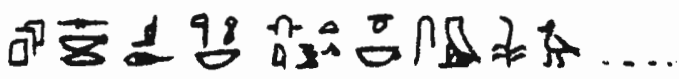

"[1] ... l'Osiris, sem, fils du roi Khamouas dit: Je suis un héritier pieux (a), un protecteur (b) vigilant, d'une intelligence excellente dans les affaires de Thot (c), logeant au coeur ... aime ... (2) ... alors qu'il était dans l'enfance. J'ai fait monter Apis vivant devant Ptah, (je l'ai (?)) fait grandir dans sa dignité de Pilier de sa mère, alors qu'il était un adolescent excellent et qu'il prenait plaisir à (ses) cornes (?). Voyez, il était ... [3] ... du (?) corps pour adorer sa beauté quand il se fut pourvu de vie, stabilité et prospérité. Le sem et fils du roi Khamouas dit: $O$ sem, grands des chefs des artisans et dignitaires du temple de Ptah, pères divins et ouab supérieurs des temples ..., prophètes et prêtres-lecteurs de ce temple, (vous) tous qui ... tout scribe compté comme sachant ... dieu grand ... [4] ... ciel qui sont devant les dieux, qui entrerez dans la demeure divine que j'ai faite pour Apis vivant et qui verrez ce que j'ai fait: j'ai gravé sur l'enceinte de pierre des choses utiles, grandes et uniques (dont) jamais le semblable n'est advenu. J'ai inscrit dans la grande salle large le tableau des offrandes devant ce temple ainsi que les 
dieux qui sont dans sa demeure divine et qui reposent (?) ... la demeure ... [5] ... dans la demeure de l'or en toutes pierres de prix excellentes. J'ai institué pour lui des offrandes divines journalières et des fêtes du ciel quand les jours arrivent pour leur temps (?) aux saisons de chaque année, en plus des gâteaux qui (lui) sont présentés et qui proviennent des offrandes de Ptah. Je lui ai donné des ouab et des prêtres-lecteurs ... qui glorifient ... ... grande ... [6] ... pour lui une "grande place" en pierre devant sa demeure divine pour y reposer en veillant quand (son ensevelissement a lieu. Je lui ai érigé, devant la "grande place", une grande stèle en belle pierre calcaire, gravée ... les offrandes divines et toutes les bonnes choses accomplies lors de l'ouverture de la bouche, toute portion (?) ..... [7] ... Il vous sera utile de regarder ce que mes prédécesseurs ont fait, en travail petit auquel on ne prête pas attention; non, vous ne verrez pas un autre se reposer, qui (?) ... Rappelez-vous mon nom au moyen de (cette ?) stèle (?) ... [8] ... remplacé (?) comme lui; il vous sera fait de même. O Apis-Sokar-Osiris, dieu grand seigneur du sanctuaire, je suis le sem, fils du roi ...".

(a) Wörterbuch, V, p. 42, 13.

(b) ibidem, II, p. 375, 15, ou: "un restaurateur", ibidem, réf. 16 ?

(c) Litt.: "excellent d'intelligence au milieu de (sic !) Thot et de ses affaires".

\section{FRAGMENT DE STATUE DE KHAMOUAS}

\section{Caire 1213}

Bibliographie:

BORCHARDT, Statuen ..., IV, 1934, p. 112 (texte);

K.A. KITCHEN, Ramesside Inscriptions, II, p. 893, n 348 B;

d'après Borchardt:

sur le socle:

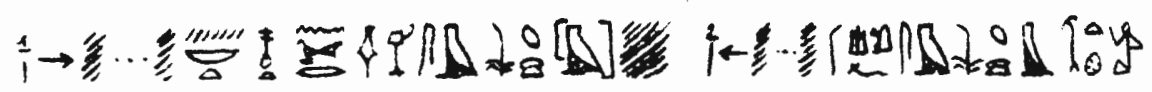

"[1] ... toutes les bonnes (choses) pour le grand des chefs des artisans, sem, fils du roi, Kham(ouas)".

[2] ... pour le Pilier de sa mère, sem, fils du roi, Khamouas". 


\section{SOCLE DE STATUE DE KHAMOUAS}

\section{Caire 17-11-24-2}

Bibliographie:

QUIBELL, Excavations at Saqqara (1907-1908), vol. III, 1909, p. 4-5 (texte);

ma copie:

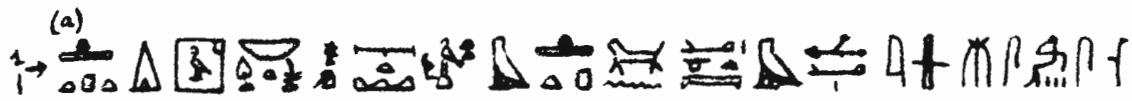

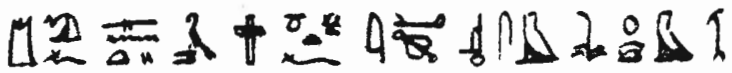 ir [

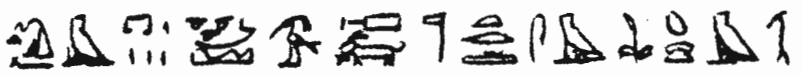

(a) Mot commun aux deux inscriptions, qui s'affrontent au milieu de la face antérieure du socle.

"[1] Une offrande que donne Hathor (a), maîtresse du Sycomore du sud, qui est à la tête du désert occidental - soit reposer (b) dans les bras (c) de Nout, en tant qu'unique parmi ses enfants ! - au Pilier de sa mère, copie (d) d'Horus assistant son père, héritier d'Osiris, sem, fils du roi, Khamouas !" "[2] Une offrande que donnent Apis vivant, Atoum et Horus de Sepa - soit reposer devant les dieux dans le voisinage d'Apis vivant ! - au Pilier de sa mère dans les demeures de Manou, au glorifié ... (?), dignitaire divin, sem, fils du roi Khamouas!"

(a) Ma traduction suppose l'omission d'un $\backsim$ après $\Lambda$, ce qui ne me paraît pas extraordinaire à l'époque de Khamouas.

(b) ḥtp, verbe, plutôt que nom; cf. 124,3.

(c) $\underline{h} n^{c}$, avec les sens de $m \underline{h} n w^{c} w y$, bien que je ne connaisse pas d'autre exemple de $\underline{h} n w$ seul signifiant "à l'intérieur de".

(d) Wörterbuch, III, 457, 9, avec l'indication "belegt Dyn. 18" et l'orthographe 
Est-ce le même mot que Wörterbuch, IV, 179, 15, cite dans l'expression 8 " $\mathcal{A}$ avec l'indication "Titel des Prinzen Cha-em-weset als Hoherpriester von Memphis" ? (Cf. 137).

\section{FRAGMENT DE STATUE DE KHAMOUAS}

\section{Lieu de conservation?}

Bibliographie:

CHABAN, Fouilles à Achmouneïn, dans Annales du Service des Antiquités, 8 (1907), p. 211- 223 (description de la trouvaille);

DARESSY, Fragment de statue du prince Khamouas, ibidem, 16 (1916), p. 255-256 (texte):

Sur l'emblème tenu par Khamouas (deux fois):

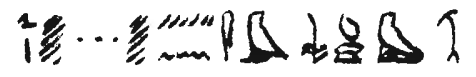

"[1] ... pour le sem, fils du roi, Khamouas !"

Autour du socle, à gauche et à droite:

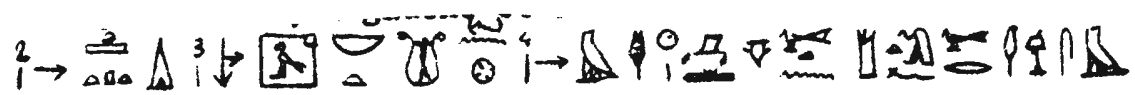

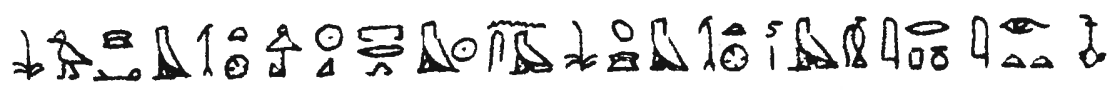

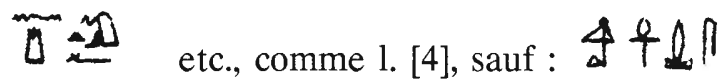
他[敦]

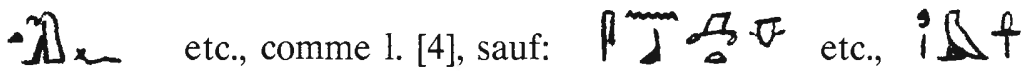

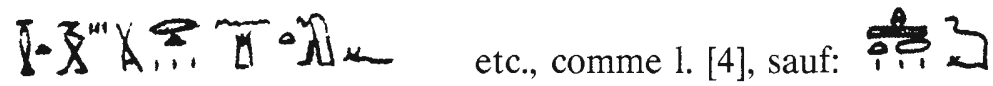


(a) Mot commun aux inscriptions de gauche et de droite.

"[2] Une offrande que donne [3] Hathor, maîtresse de Qoussîeh, - [4] (offrande) consistant en: durée de vie et grande joie! - au Pilier de sa mère, grand des chefs des artisans, sem, fils du roi, Khamouas - entrer (a) et sortir au jour - au sem, fils du roi, Khamouas ! [5] et en: eau fraîche, vin, lait ! au Pilier de sa mère, grand des chefs des artisans, sem, fils du roi, Khamouas - vie, santé, force! - au sem, fils du roi, Khamouas !"

"[6] Une offrande que donne [7] Hathor, maîtresse de Ro-Inet, [8] (offrande) consistant en: éternité du (?) premier prince (b), - au Pilier de sa mère, grand des chefs des artisans, sem, fils du roi, Khamouas - santé, joie ! au sem, fils du roi, Khamouas - [9] et en: vie, louanges, amour, - au Pilier de sa mère, grand des chefs des artisans, sem, fils du roi, Khamouas offrandes alimentaires - au sem, fils du roi, Khamouas!"

(a) Cf. 145,1,4, et remarque (b).

(b) Cf. Wörterbuch, IV, 189, 12: Titel des Osiris; Dyn. 18".

Sur le pilier dorsal (deux fois):

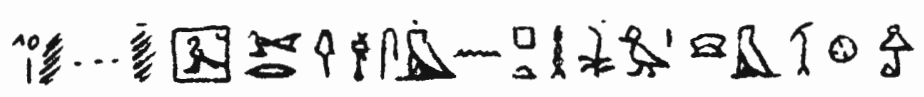

"[10] ... Hathor, le grand des chefs des artisans, sem de Ptah, fils du roi, Khamouas !"

\section{STATUE DE KHAMOUAS A KARNAK}

\section{Caire 42147}

Bibliographie:

LEGRAIN, Statues et statuettes de rois et de particuliers, tome II, p. 1213 (texte) et pl. IX-X (photos);

K.A. KITCHEN, Ramesside Inscriptions, II, p. 890-891, n 344; d'après Legrain: 
Sur le naos contenant une image de Ptah et tenu par Khamouas, face antérieure (haut, gauche et droite):

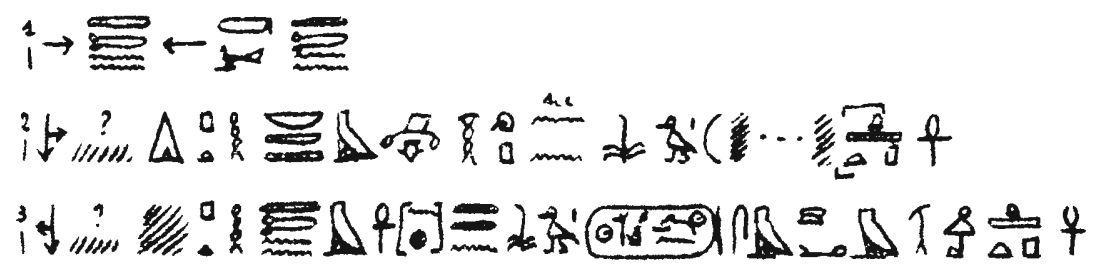

"[1] Tatenen - le grand nom - Tatenen".

"[2] (Une offrande ?) que donne Ptah, maittre des Deux Pays - (offrande) consistant en joie et durée sur (terre) - au fils du roi ......, qu'il soit content et qu'il vive!

"[3] (Une offrande (?) que donne) Ptah-Tatenen dans Ankh-taoui au fils du roi Ouser-maât-rê-Setep-en-rê, au sem Khamouas, qu'il soit content et qu'il vive!"

Sur les faces latérales du naos (gauche et droite):

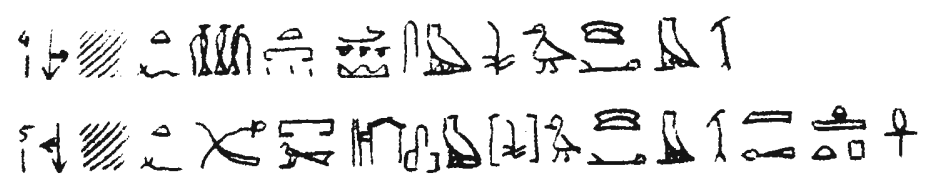

"[3] ... (?) (le Pilier de) sa (mère), celui qui est à la tête des demeures du Manou, le sem, fils du roi, Khamouas !"

"[4] ... (?) (le Pilier de) sa (mère), purificateur (a) de la Grande maison, le sem, fils du roi, Khamouas, juste de voix; qu'il soit content et qu'il vive !"

(a) cf. 124,4.

Autour du socle, à gauche et à droite:

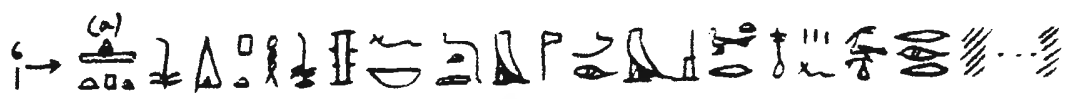

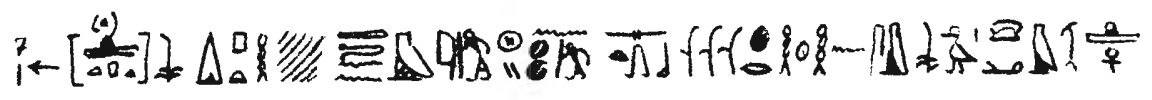


(a) Mot commun aux deux inscriptions.

"[6] Une offrande que le roi donne, et Ptah qui est au sud de son mur, consistant en: voir la beauté du dieu dans la Grande Place (a), être dans l'abondance plus que celui qui fait (?) ..."

"[7] Une offrande que le roi donne, et Ptah-Tatenen, consistant en: devenir très vieux et âgé, passer les années jusqu'à l'éternité, au sem, fils du roi, Khamouas, qu'il soit content et qu'il vive !"

(a) Litt.: voir le dieu dans la Grande Place, et sa beauté, - avec le mot "dieu" placé par respect devant le verbe "voir", mais tourné vers celui-ci.

$\mathrm{Au}$ dos de la statue:

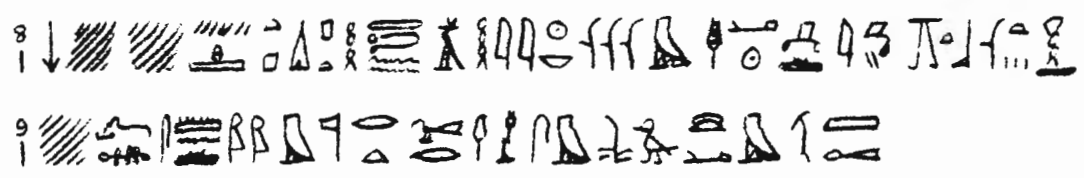

"[8] ... une offrande que donne Ptah-Tatenen, l'éternel, seigneur des années, consistant en: durée de vie, joie, être imakhou, passer les années, durer (sur ?) terre, [9] ... protéger (?), faire régner l'équité (a) en tant que (?) divine, - (au) (b) grand des chefs des artisans, sem, fils du roi, Khamouas, juste de voix !"

(a) On trouve $m 3^{c} t y$ pour $m 3^{c} t$ dès la XIXème dynastie; cf. Wörterbuch, II, p. $21,3$.

(b) En supposant l'omission du $n$ employê dans les autres inscriptions de cette statue.

\section{FRAGMENT DE COLONNE DU TEMPLE DE PTAH}

Bibliographie:

PETRIE, Memphis, I, p. 10 et pl. 25 (photo):

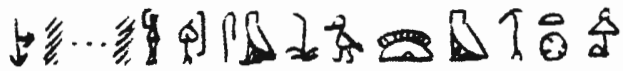

"... (grand) des chefs des artisans, sem, fils du roi, Khamouas". 


\section{AUTRE FRAGMENT DE COLONNE DU TEMPLE DE PTAH}

Bibliographie:

PETRIE, Memphis, I, pl. 25 (photo):

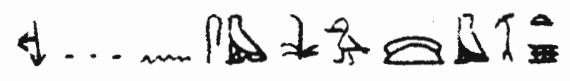

"... pour le sem, fils du roi, Khamouas".

\section{BAS-RELIEF DU TEMPLE DE PTAH}

\section{Cambridge Fitzwilliam Museum}

Bibliographie:

ENGELBACH, Riqqeh and Memphis, VI, p. 33 et pl. 56, n ${ }^{\circ} 22$ (photo):

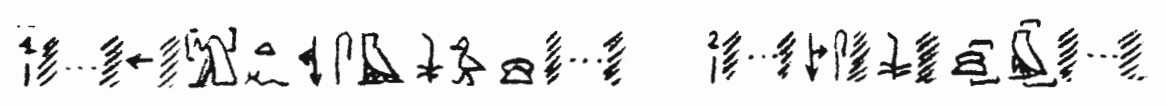

"[1] ... (Pilier) de sa mère, sem, fils du roi, Kha(mouas)".

"[2] ... s(em), (fils du) roi, Kham(ouas) ...".

\section{DEPOTS DE FONDATION DU TEMPLE DE PTAH}

Bibliographie:

PETRIE, Memphis, I, p. 8 et pl. XIX, bas gauche (photo des deux faces d'un petit bloc d'albâtre):

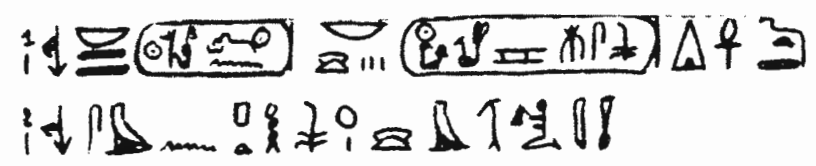

"[1] Le maître des Deux Pays, Ouser-mâ̂t-rê-Setep-en-rê, le maître des apparitions, Ramsès-Méry-imen, doué de vie éternellement".

"[2] Le sem de Ptah, fils du roi, Khamouas, juste de voix". 


\section{3a. STATUE DE KHAMOUAS}

\section{British Museum}

Bibliographie:

SHORTER, The statue of Khaemuas in the British Museum, Addendum, dans Studies presented to F. LL. GRIFFITH, London 1932, p. 132 (description et texte):

(petit bloc parallélipipédique, en grès, gravé sur deux faces)

Ière face:

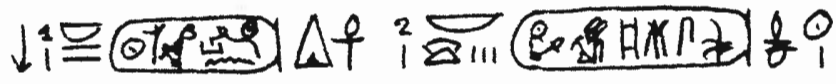

"[1] Le maître des Deux Pays, Ouser-maât-rê-Setep-en-rêe, [2] le maître des levers Ramsès-Méry-imen, doué de vie comme Rê".

2ème face (les 2 colonnes sont surmontées de

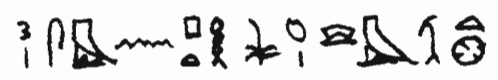

"[3] Le sem de Ptah, fils du roi, Khamouas".

\section{STATUE DE KHAMOUAS}

Vienne $21\left(N^{\circ} 5768\right)$

Bibliographie:

PORTER et MOSS, Memphis, p. 226;

WRESZINSKI, Ägyptische Inschriften aus dem K.K. Hofmuseum im Wien, 1906, p. 132-135 (texte);

K.A. KITCHEN, Ramesside Inscriptions, II, p. 883-884, n 340;

Corpus Antiquitatum Aegyptiacarum, Kunsthistorischen Museum Wien, Lieferung 6, 1990, 6,84-6,90;

d'après Wreszinski:

Sur les trois faces du pilier dorsal: 


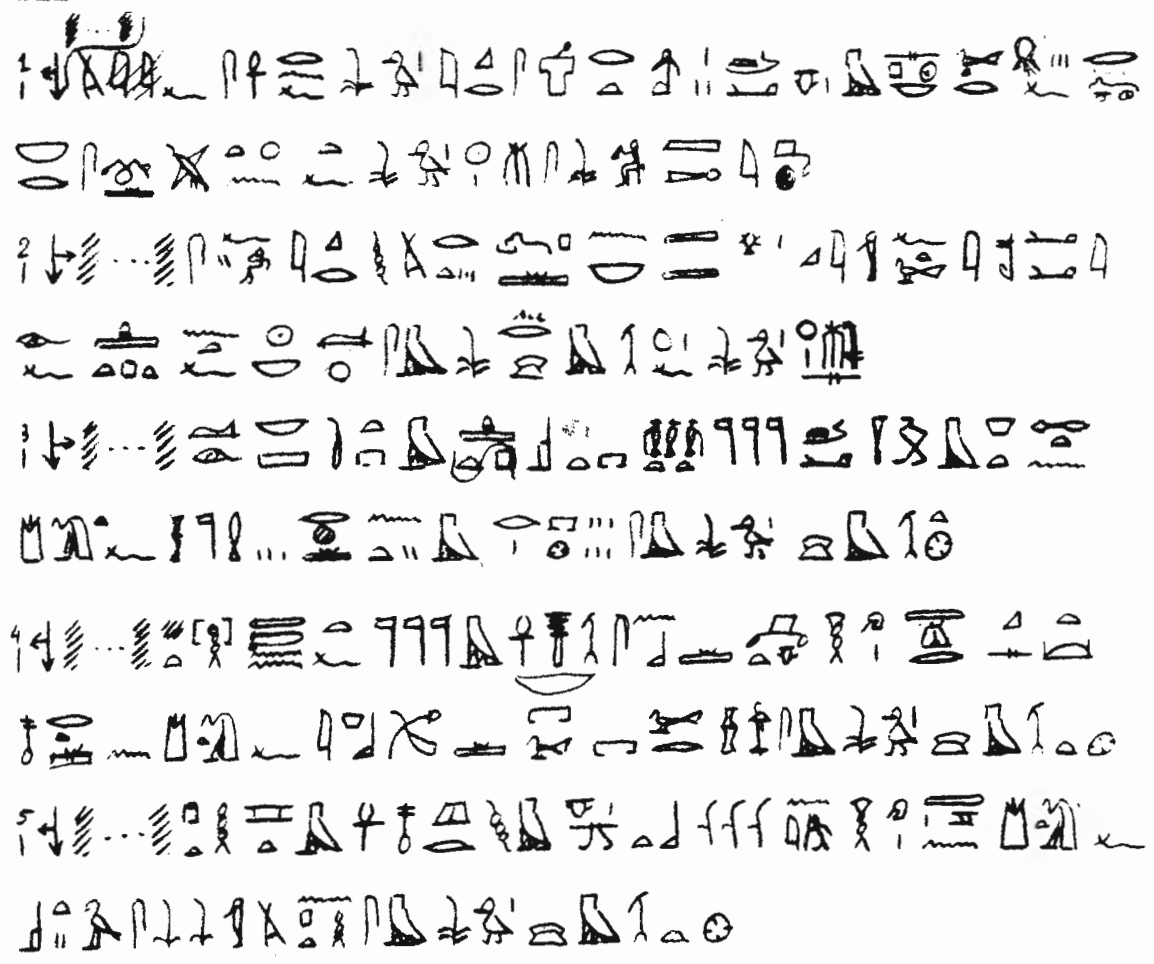

"[1] ... aimé par lui, qui fait vivre son nom, le fils royal dont l'intelligence est excellente, dont la pensée est juste en toute occasion, qui est grand ... (?) en tout temps pour maintenir les offrandes de gâteaux pour son père, le fils royal Ramsès, juste de voix et imakhou".

"[2] ... enfant excellent et aimable, choisi par le mâtre des Deux Pays à cause de sa manière, dont les doigts sont purs quand il fait chaque jour l'offrande à son père, l'Osiris, sem, porte-parole (sic) du roi, Khamouas; son fils, le fils royal Ramsès".

"[3] ... Osiris seigneur du sanctuaire, consistant à occuper la place qui est audevant des dieux, à être justifié dans la grande Salle large, au Pilier de sa mère, chef des prophètes instruit de ce qui est dans les temples, le sem et fils du roi, Khamouas !"

[4] ... Ptah-Tatenen, père des dieux, consistant en vie, stabilité, force, santé, joie, longue durée sur terre, possession d'un beau tombeau, au Pilier de sa mère, purificateur de la Grande Maison, grand des chefs des artisans, sem, 
fils du roi, Khamouas!"

[5] ... (la déesse) aimée de Ptah, consistant en une belle vie pleine d'agrément, passer les années de vieillesse, durer sur terre, au Pilier de sa mère, remplaçant de l'Horus image aimée de Ptah, sem, fils du roi, Khamouas!"

\section{CANOPE D'ITY}

\section{Louvre N 493}

Bibliographie:

CAPART, dans Chronique d'Egypte, 34 (juillet 1942), p. 256 (texte et traduction);

K.A. KITCHEN, Ramesside Inscriptions, II, p. 897, n 353 B; d'après Capart:

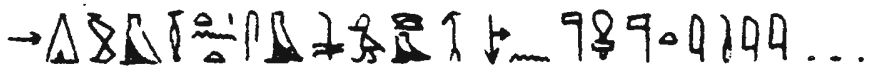

" Donné en récompense du sem, fils du roi, Khamouas, au trésorier divin et père divin Ity ..."

(Autres canopes d'Ity: PIERRET, Catalogue de la Salle historique, 1877, p. $\left.90, \mathrm{n}^{\circ} 369-371\right)$.

\section{STATUE DE KHAMOUAS D'ABYDOS}

\section{British Museum 947}

Bibliographie:

A guide to the Egyptian galleries (Sculpture), 1909, p. 170, $\mathrm{n}^{\circ} 615$ (description);

BUDGE, Egyptian Sculptures in the British Museum, pl. 36 (photo, sur laquelle les inscriptions sont illisibles);

SHORTER, The statue of Khaemuas in the British Museum, dans Studies presented to F. LL. GRIFFITH, London 1932, p. 128-132 et pl. 12-13 (texte, traduction, photos);

K.A. KITCHEN, Ramesside Inscriptions, II, p. 889-890, n 343 ;

d'après Shorter: 
I. Autour de la base (Chapitre 56 du Livre des Morts):

$i \ldots \neq i p Q \geq \square \lambda \ldots \ldots$..... (3 fois)

"[1] ... le fils du roi, sem, Khamouas ...; le fils du roi ...".

II. Sur la base (dédicace):

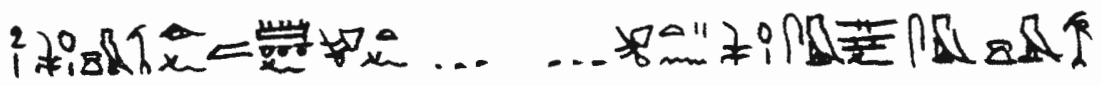

"[2] Le fils du roi, Khamouas, a fait, en tant que fondation pieuse de lui, sa statue ..... (à la fin du même texte:) ... la statue du fils aîné du roi, son aimé, le sem, Khamouas".

III. Pilier dorsal ?:

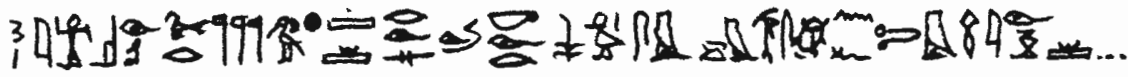

"[3] O Osiris, le plus grand des dieux, plus glorieux que Celui qui l'a créé, puisses-tu voir ce qu'a fait le fils du roi et sem Khamouas quand il t'a rendu grand de forme ...".

IV. Pilier dorsal

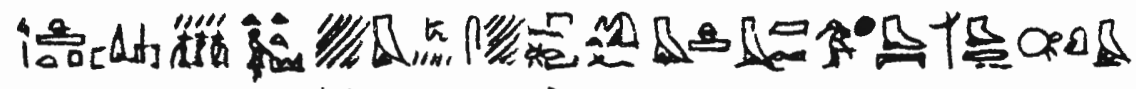

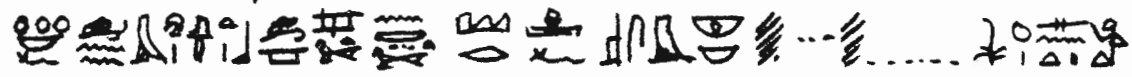

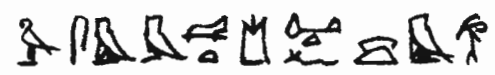

"[4] Une offrande que donne Osiris qui est à la tête de l'Occident (à) celui que le ventre de sa mère a créé en paix et en triomphe, le glorieux au ciel et puissant sur terre, le premier menuisier dans la protection de (?) son maître, celui qui est à la tête du ciseau, qui ouvre la grande route de Taour pour occuper sa (?) place à chaque fête .... fils du roi, copie de l'Horus, sem en place de Pilier de sa mère, Khamouas". 


\section{GOBELET DE KHAMOUAS DE TELL HORBEIT}

Pelizäeus-Museum

Bibliographie:

ROEDER et IPPEL, Die Denkmäler des Pelizäeus-Museum zu Hildesheim, 1921, p. 135 (description) et pl. XI a (texte):

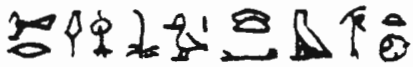

"Le grand des chefs des artisans, fils du roi, Khamouas".

\section{BAS-RELIEF DE KHAMOUAS AU GUEBEL SILSILEH}

Bibliographie:

LEPSIUS, Denkmäler, Text, IV, p. 87, o;

K.A. KITCHEN, Ramesside Inscriptions, II, p. 392, n 137 F;

d'après Lepsius:

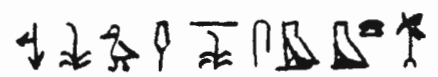

"Le fils du roi, chef de la Haute-Egypte (?), sem, Khamouas".

\section{BAS-RELIEF DE KHAMOUAS AU GUEBEL SILSILEH}

Bibliographie:

CHAMPOLLION, Monuments..., II, pl. 116;

K.A. KITCHEN, Ramesside Inscriptions, II, p. 390, n 137 B; d'après Champollion:

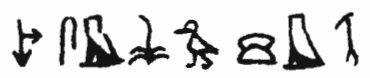

"Le sem, fils du roi, Khamouas".

130. BAS-RELIEF DE KHAMOUAS AU GUEBEL SILSILEH

Bibliographie:

CHAMPOLLION, Monuments..., II, pl. 120,1 et 
LEPSIUS, Denkmäler..., III, pl. 175 e (dessins);

K.A. KITCHEN, Ramesside Inscriptions, II, p. 891-892, n 345 A; d'après Champollion et Lepsius:

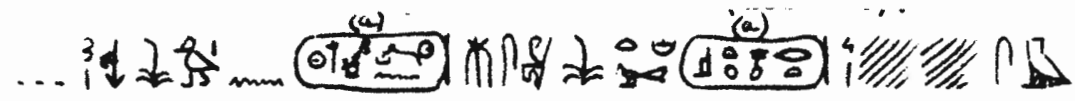

8及\}

(a) Le cartouche est gravé horizontalement.

"... [3] le fils du roi Ouser-maât-rê-Setep-en-rê, né de la grande épouse royale Isetnefert, [4] ..... sem, Khamouas".

\section{GRAFFITO DE KHAMOUAS ENTRE ASSOUAN ET PHILAE}

Bibliographie:

LEPSIUS, Denkmäler ..., III, pl. 175 h (dessin):

(Statue de Khnoum adorée par Ramsès II, suivi d'Isetnefert et:)

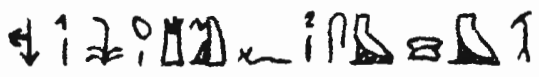

"[1] Le fils du roi, Pilier de sa mère, [2] sem, Khamouas".

\section{FIGURE DE KHAMOUAS SUR UN SISTRE}

Caire 1201 (statue fragmentaire de Ramsès II tenant un sistre) Bibliographie:

BORCHARDT, Statuen ..., IV, 1934, p. 102 (texte):

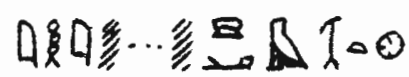

"Le musicien ..... Khamouas". 


\section{MENTION DE KHAMOUAS SUR UN PAPYRUS HIERATIQUE}

\section{P. Leiden I, 368}

Bibliographie:

CHABAS, Mélanges égyptologiques, I, 1862, p. 3 et pl. I (texte et traduction;

K.A. KITCHEN, Ramesside Inscriptions, II, p. 894-895, n 352 ;

d'après Chabas: (lettre adressée au)

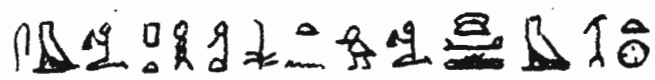

"Sem de Ptah, fils du roi, Khamouas".

\section{BASE D'UN OSIRIS AVEC INSCRIPTION DE KHAMOUAS}

Bibliographie:

QUIBELL, Excavations at Saqqara, IV, 1912, p. 16:

(extrait des fondations de l'église du monastère de l'Apa Jérémie)

"The base of an Osiris of basalt, broken off at the ankles; it bore an inscription of Khaemuas".

\section{FRAGMENT AVEC LE NOM DE KHAMOUAS}

Bibliographie:

QUIBELL, Excavations at Saqqara, IV, 1912, p. 29: (même provenance que 134)

"... on another fragment, the name of Khaemuas".

\section{BIJOUX DE KHAMOUAS AU SERAPEUM}

Louvre 2943, 2333, 2318

Bibliographie:

PIERRET, Catalogue de la Salle historique, n 527, 540, 541;

MARIETTE, Sérapéum, 1857, pl. 20 (dessins):

K.A. KITCHEN, Ramesside Inscriptions, II, p. 376, n 133 A; 
(titres)

(graphies du nom)

" Le grand des chefs des artisans, sem, fils du roi, Khamouas";

" Le Pilier de sa mère ...".

\section{7-139. STATUETTES FUNERAIRES DE KHAMOUAS}

Les statuettes funéraires de Khamouas sont commentées par J.F. et L. AUBERT, Statuettes égyptiennes, Paris, 1974, p. 85-91.

\section{Louvre 456}

Bibliographie:

BOREUX, Antiquités égyptiennes, Catalogue-guide, II, 1932, p. 324:

"(une figurine en serpentine, au nom du) "fils royal (prêtre-)sam, Khaemouast (avec un hymne au soleil)".

Ce monument est peut-être celui qui a été publié par PRISSE, Monuments égyptiens, pl. 20,1 (dessin), 2 (inscriptions);

C.-M. ZIVIE, Giza au deuxième millénaire, BdE LXX, Le Caire, 1976, p. 205-207, NE 60;

d'après Prisse:

A. 5 lignes autour du corps contiennent des voeux pour le défunt, entre autres "voir le disque du soleil et adorer Rê".

B. Au dos:

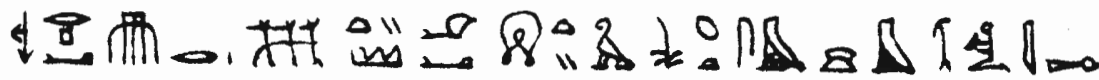

"Le noble, celui qui est à la tête de Ro-setau, le gouverneur, la copie (?) (a) d'Horus, le fils du roi, sem, Khamouas, juste de voix !".

(a) Cf. Inscription 117,1. 
137a. Louvre (?)

Bibliographie:

PRISSE, Monuments égyptiens, pl. 20, 3 (dessins), 4-5 (inscriptions):

Dans le dos:

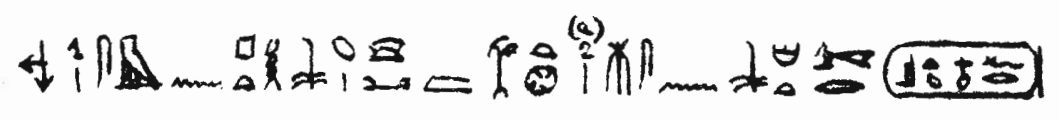

(a) La colonne [2] est répétée à droite de la colonne [1], les signes tournés vers celle-ci.

"[1] Le sem de Ptah, fils du roi, Khamouas, [2] né de la grande épouse royale Isetnefert".

Sur le devanteau du pagne:

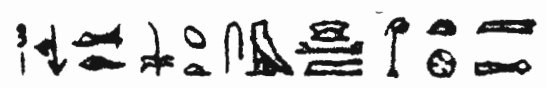

"[3] L'Osiris, fils du roi, sem, Khamouas, juste de voix".

\section{Vatican 19176}

Bibliographie:

MARUCCHI, Il Museo egizio Vaticano, 1899, p. 206-207;

K.A. KITCHEN, Ramesside Inscriptions, II, p. 898, n 354 D;

d'après Marucchi:

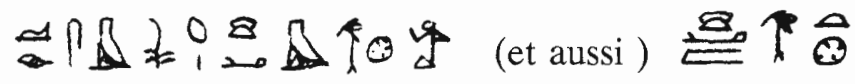

"L'Osiris, sem, fils du roi, Khamouas".

139. Berlin 306

Bibliographie:

Ägyptische Inschriften ..., II, p. 281;

K.A. KITCHEN, Ramesside Inscriptions, II, p. 898, n 354 I;

d'après Ägyptische Inschriften: 


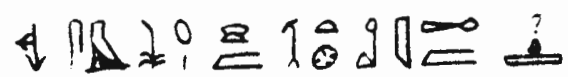

"Le sem, fils du roi, Khamouas, juste de voix, en paix !"

\section{CANOPE DE KHAMOUAS}

\section{Caire 4085}

Bibliographie:

REISNER, The dated canopic jars of the Gizeh Museum, dans Ä.Z., 37 (1899), p. 66:

MARIETTE, Monuments divers, 1889, p. 10 et pl. 36 d:

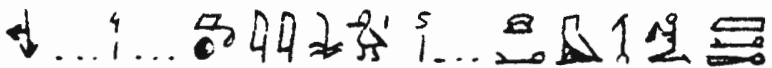

"... l'imakhy, fils du roi, Khamouas, juste de voix".

(Chaque colonne débute par 0 "à réciter").

\section{BAS-RELIEF DE KHAMOUAS AU SERAPEUM}

\section{Louvre}

Bibliographie:

BOREUX, Antiquités égyptiennes, Catalogue-guide, II, 1932, p. 481 (description).

\section{BAS-RELIEF DE KHAMOUAS}

Caire 8-10-14-1 (fragment de paroi) ma copie:

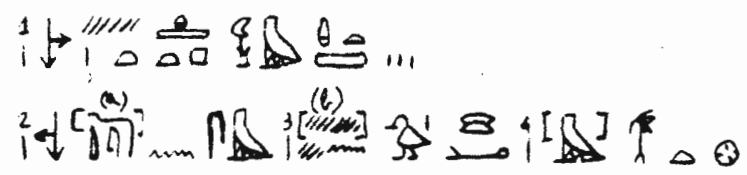

(a) Les parties entre crochets se trouvent sur un autre bloc. 
(b) Le mot nsw était sans doute écrit $¥ \stackrel{\Delta}{\text { sum }}$.

"[1] (Que le roi ?) soit gracieux (et donne ?) un millier de pains (a)". "[2] A réciter par le sem, [3] fils (du roi), Kha[4]mouas !".

(a) Fin de la formule.

\section{DEMI-TAMBOUR DE COLONNE DE KHAMOUAS}

\section{Caire 17-11-24-4}

ma copie:

(Khamouas assis devant une table d'offrandes; à droite et à gauche de la scène, qui est surmontée du signe du "ciel":)

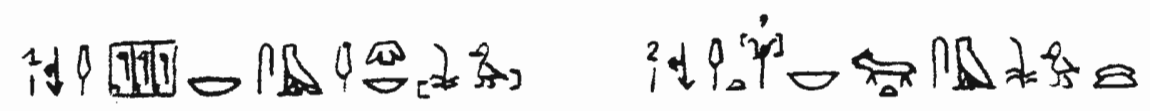

"[1] Le chef de tous les temples, sem, chef de toute la garde-robe, fils du roi ...".

"[2] Le chef de toutes (les fonctions ?) ... (?), sem, fils du roi, Kha...".

\section{STELE DE KHAMOUAS AU SERAPEUM}

Bibliographie:

MARIETTE, Notice des principaux monuments exposés ... à Boulaq, 2 ème édition, 1868 , p. 341, $\mathrm{n}^{\circ} 28$ (sous la rubrique des monuments pas encore au musée):

"Grande stèle ornée de rainures prismatiques qui forment la décoration habituelle des monuments de l'Ancien Empire. L'étude des inscriptions prouve qu'elle est du temps de Ramsès II. On n'y lit en effet que le nom et les titres du prince Sche-em-ab (= Khamouas), celui des fils de Ramsès qui fut gouverneur de Memphis. La stèle a été trouvée à l'entrée de la tombe d'Apis ...". 


\section{BASE DE STATUE DE KHAMOUAS ET D'UN APIS (?)}

Bibliographie:

QUIBELL, Excavations at Saqqara, IV, 1912, p. $45-46$ (texte):

(autour de la base, inscription en trois lignes au-dessus de figures agenouillées aux noms illisibles)

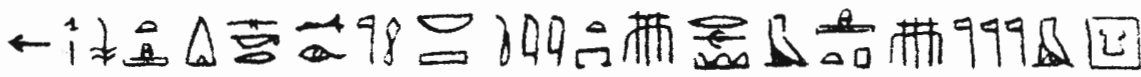

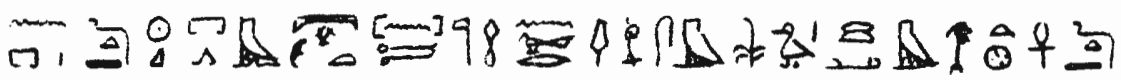

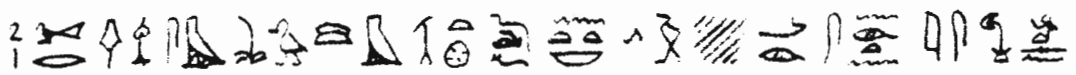

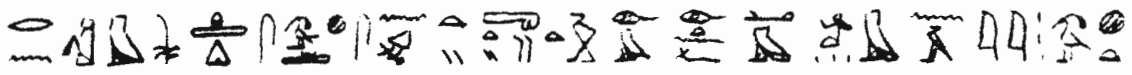

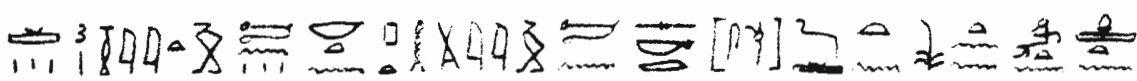

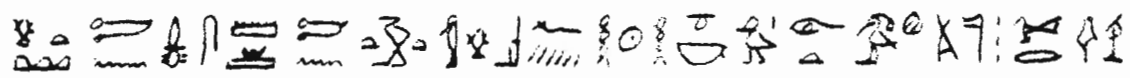

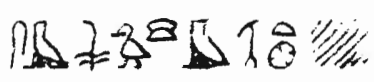

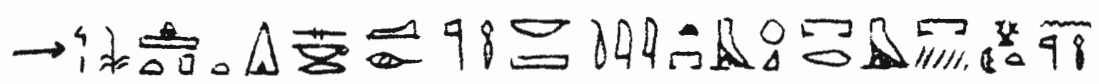

及罗

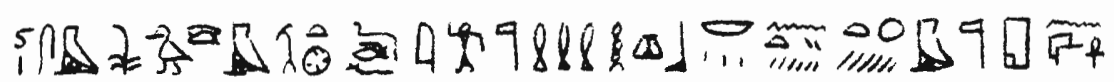

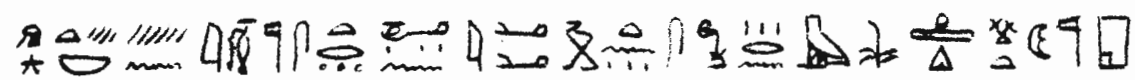

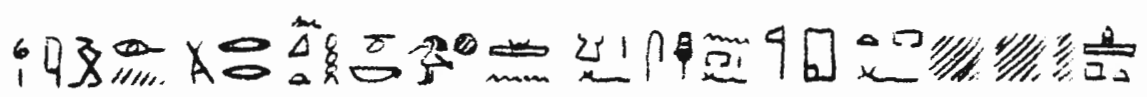

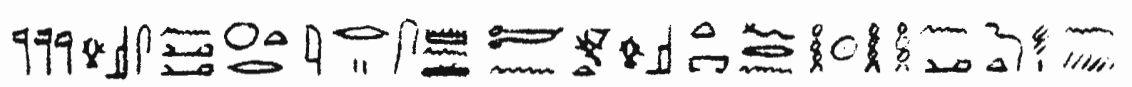

$3 \circ p f$ 
"[1] Une offrande (a) royale que donne Sokar-Osiris, le dieu grand, maitre du sanctuaire, qui est à la tête de Ro-setaou, consistant à reposer devant les dieux dans la chapelle du domaine funéraire, (ainsi qu'en) aliments (b) déposés sur l'autel (c) du dieu grand, au grand des chefs des artisans, sem, fils du roi, Khamouas, puisse-t-il vivre éternellement! [2] Le grand des chefs des artisans, sem, fils du roi Khamouas dit: $O$ vous tous qui viendrez et qui verrez ce que j'ai fait, qui rappellerez mon nom dans la formule d'offrande, qui rendrez ma statue magnifique, qui rendez témoignage à celui qui a agi en agissant pour lui (?) (d), qui savez ce qui vous est utile (e), [3] votre maître Ptah vous récompensera, Sokar vous aimera, le roi vous confiera à vos enfants (f), vous reposerez dans votre tombeau dans la mesure où vous maintiendrez ma statue à sa place d'éternité, car je suis un fils accomplissant ce qui est utile et aimé des dieux, (moi) le grand des chefs des artisans, sem, fils du roi, Khamouas ...".

"[4] Une offrande royale que donne Sokar-Osiris, le dieu grand, maittre du sanctuaire, consistant en aliments déposés (g) sur l'autel du dieu grand à chaque fête $d u$ ciel et de la terre que ramène le commencement des saisons (?) (h), au fils du roi Ouser-maât-rê-Setep-en-rê et sem Khamouas ! [5] Le sem et fils du roi Khamouas dit: O prophètes et prêtres-lecteurs qui ... dans la demeure divine d'Apis vivant chaque matin (i), ... moi de l'eau fraîche et de l'encens que vos mains m'offriront, rappelez mon nom dans la formule d'offrandes sur l'autel de la demeure divine, [6] car (j'ai ?) fait ce que Ptah (?) désire: j'ai été utile à son (k) ka, j'ai érigé sa maison et sa demeure divine ..... les dieux reposent à leur place, en échange de quoi (?) (1) vous maintiendrez ma statue à sa place pour l'éternité ... magnifique (?) Apis vivant".

(a) D'après ligne 4.

(b) Le groupe $O_{\Delta}$ se trouve aussi à la ligne 4 et dans l'inscription 118, 1. 4. Dans 118,4, où il précède "sortir au jour", j’ai supposé que j'avais affaire à un verbe de sens analogue ou contraire à "sortir"; dans 145,1, le même groupe doit être un nom désignant une ou des offrandes au dieu. Etant donné le $\Delta$, je n’hésite pas à lire le groupe

\& ${ }^{c} k$ ou ${ }^{c} k(w)$, sans pouvoir expliquer la valeur ${ }^{c}$ de 0 .

(c) Litt.: sortant en haut sur l'autel.

(d) $m$ tr.w ir $m$ irt n.f ? (mtr.w $=$ mtr.sn, mais le $t$ précédent $w$ reste inexpliqué ! ìt $=$ 
infinitif).

(e) Litt.: ils se connaissent en leurs utilités.

(f) On attendrait: "vous transmettrez vos fonctions à vos enfants". S'il n'y a pas eu erreur du graveur ancien ou du déchiffreur moderne ( $\Varangle$ à la place de $\psi$ ), j’interprète ainsi cette phrase: le roi vous mettra sous les ordres de vos enfants, c'est-à-dire qu'il les chargera de diriger tout ce qui vous concerne (sous-entendu: après votre mort ?). Si le texte a bien été composé tel que je le donne, il me semble difficile de ne pas voir un piège dans le groupe , qu'on pourrait être tenté de traduire par "Héracléopolis".

(g) Peut-être: sortis de la Maison ... (pour être déposés) sur l'autel.

(h) Litt.: aux saisons qui arrivent à leurs (= des fêtes ?) jours.

(i) Wörterbuch, V, p. 424 et 425,1.

(k) "son" et les deux "sa" de la proposition suivante paraissent désigner Ptah mais il me semble aussi possible de les rapporter à Apis, aux desservants duquel Khamouas s'adresse.

(1) Je lis: $d d$ ht $r$ iry, "donnant (?) les choses à ce qui (les ?) concerne" ? Khamouas a déclaré que les dieux (les momies des Apis ?) reposent à leur place grâce à lui (?); il me semble nécessaire d'avoir, avant la phrase "vous maintiendrez ma statue à sa place", une locution ou une proposition qui indique le rapport des deux déclarations.

\section{MENTION DE KHAMOUAS DANS LE LIVRE DES MORTS}

Bibliographie:

PLEYTE, Chapitres supplémentaires du Livre des Morts, p. 67: (transcription du hiératique)

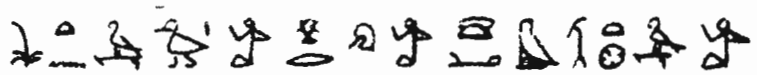

"Le fils principal du roi (a), Khamouas".

(a) Litt.: le fils du roi, placé à la tête. 


\section{STATUE NAOPHORE DE NEFERRENPET}

\section{Leyde D 44}

Bibliographie:

BOESER, Beschreibung der ägyptischen Sammlung, V, 1913, p. 7-8, n 18 (texte);

$\mathrm{PM} \mathrm{III}^{2} / 2$, p. 706-707;

cartouche de Ramsès II sur l'épaule et

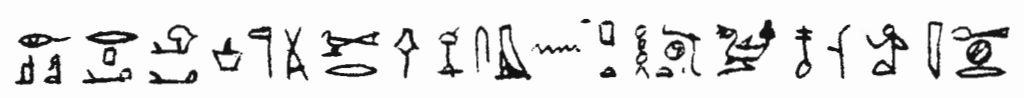

"L'Osiris, noble, gouverneur, père divin aimé du dieu, grand des chefs des artisans, sem de Ptah, préfet de la ville et vizir, Neferrenpet, juste de voix".

\section{TABLE D'OFFRANDES DE NEFERRENPET}

\section{British Museum 108}

Bibliographie:

A Guide to the Egyptian Galleries (Sculpture), 1909, p. 167, n 605 (description);

LIEBLEIN, Dictionnaire de noms, $\mathrm{n}^{\circ} 886$;

T.G.H. JAMES, The British Museum Hieroglyphic Texts from Egyptian Stelae, Part 9, p. 17-18, pl. XIII;

K.A. KITCHEN, Ramesside Inscriptions, III, p. 48-49;

d'après Lieblein;

entre autres:

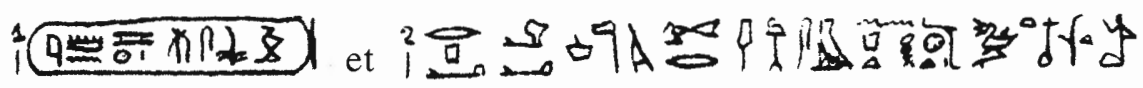

"[1] Méry-Imen-Ramsès".

"[2] Le noble, gouverneur, père divin aimé du dieu, grand des chefs des artisans, sem de Ptah, préfet de la ville et vizir, Neferrenpet". 
149. MONUMENT EN FORME DE NAOS DE NEFERRENPET

\section{Berlin 2290}

Bibliographie:

Ägyptische Inschriften ..., II, p. 316-317 (texte);

Ägyptische und Vorderasiatische Altertümer, pl. 27 (photo);

K.A. KITCHEN, Ramesside Inscriptions, III, p. 49-50;

d’après Ägyptische Inschriften:

I. Face supérieure:

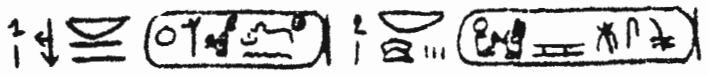

"[1] Le mâttre des Deux Pays Ouser-maât-rê-Setep-en-rê,, [2] maître des apparitions Méry-Imen-Ramsès".

II. Face antérieure:

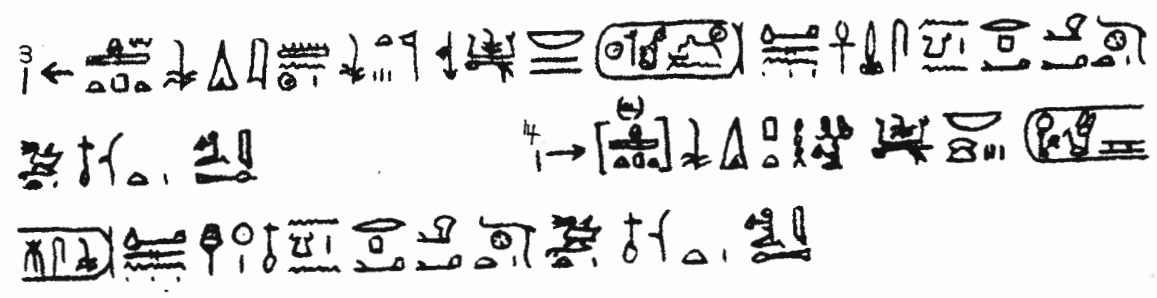

(a) Mot commun aux deux inscriptions.

"[3] Une offrande royale que donnent Amon-rê, roi des dieux, et le ka du roi, maître des Deux Pays Ouser-maât-rê-Setep-en-rê; puissent-ils donner vie, santé et force au ka du noble, gouverneur, préfet de la ville et vizir, Neferrenpet, juste de voix !"

"[4] Une offrande royale que donnent Ptah-Tatenen et le ka du roi maitre des apparitions Méry-Imen-Ramsès; puissent-ils donner une belle durée de vie au ka du noble, gouverneur, préfet de la ville et vizir, Neferrenpet, juste de voix !" 
III. Face latérale droite (par rapport à la statue):

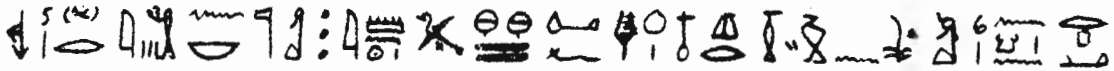

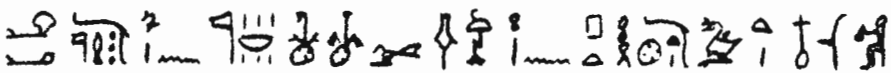

"[5] Décerner des louanges au seigneur des dieux, Amon-rê, le primordial des Deux Pays, afin qu'il donne une belle durée de vie comblée des récompenses du roi [6] au ka du noble, gouverneur, directeur des prophètes [7] de tous les dieux de Haute et Basse-Egypte, grand des chefs des artisans [8] de Ptah, préfet de la ville et vizir, Neferrenpet !"

IV. Face latérale gauche:

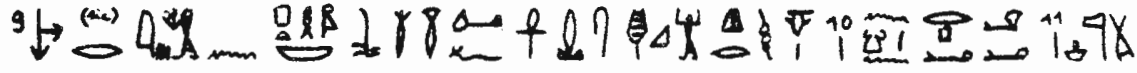

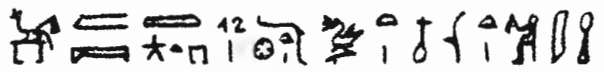

"[9] Décerner des louanges à Ptah, maître de la Vérité, roi des Deux Pays, afin qu'il donne vie, santé, force, longue durée de vie comblée d'agrément [10] au ka du noble, gouverneur, [11] père divin aimé du dieu, supérieur des secrets dans le ciel, la terre et la douat, [12] préfet de la ville et vizir, Neferrenpet, juste de voix !"

V. Face postérieure:

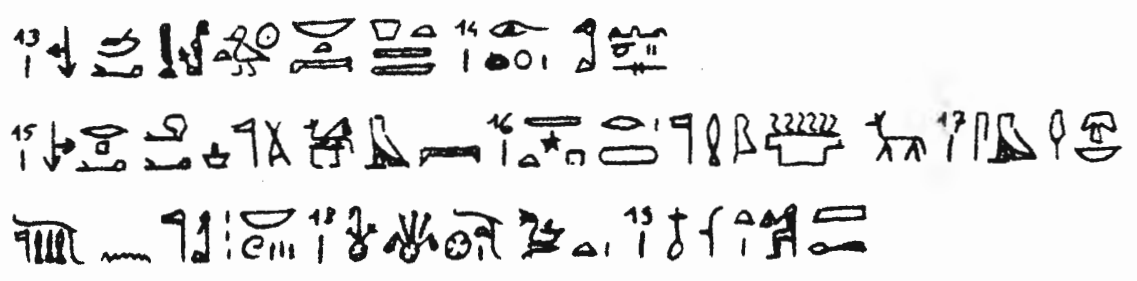

"[13] Mâat, fille de Rê, maîtresse du ciel, régente des Deux Pays, [14] celle de Rê, Celle qui n'a pas son pareil".

"[15] Le noble, gouverneur, père divin aimé du dieu, supérieur des secrets dans le ciel, [16] la terre et la douat, porte-parole de Nekhen, prophète de Maât, celui du Rideau, juge, [17] sem, chef de toute la garde-robe, directeur des prophètes de tous les dieux [18] de Haute et de Basse-Egypte, préfet de la ville et vizir, [19] Neferrenpet, juste de voix !" 


\section{STATUETTE CUBIQUE DE NEFERRENPET}

\section{Caire 713}

Bibliographie:

BORCHARDT, Statuen ..., III, p. 50-51 (texte) et pl. 132 (photo):

I. Au dos de la statue:

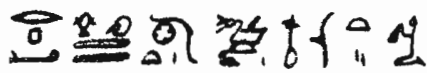

"[1] Le noble placé à la tête des Deux Pays, préfet de la ville et vizir, Neferrenpet".

II. Face antérieure du socle:

\section{匀器夆}

"[2] Le préfet de la ville et vizir, Neferrenpet, juste de voix".

III. Face latérale gauche du socle:

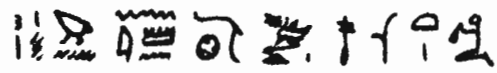

"[3] Le conducteur (de la fête ?) d'Amon, préfet de la ville et vizir, Neferrenpet".

IV. Faces latérale droite et postérieure du socle:

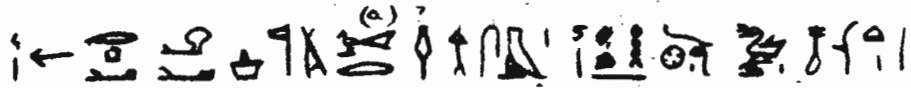

"[4] Le noble, gouverneur, père divin aimé du dieu, grand des chefs (?) des artisans (?), sem [5] de Ptah (?), préfet de la ville et vizir, Neferrenpet".

(a) Borchardt: 


\section{STATUETTE NAOPHORE DE NEFERRENPET}

Caire 1034

Bibliographie:

BORCHARDT, Statuen ..., IV, 1934, p. 33:

I. Face antérieure:

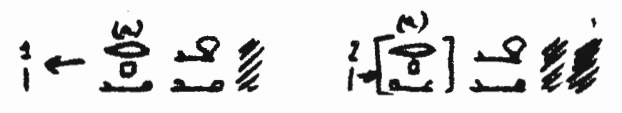

(a) Mot commun aux deux inscriptions.

"[1-2] Le noble, gouverneur ..."

II. Face latérale droite:

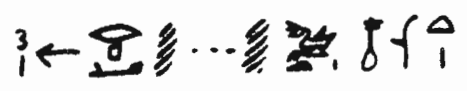

"[3] Le noble, ... vizir, Neferrenpet".

III. Face latérale gauche:

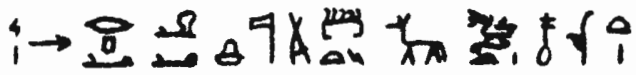

"[4] Le noble, père divin aimé du dieu, celui du Rideau, juge, vizir, Neferrenpet".

IV. Sur le pilier dorsal:

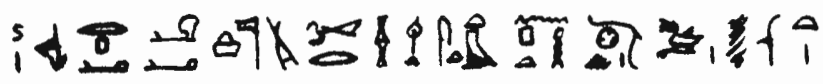

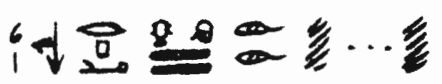

"[5] Le noble, gouverneur, père divin aimé du dieu, grand des chefs des artisans, setem de Ptah, préfet de la ville et vizir, (Nefer)renpet. [6] Le noble placé à la tête des Deux Pays, yeux .....". 


\section{PYRAMIDION DE NEFERRENPET}

\section{Liverpool M 11015}

Bibliographie:

EDWARDS, Amelia B., The provincial and private collections of Egyptian antiquities in Great Britain, dans Rec. Trav. X (1888), p. 130-132;

LIEBLEIN, Dictionnaire de noms, ${ }^{\circ} 1068$;

WEIL, Die Veziere, p. 94-95;

K.A. KITCHEN, Ramesside Inscriptions, III, p. 50;

D'après Edwards, corrigée par ANTHES, Die Verwendung ..., dans Ä.Z., 74 (1938), p. 109-110:

Face I.

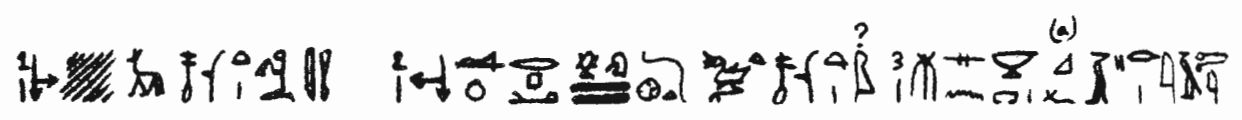

"[1] ... juge, Neferrenpet, juste de voix".

"[2] L'Osiris, noble placé à la tête des Deux Pays, préfet de la ville et vizir,

Neferrenpet, juste, [3] né de celle qui possède une maison, Qefarit (?)".

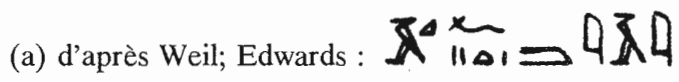

Face II:

\section{i.}

"[4] L'Osiris, grand des chefs des artisans, sem, Neferrenpet, juste de voix; [5] sa soeur, Iniouhy, puisse-t-elle vivre! [6] sa fille, Ta-our-khat".

Face III.

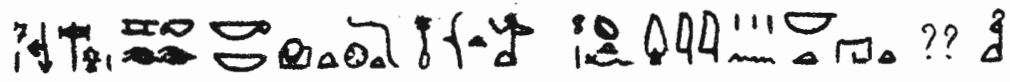 i⿺}

"[7] (Puisses-tu ?) saluer, puisses-tu voir le maître de l'horizon, préfet de la ville Neferrenpet ! Sa fille, Iamou-n(w)-nebet-(resit ?) [8] Sa fille, Henetmet". 
Face IV.

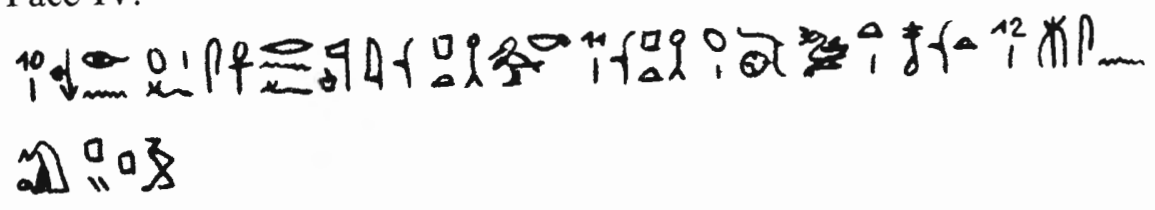

"[10] Fait par son fils qui fait vivre son nom, le père divin de Ptah, Bak[11]enptah, fils du préfet de la ville èt vizir, Neferrenpet, [12] et de Moutpypou !"

\section{PLAQUETTE DE NEFERRENPET}

\section{Collection Amherst}

Bibliographie:

NEWBERRY, Scarabs, p. 182, n 24 et pl. XXXV (dessin):

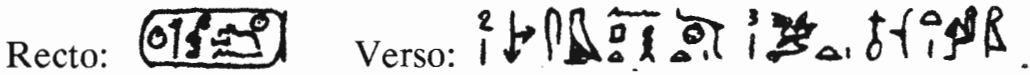

"[1] Ouser-maât-rê-Setep-en-rê". "[2] Le sem de Ptah, préfet de la ville [3] et vizir, Neferrenpet, juste".

\section{PLAQUETTE DE NEFERRENPET}

\section{British Museum 4104}

Bibliographie:

NEWBERRY, Scarabs, p. 182, n 24 et pl. XXXV (dessin); BRUGSCH, Thesaurus, p. 1456 (texte des titres):

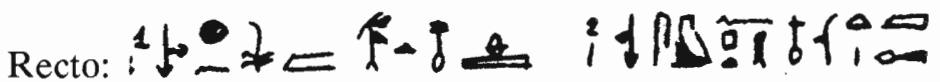

"[1] Khonsou-dans-Thèbes-Neferhotep. "[2] Le sem de Ptah, Neferrenpet, juste de voix".

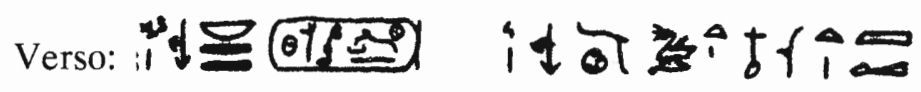


"[3] Le maître des Deux Pays, Ouser-maât-rê-Setep-en-rê". "[4] Le préfet de la ville et vizir, Neferrenpet, juste de voix".

\section{PALETTE DE PTAHMES IV}

\section{Louvre 3026}

Bibliographie:

PIERRET, Etudes égyptologiques, II, Recueil d'inscriptions inédites du Musée égyptien du Louvre, 1874, p. 93-94 (texte);

BRUGSCH, Thesaurus, V, p. 963 (texte):

Cf. aussi MASPERO, Un manuel de hiérarchie égyptienne, dans Etudes égyptiennes, II, 1er fascicule, 1888, p. 20 (fragment des épithètes avec traduction), et ANTHES, Die hohen Beamten ..., dans Ä.Z., 72 (1936), p. $63, \mathrm{n}^{\circ} \mathrm{IV}, 8$.

D'après Pierret:

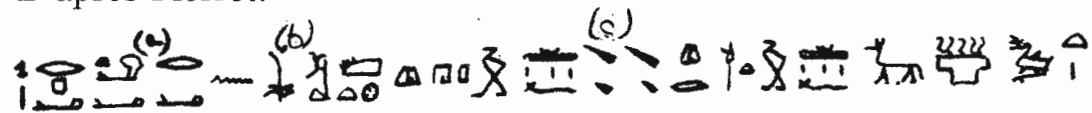

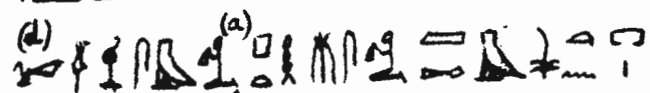

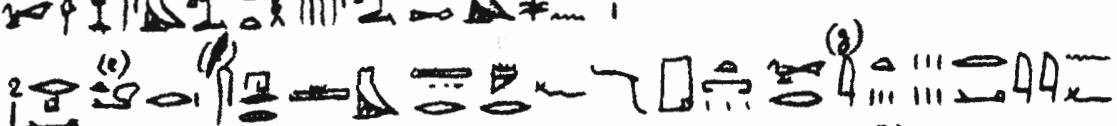

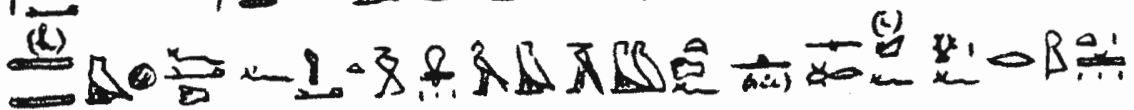

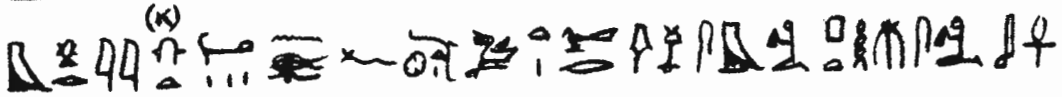

(a) Maspero.

(b) Brugsch: $\neq-1$, Maspero $\neq 09$

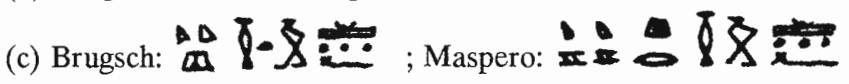

(d) Maspero $\searrow$ (sic !).

(e) à (k) variantes de Brugsch:

(e) $\$$ 
(f) $\longleftarrow$

(g) $44, \ldots$

(h)

(i)

(k) ?

"[1] Le noble et gouverneur sous les lois duquel le roi a placé l'Egypte, sous le bon plaisir duquel il a mis les Deux Régions, le juge, celui du Rideau, le vizir, grand des chefs des artisans, sem, Ptahmès, juste de voix dans la Maison du roi".

"[2] Le noble, gouverneur, porte-parole qui apaise dans le pays entier, directeur des Six Grandes Maisons, dans le poing duquel les sceaux d'Horus ont été mis, celui qui ne fait pas la sourde oreille à la vérité par crainte de son oeil Oudjat, le préfet de la ville et vizir, grand des chefs des artisans, sem, Ptahmès, renouvelant (sa) vie".

\section{STATUE CUBIQUE NAOPHORE DE IYRY}

\section{Louvre A 71}

Bibliographie:

PIERRET, Etudes égyptologiques, I, p. 10;

ma copie:

I. Face supérieure

\section{it (618)

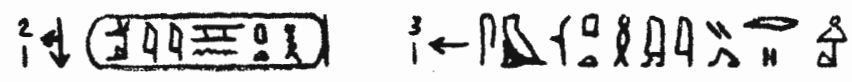

"[1] Ouser-maât-rê-Setep-en-(rê ?)". "[2] Séti-Mer-en-ptah".

"[3] Le sem de Ptah, Iyry".

II. Face antérieure, sur le naos 


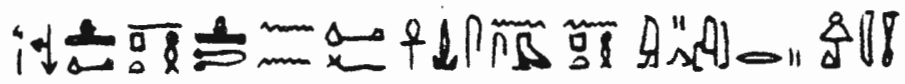

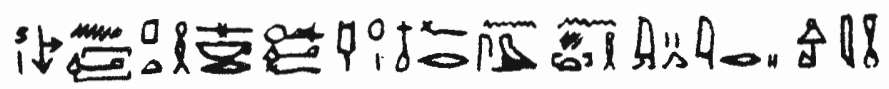

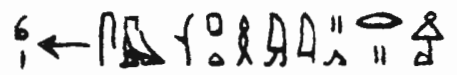

"[4] Une offrande donnée à Ptah (?), une offrande de Tenen, pour qu'il donne vie, santé et force au sem de Ptah, Iyry, juste de voix".

"[5] Une offrande donnée (à) Ptah-Sokar-Osiris, pour qu'il donne une belle durée de vie au sem de (Pt)ah, Iyry, juste de voix".

"[6] Le sem de Ptah, Iyry".

III. Face antérieure, à la droite et à la gauche du naos:

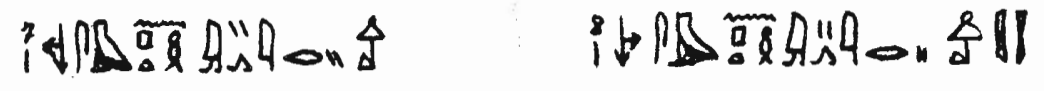

"[7] Le sem de Ptah, Iyry". "[8] Le sem de Ptah, Iyry, juste de voix".

IV. Face latérale droite de la statue:

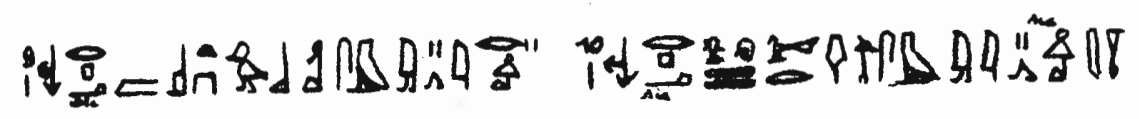

"[9] Le noble dans la place de Geb, sem, Iyry".

"[10] Le noble placé à la tête des Deux Pays, grand des chefs des artisans, sem, Iy (sic), juste de voix".

V. Face latérale gauche de la statue:

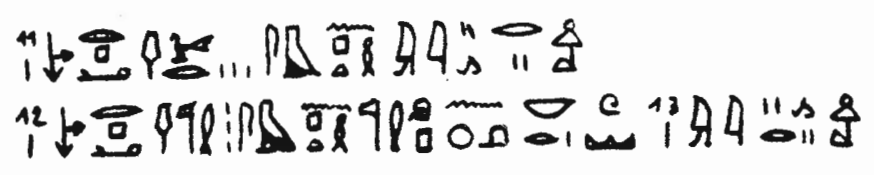

"[11] Le noble, chef des grands, sem de Ptah, Iyry".

"[12] Le noble, chef des prophètes, sem de ptah, premier prophète d'Osiris seigneur de Ro-setaou, [13] Iyry".

VI. Autour du socle (côtés droit et gauche de la statue): 


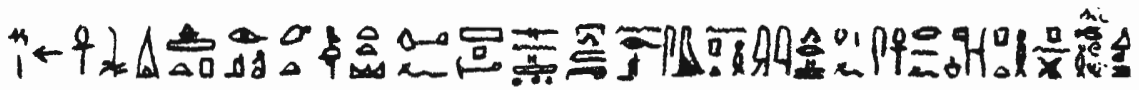

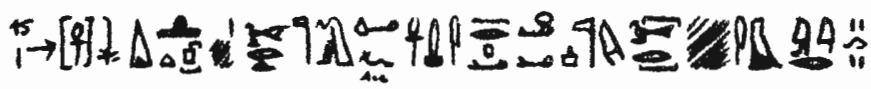

"[14] Vie! Une offrande royale (à) Osiris qui est à la tête des Occidentaux pour qu'il accorde de recevoir des gâteaux qui (lui) sont présentés, à l'Osiris, sem de Ptah, Iyry, juste de voix ! Son fils qui fait vivre son nom, le père divin de Ptah, Pen-pa-dehou, juste de voix".

"[15] (Vie !) Une offrande royale (à) (Isis) la Grande, mère du dieu, pour qu'il (sic !) accorde vie, santé et force au noble, gouverneur, père divin aimé du dieu, grand (des chefs des artisans), sem, lyry !"

VII. Sur le pilier dorsal:

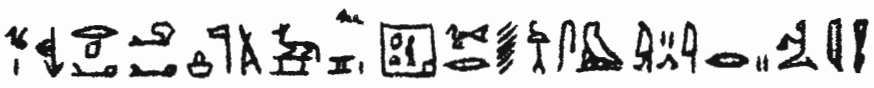

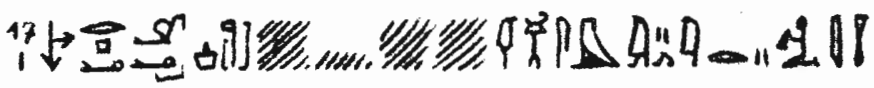

"[16] Le noble, gouverneur, père divin aimé du dieu, supérieur des secrets du temple de Ptah, grand (des chefs) des artisans, sem, Iyry, juste de voix". "[17] Le noble, gouverneur, père divin ....... des chefs des artisans, sem, Iyry, juste de voix".

\section{STATUE D'UN PERE DIVIN DE PTAH}

\section{Leyde D 45}

Bibliographie:

BOESER, Beschreibung der ägyptischen Sammlung, V, p. 11, fig. 63 (texte):

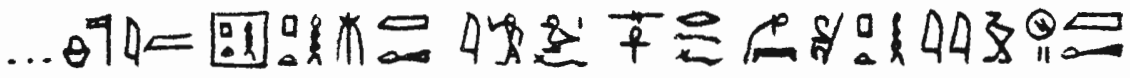

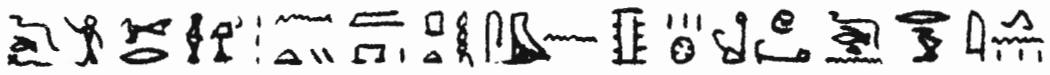

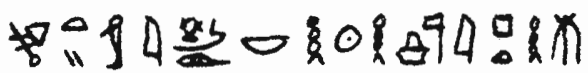


"... le père divin dans le temple de Ptah, Ptahmès, juste de voix. C'est son fils qui fait vivre son nom, le ouab supérieur de Ptah, Youyou, juste de voix; il dit: $O$ grands des chefs des artisans qui serez dans le temple de Ptah, sem des Murs (a), gardez-vous de dire que ma statue vous (b) empêche (c) de voir le Seigneur de l'éternité, (moi) le père divin Ptahmès !"

(a) $=$ Memphis.

(b) Litt.: nous.

(c) Le Wörterbuch, II, 406, sous lettre B, n'indique pas d'emploi de rwi + complément d'objet $+h r+$ infinitif avec le sens d'"empêcher quelqu'un de ...", mais seulement $r w i+$ objet $+h r+$ nom de lieu avec le sens d"éloigner quelque chose de ...". On pourrait aussi traduire: "... gardez-vous de dire: "Nous empêcherons sa (litt. ma) statue de voir..." Les Belegstellen du Wörterbuch, II, 406, 19 citent seulement: " Leid. D. 45", ce qui conduit à traduire: "nous éloignerons ma (sic) statue", mais ne donnent aucune indication sur la suite de la phrase.

\section{STELE DANS LE TEMPLE DE SAHOURE}

Bibliographie:

BORCHARDT, Das Grabdenkmal des Königs Sahure, I, 1910, p. 125 (texte des fragments de la stèle):

Fragment I:

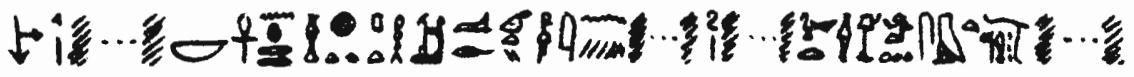

"[1] ... Seigneur d'Ankh-taoui, Sekhmet (aimée de ?) Ptah, Osiris à la tête de l'Occident, Anu(bis) (?) ... [2] ... grands des chefs des artisans, sem, directeurs des prophètes ..."

Fragment III:

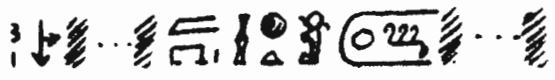

"[3] ... dans le temple de Sekhmet de Sahourê..." 
Fragment IV:

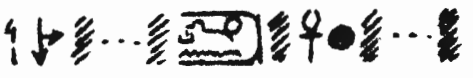

"[4] ... -Setep-en-rê, (doué de ?) vie ..."

\section{LIVRE DES MORTS, CHAPITRE I}

Bibliographie:

NAVILLE, Das ägyptische Todtenbuch, chapitre I, col. 20-22; idem, dans THEODORE M. DAVIS excavations: Bibân el-Molûk. The funeral papyrus of Iouiya, Londres 1908, chapitre I:

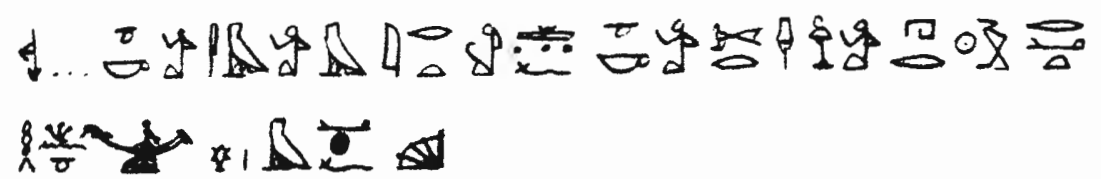

"... je suis le sem à son affaire; je suis le grand des chefs des artisans au jour où l'on met le henou (a) sur son (?) traîneau ..."

(a) = la barque de Sokar.

160. STELE DE "OUR-KHEREP-HEMOU"

\section{Leyde V 57}

Bibliographie:

BOESER, Beschreibung der ägypt. Sammlung, VI, pl. 20:

I. Au premier registre:

\section{i}

"[1] Le ouab de Ptah, Our-kherep-hemou, juste, appelé Hori, juste de voix".

II. Au deuxième registre: 


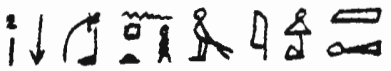

"[2] Le ouab de Ptah, Hori, juste de voix".

(Ce monument est de la XIXème dynastie, comme le montrent la graphie de l'adjectif du génitif $n(y)$, écrit $\$ \mathcal{f}$, et le style de la sculpture).

\section{STELE DE NEKHT}

\section{Munich, Antiquarium 15}

Bibliographie:

DYROFF et PORTNER, Ägyptische Grabsteine und Denksteine aus süddeutschen Sammlungen, herausgegeben von W. SPIEGELBERG, 1904, pl. 16:

En face du défunt et de sa femme, à la deuxième rangée de personnages assis, le troisième est:

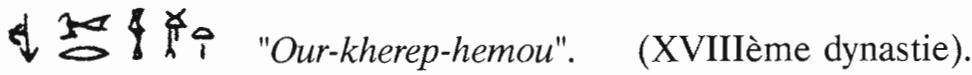

\section{STATUETTE FUNERAIRE DE OUR-KHEREP-HEMOU}

\section{Mannheim, Schlossmuseum}

Bibliographie:

signalé par RANKE, Ägyptische Personennamen, 81, 18.

\section{2a. LE GRAND PAPYRUS HARRIS}

Bibliographie:

BIRCH, Facsimile of an Egyptian Hieratic Papyrus of the reign of Ramses III, now in the British Museum, Londres, 1876 (texte);

ERICHSEN, Papyrus Harris I (Bibliotheca aegyptiaca, V), 1933, p. 57 (transcription); 
BREASTED, Ancient Records, IV, $\S 338$ (traduction);

d'après Birch et Erichsen:

planche 51a, ligne 7:

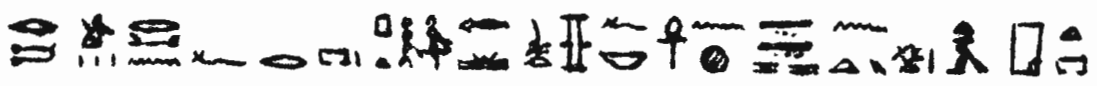

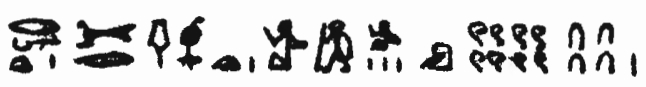

"Les gens qu'il a donnés au domaine de Ptah le grand qui est au sud de son mur, le seigneur d'Ankh-taoui, et qui sont pour (a) le temple (b) et sous le commandement du grand des chefs des artisans et des "principaux": 841 personnes".

(a) Litt.: "sur".

(b) Il s'agit du "temple de Ramsès-régent d'Héliopolis, V., S., F., dans le domaine de Ptah" qui est cité quelques lignes plus haut.

\section{2b. FRAGMENT DU TEMPLE DE PTAH}

Bibliographie:

ENGELBACH, Riqqeh and Memphis VI, pl. 58, n 32; cf. p. 33.

Légende d'un personnage agenouillé devant un roi (?) et lui tendant un vase à bout de bras:

\section{$\checkmark=9 t$}

"Le grand des chefs des artisans". 



\section{CHAPITRE XVI}

\section{LES INSCRIPTIONS DE LA BASSE EPOQUE}

\section{3-164. LINTEAUX DU ROI SIAMON}

\section{Linteau Caire 40033}

Bibliographie:

PETRIE, Memphis I, pl. XXXI (moitié droite): mes copie et photographie (moitié gauche):

I. Au milieu, deux colonnes gravées dos à dos: (gauche) it z

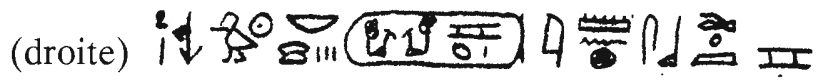

"[1] Le roi de Haute et Basse-Egypte, seigneur des Deux Pays, Neter-kheperrê-Setep-en-imen, aimé de Ptah au beau visage, [2] le fils de Rê, maitre des apparitions, Méry-imen-Siamon, aimé d'Amon du lapis-lazuli véritable".

II. A gauche, Ptah et Sekhmet reçoivent l'offrande de Siamon et de Paoupaou:

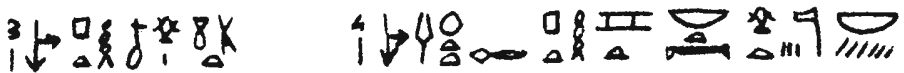

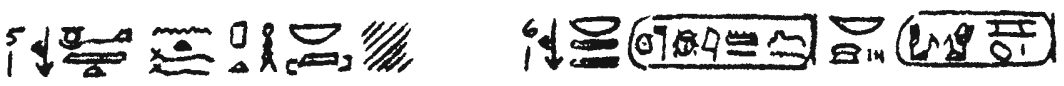

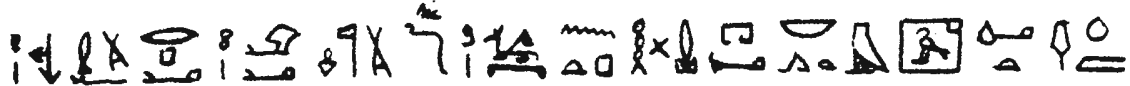




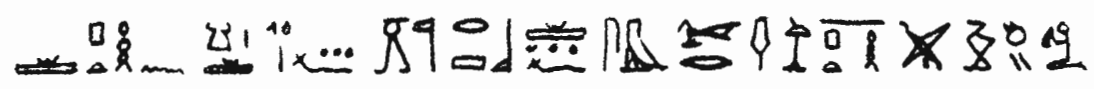

88 8)

"[3] Ptah au beau visage, l'aimable".

"[4] Sekhmet la Grande, l'aimée de Ptah, la mâttresse du ciel, supérieure de tous les dieux".

"[5] Offrir la Vérité à son père Ptah, seigneur de la vérité !"

"[6] Le maitre des Deux Pays, Neter-kheper-rê-Setep-en-imen, maître des apparitions, Méry-imen-Siamon".

"[7] L'aimé de Sa Majesté, noble, [8] gouverneur, père divin aimé du dieu, (a) [9] supérieur des secrets de Tepehet-djat, maître de ses allées et venues dans ... (b) [10] ..., celui qui conduit le dieu à sa nourriture, le sem, grand des chefs des artisans de Ptah, Paoupaou, juste de voix éternellement."

\section{(a) Cf. 164.}

(b) Litt.:"maître de ses allées et venues dans Hathor qui donne le pouvoir de Ptah à ses (= de lui) aliments (?).

III. A droite, Amon et Mout reçoivent l'offrande de Siamon et de Ankhef-en-mout :
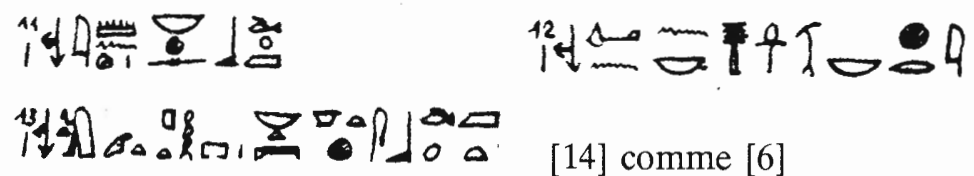

[14] comme [6]

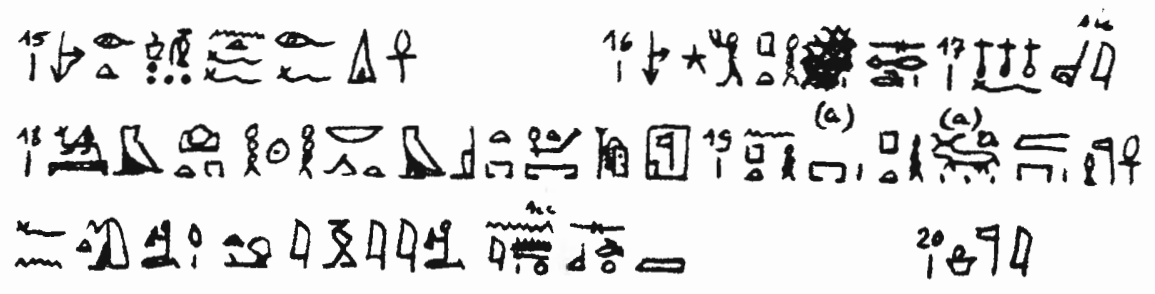

(a) Signes gravés sur d'autres signes presque entièrement effacés. 
"[11] Amon-Rê, seigneur du lapis-lazuli véritable".

"[12] Je te donne toute stabilité, vie et bien-être (venant) de moi".

"[13] Mout qui préside au temple de Ptah, maîtresse du ciel, régente du lapis-lazuli véritable".

"[14] (comme [6])".

"[15] Offrir l'encens et l'eau fraîche à son père, pour qu'il puisse être (un roi) gratifié de la vie (a)".

"[16] Adorer Ptah (sic !), ... (?) [17] sa beauté par [18] le supérieur des secrets dans l'Horizon d'éternité, le maitre de ses allées et venues dans la Place sacrée, hiérogrammate [19] du temple de Ptah, comptable des troupeaux dans le temple de Ptah, prophète, Ankhef-en-mout, fils du gouverneur Iouy, appartenant à Amon du lapis-lazuli véritable".

"[20] Le père divin (b)".

(a) Cf. LEFEBVRE, Grammaire, $\S 455$.

(b) Légende d'Ankhef-en-mout.

\section{Linteau de Copenhague}

Ny Carlsberg Glyptothèque A 746

Bibliographie:

MOGENSEN, La glyptothèque Ny Carlsberg, pl. 111 A;

KOEFED-PETERSEN, Recueil des inscriptions hiéroglyphiques de la Glyptothèque Ny Carlsberg, dans Bibliotheca Aegyptiaca, tome VI, 1936, p. 85 (texte);

PETRIE, The Palace of Apries and Memphis II, 1909, pl. XXIV, haut (dessin):

(même disposition que le linteau du Caire)

I. A gauche, Ptah et Hathor adorés par Siamon et Paoupaou:

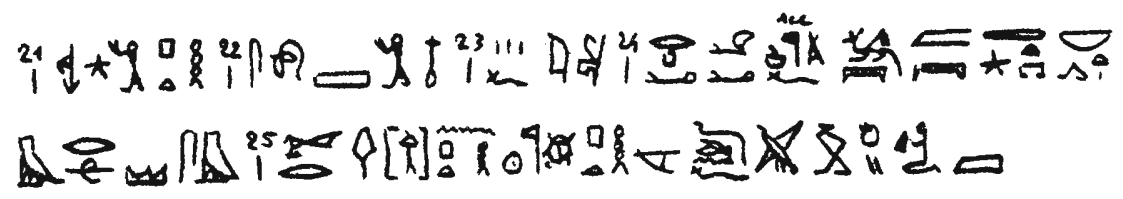


"[21] Adorer Ptah, [22] louer sa [23] beauté par [24] le noble, gouverneur, père divin aimé du dieu (a), supérieur des secrets dans le ciel, la terre et la douat, maitre de ses allées et venues dans Ro-setaou, sem, [25] grand des chefs des artisans de Ptah, Neter-kheper-rê-Méry-ptah, appelé Paoupaou, le juste".

(a) Je lis tf-ntr $m r$ (ntr) et suppose que le $f$ de $t f$ est à l'origine de l'incompréhensible $\underline{d}$ du bas de $16 \overline{3}, \mathbf{8}$.

II. A droite, Ptah et Sekhmet sont adorés par Siamon et Ankhef-en-mout, intitulé:

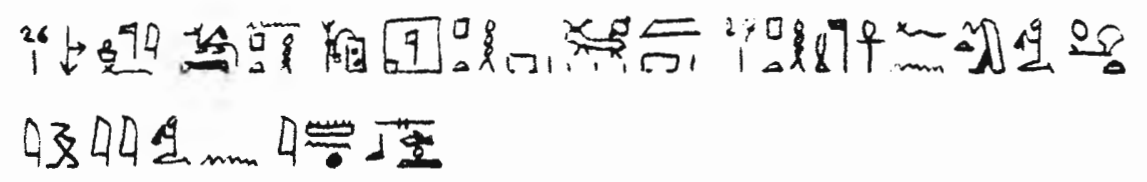

"[26] Le père divin (a), supérieur des secrets de Ptah, hiérogrammate du temple de Ptah, comptable des troupeaux dans le temple [27] de Ptah, prophète, Ankhef-enmout, fils du gouverneur Iouy, appartenant à Amon du lapis-lazuli véritable".

(a) ${ }^{2} t f-n t r$.

Quatre autres linteaux provenant du même monument montrent Ankhefen-mout porteur d'éventail agenouillé de part et d'autre des cartouches de Siamon (PETRIE, The Palace of Apries and Memphis II, 1909, pl. XXIV, et idem, Memphis I, p. 12, § 38 .

\section{STATUE D'HORSAISET DERRIERE UN NAOS}

\section{Caire 1212}

Bibliographie:

MARIETTE, Monuments divers, pl. 27, g (texte à l'envers, donc d'après estampage);

BORCHARDT, Statuen ..., IV, 1934, p. 110-112 (texte) et pl. 170 (photo); 
ma copie:

I. Au haut des faces gauche, antérieure et droite du naos:

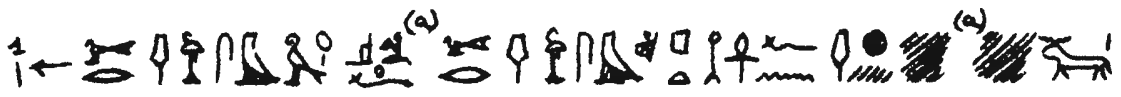

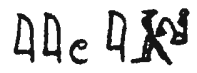

(a) Angle du naos.

"[1] Le grand des chefs des artisans, sem, Horsaïset; son fils, le grand des chefs des artisans, sem de Ptah, Ankhef-en-sekhmet, ... Ba-youia".

II. Face antérieure du naos:

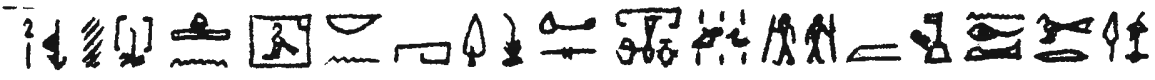

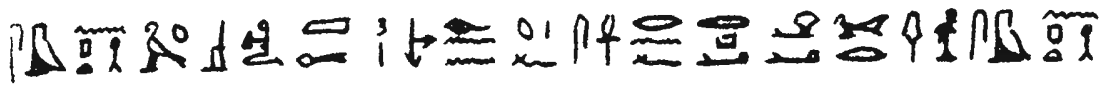 70 -}

"[2] Une offrande royale à Hathor, maîtresse du Sycomore du sud, pour qu'elle donne l'offrande (consistant en) pain, bière, boeufs et volatiles, ainsi qu'entrer dans le Kherneter et en sortir, à l'Osiris, grand des chefs des artisans, sem de Ptah, Horsaïset, juste de voix! [3] Fait par son fils qui fait vivre son nom, le noble, gouverneur, grand des chefs des artisans, sem de Ptah, Ankhef-en-sekhmet, dont la mère est la grande des concubines, Ba-Iay".

III. Face latérale gauche du naos:

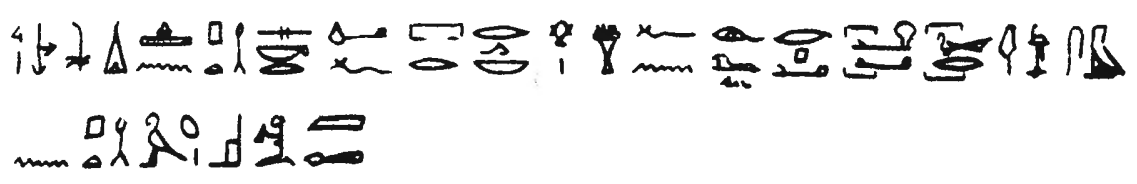

"[4] Offrande royale à Ptah-Sokar afin qu'il donne tout ce qui est déposé sur son autel à l'Osiris, noble, gouverneur, grand des chefs des artisans, sem 
de Ptah, Horsaïset, juste de voix !"

IV. Face latérale droite du naos:

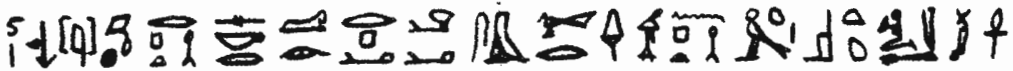

"[5] L'ịmakhou auprès de Ptah-Sokar, l'Osiris, noble, gouverneur, sem, grand des chefs des artisans de Ptah, Horsaïset, juste de voix, renouvelant sa vie !"

V. Pilier dorsal de la statue:

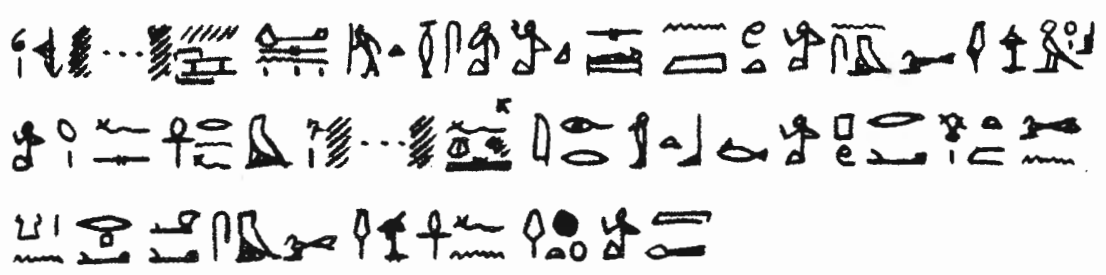

"[6] ..... aimé(e) ..., afin qu'ils donnent une vieillesse de faveur et une séprilture neuve au sem, grand des chefs artisans, Horsaïset. Son fils qui fait vivre son nom par (a) [7] ...... ce qui est à faire (?) - je (sic) déteste la partialité (b) -. Pour le ka du noble, gouverneur, sem, grand des chefs des artisans, Ankhef-en-sekhmet, juste de voix !"

(a) Ou: dans.

(b) bwt.ì pw rdit hr gs; litt.: c'est mon abomination que donner d'un côté.

\section{BLOC D'UNE OUABET D'APIS}

Mitrahineh (près de l'endroit où la route de Mitrahineh à la nécropole de Saqqara aborde le désert).

Bibliographie:

BRUGSCH, Thesaurus, p. 817 et 949 (deux fois dessin et texte):

PETRIE; Memphis I, p. 13 (description; Petrie annonce la publication du bloc pour l'année suivante, mais je n'ai pas trouvé la publication en question dans Memphis II et suivants); 
J. VERCOUTTER, Textes biographiques du Sérapéum de Memphis, p. 57 , h, plus note 2 . d'après Brugsch, p. 949:

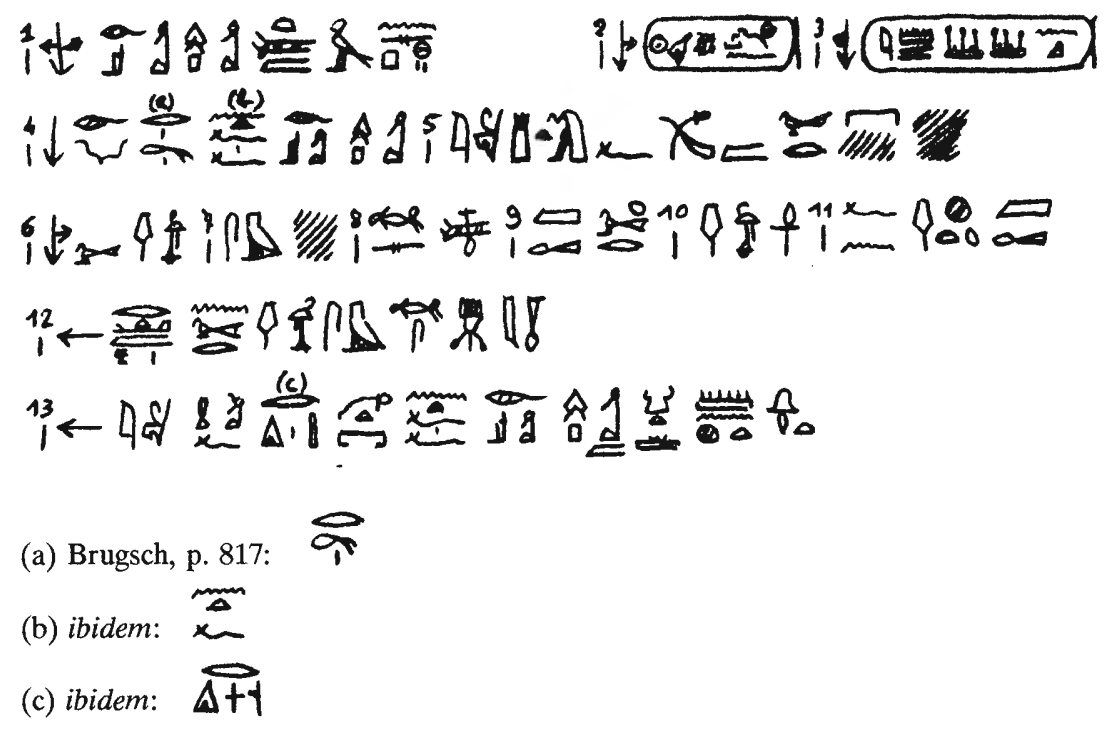

"[1] Osiris-Apis-Toum-Horus-de-Sepa".

"[2] Hedj-kheper-rê-Setep-en-rê [3] Chéchanq-Méry-Imen".

"[4] Accomplir l'Ouverture de la bouche pour son père Osiris-Apis [5] par le Pilier de sa mère, purificateur dans la Grande maison (a) ...".

"[6] Le grand des chefs des artisans, [7] sem, ..., [8] Chedsounefertoum, [9] juste de voix, fils du grand [10] des chefs des artisans, Ankh[11]ef-ensekhmet, juste de voix".

"[12] Au soin du grand des chefs des artisans, sem, Chedsounefertoum, juste de voix".

"[13] C'est Sa Majesté qui a fait construire en travail parfait un laboratoire d'embaumement pour son père Osiris-Apis".

(a) Cf. 124,4 (texte). 


\section{GROUPE DE CHEDSOUNEFERTOUM ET DE TACHEPENISE'T}

\section{Caire 741}

Bibliographie:

BORCHARDT, Statuen ..., III, 1930, p. 67-69 (texte);

DARESSY, Inscriptions inédites de la XXIIème dynastie, dans Rec. Trav., XVIII (1896) p. 46-48 (texte et traduction);

ma collation:

I. Côté gauche du siège:

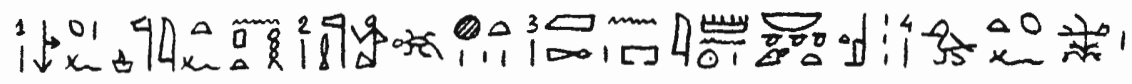

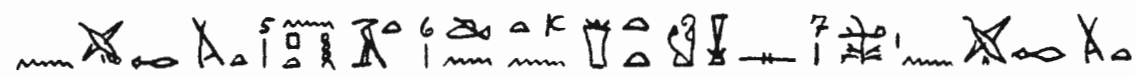

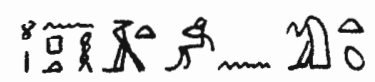

"[1] Son fils, le père divin de Ptah, [2] le prophète Acha-ikhout, [3] juste de voix, du domaine d'Amon-rê seigneur du Noun qui est devant les trônes". "[4]'Sa fille, chanteuse du choeur de l'Aimée-[5]de-Ptah, Ta[6]denit-enbastet".

"Sa soeur, [7] chanteuse du choeur de l'Aimée-[8]de-Ptah, Tacherenmout".

II. Côté droit du siège:

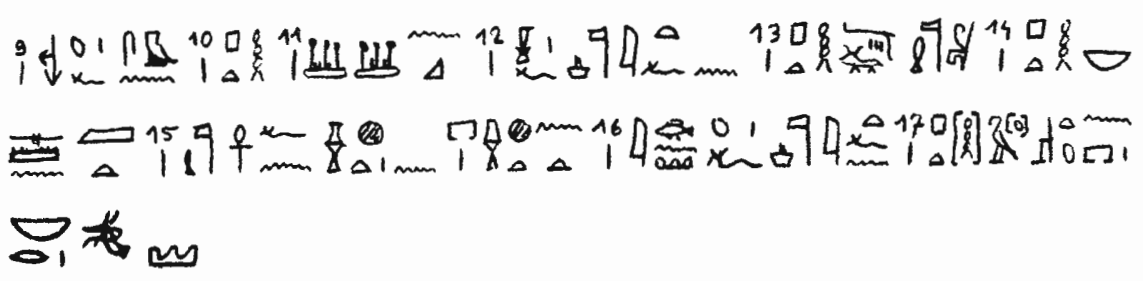

"[9] Son fils, sem de [10] Ptah, [11] Chechanq".

"[12] Son frère, père divin de [13] Ptah, directeur des troupeaux et prophète de [14] Ptah seigneur de Semen-maât, [15] prophète Ankhef-en-sekhmet, du domaine de Sekhmet-de-la-[16]Vallée".

"Son fils, père divin de [17] Ptah, Horsaïset, du domaine du Seigneur de Rosetaou". 
III. Au dos du groupe:

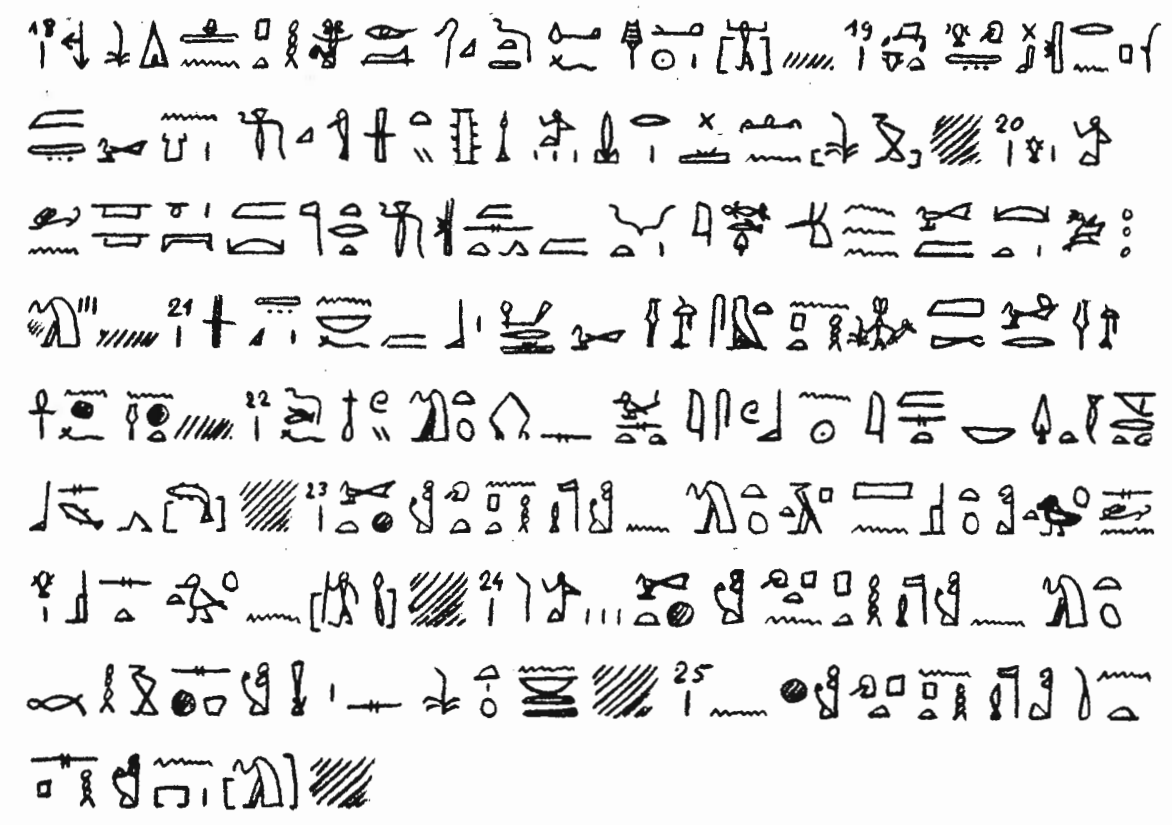

"[18] Offrande royale à Ptah-Tatenen-Osiris, régent d'éternité, pour qu'il accorde une longue durée de vie (en ?) [19] joie sur terre et un renouvellement de jeunesse dans le Grand Pays au ka de celui dont l'état (a) est florissant au milieu des Memphites, du fort (b) sans ... (?) [20] ... (?), de celui qui ouvre les portes du ciel dans le Tumulus divin et dont la course réussit (c) dans (?) le Sommet (d) de l'arbre iched, ... les hautes eaux (?) dans le tumulus de Tjamout, [21] "celui qui est dans la terre" (e) de son maître dans le lieu sacré, le grand des chefs des artisans, setem de Ptah, Chedsounefertoum, juste de voix, fils du grand des chefs des artisans Ankhef-en-sekhmet (juste de voix ?). [22] Il dit: Comme Mout est belle quand elle embrasse sa fille, et elle brille (?) en elle, la gracieuse et l'aimée, introduisant ... (?) ... (?) [23] la première grande du harem de Ptah et prophétesse de Mout, Tachepeniset - sa fille qui est à sa place - la fille du grand chef des Ma [24] et de (?) la première grande du harem de Ptah et prophétesse de Mout, Mehousekh; et son frère est le fils royal du maitre des Deux Pays ... [25] ... première concubine de Ptah et prophétesse, Tentsepeh, du domaine de Mout". 
(a) Wörterbuch, V, 16, 6-7.

(b) $d(n) r$ pour $\underline{t}(n) r$, Wört., $\mathrm{V}, 382$.

(c) litt.: "florissant de course".

(d) $w p t=$ corne, d'où le sens dérivé de "sommet" d'une montagne, ou d'un arbre (Wört. I, 298, 1, qui n'indique pas l'emploi de wpt pour un végétal).

(e) Wörterbuch, I, 75, 19, titre religieux.

\section{STELE DE CHEDSOUNEFERTOUM}

\section{Cleveland (U.S.A.)}

Bibliographie:

C. RANSOM WILLIAMS, Dans Bulletin of the Cleveland Museum of Art, oct.-nov. 1918, p. 67-69, d'après idem, The Egyptian Collection at Cleveland, Ohio, dans J.E.A., V (1918), p. 279.

(Je n'ai pas eu connaissance de cet article).

\section{STATUE DE CHEDSOUNEFERTOUM}

\section{lieu de conservation?}

(Les inscriptions ci-dessous m'ont été obligeamment communiquées par M. B. GRDSELOFF, en juin 1946):

I. Sur le vêtement:

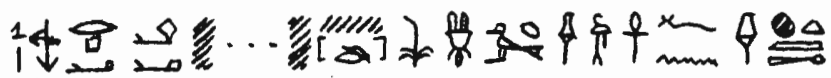

[1] Le noble, gouverneur, ...... (Che)dsounefertoum, fils du grand des chefs des artisans, Ankhef-en-sekhmet, juste de voix".

II. Faces latérale droite [2] - [6], postérieure [7] - [11], et gauche [12] [16] du siège: 


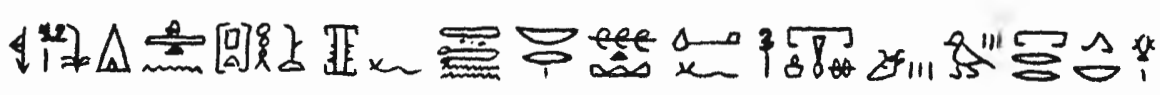

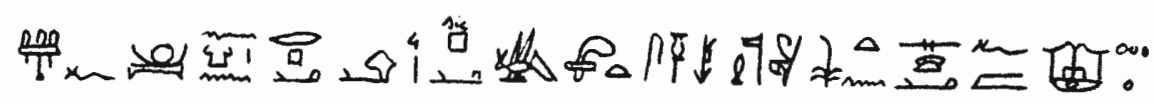

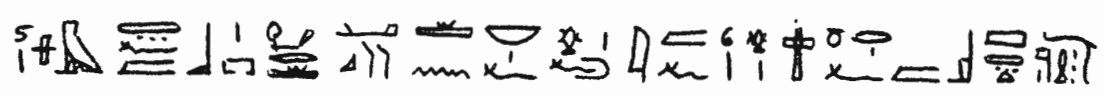

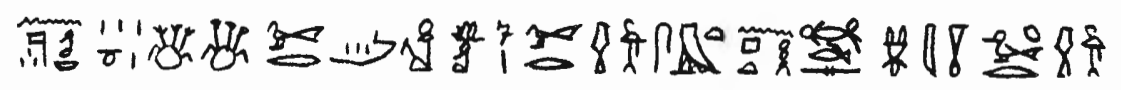

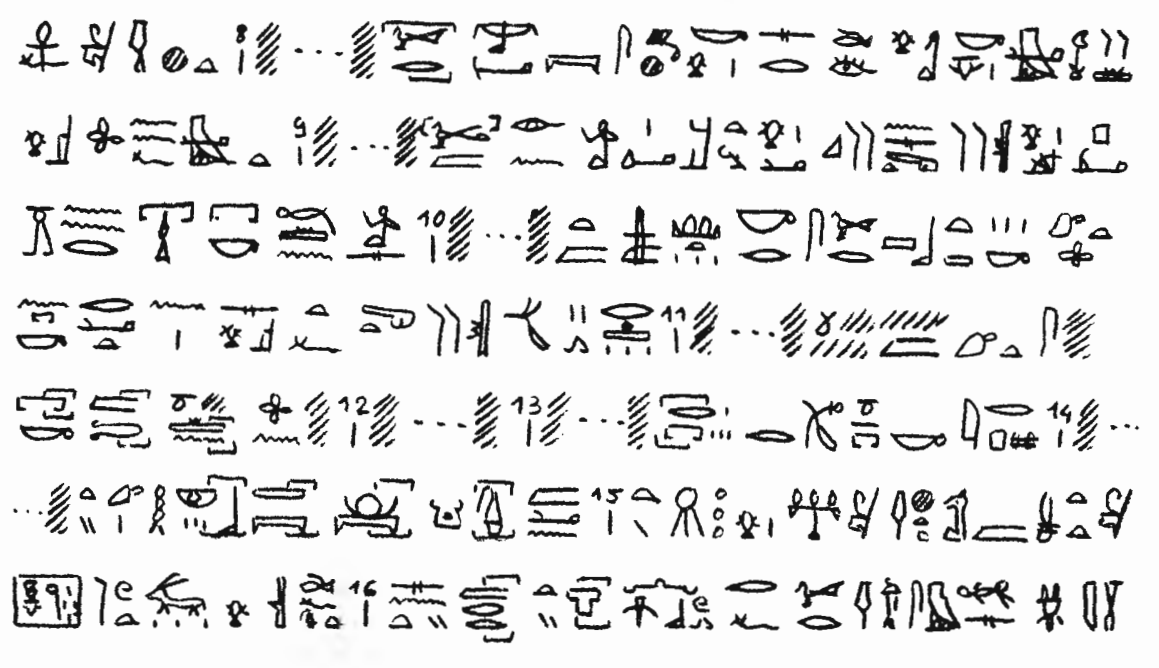
"[2] Une offrande royale à Ptah qui est au sud de son mur - Tatenen seigneur de Ro-setaou, pour qu'il donne [3] l'offrande funéraire consistant en pain, bière, boeufs, volatiles et tout ce qui parait sur son autel chaque jour (a) au ka du noble, gou [4]verneur, trésorier du roi, compagnon unique, prophète du roi quand il se montre à la fête Sed, [5] "celui qui est dans sa ( = du roi) terre" (b) dans le lieu sacré, celui qui agit droitement pour son maître en sortant de là (c) [6] et (?) en prenant conseil de lui dans la Place secrète [d], directeur des prophètes de tous les dieux de Haute et BasseEgypte, grand des voyants de Rê-Atoum, [7] grand des chefs des artisans, setem de Ptah, Chedsounefertoum, juste de voix, fils du grand des chefs des artisans Ankhef-en-sekhmet. [8] (Il dit :) (?) ..... Grand qui a fondu (= créé) le ciel ..... pour voir (?) Ton visage, le coeur étant de bonne humeur à la place (ou ?) il est avec (?) [9] ... dans ce que j'ai fait; j'ai posé le bras de balance (e) avec une précision vérifiée (?) (f) lorsque je prenais livraison (g) de ce qui est apporté à ton trésor; j'ai rempli ... [10] ... ce qui 
est dans tes greniers pour accroître tes offrandes alimentaires dans ton sanctuaire; j'ai mis cela à sa place exacte ... (?) vers tes offrandes [11] ..... dans ton ... en nombre (?) ... [12] ............. [13] ..... les temples pour purifier ta résidence, le vin [14] ..... les fêtes de la terre et du ciel chaque jour (a), allumer ... [15] ... (?) sur l'autel de Sekhmet, et aussi du temple "Qui réunit le(s) coeur(s) des dieux" (h); le petit bétail en entier (?) ... [16] ... (?) dehors (?) sans que le grand des chefs des artisans et sem Chedsounefertoum juste de voix se sépare de lui (?)".

(a) Jeu graphique analogue au $\odot \odot$ ptolémaïque?

(b) Cf. 167,21.

(c) Litt.: de lui $=$ du lieu sacré ? ou a-t-on affaire à l'expression pr ìm.f, celui qui est issu de lui $=$ son fils ?

(d) Place secrète = Cabinet du roi, Wört., IV, 551, 13.

(e) Est-ce le même mot que Wört., V, 401, 2: ه "Teil des Wagens (an Elfenbein)" ?

(f) $h r^{c} k 3 n$ smtr, litt.: en précision de vérification ?

(g) Litt.: en recevant.

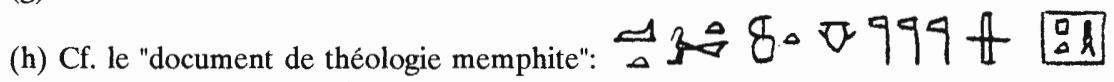
"La Grande place (= le "saint des saints") qui réunit le(s) coeur(s) des dieux dans le temple de Ptah" (SETHE, Dramatische Texte, p. 70).

\section{STELE AVEC UNE FIGURE DE PTAH}

\section{Berlin 8169}

Bibliographie:

Ägyptische Inschriften ..., II, p. 232-234 (texte);

BRUGSCH, Thesaurus, IV, 1884, p. 811-813 (texte et dessin); d'après Äg. Inschr.:

(deux personnages de chaque côté des jambes de Ptah, celui de gauche vêtu de la peau de félin)

I. Sur le personnage de gauche: 


\section{1}

"[1] ......ef-en-sekhmet, juste de voix".

II. Sur le personnage de droite :

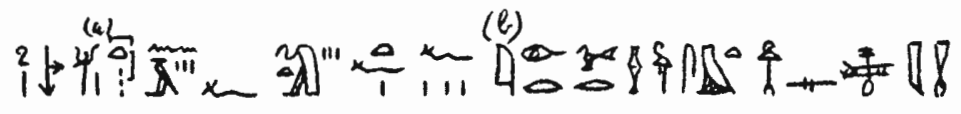

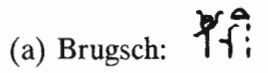

(b) idem: 40 m⿻上丨

"[2] ... les fonctions de ses mères et pères qu'accomplit le grand des chefs des artisans et setem Chedsounefertoum, juste de voix".

III. Devant le personnage de droite, en hiéroglyphes très grossièrement taillés:

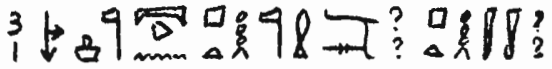

"[3] Le père divin, supérieur du territoire cultivé (a) de Ptah, prophète, Nesptah (?), juste de voix (?)".

(a) Wörterbuch, I, 409, 4.

(Le même Nesptah est nommé sur la face latérale gauche du monument, tandis que les faces latérale droite et postérieure portent une généalogie de prophètes du temple d'Apis et prophètes de Baal de Memphis).

\section{STELE DE NESPTAH}

Bibliographie:

MARIETTE, Sérapéum, 1852, pl. 25,2 (photo): 


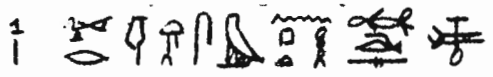

"[1] Le grand des chefs des artisans, sem de Ptah, Chedsounefertoum".

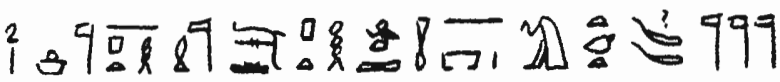

"[2] (Son descendant) (a), père divin de Ptah et prophète, Nesptah, juste de voix, du domaine de Mout qui est à la tête des Cornes des Dieux".

(a) Litt. : son "fils".

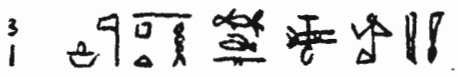

(Le fils de Nesptah) "[3] Le père divin de Ptah, Chedsounefertoum, juste de voix".

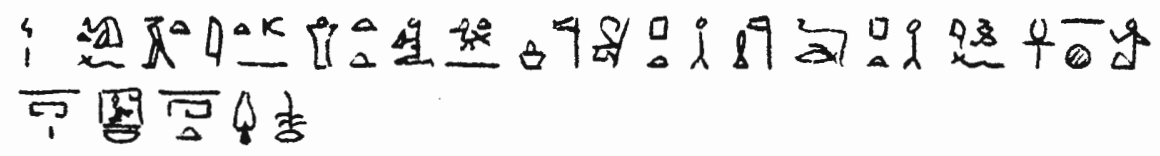

"[4] Sa (a) mère, Tadenitenbastet, fille du père divin de Ptah et prophète Djedptahiouefankh, du domaine d'Hathor, maîtresse du Sycomore du sud".

(a) $=$ du père divin Chedsounefertoum.

(Ce monument est contemporain de la mort d'un Apis en l'an 28 de Chechanq III).

\section{FRAGMENTS DE COLONNE}

Caire ?

Bibliographie:

DARESSY, Notes et remarques, dans Rec. Trav., XXII (1900), p. 143, $n^{\circ}$ CLXXVII: 


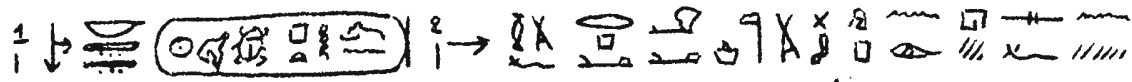

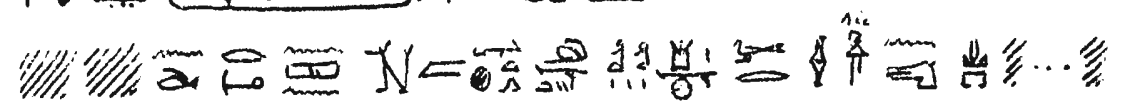

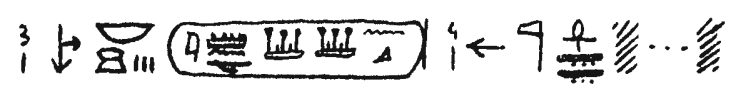

"[1] Le maitre des Deux Pays, Hedj-kheper-rê-Setep-en-ptah. [2] Son favorisé et aimé, le noble, gouverneur, père divin aimé du dieu, premier renouvelant (?) ...... de repousser la fureur derrière les Pehty d'Héliopolis, le grand des chefs des artisans (?) de Aat-Dja ... [3] Le maitre des apparitions, Chechanq-Méry-imen. [4] ... dieu d'Ankh-taoui ... (ou: Ankh-taoui est divin)".

\section{2a. TEMPLE DE BUBASTIS}

\section{Berlin 10837}

Bibliographie:

Ägyptische Inschriften, II, p. 533 (description et texte);

NAVILLE, The Festival Hall of Osorkon II, 1892, pl. 11;

(fragment de fête-Sed; en face du roi, six prêtres sur deux rangs; le second de la deuxième rangée a pour légende:)

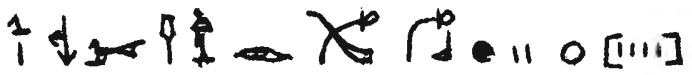

"[1] Le grand des chefs des artisans faisant la purification (et disant:) Pur, pur, (quatre) fois !"

Autre fragment:

Bibliographie:

NAVILLE, op. cit., pl. 12,7 (dessin):

(le troisième d'une procession de prêtres est le:)

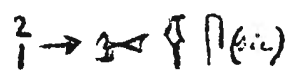

"[2] Grand des chefs des artisans". 


\section{STATUE DE CHECHANQ}

Budapest (d'après Brugsch) (provenance: Sérapéum)

Bibliographie:

BRUGSCH, Thesaurus, 1450 (texte) (avec une lacune au milieu de l'inscription):

BUDGE, Kings, II, 48, bas, sans référence (texte):

DARESSY, Notes sur les XXIIe, XXIIIe, et XXIVe dynasties, dans Rec. Trav., XXXV (1913), p. 142-143 (texte) (d'après Budge, "avec quelques corrections évidentes");

d'après Brugsch:

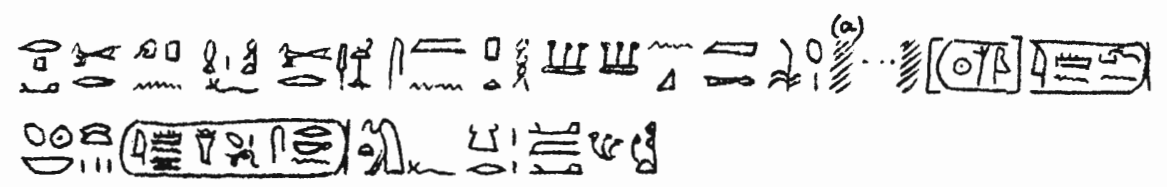

(a) Budge: $\sum_{m=1}^{2}$

"Le grand premier noble de Sa Majesté, grand des chefs des artisans, sem de Ptah, Chechanq, juste de voix, grand fils royal du maitre des Deux Pays Ouser-maât-rê-Setep-en-Imen, du fils de Rê et maître des apparitions Méryimen-Sa-bastet-Osorkon; sa mère est Karâmâ".

\section{SCARABEE DE CHECHANQ}

Bibliographie:

NEWBERRY, Scarabs, pl. XL, 8 (dessin):

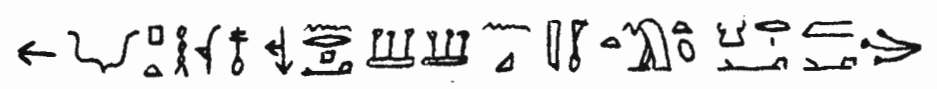

"Que Ptah ouvre une année heureuse au noble Chechanq, juste de voix ! (Sa) mère Karâmâ". 


\section{BLOC DE MERENPTAH}

\section{Louvre, Sérapéum S.N. 82}

Ma copie (ce monument est peut-être signalé dans GAUTHIER, Rois, III, 353, note 2, ou ibidem, 354, $\mathrm{n}^{\circ}$ XII; c'est un bloc à surface plane non polie avec deux colonnes et une ligne de hiéroglyphes gravés en creux; la ligne est sous les colonnes):

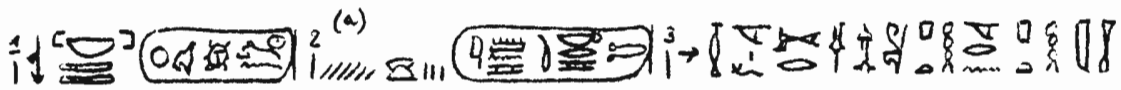

"[1] Le maître des Deux Pays, Hedj-kheper-rê-Setep-en-rê, [2] ... des apparitions Méry-imen-Takelot. [3] Son favorisé et aimé, le grand des chefs des artisans de Ptah, Merenptah, juste de voix".

(a) $\square$ était gravé sur un autre bloc.

\section{BLOC DE TAKELOT}

Bibliographie:

DARESSY, Fragments memphites, dans Annales du Service des Antiquités, 20 (1920), p. 169-170, n 5:

(Sekhmet adorée par un roi et un chef des Ma)

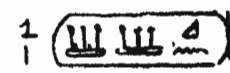

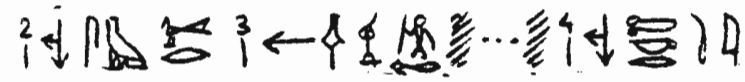

"[1] Chechanq".

"[2] Le sem, grand [3] des chefs des artisans, grand chef ... [4] Takelot".

(a) La colonne [4] est placée sous [2] et la ligne [3] à côté des premiers signes de [2]. 


\section{STELE DE PADIISET, PEFTJAOUAOUIBASTET ET TAKELOT}

\section{Louvre, Sérapéum 3749}

Bibliographie:

MARIETTE, Sérapéum, 1857, pl. 24 (photo);

CHASSINAT, Textes provenant du Sérapéum de Memphis, dans Rec. Trav., XXII (1900), p. 9-10, n०38 (texte);

M. MALININE, G. POSENER, J. VERCOUTTER, Catalogue des Stèles du Sérapéum de Memphis, I, n² 21, p. 19-20 et pl. VII;

ma collation:

I. Sous le cintre:

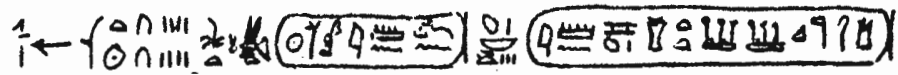

"[1] L'an 28 du roi de Haute et Basse-Egypte Ouser-mâ̂t-rê-Setep-en-imen, fils de Rê et maître des apparitions Méry-imen-Sa-bastet-Chechanq-Heqanetery-iounou".

II. Premier registre (Apis adoré par un chef des Ma et deux prêtres vêtus de peaux de félin; le second prêtre est coiffé de la tresse de cheveux tombant sur l'épaule):

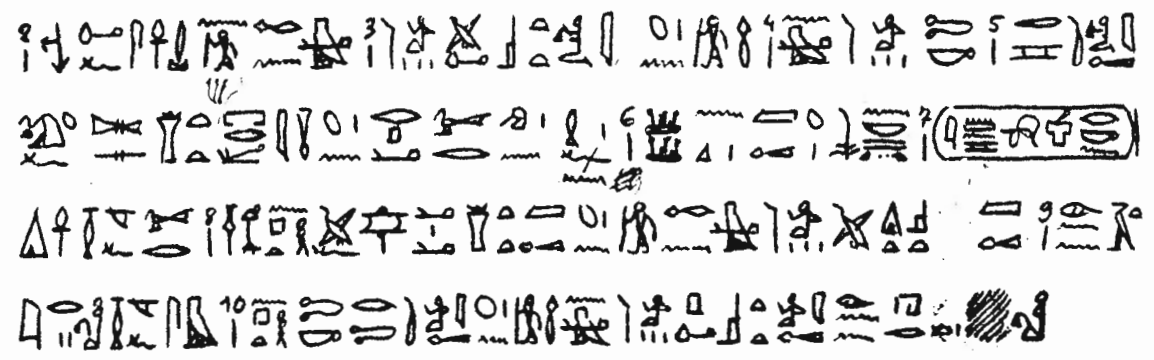

"[2] Qu'il donne santé, vie et force au grand chef des Ma [3] Padiiset, juste de voix, fils du grand chef [4] des Ma Takelot, [5] juste de voix - sa mère est Tesbastetperet, juste de voix - fils du grand premier noble de Sa Majesté, [6] Chechanq, juste de voix, fils de roi du maitre des Deux Pays [7] Méry-imenOsorkon, doué de vie".

"Son favorisé et aimé, le grand [8] des chefs des artisans de Ptah, Peftjaouaouibastet, juste de voix, fils du grand chef des Ma Padiiset, juste de voix, [9] et issu de Tairy". 
"Son favorisé et aimé, le sem [10] de Ptah Takelot, juste de voix, fils du grand chef des Ma Padiiset, juste de voix, et issu de Heret-ib... (a)".

(a) RANKE, Personennamen, 230, 23.

III. Deuxième registre:

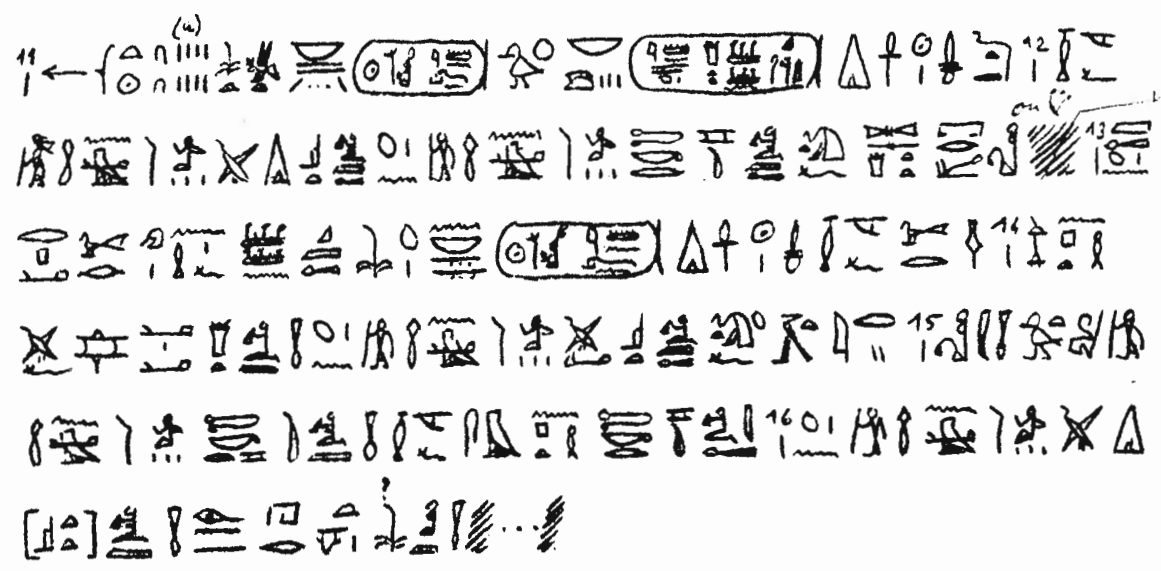

(a) et non lil, comme dans la publication de Chassinat.

"[11] L'an 28 du roi de Haute et Basse-Egypte, maître des Deux Pays Ousermaât-rê-Setep-en-imen, fils de Rê et maître des apparitions Méry-imen-Sabastet-Chechanq-Heqa-netery-iounou, doué de vie comme Rê éternellement".

"[2] Son favorisé et aimé, le grand chef des Ma Padiiset, juste de voix, fils du grand chef des Ma Takelot - sa mère est Tesbastetperet ... [13] juste de voix, fils du grand premier noble de Sa Majesté Chechanq juste de voix, fils de roi du maître des Deux Pays Ouser-maât-rê-Setep-en-imen doué de vie comme Rê".

"Son favorisé et aimé, le grand des chefs [14] des artisans de Ptah Peftjaouaouibastet, juste de voix, fils du grand chef des Ma Padiiset, juste de voix - sa mère est Tairy [15] juste de voix, fille du grand chef des Ma Takelot, juste de voix". 
"Son favorisé et aimé, le sem de Ptah Takelot, juste de voix, [16] fils du grand chef des Ma Padiiset, juste de voix et issu de Heret-ib... (a) juste de voix ...".

(a) RANKE, Personennamen, 230, 23.

\section{STELE DE PADIISET, HORSAISET ET}

\section{Louvre, Sérapéum 3697}

Bibliographie:

MARIETTE, Sérapéum, 1857, pl. 26 (photo);

CHASSINAT, Textes provenant du Sérapéum de Memphis, dans Rec. Trav., XXII (1900), p. 10-11, n 39 (texte);

M. MALININE, G. POSENER, J. VERCOUTTER, Catalogue des stèles du Sérapéum de Memphis, I, n²2, p. 21-22, pl. VIII;

ma collation:

I. Premier registre (Apis adoré par trois personnage,s un chef des Ma, un prêtre et un troisième personnage dont il ne reste que les pieds; le reste de ce personnage, y compris sa légende, a disparu dans une cassure):

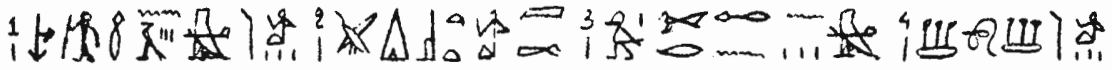

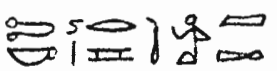

"[1] Le grand chef des Ma [2] Padiiset, juste de voix, [3] fils du grand chef des Me[4]chouech Take[5]lot, juste de voix".

"[16] Le sem de Ptah Horsa[7]iset ...".

II. Deuxième registre:

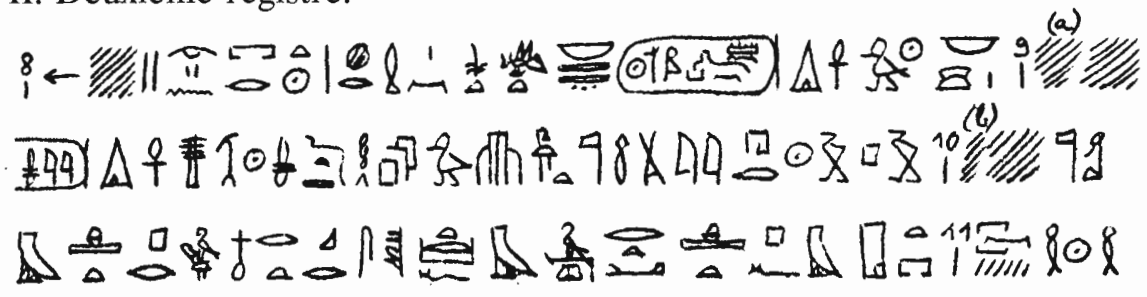




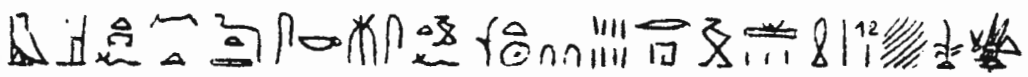

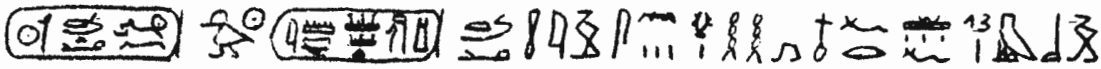

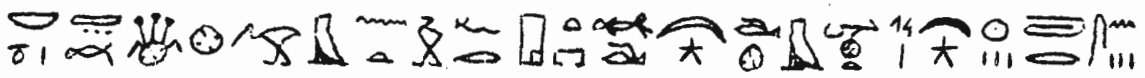

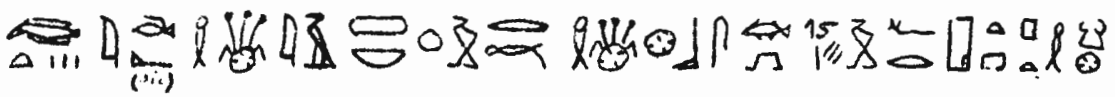

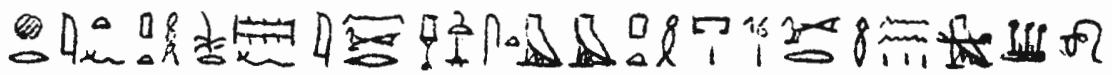

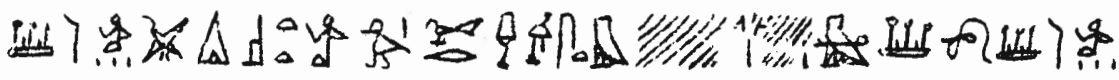

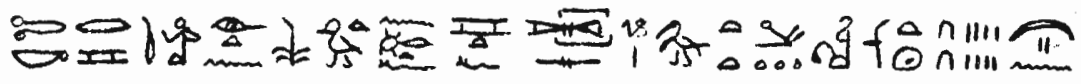

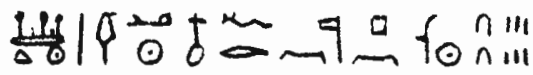

(a) Restituer: [assinat.
(b) Chassinat: [P ; actuellement, il n'y a plus que des traces illisibles.

"[8] (L'an) 2, deuxième mois de Peret, ler jour sous la Majesté du roi de Haute et Basse-Egypte, maître des Deux Pays Ouser-maât-rê-Setep-en-men (sic) doué de vie, le fils de Rê, maître de l'apparition (sic) [9] (Méry-imenPa)my doué de vie, stabilité et force comme Rê éternellement et aimé d'Apis qui est à la tête de l'Occident, le dieu grand; ce jour [10] (on a halé) le dieu en paix vers le bel Occident; on l'a enseveli dans le kher-neter; on l'a fait reposer dans la demeure [11] d'éternité à sa place de toujours. Il était né en l'an 28 au temps de la Majesté [12] (du) roi de Haute et BasseEgypte Ouser-maât-rê-Setep-en-rê, le fils de Rê Méry-imen-Chechanq-Heqanetery-iounou, juste de voix. On avait cherché sa perfection [13] en tout lieu de Basse-Egypte; il avait été trouvé à Het-ched-ibed après [14] trois mois (pendant lesquels) on avait parcouru les marécages (a) du delta et toutes les "îles" (b) ... Basse-Egypte. Il avait été introduit [15] à Memphis auprès de son père Ptah qui est au sud de son mur par le grand des chefs des artisans et setem du temple de Ptah, [16] le grand chef des Mechouech Padiiset, fils 
du grand des chefs des artisans et setem, (grand chef) [17] des Mechouech Takelot et de la fille charnelle du roi, celle qu'il aime, Tes[18]bastetperet, en l'an 28, deuxième mois d'Akhet, ler jour. La belle durée de vie de ce dieu: 26 ans".

(a) $\underline{h} 3 w t, \mathrm{~Wb} . \mathrm{I}, 155,5$.

(b) $\mathrm{Wb} \mathrm{I}, 47$, NB pour la graphie.

\section{STELE DE PADIISET ET HORSAISET}

\section{Louvre, Sérapéum 3736}

Bibliographie:

MARIETTE, Sérapéum 1857, pl. 27 (photo);

M. MALININE, G. POSENER, J. VERCOUTTER, Catalogue des stèles du Sérapéum de Memphis, I, n 23, p. 22-23, pl. VIII;

ma copie:

I. Premier registre: Apis adoré par un chef des Ma et un prêtre portant la peau de félin et coiffé de la tresse de cheveux tombant sur l'épaule.

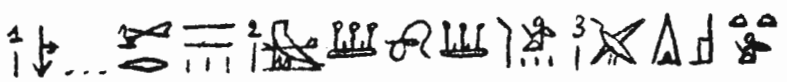

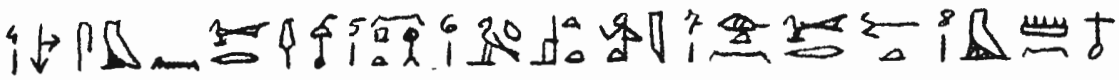

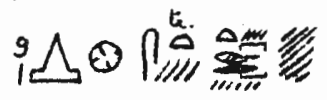

"[1] ... grand chef des [2] Mechouech [3] Padiiset.

"[4] Le sem de (sic) et grand des chefs des artisans [5] de Ptah [6] Horsaïset, juste, [7] né de la grande du harem [8] dans Memphis, [9] .... ".

II. Deuxième registre:

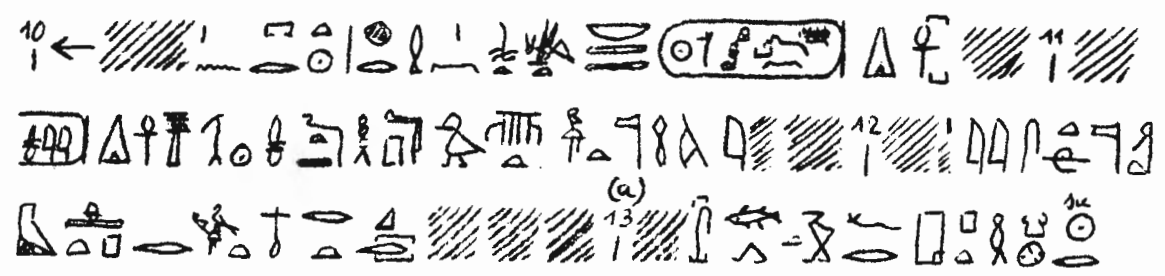




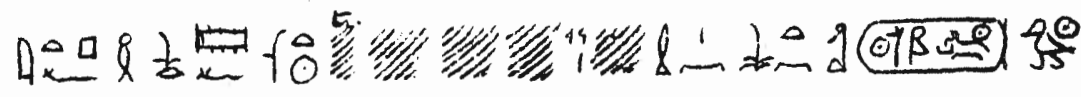

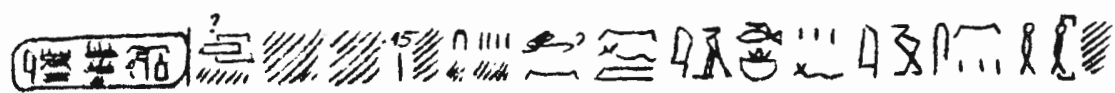

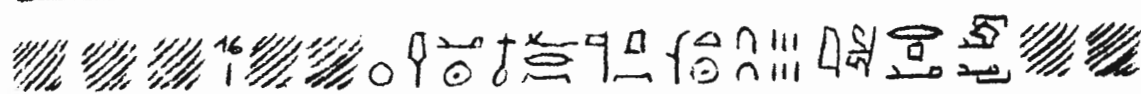

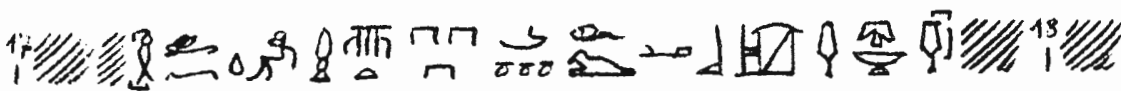

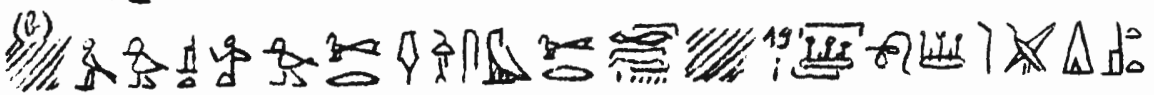

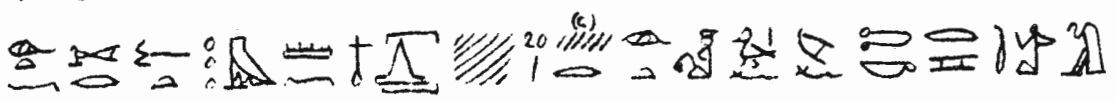

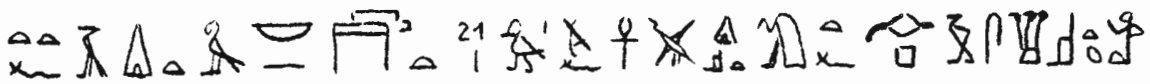

(d)

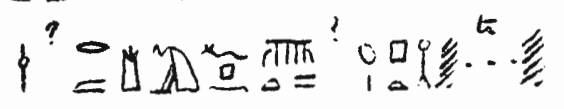

(a) La ligne 13 a été gravée par la main malhabile d'un graveur mal instruit !

(b) On lit encore la queue d'un oiseau surmontée d'un trait vertical.

(c) $\varnothing$ ou or.

(d) Deux cadrats en blanc; le reste de l'inscription en caractères plus petits.

"[10] ..... (a) de Peret, premier jour sous la Majesté du roi de Haute et Basse-Egypte et mâtre des Deux Pays Ouser-maât-rê-Setep-en-men doué de vie ... [11] ... (b) ...my doué de vie, stabilité et force comme Rê éternellement et aimé d'Apis qui est à la tête de l'Occident, le dieu grand; ... [12] ... (c) on a halé le dieu en paix vers le bel Occident, (on l'a) enseveli ... (d). [13] Il avait été introduit à Memphis auprès de son père $P(t)$ ah qui est au sud de son mur en l'an .....[14]... (e) Majesté du roi Ouser-maât-rêSetep-en-rê, fils de Rê Méry-imen-Chechanq-Heqa-netery-en-iounou, juste (?) ..... [15] ... (f) 28 (?). Il était dans son "pâturage" quand on cherchait ..... [16] ... (g). La belle durée de vie de ce dieu: 26 ans. Par (sic) (h) le noble, gouverneur, ... [17] ... le jeune orné à la tête des Perou Manou, celui qui fait (?) l'image divine (?), le purificateur de la Grande Maison (i), chef de toute la garde-robe, chef ... [18] ... Horsaïset, fils du grand des chefs des artisans, 
sem, grand chef des (Me)[19]chouech Padiiset et né de la grande des concubines dans Memphis ... [20] ...eriret. Son fils qu'il aime, Takelot, dont la mère est Tadithorneben... (?). [21] Son fils qu'il aime, Ankhpadiiset, dont la mère est Kapoueshaiset ... (?) en présence (ou: à côté) du Pilier de sa mère Penkhent...(?)... sa-ptah ... ".

(a) Restituer: (L'an 2, deuxième mois) d'après $\mathbf{1 7 8 , 8}$.
(b) " $\quad$ : (le fils de Rê, maître des apparitions [11] Méry-imen-pa.
(c) $\quad:($ ce [12] jour $)$.
(d) $\quad$ : (dans le kher-neter).
(e) $\quad$ : (28 au temps [14] $)$ de la $)$.
(f) " : (Il était né en [15] l'an).
(g) $\quad$ : (sa perfection en tout lieu de [16] Basse-Egypte).
(h) Cette stèle a donc été faite sur l'ordre de Horsaïset.
(i) Cf. 124,4 (texte).

\section{MONUMENT FAMILIAL}

Caire, sans numéro apparent (Salle 25, paroi ouest, sur le sol, contre le montant de la porte conduisant à la salle 24).

Bibliographie:

LEGRAIN, Une branche de Sheshonqides en décadence, dans Rec. Trav., XXIX (1907), p. 174:

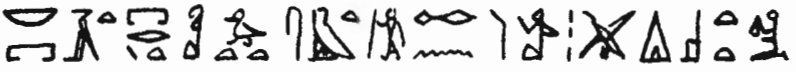

"Celle qui possède une maison, Taperet, fille du setem, grand chef des Ma, Padiiset". 
181. STATUETTES FUNERAIRES DE PEFTJAOUAOUIBASTET

Caire

Bibliographie:

DARESSY, Notes et remarques, dans Rec. Trav., XIV (1893), p. 181, $n^{\circ} 76$ :

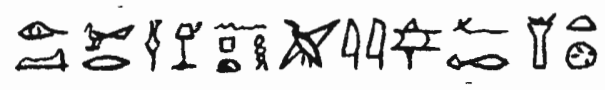

"L'Osiris, grand des chefs des artisans de Ptah, Peftjaouaouibastet".

\section{Berlin 11637}

Bibliographie:

Ausführliches Verzeichnis, 1894, p. 187 (obligeamment communiqué par M. G. Posener) (description seulement). (Pas publié dans Ägypt. Inschr.).

\section{PIERRE PLATE D'ANKHEF-EN-SEKHMET}

\section{Berlin 23673}

Bibliographie:

BORCHARDT, Die Mittel zur zeitlichen Festlegung von Punkten der ägyptischen Geschichte und ihre Anwendung, Caire 1935, p. 96-112 et pl. 2a (photo).

(Cette pierre énumère 60 générations d'une famille, en remontant de fils en père; à part les générations de "grands des chefs des artisans" et de sem, je ne donne ici que l'auteur du monument; ceux de ses ancêtres qui ne sont pas grands des chefs des artisans ou sem sont pères divins, supérieurs des secrets de Ptah, prophètes, grands prêtres d'Héliopolis, etc.).

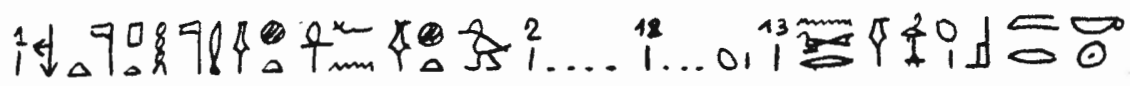

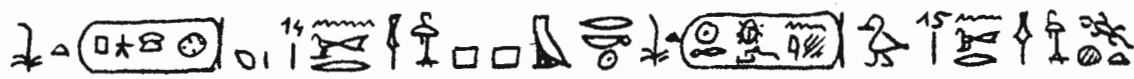

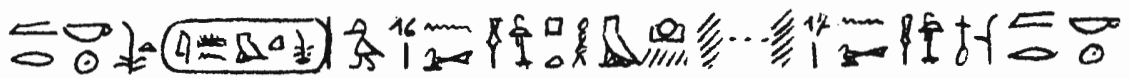




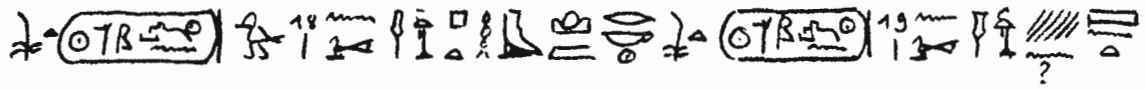

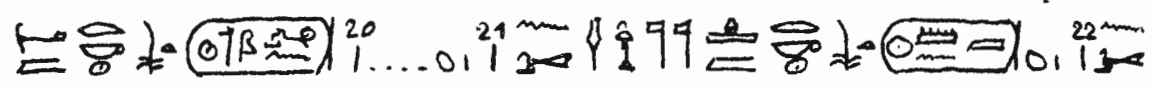

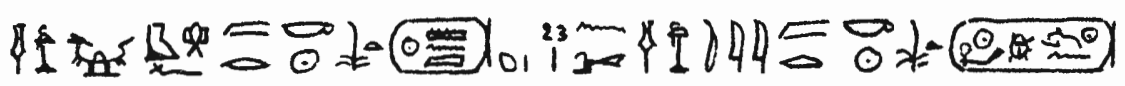

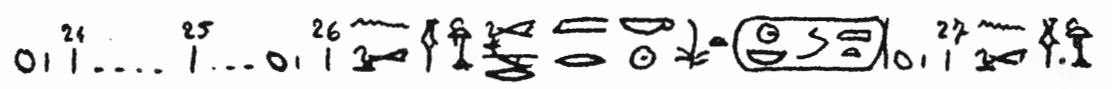

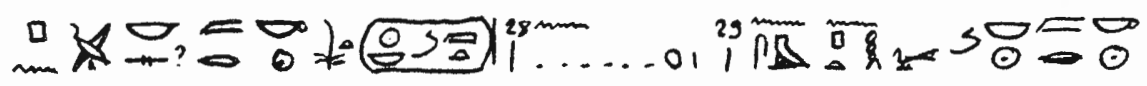

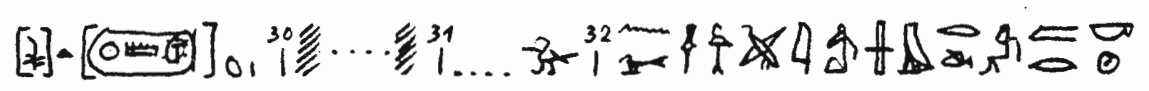

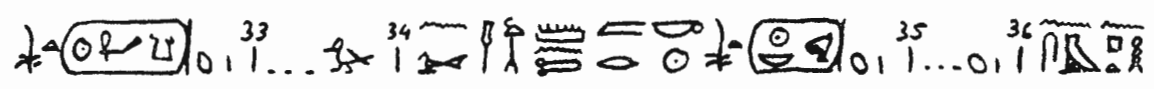

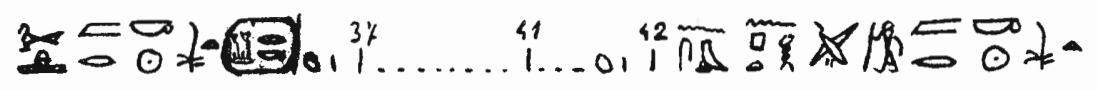

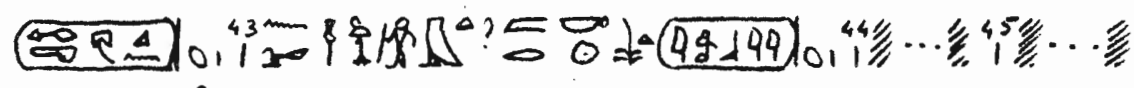

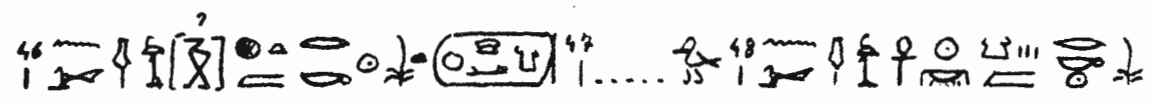
(OBU)

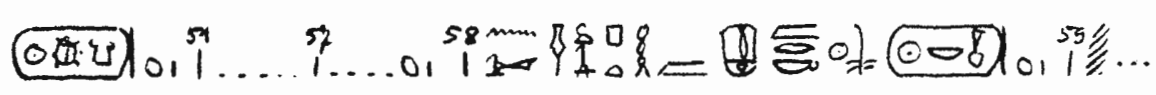
…玹 $90 . \cdots$

En face de ces 60 colonnes:

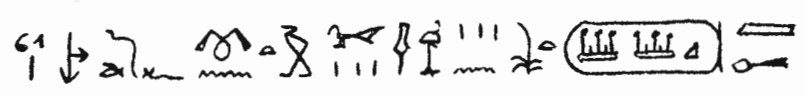

"[1] Le père divin de Ptah, prophète de Sekhmet, Ankhef-en-sekhmet, fils $d u[2]$... ... [12] ... fils $d u$ [13] grand des chefs des artisans Saiset, au temps du roi Pa-seba-kha-(en-)niout, fils du [14] grand des chefs des artisans Papa, au temps du roi Aa-kheper-rê-Setep-en-i(men), fils du [15] grand des chefs des artisans Achakhet, au temps du roi Imen-em-ipet-resit, fils du [16] grand des chefs des artisans Ptahemakhet, (au temps de ..., fils) [17] du grand des chefs des artisans Neferrenpet, au temps du roi Ouser-maât-rê-Setep-en-rê, 
fils [18] du grand des chefs des artisans Ptahemakhet, au temps du roi Ouser-mâat-rê-Setep-en-rê, (fils) [19] du grand des chefs des artisans ...enchenet (?), au temps du roi Ouser-maât-rê-Setep-en-rê, (fils) [20] du ....., fils [21] du grand des chefs des artisans Neterouy-hetep, au temps du roi Men-maât-rê, fils [22] du grand des chefs des artisans Sekeremsaef, au temps du roi Men-maât-rê, fils [23] du grand des chefs des artisans Ty, au temps du roi Djéser-kheper-rê-Setep-en-rê, fils [24] ..... [25] ... fils [26] du grand des chefs des artisans Méryour (?), au temps du roi Neb-maât-rê, fils [27] du grand des chefs des artisans Penpanebes (?), au temps du roi Neb-maât-rê, (fils) [28] du sem de Ptah et grand des voyants Nebrê, au temps du roi (Men-kheper-rê), fils [30] .... (?) [31] ... fils [32] du grand des chefs des artisans Paï-imy-(khe)red (?), au temps du roi Djeser-ka-rê, fils [33] ..., fils [34] du grand des chefs des artisans Mentou, au temps du roi Neb-peht(y)-rê,, fils [35] ..., fils [36] du sem de Ptah Ourhetep, au temps du roi Charek (?), fils [37] ... ... [41] ..., fils [42] du sem de Ptah Paser, au temps du roi Aaken(en), fils [43] du grand des chefs des artisans Sermet (?), au temps du roi Iby, fils [44] .....(?) [45] ..... (?) [46] du grand des chefs des artisans Oukhet (?), au temps du roi Kha-ka-rê, (fils) [47] ..., fils [48] du grand des chefs des artisans Ankhnoubkaourê, au temps du roi Kha-ka-rê, fils [49] du grand des chefs des artisans Ankhkhakarê, au temps du roi Noub-kaou-rê, fils [50] du grand des chefs des artisans Ankhsehetepibrê, au temps du roi Kheper-ka-rê, fils [51] ... ... [57] ..., fils [58] du grand des chefs des artisans Ptahemheb, au temps du roi Neb-kherou-rê, fils [59] ..... (?) [60] ..... (?)". "[61] Il dit: Les grands des chefs des artisans ont été rassemblés pour le roi Chechanq, juste de voix !"

\section{STELE D'ACHAOUT-AKH}

\section{Louvre, Sérapéum 3429}

Bibliographie:

MARIETTE, Sérapéum, 1857, pl. 34 (photo):

CHASSINAT, Textes provenant du Sérapéum de Memphis, dans Rec. Trav., XXII (1900), p. 16-17, n 54 (texte);

M. MALININE, G. POSENER, J. VERCOUTTER, Catalogue des stèles du Sérapéum de Memphis, I, n 52, p. 48-49, pl. XVI;

$\mathrm{PM} \mathrm{III}^{2} / 2$, p. 805;

ma copie: 
(Apis adoré par un personnage agenouillé, avec l'épaule dessinée comme si elle était déformée - imitation de l'Ancien Empire !)

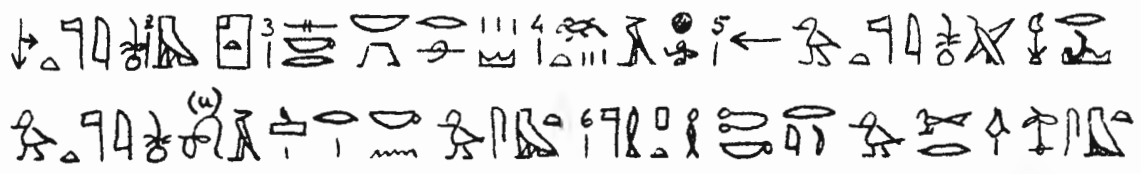

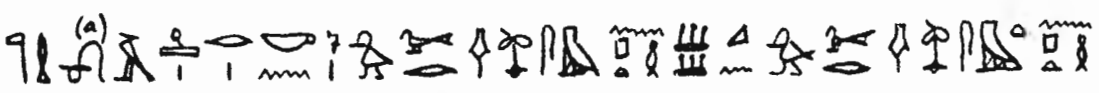

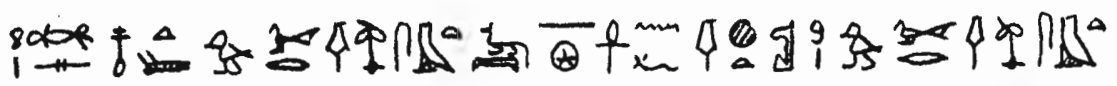

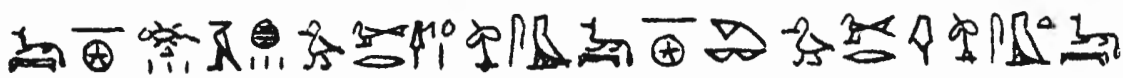

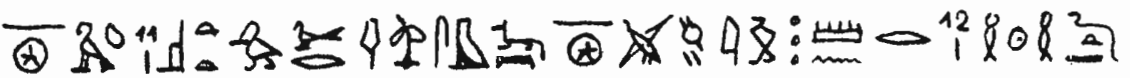

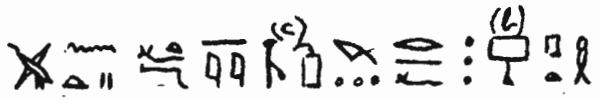

(a) A l'envers sur l'original.

(b) $\mathrm{Ou} \stackrel{\mathrm{r}}{\text { ? }}$

(c) fo ?

"[1] Le père divin, sem [2] dans le temple [3] de Sokar, maître de ses allées et venues (dans) Ro-setaou, [4] Achaout-akh, [5] fils du père divin, sem, Pakhar, fils du père divin, khe-nesou, Osorkon, fils du setem, [6] prophète de Ptah Takelot, fils du grand des chefs des artisans, setem, prophète, Osorkon, [7] fils du grand des chefs des artisans, sem de Ptah, Chechaqen (sic), fils du grand des chefs des artisans, setem de Ptah [8] Chedsounefertoum, fils du grand des chefs des artisans, setem, supérieur des secrets du ciel, de la terre et de la douat, Ankh-en-ef-Sekhmet (sic), [9] fils du grand des chefs des artisans, setem, supérieur des secrets du ciel, de la terre et de la douat, Achaout-akhou, fils du grand des chefs [10] des artisans, sem, supérieur des secrets du ciel, de la terre et de la douat Dempedjet (?), fils du grand des chefs des artisans, setem, supérieur des secrets du ciel, de la terre et de la douat Horsa[11]ïset, fils du grand des chefs des artisans, sem, supérieur des secrets du ciel, de la terre et de la douat Papa. Qu'ils 
subsistent [12] éternellement! Celui qui effacera (?) (a) (cet) écrit (?), on nommera son nom de façon impie (?) (b) ... (?) l'autel (ou: le temple) de Ptah!"

(a) Wörterbuch, I, 580, 14 ?

(b) Wörterbuch, V, 449, 19: "einen Namen frevelhaft nennen" ?

\section{3a. STATUETTES DE BAKENRENEF}

Berlin 5829 et 7997

Bibliographie:

"Ausführliches Verzeichnis", 1894, p. 228 (description):

(cette référence m'a été obligeamment communiquée par M. G. Posener); les inscriptions ne sont pas publiées dans les Ägyptische Inschriften.

\section{PAPYRUS HOOD}

Bibliographie:

MASPERO, Un manuel de hiérarchie égyptienne, dans Etudes égyptiennes, II, 1888, p. 9;

GARDINER, Ancient Egyptian Onomastica;

d'après Maspero:

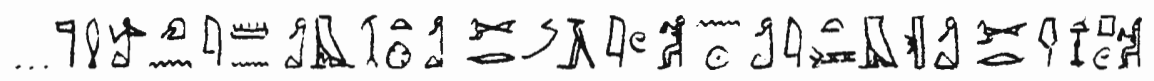

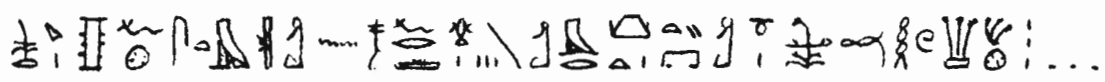

"..., le premier prophète d'Amon dans Thèbes, le grand des voyants de RêAtoum, le grand des chefs des artisans de Celui qui est au sud de son mur et setem de Celui au beau visage, le directeur des deux greniers de Haute et Basse-Egypte, ...". 


\section{PAPYRUS GOLENISCHEFF}

Bibliographie:

A.H. GARDINER, Ancient Egyptian Onomastica, Plates Volume, Oxford 1947, pl. VIII et VIII A (photo, transcription); op. cit., vol. I, p. $38^{*}$ (traduction):

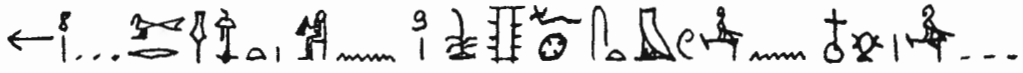

"[8] ..., le grand des chefs des artisans de [9] Celui qui est au sud de son mur, le setem de Celui dont le visage est beau (a),...".

(a) Ou: gracieux.

\section{STELE D'AHMESMEN}

\section{Louvre, Sérapéum 4213}

Bibliographie:

BRUGSCH, Thesaurus, V, p. 947, sous l'indication "stèle 485 du Sérapéum" (texte des lignes 4 (fin) à 8 (presque entière);

$\mathrm{PM} \mathrm{III}^{2} / 2$, p. 801-802;

ma copie:

(stèle cintrée peinte: au premier registre, traces d'un Apis et de sa légende; au deuxième registre:)

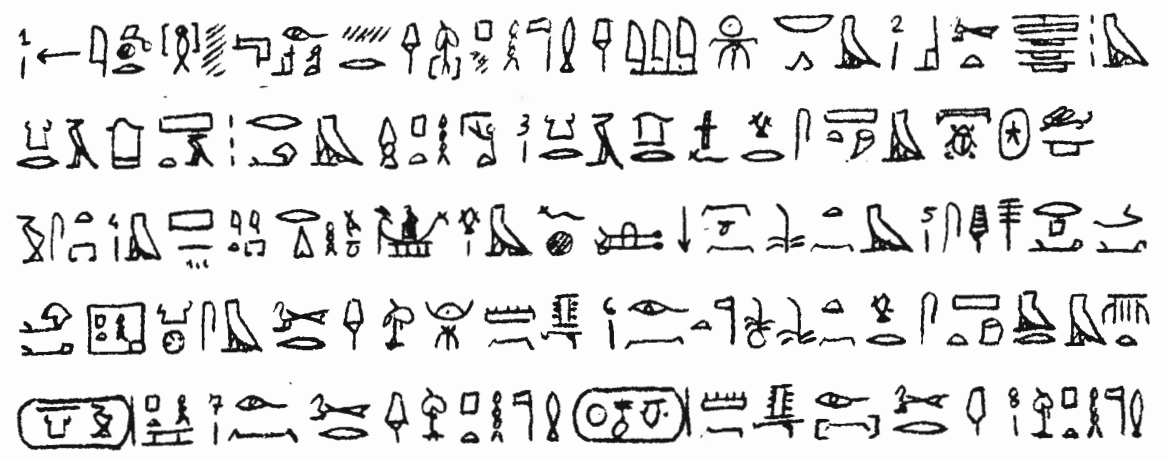




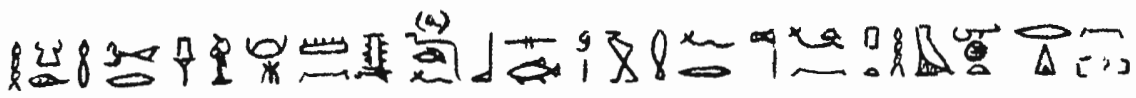

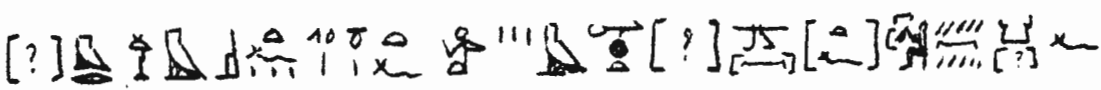

(a) Signes à l'envers sur l'original.

"[1] L'ìmakhou auprès d'Apis-Osiris, le grand des chefs des artisans, prophète de Ptah, chef des sem (a), celui qui participe à la fête de Rê, maître de ses allées et venues dans [2] la Grande Place, celui qui ouvre les portes de (b) la chapelle secrète (c), le préposé au diadème (d) quand Ptah est orné, celui qui cache (e) [3] la chapelle à Celui qui y est (?) (f), le supérieur des secrets dans le ciel, la terre et la douat, celui qui ouvre la "fenêtre" (g) [4] dans le sanctuaire, qui place la barque de Sokar sur le traîneau, le second du roi lors [5] de l'érection du pilier Djed, le noble véritable, gouverneur de Memphis, sem, grand des chefs des artisans, Ahmèsmen [6] né du père divin, sem, supérieur des secrets du roi, directeur des ìmy-khent Nekao-méryptah, [7] né du grand des chefs des artisans, prophète de Ptah, Neferibrêmen, né (du) grand des chefs [8] des artisans, prophète de Ptah, Hekairâa; le grand des chefs des artisans Ahmèsmen dit: [9] Sa Majesté m'a "intronisé" père divin de Ptah; ensuite, (elle m')a nommé directeur des artisans à la place des pères [10] de mes (?) pères, après que (mon?) père eut "passé à son ka".

(a) Sem écrit comme sous l'Ancien Empire dans "sem de Sokar", cf. 6,2.

(b) Litt. : dans.

(c) Plutôt "la chapelle qui contient du secret" que "la crypte" à cause de l'expression "ouvrir les portes"; la traduction littérale du passage serait: celui qui ouvre les portes dans la chapelle du secret (?).

(d) En restituant $n f r$ d'après 26,3 ; l'omission de $n f r$ a pu être facilitée par l'existence du nom composé iry $h 3 t$ (Wört., III, 19, 17: pilote). Le parallélisme de toute l'expression avec 26,3 me fait préfếrer $i r y$ - $(n f r)-h 3 t$ à $i r y-h 3 t$, qui peut être aussi un titre ptolémaïque de prêtre (Wört. I, 104, 9, sans traduction). 
(e) Ou: Celui ( = Ptah) dont $\ldots$ est cachée.

(f) = Ptah qui est dans la chapelle ? Le sens de toute la proposition m'est incompréhensible.

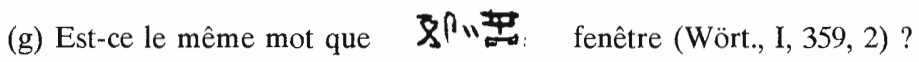

\section{STELE DE SEMATAOUITAFNEKHT}

\section{Louvre, Sérapéum 4044}

Bibliographie :

CHASSINAT, Textes provenant du Sérapéum de Memphis, dans Rec. Trav., XXI (1899), p. 66, n² 24 (texte);

ma collation:

I. Face (gravée)

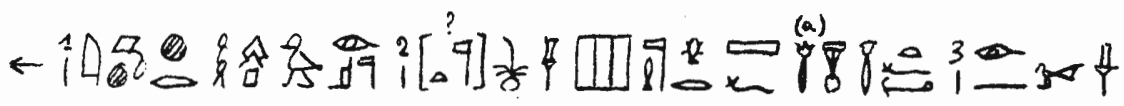

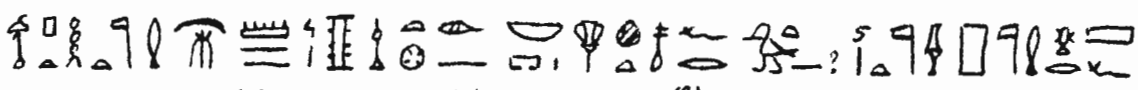

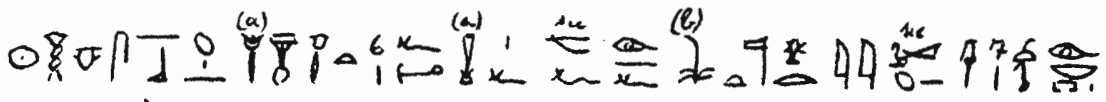

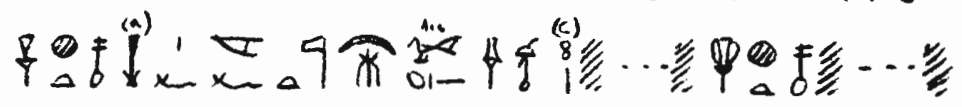

(a) En hiératique.

(b) La tige seule est gravée, sans les feuilles qui se détachent de sa base. A lire plutôt f̀ d'après la ligne 16 .

(c) La ligne 8 est écrite à l'encre noire ainsi que les autres inscriptions de ce monument.

"[1] L'ìmakhou auprès d'Apis-Osiris, [2] (le père divin ?), sem, chef des maisons et (?) prophète de Her-chefi, Semataouitafnekht, [3] né du grand des chefs des artisans, père divin et prophète de Ptah Ahmèsmen du [4] Mur Blanc, et de celle qui possède une maison, Sekhmetnefer, fille du [5] 
père divin, chef de la maison et prophète de Her-chefi Ouahibrêseneb, fils de Semataouitafnekht. Son frère qu'il aime a fait (cela) pour lui, le sem et père divin Hory, fils du grand des chefs [7] des artisans et de celle qui possède une maison Sekhmetnefer. Son frère qu'il aime, le père divin Ahmès, fils du grand des chefs des artisans [8] (et de celle qui possède une maison) (?) Sekhmetnefer ...".

II. Sur la tranche, au sommet [9] - [10], sur le côté droit de la stèle [11] [19], et sur le côté gauche de la stèle [20] - [27] (à l'encre)

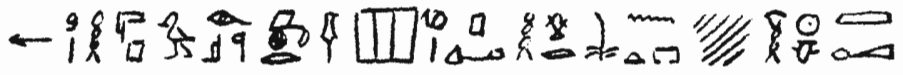

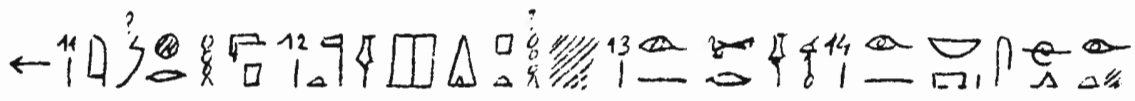

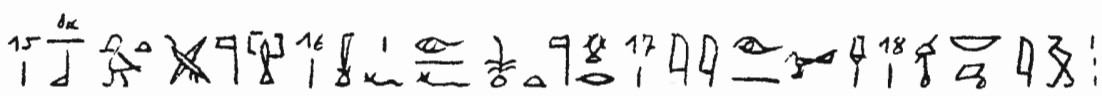

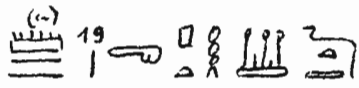

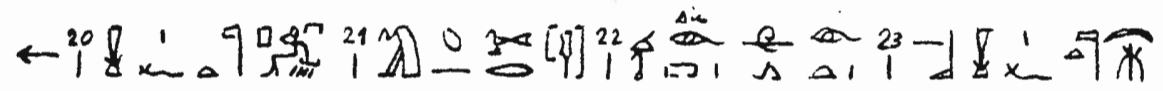

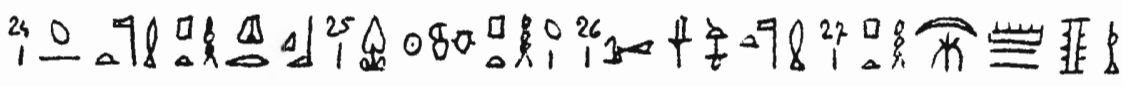

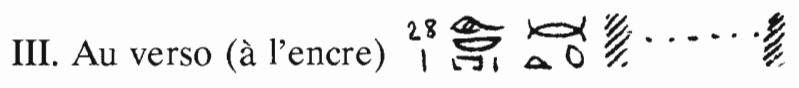

(a) $\sim$ sur l'original.

"[9] L'ỉmakhou auprès d'Apis-Osiris, chef des maisons, [10] Padihorresne ... Ouahibrê, juste de voix !"

"[11] L'imakhou auprès d'Apis, [12] chef des deux maisons, Padih... (?) [13] né du grand des chefs des artisans, [14] et de celle qui possède une maison Setairet[15]bint, fille de Pahemneter! [16] Son frère, qui a fait (cela) pour lui, le sem et père divin Hor[17ly, né du grand des chefs [18] des artisans et possesseur d'imakh ! Qu'ils demeurent [19] devant Ptah pour (a) l'éternité !" 
"[20] Son frère, le père divin Pacher-(en)[21]-mout, fils du grand des chefs [22] des artisans et de (celle qui possède) une maison Setairet[23]bint! Son frère, le père divin Ahmès, [24] fils du père divin et prophète de Ptah qui est sous son olivier [25] Khnemibrê-saptah, [26] le grand des chefs des artisans, père divin et prophète [27] de Ptah Ahmèsmen du Mur Blanc (b)".

(au verso:) "[28] né de celle qui possède une maison, Nit...... (c)".

(a) Sur la préposition "pour" (l'éternité), cf. POSENER, Première domination perse, inscription 11 , note $h$.

(b) D'après la ligne 7, j'interprète les lignes 26-27 comme une apposition du "père divin" de la ligne 24, c'est-à-dire que "le père divin et prophète de Ptah qui est sous son olivier Khnemibrê-saptah" (lignes 24-25) et "le grand des chefs des artisans, père divin et prophète de Ptah Ahmèsmen" (lignes 26-27) ne m'apparaissent que comme un seul personnage portant deux noms; cette interprétation est étayée par les inscriptions de l'Ancien Empire qui décernent le titre de "prophète de Celui qui est sous son olivier" à des grands des chefs des artisans $(4,7$ et $\mathbf{2 0 , 1 3})$.

(c) Comme la ligne 8 semble donner Sekhmetnefer pour mère au père divin Ahmès, j'incline à ne pas voir dans la ligne 28 la suite des lignes 23-27, mais plutôt celle des lignes 9-10.

\section{STELE DE PSAMMETIQUE}

\section{Louvre, Sérapéum ?}

Bibliographie:

CHASSINAT, Textes provenant du Sérapéum de Memphis, dans Rec. Trav., XXIII (1901), p. 83-84, n 141 (texte):

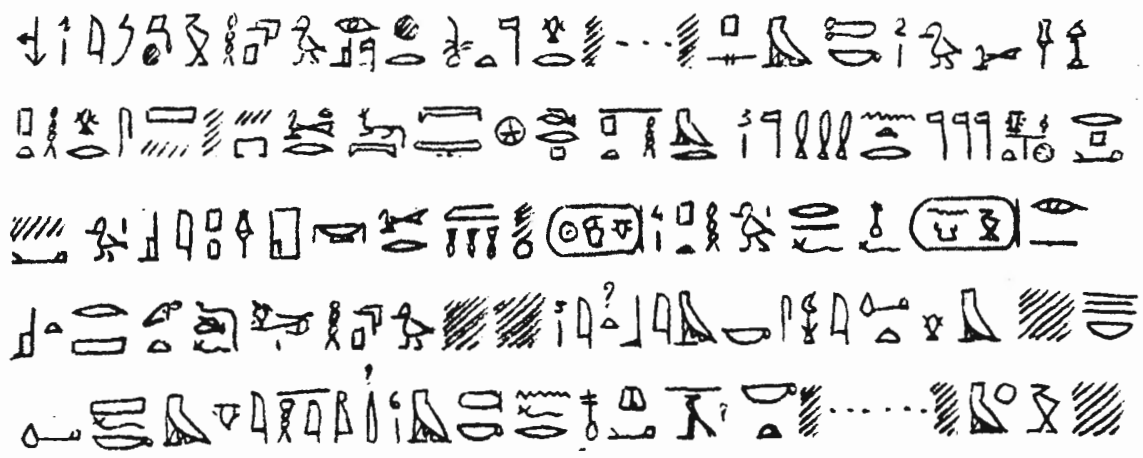




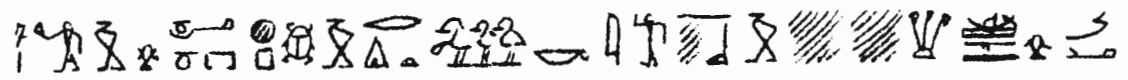

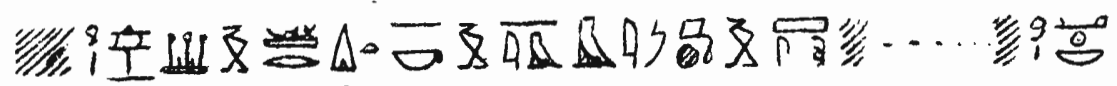

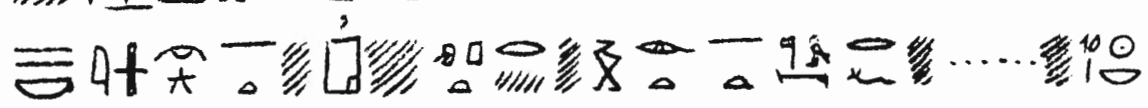

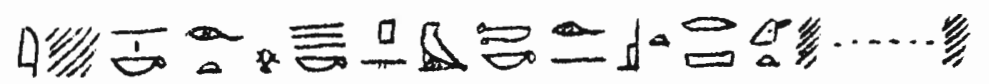

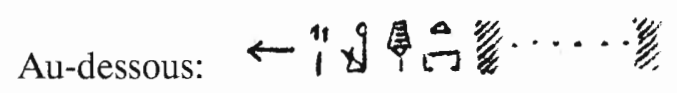

"[1] L'ìmakhou auprès d'Apis-Osiris, le sem, supérieur (des secrets ?) ... Psammétique, [2] fils du grand des chefs des artisans de Ptah, supérieur des secrets de la Grande (Place?), supérieur des secrets du ciel, de la terre et de la douat, celui qui fait les libations à Ptah, directeur [3] des prophètes des dieux du Mur Blanc, noble, gouverneur (?), .... (?), chef de la maison de l'or (a), grand ... (?), Khnemibrê-[4]saptah, dont (b) le beau nom est Nékao, né de Isetrechet. Il dit: Mon maitre (?) Apis-(Osiris), ... (c) [10] ... Psammétique né de Isetrechet .....".

(au-dessous:) "Le gardien du tombeau ...".

(a) La maison de l'or = l'atelier d'orfèvrerie.

(b) Comme 188 et 187 sont tous deux contemporains de l'ensevelissement d'un Apis en l'an 34 de Darius, je n'hésite pas à identifier les deux Khnemibrê-saptah de ces monuments. Or Khnemibrê-saptah de $\mathbf{1 8 7}$ porte aussi le nom de Ahmèsmen. Pour éviter de lui donner un troisième nom, je préfère rapporter "dont" à Psammétique, malgré la place bizarre qu'occuperait alors la mention du beau nom de ce personnage.

(c) Je ne vois pas la possibilité de tirer grand parti des restes du discours de Psammétique à Apis. 


\section{8a. VASE CANOPE DE NEKAOMEN}

\section{Berlin 11641}

Bibliographie:

Ausführliches Verzeichnis, 1894, p. 181, dit du possesseur qu'il est un "Hohepriester von Memphis".

(Pas publié dans Ägyptische Inschriften).

\section{STATUE DE ...PADIES}

\section{Caire 659}

Bibliographie:

BORCHARDT, Statuen ..., III, 1930, p. 8 (texte);

ma collation:

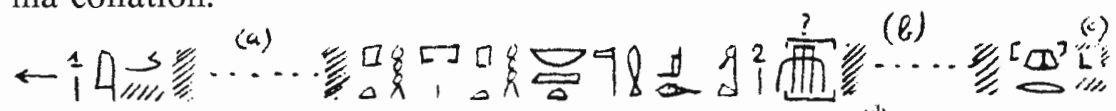

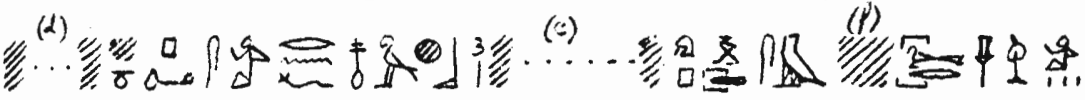

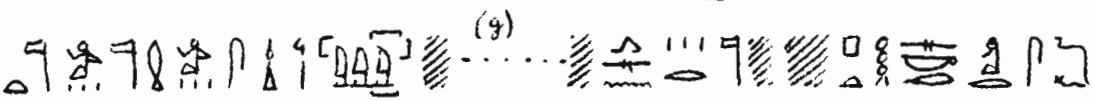

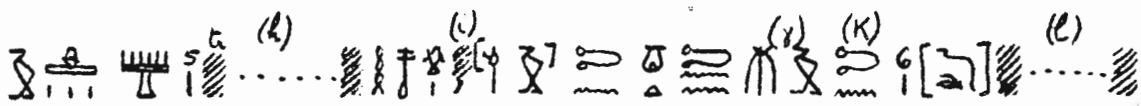

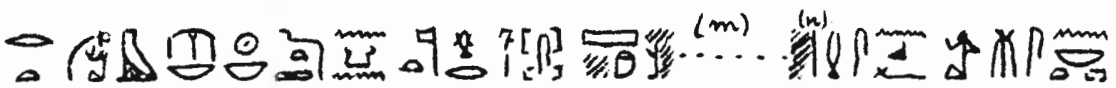

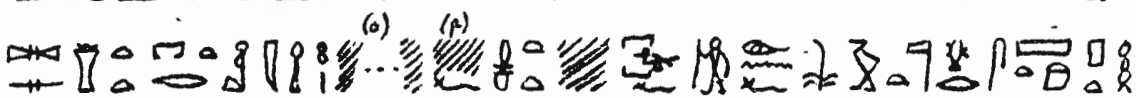

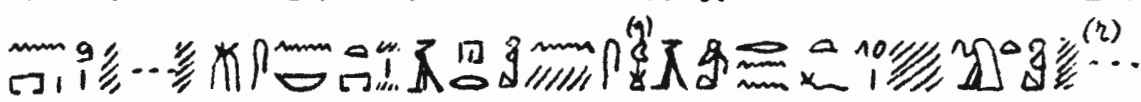

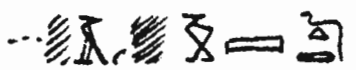

(a) Environ 8 à 10 cadrats.

(b) Environ 6 à 8 cadrats.

(c) Reste d'un $\square$, d'un $\square$ ou d'un signe de forme analogue, mais pas d'un 4 comme l'a indiqué Borchardt. 
(d) $21 / 2$ à 3 cadrats.

(e) Environ 7 cadrats.

(f) La restitution $\stackrel{\&}{11}$ me semble certaine d'après les mots suivants.

(g) 5 à 6 cadrats.

(h) 5 à 6 cadrats.

(i) Fragment d'un trait rectiligne placé de façon légèrement oblique.

(j) Pas de $\bigcap$ comme Borchardt l'a indiqué par erreur.

(k) Ce suffixe termine la ligne (Borchardt ajoute $\quad / / / /$, .)

(1) 5 à 6 cadrats.

(m) Environ 5 cadrats.

(n) Probablement w.

(o) $11 / 2$ à 2 cadrats.

(p) La queue d'un oiseau subsiste au-dessus de celle de $\measuredangle$.

(q) Gravé à l'envers.

(r) 3 à 4 cadrats.

"[1] L'ìmakhou ........, du temple de Ptah, prophète de Ptah maître de la Vérité, ..... d'Osiris [2] qui est à la tête ..... (a), porteur (?) ...... ...padies, dont le beau nom est Horkheb [3] ........ (b) qui êtes sur terre, sem (c), grands des chefs des artisans, pères divins, prophètes, surveillants [4] des champs (d) ..... qui (viendrez) au temple (e) de Ptah-Sokar pour alimenter (ses) (f) tables d'offrandes, [5] ........ (g) (Pta)h au beau visage, vous transmettrez votre "siège" à vos enfants [6] (si) (vous) (h) dites: ...... pur à chaque fête chaque jour, éternellement, au ka du père divin, supérieur [7] des secrets ....., prophète (?) Senebef, né de celle qui possède une maison, Tesbastetperet, juste de voix (i); [8] ..... son ... également ... son fils aîné qui a fait cela pour lui, le père divin, supérieur des secrets du temple de Ptah, [9] ..... né de celle qui possède une maison ...aher, ... rappeler le nom de (ses ?) [10] pères (j) et mères ..... (pour) (k) l'éternité !" 
(a) On pourrait aussi traduire ainsi la fin de la ligne 1: "... de Ptah du temple de Ptah maître de la Vérité, prophète d'Osiris etc...", mais comme ...padies est nommé, je crois, "supérieur des secrets... (?)" aux lignes 7-8, où il est fort probablement question de lui, je propose de restituer ce même titre à la ligne 1 et de lire: "... (supérieur des secrets) $d u$ temple de Ptah, prophète de Ptah maître de la Vérité, ..... d'Osiris etc.".

(b) Le début de la ligne 3 contenait la fin du beau nom de ...padies, peut-être celui de son père, et certainement les mots: "Il dit: $O$ ".

(c) Voir (f) ci-dessus.

(d) Ou: des sem, avec la graphie de ce mot sous l'Ancien Empire, puisque cette graphie se retrouve à la XXVIème dynastie $(\mathbf{1 8 6 , 1 )}$; il est vrai que c'est dans l'expression "chef des sem".

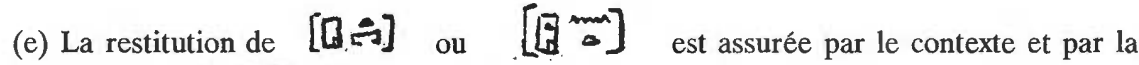
provenance (Mit-Rahineh) du monument.

(f) "Ses" était au début de la ligne 5.

(g) Le début de la ligne 5 contenait vraisemblablement une expression de même valeur grammaticale que la fin de la ligne 4: "et pour ...".

(h) (si) n'est pas exprimé. Je suppose que la formule courante dites" aura poussé Borchardt à voir un cadrat détruit à la fin de la ligne 5. (vous) est détruit.

(i) D'après les lignes 1 et 2, on peut restituer ainsi la ligne 7: "des secrets (du temple de Ptah (?) ...padies, fils du) prophète Senebef, (et) né de celle, etc.".

(j) (ses) dans le cadrat détruit à la ligne 10 ?

(k) A lire $s\left(3^{c}\right)$ ?

189a. STATUE DE ....

British Museum 25 (904)

Bibliographie:

A Guide to the Egyptian collections, Sculpture, 1909, p. 245, n 904 :

"Portion of a black granite kneeling statue of a high ecclesiastical official, whose father and grandfather were high-priests of Memphis. The pedestal is restored. Late period. Height $3 \mathrm{ft}$. 8 in." 


\section{CHAPITRE XVII}

\section{LES INSCRIPTIONS DE L'EPOQUE PTOLEMAIQUE}

\section{LA STELE DE NESQED}

\section{British Museum 379 (1029)}

Bibliographie:

YOUNG, Hieroglyphics, 1823, pl. 77-78 (dessin peu utilisable);

BRUGSCH, Thesaurus, V, 1891, p. 907-909 (texte partiel);

J. YOYOTTE, Le nom de Ramsès "souverain d'Héliopolis", dans R. ANTHES, Mit-Rahineh 1956, p. 68, n. 10. en combinant Young et Brugsch:

I. Premier registre (devant le personnage faisant offrande):

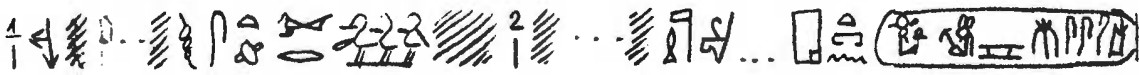

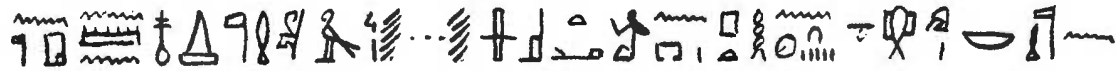

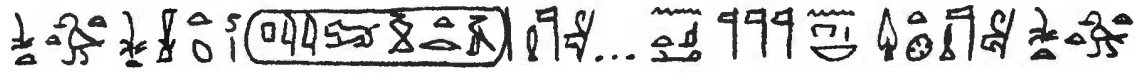

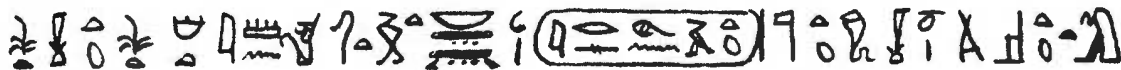

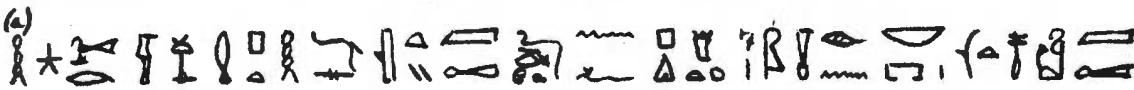

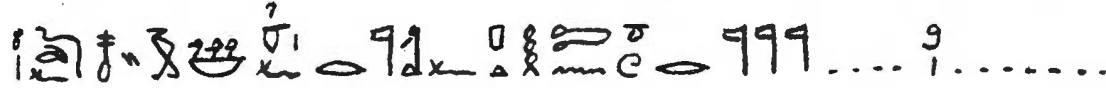

(a) Lecture de Brugsch, certainement fausse; cf. ligne 12 ci-dessous. 
"[1] ... (Ptah) le bien-odorant, dont la puissance est grande ... [2] prophète du temple de Ramsès-méry-imen-Heqa-iounou du temple de Memphis, prophète de l'Horus [4] ...... imy-set-â du temple de Ptah des quinze jours de toutes les premières phylai, prophète de la fille de roi et soeur de roi [5] Philotera prophète de ... set-â des dieux du domaine de la Maîtresse du sycomore, prophète de la fille de roi, soeur de roi, épouse de roi Tout-heqaìmen, la maîtresse des Deux Pays [6] Arsinoé, la déesse Philadelphe (aimée) d'Isis la mère du dieu, le grand des chefs des artisans, serviteur de Ptah, Nesqed, juste de voix, appelé Petobastis, juste de voix, né de celle qui possède une maison Renpetnefer, juste de voix ! [8] Il dit: Comme il est bon de remplir sa pensée de son dieu Ptah-tenen et des dieux ... [9] ...".

II. Deuxième registre:

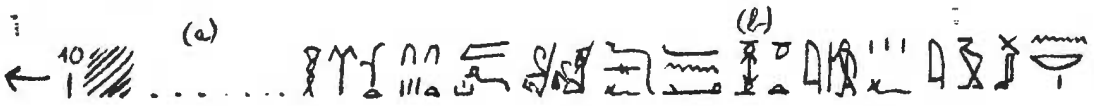

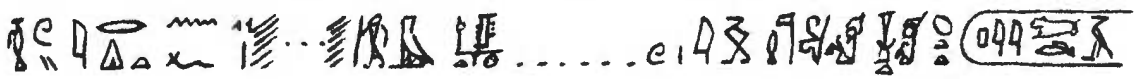

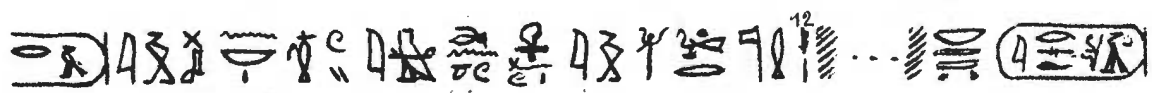

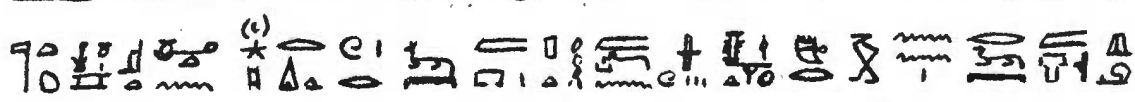

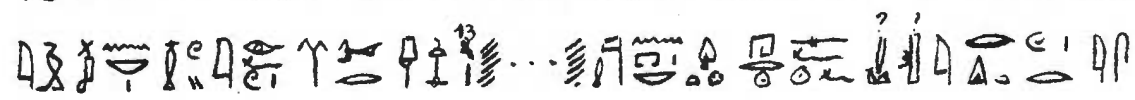

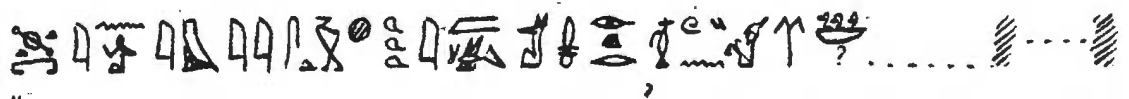

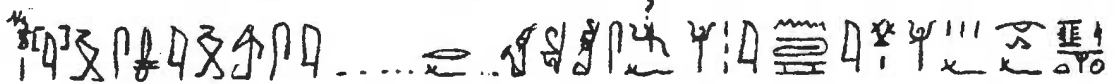

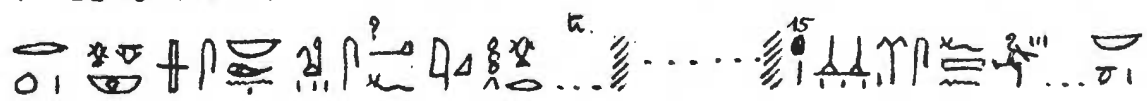

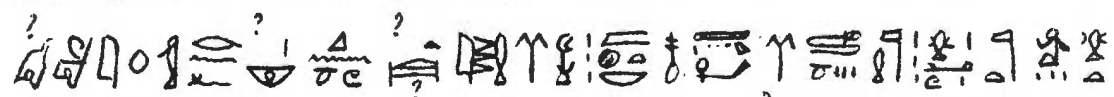

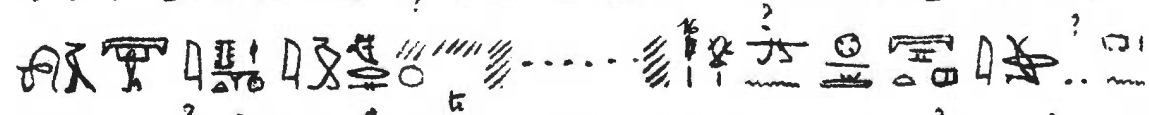

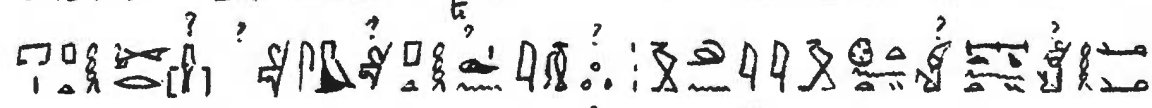

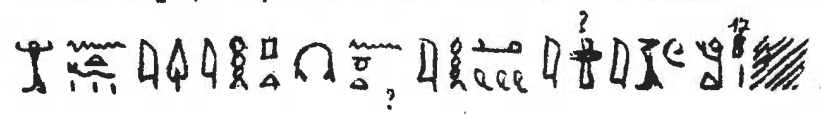




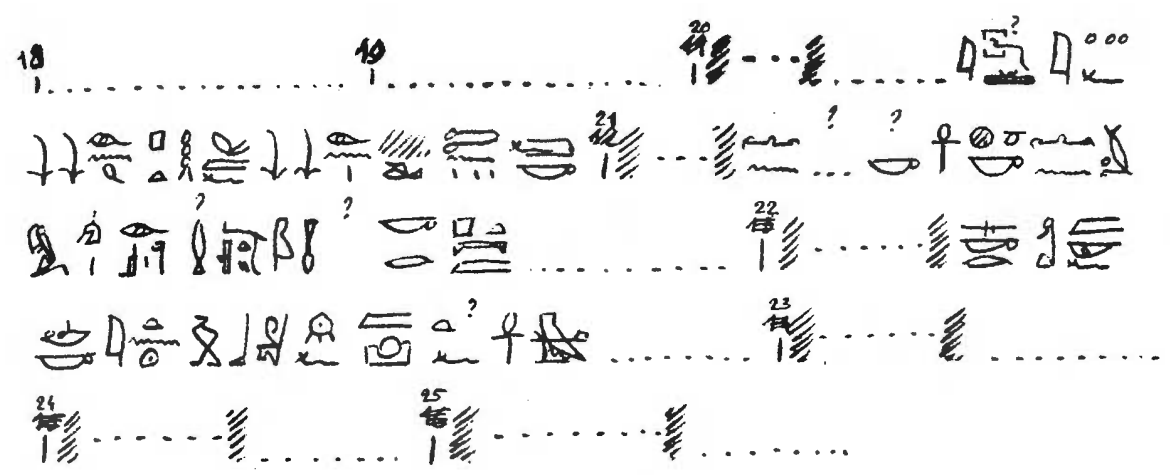

(a) Un pointillé au bas de la ligne indique un passage conservé dont je n'ai pas réussi à établir la lecture d'après Young, seul éditeur moderne de la plus grande partie de ce texte; Brugsch ne donne que la partie conservée de la ligne 10, le passage allant du milieu de la ligne 11 au milieu de la ligne 13 et une partie de la ligne 15.

(b) Lire $\downarrow$; cf. graphie ptolémaïque de snw.t, Wört. IV, 511.

(c) Lire $\star \simeq$.

"[10] ..... en l'an 23, en tant qu'élu du roi lui-même, avec ses courtisans. Mon maître redoubla la faveur dont j'étais l'objet en me nommant [11] ..... grand (?) dans le Mur Blanc. .... me (nommant) prophète de la soeur de roi et fille de roi Philotera. Mon maître redoubla la faveur dont j'étais l'objet en me faisant cadeau (a) de la grande fonction de prophète [12] maîtresse des Deux Pays Arsinoé, la déesse Philadelphe aimée d'Isis mère du dieu. Je fus nommé (c) süpérieur des secrets dans le temple de Ptah, ayant été choisi parmi les habitants du Mur Blanc ..... et supérieur des secrets dans Ro-qedit auparavant. Mon maître redoubla la faveur dont j'étais l'objet en me faisant grand des chefs des artisans [13] ..... et prophète du domaine de la Maîtresse du sycomore. Le jour de ..., j'ai été déposé dans l'atelier m'appartenant; mon corps a été embaumé (d) en travail d'Anubis, comme on traite quelqu'un qui a la faveur du roi, dans .... [14] ... annonce ... au (ou: du) roi; il transmet (?) mes fonctions à ........ (?) ses fonctions; il atteint le Mur Blanc le jour de (?) la fête Her-ib; tous ceux qui y sont me font (?) .............. [15] des étoffes (e) en ... (?), tous ... de Haute et BasseEgypte .............(?), des offrandes par milliers de toutes bonnes choses. Les gens de la nécropole (f) faisaient des offrandes, les prophètes m'ont 
porté $(\mathrm{g})$, les pères divins m'ont porté $(\mathrm{h}) ;$ le Mur Blanc tout entier ........ [16] ........ (?) domaine du temple de Ptah, le grand des chefs (?) (des artisans ?) de (??) et (?) sem de (?) Ptah m'a fait (?) des libations. J'ai atteint la ville du roi de Haute-Egypte (?) et le nome du roi de Basse-Egypte (?) (i); mes pères se réjouissent, mes (mères ?) sont accueillantes (?) et (?) embrassent mes membres; ... louanges [17] .... [18] .... [19] .... [20] ..... ce que Ptah fait (?) pour lui quand il entend ce que j'ai fait. Vous (direz:) (?) Puisses-tu sortir [21] ... sans ..., puisses-tu avoir la vie et que la mort ne soit pas sur (toi), l'Osiris et serviteur Nesqed, juste de voix ! Puisses-tu ... au ciel en ..... [22] ... Sokar à sa fête, puisses-tu voir le soleil quand il se lève à son horizon, vivant (?) de ...... [23] [24] [25] ..(j)".

(a) $m t n$, Wört., II, 170, 11 qui ne signale qu'une construction avec $m$.

(b) La ligne 5 permet de restituer le début détruit de la ligne 12.

(c) Ici et à la ligne suivante, la disposition des signes me fait préférer la lecture rdít (infinitif) $+w i$ à la forme passive rdì.tw.l.

(d) $s d w h$, Wört., IV, 368, 6.

(e) mnh.t.

(f) Litt.: le Pays sacré, c'est-à-dire ses habitants.

(g) Litt.: épaulé, $r m n$.

(h) Litt.: soulever, tw3.

(i) Est-ce un rappel des anciens pélerinages funèbres ?

(j) Une fin de ligne en démotique subsiste sous la ligne 25.

\section{STELE DE KHONSOUIOU}

British Museum 375 (1032)

Bibliographie:

YOUNG, Hieroglyphics, 1823, pl. 79 (dessin);

BRUGSCH, Thesaurus, V, 906-907 (texte de la titulature);

P. MUNRO, Die spätägyptischen Totenstelen, Äg. Forsch. 25 (1973), 
p. 339 , pl. 62 , Abb. 209 ;

d'après Young et Brugsch:

I. Premier registre; en face d'Osiris (d'après Young):

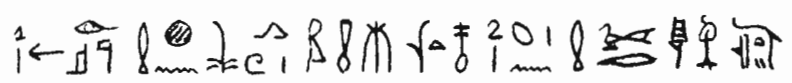

"[1] L'Osiris, serviteur, Khonsouiou, juste de voix, né de Renpetnefer [2] (et) fils du serviteur et grand des chefs des artisans Nesqed".

II. Deuxième registre:

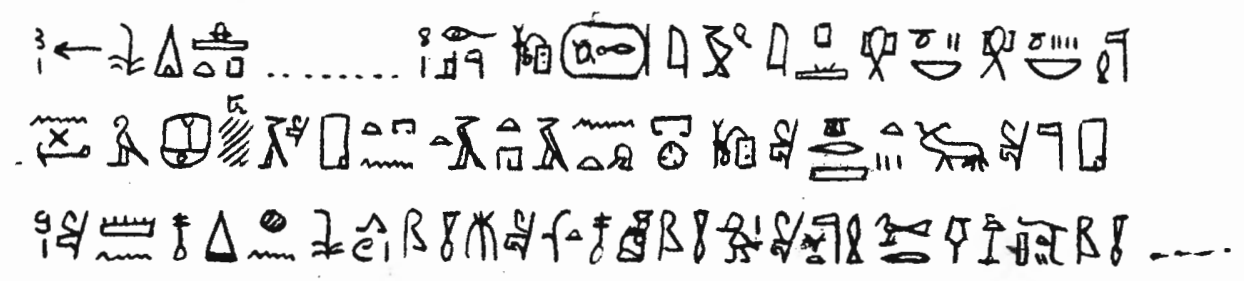

"[3] Offrande royale

[8] l'Osiris, scribe de Pharaon, celui qui compte toutes les deuxièmes phylai et toutes les quatrièmes phylai, prophète de Nectanébo, ... de la demeure de Ta-dehenet (a), scribe de l'alimentation des troupeaux du temple [9] de Memphis, Khonsouiou, juste de voix, né de Renpetnefer, juste de voix et fils du père divin et prophète, grand des chefs des artisans Nesqed, juste de voix. ... (b)".

(a) Ta-dehenet $=$ nom de déesse (Wört., V, 478, 13); dhnt = front et aussi falaise du désert (qui est comme un "front" pour la vallée du Nil).

(b) Quatre lignes en démotique suivent la ligne 9.

\section{STELE D'ANEMHER}

\section{Vienne 153}

Bibliographie:

BRUGSCH, Thesaurus, 902-906 (texte et traduction);

WRESZINSKI, Ägyptische Inschriften aus dem K.K. Hofmuseum in Wien, 1906, p. 96-98 (texte) et pl. III (photo);

P. MUNRO, Die spätägyptischen Totenstelen, Äg. Forsch. 25 (1973), 
p. 342 , pl. 61 , Abb. 207;

d'après Wreszinski:

I. Premier registre, de part et d'autre du signe nefer:

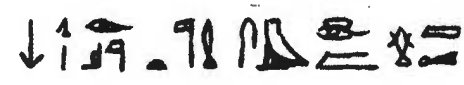

"[1] L'Osiris, père divin et prophète, sem, Anemher, juste de voix".

II. Deuxième registre:

2

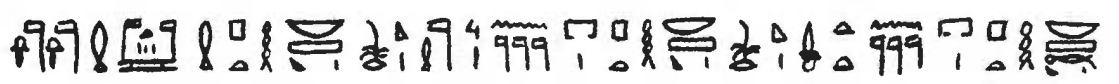

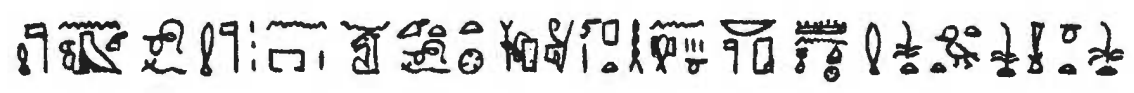

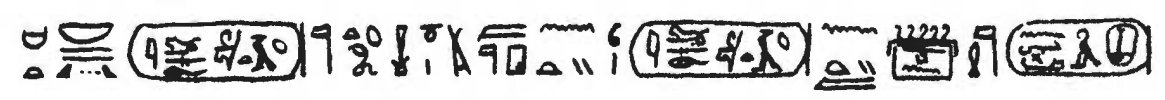

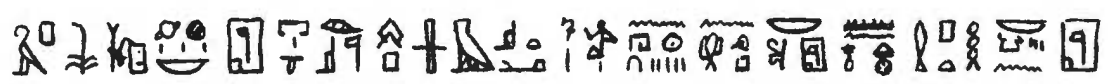

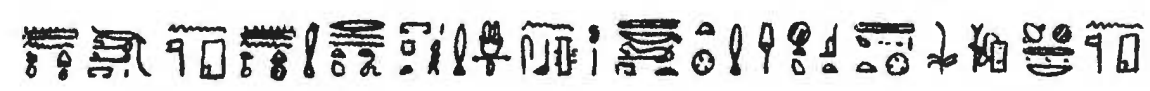

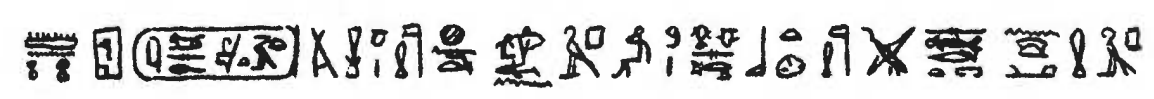

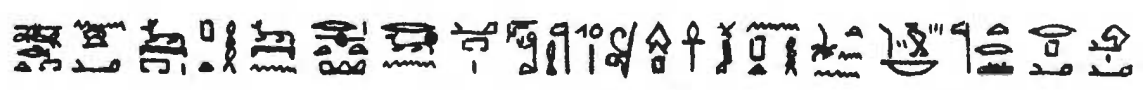

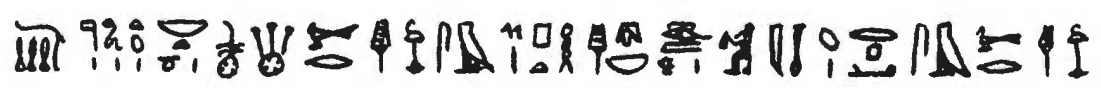

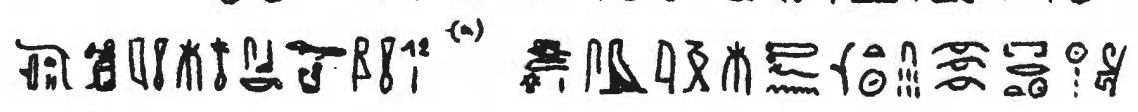

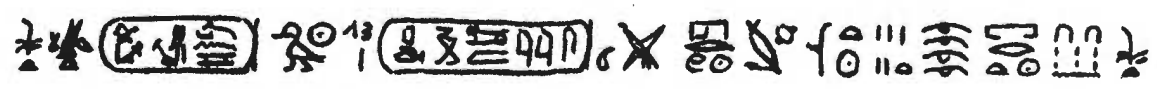

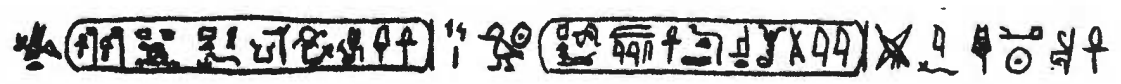

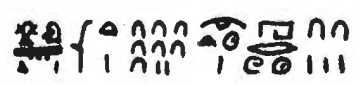


(a) $11 / 2$ cadrat en blanc.

"[2] Offrande royale à Osiris qui est à la tête de l'Occident pour qu'il donne etc... [3] ... au ka de l'Osiris, père divin aimé du dieu, khe-nesou de (?) Ptah, serviteur du Puissant, de Celui qui prête l'oreille, du Bien-odorant, de Celui dont la parole se manifeste, (serviteur) (a) des dieux Evergètes, serviteur des dieux Philopators, serviteur de Ptah seigneur du lac du sud prophète [4] des dieux du temple de Ptah seigneur du lac du sud, également des dieux du temple de Ptah seigneur du lac du nord, prophète de Khnoum qui est à la tête de Ouaf, prophète des dieux du temple de Khnoum qui est à la tête de Ouaf, scribe de [5] Ptah de toutes les troisièmes phylai du temple de Memphis, serviteur de la fille de roi, soeur de roi et épouse de roi, maitresse des Deux Pays, Arsinoé, la déesse Philadelphe, dans le temple d'[6]Arsinoé qui est dans ta Porte de ce Pays (?) (b), prophète de Nectanébo et (?) d'Horus de Pe (?), scribe royal décomptant toutes choses de la demeure divine d'Osiris-Apis, ìmy-set-â [7] des quinze jours de toutes les premières phylai du temple de Memphis, serviteur de Ptah seigneur des aliments du temple de Memphis, directeur de l'ergastule du temple de Memphis, serviteur de Renenet du temple de Ptah, serviteur de Nefertoum de la porte fortifiée [8] de Takelehet, serviteur de Sekhmet et d'Isis (?) de Ta, scribe royal décomptant toutes choses du temple de Memphis et du temple d'Arsinoé-Philadelphe, prophète et gardien d'Harpocrate [9] qui est dans Sekhtebet, prophète de la Fenêtre de l'apparition, serviteur de l'Horus de la Fenêtre de l'apparition, supérieur des secrets du temple de Ptah, supérieur des secrets de -Ro-setaou, connaissant les secrets de la Chambre cachée, prophète [10] d'Apis vivant, l'intermédiaire de Ptah et roi de tout le bétail divin, noble, gouverneur, directeur des prophètes de tous les dieux et déesses de Haute et Basse-Egypte, grand des chefs des artisans, sem [11] de Ptah, chef de toute la garde-robe, Anemher, juste de voix, fils du noble, sem, grand des chefs des artisans Nesqed, juste de voix et né de Nefersebek, juste de voix. [12] Anemher, le sem, est né en l'an 16, troisième mois de Peret, troisième jour du roi de Haute et Basse-Egypte, Setep-en-rê-Méry-imen, fils de Rê [13] Ptolémée. Son jour de mort: an 5, quatrième mois de Peret, vingt-sixième jour du roi de Haute et Basse-Egypte 'Iâou-en-neterouìmenkhoui-Setep-en-Ptah-Ouser-ka-rê-Sekhem-ankh-imen, [14] fils de Rê Ptolémée-Ankh-djet-Méry-íset. Sa durée de vie sur terre: 72 ans, 1 mois, 23 jours (c)". 
(a) Le Wört., III, 242, 17, voit dans $\&$ un titre. WRESZINSKI, Ägyptische Inschriften ..., p. 99-100, y voit une épithète de Ptah. J'adopte cette dernière interprétation parce que, dans les quatre exemples que Wreszinski a réunis de cette expression (trois sont dans des inscriptions de grands prêtres: 192,3, 193,3 et 194,1; une quatrième se trouve sur une cuve de sarcophage ptolémaïque provenant aussi de Memphis, cf. WRESZINSKI, op. cit., p. 179, V,2), 绾 suit chaque fois l'épithète

"Bien-odorant" décernée à Ptah, tandis qu'elle précède des termes dissemblables: "les dieux Evergètes" (192,3), "prêtre-ouab" $(\mathbf{1 9 3 , 3})$, "scribe de Ptah" $(\mathbf{1 9 4 , 1 )}$, "nourricier d'Harpocrate" (WRESZINSKI, op. cit.). D'après les diverses graphies: $2 \quad(192,3)$, \& $(193,3)$, os 20 (194,1), il semble bien que le premier élément est le verbe $h^{c} y$ et le second le nom $r 3 ; h^{c} y$ signifiant "se lever, apparaître", en parlant des astres, puis des dieux et enfin du roi, et $r 3$ signifiant "la bouche", puis "ce que la bouche dit", $r 3$ ne peut guère avoir que le rôle d'un "accusatif de relation" dépendant du participe $h^{c}(w$ ?)". Je propose donc la traduction littérale:"qui apparaît quant à ce que la bouche dit", soit "dont la parole se manifeste", par allusion au "verbe créateur" de Ptah. Quant à la stèle d'Anemher, on peut admettre que "des dieux Evergètes" dépend de "serviteur", écrit à leur suite comme sur le sarcophage d'Harmachis (197 ci-dessous), de même pour "des dieux Philopators", et que le "serviteur" dont dépend "des dieux Philopators" s'est fondu avec le "serviteur" dont dépend "Ptah seigneur du lac du sud". Même particularité: 206,3 (remarque (b).

(b) Sur le sarcophage d'Harmachis, peut-être fils d'Anemher (197), le temple d'ArsinoéPhiladelphe avec lequel le grand prêtre de Ptah est en rapport se trouve "dans le Mur Blanc".

serait-il une graphie ptolémaique de "Mur Blanc", de même que est une graphie ptolémaïque de $h n w$, "résidence" ? WRESZINSKI, op. cit., interprète: "Tempel ..., der in der Residenz (Alexandria ?) ist", et cite deux autres emplois des mêmes signes: Lepsius, Kg. Buch 690 et un sarcophage de Vienne (de provenance indéfinie).

(c) $11 / 2$ ligne démotique suit la ligne 14. Cf. BRUGSCH, Thesaurus 906, et SPIEGELBERG, Demotische Miscellen, dans Rec. Trav., XXX, p. 147-148.

\section{STELE DE TEOS}

\section{Vienne 154}

BRUGSCH, Thesaurus, p. 912-915 (texte et traduction);

WRESZINSKI, Ägyptische Inschriften aus dem K.K. Hofmuseum in Wien, 1906, p. 104-105 (texte) et pl. IV (photo);

P. MUNRO, Die spätägyptischen Totenstelen, Äg. Forsch. 25 (1973),

p. 342 (pas de pl.);

d'après Wreszinski: 


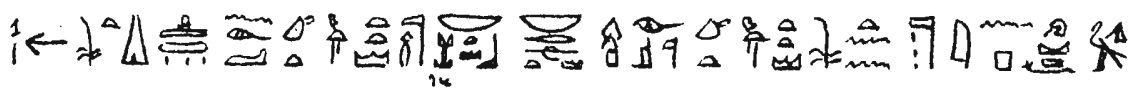

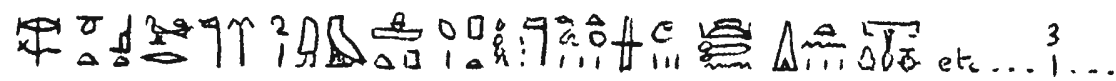

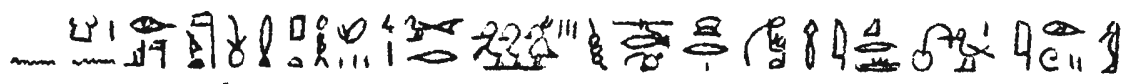

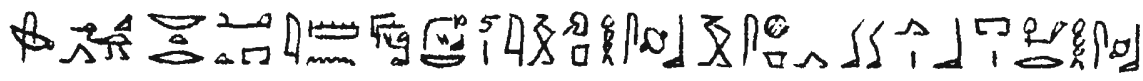

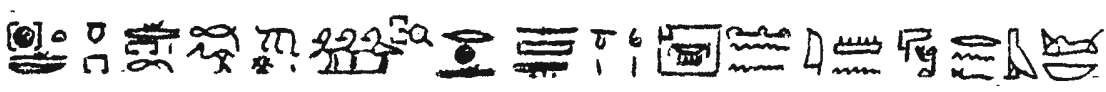

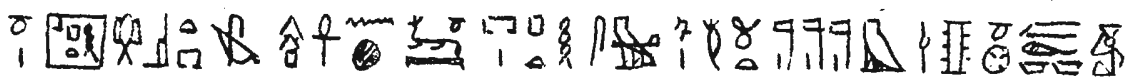

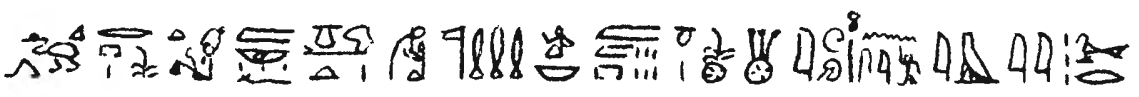

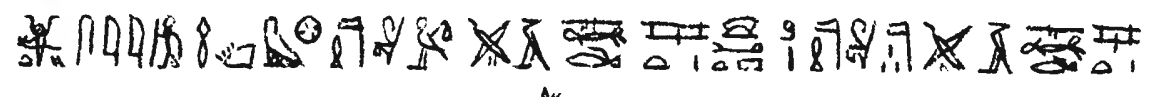

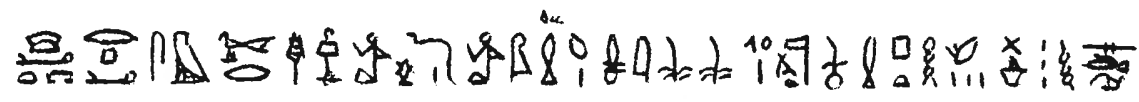

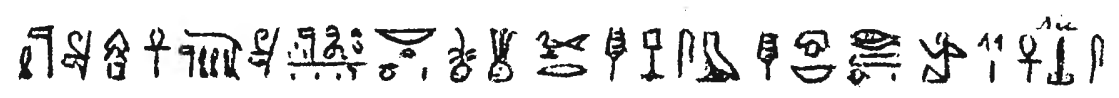

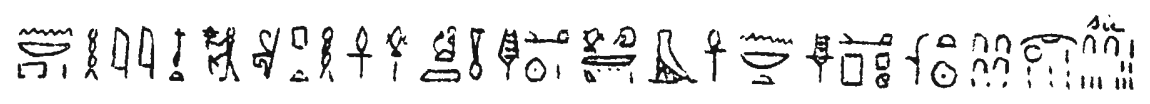

"[1] Que le roi donne une offrande à Osiris qui est à la tête de l'Occident, le dieu grand, seigneur d'Abydos, seigneur de Ro-setaou, (à) Apis-Osiris qui est à la tête de l'Occident, le roi des dieux, (à) Anubis qui est sur sa montagne, (à) Horus assistant de son père, (à) Isis la grande, mère du dieu, [2] (à) Imhotep, fils de Ptah, (aux) dieux et déesses qui sont dans ce tertre (a), afin que vous (sic) donniez la "sortie de voix" - pain, bière, etc...[3]... au ka de l'Osiris, père divin aimé du dieu, khe-nesou, serviteur de Ptah qui prête l'oreille, [4] le Puissant, le Bien-odorant, Celui dont la parole se manifeste (b), le ouab très capable (c), ...(d) (?) de forme (d), qui conduit dans la chambre cachée quiconque doit y entrer et a été purifié [5] selon la règle (e), qui peut marcher à grandes enjambées (dans) le lieu sacré, comptable de toutes les choses de la bibliothèque, qui sait combler les lacunes dans les écrits sacrés, qui connaît les secrets de l'atelier $d$ '6]orfèvrerie, (pour lequel) il n'y a rien dont le nom soit caché parmi tous les objets (?) sacrés du temple de Ptah, le protecteur magique (f) et 
conducteur d'Apis vivant, supérieur des secrets du temple de Ptah, qui habille (g) [7] les dieux dans le Mur Blanc avec ce que ses mains ont fait (h), qui entre dans la maison du roi, en ses fêtes, qui est à la tête de tous les ouab et prophètes dans les temples de Haute et Basse-Egypte et à la tête [8] de ceux qui leur appartiennent (?) (i), dont la fonction est grande, grand prince (j) d'Egypte, prophète de l'Horus de la Fenêtre de l'apparition, [9] prophète des dieux de la Fenêtre de l'apparition, noble, sem, grand des chefs des artisans, Teos, juste (de voix), fils de celui qui porte les mêmes titres, [10] le père divin aimé du dieu, sem, serviteur de Ptah qui prête l'oreille, le Puissant et Bien-odorant, prophète d'Apis vivant, directeur des prophètes de tous les dieux et déesses de Haute et Basse-Egypte, grand des chefs des artisans, sem, chef de toute la garde-robe, Anemher, [11] V.,S.,F., et de (k) celle qui possède une maison, la musicienne accomplie de Ptah, Ankhher (l), juste de voix. La durée sur terre, en vie, du possesseur de cette stèle: 43 ans, 6 mois, 29 (jours) (m)".

(a) Cette stèle provient du Sérapéum.

(b) Cf. 192,3.

(c) Litt.: grand de capacité.

(d) $\operatorname{sh} 3=$ SCHARFF, Z̈̈S, 62 (1927), p. 99.

(e) Litt.: conduisant tout (nbt pour $n b$ comme à la fin de la ligne 11) entrant dans la chambre cachée (et) tout purifié (ou, comme l'interprète WRESZINSKI, p. 106: tout $o u a b)$ selon la règle.

(f) Cf. GARDINER, JEA, 24 (1938), p. 179.

(g) $s m^{c} r$ (Wört., IV, 131, 5 et 6).

(h) ou: "en tant qu'un dont les mains agissent" ?

(i) WRESZINSKI, p. 107: "lies $r$ tp (?) nìs ì j̉.w mir unverständlich."

(j) $s r$.

(k) WRESZINSKI, op. cit., p. 108 corrige: "fu für औी ". Comme Teos n'a vécu que 43 ans tandis que son père Anemher a atteint 72 ans, Teos a pu mourir avant Anemher, ce qui expliquerait l'emploi de fu après le nom d'Anemher. Le $n$ est évidemment une difficulté; on peut y voir, peut-être, un $n(y)$ reliant $s 3$ et $n b t$ pr. 
(l) Sur les monuments d'Harmachis, frère de Teos, la femme d'Anemher est toujours appelée Herankh (forme grecque: Harunchis).

(m) Trois lignes démotiques suivent la ligne 11; cf. BRUGSCH, Thesaurus, p. 912-913, et SPIEGELBERG, Demotische Miscellen, dans Rec. Trav., XXX, p. 148.

\section{STELE D'HARMACHIS}

\section{Vienne 155}

Bibliographie:

BRUGSCH, Thesaurus, p. 915 (texte);

WRESZINSKI, Ägyptische Inschriften aus dem K.K. Hofmuseum in Wien, 1906, p. 108-110 (texte) et pl. II, bas, droite (photo);

P. MUNRO, De spätägyptischen Totenstelen, Äg. Forsch. 25 (1973), p. 342 (pas de pl.); d'après Wreszinski:

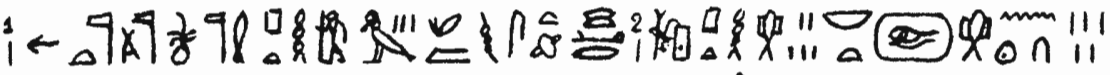

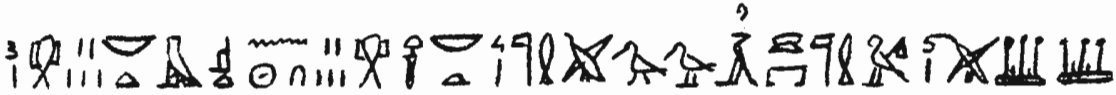

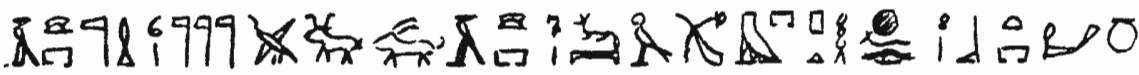

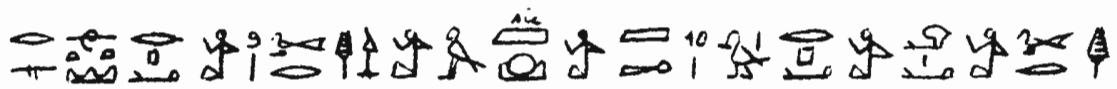

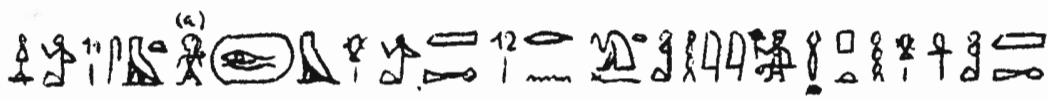

(a) Personnage vêtu de la peau de félin et coiffé de la tresse de cheveux tombant sur l'épaule.

"[1] Le père divin aimé du dieu, sem, prophète de Ptah le Puissant, Celui qui prête l'oreille, le Bien-odorant, Celui dont la parole se manifeste (a), [2] scribe de Ptah de toutes les cinquièmes phylai, le ân de phylê des quinze jours [3] de toutes les cinquièmes phylai, le imy-set des quinze jours de toutes les cinquièmes phylai, [4] le prophète de la Fenêtre de l'apparition, prophète de l'Horus [5] de la Fenêtre de l'apparition, le prophète [6] des 
dieux de la Fenêtre de l'apparition, [7] supérieur des secrets de l'Horus, purificateur dans le temple de Ptah, celui qui a le droit de regarder [8] le lieu sacré de Ro-setaou, le noble, [9] grand des chefs des artisans, Harmachis, juste de voix, [10] fils du noble, gouverneur, grand des chefs des artisans, [11] setem, Anemher, juste de voix; [12] le nom de sa mère, la grande musicienne de Ptah: Harunchis, juste de voix (b)".

(a) Cf. 192,3.

(b) Une courte inscription démotique suit la ligne 12; cf. BRUGSCH, Thesaurus, p. 915916 et SPIEGELBERG, Demotische Miscellen, dans Rec. Trav., XXX, p. 150.

\section{STELE D'HARMACHIS}

British Museum, 391 (1000)

Bibliographie:

A Guide to the Egyptian Collection (Sculpture), 1909, p. 267-268:

P. MUNRO, Die spätägyptischen Totenstelen, Äg. Forsch. 25 (1973), p. 340 , pl. 62 , Abb. 212 ;

d'après le Guide:

"Limestone sepulchral stele, with rounded top, of Heru-em-Khut 35 an Erpa, Ha prince, and high-priest of Memphis, the son of Anem-hra and Neb-hra-ankh, sculptured with a scene representing Anemhera pouring out a libation before Osiris. The text states that the funerary ceremonies were performed by Nes-qeti 7 \&, , "his eldest son", the son of the lady Nefer-itet. In the text spaces are left blank for the date of the death of the deceased, and his age, etc.; his son was a priest of Philopator (Ptolemy IV Salt Collection. From Sakkârah."

\section{STELE DE HATHOR-NEFERTIYTI}

\section{Lieu de conservation?}

Bibliographie:

DARESSY, Notes et remarques, dans Rec. Trav., XIV (1893), p. 184, n 82 (premier registre: une femme adore Osiris, Isis et Nephthys); 
Deuxième registre:

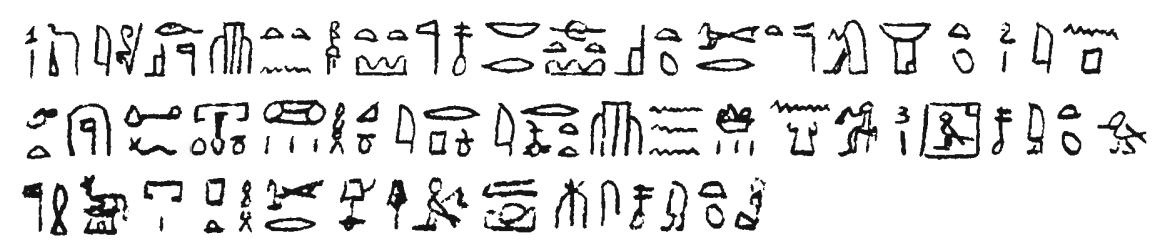

"[1] A réciter par (sic) Osiris qui est à la tête de l'Occident, le dieu bon, seigneur de Ro-setaou, Isis la grande, mère du dieu, Nephthys, [2] Anubis qui est à la tête du seh-neter, pour qu'il (sic) donne la "sortie de voix" pain, bière, vin, lait, libation et résine de térébinthe au ka auguste (3) de Hathor-Nefertiyti, fille du prophète et supérieur des secrets du temple de Ptah, grand des artisans-chefs Harmachis et née de Nefertiyti".

\section{SARCOPHAGE D'HARMACHIS}

\section{Leyde L 9}

Bibliographie:

BOESER, Beschreibung des ägyptischen Sammlung, VII, 1915, pl. IX, a, b, c (photo):

I. Sur le couvercle:

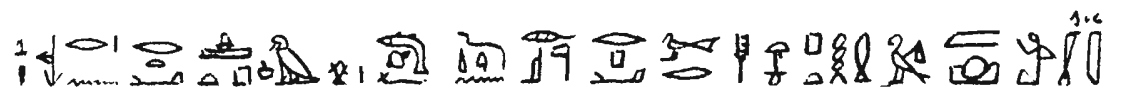

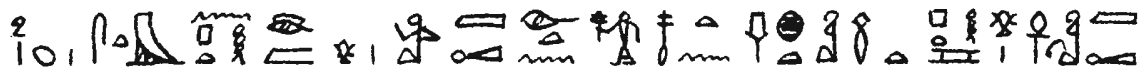

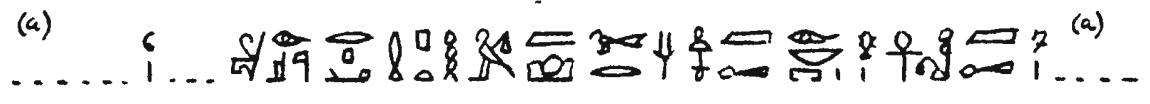

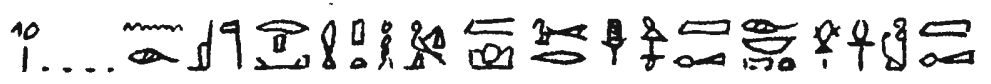

(a) Suite du chapitre 89 du Livre des Morts. 
"[1] Chapitre pour faire reposer l'âme sur son corps. A réciter par l'Osiris, noble, grand des chefs des artisans, serviteur de Ptah, Harmachis, juste de voix, [2] fils du setem de Ptah Anemher, juste de voix et né de la musicienne accomplie de Sekhmet la grande et l'aimée de Ptah, Harunchis, juste de voix: ... [6] ... de l'Osiris, noble, serviteur de Ptah, Harmachis, le grand des chefs des artisans, juste de voix, né de celle qui possède une maison, Harunchis, juste de voix; [7] ... ...[10]... de l'Osiris, noble, serviteur de Ptah Harmachis, le grand des chefs des artisans, juste de voix, né de celle qui possède une maison, Harunchis, juste de voix".

II. A l'extérieur de la cuve, sur la paroi de tête: en deux représentations symétriques, Harmachis adore, à gauche pour le spectateur, Horakhti, et à droite Atoum.

Légende d'Harmachis à gauche:

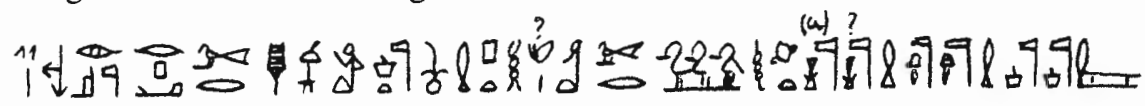

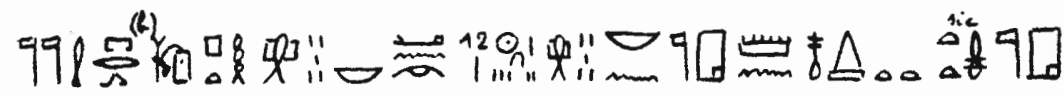

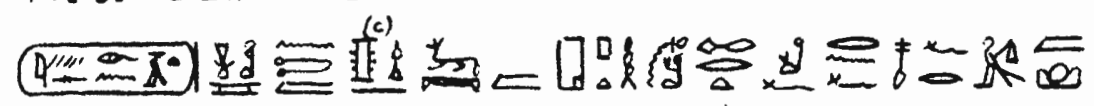

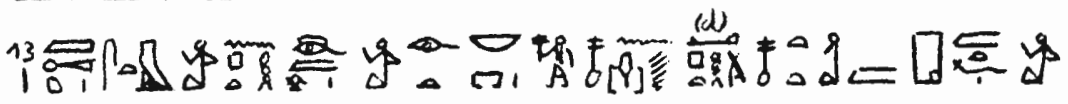

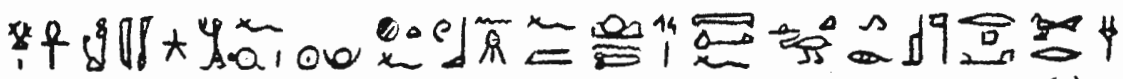

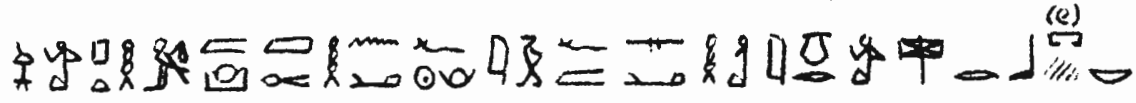

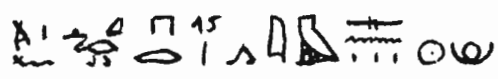

(a) - (b) Ce passage est laissé en blanc dans l'inscription symétrique.

(c) Idem dans l'inscription symétrique.

(d) $\infty$ au lieu de

(e) $d \xi^{\prime}$ dans l'inscription symétrique. 
"[11] L'Osiris, noble, grand des chefs des artisans, père divin, sem, serviteur de Ptah qui prête l'oreille, le Puissant et Bien-odorant, serviteur des dieux Adelphes, serviteur des dieux Evergètes, senviteur des dieux Philopators, serviteur des dieux Epiphanes, scribe de Ptah de toutes les cinquièmes phylai, ân du mois [12] des quinze jours de toutes les cinquièmes phylai du temple de Memphis et également du temple d'Arsinoé-Philadelphe qui est dans le Mur Blanc, supérieur des secrets dans le temple de Ptah, ouab dont le rôle est grand (a), de son beau nom Harmachis, [13] juste de voix, fils du setem de Ptah Anemher et né de celle qui possède une maison, la musicienne accomplie de Sekhmet la grande et l'aimée de Ptah, (la femme) belle de formes dans le temple à côté de mon père (b), Harunchis, juste de voix ! Il adore Rê chaque jour quand il se lève à l'horizon du [14] ciel, afin qu'il fasse entrer l'Osiris, noble, grand des chefs des artisans, serviteur de Ptah Harmachis, juste de voix avec lui chaque jour, et être un dignitaire, certes (?), allant (?) en tout lieu où il désire entrer et dont il désire sortir, chaque jour".

(a) Ou faut-il voir un nom propre dans Ouab-âa-irit-ef ?

(b) Cf. ligne 20 ci-dessous.

La légende d'Harmachis à droite (lignes 15 à 20) répète d'abord, de la ligne [15] au milieu de la ligne [19], les lignes [11] à [14], à quelques exceptions près; celles-ci ont été signalées dans l'appareil critique des lignes [11] à [14]; cette légende de droite se termine par:

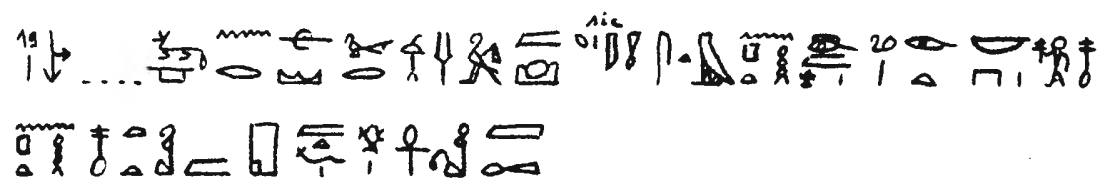

"[19] (allant (?) en tout lieu où il désire entrer et dont il désire sortir, chaque jour); le supérieur des secrets de Ro-setaou, grand des artisans-chefs, Harmachis, juste de voix, fils du setem de Ptah Anemher [20] et né de celle qui possède une maison, la musicienne accomplie de Ptah, la belle de formes dans le temple à côté de mon père (a), Harunchis, juste de voix". 
(a) Les termes de la ligne [20] montrent que l'épithète "belle de formes" n'est pas décernée à Sekhmet comme les lignes [13] et [18] (semblable à [13]) pourraient le faire croire.

III. A l'extérieur de la cuve, sur la paroi de pied: en deux représentations symétriques, Harmachis adore, à gauche pour le spectateur, Oupouaout de Basse-Egapte, et, à droite, Oupouaout de Haute-Egypte, sous forme de deux animaux d'Oupouaout sur pavois, dessinés devant le naos dont ils ont été sortis; la légende de gauche:

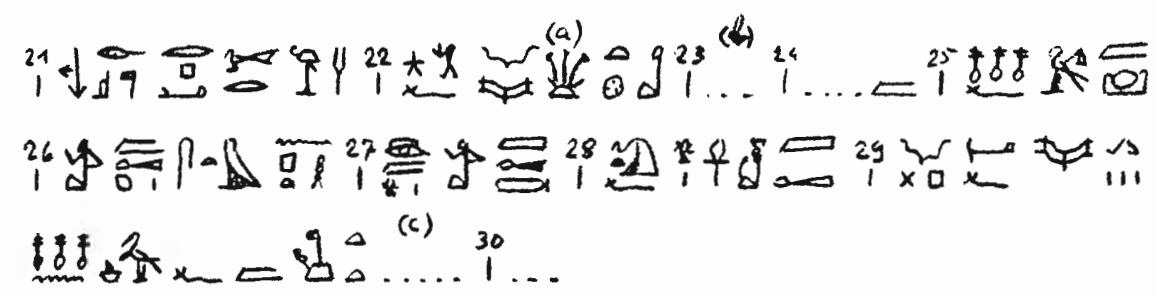

(a) La légende de la scène de droite a "Haute-Egypte".

(b) Les épithètes décernées à Oupouaout varient d'une légende à l'autre.

(c) La fin de la colonne [29] et la colonne [30] contiennent des voeux pour l'au-delà.

"[21] L'Osiris, noble, grand des artisans-chefs [22] adore Oupouaout de Basse-Egypte, [23] ... [24] ..., dans [25] sa beauté, Harmachis, [26] juste de voix, fils du setem de Ptah, [27] Anemher, juste de voix, le nom [28] de sa mère étant Harunchis, juste de voix. [29] Puisse-t-il ouvrir de beaux chemins à son âme dans le kher-neter etc...".

La légende de droite est identique à celle de gauche, sauf dans les épithètes décernées à Oupouaout.

\section{DEUX STATUES DE PSENPTAIS}

\section{Alexandrie ?}

Bibliographie: 
BRECCIA, Les fouilles dans le Sérapéum d'Alexandrie, dans Annales du Service des Antiquités, 8 (1907), p. 65:

(deux statues fragmentaires identiques avec inscription dorsale) (texte et traduction, les deux par Maspero):

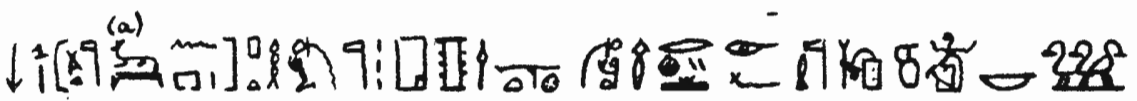

К스스.

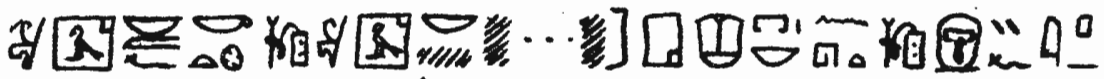

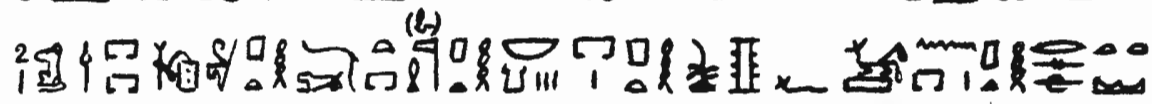

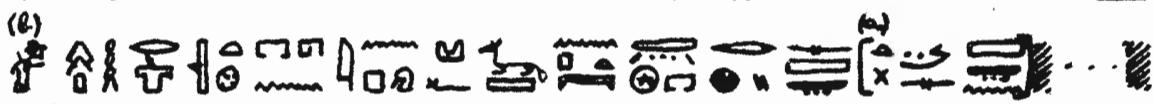

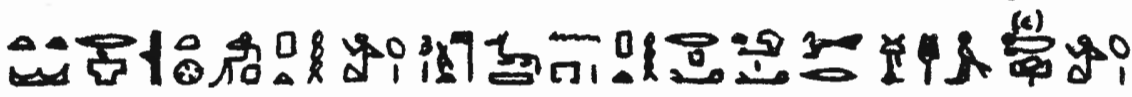

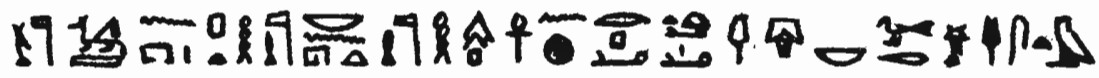

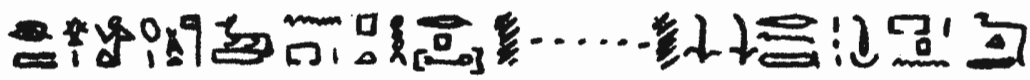

(a) Passage restitué d'après la seconde statue.

(b) Manque sur la seconde statue.

(c) Seconde statue:

"[1] (Le père divin aimé du dieu, supérieur des secrets du temple) de Ptah, ouab des dieux du temple (a) du Mur Blanc, ouab ayant une grande science de ce qu'il fait (b), prophète et scribe de Khnoum, seigneur de Semen-Hor, scribe de Min, seigneur de Sekeret, scribe (c) et prophète d'Horus-Rê seigneur de Sekhebet et de Sokar seigneur des Châteaux d'Horus, scribe d'Hathor, maîtresse du pays de la mefekat, scribe d'Hathor maîtresse ........... demeure de fête du domaine de la Maîtresse du Sycomore, scribe de son Pharaon, comptable (?) [2] et scribe du trésor, scribe de Ptah, directeur de 
... (d) et prophète de Ptah seigneur des aliments du temple de Ptah qui est au sud de son mur, supérieur des secrets du temple de Ptah, de Ro-setaou, d'Osiris et d'Apis (e) de Ro-qedit et du domaine du coffre d'Anubis qui est sur sa montagne, supérieur des secrets du ciel, de la terre et de la douat, celui qui connaît les secrets, (celui qui voit les secrets) ...... Ro-qedit, Psenptaïs (f), fils [3] du père divin aimé du dieu, supérieur des secrets du temple de Ptah, noble gouverneur, grand des artisans-chefs, Harmachis, fils du père divin aimé du dieu, supérieur des secrets du temple de Ptah, prophète de la Maîtresse du Sycomore, prophète d'Apis vivant, noble, gouverneur, chef de toute la garde-robe, grand des artisans-chefs, setem, Anemher, fils du père divin aimé du dieu, supérieur des secrets du temple de Ptah, noble, ............... que ces noms demeurent (dans) ce temple éternellement!"

(a) Ou "ouab des temples du Mur Blanc" ?

(b) Litt.: grand quant au savoir ce qu'il fait.

(c) Je ne sais s'il faut voir dans un mot séparé ou plus simplement un complément

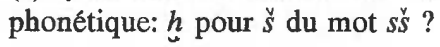

(d) Cf. 203,a, note (a).

(e) Plutôt qu’Osiris-Apis; cf. 203a,1.

(f) P3-sr-n-pth, en grec: $\Psi$ ere $\Psi^{\text {Tas. }}$. Wevrrats

199. STELE DE PETOBASTIS II

\section{Vienne}

Bibliographie:

KRALL, Studien zur Geschichte des alten Ägypten, dans Sitzungsberichte d. Wien. Akad., Phil.-hist. Klasse, Band CV (1883), p. 373-379 (traduction); (Cette stèle, entièrement en démotique, comporte 26 lignes à l'encre noire, très effacées, constate son éditeur). 


\section{STELE DE PSENPTAIS II}

\section{British Museum 886 (1026) (Stèle "Harris")}

Bibliographie:

REINISCH, Aegyptische Chrestomathie, 1873, pl. 21, (texte et bas-relief du premier registre);

BRUGSCH, Thesaurus, V, p. 940-944 (texte) et p. VIII-IX (traduction en allemand);

BRUGSCH, Dictionnaire géographique, p. 654-657 (texte et traduction en français, des lignes 18 à 22);

BEVAN, Histoire des Lagides, p. 385-387 (traduction);

J. QUAEGEBEUR, Contribution à la prosopographie des prêtres memphites à l'époque ptolémaïque, Ancient Society 3 (1972), p. 97-99: traduction et commentaires;

P. MUNRO, Die spätägyptischen Totenstelen, Äg. Forsch. 25 (1973), p. 341, pl. 63;

d'après Brugsch:

I. Premier registre: Psenptaïs, agenouillé, présente une table d'offrandes à huit divinités:

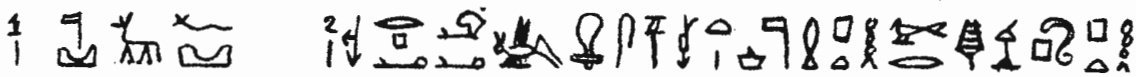

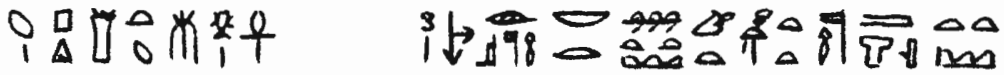

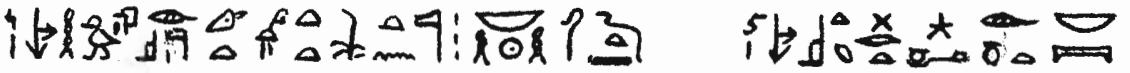

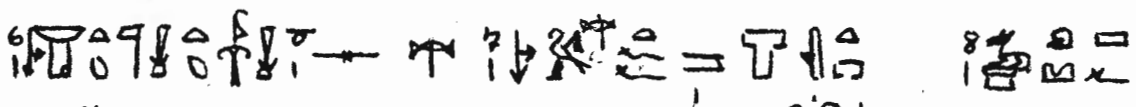

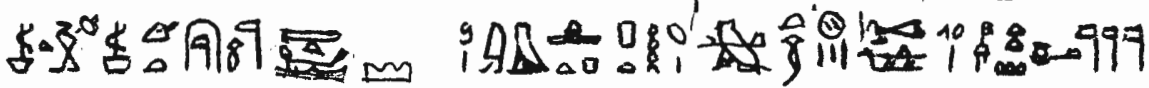

"[1] Adoration répétée quatre fois (a)".

"[2] Le noble, gouverneur, trésorier du roi, compagnon unique, père divin et prophète de Ptah, grand des chefs des artisans Psenptaïs, fils de Petobastis et né de Harunchis".

"[3] Osiris, dieu grand seigneur de Ro-setaou, qui préside à l'Occident, dieu grand dans Ro-qedit".

"[4] Apis-Osinis, qui préside à l'Occident, roi des dieux, seigneur de l'éternité, 
régent du toujours".

"[5] Isis la grande, mère du dieu, oeil de Rê, maîtresse du ciel".

"[6] Nephthys, soeur du dieu, protectrice de son frère".

"[7] Horus assistant de son père dans Ro-qedit".

"[8] Anubis qui est sur sa montagne, qui est dans les bandelettes, grand (?) de bandelettes, qui préside au seh-neter, seigneur de la Terre sacrée".

"[9] Imhotep fils de Ptah, "[10] L'Occident mère des dieux (c)".

(a) $d w 3-n t \underline{r} s p f d w$.

(b) Cf. 201,9 et note (c).

(c) Chacune des divinités adresse la parole à Psenptaïs sous la forme: je te donne + un avantage dans l'au-delà.

II. Deuxième registre (d'après Brugsch):

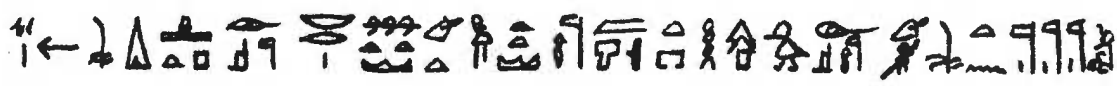

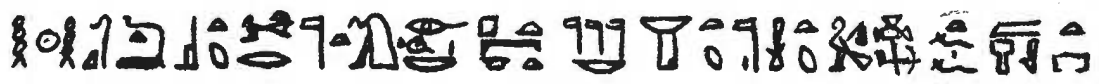

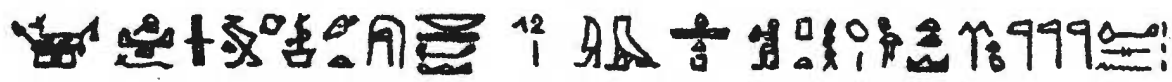

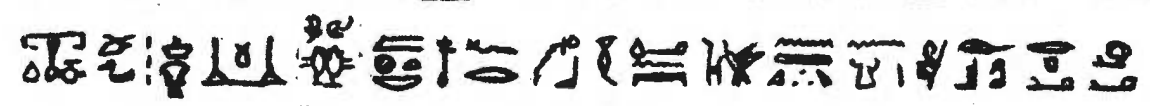

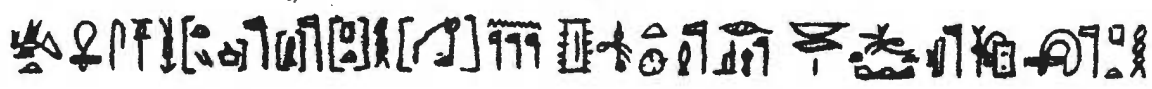

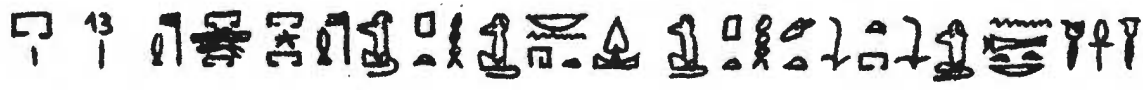

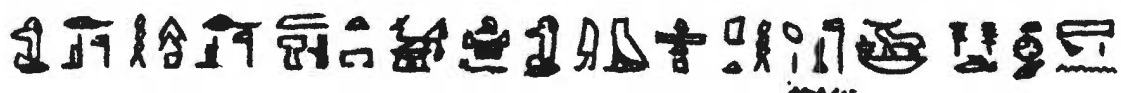

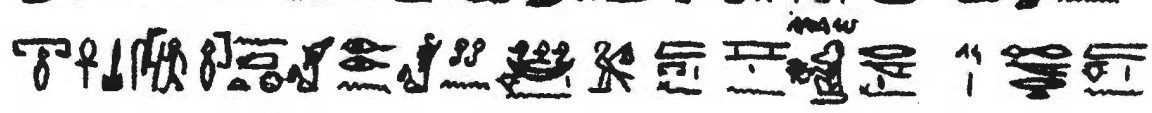

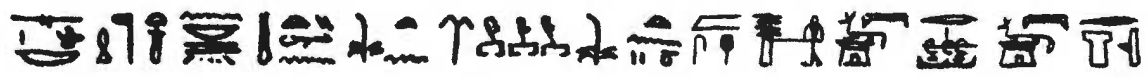




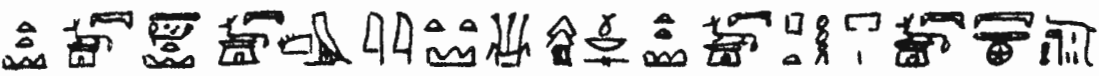

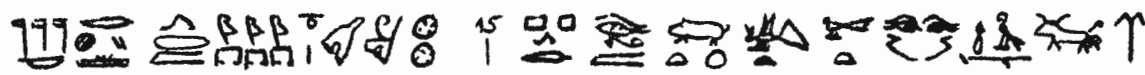

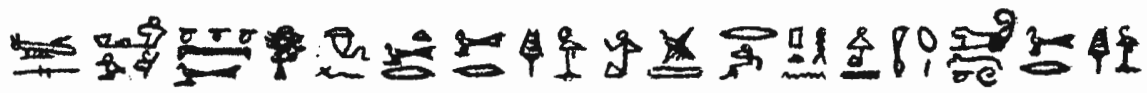

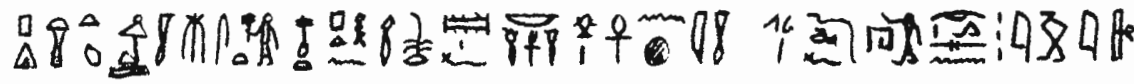

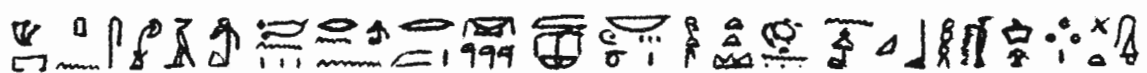

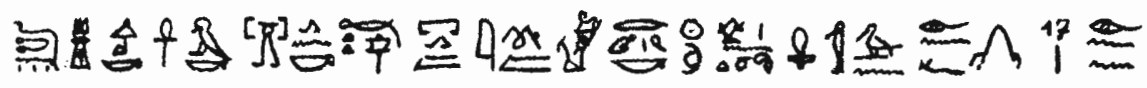

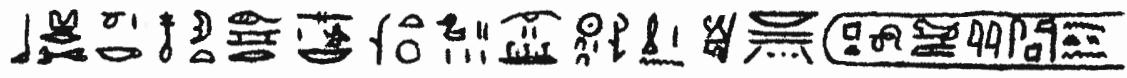

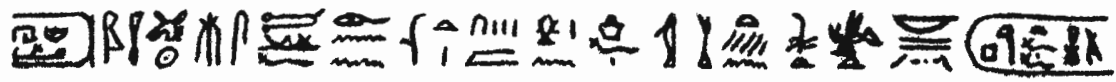

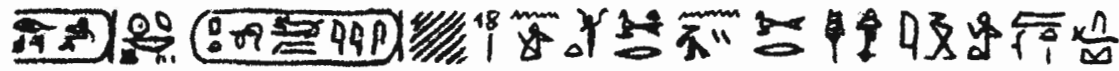

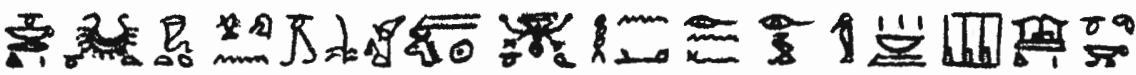

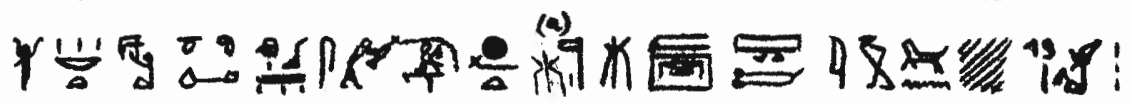

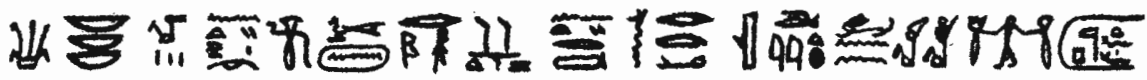

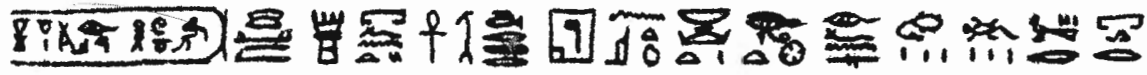

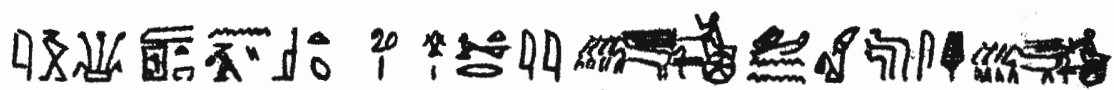

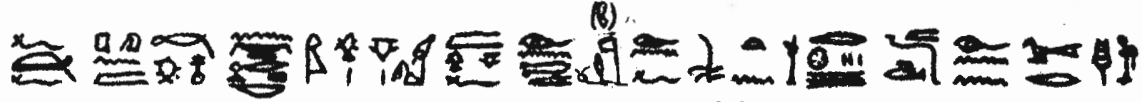

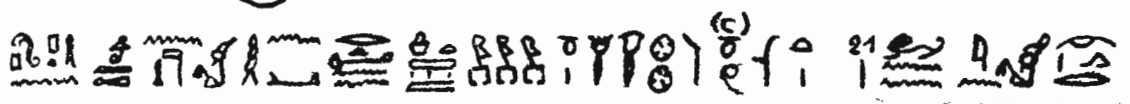

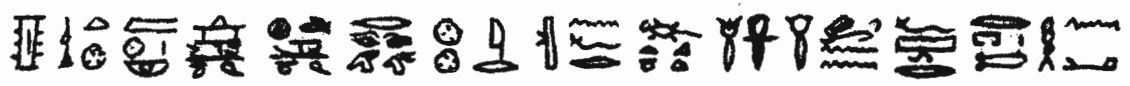

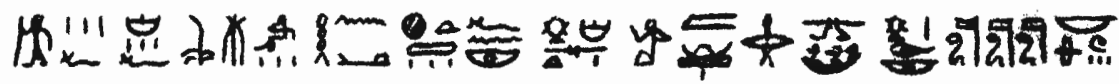


ㅅำ

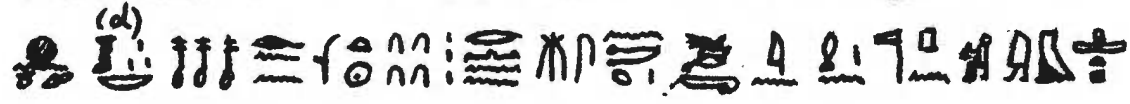

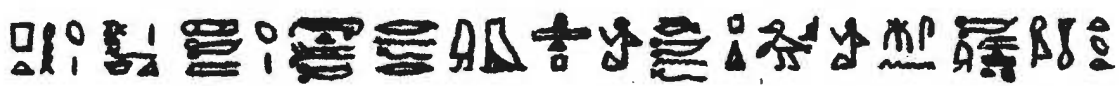
군

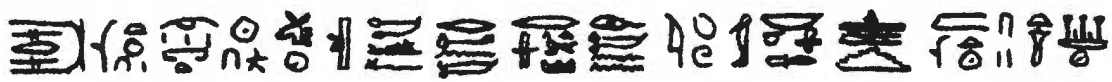

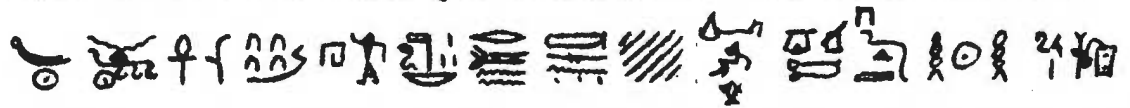

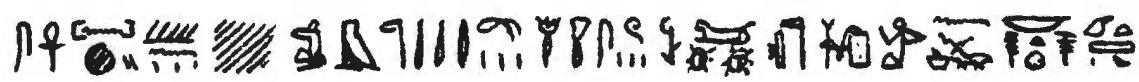

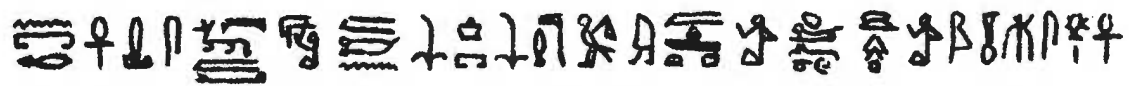

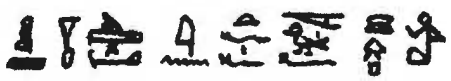

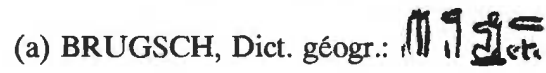

(b) ibid: : II

(c) ibid: :

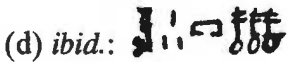

"[11] Offrande royale d'Osiris seigneur de Ro-setaou, qui est à la tête de l'Occident, dieu grand dans Ro-qedit, d'Apis-Osiris, dieu grand qui est à la tête de l'Occident, roi des dieux, roi d'étermité, régent du toujours, d' I'sis la grande, mère du dieu, oeil de Rê, maitresse du ciel, dame de tous les dieux, de Nephthys, soeur du dieu, d'Horus assistant de son père dans Ro-qedit, d'Anubis qui est sur sa montagne, qui est dans les bandelettes, qui est à la tête du seh-neter, seigneur de la Terre sacrée, [12] d'Imhotep, fils de Ptah, de l'Occident, mère des dieux, afin qu'ils donnent la "sortie de voix" - pain, bière, boeufs, volatiles, résine de thérébinthe huile, vases d'albâtre, pièces d'étoffes, des milliers de toutes choses bonnes, pures et douces que le ciel donne et que la terre crée - au ka de l'Osiris, noble, gouverneur, trésorier du roi, compagnon unique, père divin, prophète de Ptah, ouab des dieux du 
Mur Blanc, prophète d'Osiris, dieu grand seigneur de Ro-setaou, hiérogrammate, trésorier divin du temple de Ptah, [13] prophète de la bibliothèque et de la Maison du matin, prophète et scribe de Ptah, scribe de la Maîtresse du Sycomore, scribe de Ptah qui est à la tête de Tanenet, scribe de Bastet maitresse d'Ankh-taoui, scribe d'Osiris-Apis et d'Osiris (a) dans Ro-qedit, d'Anubis qui est sur sa montagne, scribe d'Imhotep fils de Ptah, prophète du Bélier seigneur de Mendès, khenti-ché de Pharaon, V.S.F., grand prince de l'Egypte, yeux du roi du sud, oreilles du roi du nord, confident de l'Horus dans son Palais, aimé du roi (b) plus que son aimé, [14] celui dont l'amour est grand dans le coeur de chaque homme, premier prophète du maître des Deux Pays, bâton du roi dans les temples, second du roi quand on dresse le Pilier Djed, supérieur des secrets de Ro-setaou, supérieur des secrets de Ro-qedit, supérieur des secrets de la Terre sacrée, supérieur des secrets de Kêmit et de Hapnebes, supérieur des secrets du temple de Ptah, supérieur des secrets du ciel, de la terre et de la douat, directeur des prophètes de tous les dieux et connaissant ... pas (?) dans les temples de Haute et de Basse-Egypte, [15] celui qui a accès (?) auprès de l'Oudjat au moment du grand travail (?), celui qui voit le dieu grand (?) enfanté par son travail (?), celui qui fait offrande au (?) fils du grand Nouou et (?) à (?) Sekhmet (?), sa grande mère, le grand des chefs des artisans, Psenptaïs, juste de voix, fils de celui qui porte les mêmes titres, le grand des chefs des artisans Pétobastis, juste de voix, et né de la musicienne accomplie de Ptah le grand qui est au sud de son mur, seigneur d'Ankhtaoui, Harunchis, juste de voix!

[16] Il dit: $O$ vous qui viendrez jusqu'à ce tombeau, rappelez mon (c) nom au seigneur des dieux dans toutes les fêtes de l'Occident, offrez(d)-moi de l'eau fraîche et de la résine de térébinthe sur le feu, et dites: Que ton ...(e) (?) soit divin, que ton âme vive, que le vent du nord sorti d'Atoum (f) soit porté chaque jour à ton nez! Parce que je suis un mort glorieux auquel on doit faire des offrandes (g), car je n'ai pas (17) fait de mal mais j'ai eu bon caractère, faisant ce que chacun aime!

L'an 25 (h), deuxième mois d'Akhet, 21ème jour sous la Majesté du roi de Haute et Basse-Egypte Ptolémée-le dieu Sauveur juste de voix est le jour où je suis né. J'ai passé 13 années en présence de mon père. Le roi de Haute et de Basse-Egypte, seigneur des Deux Pays, Le dieu Philopator-PhiladelpheNeos-Dionysos (i), le fils de Rê, maitre des apparitions Ptolémée ordonna que me fût (donnée) (j) [18] la grande fonction de grand des chefs des artisans, alors que j'étais dans ma quatorzième année. J'ai mis le large collier divin au cou du roi le jour où il réunit les Deux Pays et accomplit 
toutes les cérémonies dans les demeures du Sed. J'ai rempli toutes les fonctions secrètes. J'ai donné l'ordre d'asperger l'Horus quand le dieu naît dans la Maison de l'Or.

Je me suis rendu $(\mathrm{k})$ à la résidence [19] des rois grecs qui est au bord (1) de la mer à l'ouest de Aqâ et dont le nom est Râqôtis (m). Le roi de Haute et Basse-Egypte, seigneur des Deux Pays, Le dieu Philopator-Philadelphe-NeosDionysos, étant sorti de son palais vivant et florissant, arriva au (n) temple d'Isis maîtresse de Iat-Oudjat. Il lui fit des offrandes (o) nombreuses et grandes. Etant sorti du (p) temple d'Isis [20] sur son char, le roi arrêta luimême son char. Il coiffa ma tête (q) d'une belle couronne d'or et de toutes sortes de pierres précieuses véritables avec (?) un coeur du roi (r) en son milieu (s). Je fus nommé son prophète. Il adressa un ordre royal aux villes et aux nomes, disant: J'ai fait du grand des chefs des artisans Psenptaïs, juste de voix ( $\mathrm{sic}$ ) mon prophète (t), et des pains provenant des temples de Haute et Basse-Egypte m'ont été donnés chaque année.

[21] Le roi arriva au Mur Blanc en un jour de fête. Il vogua en amont et en aval pour voir ... (u). Quand il eut abordé à ... (?) d'Ankh-taoui, il alla au temple de Tje (?) avec ses grands, ses femmes et les enfants royaux, ainsi que tout son appareil de fête, puis il s'assit dans ma barque et parcourut les marais (?), puis il célébra la fête de tous les dieux et déesses qui sont dans Khâ-nefer, à cause de la grandeur de l'amour [22] qui est au coeur du maitre des Deux Pays, sa divine couronne blanche étant à son front.

J'ai été un grand riche (v) en toutes sortes de biens. J'ai eu de belles "concubines". J'ai passé 43 ans sans qu'il (me) naquît un enfant mâle. C'est la Majesté de ce dieu auguste Imhotep fils de Ptah qui m'a fait cadeau d'un fils (w) mâle dont le nom est Imouthès, appelé Petobastis, qu'a enfanté Taimouthès, juste de voix, fille du père divin, [23] prophète d'Horus seigneur de Létopolis, Khâhapi.

Sous la Majesté de la Régente et maîtresse des Deux Pays Cléopâtre et de son fils César, l'an 11, 3ème mois de Peret, 15ème jour est le jour où je suis mort. J'ai été déposé dans la nécropole et l'on a accompli pour moi tous les rites d'une momie parfaite. Mise au tombeau en l'an 12, 1er mois d'Akhet, dernier jour. Total de la durée de vie: 49 ans.

$O$ vous tous, dieux et déesses dont j'ai dit les noms au complet (x) ... faites que (je) sois jeune (?) sur le trône de Tje (?) éternellement!

[24] Le scribe qui fait vivre leurs noms, ... parlant (?) d'après les livres des paroles divines des Deux Pays, ... (?) auprès du maître des Deux Pays, hiérogrammate du Bélier seigneur de Mendès, khenty-ché de Pharaon V.S.F., supérieur des secrets dans la chambre cachée dans Tenenet, 
prophète d'Horus, Imouthès, fils de celui qui porte les mêmes titres, Khâhapi, juste de voix, et né de Harunchis, juste de voix !

Gravé par son père, qui aime son fils, Khâhapi !"

(a) Plutôt que "d'Osiris et d'Apis-Osiris", par comparaison avec 192,6, "La demeure divine d'Osiris-Apis".

(b) Wört. II, 325 n'indique pas cette graphie de $n s w t$, mais elle peut être déduite de \$1 "roi de Haute et Basse-Egypte" signalé dans Wört. II, 330; on retrouve une graphie analogue sur la stèle de Taimouthès, la femme de Psenptaïs.

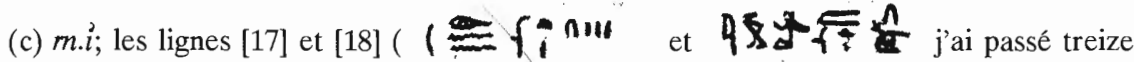
années, j'étais dans ma quatorzième année) montrent clairement que le pronom suffixe

de la lère personne du singulier peut être écrit mur sur cette stèle. Un examen des cas où le suffixe de la lère personne du singulier a été employé révèle que celui-ci est rendu soit par un . , soit par soit peut-être par I (ligne [21]: "ma (?) barque"), soit encore qu'il n'est pas écrit (ligne [23]: cet examen ne permet pas de trouver une raison à l'emploi de l'un de ces modes d'expression plutôt que l'autre. L'origine de l'équivalence inmm $=i$ reste aussi une énigme pour moi. Je ne vois que l'hypothèse d'un jeu graphique à proposer pour la solution de ce problème, jeu qui aurait consisté à tirer mar suppression des trois traits du pluriel dans le suffixe $m$ î de la 1ère personne du pluriel; le seul appui que je puisse donner à cette explication est le suivant: nמm n'est employé avec la valeur $\vec{i}$ que dans des cas où $\hat{l}$ est le suffixe de la 1ère personne du singulier. Cet emploi de appartient peut-être au Nouvel Empire; cf. G. NAGEL, Un papynus funéraire ..., dans

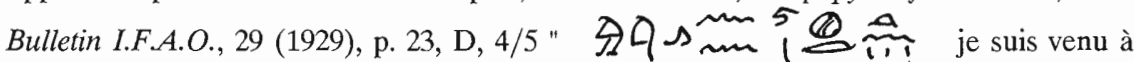
vous", où l'auteur de l'article ne voit qu'une "orthographe fautive" (ibidem, p. 25).

(d) wšn, Wört., I, 374, 7 .

(e) $\dot{\hat{\leftrightarrow}}=$ "corps" (hl3t) ? Ou bien faut-il comparer ce passage à Wörterbuch, II, Belegstellen de la page 364, 10: "Que mon âme vive, que mon esprit (3h) soit divin" (Urk., IV, 945)?

(f) Bra $_{1}=t$ par affaiblissement de $w$ et $r$ ?

(g) hr-nt(t) ink $s^{c} h$ ?h $n(y) \operatorname{lr}(t) n . f$

(h) $\mathcal{Z}=d(w) t$

(i) Litt.: Osiris le Jeune. 
(j) Restituer [ is i à la fin de la ligne [17].

(k) $\breve{s} m t .(i)$, infinitif de narration.

(l) $i b d$ pour $l d b$, rive, rivage ?

(m) nty $r n . f r R^{c}-k d y t$

(n) $d r t 3 r$, Wört. I, 387, 17.

(o) w̌̌nw, substantif formé du verbe signalé sous (d) ?

(p) $p r r-h 3 m$

(q) $m \underline{h}$ h.f tp.l (voir (k) ci-dessus).

(r) Un scarabée, ou plus simplement une amulette en forme de coeur avec le nom du roi ?

(s) Au milieu de la couronne?

(t) ir.n.l wr hrp ḥmw P-š-n-pth $n$ (pour $m$, cf. JUNKER, Grammatik des Dendera-Texte, § 25) hm.i-ntr.

(u) $s s, s 3 s$ ? Serait-il trop hasardé de voir dans "Il vogue en amont et en aval pour voir ..." le nom de la fête à laquelle le roi est arrivé ?

(v) spss

(w) $h r f k 3 t(. i) m$

(x) Litt.: j'ai complété leurs noms, ou: leurs noms sont complets pour moi. Il s'agit des divinités invoquées au premier registre et au début du second.

\section{STELE DE TAIMOUTHES}

British Museum, 147 (1027)

Bibliographie:

A general introductory guide to the Egyptian collections, 1930, p. 216 (bonne photographie médiocrement reproduite); ibidem, p. 214 et 217, traduction des lignes 15-21;

REINISCH, Aegyptische Chrestomathie, 1873, pl. 20 (avec la fausse indication "stèle in Paris") (dessin); 
BRUGSCH, Thesaurus, p. 918-927 (texte; traduction des lignes 1-15 et 21; la traduction des lignes $15-21$, annoncée à la p. 926, ne se trouve pas dans la partie imprimée du Thesaurus, p. VIII);

MASPERO, dans Journal Asiatique, 7ème série, tome XV (1880), p. 411416 (traduction des lignes 5-21);

J. QUAEGEBEUR, Contribution à la prosopographie des prêtres memphites à l'époque ptolémaïque, Ancient Society 3 (1972), p. 93-96, traduciton et commentaires;

P. MUNRO, Die spätägyptischen Totenstelen, Äg. Forsch. 25 (1973), p. 338 (pas de pl.);

d'après Brugsch:

I. Premier registre: Taimouthès adore Sokar-Osiris, Apis-Osiris, Isis, Nephthys, Horus assistant de son père, Anubis et le Beau Désert Occidental.

II. Deuxième registre: (d'après Brugsch):

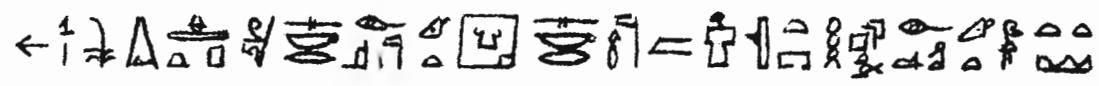

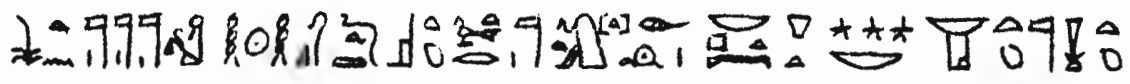

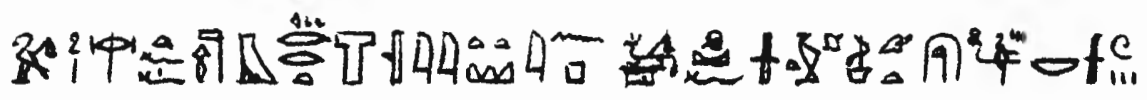

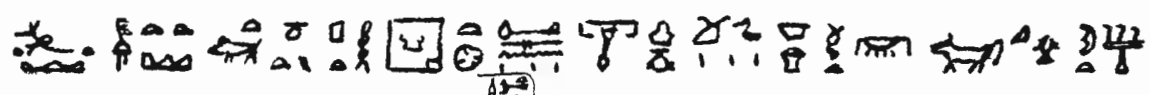

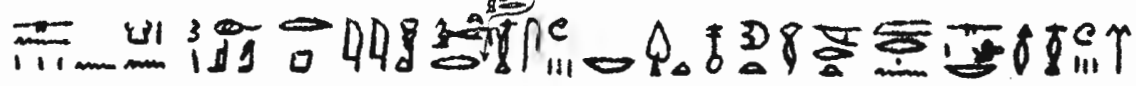

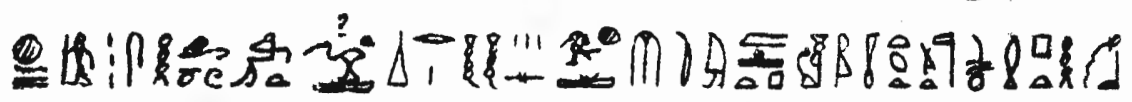

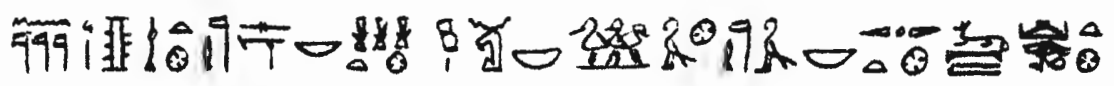

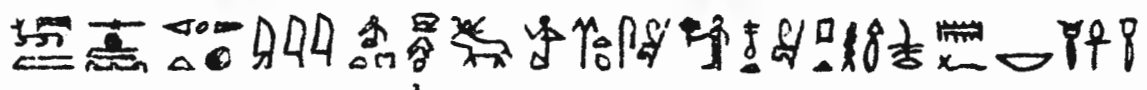

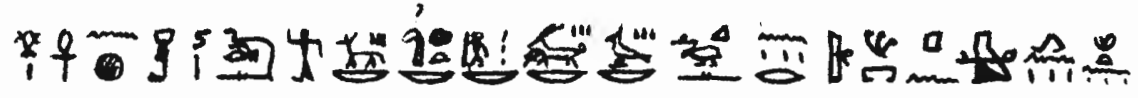

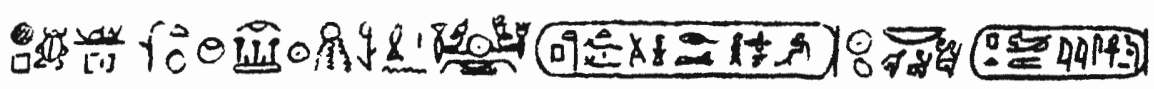




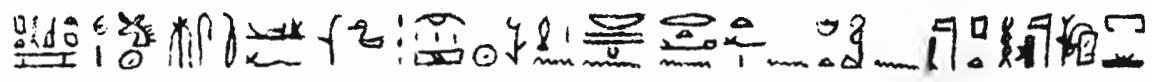

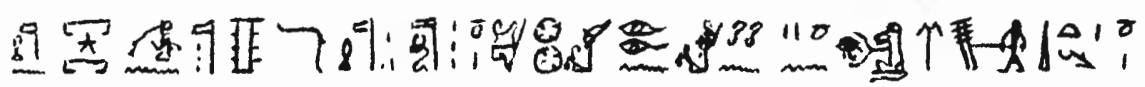

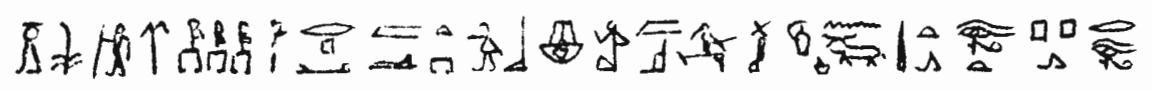

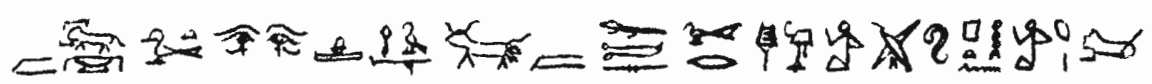

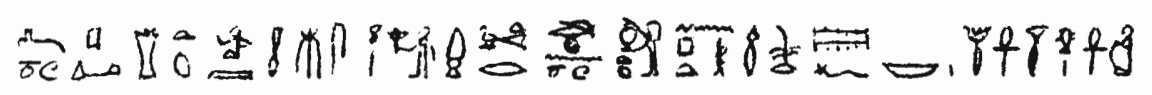

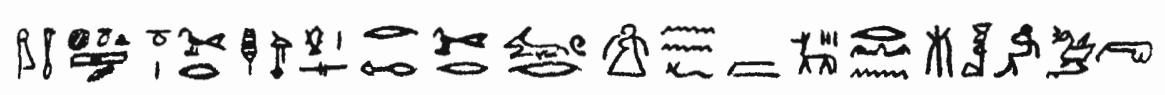

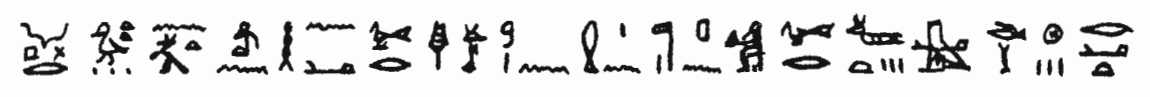

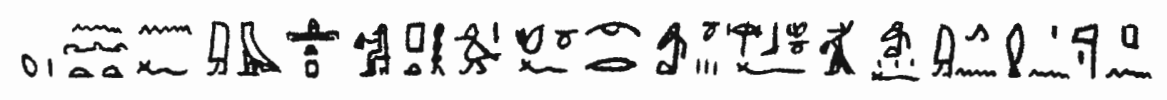

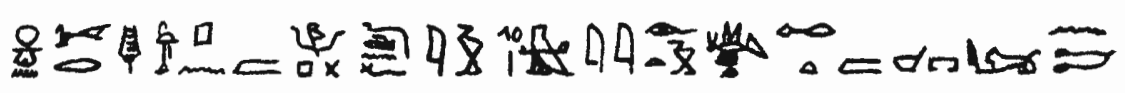

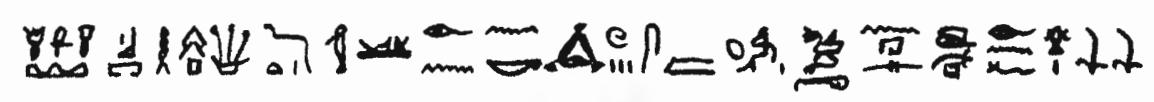

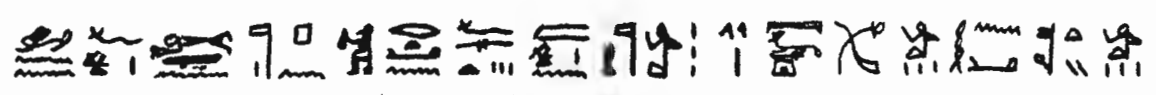

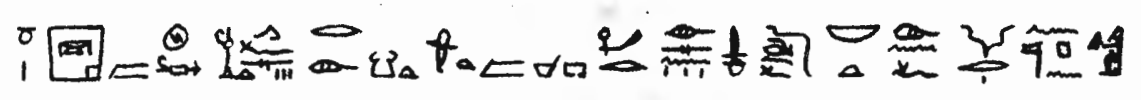

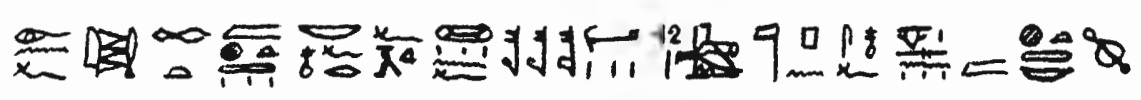

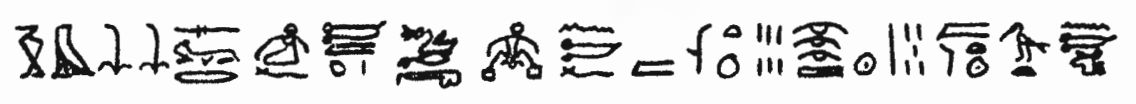

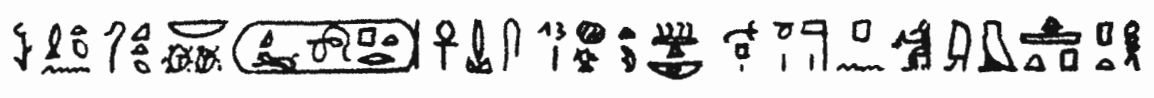

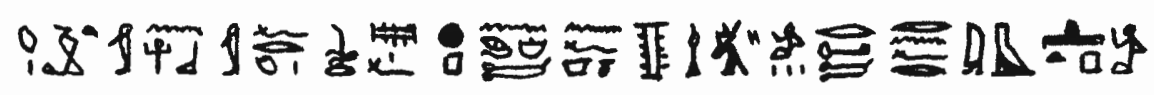

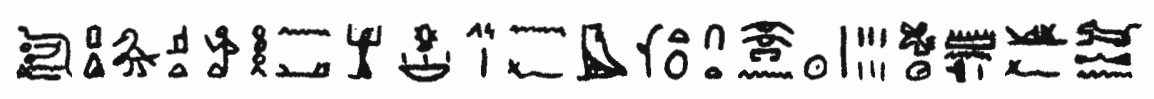

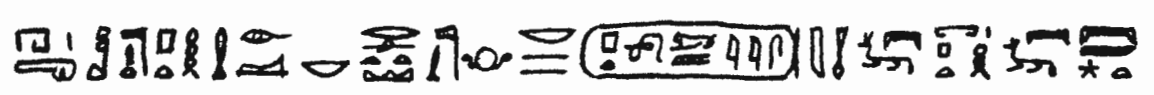




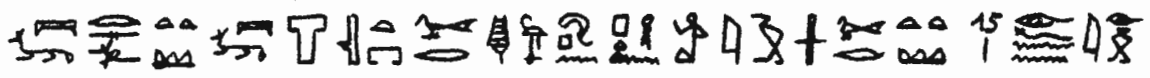

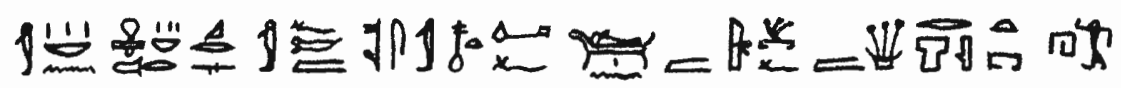

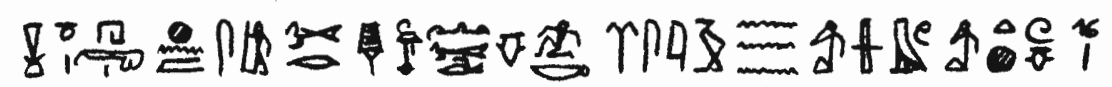

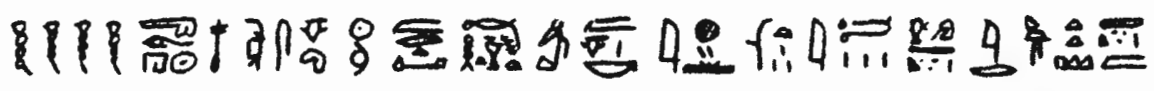

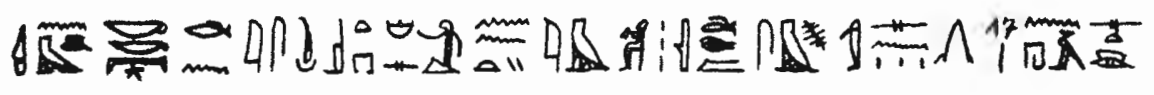

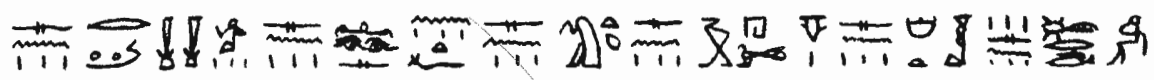

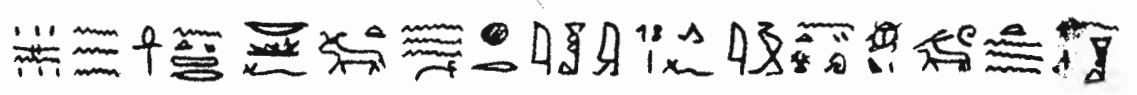

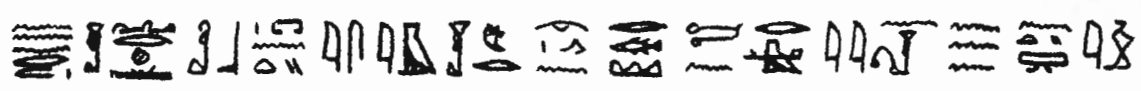

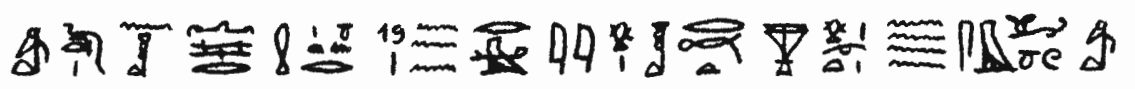

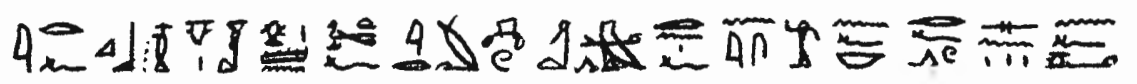

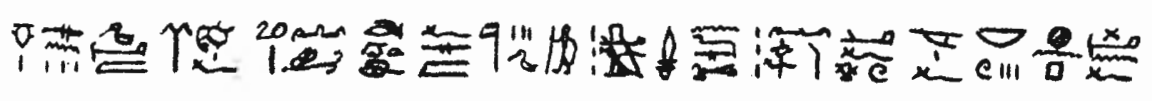

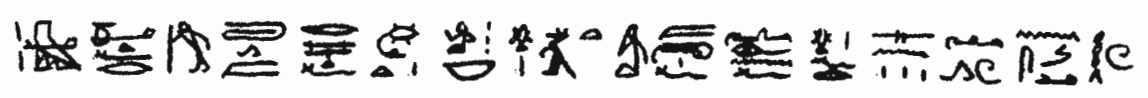

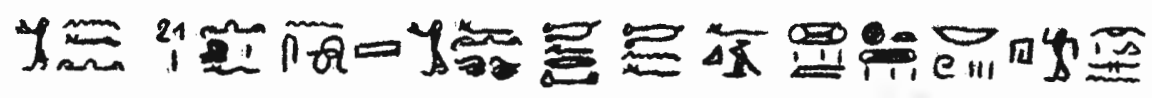

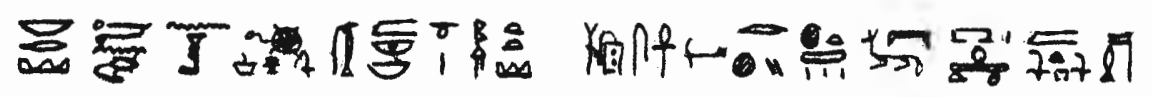

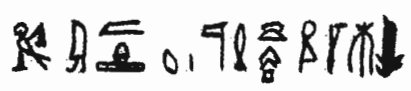


"[1] Offrande royale à Sokar-Osiris qui est à la tête du Château de ka de Sokar, dieu grand dans Ro-qedit, (à) Apis-Osiris qui est à la tête de l'Occident, roi des dieux, roi de l'éternité et régent de toujours, (à) Isis la grande, mère du dieu, oeil de Rê, maîtresse du ciel et souveraine de tous les dieux, (à) Nephthys soeur du dieu, (à) Horus [2] assistant de son père, dieu grand dans Ro-qedit, (à) Anubis qui est sur sa montagne, qui est dans les bandelettes, qui est à la tête du Seh-neter, (à) tous les dieux et déesses qui sont dans le Beau Désert Occidental de Het-ka-ptah, pour qu'ils donnent la "sortie de voix" - pain, bière, boeufs, volatiles, résine de térébinthe, huile et toutes les bonnes (?) choses (?) qui paraissent (?) sur leurs autels - au ka de [3] l'Osiris, la noble, grande par les ornements et grande par les récompenses, la maîtresse de grâce, parfaite de caractère et douce d'amour dans la bouche de tout le monde, grande par les louanges (qu'elle reçoit) de ses amis, la jeune qui sait trouver (?) et habile à parler agréablement (?), qui fait resplendir (?) la salle du conseil (?), Taimouthès, juste de voix, fille du père divin aimé du dieu, sem, serviteur de Ptah, ouab des dieux [4] du Mur Blanc, prophète de Min seigneur de Senout et de Khnoum seigneur de Semenou-hor, prophète d'Horus seigneur de Létopolis, supérieur des secrets dans Iat-Oudjat, supérieur des secrets dans Létopolis et dans Iyt, Khâhapi, et née de la musicienne accomplie de Ptah le grand qui est au sud de son mur, seigneur d'Ankh-taoui, Harunchis.

[5] Elle dit: $O$ juges, ... (a), grands, dignitaires, gens du peuple, vous tous qui entrerez dans ce tombeau, venez et écoutez ce qui m'est advenu! L'an 9, premier mois d'Akhet, neuvième jour, sous la Majesté du roi de Haute et Basse Egypte et maître des Deux Pays, le dieu PhilopatorPhiladelphe-Neos-Dionysos, fils de Rê, maître des apparitions, Ptoléméevivant-éternellement, aimé de Ptah et d'Isis [6] est le jour où je suis née. L'an 23, troisième mois de Chemou, premier jour, sous la Majesté de ce Maître des Deux Pays, mon père me donna en mariage au prophète de Ptah, hiérogrammate, prophète de la bibliothèque et de la Maison du Matin (b), ouab des dieux du Mur, directeur des prophètes des dieux et déesses de Haute et Basse-Egypte, yeux du roi du sud, oreilles du roi du nord, second du roi quand on dresse le Pilier Djed, bâton du roi dans les temples, [7] noble dans la Place de Geb, prêtre-lecteur dans la Place de Thot, celui qui renouvelle le façonnage (?) du Bélier, celui qui ... (?) l'Oudjat, celui qui a accès auprès de l'Oudjat au moment (?) du grand ... (?), celui qui voit reposer le dieu grand enfanté par son travail, le grand des chefs des artisans Psenptaïs, fils de celui qui porte les mêmes titres, Petobastis, juste de voix, et né [8] de la musicienne très ornée, donneuse de nythme de Ptah le grand 
qui est au sud de son mur, seigneur d'Ankh-taoui, Harunchis, juste de voix. Le grand des chefs des artisans en fut extrêmement heureux. Je conçus pour lui par trois fois, sans mettre au monde un enfant masculin, mais trois filles. J'implorai, avec le grand des chefs des artisans, [9] la Majesté de ce dieu auguste, grand par ses miracles, ... (c), qui donne un fils à celui qui n'en a pas, Imhotep fils de Ptah. Il entendit notre prière, il écouta les supplications à son adresse. La Majesté de ce dieu vint à la demeure (?) de ce grand des chefs des artisans en songe (d) et lui dit: [10] Fais faire le grand travail dans le Lieu Sacré d'Ankh-taoui, le lieu où mon corps est caché, et je t'en récompenserai par un enfant mâle. (e) Là-dessus, il s'éveilla. Il se prosterna pour ce dieu auguste. Il fit part de ces choses aux prophètes, [11] aux supérieurs des secrets, aux ouab et aux sculpteurs de l'atelier d'orfèvrerie à la fois. Il leur ordonna d'accomplir le travail parfait dans le Lieu Sacré et ils firent selon tout ce qu'il avait dit. Il accomplit l'ouverture de la bouche pour ce dieu auguste. Il lui fit une grande offrande de toutes sortes de bonnes choses. Il récompensa les sculpteurs [12] de la part de (f) ce dieu et réjouit leur coeur avec toutes sortes de choses. En contre-partie de cela, il me (g) rendit enceinte d'un enfant masculin. Il naquit en l'an 6, troisième mois de Chemou, quinzième jour, à la huitième heure du jour, sous la Majesté de la Régente et Maîtresse des Deux Pays Cléopâtre, V.S.F.: [13] c'est la fête des Offrandes-sur-l'autel de ce dieu auguste Imhotep fils de Ptah. Sa forme (h) ressemblait à celle du fils de Celui qui est au sud de son mur. Il y eut ... (i) pour lui de la part (k) des habitants du Mur Blanc. On lui donna le nom d'Imouthès, appelé Petobastis et tout le monde se réjouit [14] à son sujet. L'an 10, deuxième mois de Peret, seizième jour, fut le jour où je mourus. Mon mari, le père divin et prophète de Ptah, serviteur d'Osiris seigneur de Ro-setaou, prophète du roi de Haute et Basse-Egypte, maître des Deux Pays Ptolémée, juste de voix, supérieur des secrets du temple de Ptah, supérieur des secrets du ciel, de la terre et de la douat, supérieur des secrets de Ro-setaou, supérieur des secrets de Ro-qedit et grand des chefs des artisans Psenptaïs (me) déposa (1) dans la nécropole. [15] Il accomplit pour moi tous les rites de toutes les momies parfaites. Il m'ensevelit dans une belle sépulture. Il me fit reposer dans son tombeau derrière Ro-qedit.

$O$ frère, mari, ami, grand des chefs des artisans, ne t'arrête de boire, de manger, de t'enivrer [16] de pratiquer l'amour, de passer heureusement le jour et de suivre ton désir chaque jour! Ne mets pas le chagrin dans ton coeur! Que sont ces (?) années sur terre ? (m) L'Occident est une terre dans le sommeil et l'obscurité. La demeure est écrasante pour ceux qui y sont ! Les morts augustes dorment en leurs formes de momies. Ils [17] ne 
s'éveillent pas pour voir leurs frères. Ils ne voient ni leurs pères ni leurs mères. Leurs coeurs oublient (n) leurs femmes et leurs enfants. L'eau vive que la terre a pour quiconque est en elle, c'est de l'eau ...(o) chez moi! Elle [18] vient vers ceux qui sont sur terre, (mais) elle est ... (o) pour moi, l'eau près de moi! Je ne sais plus où j'en suis (p) depuis que je suis arrivée dans cette vallée. Donnez-moi de l'eau courante en me disant: N'écarte pas ton vase (?) de l'eau! Toumez mon visage vers le vent du nord au bord de l'eau et, sûrement, mon coeur sera délivré (q) de son tourment! Quand celui dont le nom est "Mort complète, viens !" (r) les a tous appelés à lui, ils viennent à lui avec leurs coeurs effrayés par la crainte qu'il inspire. [20] Il n'est personne qui ose le regarder parmi les dieux et les hommes. Les grands sont pour lui comme les petits et il n'y a de résistance contre lui chez aucun de ceux qu'il désire. Il enlève l'enfant (s) à sa mère, aussi bien que le vieillard, en parcourant son chemin de terreur. Tous les hommes le supplient, mais il ne fait pas attention à eux (t). On ne vient pas le supplier [21] car il n'écoute pas quand il est imploré. On ne le voit point (même quand ?) toutes sortes de cadeaux lui sont faits $(\mathrm{u})$.

O vous tous qui viendrez dans ce désert, offrez-moi ... (v), de la résine de térébinthe sur le feu et de l'eau fraîche à toutes les fêtes de l'Occident!

Le scribe graveur, le savant, supérieur des secrets de la maison ...(w) dans la Tenenet, prophète d'Horus, Imouthès, fils du prophète Khâhapi, l'a gravé !"

(a) Brugsch: "hiérogrammates".

(b) En interprétant les deux noms composés dont l'un précède et l'autre suit "prophète" comme des génitifs dépendant de ce dernier.

(c) Brugsch: "glückbringend"; SETHE, Imhotep, ..., p. 20 (Untersuchungen, II, 1902, p. 112) transcrit: " $m^{c} d($ ?)", mais ne traduit pas. Cf. aussi 200,9.

(d) $m$ wpwt $m 3^{c} t$, litt.: en message de vérité (révélée en songe); cf. B. GUNN, Notes on Ammenemes I, dans J.E.A., 27 (1941), p. 2-6.

(e) De "Fais faire ..." à "mâle", traduction dans SETHE, Imhotep, p. 7 (Untersuchungen, II, 1902, p. 99).

(f) Le hiéroglyphe de la patte antérieure de bovidé est employé ici pour l'avant-bras humain; il faut transcrire $m^{-}$; pour la valeur ${ }^{c}$ de la patte de bovidé, cf. LORET, Manuel de la langue égyptienne, 1889, signe $\mathrm{n}^{\circ} 264$. 
(g) $=$ wì, pronom dépendant, 1ère personne (féminin) singulier; la même graphie à la ligne 15. Quant au suffixe .i, 1ère personne féminin singulier, il est tantôt écrit (lignes 5, 14), tantôt (lignes 8, 14, 18, 19, 21) tantôt IJ (ligne 18) ou 9] (ligne 17), tantôt il est rendu par mm comme sur la stèle de Psenptaïs (200,16, et remarque b) (ligne 15 ir.f $n . i$ et $s d r . i$; ligne 18 spr.ì); enfin il s'amalgame avec le $t w$ du passif dans $\pi$ ? $m s . t(w) . i$ (ligne 6). Ailleurs, on peut se demander s'il est exprimé ou rendu par man :ligne 8 iwr.n(.ì) n.f ou iwr.i n.f; nht $(y) . n(i)$ ou $n h t(y) . \hat{i}$.

(h) La forme du fils de Taimouthès.

(i) nhmt ? Maspero saute ce passage; Brugsch: "Es war ein jubel um ihn von den Bewohnern der weissen Mauer".

(j) ${ }^{c}-n$ ?

(k) Le lion couché a la valeur $r$ et la patte antérieure de bovidé remplace l'avant-bras humain qui peut avoir la valeur $d \vec{l}$, donc se transcrit $r d i$.

(l) Maspero: "Qu'est-ce que les années, si nombreuses fussent-elles, qu'on passe sur terre ?"; Brugsch ne traduit pas toute cette partie de la stèle.

(m) Ce sens dérivé n'est pas signalé par le Wörterbuch au mot wh, "manquer".

(n) Maspero: "croupie".

(o) La traduction littérale de cette proposition principale serait en contradiction avec la subordonnée; c'est pourquoi, bien que le Wörterbuch ne mentionne pas de sens figuré au mot $b w$, l'interprétation de Maspero me semble la meilleure et je l'ai reproduite.

(p) $k b$ aurait ici un sens analogue à celui qui est signalé dans Wörterbuch, V, 23, 9: "vom Herzen, das von etwas befriedigt wird".

(q) Maspero: "La mort complète vient".

(r) Peut-on se hasarder à transcrire $n(\underset{v}{h}) n$ ?

(s) $l 1$ y a lieu de noter l'expression ir hr $r$ au lieu de $r d i$ h $r r$.

(t) Maspero: "Il ne voit point qui lui donne des présents de toute sorte de gâteaux".

(u) A lire $\stackrel{v}{v}(y w)=h t$ ? Maspero: "des provisions".

(v) Cf. 200,24, où Imouthès fils de Khâhapi est intitulé "supérieur des secrets dans la chambre cachée de la Tenenet". Par ailleurs, le texte de 201,21 (le scribe graveur, le savant) laisse supposer que le passage correspondant de 200,24 n'a pas été lu tout à fait 
correctement par Brugsch.

\section{STELE DE KHEREDOUANKH}

\section{Londres, University College 14357}

Bibliographie:

WEIGALL, Some inscriptions in Prof. Petrie's collection of Egyptian antiquities, dans Rec. Trav., XXIX (1907), p. 221-222 (texte; traduction des dates);

J. QUAEGEBEUR, Contributions à la prosopographie des prêtres memphites à l'époque ptolémaïque, Ancient Society 3 (1972), p. 99-100, traduction partielle et commentaires;

H.S. STEWART, Egyptian Stelae, Reliefs and Paintings from the Petrie Collection, Part III, Late Period, 1983, p. 8-9, n 20, pl. 13;

d'après Weigall:

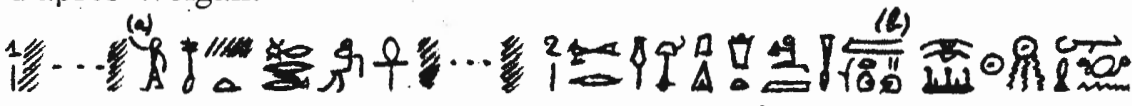

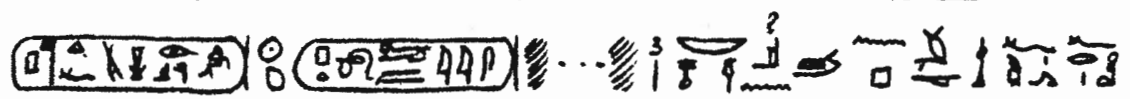

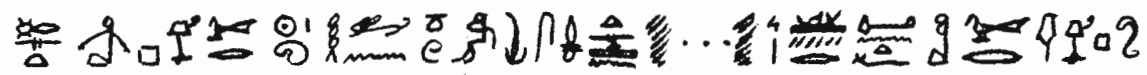

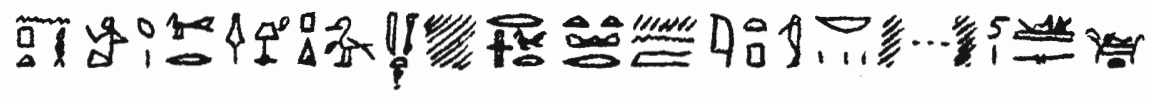

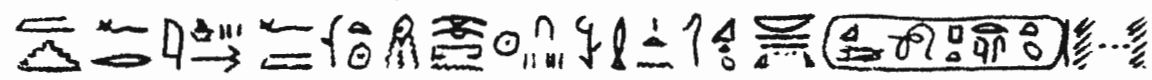

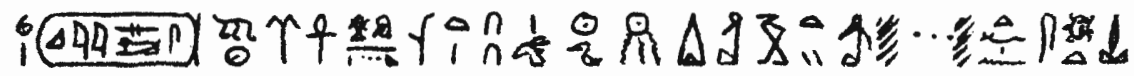

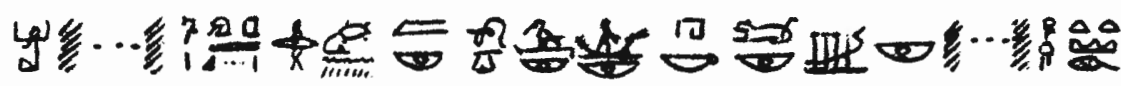

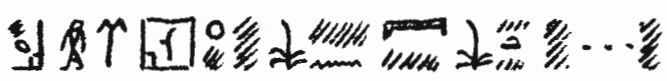

(a) Etant donné le geste du personnage, l'épithète nfr et ce que nous savons de la parenté féminine des grands prêtres de Ptah, on peut restituer un sistre au bout du bras levé.

(b) GAUTHIER, Rois, IV, 1916, p. 392, citant cette date, place un point d'interrogation après le signe de la tête. Il est en effet bizarre que ce signe, représentant le chiffre "7" 
soit écrit au-dessous du chiffre "2" écrit au moyen de deux traits. Mais comme Weigall déclare: "An inscription of this period requires to be read by a specially trained student" (op. cit., p. 222) et reconnaît ainsi qu'il n'est pas entraîné aux textes ptolémaïques, je pense qu'il a correctement lu le signe de la tête sans le comprendre, puisque dans sa traduction, il écrit: "in the second year". Si le chiffre "7" a été écrit au moyen du signe de la tête, je pense qu'il représentait le nombre exact d'unités; au-dessus de lui, je m'attendrais à trouver un nombre de dizaines; c'est pourquoi je propose de voir dans les deux traits qui ont passé jusqu'ici pour le chiffre "2" les extrémités du chiffre n "10".

"[1] ... la musicienne (?) accomplie, Kheredou-ankh ... (a) [2] grand des chefs des artisans Petobastis, juste de voix. L'an dix-(?)sept, deuxième mois d'Akhet, neuvième jour sous la Majesté du roi de Haute et Basse-Egypte Le dieu Philopator-Philadelphe-Neos-Dionysos, fils de Rê Ptolémée ... [3]

(b) [4] dans ... Mon père, le grand des chefs des artisans Psenptaïs fils du grand des chefs des artisans ...... (c) [5] en elle (d). J'ai été mise dans son tombeau (e) près de ses pères et mères en l'an neuf, deuxième mois de Peret, quinzième jour sous la Majesté de la Régente et Maitresse des Deux Pays Cléopâtre (et de son fils) (f) [6] César. Durée de vie (g) sur terre: 21 ans, 1 mois (?), 29 jours (h). ... (?) ... ...(?) ....7] sur terre, et faites-moi des offrandes à la fête Ouag, à la fête de Thot, à la fête de Sokar, à la fête de Heker, à la fête de la Moisson, (et à toutes les fêtes) (?) de l'Occident! Qu'Isis la grande dans Het-renpet fasse (?) .....".

(a) La ligne 4 permet de restituer "(juste de voix, fille du grand des chefs des artisans Psenptais, fils du)".

(b) D'après 201,6 et 14, on peut conjecturer qu'une partie de la fin de la ligne 2 était ainsi rédigée: "(juste de voix, est le jour où je suis née)".La ligne 3 me semble contenir le récit des circonstances de la mort de Kheredouankh.

(c) Comparer ce passge à 200,23 et 201,14-15 (le pronom "me" est également passé sous silence dans 201,14); on peut se demander si, après le mot "nécropole", la stèle de Kheredouankh ne continue pas par "(il accomplit pour moi tous les rites ...)" (?).

(d) elle = la nécropole ?

(e) Les passages parallèles, dans $\mathbf{2 0 0 , 2 3}$ et $\mathbf{2 0 1 , 1 5}$ livrent la transcription: htp.i (en admettant que $i$, ici féminin, soit écrit au moyen de $t$ ) $m$ is.f.

(f) La restitution, déjà proposée par GAUTHIER, Rois, IV, 1916, p. 412, note 2 fin) est confirmée par $\mathbf{2 0 0 , 2 3}$. 
(g) Litt.: "durée en vie sur terre".

(h) Je lis la tête de chevreau: $i b$ pour ibd $=1$ mois. Il me semble peu vraisemblable que ce signe puisse représenter un nombre d'unités s'ajoutant au "1" qui le surmonte, et je considère comme certain que Kheredouankh a vécu 21 ans et une fraction d'année. D'autre part la date de l'ensevelissement est claire: Kheredouankh a été mise au tombeau le 15 du 2ème mois de Peret de l'an 9 de Cléopâtre, ce qui correspond au 14 février 43 av. J.-C. (d'après GAUTHIER, Rois, IV, 1916, p. 412, V et note 2). Vingt et un ans plus tôt nous reportent en 64-65 av. J.-C. Or Ptolémée-Neos-Dionysos, sous lequel je suppose que Kheredouankh est née (voir note (b) a commencé son règne en 80 av. J.-C.; Gauthier écrit en effet à son sujet (Rois, IV, 1916, p. 392, note 4): "L'avènement du roi parait, en effet avoir eu lieu dans le courant de l'été 80 , son an 1er a donc duré jusqu'au 11 septembre de cette même année, correspondant au dernier des jours épagomènes, et son an 2 a commencé le 12 septembre 80 (= 1er Thot)". En continuant ce calcul, nous arrivons au résultat suivant: l'an 65-64 av. J.-C. est l'an 17 de Neos-Dionysos. Ansi me semblent étayées l'hypothèse que j'ai émise dans la note (b) ci-dessus sur la lecture de la date de la ligne 2, et la lecture de la tête d'animal dans la date de la ligne 6 . En examinant de plus près ce dernier point, je constate qu'il y a 126 jours, soit 4 mois et 6 jours entre le 9 du 2ème mois d'Akhet (date de la ligne 2) et le 15 du 2ème mois de Peret (ligne 6); si, de ces 126 jours, je soustrais les 1 mois et 29 jours, soit 59 jours que je lis dans la durée de vie de la ligne 6 , il me reste 67 jours pour la momification de Kheredouankh.

\section{STELE D'IMOUTHES-PETOBASTIS III}

\section{British Museum 188 (1030)}

Bibliographie:

YOUNG, Hieroglyphics, 1823, pl. 70, 71, 74 (dessin; il n'y a guère que la représentation du haut de la stèle qui soit utilisable);

BRUGSCH, Thesaurus, V, p. 928 sq. (texte);

J. QUAEGEBEUR, Contribution à la prosopographie des prêtres memphites à l'époque ptolémaïque, Ancient Society 3 (1972), p. 83-88, traduction du texte démotique et commentaires;

P. MUNRO, Die spätägyptischen Totenstelen, Äg. Forsch. 25 (1973), p. 339 (pas de pl.);

d'après Brugsch:

I. Premier registre (d'après Young): Osiris, Isis et Nephthys adorés par Petobastis entouré par Horus, Anubis et Imhotep;

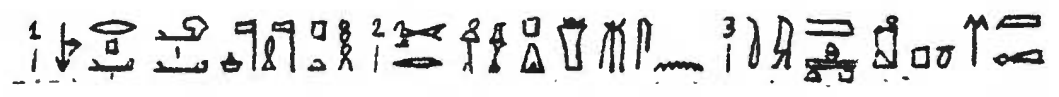


"[1] Le noble, gouverneur, père divin et prophète de Ptah, [2] grand des artisans-chefs Petobastis, né de [3] Taimouthès, juste de voix".

II. Deuxième registre (d'après Brugsch):

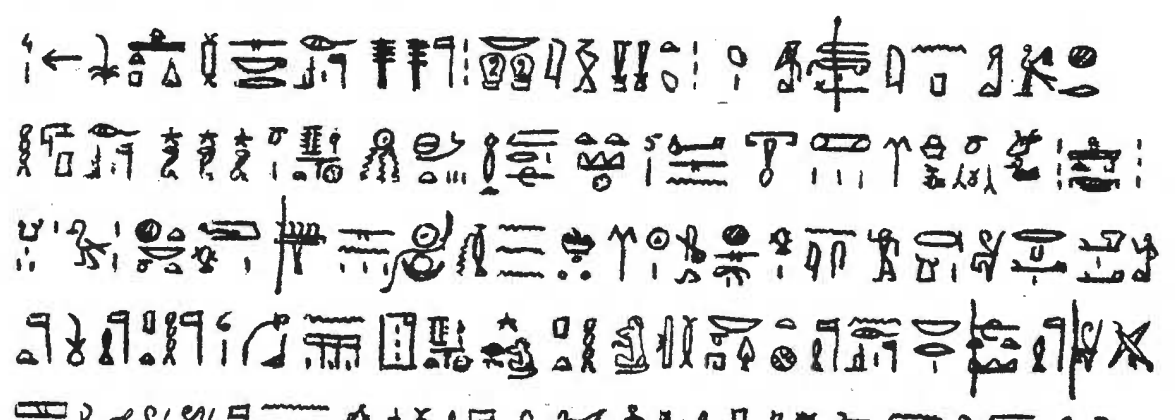

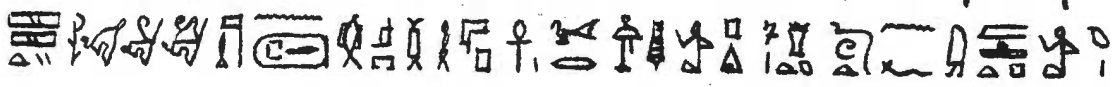

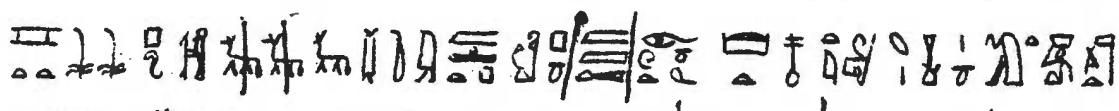

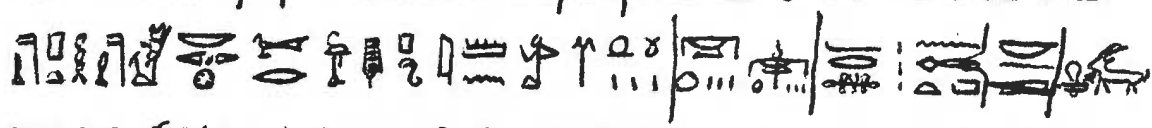

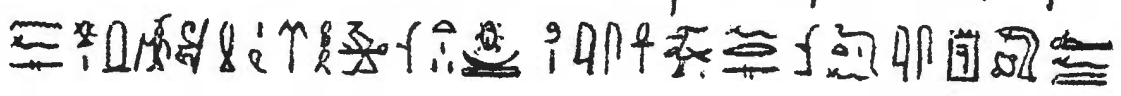

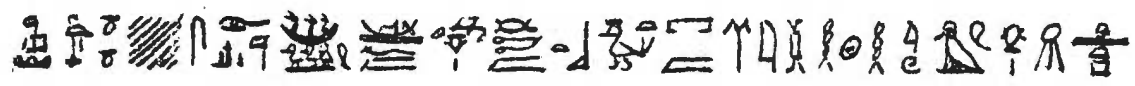

"[4] Offrande royale à Sokar-Osiris, aux divinités (a) maîtresses des deux sanctuaires (b), aux deux soeurs qui sont sa protection (c) devant lui, à Anubis et Horus auprès d'Apis-Osiris, aux dieux et déesses du Mur Blanc et à la grande énéade dans Ro-setaou (5) pour qu'ils donnent l'offrande "sortie de voix" consistant en pain, bière (?), vases d'albâtre (?), étoffes, boeufs et volatiles, toutes (d) les offrandes, aliments, nourriture (e), et toutes choses qui paraissent sur leurs autels chaque jour, (et aussi) de l'eau fraîche et de la résine de térébinthe au jour de "diriger les rites" (f) à l'appel du nom (g) du noble, gouverneur, père divin, khe-nesou, prophète de Ptah, [6] ouab des temples du Mur Blanc, hiérogrammate (?) (h) de Ptah, hiérogrammate de la Maîtresse du Sycomore, prophète d'Osiris, seigneur de Ro-setaou, prophète de la Fenêtre de l'apparition, prophète de Pharaon, protecteur magique (i) d'Apis vivant, grand des artisans-chefs, Peto[7]bastis 
appelé Imouthès (j), fils de celui qui porte les mêmes titres, Psenptaïs (k), et né de Taimouthès qui est juste de voix!

Un bel ensevelissement lui a été fait par le fils du frère de sa mère (l), le père divin et prophète de Ptah, prophète d'Horus seigneur de Létopolis, grand des artisans-chefs Psenamounis (m), avec (n) des anneaux (?) d'or et d'argent et (o) des amulettes de toutes sortes de véritables pierres précieuses. Il l'a momifié avec le grand nom de Sa Majesté en ... (?) années d'éternité (?). Que son âme vive (p) et (?) que son corps soit rajeuni! Que son cadavre soit divin dans le Kher-neter ... (?) ... Osiris complétant sa puissance (?) (q) par lui en joie! Celui qui est dans le sarcophage (r) ... (?) éternité, que son âme se lève (s) et se couche!"

(a) $\underline{d} d w$, Wörterbuch, V, 627, 13: belegt gr.

(b) itr.ty

(c) Litt.: en qualité de protection de lui.

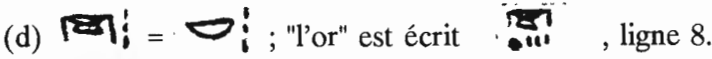

(e) 'fos' $d f 3 w$; remarquer la disposition singulière des trois traits.

(f) = jour de la mise au tombeau (Wört., III, 332, 3).

(g) k3; on attendrait cependant $\xi^{3} \Leftrightarrow \quad$, Wört., V, 92.

(h) sśm $m d 3 t$ (?) ntr, ou "prophète et scribe", hm(?)-ntr sśs (cf. 200,13)?

(i) Cf. 193,6.

(j) Sur les stèles de ses parents $(\mathbf{2 0 0 , 2 2}$ et 201,13), l'expression est renversée et l'on a: "Imouthès appelé Petobastis".

(k) Psenptaïs, décédé avant son fils, devrait, comme Taimouthès, être qualifié de "juste de voix". Serait-ce la graphie de son nom qui aurait empêché le scribe de mettre "juste de voix" à côté de l'image de Ptah ? Les trois signes suivants se lisent certainement $m s w$ (trois animaux vivants au lieu de leurs trois peaux formant if $\uparrow$ - cf. RANKE, dans Ä.Z. 45 (1908), p. 92.

(l) $s 3$ sn $n$ mwt.f. Imouthès a donc été enseveli par son cousin, neveu de sa mère, ou par son demi-frère, si frère a le sens de mari. 
(m) P3-šr- $(n)-i m n$.

(n) "L'ensevelissement" dont il est question est donc la préparation de la mise au tombeau, c'est-à-dire la momification et la préparation de l'attirail funèbre.

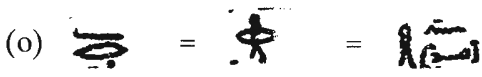

(p) is ${ }^{c} n h$ b3.f

(q) $m h b 3 w \cdot f$

(r) $d b 3 t$, Wörterbuch, V, 561, 12.

(s) $w b n$

Le texte hiéroglyphique est suivi d'un texte démotique en 9 lignes; cf. REVILLOUT, Un prophète d'Auguste, dans Revue Egyptologique, II (1882), p. 101-102 (traduction du début); BRUGSCH, Thesaurus, V, p. 929-933 (texte et traduction mot à mot du début).

\section{3a. STATUE DE PETOBASTIS III}

Musée de Cherchel (Algérie)

Bibliographie:

G. BENEDITE, dans une note adressée à M. R. CAGNAT (Bulletin archéologique du Comité des travaux historiques et scientifiques, 1908, p. CCLIV-CCLVI et pl. XLVII (traduction et photo) (ce monument m'a été obligeamment signalé par M. Jean Sainte Fare Garnot).

D'après la photo; inscription dorsale.

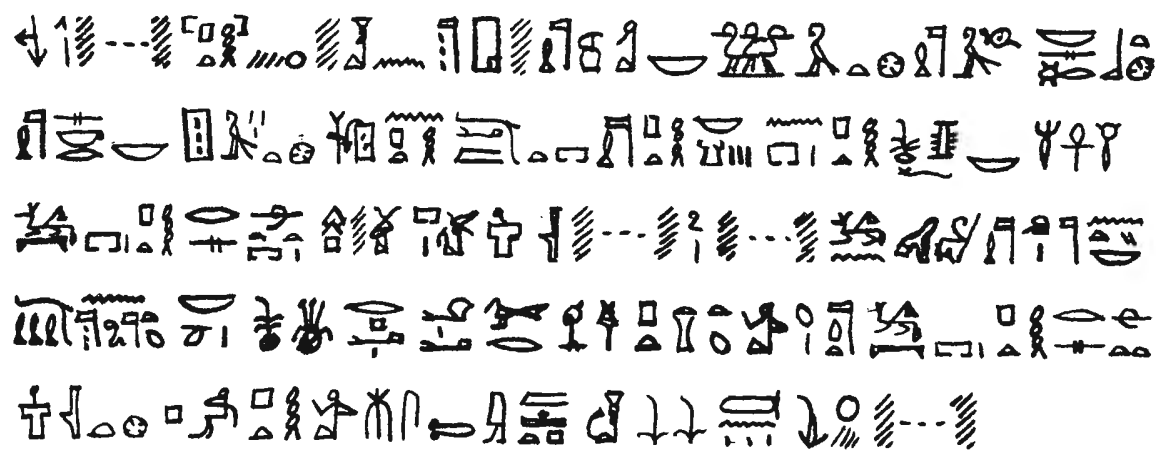


"[1] ...... Ptah .... des temples ..., prophète de Khnoum, seigneur de SemenHor, prophète d'Horus-Rê seigneur de Sekhebet, prophète de Sokar seigneur des Châteaux d'Horus, scribe de Ptah, directeur de ... (a), prophète de Ptah seigneur des aliments du temple de Ptah qui est au sud de son mur et seigneur d'Ankh-taoui, supérieur des secrets du temple de Ptah, de Rosetaou, d'Apis et d'Osiris (b) du temple d'Osiris de Ro-qedit, ..... [2] ..... supérieur des secrets du roi de Haute et Basse-Egypte, premier prophète du dieu de tout ce qui est (c), directeur des prophètes de tous les dieux et déesses de Haute et Basse-Egypte, noble, gouverneur, grand des artisanschefs Petobastis, fils du père divin et supérieur des secrets du temple de Ptah, de Ro-setaou et de Ro-qedit (d), Psenptaïs, et né de Taimouthès. Que ces noms demeurent (e) .....".

(a) Cf. Wörterbuch, I, p. 187, 2:(Nr, Sp) = "partie de l'administration du temple".

(b) Le parallélisme entre tout le passage et $\mathbf{1 9 8 , 2}$ invite à dissocier Apis et Osiris dans un cas et Osiris et Apis dans l'autre; cf. aussi 200,13.

(c) = le roi; il s'agit ici de la reine Cléopâtre.

(d) Cf. 200,14.

(e) Restituer: "dans ce temple éternellement" d'après $\mathbf{1 9 8 , 3}$.

\section{STELE DE TANEPHEROS}

\section{British Museum 184 (983)}

Bibliographie:

YOUNG, Hieroglyphics, 1823, pl. $72-73$ et 74 (dessin);

BRUGSCH, Thesaurus, V, p. 934 (texte, du milieu de la ligne 6 à la fin de la ligne 7 , qui termine le texte hiéroglyphique);

P. MUNRO, Die spätägyptischen Totenstelen, Äg. Forsch., 25 (1973), p. 338-339, détail pl. 64, Abb. 218;

d'après Brugsch:

I. Premier registre:

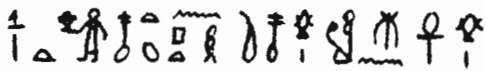


"[1] La musicienne accomplie de Ptah, Tanepherôs, née de Harunchis".

II. Deuxième registre: (partiellement détruit). Le dessin de Young permet de lire quelques bribes d'une "[2] Offrande royale à Ptah ..... [4] toutes les choses bonnes et pures ....."

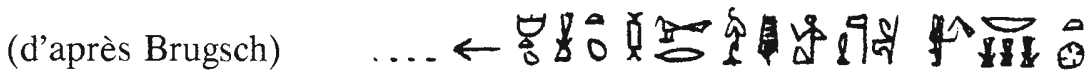

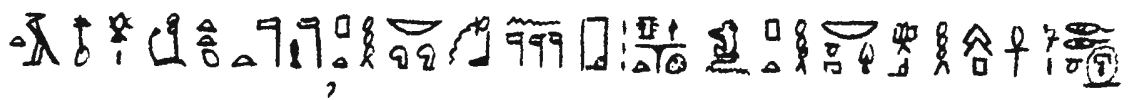

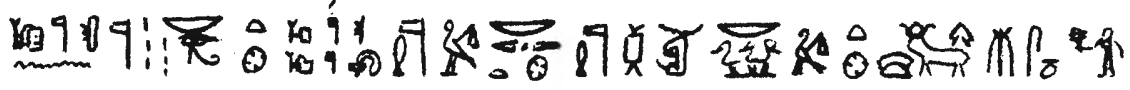

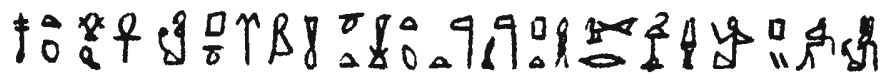

"[6] ...... l'épouse, la soeur du grand des artisans-chefs et prophète de Min seigneur de Senout, Tanepherôs, fille du père divin et prophète de Ptah le puissant, ouab des temples du Mur Blanc, scribe de Ptah seigneur du Sycomore (a), protecteur magique (b) d'Apis vivant, [7] yeux de Pharaon, hiérogrammate de tous les dieux de l'Egypte (c), hiérogrammate, trésorier divin et (?) prophète d'Horus seigneur de Létopolis, prophète de Khnoum seigneur de Semen-Hor, Khâhapi, et née de la musicienne accomplie Harunchis, qui est juste de voix, la soeur (d) du père divin et prophète de Ptah, grand des artisans-chefs, Psenamounis".

(a) Ou peut-être: "scribe de Ptah et de la Maîtresse du Sycomore" ?

(b) Cf. 193,6.

(c) b3kt, Wörterbuch, I, 425, 18.

(d) Le texte démotique de cette stèle indique clairement que Tanepherôs a été la femme de Psenamounis; l'épithète "la soeur" ne peut donc se rapporter qu'à Tanepherôs et non à Harunchis. Il est d'ailleurs curieux que le nom du mari de Tanepherôs ne soit pas mentionné à la ligne 6 après" ... et prophète de Min seigneur de Senout". Y a-t-il là une omission du lapicide réparée à la fin de la ligne 7, ou le texte a-t-il été composé tel que nous l'avons, peut-être de façon que le nom du grand prêtre Psenamounis vint à la fin du texte hiéroglyphique?

Le texte hiéroglyphique est suivi d'un texte démotique; cf. REVILLOUT, Un prophète d'Auguste, dans Revue Egyptologique, II (1881), p. 100-101 
(traduction), et idem, Le Comput de Ptolémée Denys et le canon des Rois, dans Revue Egyptologique, V, p. 130, note 2 et p. 131 (rectifications de lapsus calami de "Un prophète d'Auguste");

BRUGSCH, Thesaurus, V, p. 935-940 (texte et traduction mot à mot).

\section{TEMPLE D'EDFOU}

Bibliographie:

CHASSINAT, Le temple d'Edfou, I, 1897 (Mémoires de l'Institut Français d'Archéologie Orientale, tome X), p. 329:

(texte relatif au nome memphite)

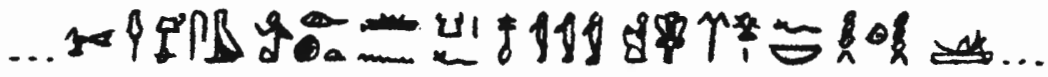

"...Le grand des chefs des artisans-sem accomplissant les rites pour sa (a) nourriture; la "belle de formes" (b) jouant du sistre devant lui (c); Seigneur d'éternité (d) ...".

(a) Le possessif se rapporte à Ptah.

(b) "formes", sens dérivé de "statue", Wörterbuch, 256, 20. Sur le sarcophage d'Harmachis, la mère de ce grand prêtre, elle-même femme du grand prêtre Anemher, est intitulée: $\mathbf{J}[\mathrm{vm}: 3]=0 \mathbf{S}[\mathbf{S}]$ "la belle de formes (?) dans le temple à côté de (mon) père" (197,13 et 20). Le rapprochement des deux expressions permet d'affirmer que "la belle de formes" est un titre ou une épithète de l'épouse du grand prêtre, celle-ci étant en même temps à la tête du clergé féminin de Ptah.

(c) Lui = Ptah.

(d) C'est le nom de la barque sacrée.

\section{STELE DE KHAHAPI}

Berlin 2118

Bibliographie:

STERN, Die bilingue Stele des Châhap im ägyptischen Museum zu Berlin, dans Ä.Z., 22 (1884), p. 101-109 (traduction);

SCHAEFER, Ein Phönizier auf einem ägyptischen Grabstein der 
Ptolemäerzeit, dans Ä.Z., 40 (1902), p. 31-35 (photo p. 32);

SETHE, Hieroglyphische Urkunden der griechisch-römischen Zeit, Heft 3, 1916, p. 164, 4 et 12 (texte);

P. MUNRO, Die spätägyptischen Totenstelen, Äg. Forsch., 25 (1973), p. 336 (pas de pl.);

(un estampage en ma possession):

Troisième registre (texte hiéroglyphique):
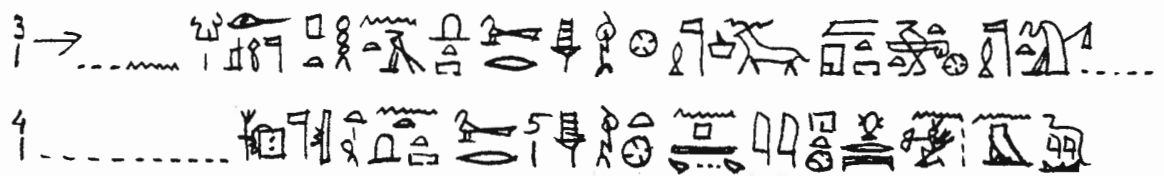

$\sum_{1 . . .}^{9}$

"[1] (Offrande royale) ....... [3] ...... au ka de l'Osiris (a), (prophète) (b) de Ptah de Chenout-Our-kherep-hemou(t) (c), prophète du Bélier dans HetOutet (d), prophète de Mout ... [4] .......... hiérogrammate de Chenout-Our[5]kherep-hemout de Pa-ta-yhet, supérieur des troupes de Medjayou ......"

(a) SETHE, op. cit., note a: "lies " = L'Osiris, prophète.

(b) Le terme "prophète" qui suit "Ptah de Chenout-..." et commande "Bélier ..." pourrait aussi avoir pour complément "Ptah de Chenout-...". Cf. un cas analogue dans 192,3 et remarque (a), fin.

(c) Mot à mot: "Ptah du Magasin-du-grand-des-chefs-des-artisans".

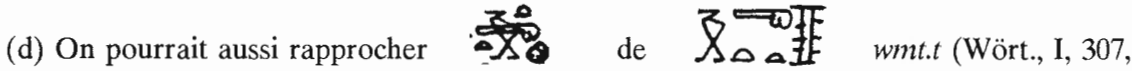
6-7; belegt D. 18, dans une expression composée !) et proposer la lecture "Het-Oumetet" (= Temple d'enceinte). 



\section{A P P E N D I C E}

\section{APERCU DU PERSONNEL DE PTAH}

En dépouillant les publications égyptologiques pour l'étude des grands prêtres de Ptah, nous avons noté un certain nombre de titres portés par d'autres membres du clergé memphite à différentes époques. Les listes que nous établissons sur cette base sont sans doute incomplètes, mais elles permettent quelques observations non dépourvues d'intérêt. C'est pourquoi il nous paraît utile de les publier malgré leurs défauts.

Les titres énumérés semblent bien appartenir à des personnages memphites, sauf dans les cas où une réserve est faite à ce sujet.

\section{A. ANCIEN EMPIRE}

La titulature des grands prêtres enseigne l'existence de "prophètes de Ptah" et de "prêtres-ouab de Ptah".

D'autres membres du haut clergé ont été

"directeur des artisans, prophètes de Ptah, prophètes de Sokar"1; il en est qui précisent le premier de ces titres en s'appelant "directeurs des artisans de la ouabet, etc..."2.

On note aussi à cette époque un

${ }^{1}$ Caire $1359=$ BORCHARDT, Denkmäler, p. 27 (Fefy); Caire $1295=$ ibidem, p. 1-2 (Khouenptah).

2 MARIETTE, Mastabas, E 4, p. 392 (Nefersechemptah); SELIM HASSAN, Excavations at Giza, 1930-1931 (1936), p. 4 (Ouachptah). 
"prophète de Ptah, prophète de Sokar, prêtre-sem"1, et un "surveillant du prophète de Ptah"'.

Les textes des pyramides mentionnent enfin ${ }^{3}$ un "ancien du palais" ou "ancien de la cuisine de Ptah"4.

\section{B. MOYEN EMPIRE}

Le personnel de Ptah a probablement compris entre autres les fonctions suivantes:

"directeur des prophètes"15;

"prophète de Ptah"6; "père divin de Ptah"7;

"prêtre-ouab de Ptah"8,

"prêtre-lecteur de Ptah"';

"directeur des joueurs de tambourin de Ptah-Sokar"10;

"directeur des maçons de Ptah"11;

"constructeur des bateaux de Ptah"12;

"directeur des fermiers de Ptah"13.

Seul le premier de ces titres a été porté certainement par quelqu'un de Memphis.

${ }^{1}$ MARIETTE, Mastabas, D 24, p. 250 (Nymaâtptah).

${ }^{2}$ Berlin 1108 = Ägyptische Inschriften, I (1901), p. 101-118 (Manefer).

3 Pyr. \& 560 b, 566 b.

4 KEES, Totenglauben, p. 121-122 (Palastälteste"); SETHE, Pyr., Kommentar (Küchenältester).

5 PETRIE, Memphis I, pl. 4.

${ }^{6}$ BOESER, Ägypt. Sammlung ... Leiden, II, pl. 22.

${ }^{7}$ Caire 20520, d'Abydos = LANGE-SCHÄFER, Cat. II, p. 120, e.

${ }^{8}$ Caire 20030, d'Abydos = LANGE-SCHÄFER, Cat. I, p. 39, h4.

${ }^{9}$ Berlin 1203 = Ägypt. Inschriften, II, p. 256.

${ }^{10}$ BOESER, Ägypt. Sammlung ... Leiden, II, pl. 14, n 13; pl. 15, $n^{\circ} 15$.

11 Brit. Mus., d'Abydos = Hierogl. Texts from Egyptian Stelae, II, pl. 14, n 209.

${ }^{12}$ Caire 20528, d'Abydos = LANGE-SCHÄFER, Cat. II, p. 130, h.

13 BOESER, Ägypt. Sammlung ... Leiden, II, pl. 22. 


\section{NOUVEL EMPIRE ${ }^{1}$}

Le clergé de Ptah comprend les grades de:

"père divin de Ptah"2;

les pères divins ou prophètes de $\mathrm{Ptah}^{3}$ dépendent, en ce qui concerne les questions administratives, d'un "directeur des prophètes de Ptah"4. Le titre de "père divin aimé du dieu" est porté par les grands prêtres Ptahmès II et III, Neferrenpet et Rêhetep; ce sont aussi des grands prêtres qui sont qualifiés de "supérieurs des secrets du saint des saints (Grande Place)" (Ptahmès II), ou "supérieur des secrets dans le ciel, la terre et la douat" (Neferrenpet), ou encore "supérieur des secrets ... le temple de Ptah" (Iyry); par contre, le grand prêtre partage l'épithète de

"conducteur de la fête de Ptah" avec d'autres hauts personnages du clergé memphite: un "directeur des prophètes de Ptah"s, un "majordome du temple de Nebmaâtrê"6;

"prêtre-ouab de Ptah";

les ouab ne sont pas tous de même rang; il y a des "ouab supérieurs de

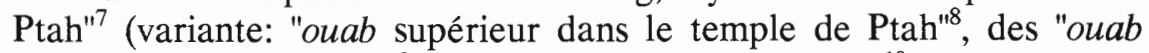
prêtres-lecteurs de Ptah"1, et de simples "ouab de Ptah"10.

${ }^{1}$ Dans le classement des différentes catégories du personnel de Ptah, nous suivons l'ordre adopté par G. Lefebvre pour le personnel d'Amon.

${ }^{2}$ BOESER, Ägypt. Sammlung ... Leiden, V, p. 11. Des fils des trois grands prêtres Ty, Neferrenpet et Iyry remplissent cette fonction.

${ }^{3}$ STOLK, Ptah, p. 36, signale des "premiers prophètes de Ptah" au Nouvel Empire. Ce sont, à notre avis, des membres du clergé de Ptah de Karnak; voir ci-dessus, § 57, dernière note.

${ }^{4}$ Berlin 21595 = Ägypt. Inschriften, II, p. 398.

5 Berlin 21595 = Ägypt. Inschriften, II, p. 398.

${ }^{6}$ BOESER, Ägypt. Sammlung ... Leiden, V, p. 1.

${ }^{7}$ Berlin 2297 = Ägypt. Inschriften, II, p. 6-7.

${ }^{8}$ Leyde, d'après SCHIAPARELLI, Cat. Firenze, p. $330=$ LIEBLEIN, Dict. $n^{\circ} 708$.

${ }^{9}$ BOESER, Ägypt. Sammlung ... Leiden, VI, pl. 20.

${ }^{10}$ Berlin 12410 = Ägypt. Inschriften, II, p. 178; J.E.A., 4 (1917), pl. 10,2; un contemporain de Ramsès II est "ouab et orfèvre de Ptah" (MARIETTE, Sérapéum, 1857, pl. 14, bas, gauche). 
Un texte de Ramsès II emploie l'expression "les ouab et les prophètes" pour désigner l'ensemble du clergé masculin de Ptah; le même document mentionne les "grandes dames du temple de Ptah"1.

C'est encore au clergé que se rattache le "supérieur des chanteurs de Ptah" (variante: "du temple de Ptah") (59; 60; 61).

Dans le personnel du domaine de Ptah, nous trouvons des;

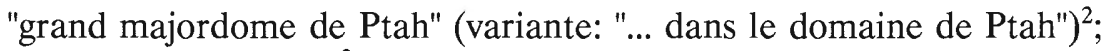
"majordome de Ptah"3;

"supérieur de l'autel de Ptah"4;

"scribe de Ptah";

"scribe copiste du domaine de Ptah";,

"supérieur de l'équipage du domaine de Ptah" (variante:

"supérieur des bateaux de transport du domaine de Ptah") ${ }^{7}$; "commandant (?) du domaine de Ptah"8;

"serviteur dans le domaine de Ptah";

"scribe des offrandes du domaine de Ptah"10;

"scribe du domaine de Ptah"11;

"scribe du temple dans le domaine de Ptah"12;

1 E. NAVILLE, dans T.S.B.A., 7 (1882), p. 119 sq. Sur un ostracon datant vraisemblablement du règne de Ramsès IX et portant un hymne à Thot, il est question de Maât qui est "parmi les grandes dames qui sont dans le temple de Ptah" (A. ERMAN, Gebete eines ungerecht Verfölgten und andere Ostraka aus den Königsgräbern, dans $\ddot{A}$. Z., 38 (1900), p. 35).

${ }^{2}$ BOESER, Ägypt. Sammlung ... Leiden, IV, pl. 26.

${ }^{3}$ Caire 671 = BORCHARDT, Statuen .... III (1930), p. 18.

${ }^{4}$ Berlin 12411 = Ägypt. Inschriften, II, p. 179.

5 BOESER, Ägypt. Sammlung ... Leiden, XIII, p. 16 n 90.

${ }^{6}$ NAVILLE, Todtenbuch, I, pl. 7 (Aa).

${ }^{7}$ idem, ibid., Einleitung (Ha et $\mathrm{Ib}$ ).

${ }^{8}$ Caire 45544.

${ }^{9}$ Caire 26-11-24-7.

${ }^{10}$ Berlin = Ägypt. Inschriften, II, p. 172-173.

11 Caire 1210 = BORCHARDT, Statuen ..., IV, p. 109.

12 Florence 2557 = SCHIAPARELLI, Cat. p. 326. 
"hiérogrammate de Ptah"1;

"supérieur de l'ergastule de Ptah"2;

"supérieur des portiers de Ptah";

puis le personnel de bouche;

"brasseur du domaine de Ptah"4;

"pâtissier de Ptah";

les corps de métier:

"directeur des corps de métier de Ptah"6;

"directeur des artisans dans l'atelier d'orfèvrerie de Ptah"7;

"scribe de l'atelier d'orfèvrerie de Ptah"8;

"orfèvre de Ptah";

"fabricant de ... (?) de Ptah"10;

ceux qui s'occupaient des bestiaux:

"supérieur de l'étable"11.

"directeur des troupeaux de Ptah"12;

"chef de la nouvelle basse-cour du domaine de Ptah"13;

"gardien des oies de Ptah"14;

${ }^{1}$ Berlin = Ägypt. Inschriften, II, p. 172-173.

${ }^{2}$ Louvre, Sérapéum 5270 (nom propre dans RANKE, 209,2).

3 BOESER, Ägypt. Sammlung ... Leiden, V, pl. 1, 2 a.

${ }^{4}$ Berlin 7321 = Ägypt. Inschriften, II, p. 167.

${ }^{5}$ Berlin 4397 = ibid., p. 579.

${ }^{6}$ Caire 26-11-24-7; BOESER, Ägypt. Samml., VI, pl. 23, n 38.

${ }^{7}$ BRUGSCH, Dict. hiérogl., V (Supplément), p. 206.

${ }^{8}$ Caire 26-11-24-7.

${ }^{9}$ MARIETTE, Sérapéum, 1857, pl. 14, bas, gauche.

${ }^{10}$ Berlin, statuette funéraire 572 = Ägypt. Inschriften, II, p. 586.

11 BERGMAN, Inschriftliche Denkmäler ..., dans Rec. Trav., VII, p. 196.

12 Florence 2541 = BEREND, Principaux monuments ... (Bibl. Ecole des Hautes Ecoles, Sciences phil. et hist., fasc. 51; BOESER, Ägypt. Sammlung ... Leiden, XII, p. 13, $\mathbf{n}^{\circ} 72 ;$ p. $14, \mathbf{n}^{\circ} 85$.

${ }^{13}$ Berlin 7289 = Ägypt. Inschriften, II, p. 165.

14 ibidem, p. 166. 
enfin, pour le trésor, le:

"scribe du trésor de Ptah"1.

\section{XXIème A XXVème DYNASTIES}

Le clergé de Ptah est composé de:

"pères divins de Ptah"2;

quand leur appartenance à Ptah est suffisamment indiquée par d'autres titres, ils s'appellent simplement "pères divins"; exemples: "père divin et supérieur des secrets de $\mathrm{Ptah}^{\prime \prime}$, "père divin, prophète et supérieur des secrets du domaine de Ptah"4. Le titre de "père divin" est d'ailleurs souvent accompagné de "prophète", ainsi que dans ce dernier exemple, mais nous n'avons pas rencontré de "prophète de Ptah" à cette époque et, comme nous avons vu à l'époque précédente un "directeur des prophètes de Ptah", il nous semble qu'on peut se demander si "prophète de Ptah" n'a pas été employé pour désigner le rang administratif d'un père divin de Ptah. Le titre "père divin aimé du dieu" et l'épithète "supérieur des secrets dans le ciel, la terre et la douat" n'appartiennent qu'au grand prêtre, comme sous le Nouvel Empire ${ }^{6}$.

"prêtre-sem de Ptah"7;

"prêtre-ouab de Ptah" de divers rangs; il y a des :

"ouab supérieurs du domaine de Ptah",

${ }^{1}$ Berlin 7315 = Ägypt. Inschriften, II, p. 172-173; NICHOLSON, Aegyptiaca, 1891, pl. 4, E 4; Caire 17-6-25-5.

2 167,1,12-13,16; GAUTHIER, A travers la Basse-Egypte, dans Annales du Service des Antiquités, 21 (1922), p. 205.

${ }^{3}$ Caire $667=$ BORCHARDT, Statuen ..., III, p. 15; 164,26.

${ }^{4}$ Caire $45327=$ Ann. Serv. Ant., 15, p. 141 et IVERSEN, Two inscriptions concerning private donations to temples, Copenhague, 1941.

5 167,13-14 est une exception apparente: il s'agit du prophète d'un culte particulier du dieu memphite, celui de "Ptah seigneur de Semenmaât".

6164,24 et 163,7 .

7 167, 9-10.

${ }^{8}$ Berlin 8169 = Ägypt. Inschriften, II, p. 232-234. 
"ouab du saint des saints"1,

"ouab prêtres-lecteurs de Ptah"2, enfin de simples

"ouab de Ptah"3.

Les grandes dames du temple de Ptah de l'époque précédente portent maintenant des titres:

"grande des concubines" ou "première grande du harem de Ptah" ou encore "grande du harem de Ptah"4;

"chanteuse du choeur de Ptah"5.

Dans le personnel du domaine de Ptah, on trouve des:

"supérieur du territoire cultivé de Ptah"6;

"scribe du temple"7 ou "scribe du temple du domaine de Ptah" "portier du domaine de Ptah"";

"supérieur des artisans du bronze (?) ${ }^{10}$ de Ptah"11;

"directeur des troupeaux et prophète de Ptah seigneur de Semenmaât"12; "directeur des troupeaux du terrain marécageux (?) méridional du domaine de Ptah"13;

"comptable des troupeaux dans le domaine de Ptah"14.

${ }^{1}$ Caire 1106 = BORCHARDT, Statuen ..., IV (1934), p. 59.

${ }^{2}$ Caire 667 = ibidem, III (1930), p. 15.

3 Caire 18-2-25-3.

${ }^{4}$ 165,3; 167,23 (femmes de grands prêtres); 167,24; DARESSY, Fragments memphites, dans Annales Serv. Ant., 20 (1920), p. 170, n 6 (voir ci-dessus, § 66, note finale).

${ }^{5}$ Musée Guimet E 2869 = WEIL, Veziere, p. 157.

${ }^{6} \mathbf{1 7 0 , 3}=$ Berlin 8169 .

7 Caire 45327 = IVERSEN, Two inscriptions concerning private donations to temples, Copenhague, 1941.

8 PETRIE, Memphis II, pl. 24 = 164,26; 163,18-19.

9 CHASSINAT, dans Rec. Trav., XXIII, p. 82.

${ }^{10}$ Wörterbuch, I, p. 477,7.

${ }^{11}$ Louvre Sérapéum 3010 = CHASSINAT, dans Rec. Trav., XXIII, p. 81-82, n 137 = MARIETTE, Sérapéum, 1857, pl. 35.

12 167,13-14.

${ }^{13}$ PETRIE, Memphis II, pl. 23, droite.

14 163,19; 164,26-27; IVERSEN, op. cit. 


\section{E. XXVIème A XXXème DYNASTIES}

Le clergé comprend des:

"pères divins de Ptah"1;

le titre "père divin aimé du dieu" est porté par d'autres membres du haut clergé que le grand prêtre ${ }^{2}$;

"prophètes de Ptah"3; ce titre apparaît aussi sous la forme désormais fréquente de "serviteur de Ptah"4;

les deux titres, père divin de Ptah et prophète de Ptah se combinent aussi en "père divin et prophète de Ptah"5, ce qui semble indiquer de nouveau qu'ils ne sont pas absolument identiques;

"prêtres-sem de Ptah" sous des formes diverses: "prêtre-sem"6 (variante: Setem7, "Sem dans le temple de Ptah"8, "Sem dans Het-ka-Ptah"; "connus du roi (?)"10 et "divins connus du roi (?)"11;

"prêtres-ouab": "ouab dans le (variante: qui est dans $l \mathrm{e}^{12}, \mathrm{du}^{13}$ temple de Ptah"14; "ouab de la cour du temple de Ptah"15; "ouab du saint des saints de Ptah"16.

1 189,8 (père divin et supérieur des secrets du domaine de Ptah); 189,3 (ô ... pères divins, ..).

${ }^{2}$ Louvre D 8 = BRUGSCH, Thesaurus, p. 948.

${ }^{3} 189,3$ (o ... pères divins, prophètes ...); CHASSINAT, dans Rec. Trav., XXI (1899), p. $60, \mathrm{n}^{\circ} 7$.

${ }^{4}$ CHASSINAT, dans Rec. Trav., XXI, p. 69, n 32; LIEBLEIN, Dict. noms, $\mathrm{n}^{\circ} 1207$.

${ }^{5}$ Caire 669 = BORCHARDT, Statuen, III, p. 16; CHASSINAT, dans Rec. Trav., XXI (1899), p. 63, n 16 ; ibidem, XXII (1900), p. 172, n 106; p. 25, n $^{\circ} 78$ (plusieurs générations); ibidem, XXIII (1901), p. 79, ligne 4; LIEBLEIN, Dict. noms, n 1234.

${ }^{6}$ WEIL, Veziere, p. 145; LIEBLEIN, Dict., $\mathrm{n}^{\circ} 1207$.

${ }^{7}$ CHASSINAT, dans Rec. Trav., XXI (1899), p. 63, n 16.

${ }^{8}$ WEIL, Veziere, p. 152.

${ }^{9}$ DARESSY, Notes, dans Rec. Trav., XIX (1897), p. 21, n 144.

${ }^{10}$ MARUCCHI, Il museo Vaticano, p. 172; LIEBLEIN, $n^{\circ} 1254$.

${ }^{11}$ Rec. Trav., XXIII (1901), p. 89; ibidem, XXI (1899) p. 69, $\mathrm{n}^{\circ} 32$; LIEBLEIN, $\mathrm{n}^{\circ}$ 1055, 1192, 1207, 1231.

12 CHASSINAT, dans Rec. Trav., XXII (1900), p. 174, n 113; LIEBLEIN, nº 1140.

${ }^{13}$ CHASSINAT, op. cit., p. $164, \mathrm{n}^{\circ} 84$.

14 ibidem, p. $19, \mathrm{n}^{\circ} 62$.

15 Ä.Z., 18 (1880), p. 52.

${ }^{16}$ LIEBLEIN, $\mathrm{n}^{\circ} 1197$. 
On peut rattacher au clergé les:

"directeur des chanteurs de Ptah"1;

"chanteur du domaine de Ptah"2, titre complété aussi par "celui de Sokar qui rythme" .

Parmi le personnel du domaine, nous notons les:

"hiérogrammate du (variante: dans le) domaine de Ptah";

"scribe de la cour du domaine de Ptah"5;

"portier du domaine de Ptah"6;

"directeur des artisans dans le temple de Ptah"7;

"... du domaine de Ptah"8;

"scribe comptable de toutes choses dans le trésor du temple de Ptah"'.

\section{F. EPOQUE PTOLEMAIQUE}

Les inscriptions hiéroglyphiques ne mentionnent que des membres du haut clergé et du personnel supérieur de Ptah. Pour le clergé, on trouve les titres de:

"père divin"10; le titre "père divin aimé du dieu" et l'épithète "supérieur des secrets du (variante: dans le) domaine (variante: temple) de Ptah"

${ }^{1}$ CHASSINAT, dans Rec. Trav. XXI (1899), p. 65, n²0.

2 ibidem.

3 op. cit., p. 61, n 9; tome XXII (1900), p. 24, n 73 .

${ }^{4}$ LIEBLEIN, n 1207; Caire $867=$ BORCHARDT, Statuen, III (1930), p. 129.

${ }^{5}$ CHASSINAT, dans Rec. Trav., XXIII (1901), p. 79 et p. 89.

${ }^{6}$ op. cit., XXII (1900), p. 26, n 80; p. 165, n 86.

${ }^{7}$ Louvre D 8 = BRUGSCH, Thesaurus, p. 948.

${ }^{8}$ Louvre Sérapéum 257,258 et $264=$ CHASSINAT, dans Rec. Trav. XXII (1900), p. $170, \mathrm{n}^{\circ} 99$ et 100 , et p. $171, \mathrm{n}^{\circ} 103$.

${ }^{9}$ Louvre D 8 = BRUGSCH, Thesaurus, p. 948.

${ }^{10}$ SPIEGELBERG, dans Rec. Trav. XXX, (1908), p. 145 et 152; 204,6; cf. aussi 190,15 . 
s'appliquent à des grands prêtres ${ }^{1}$ et à d'autres dignitaires ${ }^{2}$; "prophète de Ptah" ${ }^{\prime 3}$, le plus souvent abrégé en "serviteur de Ptah"4; "connu du roi (?)"

\title{
Le clergé féminin comprend des:
}

\author{
"musiciennes de Ptah"6.
}

Pour le personnel de Ptah, nous pouvons citer les titres de: "imy-khet du domaine de Ptah"; "scribe de Ptah et imy-set-â de la troisième phylê"';

\footnotetext{
${ }^{1}$ Par exemple Anemher $(\mathbf{1 9 2 , 3}$ et 9$)$ et Teos $(193,3$ et $\mathbf{6})$.
}

${ }^{2}$ Berlin 18 = BRUGSCH, Thesaurus, p. 910; on a vu dans cet Amasis un grand prêtre de Memphis (BRUGSCH, op. cit., et après lui OTTO, Priester I, p. 204, note 2), mais ce personnage ne porte pas le titre de "grand des chefs des artisans". SPIEGELBERG l'a d'ailleurs désigné simplement comme grand prêtre de Létopolis (Varia 72, dans Rec. Trav. XXVI (1904), p. 42, d'après OTTO, Priester I, p. $415=$ Complément à la p. 204).

${ }^{3}$ 204,6; cf. aussi 190,15.

4 201,3; SPIEGELBERG, op. cit.; Caire 31099 = SPIEGELBERG, Die demot. Inschriften (Cat. gén.) p. 28-30 et 36-37, qui a vu dans le personnage en question (Pahemneter-Padihor) un grand prêtre de Memphis (p. 36) quoiqu'il ne porte pas le titre de grand des chefs des artisans.

${ }^{5}$ SPIEGELBERG, dans Rec. Trav, XXX (1908), p. 145 et 152.

${ }^{6}$ Dans ce rôle, nous connaissons une fille de grand prêtre (Kheredouankh $=\mathbf{2 0 2 , 1}$ ), des épouses de grands prêtres (Harunchis femme d'Anemher $=193,11$ et 194,12; Harunchis femme de Petobastis II $=\mathbf{2 0 0 , 1 5}$ et $\mathbf{2 0 1 , 8}$ où elle est appelée la musicienne très ornée et donneuse du rythme de Ptah; Taimouthès femme de Psenptaïs II = REINISCH, Chrestomathie, pl. 20, 1er registre; Tanepherôs femme de Psenamounis = 204,1), enfin une belle-mère de grand prêtre (Harunchis femme de Khahapi $=\mathbf{2 0 1 , 4}$ et 204,7). D'après le texte démotique de sa stèle, Tanepherôs a été "intronisée grande femme de Ptah" après avoir passé par le rang de musicienne de Ptah (voir ci-dessus, § 83).

${ }^{7}$ Rec. Trav. XIV, p. 184.

${ }^{8}$ Berlin 18 = BRUGSCH, Thesaurus, p. 910. On rencontre chez les grands prêtres les titres de "ìmy-set-â du domaine de Ptah des 15 jours de toutes les premières phylai" (Nesqed, = 190,4), "scribe de Ptah de toutes les troisièmes phylai du temple de Memphis" (Anemher = 192,4-5; Harmachis, avec cinquièmes au lieu de troisièmes = 194,2), "ân de phylê des 15 jours de toutes les cinquièmes phylai" (Harmachis = 194,2-3), et "imy-set des 15 jours de toutes les premières phylai" (Harmachis $=194,3$ ). Il semble que chaque 
"orfèvre du domaine de Ptah"1.

sanctuaire avait ses phylai et que le grand prêtre avait affaire avec toutes les phylai memphites portant le même numéro d'ordre que celle du temple de Ptah à laquelle il appartenait.

${ }^{1}$ Louvre, Sérapéum 3199. 



\section{ADDENDUM}

Une liste des grands prêtres de Ptah connus jusqu'en 1977 a été publiée par:

- D. WILDUNG, Hoherpriester von Memphis, Lexikon der Ägyptologie II, 1977, col. 1256-1263.

- Voir également l'appendice de $\mathrm{PM} \mathrm{III}^{2} / 3$, F. High Priests of Memphis, p. 916-918.

Pour la lecture hmw wr shm du titre du grand prêtre de Ptah de Memphis, proposée par $\vec{H}$. De Meulenaere, voir son article dans Festschrift zum 150 jährigen Bestehen des Berliner Museum, p. 183-184.

\section{Moyen Empire}

- $\quad$ Musée du Louvre, E. DELANGE, Catalogue des statues égyptiennes du Moyen-Empire, Paris, 1987, p. 59-62: sur la statue A 123 de Montouhotep, figure la mention, ligne 1: "grand prêtre de Ptah (wr hrp ...) de la double maison, Imenhotep neb imakh."

- PM IV, 75, El Maasoura: autel en pierre de Sehetepibrê-ankh, "high priest of Ptah", Dyn. XII, Wilkinson, Mss XVII, F.d 7,8 (haut gauche) (Bodleian Library, Oxford).

\section{Nouvel Empire}

- Statue naophore d'un homme en costume de grand prêtre de Ptah, ÄS 5773, Corpus Antiquitatum Aegyptiacarum, Kunsthistorisches Museum, Wien, Lieferung 6, 1990, 6,164-172. 
- Plaque BM 59259 - avec nom et titres: M.L. BIERBRIER, The British Museum Hieroglyphic Texts from Egyptian Stelae, Part 10, 1982, p. 19, pl. 38-39.

- Simulacre de vase, albâtre, au nom du prêtre-sem et wr ḩrp h̆mw, Ptahmès, XVIIIe dyn., ex-coll. Koller 241: J. MONNET SALEH, Les antiquités égyptiennes de Zagreb, 1970, n 567, p. 126;

- Gros vase avec bouchon d'albâtre, ex-coll. Koller 236, personnage idem, op. cit., $\mathrm{n}^{\circ} 548$, p. 123.

- Peter LASZLO, Statue de Ptahankh au Musée des Beaux-Arts, Bulletin du Musée Hongrois des Beaux-Arts, Budapest n 56-57 (1981), p. 3-16 : discute les deux Ptahmès.

- Montant de porte d'un Ptahmès, XIXe dyn., University College 14477: H.M. STEWART, Egyptian Stelae, Reliefs and Paintings from the Petrie Collection, Part I, The New Kingdom, 1976, p. 53, pl. 43.2.

- Ouchebti d'un Ptahmès sm wr h̆rp h̆mw: La collezione egiziana del Museo Archeologico Nazionale di Napoli, s. date, n 17.17, pl. XII.

- Fragment de bas-relief aux noms des grands prêtres Hori et Pahemneter fils de Meh: C.M. ZIVIE, Giza au deuxième millénaire, BdE LXX, Le Caire, 1976, p. 191, n NE 52.

- Fragments appartenant au prince Khaemouas = ibidem, p. 201-207, $n^{\circ}$ NE 57-60.

- Statue Caire JE 89046: G.A. GABALLA, Two dignitaries of the XIXth dynasty, MDAIK 30 (1974), p. 21-23, pl. 2.

- Sur stèle BM 167, de Ptahemwia, 19e dyn. 2e. reg.: Hori, grand prêtre de Ptah; T.J.H. JAMES, The British Museum Hieroglyphic Texts from Egyptian Stelae, Part 9, p. 29-30, pl. XXV.

- Monuments du grand prêtre Rêhetep: dans K.A.KITCHEN, Ramesside Inscriptions, III, p. 52-66, cités sous "(P)rahotep B, Vizir". 
- $\quad \mathrm{PM} \mathrm{III}^{2} / 2$, 858: statue en grès, N.E., Philadelphia Univ. Museum, E 12038: Khaemouas, prêtre-ouab de Ptah, etc.,... dédiée par son fils Our-kherep-hemout, scribe.

- Chaouabtis de Hori I, Iyry I et Nebmehyt = J. et L. AUBERT, Statuettes funéraires, Paris, p. 91.

- Canope en albâtre de Hori au Musée Archéologique de Liège (Maison Curtius), signalé par Ph. Derchain par lettre du 14 octobre 1952.

\section{Basse-Epoque}

H. DE MEULENAERE, Les grands prêtres de Ptah à l'époque saïto-perse, dans Mélanges offerts à Jean Vercoutter, Paris 1985, p. 263-266.

- Le tombeau du prince Chechanq publié par A. BADAWI, Das Grab des Kronprinzen Scheschonk, Sohnes Osorkon's II und Hohenpriester von Memphis, ASAE 54 (1956), p. 153-177 + 16 pl.

- Statue ayant peut-être appartenu au prince Chechanq = Helen JACQUET-GORDON, $A$ statue of a Son of Karoma, The Brooklyn Museum Annual VI (1964-65), p. 43-49.

- Statue fragmentaire du grand-prêtre Chedsounefertoum, Louvre E $25680=$ La Revue du Louvre et des Musées de France, n ${ }^{\circ}-5$ (1966), p. 242-244.

- Fragment d'inscription RB 18391 du Sérapéum de Memphis, avec reste de texte: "son fils, le grand prêtre de Ptah, le prophète ... fils de l'homologue Nekao ..." = Mohammed Ibrahim ALY, Ramzy NAGEB, Didier DEVAUCHELLE, François-René HERBIN, Présentation des stèles nouvellement découvertes au Sérapeum, BSFE 106 (juin 1986), p. 36.

- Stèle de Hory, fils de Ahmesmèn Iraheka "greatest of the Directors of craftsmen" et de Sekhmet-nefert" = $\mathrm{PM} \mathrm{III}^{2} / 3$, p. 801; ép. de Darius. 
448

- Liste de hauts personnages, prêtres et fonctionnaires = J. YOYOTTE, A propos d'un monument copié par G. Daressy (Contribution à l'histoire littéraire), BSFE 11 (octobre 1952), p. 67-72: photo du monument et commentaires aimablement fournis par J. Yoyotte, lettre du 1er juillet 1952. Voir aussi Annuaire EPHE, Ve Section, Tome 89 (1980-81), p. 72. 


\section{INDEX DES NOMS PROPRES EGYPTIENS DE PERSONNES}

Les chiffres renvoient aux pages. Abréviations: $\mathrm{n}=$ note; $\mathrm{gp}=$ grand prêtre.

Aakenen, roi; p. 94

Achakhet: p. 94, 95

Achaout-akh: p. 97, 98, 99, 99

n. 3,100 n. $1,101,102,167$, 175,176 n. 2

Adelphes (dieux): p. 43, 188

Ahmès Ier, roi: p. 94, 127

Ahmès: p. 94, 95, 127, 175

Ahmèsmen, gp: p. 16 n. 11, 17, 21 n. $2,173,174,175,195$ n. 2

Akhenaton, roi: p. $76,78,80,80$

n. $4,81,88,138$

Alexandre, roi: p. 179

Amasis, roi: p. 174

Amenemope: p. 85, 125

Amenemhat Ier, roi: p. 121, 123

Amenemhat II, roi: p. 93

Amenemhat, gp d'Amon: p. 77

Amenhotep: p. 80 n. 4

Aménophis Ier, roi: p. 94, 127

Aménophis II, roi: p. 77

Aménophis III, roi: p. 21, 76, 77, 77 n. 3 , n. 4, 78, 80, 81, 86, 88,

$94,96,128,129,130,131,132$, $133,136,151,154-5$ n. 6,160 , 166

Aménophis IV = Akhenaton:

p. $137,154-5$ n. 6

Amenophthis, roi: p. 94, 95
Amyrtée, roi: p. 153 n. 1

Anemher, gp: p. 15, 16 n. 12, 22, 23,39 n. $1,47,50$ n. $1,69,78$, $82,85,85$ n. $2,87,100,101,101$ n. $3,102,103,179,181,182$, 183, 184, 184 n. $1,185,185$ n. 2 , $186,187,188,189,189$ n. 5,191 n. 5 , n. 6, n. 7,201 n. 1

Anemher: p. 180

Ankhef-ny-mout: p. 163, 164

Ankhef-ny-sekhmet, gp: 16 n. 9, $69,73,93,94,95,97,98,99,99$ n. 3,100 n. $1,123,140,156$, 163, 164, 165, 176, 176 n. 2 Ankh-en-ef-shmt: p. 98 Ankhkhakarề: p. 93, 96 Ankhnoubkaourê: p. 93, 95, 96 Ankhpadiiset: p. 176

Ankhsehetepibrê: p. 93, 94, 95 Ankhty: p. 161

Aoupout, gp d'Amon: p. 166 Apriès, roi: p. 170 n. 2

Arcadius, empereur: p. 214

Arsinoé: p. 17, 43, 180, 187, 188, 193

Bakenkhonsou II, gp d'Amon: p. 15,75

Bakenptah: p. 157

Bakenrenef, gp: p. 76 n. 1, 172, 
172 n. 1,174 n. 1

Bakenrenef: p. 76 n. 1

Bérénice, femme de gp: p. 191

Cambyse, roi: p. 175

César-Auguste, empereur: p. 20, 201, 202, 202 n. 2, 203 n. 2, 211, 211 n. 4,213

Césarion $=$ César, fils de Cléopâtre VII: p. 198

Chabaka, roi: p. 88, 89, 172, 173

Chabataka, roi: p. 172

Chareq, roi: p. 94

Chechanq Ier, roi: p. 46, 47, 98, 98 n. $1,99,165,166,167,167$

n. 1,176

Chechanq II, roi: p. 168 n. 2 , n. 4

Chechanq III, roi: p. 168, 169, 170, 171

Chechanq V, roi: p. 93,93 n. 1 , 176

Chechanq, prince et gp: p. 16

n. $2,10,98,167,168,168$ n. 2 , n. 3,169

Chechanq, gp: p. 98, 99, 102, 103, 167

Chedsounefertoum, gp: p. 16 n. $9,31,39$ n. $1,43,46,47,50$ n. 1,75 n. $1,78,81,82$ n. 1,85 , $98,99,165,166,166$ n. 3,167 , 167 n. $1,175,176$

Chepesipouptah: p. 115, 116

Chepseskaf, roi: p. 105

Cléopâtre Ière, (femme de Ptolémée V): p. 188

Cléopâtre (femme de Ptolémée VII Evergète II): p. 191 n. 3

Cléopâtre VII: p. 32, 43, 196, 197, 199, 200, 202, 202 n. 2, 203, 203 n. $2,205,209$ n. 1, 211 n. $4,212,213,214$
Darius, roi: p. 146, 173, 175 n. 1 Debehen: p. 4, 5, 10, 51, 52, 53, 57

Dedia, gp: p. 81 n. 3, 5, 142, 143

Dempedjet (?): p. 98, 99, 176

Djedbastetiouesankh: p. 169 n. 2

Djedkarê-Isesi, roi: p. 44 n. 4, 115

Djeser, roi: p. 197

Epiphanes, dieux: p. 43, 188

Evergètes, dieux (Evergète Ier et son épouse): p. 43, 182, 182 n. $1,187,188$

Evergètes, dieux (Evergète II et son épouse): p. $191 \mathrm{n} .2$

Hapidjefa, gp: p. 23, 71, 72, 121, 122

Hapouseneb, gp d'Amon: p. 76

Harmachis, gp: p. 16 n. 12, 26, 43, 102, 103, 181, 182, 183, 186, $187,188,189,206$

Harunchis, femme d'Anemher:

p. 181,184

Harunchis, femme de Petobastis: p. 193

Harunchis, femme de Khahapi, mère de Tanepherôs: p. 210

Hatchepsout, reine: p. 77, 127

Hekairâa, gp: p. 16 n. 11, 172, 174

Hekenou: p. 110

Hemioun: p. 115

Horemheb, roi: p. 68, 75, 94, 96, 140

Horemheb: p. 96, 138

Hori I, gp: p. 16 n. 8, 24, 27-28 n. 5,81 n. 4,82 n. 2 , n. 4,141 , 142

Hori II, gp: p. 16 n. 6, 24, 27, 27 28 n. 5,81 n. 4,82 n. 4,141142 
Hori: p. 86 n. 1, n. 2, 143, 144

Hormin: p. 67,84

Horsaïset I, gp: p. 16 n. 1, n. 9, $85,86,96,98,99,100,100$ n. 1 , $103,164,164,176$

Horsaïset II, gp: p. 16 n. 10, 82 , 85 n. $1,87,169,170,170$ n. 1 , $171,172,175,176,176$ n. 2,177 n. 1,195 n. 2

Hory: p. 174, 175

Houel: p. 146

Hy, gp: p. 81 n. 5, 84, 145, 146, 147

Iby, roi: p. 94

Imhotep, architecte de Djeser: p. 48 n. 1,146

Imouthès $=$ Petobastis III: p. 20

n. $3,22,26,37$ n. $4,49,103$, 197, 197 n. 1, 199, 199 n. 2, 202, 202 n. 2, 203, 203 n. 1, 2, 204, 205, 205 n. 1, 207, 211, 212, 213, 214

Iny-ankh: p. 9, 10, 10 n. 2, 119

Inti: p. $64,65,65$ n. $1,116,117$

Iouy: p. 163

Iry: p. 116

Isetemkheb; p. 169

Isetnefert, reine: p. 147

Isetrechet: p. 175

Ity: p. 152

Iyry, gp: p. $15,49,81$ n. 4,82 n. 2 , n. $3,84,86,158,159,160$, $163,165,172$ n. 6

Khabaouseker: p. 27, 27-28 n. 5, 28,28 n. $1,30,30$ n. 4 , n. 5,31 n. $2,59,59$ n. $2,72,119$

Khahapi: p. 195, 207, 210, 211 n. 1,213

Khamouas I, gp: p. 16 n. 3, 8, 82 n. $2,141,141$ n. 4,142
Khamouas II, prince et gp: p. 12, 15,16 n. $2,17,24,24$ n. 4,27 , 27 n. $2,27-28$ n. $5,36,36$ n. 2 , 43 n. $1,46,47,48,79,82$ n. 4 , $84,86,97$ n. $1,132,133,145$, $146,147,147$ n. $2,148,149,149$ n. 1 , n. 4 , n. $5,150,151,152$, 153,153 n. $1,154,154$ n. 6,155 , 156, 157, 157 n. 1,168 n. 1,172 n. 4

Khamouas, prince (fils de

Ramsès III): p. 15 n. 3, 160

Khamouas: p. $17,154-55$ n. 6

Khatnesout: p. 144

Khendjer: p. 151

Khenou: p. 54, 115

Khentetka: p. 108

Kheredouankh: p. 195, 198, 214

Khnemibrê: p. 97 n. 3, 146, 147

Khnemibrê-saptah $=$

Ahmèsmen: p. 48, 174

Khonsouiou: p. 180, 181, 181

n. 1,182

Maï: p. 129 n. 2

Meh: p. 143

Mehousekht: p. 152

Mehousekh: p. 166

Memphitès, fils d'Evergète II:

p. 194-195, n. 3

Meneptah, roi: p. 78, 158

Menkaouhor, roi: p. 44, 54, 113, 115

Menkaourê, roi : p. 105

Menkheper: p. 128, 134, 135 n. 4

Menkheperrêseneb, gp d'Amon: p. 77

Mentou: p. 94, 95

Mentouhetep III, roi p. 94

Merenptah, gp: p. 16 n. 10, 168, 169 
Merenrê, roi : p. 52

Méry, gp d'Amon: p. 77

Méry: p. 144

Méryour (?): p. 94, 96

Méryptah, gp: p. 15 n. 3, 23, 27, 28 n. $5,29,30$ n. $1,81,130,130$

n. $3,134,135,135$ n. 3,138

Méryptah, gp d'Amon: p. 77

Méryptah: p. 15 n. 3

Mes: p. 131

Minmès, gp d'Amon: p. 86, 144, 147

Mycérinus, roi: p. 4, 11, 35, 51, $53,55,59$

Nebkherourê = Mentouhetep III: p. 93, 94

Nebmehyt, gp: p. 81 n. 5, 141

Nebneterou, gp d'Amon: p. 15 n. $3,75,78$

Nebou: p. 119

Nebounnef, gp d'Amon: p. 138 n. 2

Nebpou, gp: p. 16 n. 7, 27-28

n. $5,72,96,123$

Nebrê: p. 94, 94 n. 2

Nectanébo, roi: p. 43

Neferef-rê-ankh, gp: p. 5, 13, 37 n. $1,45,53,109,111,152$

Neferhotep II, roi: p. 94, 95

Neferibrêmen, gp: p. 16 n. 11, 173,174

Neferirkarê, roi: p. 44, 106

Neferrenpet, gp: p. 25 n. 1, 50

n. $1,76,78,81$ n. $4,84,86,86$

n. 1, n. $2,94,95,96,156,157$, 157 n. $1,158,159$

Neferrenpet: p. 15 n. 4, 82 n. 2, n. $4,139,158$ n. 2

Nefersebek: p. 180, 184 n. 1

Nefertari-Merymout, reine: p. $154-155$ n. 6

Nefertiti, femme d'Harmachis: p. 187

Nekao, roi: p. 173

Nekaomen, gp: p. 173, 174, 174

n. 1

Nekao-méryptah: p. 173, 174

Nemrod: p. 166

Nena: p. 143

Nesptah: p. 167

Nesqed, gp: p. 16 n. 12, 17, 20, 21 n. 1, 26, 31, 102, 103, 179, 180, 181, 182 n. $1,185,193,195$ n. 2,205

Nesqed: p. 43, 187, 188, 189

Neter-kheperrê-méryptah $=$ Paoupaou: p. 16 n. 1, 96, 164, 164 n. 2

Neterouy-hetep: p. 94

Netery-khet, roi: p. 10 n. 1

Netery-metout (?): p. 10 n. 1

Netery-mou, roi: p. 10 n. 1

Noubrê, roi (?): p. 124

Ny-ankh-sekhmet: p. 4, 5, 10, 11, 51, 52, 53, 56 n. 4, 57, 106

Nymaâtrê: p. 119

Nyouserrê: p. 44, 106, 106 n. 1, $113,115,150$

Octave $=$ César-Auguste: p. 205, 213

Osorkon II, roi: p. 67, 98, 98 n. 1, 167, 168, 169

Osorkon, gp: p. 98, 99, 103, 167, 177

Ou(?)khet: p. 94, 95

Ounas, roi: p. $18,43,44$ n. 4 , $112,113,114,115,116,133$, 148,150

Ourhetep: p. 94

Ouserkaf, roi: p. 44, 196 
Pacherenmout: p. 175

Padihor-Pahemneter: p. 206

Padihorresne: p. 175

Padiiset, gp: p. 16 n.10, 46, 47, $168,169,170,170$ n. $3,171,177$ n. 1,195 n. 2

Pahemneter, gp: p. 16 n. 6 , n. 8 , 24, 27, 28 n. 5,81 n. 4,82 n. 1 , n. $4,84,84$ n. 4,86 n. 1,97

n. $1,137,142,143,144,144$

n. $5,145,151$

Pahemneter-Padihor: p. 206

Paiimy-(khe)red (?): p. 94

Pamy, roi: p. 170, 176

Paoupaou, gp: p. 16 n. 9, 25, 27 28 n. 5,82 n. $3,85,96,98,99$, 99 n. $2,100,103,163,163$ n. 1 , 164, 176

Papa: p. 94, 96, 98, 99, 100 n. 1

Paser: p. 78, 94

Peftjaouaouibastet, gp: p. 16

n. $10,27-28$ n. $5,79,169,170$, 171, 177 n. 1

Penpanebes (?): p. 94, 96

Pépi Ier, roi: p. 52, 53, 55, 61, 62, $65,66,67,69,71,74$ n. 3

Pépi II, roi: p. 52, 118

Petobastis I = Nesqed, gp:

p. 101, 101 n. 3, 179, 191 n. 1, 195 n. 2

Petobastis, gp: p. 100, 101, 102, 103, 103 n. 1, 104, 188, 190

Petobastis II, gp: p. 16 n. 12, 17, 18, 22, 103, 191, 191 n. 1, 192, 193, 193 n. 2, 195

Petobastis III - Imouthès, gp:

p. 16 n. $12,43,199,200,201$

Philadelphes, dieux = Adelphes

Philopators, dieux: p. 43, 182,

182 n. $1,183,187,188$
Philopators et Philadelphes:

p. 192

Philotéra: p. 17, 180

Piankhi, roi: p. 172

Psammétique Ier, roi: p. 175

Psammétique II, roi: p. 173

Psammétique: p. 150

Psenamounis, gp: p. 16 n. 12, 18, 20, 22, 37 n. 4, 103, 207, 210

n. 2, 211, 211 n. 3, 212, 213, 214

Psenamounis (ou Psenptaïs ?),

fils du gp Psenamounis: p. 213, 214 n. 2

Psenptaïs I, gp: p. 16 n. 12, 100, 101, 102, 103, 104, 183, 187, $188,189,189$ n. $2,190,191$, 193, 193 n. 3, 204, 205, 205 n. 1

Psenptaïs, gp: p. 16 n. 12,103

n. 1,104

Psenptaiis II, gp: p. 16 n. 12, 17, 20,20 n. $3,22,26,31,32,37$

n. $4,43,47,48,48$ n. $1,49,50$

n. $1,78,81,82$ n. $1,100,103$, 115 n. $2,190,193,194,194$ n. 1 , n. $3,195,195$ n. 1 , n. 2,196 , 196 n. 1, 197, 198, 198 n. 1, 199, 200, 203, 203 n. 3, 204, 205 ., 209 n. 1,211 n. $3,212,213,214$, 214 n. 2

Psenptaïs (ou Psenamounis), fils du gp Psenamounis: p. 213, 214 n. 2

Psintaès: p. 190

Psousennès II, roi: p. 93 n. 1, 94, 96

Ptahankh: p. 33, 136, 136 n. 2, 139

Ptahchepses: p. $7,17,19$ n. 8, 54, 62 n. 5, 112, 115, 116, 118

Ptahchepses I, gp: p. 7, 17, 18, 
$21,22,35$ n. 1,36 n. 3,39 n. 1 , 44,44 n. $4,45,45$ n. $1,48,53$, $55,63,105,105$ n. $2,106,106$ n. $1,107,109,111,113,113$ n. 4,114

Ptahchepses II, gp: p. 18, 53, 105 n. 2, 109, 111

Ptahchepses III, gp: p. 17, 53, 105 n. 2, 109, 111, 111 n. 2, 112

Ptahchepses IV, gp: p. 17, 19, 19 n. $7,23,43,45,52,53,54,55$, 65,105 n. $2,107,107$ n. 3,108 , $109,111,112,113,114$ n. 2 , 115, 116, 116 n. 4, 117, 118

Ptahchepses-Impy, gp: p. 19, 37

n. 1,62 n. $1,96,114,116,118$, 118 n. 4

Ptahchepses-Impy: p. 62 n. 5

Ptahchepses-pakem: p. 115

Ptahiemakhet: p. 94

Ptahemhat-Ty, gp: p. 16 n. 5, 73, 80,80 n. 2, 81, 96, 138, 141

Ptahemheb: p. 93, 94

Ptahhotep: p. 186 n. 3

Ptahmès Ier, gp: p. $73,74,128$, 129, 129 n. 3, 103

Ptahmès II, gp: p. 16 n 3, 26, 27 28 n. 5, 29, 50 n. 1, 73, 74, 76, 84,84 , n. 1 , n. 4, 129, 130, 131, $132,133,134,134$ n. $1,135,136$ n. $2,137,142$

Ptahmès III, gp: p. 16 n. 5, 20, 21, 26, 27-28 n. 5, 31, 32, 73, 74, $80,133,134,135,135$ n. 3,136 , 136-137 n. 4, 137, 139, 142

Ptahmès IV, gp: p. 18, 23, 158, 159, 159 n. 1,163

Ptahmès, gp d'Amon: p. 77

Ptahmès: p. 17, 96, 135 n. 4, 161

Ptolémée Ier Sôter: p. 100, 101,
184, 185 n. 2

Ptolémée II Philadelphe: p. 21

n. $1,100,179,180,181,181$

n. 1, n. $2,184,185,185$ n. 2 , 205, 206

Ptolémée III Evergète Ier:

p. 100,150 n. 2, 182, 183, 185, 187, 189

Ptolémée IV Philopator: p. 100, $101,180,181,181$ n. 1 , n. 2 , 184, 185, 185 n. 2, 205, 206

Ptolémée V Epiphane: p. 100, 188, 194 n. 1

Ptolémée VI Philomêtor: p. 100, 190

Ptolémée VII Evergète II:

p. $100,186,192,194$ n. 4

(voir aussi sous:

Adelphes, Evergètes,

Philopators, Epiphanes)

Ptolémée Sôter II: p. 192, 193

n. 3

Ptolémée Alexandre Ier: p. 100, 192, 193, 193 n. 3

Ptolémée Neos Dionysos

(Aulète): p. 31, 192 n. 5, 194, 194 n. 1, 195, 195 n. 2, 196, 207, 211, 211 n. 4, 213

Ptolémées (les): p. 37, 39 n. 1, 43, 44, 47, 49, 78, 79, 82, 85, 87, 89,168 n. 6, 184, 190, 205, 213

Ramôse: p. 77 n. 3, 78

Ramsès II, roi: p. 36 n. 2, 46, 75, 75 n. $1,78,81,82,84,85,86$, $87,88,94,95,96,132,142,143$, $144,146,147,151,154-55$ n. 6 , $156,157,157$ n. 1,158 n. 2,159 , 161, 166, 168 n. 1, 172, 179

Ramsès III, roi: p. 32 n. 2, 33

n. $1,38,39,43$ n. 2,44 n. 2,67 , 
160

Ramsès IV, roi: p. 158 n. 2

Ramsès IX, roi: p. 141 n. 4

Ramsès: p. 152

Rêhetep, gp: p. 16 n. 8, 20 n. 1 , 23,25 n. $1,46,75$ n. 1,81 n. 4 , 82 n. $4,84,84$ n. $4,86,144$, $145,147,147$ n. 1,148 n. 1,157 , 159, 172

Rêhetep: p. 82 n. 2

Rênefer, gp: p. 26, 26 n. 1, 27-28 n. $5,53,109,110,111,189$ n. 2

Renpet-nefer, mère de Nesqed: p. 26, 179

Renpet-nefer, femme de Nesqed: p. 180

Romê-Roy, gp d'Amon: p. 78, 157

Sabou-Ibebi, gp: p. 14, 17, 18, 21, $23,32,43,44,44$ n. $4,45,45$

n. $1,48,52,53,54,55,62,62$ n. $5,63,65,96,107,109,111$, $112,113,113$ n. $4,114,114$

n. $2,115,115$ n. 2,116 n. 4,118 n. 5

Sabou-kem, gp: p. 26, 53, 109, 111,112

Sabou-Tjéti, gp: p. 4, 5, 14, 19, $20,21,21$ n. $3,25,27-28$ n. 5 , 36 n. 3,37 n. 1,42 n. $4,51,52$, 53,55 n. 2,56 n. $4,57,62,63$, 63 n. $3,64,65,67,71,72,73,74$ n. $3,81,82,83,83$ n. $4,96,110$, $111,116,117,118$

Sabou: p. 7, 9, 17, 19 n. 8, 54, 62, $96,112,115,116$

Sahourê, roi: p. 4, 51, 106, 150, 161

Saïset: p. 94, 96, 99, 100 n. 1

Sarenpout: p. 124,124 n. 3
Say: p. 140

Sebekhotep: p. 116

Sehetepibrê-ankh-nedjem: p. 16 n. $7,27-28$ n. $5,71,72,73,74$, 95, 123

Sehetepibrê-cheri: p. 16 n. 7 , $73,96,123$

Sekeremsaef: p. 94, 95, 140

Sekhmet-nefer: p. 175

Semataouitefnakht: p. 173, 175

Senimen: p. 111, 152

Sennefer, gp: p. 81 n. 5,84 n. 4 , $86,142,144$

Senousert-ankh, gp: p. 16 n. 7 , $32,36,59$ n. $2,71,71$ n. 1,72 , 73,74 n. $3,122,123$

Ser-met (?): p. 94

Sésostris Ier, roi: p. 71, 73, 93, $94,95,121,122,123$

Sésostris II, roi: p. 93, 95

Setairetbint: p. 175

Séti Ier, roi: p. 41, 41 n. 3, 67, $83,994,95,145,146,159$

Séti II, roi: p. 159,172 n. 6

Setjou, gp: p. 45, 33, 107, 107

n. 1,108

Siamon, roi: p. $25,76,82$ n. 3, 98 , 99, 99 n. 2, 163, 176

Snefrou, roi: p. 59

Tachepeniset: p. 165, 166, 195

n. 2

Taimouthès: p. 195, 196, 197 , 198, 199, 299, 203, 203 n. 1 , n. $2,210,211,211$ n. 2 , n. 3 , 213, 214

Tairy: p. 169

Takelot II, roi p.168, 169

Takelot, gp: p. 16 n. 10,85 n. 1 , 168, 168 n. 3, 169

Takelot: p. 167, 169, 169 n. 2, 
170 n. $3,171,176,177,177$ n. 1 Tanepherôs: p. 20, 199 n. 2, 207, 210, 211, 211 n. 3, 212, 212 n. 4, 213,214

Taoui: p. 130, 134

Taperet: p. 170

Tefnakht, roi: p. 172, 186

Teos, roi: p. 186

Teos, gp: p. 16 n. 12, 22, 69, 85, $102,103,168$ n. $6,180,181$, $182,183,184,185,185$ n. 2 , 186, 187, 188, 189

Tesbastetperet: p. 169

Teti, roi: p. 18, 43, 44 n. 4, 52, $65,112,113,115,116$

Thoutmôsis Ier, roi: p. 127

Thoutmôsis II, roi: p. 127

Thoutmôsis III, roi: p. 73, 75, 77, $94,127,128,129,129$ n. 3,152 n. 4

Thoutmôsis IV, roi: p. 77, 127, 128, 129, 132 n. 1

Thoutmôsis, prince et gp: p. 14, 15,16 n. $2,46,47,50$ n. 1,73 , 77,77 n. 4,80 n. $4,84,86,132$, $133,134,134$ n. 1,135 n. 3 , 136-37 n. 4

Thoutmôsis: p. 29, 76, 77 n. 4 , 130

Thoutankhamon, roi: p. 80,80 n. $2,96,132,138$

Tryphaena, femme de Ptolémée

Neos Dionysos: p. 192, 192 n. 3

Ty = Ptahemhat: p. 94, 96, 140

(...)enchenet (?): p. 94

(...)ka: p. 107 


\section{LISTES ALPHABETIQUES}

\section{DES NOMS DE "GRANDS DES CHEFS DES ARTISANS"}

(Les chiffres renvoient aux inscriptions)

I. Grands Prêtres dont l'existence est certaine

\begin{tabular}{|c|c|c|}
\hline 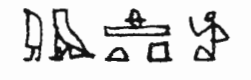 & $' l i-m-h t p$ & 200,$22 ; 201,12-13 ; 203$ \\
\hline h4"s & 'Ty-iry & 156. \\
\hline o t & ${ }^{\prime} T^{c} h-m s-m n(w)$ & 186; 187,3,27. \\
\hline & ${ }^{2} I b b i$ & $19 ; 20$ \\
\hline$B \approx 44$ & 'Impy & 29. \\
\hline & ${ }^{c} n-m-h r$ & $\begin{array}{l}192 ; 193,10 ; 194,11 ; 195 \\
197,2,12,19,21 ; 198,3 .\end{array}$ \\
\hline $\min x \& 8$ & ${ }^{c} n h . f-n-s \underline{h} m t$ & $\begin{array}{l}165,1,3,7 ; 166,10-11 ; 167,21 ; \\
169,1,7 ; 170,1 ; 183,8\end{array}$ \\
\hline to $\sum_{\infty} x^{9}$ & $W^{c} b-{ }^{c} 3$-iryt. $f$ & (ou titre ?) $\mathbf{1 9 7 , 1 2 .}$ \\
\hline Bakenrenef & $B 3 k-n-r n . f$ & $183 a$. \\
\hline 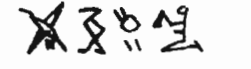 & $P 3 w-p 3 w$ & $163 ; 164 ; 182,14 ; 183,11$ \\
\hline 98 & $\begin{array}{l}\text { P3y.f-t3w-(m- } \\
\text { P3-hm-ntr }\end{array}$ & $\begin{array}{l}177,8,14 ; 181 ; 181 a . \\
69,11 ; 89,8 ; 94 \text { à } 102 ; 104,7 .\end{array}$ \\
\hline
\end{tabular}


믄 4 m 2 d $\quad P 3-\dot{s} r(i)-(n)-i m n \quad$ 203,8; 204,7.

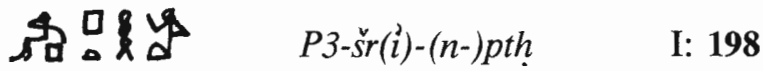

II: $200 ; 201,6-15 ; 202,4 ; 203,7$; 203a,2.

Ka-dån P3-díst

177 à 180.

$\square \Lambda \backsim 0 \quad P 3-d i-b 3 s^{\prime} t t$

I: 190,6 .

II: $199 ; 200,15 ; 201,7 ; 202,2,4$.

III: 200,22; 201,12-13; 203; $203 a$.

$\Delta 8=-\frac{0}{\Delta 1} \quad$ Pth-m-h3t

73.

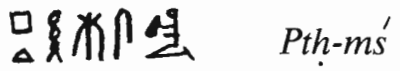

I: 36.

II: $37 ; 38 ; 39,2,3 ; 40$ à $58 ; 59$ à $61(?)$.

III: 38; 59 à 61 (?); 67 à 69.

IV: 155.

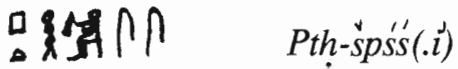

I: $3 ; 4 ; 6(?) ; 7$.

II: $5 ; 6(?) ; 7$ (?).

III: 11.

IV: 21 à 25.

-Impy 29.

口\&

79 à 81 .

$\pm \operatorname{\square q} \quad \operatorname{Mr}(y)-n(y)-p t h$

175.

$\rightarrow \square 8$

$N b(. i) p w$

31,2-4.

$\multimap \infty 9$ 내후새 $N b-m h y t$

82.

Ob $N f r-i b-r^{c}-m n(w)$

186,7 .

otw $\quad$ of $-f-r^{c}-{ }^{c} n h(w)$

8. 
然里

$\rightarrow$ t

Nekao-men

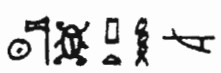

의

ig 9 兽果

프 49 웡

织员包

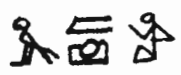

久이엉

3ㄴ영

88

是乐果
Nfr-rmpt

$N s-k d$

$N(y)-k 3-w(i)-m n(w)$

$N \underline{t r}(i)-h p r-r^{c}-m r(y)$-pth 164.

$R^{c}-n f r(w)$

$R^{c}-h t p(w)$

Hy

$H^{c} p(i)-d f(3 . i)$

Hr-m-3ht

$H r-s 3-s t$

$H r^{\prime}$

$H k 3-i r(w ?)-{ }^{c} 3$

$H^{c}(y)-m-w 3 s t$

०8रण

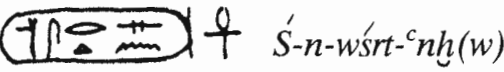

节倠子 St

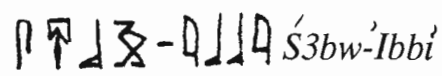

丑」る己
147 à $154 ; 182,17$.

$190 ; 191,2,9 ; 192,11$.

$188 \mathrm{a}$.

9; 10.

103 à $105 ; 102,3$ (?).

106.

30.

$194 ; 195 ; 196,3 ; 197 ; 198,3$.

I: $165 ; 182,13$ (?); 183,10-11 (?).

II: $178,6-7 ; 179,6,18$.

I: $86 ; 87$.

II: 88.

I ou II: 89 à 92.

186,8 .

I: 85 à 87.

II: 107 à 146.

187,$25 ; 188,3-4$.

33 à 35.

$14 ; 15$.

16 à 20.

$12 ; 13$. 


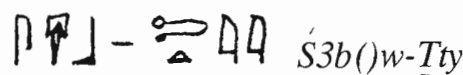

是提

op + f

of $\frac{A}{5}$ 负

패 4

梠

DDA

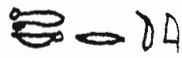

$\because 44$

殒口更里

慗的

的里

Sn-nfr(w)

Ssink

Ty

$\underline{T} k r t$

Tty

$D d y$

Dhwty-mś
$S h t p-i b-r^{c}-{ }^{c} n h(w)-n d m \quad 31,1,2 ; 32,1 ; 182,50(?)$.

$\stackrel{\prime}{S} h t p-i^{i} b-r^{c}-s r(i)$

Sd-sw-nfr-tm

$D(d)-h r$
26; 27 (?); 28 (?).

$83 ; 84 ; 98,6$.

31,4 .

$173 ; 174 ; 177,6,13$.

166 à $169 ; 170,2 ; 171,1 ; 183,8$.

72 à $75 ; 182,23$ (?).

$176 ; 177,4,5,12,15 ; 178,4-5,17$.

26 à 28.

102,2 .

62 à $65 ; 66(?)$.

193.

II. Grands Prêtres cités seulement dans les généalogies

$f \circ \sum_{11}^{v_{11}} n h-n w b-k 3 w-r^{c} \quad 182,48$.

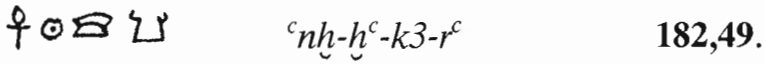

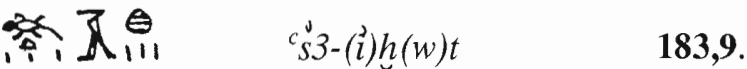

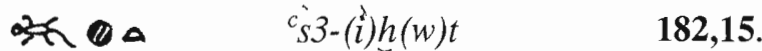

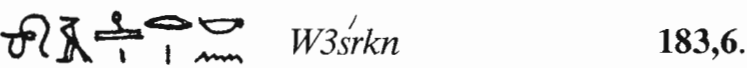

$[8] \oplus \infty \quad W(?)-(i) h(w) t \quad 182,46$. 


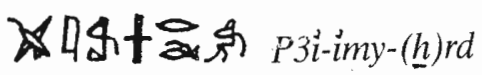

m $\nVdash$ ? Pn-p3-nb.s' (?)

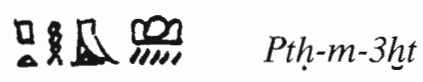

口马्य Pth-m-3ht

$\Delta \& \Leftarrow \bigoplus \quad P t h-m-h b$

Mnt

$3 \approx \quad \operatorname{Mr}(y)-w r$

99 Ntrwy-htp

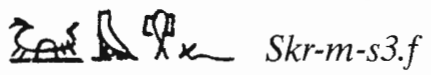

HA口? Sr(?)-mt(?)

IIII $\Delta(\Delta i c) \stackrel{v}{S}$ Sink

$\infty$

mirr" ?
182,32 .

$182,27$.

$182,16$.

182,18 .

182,58 .

182,34 .

182,26 .

$182,21$.

182,22 .

182,43 .

183,7 .

183,10.

$182,19$. 



\section{EXPLICATIONS DES PLANCHES I ET II}

(L'étude des insignes pectoraux est faite au $\$ 9$ ci-dessus).

Pl. I, fig. 1. Insigne pectoral et douze cordons à pendeloques de Khabaouseker. Ancien Empire. Bas-relief. Musée du Caire. D’après M.A. MURRAY, Saqqara Mastabas, II, pl. I. Cette représentation de Khabaouseker est sur la paroi gauche de sa chapelle; sur la paroi droite, l'insigne rigide est dans l'autre sens, avec la tête de l'animal se profilant sur l'épaule droite.

Mêmes ornements chez le grand prêtre Ptahmès III, fils de Menkheper. XVIIIème dynastie. Ronde-bosse (Ptahmès a la position accroupie des "statues cubiques" mais ses membres sont bien marqués). Florence 1790. D'après description de SCHIAPARELLI, Catal. Firenze, p. 197-198.

Pl. I, fig. 2. Rênefer (grand prêtre memphite). Ancien Empire. Rondebosse, peinte. Caire 19. D'après notre relevé; cf. aussi photo dans BORCHARDT, Statuen, I, pl. 5. Le fragment de pectoral visible est dessiné au trait à l'encre rouge (première ébauche); une peinture blanchâtre marque encore le "collier large"; la peau est ocre.

PI. I, fig. 3. Sabou-Tjéti (grand prêtre memphite). Ancien Empire. Basrelief. Caire 1756. Notre relevé. La ligne des épaules est ininterrompue; les parties du pectoral qui la dépassent sont sculptées sur le champ et paraissent ainsi coupées du reste du pectoral.

PI. I, fig. 4. Nebpou (grand prêtre memphite). Moyen Empire. Rondebosse. Louvre A 47. Notre relevé; cf. aussi dessin, avec une inexactitude, d'ERMAN, Aus dem Grabe ..., dans $\ddot{A} . Z$., 33 (1895), p. 22, fig. c. Louvre A 47 représente aussi le père de Nebpou, le grand prêtre Sehetepibrêankh-nedjem, avec un insigne identique à celui de son fils. 
Pl. I, fig. 5 ( = Pl. II, fig. 5, a-b). (Nom et partie des titres détruits). Fin du Moyen Empire (?). Ronde-bosse. Caire 14-6-24-12. Notre relevé. Le "collier" fait le tour du cou du personnage. Ce monument nous a été obligeamment signalé par M. Leibovitch.

PI. I, fig. 6 (= Pl. II, fig. 6). Méryptah (majordome du temple memphite de Nebmaâtrê). XVIIIème dynastie. Haut-relief. Leyde V 14. D'après photo de BOESER, Ägypt. Sammlung ... Leiden, tome 6, pl. 15. Leyde V 14 représente également les grands prêtres Ptahmès II fils du vizir Thoutmôsis et Ptahmès III fils de Menkheper ornés comme Méryptah.

Pl. I, fig. 7. (Nom et titres détruits). XVIIIème dynastie (?). Ronde-bosse (fragment). Caire (?). D’après M.A. MURRAY, Saqqara Mastabas, I, pl. 36,3 .

PI. I, fig. 8. (Nom et titres détruits). XVIIIème dynastie (?). Ronde-bosse (fragment). Caire 870. D'après BORCHARDT, Statuen, III, p. 131.

Pl. I, fig. 9. (Nom détruit) (grand prêtre memphite, inscription 78). XVIIIème dynastie (?). Ronde-bosse (fragment). Caire 852. D'après BORCHARDT, Statuen, III, p. 122.

PI. I, fig. 10. (Ni nom ni titres). XVIIIème dynastie (?). Ronde-bosse (fragment placé autrefois devant les genoux d'une statue plus grande (dieu ?) dont les mains subsistent sur la tête du fragment). Grenoble. D'après photo d'A. MORET, dans Revue égyptologique, I (1919), pl. 5 opp. p. 168 (ibidem, p. 174, l'éditeur attribue ce monument à la XIXème dynastie; le dessin du pectoral nous fait proposer la XVIIIème).

PI. I, fig. 11 a. (Nom détruit) (grand prêtre memphite ?). XVIIIème dynastie. Bas-relief. Berlin 12410. D'après dessin d'ERMAN, Aus dem Grabe ..., dans Ä.Z., 33 (1895), p. 23. Les parties dépassant l'épaule n'apparaissent pas sur la photo (ibidem, pl. II b), sans doute parce qu'elles sont gravées sur la tranche supérieure du creux qui dessine les épaules; ce creux est profond.

Même ornement chez le grand prêtre Peftjaouaouibastet. XXIIème dynastie. Berlin 11637. Statuette funéraire. D'après description dans Königl. Museen zu Berlin, Ausführliches Verzeichnis der ägypt. Altertümer (1894), p. 187. Obligeamment communiqué par M. G. Posener. 
Pl. I, fig. 11 b. (même monument que 11 a). Signe hiéroglyphique. Cf. inscription 71,7 .

PI. I, fig. 12. Hori I (grand prêtre memphite). XIXème dynastie. Basrelief (pilier à base carrée). Caire Journal 43271. D'après dessin dans QUIBELL, Saqqara, 4, pl. 70,3.

Même ornement chez le grand prêtre et prince Khamouas. XIXème dynastie. Bas-relief (paroi). Louvre. D'après description de $\mathrm{CH}$. BOREUX, Antiquités égypt. II, p. 481.

Même ornement chez le grand prêtre Paoupaou. XXIème dynastie. Bas-relief (linteau). Copenhague. D'après dessin de PETRIE, Memphis II, pl. 24, haut.

PI. I, fig. 13. Hori II (grand prêtre memphite). XIXème dynastie. Basrelief (colonne). Caire 20-1-25-4. Notre relevé. Les parties dépassant les épaules sont gravées peu profondément dans le champ non-levé.

Pl. I, fig. 14 (= PI. II, fig. 14). Pahemneter (grand prêtre memphite). XIXème dynastie. Ronde-bosse. Louvre A 72. Notre relevé; cf. aussi dessin d'ERMAN, Aus dem Grabe ..., dans Ä.Z., 33 (1895), p. 23, fig. g. Les extrémités supérieures sont cachées par la tresse de cheveux et la peau de félin.

Pl. I, fig. 15. Khamouas (prince et grand prêtre memphite). XIXème dynastie. Bas-relief (sur demi-tambour de colonne). Caire 17-11-24-4. Notre relevé. 


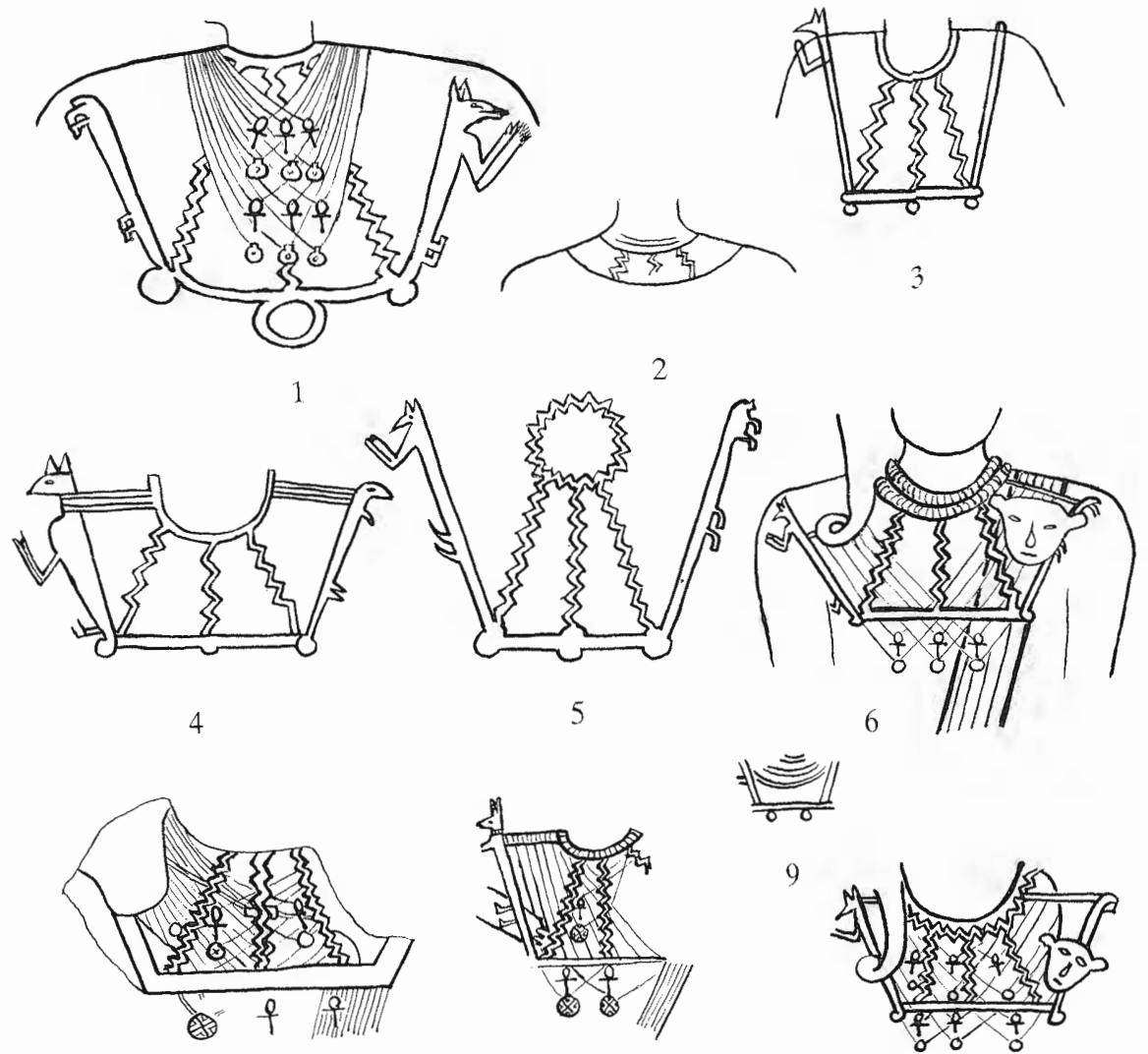

8
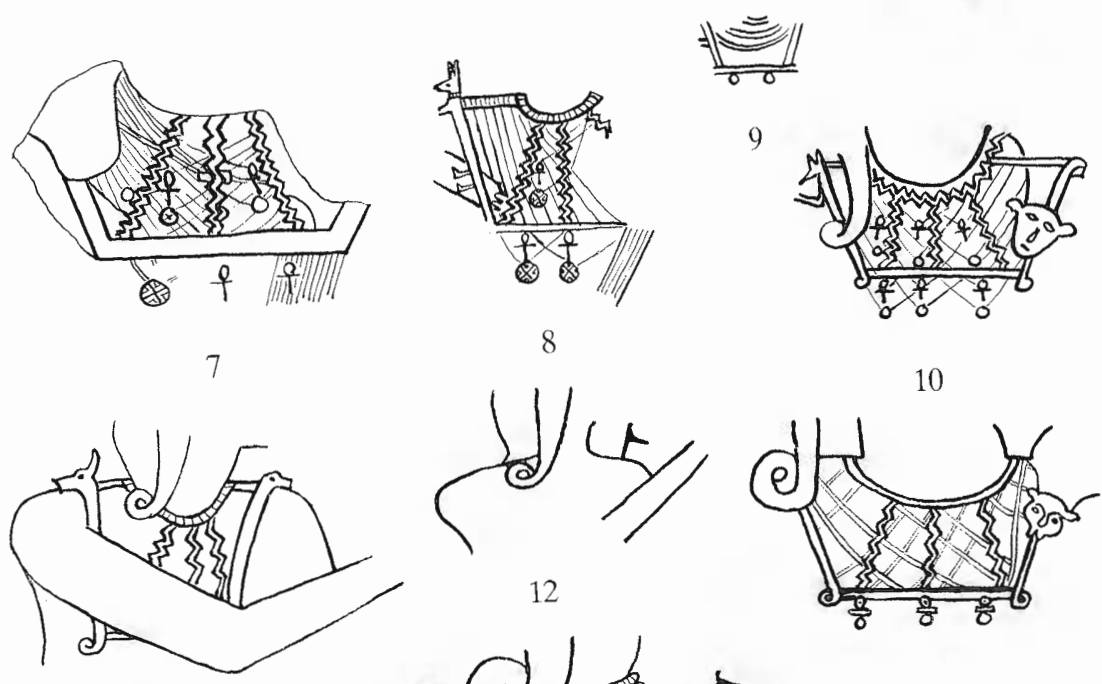

10
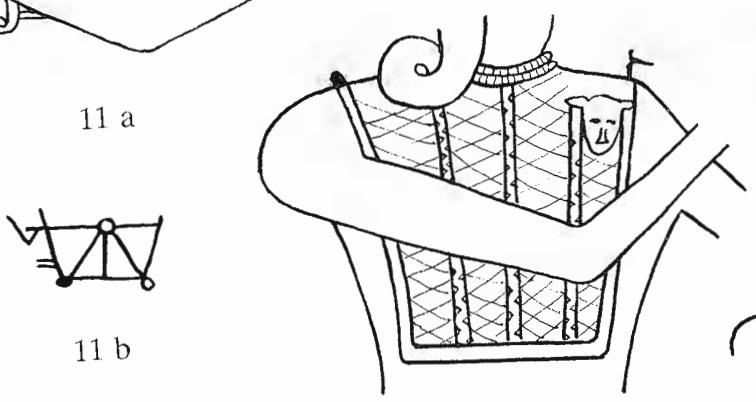

14

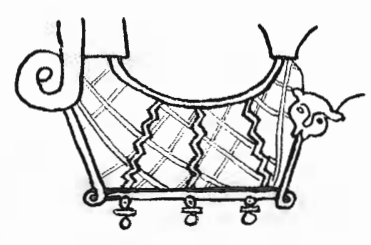



PLANCHE II

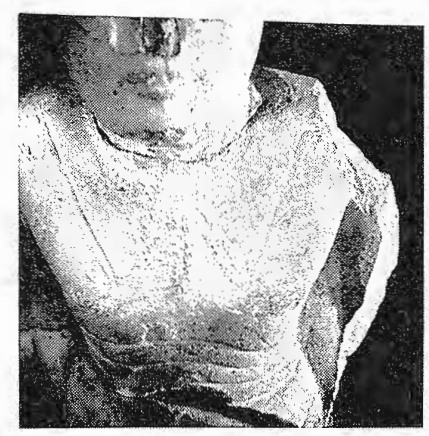

5

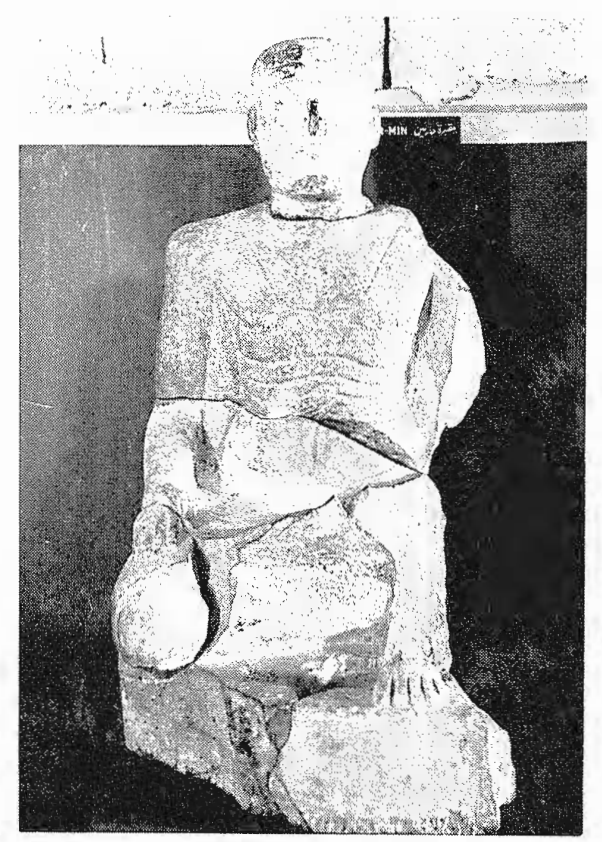

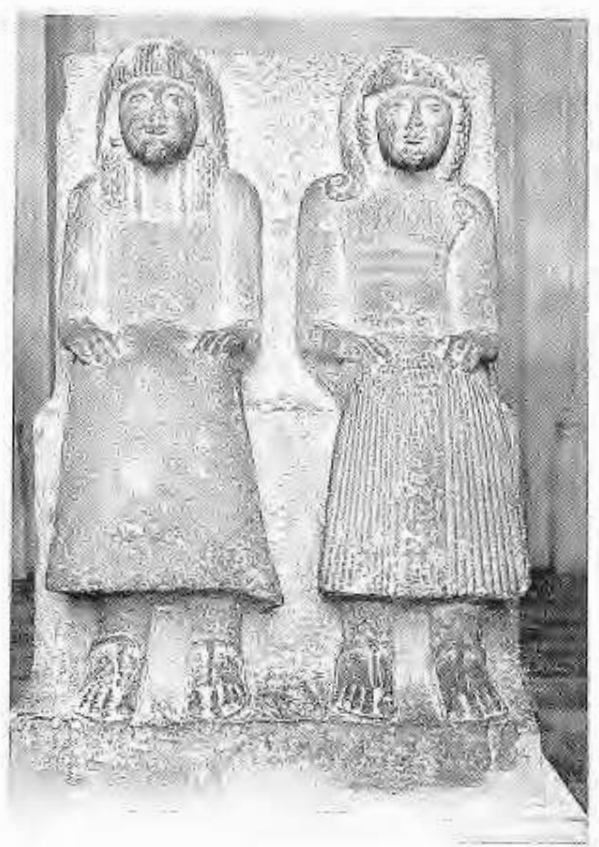

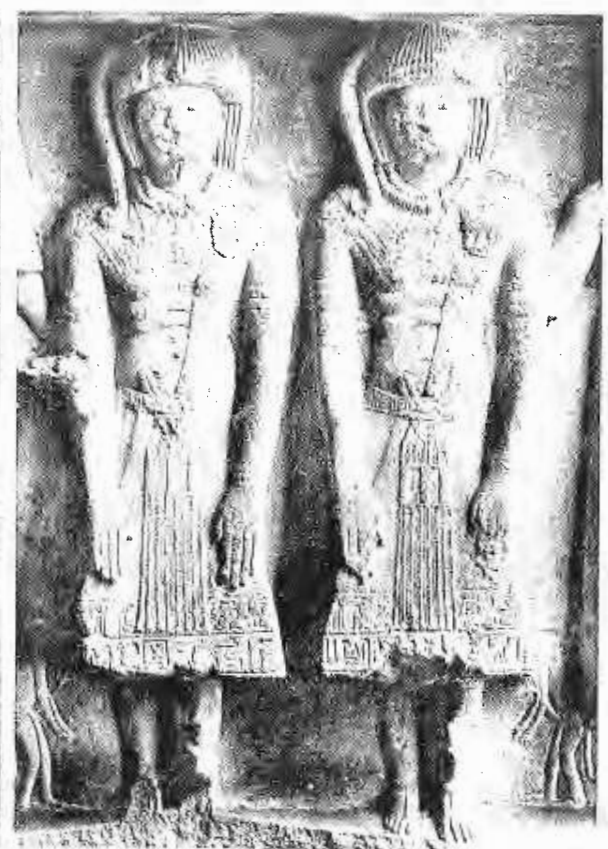


ORBIS BIBLICUS ET ORIENTALIS

Bd. 1 OTTO RICKENBACHER: Weisheitsperikopen bei Ben Sira. X-214-15* Seiten. 1973. Vergriffen.

Bd. 2 FRANZ SCHNIDER: Jesus der Prophet. 298 Seiten. 1973. Vergriffen.

Bd. 3 PAUL ZINGG: Das Wachsen der Kirche. Beiträge zur Frage der lukanischen Redaktion und Theologie. 345 Seiten. 1974. Vergriffen.

Bd. 4 KARL JAROS: Die Stellung des Elobisten zur kanaanäischen Religion. 294 Seiten, 12 Abbildungen. 1982. 2. verbesserte und überarbeitete Auflage.

Bd. 5 OTHMAR KEEL: Wirkmächtige Siegeszeichen im Alten Testament. Ikonographische Studien zu Jos 8, 18-26; Ex 17,8-13; 2 Kön 13, 14-19 und 1 Kön 22, 11. 232 Seiten, 78 Abbildungen. 1974. Vergriffen.

Bd. 6 VITUS HUONDER: Israel Sobn Gottes. Zur Deutung eines alttestamentlichen Themas in der jüdischen Exegese des Mittelalters. 231 Seiten. 1975.

Bd. 7 RAINER SCHMITT: Exodus und Passa. Ibr Zusammenbang im Alten Testament. 124 Seiten. 1982. 2. neubearbeitete Auflage.

Bd. 8 ADRIAN SCHENKER: Hexaplarische Psalmenbruchstücke. Die hexaplarischen Psalmenfragmente der Handschriften Vaticanus graecus 752 und Canonicianus graecus 62. Einleitung, Ausgabe, Erläuterung. XXVIII-446 Seiten. 1975.

Bd. 9 BEAT ZUBER: Vier Studien zu den Ursprüngen Israels, Die Sinaifrage und Probleme der Volks- und Traditionsbildung. 152 Seiten. 1976. Vergriffen.

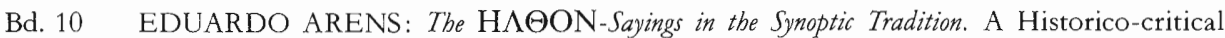
Investigation. 370 Seiten. 1976.

Bd. 11 KARL JAROS : Sichem. Eine archäologische und religionsgeschichtliche Studie, mit besonderer Berücksichtigung von Jos 24. 280 Seiten, 193 Abbildungen. 1976.

Bd. 11 a KARL JAROS/BRIGITTE DECKERT: Studien zur Sichem-Area. 81 Seiten, 23 Abbildungen. 1977.

Bd. 12 WALTER BÜHLMANN: Vom rechten Reden und Schweigen. Studien zu Proverbien $10-31$. 371 Seiten. 1976. Vergriffen.

Bd. 13 IVO MEYER: Jeremia und die falschen Propheten. 155 Seiten. 1977. Vergriffen.

Bd. 14 OTHMAR KEEL: Vögel als Boten. Studien zu Ps 68, 12-14, Gen 8, 6-12, Koh 10, 20 und dem Aussenden von Botenvögeln in Ägypten. - Mit einem Beitrag von Urs Winter zu Ps 56, 1 und zur Ikonographie der Göttin mit der Taube. 164 Seiten, 44 Abbildungen. 1977. Vergriffen.

Bd. 15 MARIE-LOUISE GUBLER: Die frühesten Deutungen des Todes Jesu. Eine motivgeschichtliche Darstellung aufgrund der neueren exegetischen Forschung. XVI-424 Seiten. 1977. Vergriffen.

Bd. 16 JEAN ZUMSTEIN: La condition du croyant dans l'Evangile selon Mattbieu. 467 pages. 1977. Epuisé.

Bd. 17 FRANZ SCHNIDER: Die verlorenen Söbne. Strukturanalytische und historisch-kritische Untersuchungen zu Lk 15. 105 Seiten. 1977.

Bd. 18 HEINRICH VALENTIN: Aaron. Eine Studie zur vor-priesterschriftlichen Aaron-Überlieferung. VIII-441 Seiten. 1978. 
Bd. 19 MASSÉO CALOZ: Etude sur la LXX origénienne du Psautier. Les relations entre les leçons des Psaumes du Manuscrit Coislin 44, les Fragments des Hexaples et le texte du Psautier Gallican. 480 pages. 1978 .

Bd. 20 RAPHAEL GIVEON: The Impact of Egypt on Canaan. Iconographical and Related Studies. 156 Seiten, 73 Abbildungen. 1978.

Bd. 21 DOMINIQUE BAR'THÉLEMY: Etudes d'bistoire du texte de l'Ancien Testament. XXV419 pages. 1978. Epuisé.

Bd. 22/1 CESLAS SPICQ: Notes de Lexicograpbie néo-testamentaire. 'Tome I: p. 1-524. 1978. Epuisé.

Bd. 22/2 CESLAS SPICQ: Notes de Lexicograpbie néo-testamentaire. 'Tome II: p. 525-980. 1978. Epuisé.

Bd. 22/3 CESLAS SPICQ: Notes de Lexicograpbie néo-testamentaire. Supplément. 698 pages. 1982.

Bd. 23 BRIAN M. NOLAN: The Royal Son of God. The Christology of Matthew 1-2 in the Setting of the Gospel. 282 Seiten. 1979. Out of print.

Bd. 24 KLAUS KIESOW: Exodustexte im Jesajabuch. Literarkritische und motivgeschichtliche Analysen. 221 Seiten. 1979. Vergriffen.

Bd. 25/1 MICHAEL LAT'TKE: Die Oden Salomos in ibrer Bedeutung für Neues Testament und Gnosis. Band I. Ausführliche Handschriftenbeschreibung. Edition mit deutscher Parallel-Übersetzung. Hermeneutischer Anhang zur gnostischen Interpretation der Oden Salomos in der Pistis Sophia. XI-237 Seiten. 1979.

Bd. 25/1a MICHAEL LATTKE: Die Oden Salomos in ibrer Bedeutung für Neues Testament und Gnosis. Band Ia. Der syrische Text der Edition in Estrangela Faksimile des griechischen Papyrus Bodmer XI. 68 Seiten. 1980.

Bd. 25/2 MICHAEL LATTKE: Die Oden Salomos in ihrer Bedeutung für Neues Testament und Gnosis. Band II. Vollständige Wortkonkordanz zur handschriftlichen, griechischen, koptischen, lateinischen und syrischen Uberlieferung der Oden Salomos. Mit einem Faksimile des Kodex N. XVI-201 Seiten. 1979.

Bd. 25/3 MICHAEL LATTKE: Die Oden Salomos in ibrer Bedeutung für Neues Testament und Gnosis. Band III. XXXIV-478 Seiten. 1986.

Bd. 26 MAX KÜCHLER: Frübjüdische Weisheitstraditionen. Zum Fortgang weisheitlichen Denkens im Bereich des frühjüdischen Jahweglaubens. 703 Seiten. 1979. Vergriffen.

Bd. 27 JOSEF M. OESCH: Petucha und Setuma. Untersuchungen zu einer überlieferten Gliederung im hebräischen Text des Alten Testaments. XX-392-37* Seiten. 1979.

Bd. 28 ERIK HORNUNG / OTHMAR KEEL (Herausgeber): Studien zu altägyptischen Lebenslebren. 394 Seiten. 1979.

Bd. 29 HERMANN ALEXANDER SCHLÖGL: Der Gott Tatenen. Nach Texten und Bildern des Neuen Reiches. 216 Seiten, 14 Abbildungen. 1980.

Bd. 30 JOHANN JAKOB STAMM : Beiträge zur Hebräischen und Altorientalischen Namenkunde. XVI264 Seiten. 1980.

Bd. 31 HELMUT UTZSCHNEIDER: Hosea - Prophet vor dem Ende. Zum Verhältnis von Geschichte und Institution in der alttestamentlichen Prophetie. 260 Seiten. 1980.

Bd. 32 PETER WEIMAR: Die Berufung des Mose. Literaturwissenschaftliche Analyse von Exodus 2, 23-5, 5. 402 Seiten. 1980. 
Bd. 33 OTHMAR KEEL: Das Böcklein in der Milch seiner Mutter und Verwandtes. Im Lichte eines altorientalischen Bildmotivs. 163 Seiten, 141 Abbildungen. 1980.

Bd. 34 PIERRE AUFFRET: Hymnes d'Egypte et d'Israël. Etudes de structures littéraires. 316 pages, 1 illustration. 1981.

Bd. 35 ARIE VAN DER KOOIJ: Die alten Textzengen des Jesajabuches. Ein Beitrag zur Textgeschichte des Alten Testaments. 388 Seiten. 1981.

Bd. 36 CARMEL McCARTHY: The Tiqqune Sopherim and Other Theological Corrections in the Masoretic Text of the Old Testament. 280 Seiten. 1981.

Bd. 37 BARBARA L. BEGELSBACHER-FISCHER: Untersucbungen zur Göttervelt des Alten Reiches im Spiegel der Privatgräber der IV. und V. Dynastie. 336 Seiten. 1981.

Bd. 38 MÉLANGES DOMINIQUE BARTHÉLEMY. Etudes bibliques offertes à l'occasion de son $60^{e}$ anniversaire. Edités par Pierre Casetti, Othmar Keel et Adrian Schenker.

724 pages, 31 illustrations. 1981.

Bd. 39 ANDRÉ LEMAIRE: Les écoles et la formation de la Bible dans l'ancien Israël. 142 pages, 14 illustrations. 1981.

Bd. 40 JOSEPH HENNINGER: Arabica Sacra. Aufsätze zur Religionsgeschichte Arabiens und seiner Randgebiete. Contributions à l'histoire religieuse de l'Arabie et de ses régions limitrophes. 347 Seiten. 1981.

Bd. 41 DANIEL VON ALLMEN: La famille de Dieu. La symbolique familiale dans le paulinisme. LXVII-330 pages, 27 planches. 1981.

Bd. 42 ADRIAN SCHENKER: Der Mäcbtige im Scbmelzofen des Mitleids. Eine Interpretation von 2 Sam 24. 92 Seiten. 1982.

Bd. 43 PAUl DESELAERS: Das Buch Tobit. Studien zu seiner Entstehung, Komposition und Theologie. 532 Seiten + Übersetzung 16 Seiten. 1982.

Bd. 44 PIERRE CASETTI: Gibt es ein Leben vor dem Tod? Eine Auslegung von Psalm 49. 315 Seiten. 1982.

Bd. 45 FRANK-LOTHAR HOSSFELD: Der Dekalog. Seine späten Fassungen, die originale Komposition und seine Vorstufen. 308 Seiten. 1982. Vergriffen.

Bd. 46 ERIK HORNUNG: Der ägyptische Mythos von der Himmelskuh. Eine Ätiologie des Unvollkommenen. Unter Mitarbeit von Andreas Brodbeck, Hermann Schlögl und Elisabeth Staehelin und mit einem Beitrag von Gerhard Fecht. XII-129 Seiten, 10 Abbildungen. 1991. 2. ergänzte Auflage.

Bd. 47 PIERRE CHERIX: Le Concept de Notre Grande Puissance (CG VI, 4). Texte, remarques philologiques, traduction et notes. XIV-95 pages. 1982.

Bd. 48 JAN ASSMANN/WALTER BURKERT/FRITZ STOLZ: Funktionen und Leistungen des Mythos. Drei altorientalische Beispiele. 118 Seiten, 17 Abbildungen. 1982. Vergriffen.

Bd. 49 PIERRE AUFFRET: La sagesse a bâti sa maison. Etudes de structures littéraires dans l'Ancien Testament et spécialement dans les psaumes. 580 pages. 1982.

Bd. 50/1 DOMINIQUE BARTHÉLEMY: Critique textuelle de l'Ancien Testament. 1. Josué, Juges, Ruth, Samuel, Rois, Chroniques, Esdras, Néhémie, Esther. Rapport final du Comité pour l'analyse textuelle de l'Ancien Testament hébreu institué par l'Alliance Biblique Universelle, établi en coopération avec Alexander R. Hulst $\dagger$, Norbert Lohfink, William D. McHardy, H. Peter Rüger, coéditeur, James A. Sanders, coéditeur. 812 pages. 1982. 
Bd. 50/2 DOMINIQUE BARTHÉLEMY: Critique textuelle de l'Ancien Testament. 2. Isaïe, Jérémie, Lamentations. Rapport final du Comité pour l'analyse textuelle de l'Ancien Testament hébreu institué par l'Alliance Biblique Universelle, établi en coopération avec Alexander R. Hulst †, Norbert Lohfink, William D. McHardy, H. Peter Rüger, coéditeur, James A. Sanders, coéditeur. 1112 pages. 1986.

Bd. 50/3 DOMINIQUE BARTHÉLEMY: Critique textuelle de l'Ancien Testament. Tome 3. Ézéchiel, Daniel et les 12 Prophètes. Rapport final du Comité pour l'analyse textuelle de l'Ancien Testament hébreu institué par l'Alliance Biblique Universelle, établi en coopération avec Alexander R. Hulst $f$, Norbert Lohfink, William D. McHardy, H. Peter Rüger, coéditeurf, James A. Sanders, coéditeur. 1424 pages. 1992.

Bd. 51 JAN ASSMANN: Re und Amun. Die Krise des polytheistischen Weltbilds im Ägypten der 18.-20. Dynastie. XII-309 Seiten. 1983.

Bd. 52 MIRIAM LICHTHEIM : Late Egyptian Wisdom Literature in the International Context. A Study of Demotic Instructions. X-240 Seiten. 1983.

Bd. 53 URS WINTER: Frau und Göttin. Exegetische und ikonographische Studien zum weiblichen Gottesbild im Alten Israel und in dessen Umwelt. XVIII-928 Seiten, 520 Abbildungen. 1987. 2. Auflage. Mit einem Nachwort zur 2. Auflage.

Bd. 54 PAUL MAIBERGER: Topographische und bistorische Untersucbungen zum Sinaiproblem. Worauf beruht die Identifizierung des Ğabal Mūsā mit dem Sinaì? 189 Seiten, 13 Tafeln. 1984.

Bd. 55 PETER FREI/KLAUS KOCH: Reicbsidee und Reichsorganisation in Perserreich. 119 Seiten, 17 Abbildungen. 1984. Vergriffen. Neuauflage in Vorbereitung

Bd. 56 HANS-PETER MÜLLER: Vergleich und Metapher im Hobenlied. 59 Seiten. 1984.

Bd. 57 STEPHEN PISANO: Additions or Omissions in the Books of Samuel. The Significant Pluses and Minuses in the Massoretic, LXX and Qumran Texts. XIV-295 Seiten. 1984.

Bd. 58 ODO CAMPONOVO: Königtum, Königsherrschaft und Reich Gottes in den Frübjüdischen Scbriften. XVI-492 Seiten. 1984.

Bd. 59 JAMES KARL HOFFMEIER: Sacred in the Vocabulary of Ancient Egypt. The Term DSR, with Special Reference to Dynasties I-XX. XXIV-281 Seiten, 24 Figures. 1985.

Bd. 60 CHRISTIAN HERRMANN: Formen für ägyptische Fayencen. Katalog der Sammlung des Biblischen Instituts der Universität Freiburg Schweiz und einer Privatsammlung. XXVIII-199 Seiten. 1985.

Bd. 61 HELMUT ENGEL: Die Susanna-Erzäblung. Einleitung, Übersetzung und Kommentar zum Septuaginta-Text und zur Theodition-Bearbeitung. 205 Seiten + Anhang 11 Seiten. 1985.

Bd. 62 ERNST KUTSCH: Die cbronologischen Daten des Ezecbielbuches. 82 Seiten. 1985.

Bd. 63 MANFRED HUTTER: Altorientalische Vorstellungen von der Untervelt. Literar- und religionsgeschichtliche Überlegungen zu «Nergal und Ereškigal». VIII-187 Seiten. 1985.

Bd. 64 HELGA WEIPPERT/KLAUS SEYBOLD/MANFRED WEIPPERT: Beiträge zur prophetischen Bildspracbe in Israel und Assyrien. IX-93 Seiten. 1985.

Bd. 65 ABDEL-AZIZ FAHMY SADEK: Contribution à l'étude de l'Amdouat. Les variantes tardives du Livre de l'Amdouat dans les papyrus du Musée du Caire. XVI-400 pages, 175 illustrations. 1985.

Bd. 66 HANS-PETER STÄHLI: Solare Elemente im Jahweglauben des Alten Testamentes. X-60 Seiten. 1985. 
Bd. 67 OTHMAR KEEL/SILVIA SCHROER: Studien zu den Stempelsiegeln aus Palästina/Israel. Band I. 115 Seiten, 103 Abbildungen. 1985.

Bd. 68 WALTER BEYERLIN: Weisheitliche Vergewisserung mit Bezug auf den Zionskult. Studien zum 125. Psalm. 96 Seiten. 1985.

Bd. 69 RAPHAEL VENTURA: Living in a City of the Dead. A Selection of Topographical and Administrative Terms in the Documents of the Theban Necropolis. XII-232 Seiten. 1986.

Bd. 70 CLEMENS LOCHER: Die Ehre einer Frau in Israel. Exegetische und rechtsvergleichende Studien zu Dtn 22, 13-21. XVIII-464 Seiten. 1986.

Bd. 71 HANS-PETER MATHYS: Liebe deinen Nächsten wie dich selbst. Untersuchungen zum alttestamentlichen Gebot der Nächstenliebe (Lev 19,18). XIV-196 Seiten. 1986. Vergriffen. Neuauflage in Vorbereitung.

FRIEDRICH ABITZ: Ramses Ill. in den Gräbern seiner Söbne. 156 Seiten, 31 Abbildungen. 1986.

Bd. 73 DOMINIQUE BARTHÉLEMY/DAVID W. GOODING/JOHAN LUST/EMANUEL TOV: The Story of David and Goliath. 160 Seiten. 1986.

Bd. 74 SILVIA SCHROER: In Israel gab es Bilder. Nachrichten von darstellender Kunst im Alten Testament. XVI-553 Seiten, 146 Abbildungen. 1987.

Bd. 75 ALAN R. SCHULMAN: Ceremonial Execution and Public Rewards. Some Historical Scenes on New Kingdom Private Stelae. 296 Seiten, 41 Abbildungen. 1987.

Bd. 76 JOŽE KRAŠOVEC: La justice $(S d q)$ de Dieu dans la Bible bébrä̈que et l'interprétation juive et chrétienne. 456 pages. 1988.

Bd. 77 HELMUT UTZSCHNEIDER: Das Heiligtum und das Gesetz. Studien zur Bedeutung der sinaitischen Heiligtumstexte (Ez 25-40; Lev 8-9). XIV-326 Seiten. 1988.

Bd. 78 BERNARD GOSSE: Isaie 13,1-14,23. Dans la tradition littéraire du livre d'Isaïe et dans la tradition des oracles contre les nations. 308 pages. 1988.

Bd. 79 INKE W. SCHUMACHER: Der Gott Sopdu - Der Herr der Fremdländer. XVI-364 Seiten, 6 Abbildungen. 1988.

Bd. 80 HELLMUT BRUNNER: Das börende Herz. Kleine Schriften zur Religions- und Geistesgeschichte Agyptens. Herausgegeben von Wolfgang Röllig. 449 Seiten, 55 Abbildungen. 1988.

Bd. 81 WALTER BEYERLIN: Bleilot, Brecheisen oder was sonst? Revision einer Amos-Vision. 68 Seiten. 1988.

Bd. 82 MANFRED HUTTER: Bebexung, Entsübnung und Heilung. Das Ritual der Tunnawiya für ein Königspaar aus mittelhethitischer Zeit (KBo XXI 1 - KUB IX 34 - KBo XXI 6). 186 Seiten. 1988.

Bd. 83 RAPHAEL GIVEON: Scarabs from Recent Excavations in Israel. 114 Seiten, 9 Tafeln. 1988.

Bd. 84 MIRIAM LICHTHEIM : Ancient Egyptian Autobiograpbies chiefly of the Middle Kingdom. A Study and an Anthology. 200 Seiten, 10 Seiten Abbildungen. 1988.

Bd. 85 ECKART OTTO: Rechtsgeschicbte der Redaktionen im Kodex Ešnunna und im "Bundesbuch». Eine redaktionsgeschichtliche und rechtsvergleichende Studie zu altbabylonischen und altisraelitischen Rechtsüberlieferungen. 220 Seiten. 1989.

Bd. 86 ANDRZEJ NIWINSKI: Studies on the Illustrated Theban Funerary Papyri of the 11th and 10th Centuries B.C. 488 Seiten, 80 Seiten Tafeln. 1989.

Bd. 87 LRSULA SEIDL: Die babylonischen Kudurru-Reliefs. Symbole mesopotamischer Gottheiten. 236 Seiten, 33 Tafeln und 2 Tabellen. 1989. 
Bd. 88 OTHMAR KEEL/HILDI KEEL-LEU/SILVIA SCHROER : Studien zu den Stempelsiegeln aus Palästina/Israel. Band II. 364 Seiten, 652 Abbildungen. 1989.

Bd. 89 FRIEDRICH ABITZ: Baugeschichte und Dekoration des Grabes Ramses' VI. 202 Seiten, 39 Abbildungen. 1989.

Bd. 90 JOSEPH HENNINGER SVD : Arabica varia. Aufsätze zur Kulturgeschichte Arabiens und seiner Randgebiete. Contributions à l'histoire culturelle de l'Arabie et de ses régions limitrophes. 504 Seiten. 1989.

Bd. 91 GEORG FISCHER : Jahwe unser Gott. Sprache, Aufbau und Erzähltechnik in der Berufung des Mose (Ex. 3-4). 276 Seiten. 1989.

Bd. 92 MARK A. O'BRIEN: The Deuteronomistic History Hypothesis: A Reassessment. 340 Seiten. 1989.

Bd. 93 WALTER BEYERLIN: Reflexe der Amosvisionen im Jeremiabuch. 120 Seiten. 1989.

Bd. 94 ENZO CORTESE: Josua 13-21. Ein priesterschriftlicher Abschnitt im deuteronomistischen Geschichtswerk. 136 Seiten. 1990.

Bd. 95 ERIK HORNUNG (Herausgeber): Zum Bild Ägyptens im Mittelalter und in der Renaissance. Comment se représente-t-on l'Egypte au Moyen Age et à la Renaissance. 268 Seiten. 1990.

Bd. 96 ANDRÉ WIESE: Zum Bild des Königs auf ägyptischen Siegelamuletten. 264 Seiten. 1990.

Bd. 97 WOLFGANG ZWICKEL: Räucherkult und Räuchergeräte. Exegetische und archäologische Studien zum Räucheropfer im Alten Testament. 372 Seiten. 1990.

Bd. 98 AARON SCHART: Mose und Israel im Konflikt. Eine redaktionsgeschichtliche Studie zu den Wüstenerzählungen. 296 Seiten. 1990.

Bd. 99 THOMAS RÖMER: Israels Väter. Untersuchungen zur Väterthematik im Deuteronomium und in der deuteronomistischen Tradition. 664 Seiten. 1990.

Bd. 100 OTHMAR KEEL/MENAKHEM SHUVAL/CHRISTOPH UEHLINGER: Studien zu den Stempelsiegeln aus Palästina/Israel. Band III. Die Frühe Eisenzeit. Ein Workshop. XIV-456 Seiten. XXII Tafeln. 1990.

Bd. 101 CHRISTOPH UEHLINGER: Weltreich und «eine Rede». Eine neue Deutung der sogenannten Turmbauerzählung (Gen 11,1-9). XVI-654 Seiten. 1990.

Bd. 102 BENJAMIN SASS: Studia Alphabetica. On the Origin and Early History of the Northwest Semitic, South Semitic and Greek Alphabets. X-120 Seiten. 16 Seiten Abbildungen. 2 Tabellen. 1991.

Bd. 103 ADRIAN SCHENKER: Text und Sinn im Alten Testament. Textgeschichtliche und bibeltheologische Studien. VIII-312 Seiten. 1991.

Bd. 104 DANIEL BODI: The Book of Ezekiel and the Poem of Erra. IV-332 Seiten. 1991.

Bd. 105 YUICHI OSUMI: Die Kompositionsgescbichte des Bundesbuches Exodus 20,22b-23,33. XII-284 Seiten. 1991.

Bd. 106 RUDOLF WERNER: Kleine Einfübrung ins Hieroglyphen-Luwische. XII-112 Seiten. 1991.

Bd. 107 THOMAS STAUBLI: Das Image der Nomaden im Alten Israel und in der Ikonograpbie seiner sesshaften Nacbbarn. XII-408 Seiten. Mit 145 Abb. und 3 Falttafeln. 1991.

Bd. 108 MOSHÉ ANBAR: Les tribus amurrites de Mari. VIII-256 Seiten. 1991.

Bd. 109 GÉRARD J. NORTON/STEPHEN PISANO (eds.): Tradition of the Text. Studies offered to Dominique Barthélemy in Celebration of his 70th Birthday. 336 Seiten. 1991.

Bd. 110 HILDI KEEL-LEU: Vorderasiatische Stempelsiegel. Die Sammlung des Biblischen Instituts der Universität Freiburg Schweiz. 180 Seiten. 24 Tafeln. 1992.

Bd. 111 NORBERT LOHFINK: Die Väter Israels im Deuteronomiun. Mit einer Stellungnahme von Thomas Römer. 152 Seiten. 1991. 
Bd. 112 EDMUND HERMSEN: Die zwei Wege des Jenseits. Das altägyptische Zweiwegebuch und seine Topographie. XII-282 Seiten, 1 mehrfarbige und 19 Schwarzweiss-Abbildungen. 1992.

Bd. 113 CHARLES MAYSTRE: Les grands prêtres de Ptab de Memphis. XIV-474 pages, 2 planches. 1992. 
Résumé

Considéré dans son ensemble, le présent livre est une recherche sur les grands prêtres du dieu égyptien Ptah. Une première partie comprend l'étude du pontificat memphite, une seconde présente l'histoire des pontifes memphites, tandis qu'une dernière partie donne les sources. Pour chacune d'elles, le lecteur rencontre d'abord le texte hiéroglyphique dans la grande majorité, puis la traduction en français et un commentaire. Les hiéroglyphes ont été dessinés par l'auteur. 